Tobias Just

\title{
Globalisierung und Ideologie
}




\section{Tobias Just}

\section{Globalisierung und Ideologie}

Die Arbeit untersucht, inwiefern es im Zuge fortschreitender Offnung der Güterund Faktormärkte für nationale Regierungen möglich ist bzw. möglich bleibt, der Wirtschaftspolitik einen ideologisch motivierten Stempel aufzudrücken. Dafür wird zunächst der Begriff der Ideologie für das ökonomische Analyseinstrumentarium zugänglich gemacht. Anschließend wird versucht, wirtschaftspolitisch unterscheidbare und für die empirische Analyse operationalisierbare Ideologien zu kennzeichnen. Dies bildet die Grundlage für den empirischen Teil, in dem geprüft wird, ob es stärkere ideologische Prägemöglichkeiten nationaler Regierungen in offenen oder geschlossenen Volkswirtschaften gibt.

Nach einer kaufmännischen Ausbildung beim Otto-Versand studierte Tobias Just von 1992 bis 1997 in Hamburg und Uppsala Volkswirtschaftslehre. Nach Abschluss des Studiums war er als wissenschaftlicher Mitarbeiter am Institut für Finanzwissenschaft der Universität der Bundeswehr tätig, wo er im Jahr 2001 promoviert wurde.Von 1998 bis 2001 war er gleichzeitig freiberuflicher Dozent für das Europa-Kolleg Hamburg und für das Haus Rissen. 
Globalisierung und Ideologie

Tobias Just - 978-3-631-75139-8

Downloaded from PubFactory at 01/11/2019 07:52:01AM

via free access 


\title{
SCHRIFTEN ZUR \\ WIRTSCHAFTSTHEORIE UND WIRTSCHAFTSPOLITIK
}

\author{
Herausgegeben von \\ Rolf Hasse, Jörn Kruse, Wolf Schäfer, Thomas Straubhaar \\ und Klaus W. Zimmermann
}

Band 21

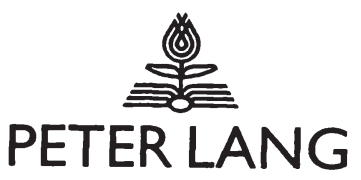

Frankfurt am Main - Berlin - Bern - Bruxelles - New York - Oxford - Wien 


\section{Tobias Just}

\section{Globalisierung und Ideologie Eine Analyse der Existenz und Persistenz von Partisaneffekten bei zunehmender Internationalisierung der Märkte}

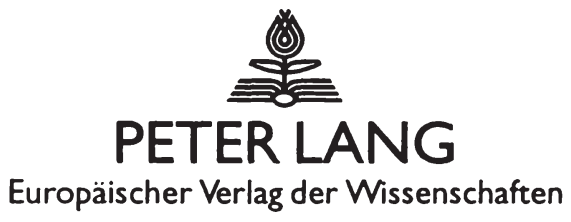




\section{Die Deutsche Bibliothek - CIP-Einheitsaufnahme}

Just, Tobias:

Globalisierung und Ideologie : Eine Analyse der Existenz und Persistenz von Partisaneffekten bei zunehmender Internationalisierung der Märkte / Tobias Just. - Frankfurt am Main ; Berlin ; Bern ; Bruxelles ; New York ; Oxford ; Wien : Lang, 2001

(Schriften zur Wirtschaftstheorie und Wirtschaftspolitik ;

Bd. 21)

Zugl.: Hamburg, Univ. der Bundeswehr Hamburg, Diss., 2001 ISBN3-631-37857-2

Open Access: The online version of this publication is published on www.peterlang.com and www.econstor.eu under the international Creative Commons License CC-BY 4.0. Learn more on how you can use and share this work: http://creativecommons. org/licenses/by/4.0.

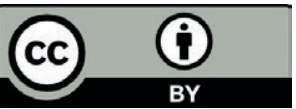

This book is available Open Access thanks to the kind support of ZBW - Leibniz-Informationszentrum Wirtschaft.

Gedruckt mit Unterstützung der Universität der Bundeswehr Hamburg.

Gedruckt auf alterungsbeständigem, säurefreiem Papier.

D705

ISSN 1433-1519

ISBN3-631-37857-2

ISBN 978-3-631-75139-8 (eBook)

(C) Peter Lang $\mathrm{GmbH}$

Europäischer Verlag der Wissenschaften

Frankfurt am Main 2001

Alle Rechte vorbehalten.

Das Werk einschließlich aller seiner Teile ist urheberrechtlich geschützt. Jede Verwertung außerhalb der engen Grenzen des

Urheberrechtsgesetzes ist ohne Zustimmung des Verlages unzulässig und strafbar. Das gilt insbesondere für Vervielfältigungen, Übersetzungen, Mikroverfilmungen und die Einspeicherung und Verarbeitung in elektronischen Systemen.

Printed in Germany 123467

www.peterlang.de 


\section{Vorwort}

Die vorliegende Arbeit entstand während meiner Zeit als wissenschaftlicher Mitarbeiter am Institut für Finanzwissenschaft an der Universität der Bundeswehr Hamburg. Ich möchte an dieser Stelle die Gelegenheit nutzen, allen denjenigen zu danken, die durch ihre rege fachliche und/oder freundschaftliche Anteilnahme an der Arbeit und mir halfen, die Dissertation fertigzustellen.

An erster Stelle möchte ich meinem Doktorvater Herrn Prof. Dr. Klaus W. Zimmermann dafür danken, dass er die Arbeit thematisch motivierte und durch konstruktive Kritik bereicherte. Insbesondere möchte ich ihm aber dafür danken, dass er eine angenehme Arbeitsatmosphäre schaffte, die mir nie die Freude an der Wissenschaft nahm. Des weiteren möchte ich auch meinem vorübergehenden Vorgesetzten Herrn Prof. Dr. Michael Carlberg danken, der mir zum einen genügend Freiräume für die Anfertigung der Arbeit ließ, zum anderen in zahlreichen Gesprächen manche Unklarheit der Argumentation beseitigte. Herr Prof. Dr. Wolf Schäfer übernahm nicht nur das Zweitgutachten, er nahm auch aktiv Anteil an der Arbeit, und ich bin froh, einige wichtige Aspekte mit ihm diskutiert zu haben.

Außerdem möchte ich ausdrücklich Herrn PD Dr. Michael Bräuninger danken, dessen ausgeprägtes Interesse und dessen kritische Zwischenfragen dafür sorgten, dass einige Passagen der Arbeit, v.a. im empirischen Teil, verbessert werden konnten. Herr PD Dr. Frank Decker erleichterte mir durch seine Kommentare und Diskussionen das Verständnis der politikwissenschaftlichen Literatur. Jedoch all diese Hilfe wäre nur halb so viel wert, hätten mir nicht Herr Prof. Dennis $P$. Quinn, Ph.D. und Herr Thomas R. Cusack, Ph.D. Zugang zu Teilen ihrer Datensätze verschafft. Bei den selbst erhobenen Daten leistete Frau Dipl. Kffr. Laurence Miraillès in der mühevollen Phase der Datenerfassung wertvolle Unterstützung. Ferner möchte ich meinem Kollegen Herrn Dipl. Vw. Eckhard Zirkmann für seine klärenden Diskussionen danken. Frau Dr. Antje M. Gerhold las weite Teile der Arbeit kritisch Korrektur. Ihr, meinen Eltern Christine und Hans-Günter Just sowie vielen Freunden, die immer wieder bemüht waren, mir zu beweisen, dass es ein Leben außerhalb der Universität gibt, ist es zu verdanken, dass ich die Promotionszeit nicht nur als arbeitsreichen Lebensabschnitt in Erinnerung behalten werde.

Schließlich möchte ich der Universität der Bundeswehr für die finanzielle Unterstützung bei der Drucklegung danken. 
Tobias Just - 978-3-631-75139-8

Downloaded from PubFactory at 01/11/2019 07:52:01AM

via free access 


\section{Inhaltsübersicht}

Abbildungsverzeichnis ........................................................................ 13

Tabellenverzeichnis ........................................................................... 14

Abkürzungsverzeichnis ....................................................................... 17

KAPITEL 1 EINLEITUNG ……………………………………......... 19

1.1 Forschungskontext und Problemstellung................................................. 19

1.2 Gang der Untersuchung ................................................................ 21

\section{KAPITEL 2 DAS IDEOLOGIEKONZEPT IN DER ÖKONOMISCHEN} THEORIE

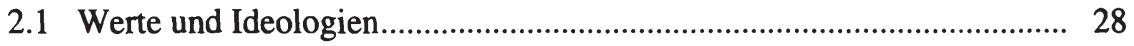

2.1.1 Definition des Wertbegriffs ..................................................... 28

2.1.2 Die Bildung von Werten ......................................................... 30

2.1.3 Ideologie als konsistentes Wertesystem ..................................... 32

2.1.4 Funktionen von Werten und Ideologien ...................................... 34

2.2 Ökonomische Theorie der Politik ........................................................... 36

2.2.1 Der homo oeconomicus ........................................................... 36

2.2.2 Die zentralen Akteure der Politik ............................................... 39

2.2.2.1 Die Wähler....................................................................... 40

2.2.2.2 Die Politiker..................................................................... 42

2.2.3 Konvergenz oder Divergenz der Wahlprogramme...................... 43

2.2.3.1 Die Medianwählerhypothese .......................................... 43

2.2.3.2 Langfristige Divergenz der Parteiprogramme .................. 45 
2.3 Notwendigkeit einer Berücksichtigung von Werten und Ideologien in der Ökonomie ................................................................................ 52

2.3.1 Individuelle Rationalität und kollektive Unvernunft.................. 52

2.3.2 Ist der Mensch tatsächlich ein homo oeconomicus? .................... 55

2.3.2.1 Die Überprüfung der Freifahrerhypothese ..................... 55

2.3.2.2 Das Wahlparadoxon ...................................................... 59

2.3.2.3 Der Politiker als Ideologe ............................................... 66

2.4 Werte und Ideologien in Rational Choice Modellen ............................... 69

2.4.1 Das Selbstinteresse und das Interesse am eigenen Selbst ........... 71

2.4.2 Konkurrierende individuelle Präferenzordnungen ....................... 75

2.4.3 Werte als Kapitalbestände des Menschen .................................. 81

2.4.3.1 Begrenzte Problemlösungskompetenz .......................... 82

2.4.3.2 Theorie des Humankapitals ........................................... 84

2.4.3.3 Werte und Humankapital............................................... 88

2.4.4 Zusammenfassung und kritische Würdigung der Theorien ......... 94

\section{KAPITEL 3 KONZEPTIONEN IDEOLOGISCHER WIRTSCHAFTSPOLITIK ................................................. 97}

3.1 Politisch relevante Konfliktlinien ......................................................... 97

3.2 Links und rechts - un clivage dépassé? .................................................. 101

3.2.1 Geschichtliche Entwicklung der räumlichen Parteiverortung...... 102

3.2.2 Was ist links und was ist rechts? .................................................. 103

3.2.3 Anzahl relevanter ideologischer Dimensionen........................... 110

3.2.3.1 Die Konfliktlinien Regimeunterstützung und Außenpolitik ........................................................ 110

3.2.3.2 Die Konfliktlinie Postmaterialismus ................................. 111

3.3 Operationalisierung des Links-Rechts-Schemas ................................... 115

3.3.1 Anforderungen an einen Links-Rechts-Indikator................... 116 


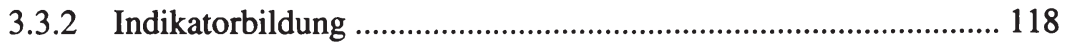

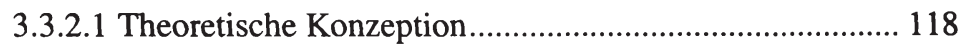

3.3.2.2 Praktische Umsetzung ................................................. 119

3.3.3 Bedarf es einer dynamischen Flexibilisierung des Index? ........... 122

3.4 Idealtypische linke und rechte Wirtschaftspolitik .................................. 123

3.4.1 Determinanten der personellen Einkommensverteilung .............. 123

3.4.2 Das wirtschaftspolitische Instrumentarium ................................. 128

3.4.3 Verteilungswirkungen einzelner prozesspolitischer Elemente .... 130

3.4.3.1 Verteilungswirkungen der Arbeitslosigkeit .................... 130

3.4.3.1.1 Die individuellen Kosten der Arbeitslosigkeit. 130

3.4.3.1.2 Die gesamtwirtschaftlichen Kosten der Arbeitslosigkeit ............................................... 133

3.4.3.1.3 Verteilungseffekte ............................................ 134

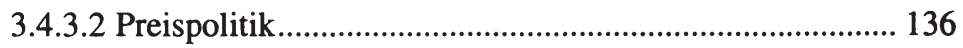

3.4.3.2.1 Die Kosten der Inflation................................. 136

3.4.3.2.2 Inflation und die personelle Einkommensverteilung....................................................... 138

3.4.3.3 Budgetpolitik ................................................................. 141

3.4.3.4 Zusammenfassung ........................................................ 145

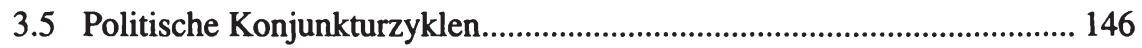

3.5.1 Überblick über den Stand der Konjunkturtheorie ....................... 146

3.5.2 Wiederwahlorientierte politische Akteure..................................... 151

3.5.2.1 Das Nordhaus / MacRae-Modell ....................................... 151

3.5.2.2 Rationale Erwartungen und wiederwahlorientierte

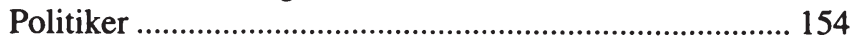

3.5.3 Ideologieorientierte politische Akteure ..................................... 158

3.5.3.1 Die Partisantheorie von Hibbs.......................................... 158

3.5.3.2 Die rationale Partisantheorie von Alesina. 
3.5.4 Ideologie- und wiederwahlorientierte Politiker............................ 164

3.5.5 Empirischer Befund politischer Konjunkturzyklen..................... 168

3.5.5.1 Wiederwahlorientierte Politiker ..................................... 169

3.5.5.2 Ideologisch gebundene Politiker .................................. 170

\section{KAPITEL 4 DEFINITION UND MESSUNG DES GRADS DER} GLOBALISIERUNG

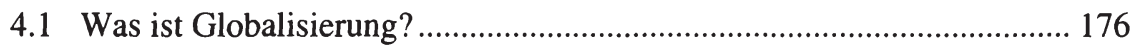

4.2 Begründung des Globalisierungsprozesses............................................ 177

4.2.1 Wirtschaftliche Komponenten der Globalisierung ....................... 178

4.2.2 Technologische Komponenten der Globalisierung ..................... 178

4.2.3 Politische und institutionelle Komponenten der Globalisierung.. 180

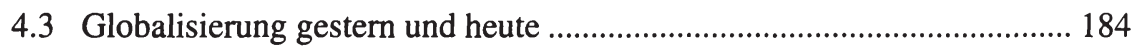

4.4 Race to the Bottom oder Race to the Top? ........................................... 188

4.5 Politische Ökonomie der Gütermarktliberalisierung .............................. 193

4.6 Globalisierungscluster .......................................................................... 201

4.6.1 Ökonomische Globalisierungsindikatoren ................................ 202

4.6.1.1 Umfang von Handels- und Kapitalströmen als Globalisierungsindikatoren ........................................... 202

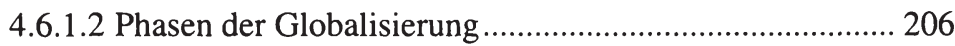

4.6.1.3 Ländercluster im Globalisierungsprozess ........................ 208

4.6.2 Politische Globalisierungscluster .............................................. 212

4.6.2.1 Indikatoren für Kapitalmarkt- und Handelsliberalisierung................................................... 213

4.6.2.2 Phasen im Globalisierungsprozess .................................. 216

4.6.2.3 Ländercluster im Globalisierungsprozess ....................... 217

4.6.3 Vergleich der politischen und ökonomischen

Clusterergebnisse 220 
KAPITEL 5 GLOBALISIERUNG UND NATIONALE

WIRTSCHAFTSPOLITIK - EMPIRIE .............................. 223

5.1 Handlungsspielräume ideologisch motivierter Arbeitsmarktpolitik ........ 224

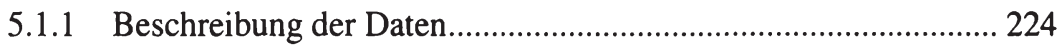

5.1.2 Traditionelle Partisantheorie........................................................ 226

5.1.2.1 Cluster gemäß den ökonomischen Globalisierungsindikatoren .......................................... 230

5.1.2.2 Cluster gemäß den politischen Globalisierungsindikatoren ........................................... 233

5.1.2.3 Zusammenfassung ........................................................ 237

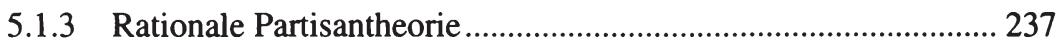

5.1.3.1 Cluster gemäß den ökonomischen Indikatoren ................ 240

5.1.3.2 Cluster gemäß den politischen Indikatoren ....................... 243

5.1.3.3 Zusammenfassung ....................................................... 246

5.2 Partisanpolitik, Inflation und Globalisierung ........................................ 247

5.2.1 Datenbeschreibung und Modellspezifikation .............................. 248

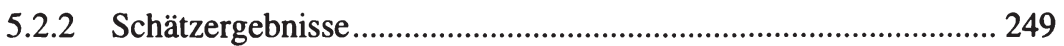

5.2.2.1 Cluster gemäß den ökonomischen Indikatoren ................ 250

5.2.2.2 Cluster gemäß den politischen Indikatoren....................... 253

5.3 Zusammenfassung der Ergebnisse bezüglich Inflation und

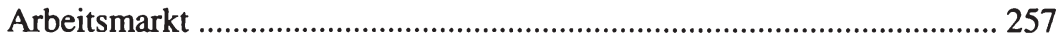

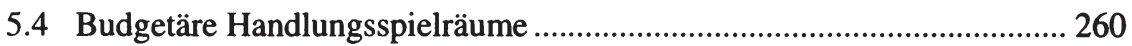

5.4.1 Methodische Vorbemerkungen und Beschreibung der Daten...... 261

5.4.2 Verteilungsziele als ideologisches Trennkriterium ....................... 262

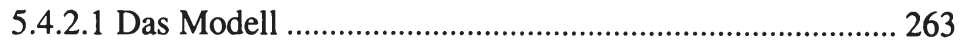

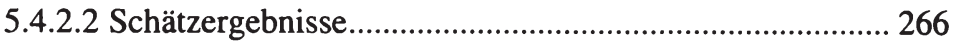

5.4.2.2.1 Cluster gemäß den ökonomischen Indikatoren ................................................. 270

5.4.2.2.2 Cluster gemäß deimpditischen3Indikatoßen?.... 274 
5.4.3 Progressivität als ideologisches Unterscheidungsmoment........... 277

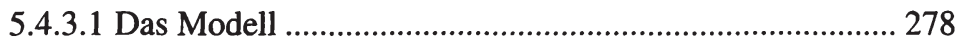

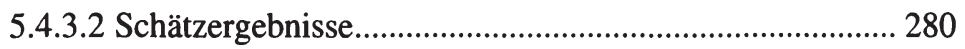

5.4.3.2.1 Cluster gemäß den ökonomischen Indikatoren ...................................................... 283

5.4.3.2.2 Cluster gemäß den politischen Indikatoren...... 286

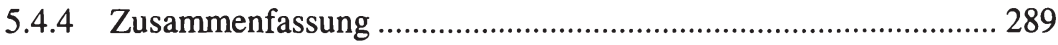

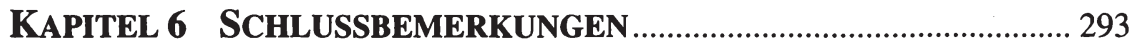

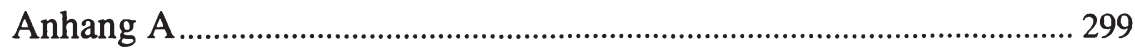

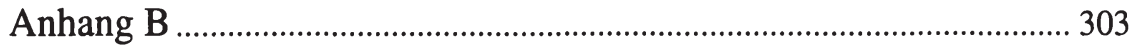

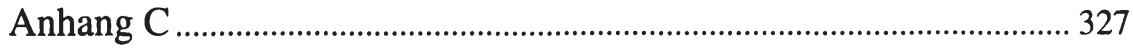

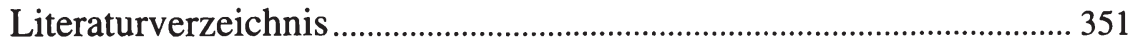




\section{Abbildungsverzeichnis}

Abbildung 2.1 : Modell zur (politischen) Sozialisation ................................ 32

Abbildung 2.2 : Die Medianwählerhypothese ............................................. 44

Abbildung 2.3 : Divergenz der Programme bei Parteiideologie und Unsicherheit ....................................................................... 50

Abbildung 2.4 : Verteilungsergebnis als Dimension im moral space .............. 73

Abbildung 2.5 : Das Fair Share Gleichgewicht und der Einkommenspfad .... 80

Abbildung 2.6 : Eine Erhöhung des Sozialkapitals führt zu höherem Nutzenniveau .................................................................. 90

Abbildung 2.7 : Eine Erhöhung des Sozialkapitals senkt das Nutzenniveau... 91 Abbildung 3.1 : Das Links-Rechts-Verortungsschema .................................. 109

Abbildung 3.2 : Parteienverortung in Klassen............................................ 117

Abbildung 3.3 : Positionierung der Parteien von 19 OECD-Staaten im Links-Rechts-Kontinuum .................................................. 121

Abbildung 3.4 : Eurobarometer-Befragung und Castles-Mair-Studie im Vergleich

Abbildung 3.5 : 100 Perioden-Simulation der Entwicklung der

Einkommensverteilung.

Abbildung 4.1 : Entwicklung der Transport- und Kommunikationskosten ..... 179

Abbildung 4.2 : Bestimmung der stimmenmaximierenden Zollsätze ............... 195

Abbildung 4.3 : Ländercluster der politischen Indikatoren .............................. 219 


\section{Tabellenverzeichnis}

Tabelle 2.1 : Werte, Normen und Bedürfnisse .......................................... 30

Tabelle 2.2: Das kritische Parteidifferential PD* ..................................... 42

Tabelle 2.3: Auszahlungen in einem einfachen Gefangenendilemma ........ 53

Tabelle 2.4: Durchschnittliche Wahlbeteiligung in der EU-12 .................. 59

Tabelle 2.5: Wahlentscheidung bei expressiven Nutzen .............................. 65

Tabelle 2.6 : Die Modelle konkurrierender Nutzenfunktionen ..................... 77

Tabelle 3.1 : Konfliktlinien in westlichen Demokratien .............................. 99

Tabelle 3.2: Konfliktlinien in den Parteiensystemen der Industriestaaten... 100

Tabelle 3.3 : Durchschnittliche Einkommensverteilung in Deutschland von 1950-1988 ....................................................................... 124

Tabelle 3.4 : Ordnungs-und Prozesspolitik als Komponenten der Wirtschaftspolitik

Tabelle 3.5 : Arbeitslosigkeit nach der Berufsausbildung in Deutschland ... 135

Tabelle 3.6 : Der Einfluss der Inflation auf die Einkommensquintile in

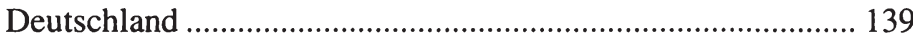

Tabelle 3.7 : Linke und rechte Wirtschaftspolitik im Vergleich .................... 145

Tabelle 3.8 : Übersicht der verschiedenen Modelle politischer Konjunkturzyklen .................................................................... 150

Tabelle 3.9 : Synopse der wichtigsten Modelle politischer Konjunkturzyklen ................................................................... 167

Tabelle 4.1: Wichtige Integrationsabkommen der führenden

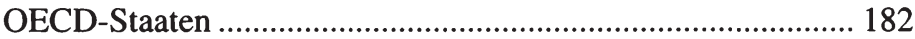

Tabelle 4.2: Exporte von Handelswaren als Prozentanteil vom BIP............ 185

Tabelle 4.3 : Absolute Höhe der Leistungsbilanz relativ zum BIP von 1970-1996.

Tabelle 4.4 : CG-Werte während der GATT-Runden und in den Zwischenphasen 200

Tabelle 4.5 : Entwicklung des Offenheitsgrads von 1963-1997 ................... 203

Tabelle 4.6 : Entwicklung von Direkt- und Portfolioinvestitionen

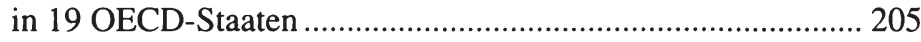

Tabelle 4.7 : Clusteranalyse der ökonomischen Indikatoren für den Zeitraum 1974-1997 (nach Jahren)

Tabelle 4.8 : Clusteranalyse der ökonomischen Indikatoren für den Zeitraum 1990-1997 (nach Ländern)

Tabelle 4.9 : Kennzeichnung der ökonomischenda Culust - 978-3-631-75139-8 
Tabelle 4.10 : Kapitalmarktliberalisierung .................................................... 213

Tabelle 4.11: Gütermarktliberalisierung................................................. 215

Tabelle 4.12: Clusteranalyse der politischen Indikatoren für den Zeitraum 1960 bis 1993 (nach Jahren).................................................. 216

Tabelle 4.13 : Clusteranalyse der politischen Indikatoren für den Zeitraum 1960 bis 1993 (nach Ländern) .................................................. 218

Tabelle 4.14 : Vergleich der Clusterergebnisse .............................................. 221

Tabelle 5.1 : Stützzeiträume und Erhebungsmodi der nationalen Arbeitslosenquoten

Tabelle 5.2: Partisaneffekte auf dem Arbeitsmarkt im Gesamtpanel 1960-1997

Tabelle 5.3 : Arbeitsmarkteffekte für die ökonomischen Cluster 1960-1997

Tabelle 5.4 : Arbeitsmarkteffekte in der Clusterung gemäß ökonomischen Globalisierungsindikatoren (alle Ländercluster)

Tabelle 5.5: Arbeitsmarkteffekte in der Clusterung gemäß politischen Globalisierungsindikatoren 1960-1997

Tabelle 5.6 : Arbeitsmarkteffekte in der Clusterung gemäß politischen Globalisierungsindikatoren (alle Länder).

Tabelle 5.7 : Rationale Arbeitsmarkteffekte im Gesamtpanel 1960-1997 ... 239

Tabelle 5.8: Arbeitsmarkteffekte für die ökonomischen Cluster 1960-1997

Tabelle 5.9: Arbeitsmarkteffekte in der Clusterung gemäß ökonomischen Globalisierungsindikatoren (alle Ländercluster)........ 242

Tabelle 5.10 : Arbeitsmarkteffekte in der Clusterung gemäß politischen Globalisierungsindikatoren 1960-1997

Tabelle 5.11 : Arbeitsmarkteffekte in der Clusterung gemäß politischen Globalisierungsindikatoren (alle Länder).

Tabelle 5.12 : Schätzergebnisse für die Inflationsperformance im Gesamtpanel 1960-1997. 250

Tabelle 5.13 : Inflationsperformance für die ökonomischen Cluster 1960-1997 251

Tabelle 5.14 : Inflationsperformance in der Clusterung gemäß ökonomischen Globalisierungsindikatoren (alle Ländercluster)....

Tabelle 5.15 : Inflationsperformance in der Clusterung gemäß politischen Globalisierungsindikatoren 1960-1997 254

Tabelle 5.16 : Inflationsperformance in der Clusterung gemäß politischen Globalisierungsindikatoren (alle Länder)............................... 255

Tabelle 5.17 : Übersicht über die Koeffizienten der Ideologievariablen........ 258 
Tabelle 5.18 : Simultane Gültigkeit der rationalen Partisantheorie in bezug auf den Arbeitsmarkt und die Inflation...................... 258

Tabelle 5.19 : Zusammenfassung der Ergebnisse hinsichtlich der

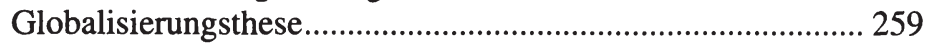

Tabelle 5.20 : Budgetäre Partisaneffekte im Gesamtpanel 1960-1996 ............ 268

Tabelle 5.21 : Partisaneffekte einzelner Budgetteile im Gesamtpanel

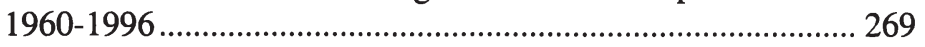

Tabelle 5.22 : Partisaneffekte im Budget in der Clusterung gemäß ökonomischen Globalisierungsindikatoren 1960-1996............ 272

Tabelle 5.23 : Partisaneffekte im Budget in der Clusterung gemäß ökonomischen Globalisierungsindikatoren (alle Länder) ......... 273

Tabelle 5.24 : Partisaneffekte im Budget in der Clusterung gemäß politischen Globalisierungsindikatoren (1960-1996) ............... 275

Tabelle 5.25 : Partisaneffekte im Budget in der Clusterung gemäß politischen Globalisierungsindikatoren (alle Länder) .............. 276

Tabelle 5.26 : Budgetäre Variabilität im Gesamtpanel 1960-1996.................. 281

Tabelle 5.27 : Ausgabenvariabilität in den ökonomischen Clustern 1960-1996

Tabelle 5.28 : Ausgabenvariabilität in den ökonomischen Clustern (alle Länder) ................................................................... 285

Tabelle 5.29 : Ausgabenvariabilität in der Clusterung gemäß politischen Globalisierungsindikatoren 1960-1997 287

Tabelle 5.30 : Ausgabenvariabilität in den politischen Clustern (alle Länder) 288 


\section{Abkürzungsverzeichnis}

ADA

ANZCERTA

Art.

ASEAN

BIP

BVerfGE

C-D-Gap

CG

CUSTA

DW

EB

EFTA

EGKS

EG-Vertrag

EU

EWS

GATT

GfdS

GG

GMM

IMF

i.V.m.

LSDV

MIPS

NAFTA

NATO
Americans for Democratic Action

Australian New Zealand Free Trade Area

Artikel

Association of Southeast Asian Nations

Bruttoinlandsprodukt

Bundesverfassungsgerichtsentscheid

Competence-difficulty-gap

(Ideological) center of gravity

Canada-United States Free Trade Area

Durbin-Watson Test

Eurobarometer

European Free Trade Area

Europäische Gemeinschaft für Kohle und Stahl

Vertrag der Europäischen Gemeinschaften

Europäische Union

Europäisches Währungssystem

General Agreement on Tariffs and Trade

Gesellschaft für deutsche Sprache

Grundgesetz

Generalized method of moments

International Monetary Fund

in Verbindung mit

Least squares dummy variable

Million instructions per second

North American Free Trade Area

North Atlantic Treaty Organization - 978-3-631-75139-8 
OECD Organization of Economic Cooperation and Development

OLS Ordinary least squares

PD Parteidifferential

REMM Resourceful evaluating maximizing man

SVR Sachverständigenrat zur Begutachtung der gesamtwirtschaftlichen Lage

UN United Nations

WTO World Trade Organization

\section{Länderkürzel}

$\mathrm{AU}$

AUS

BEL

CAN

$\mathrm{CH}$

D

DK

$\mathrm{E}$

$\mathrm{F}$

IRL

ITA

J

NL

NOR

NZL

$\mathrm{S}$

SF

UK

USA
Österreich

Australien

Belgien

Kanada

Schweiz

Deutschland

Dänemark

Spanien

Frankreich

Irland

Italien

Japan

Niederlande

Norwegen

Neuseeland

Schweden

Finnland

Großbritannien

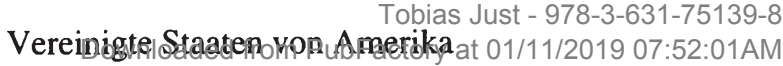




\section{Kapitel 1 Einleitung}

\subsection{Forschungskontext und Problemstellung}

In den Sozialwissenschaften wird in beeindruckender Regelmäßigkeit die Frage diskutiert, inwiefern Parteien zum einen in ihren Programmen, also ihren ideologischen Zielvorstellungen, und zum anderen in den realisierten Politikergebnissen konvergieren oder divergieren. In der Ökonomie gehen die theoretischen Grundüberlegungen auf Schumpeter (1950, S. 427ff) zurück, der in seiner Demokratietheorie das Prinzip des Wettbewerbs auf den politischen Markt übertrug, wo als „Nebenerscheinung“ der Erlangung der politischen Macht die soziale Funktion der Produktion öffentlicher Güter wahrgenommen wird. Auf der Grundlage dieses Demokratiemodells begründete Downs (1957, S. 114ff) die zu erwartende Konvergenz der Politikprogramme und -ergebnisse, falls die Politiker allein wahlsiegorientiert sind.' Sowohl bei Schumpeter als auch bei Downs hat die Ideologie für die Parteien allein eine Instrumentfunktion zur Gewinnung von Wählerstimmen.

Nahezu zeitgleich wurde in den benachbarten Sozialwissenschaften unter dem von Bell (1960) vorgebrachten Diktum des ,End of Ideology“ diskutiert, ob der weitgehende Konsens über das Sozialstaatsmodell in den Industrienationen der späten 50er und 60er Jahre den Boden für ideologische Differenzierung austrocknete. In der Argumentation Bells besitzen Ideologien nicht nur Instrumentfunktion, sondern sie sind für die Parteien quasi Zielvorgaben. Diese Zielfunktionen können Ideologien allerdings nur solange erfüllen, solange sie nicht im Widerspruch zum Stand des verfügbaren Wissens und der wissenschaftlichen und politischen Erfahrungen stehen. Gibt es also einen parteiübergreifenden Wissenskanon, dann bieten die zur Wahl stehenden Parteien dasselbe Programm an, nicht um dadurch Wahlen zu gewinnen, sondern aufgrund der Überzeugung, dass es keine Alternative dazu gibt. ${ }^{2}$

1 Downs (1957) selber prognostizierte keine Konvergenz der Wahlprogramme, falls mehr als zwei Parteien zur Wahl stehen und/oder die Wählerverteilung nicht unimodal ist.

2 Ähnlich begründet auch Fukuyama (1992) seine These vom „Ende der Geschichte“ damit, dass die liberale Demokratie die erste widerspruchsfreie Regierungsform ist und daher das Ende der Evolution der menschlichen Ideologiegeschichte kennzeichnet. Um möglichen Missverständnissen vorzubeugen, sei darauf hingewà่esedn, 
Sowohl die Theorien von Schumpeter und Downs als auch die These von Bell haben die weitreichende empirische Implikation, dass zu beobachten sein sollte, dass Parteien ihre Unterscheidbarkeit verlieren. Folgerichtig wurden in den 70er und 80er Jahren zahlreiche Studien mit dem Ziel durchgeführt, die Hypothese der Parteienkonvergenz zu überprüfen. Dabei standen im Zentrum der Analysen zum einen makroökonomische Variablen wie z.B. die Inflationsrate, die Wachstumsrate des Bruttoinlandsprodukts oder die Arbeitslosenquote, und zum anderen Instrumentvariablen wie z.B. die Höhe der Einnahmen und Ausgaben eines Staates. Zwar gab es weitgehenden Dissens über den Umfang des Einflusses, den Parteien auf diese Parameter haben, jedoch konnten die meisten Studien zeigen, dass Parteien in ihrer Politikgestaltung differieren. ${ }^{3}$ Parteien boten nicht nur gemäß ihrer ideologischen Positionierung systematisch unterschiedliche Programme an, sondern unterschieden sich darüber hinaus auch systematisch in bezug auf die Höhe der Staatsausgaben, der Struktur der Staatsausgaben und der Art der Ausgabenfinanzierung.

In den letzten Jahren zog die Frage nach den wirtschaftspolitischen Einflussmöglichkeiten von Parteien neues Interesse auf sich. Als zentrale Ursache für den möglichen Bedeutungsverlust von Parteien und den von ihnen repräsentierten Ideologien gilt das Phänomen, das unter dem Begriff „Globalisierung“ diskutiert wird. Unter Globalisierung wird dabei im folgenden ausschließlich die zunehmende internationale Verflechtung der Güter- und Faktormärkte verstanden. Dabei scheint es sehr weitgehenden Konsens zu geben, dass die Kräfte der Globalisierung wie ein externes Zwangsinstrument wirken, welches die nationalstaatlichen Akteure alternativenlos zur Durchführung umfangreicher ordnungspolitischer Reformen nötigt (Theurl, 1999, S. 24). Allerdings lassen sich die Wissenschaftler, Journalisten und Politiker, die zu diesem Urteil kommen, zwei Gruppen zuordnen: Die Pessimisten sind der Ansicht, dass die Globalisierung zu einer stärkeren Differenzierung der Einkommen, einem Anstieg der Arbeitslosigkeit, v.a. aber zu einer Reduktion der Steuerbasis der Industriestaaten und damit letztlich zu einem Ende der sozialen Umverteilungsinstrumente führen muss, falls sich nicht die Einsicht durchsetzt, dass es einer globalen Ordnungsinstanz bzw. internationaler wirtschaftspolitischer Koordination und Harmonisierung bedarf. Die Optimisten äußern hingegen die Erwartung, dass im Zuge der Internationalisierung der Märkte lediglich die Ineffizienzen nationalstaatlicher Wirtschafts-, v.a. Verteilungspolitik abgebaut werden.

der Ideologie sinnvollerweise nicht auf die Wahl der Regierungsform eingeengt wird, da alle untersuchten Staaten während des Untersuchungszeitraums liberale Demokratien besaßen. Wie später noch ausführlich darzustellen sein wird, kennzeichnet eine Ideologie jede Verdichtung von Wertvorstellungen mit konsistenten Umsetzungsstrategien (vgl. Abschnitt 2.1).

3 Vgl. neben vielen anderen Veröffentlichungen die Beiträge in dem Sammelband von Castles (1982) sowie Hibbs (1987). 
Die Pessimisten kommen zu dem Urteil, dass ein race to the bottom unausweichlich ist; die politischen Akteure sind gezwungen, ihre Politik an dem untersten Standard im internationalen Vergleich anzupassen, da jede zusätzliche wohlfahrtsstaatliche Aktivität nicht mehr zu finanzieren wäre. Das hätte zur Folge, dass jede Regierung, egal in welchem Land, dieselbe Wirtschaftspolitik durchsetzen müsste.

Da die Interpretation der Globalisierungsoptimisten lediglich impliziert, dass frühere wohlfahrtsstaatliche Fehlentwicklungen revidiert werden müssen, könnte es nach ihrer Einschätzung auch in der Zukunft gemäß landesspezifischen Präferenzunterschieden zwischen den einzelnen Staaten unterschiedliche Regulierungstiefen geben. Allerdings könnte in einem Land keine Regierung von dem landesspezifischen Regulierungsniveau auf Dauer abweichen, sodass die Parteien innerhalb eines Landes gezwungen wären, dieselbe Politik anzubieten.

Daraus leitet sich direkt die Problemstellung dieser Arbeit ab. Es wird untersucht, ob der beobachtete Globalisierungstrend in der Wirtschaft dazu geführt hat, dass sich die wirtschaftspolitischen Handlungsspielräume nationaler Regierungen nachweislich verengt haben, sodass es nicht mehr möglich ist, parteispezifische, ideologiegeleitete Wirtschaftspolitik zu realisieren. Dem liegt zugrunde, dass zuvor deutliche Partisanunterschiede zu erkennen sein müssen. ${ }^{4}$ Deshalb ist es zunächst erforderlich, die bisherige Unterschiedlichkeit der parteilichen Wirtschaftspolitik zu begründen und zu kennzeichnen. Da das Spektrum möglicher Unterscheidungspunkte der Wirtschaftspolitik sehr breit ist und folglich Akzentsetzung erfordert, wird in dieser Arbeit ausschließlich auf drei Kernaspekte der Wirtschaftspolitik eingegangen. Zum ersten wird geprüft, ob die Globalisierung die Handlungsspielräume auf dem Arbeitsmarkt eingeschränkt hat. Zum zweiten wird untersucht, ob es je nach Ideologie der Regierungspartei systematische Unterschiede in den realisierten Preissteigerungsraten gab und noch immer gibt. Den dritten Untersuchungsgegenstand stellen die öffentlichen Ausgaben dar: Es wird geprüft, inwiefern durch die fortschreitende Internationalisierung der Märkte die parteispezifische Profilierung in den öffentlichen Haushalten reduziert wird.

\subsection{Gang der Untersuchung}

Die Arbeit ist in vier zentrale Kapitel unterteilt: In Kapitel 2 wird der Ideologiebegriff in die ökonomische Theorie der Politik eingebunden. Grundsätzlich

4 Hier bedeutet Partisan natürlich in enger Anlehnung an die lateinische Herkunft Anhängerschaft (von einer politischen Partei und deren-Ideglggie) - 978-3-631-75139-8 
könnte man der Vorgehensweise von Schumpeter und Downs folgen, in deren Modellen Ideologie für die Parteien allein eine instrumentelle Funktion als Mittel zur Gewinnung von Wählern besitzt. Dieser Ansatz greift aber dann zu kurz, wenn das dabei zugrunde liegende Menschenbild zu derart großen Brüchen im Vergleich zur Realität führt, dass es gravierend an Prognosekraft verliert. Es wird argumentiert, dass gerade davon auszugehen ist. Daher werden zunächst die Elemente eines allgemeinen Ideologiebegriffs gekennzeichnet, der beinhaltet, dass Ideologien für den Menschen auch Zielfunktionen im Sinne eines übergeordneten Leitbildes sein können. Diese soziologische Ideologiekonzeption gilt es im folgenden mit der ökonomischen Theorie der Politik zu verbinden. Es lässt sich zeigen, dass diese Integration auch in ökonomischen Modellen des Parteienwettbewerbs dazu führen kann, dass politische Gleichgewichte ohne Konvergenz der Parteiprogramme existieren können.

Anschließend wird argumentiert, dass eine Integration des Ideologie- und des damit einhergehenden soziologischen Wertbegriffs nicht nur ein nützlicher Trick zur Überwindung der Theorielücke des nicht (vollständig) stattfindenden Konvergenzprozesses angebotener Parteiprogramme darstellt, sondern sich aus dem Erklärungsnotstand des engen ökonomischen Menschenbildes für zahlreiche Aspekte des politischen Lebens ergibt. ${ }^{5}$ Dies soll anhand von drei Tatbeständen veranschaulicht werden: Erstens scheinen viele Menschen bereit zu sind, freiwillig in ein öffentliches Gut zu investieren, selbst wenn dies weder rational noch eigennutzorientiert scheint. Zweitens beteiligen sich weite Teile der Bevölkerung an öffentlichen Wahlen, obwohl es höchst unwahrscheinlich ist, dass eine einzige Stimme wahlentscheidend ist. Drittens gibt es Anhaltspunkte dafür, dass zumindest ein Teil der Politiker nicht nur monetären Anreizen oder Wählerstimmen folgt. Im letzten Abschnitt des zweiten Kapitels werden drei Modelle präsentiert, die in der Lage sind, den ökonomischen Rational Choice Ansatz mit der soziologischen Werte- und Ideologiekonzeption auszusöhnen und die darüber hinaus einige interessante Implikationen aufweisen. Diese Modelle bieten also die Mikrofundierung für polit-ökonomische Ansätze, in denen Ideologie nicht nur Instrumentfunktion, sondern auch Zielfunktion für die politischen Akteure hat. Dadurch wird letztlich erst die Modellierung der beobachtbaren Divergenz von Parteiprogrammen und implementierter Politik möglich.

Nachdem also gezeigt wurde, dass es sinnvoll ist, von einer Unterschiedlichkeit der Parteiprogramme auszugehen, soll im dritten Kapitel die Art der Unterschiedlichkeit näher bestimmt werden. Dabei kommt es darauf an, die für die Fragestellung relevanten Unterscheidungsmerkmale zu isolieren und zu operati-

5 Der homo oeconomicus wird üblicherweise dadurch definiert, dass er rational und allein eigennutzmaximierend entscheidet. Werte im soziologischen Wortverständnis, die vom Einzelnen zumindest zu großen Teilen nicht beeinflusst werden und deren Befolgung nutzenmindernd wirken kann, haben in diesem Menschenbild offensichtlich keinen 3 glatz. 
onalisieren. Es wird argumentiert, dass für wirtschaftspolitische Fragestellungen durchaus ein einziges Unterscheidungsmerkmal ausreicht und dass dieses Merkmal durch das traditionelle Links-Rechts-Schema abgebildet werden kann. Dabei ist allerdings zu beachten, dass das Links-Rechts-Schema in den über 200 Jahren seit seiner Einführung als Bewertungsgrundlage für (fast) alle Politikfelder und Politikinstrumente angewandt wurde, sodass das Begriffspaar an Definitionsklarheit verloren hat. Da es jedoch möglich ist, die Abgrenzung linker und rechter Politik anhand der Grundfrage der „Reduktion von Ungleichheit“ vorzunehmen, und es zur Zeit keine bessere Alternative gibt, wird auf das LinksRechts-Schema zurück gegriffen. Dies geschieht in Abschnitt 3.3. Auf der Grundlage von Expertenbefragungen werden alle Regierungen von 19 OECDStaaten für den Zeitraum von 1960 bis 1997 in einem Links-Rechts-Kontinuum verortet. Außerdem muss geklärt werden, welche konkreten wirtschaftspolitischen Ziele und Instrumente aus der gewählten Unterscheidung zwischen links und rechts deduziert werden können. Es wird gezeigt, dass auf der Grundlage des grundsätzlichen Unterscheidungsmerkmals "Gleichheitskonzept" tatsächlich verschiedene idealtypische wirtschaftspolitische Ziele bezüglich der Arbeitsmarktpolitik, der Preispolitik und der Budgetpolitik formuliert werden können.

Die Differenz der Ziele in der Wirtschaftspolitik bedeutet allerdings noch nicht, dass die ideologisch geprägten Parteien ihre Ziele auch tatsächlich verfolgen und realisieren können. Die Möglichkeit der ideologischen Prägung von Wirtschaftspolitik wird in der wirtschaftstheoretischen Literatur nicht einheitlich beantwortet. Im wesentlichen wird die Diskussion durch zwei Theorien bestimmt. Auf der einen Seite geht die traditionelle Partisantheorie, die auf Hibbs (1977) basiert, davon aus, dass es permanente Unterschiede linker und rechter Regierungen auf dem Arbeitsmarkt und hinsichtlich der realisierten Inflationsraten geben muss. Auf der anderen Seite kommen die Modelle der rationalen Partisantheorie, die v.a. Alesina (1987) folgen, zu dem Ergebnis, dass es lediglich kurzfristig zu unterschiedlichen Arbeitsmarkteffekten kommen kann. ${ }^{6}$

Da das Ziel der Arbeit ist, die Bedeutung der Globalisierung für die politischen Handlungsspielräume abzuschätzen, bedarf es eines Parameters, der den Grad der Globalisierung einer Volkswirtschaft abbildet. Diesen zu konstruieren, ist zunächst Inhalt des folgenden vierten Kapitels. Nach einer kurzen Begriffsdefinition wird erläutert, was die Ursachen für die Entwicklung zu einer stärkeren weltwirtschaftlichen Integration waren. Anschließend werden die zu testenden Hypothesen formuliert und begründet, warum dieser Globalisierungsprozess gegebenenfalls die Handlungsspielräume nationaler Wirtschaftspolitik verkleinert.

6 Einzelheiten werden in Abschnitt 3.5 erörtert. Das zentrale Moment der beiden Theorien ist die Modellierung der Erwartungsbildung hinsichtlich der Preisentwicklung, letztlich al-

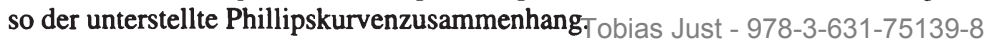


Kern des vierten Kapitels sind aber die Clusteranalysen des Abschnitts 4.6. Es werden anhand von Globalisierungsindikatoren Ländergruppen gemäß ihrem Integrationsgrad in die internationale Arbeitsteilung gebildet. Hierbei wird nicht nur der häufig benutzte Offenheitsgrad (Exporte plus Importe in Relation zum Bruttoinlandsprodukt) einer Volkswirtschaft benutzt, sondern es werden darüber hinaus die heute wesentlich umfangreicher gewordenen Kapitaltransfers und die Integration einer Nation in supranationale Zusammenschlüsse herangezogen. Es werden zwei Clusteranalysen durchgeführt. In der ersten werden „ökonomische“ Indikatoren als Variablen für die Clusteranalyse verwendet. Die Gruppenbildung erfolgt also gemäß dem tatsächlich stattfindenden Handelsvolumen und den tatsächlich vorgenommenen Auslandsinvestitionen. In der zweiten Clusterung werden ,politische“ Indikatoren (z.B. Integration in regionale Freihandelszonen, Ratifizierung von Freihandelsabkommen etc.) als Variablen verwendet. Hier erfolgt die Gruppenbildung also nicht nach den realiter stattgefundenen Marktergebnissen, sondern gemäß dem durch die Liberalisierung ermöglichten Austauschpotential. Es lässt sich zeigen, dass beide Clusterungen zu unterschiedlichen Ländergruppierungen führen. Es muss im folgenden also stets unterschieden werden, ob die Länder den Außenhandel deklaratorisch zulassen oder ob dieser tatsächlich eintrat.

In einem weiteren Schritt wird geprüft, ob die ökonomischen und politischen Variablen den Globalisierungsprozess in unterschiedliche Zeitperioden unterteilen. Dafür werden erneut zwei Clusteranalysen durchgeführt. Allerdings sind dabei die zu clusternden Objekte nicht die Länder, sondern die Jahre. Auch hier führen beide Clusteranalysen zu unterschiedlichen Ergebnissen. Möchte man also den Globalisierungsprozess und den Integrationsgrad eines Landes beschreiben, macht es einen großen Unterschied, ob man über das tatsächliche Ausmaß der Güter- und Faktorströme oder über die Liberalisierung der Güterund Faktormärkte spricht. Zwar hängen beide eng miteinander zusammen, jedoch kann eine differenziertere Betrachtung interessante Details offen legen.

Im fünften Kapitel wird dann mit Hilfe von Regressionsanalysen geprüft, ob die Globalisierung tatsächlich die wirtschaftspolitischen Handlungsspielräume der nationalen Regierungen reduzierte. In der Analyse werden 19 OECD-Staaten im Zeitraum von 1960-1997 betrachtet. ${ }^{7}$ Da es nicht möglich ist, alle Aspekte nationalstaatlicher Wirtschaftspolitik zu untersuchen, wurden nur jene Bereiche berücksichtigt, für die relativ gut quantifizierbare Indikatoren gebildet werden können. Dies sind zum einen die Arbeitsmarktperformance, zum zweiten die Inflationsraten und schließlich die realisierten Budgetstrukturen eines Landes. Es wird also untersucht, ob linke Regierungsparteien grundsätzlich bessere Leistungen auf dem Arbeitsmarkt in Form niedrigerer Arbeitslosenraten erzielen

7 Zum Teil werden einzelne Regressionen mit einer etwas kleineren Stichprobe durchgeführt. Das Auswahlkriterium war dann stets die Verfügbarkeit dgy geleyantep5Daten. 
und dafür höhere Inflationsraten in Kauf nehmen als rechte Regierungen. Anschließend wird geprüft, ob dieser Partisaneffekt besonders stark in jenen Ländern vorzufinden ist, die weniger stark international integriert sind. Es werden also für die zuvor in Kapitel vier gewonnenen Cluster unterschiedlicher Integrationstiefe separate Regressionen geschätzt. Diese Prüfung erfolgt sowohl für die ökonomischen Cluster als auch für die politischen Cluster.

Auf dem Arbeitsmarkt und hinsichtlich der Inflationsperformance lassen sich deutliche Effekte isolieren, die mit der rationalen Partisantheorie im Einklang stehen. Dies gilt jedoch nicht für alle Länder, die in der Untersuchung berücksichtigt wurden. Der Integrationsgrad, sowohl nach den ökonomischen als auch nach den politischen Variablen, scheint allerdings kein gutes Trennkriterium zu sein, für welches Land die rationale Partisantheorie gilt und für welches nicht. Auch gibt es keine überzeugenden Belege dafür, dass die Partisaneffekte in den 90er Jahren nicht mehr existieren. Die Integration eines Landes beschränkte die nationalen Steuerungsmöglichkeiten eines Landes zumindest bis zum Jahr 1997 nicht sehr nachhaltig.

Hinsichtlich der nationalen Budgets wird geprüft, ob linke Parteien tatsächlich eher verteilungspolitisch regressiv wirkende Ausgabengruppen stärken oder nicht. Ferner wird untersucht, ob sich dieser Zusammenhang in den letzten Jahren verkleinert hat und ob das budgetäre Profilierungspotential besonders in den eher geschlossenen Ländern existiert. Auch hier scheinen die Kräfte der offenen Märkte zumindest bis 1997 noch nicht so stark gewesen zu sein, dass von einer vereinheitlichten Haushaltspolitik in allen Ländern unabhängig von der jeweiligen Regierungspartei gesprochen werden kann. Es gibt allerdings deutlichere Anzeichen für einen Konvergenzprozess als bei den Makrovariablen Arbeitslosigkeit und Inflation.

Schließlich wird untersucht, ob linke Parteien eher zu budgetären Strukturbrüchen neigen, bzw. ob rechte Parteien in dem Sinne konservativ sind, dass sie die übernommenen Budgetstrukturen konservieren. In der Tat gibt es deutliche Anzeichen dafür, dass insbesondere in den Jahren nach einem Regierungswechsel nach links die deutlichsten Strukturbrüche in den nationalen Budgets anzutreffen sind. Es lässt sich aber nicht abschätzen, inwiefern dieses Ergebnis tatsächlich auf eine unterschiedliche Reformneigung der Parteien zurückzuführen ist oder eher durch einen strategischen Vorteil linker Parteien begründet werden kann. Es gibt gute Gründe anzunehmen, dass linke Parteien einen Signalingvorteil haben, wenn Ausgabenkürzungsprogramme durchgesetzt werden müssen. Das sechste und letzte Kapitel fasst die zentralen Ergebnisse der Arbeit zusammen. 
Tobias Just - 978-3-631-75139-8

Downloaded from PubFactory at 01/11/2019 07:52:01AM

via free access 


\section{Kapitel 2 \\ Das Ideologiekonzept in der ökonomischen Theorie}

"Economics is all about choices while sociology is about why people have no choices. " Duesenberry (1960, S. 233)

Seit über dreißig Jahren muss sich die Ökonomie von anderen Sozialwissenschaften den Vorwurf des Wissenschaftsimperialismus gefallen lassen. ${ }^{8}$ Dass dieser Vorwurf nicht völlig ungerechtfertigt ist, muss hier nicht besonders ausgeführt werden. Ökonomen untersuchen schon lange nicht mehr allein das Erkenntnisobjekt Wirtschaft (economy), sondern dringen mit der Analysemethode der Ökonomie (economics) in nahezu alle Lebensbereiche des Menschen vor. ${ }^{9}$ Ein solches Vorgehen erweist sich stets dann als sinnvoll, wenn die Ergebnisse aus der Anwendung der ökonomischen Methode in anderen Wissenschaftsgebieten dort zu neuen Erkenntnissen führen. Der Vorwurf, dass das Menschenbild der Ökonomie der Komplexität des Menschen nicht gerecht wird, greift dann nicht, wenn es mit Hilfe dieses reduzierten Zerrbilds des Menschen möglich ist, exaktere Verhaltensprognosen abzuliefern als mit komplexeren Menschenbildern, die ja trotzdem immer nur als Modell gestaltbar sind. Andererseits muss auch der Analyserahmen der Ökonomie flexibel genug sein, um stets dann Erweiterungen zulassen zu können, wenn durch Ergänzungen von außen die Vorhersagekraft des Modells zunimmt. Eine solch dringend erforderliche Ergänzung sehen nun einige Ökonomen in einer Berücksichtigung von Normen, Werten und Ideologien innerhalb des Konzepts des homo oeconomicus (North, 1988).

In diesem Kapitel wird zunächst der Begriff Ideologie definiert. Da in der politikwissenschaftlichen und soziologischen Literatur dafür auf den (soziologischen) Wertbegriff rekurriert wird, der in der Ökonomie (noch) keine starke Verbreitung erfahren hat, wird dafür zunächst auch eine Kennzeichnung des Wertbegriffs benötigt. In Abschnitt 2.2 werden Grundzüge der Ökonomie der Politik dargestellt, die als Grundlage für die Argumentation in Abschnitt 2.3

8 Erstmals kam dieser Vorwurf sogar aus den eigenen Reihen. Allgemein gilt Boulding (1969) als den Namensgeber des Ausdrucks ,economic imperialism" (Engelhardt, 1989, S. 20).

9 Natürlich gehört zu den prominentesten Arbeiten die ökonomische Analyse der Politik seit Downs (1957) und die ökonomische Analyse des Rechts seit Posner (1973). Insbesondere auf den Einfluss der Arbeiten von Becker (1976,1981) sei yerviesen631-75139-8 
dienen. Dieser Abschnitt zeigt auf, warum eine Integration des soziologischen Wert- und Ideologiebegriffs Erklärungsdefizite der engen ökonomischen Analyse beheben könnte. Schließlich werden in Abschnitt 2.4 Lösungsvorschläge vorgestellt, mit denen es möglich ist, den Wertbegriff mit dem ökonomischen Menschenbild zu versöhnen, sodass der Hypothese von North gefolgt werden kann:

„Die Einführung des Begriffs der Ideologie nimmt der ökonomischen Theorie nichts von ihrer Wissenschaftlichkeit in dem Sinne, dass sie Verfahren zur Prüfung der Widerlegbarkeit konkurrierender Hypothesen entwickeln kann." (North, 1988, S. 56)

\subsection{Werte und Ideologien}

Um den Wert- und Ideologiebegriff angemessen in die ökonomische Theorie einbinden zu können, ist es sinnvoll, zunächst darauf einzugehen, wie Werte und Ideologien in der Soziologie und der Politikwissenschaft behandelt werden. Es soll hier allerdings explizit darauf hingewiesen werden, dass es auch in anderen Disziplinen der Sozialwissenschaften keine verbindliche Definition dieser zwei Begriffe gibt. ${ }^{10}$

\subsubsection{Definition des Wertbegriffs}

Der Wertbegriff steht im Zentrum fast jeder Definition von politischer Ideologie und verdient daher etwas Aufmerksamkeit. Dass eine klare Definition des Begriffes ,Wert" für die weitere Arbeit notwendig ist, wird dadurch deutlich, dass in der Literatur, je nach Untersuchungsschwerpunkt, verschiedene Wertdefinitionen benutzt werden. Lautmann (1969) schätzte die Anzahl unterschiedlicher Definitionen des Begriffes auf ungefähr 200." Aus dieser unübersehbaren Vielfalt an Abgrenzungen verdichtete Lautmann drei Aspekte, die eine geeignete Definition enthalten muss, um den Begriff Wert sinnvoll zu erfassen.

Demnach gibt es erstens ein Formelement, das besagt, dass ein gültiger Wert als Vergleichsmaßstab oder Standard dienen können muss. Dieser Maßstab muss zweitens an ein Objekt oder einen Gegenstand angelegt werden. Lautmann nennt diese Objektivierbarkeit das Sachverhaltselement. Hierbei ist Objekt nicht rein materiell zu fassen; es kann auch menschliches Handeln als Betrachtungsgegenstand darunter subsumiert werden. Der dritte Aspekt ist das normative E-

10 Die Darstellung gibt im wesentlichen die herrschende Meinung wieder und verweist lediglich vereinzelt auf Minderheitsmeinungen.

1 Auch Kmieciak (1976) zählt knapp 180 unterschiedliche, wenn auch teilweise überschneidende Definitionen. 
lement eines Wertes (Lautmann, 1969, S. 28). ${ }^{12}$ Ein Wert ist demnach für ein beliebiges Untersuchungsobjekt ein zu erstrebender Maßstab im Sinne einer normativen Zielvorgabe. Gerade für die Ökonomie ist es jedoch sinnvoll, das Sachverhaltselement auf gesellschaftlich relevante Sachverhalte zu fokussieren, um den Wertbegriff vom individuellen Bedürfnisbegriff der Ökonomie zu trennen (Murck, 1982, S. 139).

Für die weitere Arbeit ist die Definition von Kluckhohn (1951) zweckdienlicher, wonach Werte "...Vorstellungen vom gesellschaftlich Wünschenswerten..." darstellen. Auch hier sind das normative und das formale Maßstabselement enthalten, jedoch ist das Sachverhaltselement auf gesellschaftliche Objekte verengt. ${ }^{13}$ Wichtig ist darüber hinaus, dass das Individuum auf den Inhalt der Werte nicht maßgeblich einwirken kann, da Werte historisch durch gesellschaftliche Interaktion außerhalb des Individuums gebildet werden. Für das einzelne Individuum ist der Wert damit zumindest zu Teilen ,fremdindividuell fundiert" (Wiehn, 1972, S. 34). Nur durch unterschiedliche Interpretation durch das Individuum und die Sozialisationsgruppe kommt es zu Abweichungen in der Wertestruktur zwischen Menschen.

Das normative Element innerhalb der Wertdefinition legt es nahe, Werte mit Normen gleichzusetzen. Diese Übereinstimmung trifft aber lediglich auf der formalen und normativen Ebene zu, das Objektelement der Normen hingegen umfasst ausschließlich das Handeln; Normen sind durch Konsens legitimierte Verhaltensverpflichtungen. Sie sind allgemeingültig und vor allem sanktionierbar. Dabei zielen spezielle Normen darauf ab, allgemeine Werte zu erreichen Normen werden also durch Werte gerechtfertigt (Reichart-Dreyer 1976, S. 10f und Maag 1991, S. 22). ${ }^{14}$

So stehen fremdindividuell mitbestimmte Werte, die das gesellschaftlich Wünschenswerte abbilden und die allein durch die individuelle Selbstsanktionierung durchgesetzt werden, neben den eigenbestimmten Bedürfnissen und den konsensualen, von außen sanktionierbaren Normen, die nicht unbedingt das gesellschaftlich Wünschenswerte als Ziel haben, und führen gemeinsam zu einer Wahlhandlung, die dann in der Ökonomie seit Samuelson (1938) üblicherweise als die bekundete Präferenz erfasst wird. Anders jedoch als in ökonomischen Standardmodellen gibt es zwei trennbare Verhaltensmotivationen. ${ }^{15}$ Tabelle 2.1 rie der Wahlhandlung über den Bedürfnisbegriff himatasgehatn 
fasst die Unterscheidung zwischen Werten, Normen und Bedürfnissen, wie sie dieser Arbeit zugrunde liegt, zusammen. ${ }^{16}$

Tabelle 2.1 : Werte, Normen und Bedürfnisse

\begin{tabular}{lccc}
\hline & Werte & Normen & Bedürfnisse \\
\hline $\begin{array}{l}\text { Allgemeinheits- } \\
\text { grad }\end{array}$ & hoch & mittel & niedrig \\
$\begin{array}{l}\text { Durchsetzungs- } \\
\text { instanz }\end{array}$ & intern & extern & intern \\
Ziel & $\begin{array}{c}\text { das gesellschaftlich } \\
\text { Wünschenswerte }\end{array}$ & $\begin{array}{c}\text { das von Dritten } \\
\text { gewünschte Han- } \\
\text { deln }\end{array}$ & $\begin{array}{c}\text { das persönlich } \\
\text { Wünschenswerte }\end{array}$ \\
\hline
\end{tabular}

Quelle: Eigene Darstellung

\subsubsection{Die Bildung von Werten}

In der soziologischen Forschung geht man davon aus, dass Werte während der Sozialisation des Menschen ausgebildet werden. Sozialisation bezeichnet dabei den „...Prozess der Entstehung und Entwicklung der menschlichen Persönlichkeit in Abhängigkeit von und in Auseinandersetzung mit den sozialen und den dinglich-materiellen Lebensbedingungen..." (Hurrelmann, 1998, S. 14), also den Prozess, der den Menschen zu einem in einer komplexen Welt handlungsfähigen und eigenständigen Wesen werden lässt. Dies geht über das veraltete Wortverständnis der Sozialisation hinaus, nach dem Sozialisation die Übertragung von Kenntnissen, Einstellungen, Werten und Normen von so genannten Sozialisationsagenten (Familie, Gleichaltrige, Lehrer...) auf den Sozialisanden beschreibt (Zängle, 1978, S. 18). Dieses Verständnis des Sozialisationsprozesses musste erweitert werden, da der dadurch gekennzeichnete, passiv Rollenbilder annehmende homo soziologicus nicht dem Individualisierungsvermögen des Menschen gerecht wird.

Im politischen Bereich beschreibt „politische Sozialisation“ analog einen lebenslangen Lernprozess des Menschen, der diesem für politisches Handeln notwendige Werte, Normen und Wissen vermittelt (Pawelka, 1977, S. 12). Auch im politischen Bereich gilt mittlerweile die Kristallisationsthese von Hess und Torney (1966), die besagt, dass politische Einstellungen sehr früh - bis zum 13. Lebensjahr - und unumkehrlich durch Sozialisationsagenten festgeschrieben wer-

16 Vgl. zu einer Gegenüberstellung von Werten, Normen und Präferenzen (sic!) auf der Basis anderer Abgrenzungskriterien Hechter (1994, S. 322)bias Just - 978-3-631-75139-8 
den, als widerlegt. ${ }^{17}$ Zwar wird die Bedeutung von Sozialisationsagenten, insbesondere der Familie und der Schule nicht bestritten, jedoch geht die heutige Forschung davon aus, dass die Bildung von Werten (und damit auch von politischer Ideologie) durch ein kompliziertes Wirkungsgeflecht aus teilweise konfligierenden Institutionen und einem sich selbst reflektierenden Individuum entsteht und zu keinem Zeitpunkt abgeschlossen ist.

Werte entstehen also im Individuum durch drei interagierende Prozesse: Erstens durch die Übertragung durch Sozialisationsagenten (Sozialisation im traditionellen Sinne). Eltern, Lehrer, Freunde etc. haben ein Interesse daran, dass der Sozialisand dieselben Werte besitzt und werden diese ganz gezielt anerziehen. Zweitens können Sozialisationsagenten nicht geplante Einflüsse ausüben oder die sozio-ökonomische Umwelt wirkt auf das Individuum ein. Auf keinen dieser Einflüsse hat der Sozialisand zunächst einen Einfluss. Allerdings ist auch in der soziologischen Literatur die Bedeutung der einzelnen Einflussfaktoren strittig..$^{18}$ Es ist nicht unplausibel, die von Geißler (1996) festgestellte Abschwächung des Prägeeinflusses der Familie dahingehend zu verallgemeinern, dass zu jeder Zeit einzelne Einflussfaktoren auf die individuelle Persönlichkeitsbildung unterschiedliches Gewicht hatten und haben, das Individuum aber zu keinem Zeitpunkt frei von äußeren Prägungen war. Drittens ist das vernunftbegabte Individuum in der Lage, sich selbst zu reflektieren und dementsprechend auch prägende Einflüsse zu erkennen und zu bewerten, gegebenenfalls auch abzulehnen und zu verwerfen. Dies ist jedoch mit Opportunitätskosten verbunden, wie Arno Schmidt festhält:

„Den meisten von uns vergeht das Leben damit, die in der Jugend verkehrt eingestellten Maßstäbe mühsam wieder zu adjustieren." (zit. nach Weise, 1995, S. 101).

Abbildung 2.1 fasst diese Wirkungstrias graphisch zusammen. Natürlich ist die Trennung in dominantes Subsystem, peripheres Subsystem und direkt im Kontakt stehende Sozialisationsagenten schematisch. In der Realität sind die Unterschiede zwischen diesen drei Bereichen fließend; so ist der Lehrer in der Schule einerseits Repräsentant des Staates und an curriculare Verordnungen gebunden, hat daneben jedoch weite erzieherische Handlungsfreiräume.

17 Jennings (1990, S. 346f) kommt allerdings für Deutschland, die USA und die Niederlande in seiner Studie zu dem Ergebnis, dass man den Sozialisationsprozess differenzierter betrachten muss. Er unterscheidet zwischen subjektiven Orientierungen, die fast niemals ein stabiles Niveau erreichen und konsensual gesellschaftlichen Orientierungen, die bis zum frühen Erwachsenenstadium weitgehend verfestigt sind und nur durch ,epochale“ Ereignisse wandelbar werden.

18 Inglehart (1971, 1990a) betrachtet z.B. die materielle Versorgung während der formativen Jahre im Sinne der Kristallisationsthese als zentral, während Pawelka (1977) dem Staatsapparat und den herrschenden Eliten (Pawelka spricht vom dominanten Subsystem) eine besondere Stellung einräumt. 


\section{Abbildung 2.1 : Modell zur (politischen) Sozialisation}
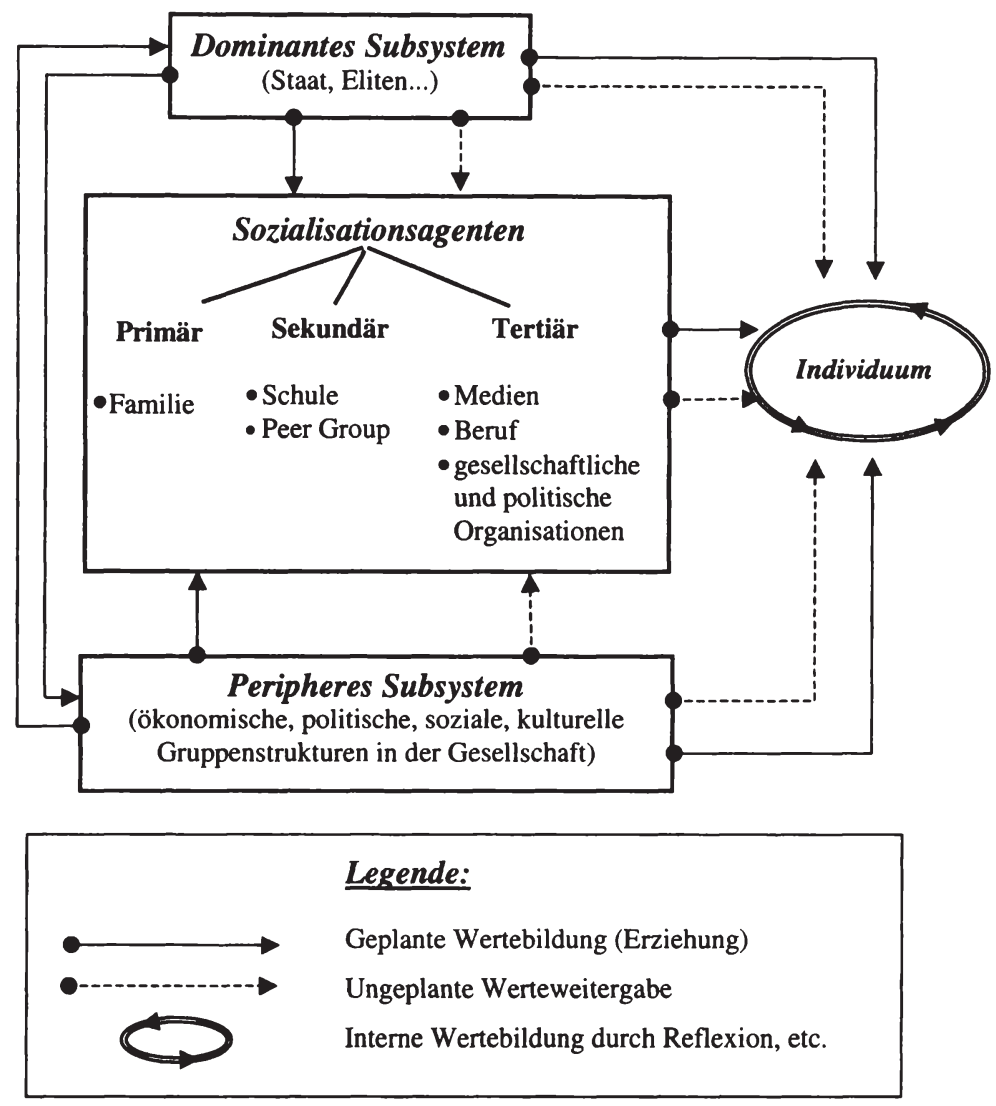

Quelle: In Anlehnung an Pawelka (1977, S. 31) und Hurrelmann (1998, S. 14ff).

\subsubsection{Ideologie als konsistentes Wertesystem}

Der Ideologiebegriff ist ähnlich wie der Wertbegriff von Autoren durch individuelle Akzentsetzung sehr unterschiedlich definiert worden. So ist bei Aron (1968) sowie Haber (1968) der Ideologiebegriff v.a. durch eine kognitive Dimension gekennzeichnet: ,...ideology [...] is a pseudo-systematic formulation of a total vision of the historical world." (Aron, 1968, S. 3). Für LaPalombara (1968, S. 320) ist diese Weltwahrnehmung erst dann Ideologie zu nennen, wenn ein kohärentes Wertesystem die Erkenntnis bestimmt und zu einer als moralisch 
höherwertig empfundenen Wunschvorstellung über die Welt führt. ${ }^{19}$ Bell (1968, S. 96) weist in seiner Definition politischer Ideologie auf zwei weitere bedeutsame Sachverhalte hin, dass nämlich erstens eine Ideologie einer deutlichen $\mathrm{Zu}$ kunftsorientierung und demnach einer programmatischen Komponente bedarf und dass sie zweitens eine langfristige Absicherung der Unterstützung durch Anhänger (Wähler) und emotionale Aufladung als Vehikel benötigt.

Rejai (1991, S. 11) amalgamiert diese Elemente in seiner Definition von Ideologie. Aufgrund ihrer strukturellen Geschlossenheit wird dieser Definition im weiteren gefolgt. Das Ideologiekonzept umschließt danach fünf Dimensionen:

1. Die kognitive Dimension: Diese beinhaltet das Element der Weltwahrnehmung auf der Grundlage von Wissen und Glauben, wie es von Aron und $\mathrm{Ha}$ ber betont wurde.

2. Die Wertungsdimension: Sie umfasst negative Werturteile über das, was den Status quo darstellt und verändert werden sollte, bzw. darüber, was andere fälschlicherweise zu ändern gedenken. ${ }^{20}$ Dies impliziert gleichzeitig ein System positiver Werturteile hinsichtlich der eigenen moralischen Überlegenheit.

3. Die affektive Dimension: Sie ist nicht formal konstituierender Teil einer Ideologie, sondern ein wiederholt beobachtetes Instrument zu deren Verbreitung.

4. Programmatische Dimension: Sie stellt dar, wie die durch die Werte festgelegten Ziele erreicht werden können, falls das Werturteil nicht in einem historischen Determinismus besteht, wie es beispielsweise dem historischen Materialismus der marxistischen Ideologie zugrunde liegt.

19 Auf das Moment der Bewertung rekurrieren auch Prätorius (1987, S. 139) und Inglehart und Klingemann (1979, S. 205).

20 Mannheim (1955) unterschied Ideologie als Ideensystem, das dem Erhalt des bestehenden Systems dient, von der Utopie, worunter er ein Ideensystem verstand, das einen Regimewechsel befürwortet. In westlichen Demokratien ist allerdings davon auszugehen, dass systemablehnende Parteien entweder keine Bedeutung haben oder aber keine Bedeutung erhalten können (z.B. ist es in Deutschland zulässig, verfassungsfeindliche Parteien gemäß Art. 21 GG zu verbieten, wenn sie sich gegen die durch das Bundesverfassungsgerichtsurteil BVerfGE 2,1 S. 12ff umschriebene Grundordnung der Verfassung richten). Gerade wenn man die in westlichen Demokratien beobachtbaren Ideologien als subsidiär zu der Ideologie Demokratie sieht (vgl. Rejai, 1991, S. 166f), wird deutlich, dass diese Unterscheidung Mannheims für die weitere Untersuchung ohne Bedegtung \&̇s4 
5. Mobilisierungsdimension: Damit eine Ideologie Aussicht auf Erfolg hat, benötigt sie die Unterstützung weiter Teile der Bevölkerung. Hierzu sind eine Organisation und koordinierte Kommunikation erforderlich. ${ }^{21}$

Die ersten beiden Dimensionen beschreiben eine allgemeine Definition von Ideologie, worunter auch private Ideologien ohne politische Motivation subsumierbar sind. Für den Begriff der politischen Ideologie, bei dem die potentielle politische Durchsetzung wichtig ist, stellen die drei instrumentellen Dimensionen (3)-(5) auch konstituierende Merkmale dar. Im weiteren Verlauf wird ausschließlich von politischen Ideologien ausgegangen und demzufolge auf das Adjektiv ,politisch“ verzichten.

\subsubsection{Funktionen von Werten und Ideologien}

Gemäß der Vielschichtigkeit des Entstehungsprozesses von Werten und Ideologien sind die Funktionen, also die Rationalisierung ihrer Zweckmäßigkeit, je nach Beteiligtenkreis verschieden. Grundsätzlich kann zwar mit North (1988, S. 50) resümiert werden: „Ideologie ist eine Sparmaßnahme“, es ist jedoch sinnvoll, diese Sparfunktion für die einzelnen Akteure zu konkretisieren.

Die Akteure, die bewusst an der Bildung von Werten und Ideologien arbeiten, tun dies, da sie ein begründetes Interesse an einem konkreten Verhalten des Sozialisanden haben. Um dies herbeizuführen, haben sie grundsätzlich die Wahl, dieses Verhalten durch Sanktionen (positive oder negative selektive Anreize) zu motivieren oder das Individuum so zu erziehen, dass es sich freiwillig für das gewünschte Verhalten entscheidet. Das Individuum entwickelt also ein inneres Sanktionssystem (Coleman, 1991, S. 380). Der Sozialisationsagent, respektive der Vertreter der prägenden Subsysteme, investiert dann in die Wertebildung, wenn die Grenzkosten einer solchen Investition die Grenznutzen unterschreiten und zudem geringer sind als die Grenzkosten externer Sanktionen.

Gerade bei Regeln des öffentlichen Lebens ist eine vollständige Kontrolle der Regelbefolgung sehr kostenintensiv, sodass der Versuch eines ausschließlichen Anwendens extrinsischer selektiver Anreize zur Funktionsunfähigkeit der politischen und wirtschaftlichen Ordnung führen muss (North, 1988, S. 19). Die logische Konsequenz dieser Tatsache ist für North dann auch die beobachtbare, enorme Anstrengung staatlicher Institutionen (und auch solcher Institutionen anderer gesellschaftlicher Teilgruppen), den Einzelnen von der Rechtmäßigkeit der vorherrschenden Systeme zu überzeugen. Dies wäre dann z.B. in den Ausgaben

21 In totalitär regierten Gesellschaften besteht die Kommunikation im wesentlichen aus Manipulation der Massen. Da in dieser Arbeit ausschließlich relativ verfestigte Demokratien untersucht werden, findet das bei Rejai aufgezeigte Kriterium der Manipulation hier abgeschwächt als Kommunikation Eingang. 
für politische Bildung eines Staates abzulesen. Hier erfüllen Werte dann neben der Reduktion der Transaktionskosten auch die Funktion der System- und Kollektivstabilisierung. ${ }^{22}$

Auch für die konkreten Sozialisationsagenten (Familie, Lehrer) besteht die Wahl, Wohlverhalten durch extrinsische Sanktionen oder die Erziehung intrinsischer Sanktionen zu motivieren. Hier gilt oft, dass die Anerziehung allgemeiner Werte gerade aufgrund ihrer Allgemeinheit Skalenvorteile gegenüber extrinsischen Sanktionen besitzt, da gleichzeitig eine Vielzahl von verschiedenen Handlungstypen kontrolliert werden kann (Coleman, 1991, S. 296) ${ }^{23}$

Für den Sozialisanden sparen Werte und Ideologien Informationskosten sowohl bei der Selektion der zu berücksichtigenden Alternativen als auch bei der konkreten Handlungswahl, da systematisch nicht alle Optionen ins Kalkül gezogen werden müssen. Dies könnte die Steuerungs- oder Orientierungsfunktion von Werten genannt werden. ${ }^{24}$ Daneben haben Werte eine der Entscheidung nachgelagerte Legitimationsfunktion. Sowohl für den Entscheider als auch für Dritte bieten Werte später eine Begründungshilfe für die getroffene Wahl. Wird es für Individuen einfacher, ihre Entscheidungen zu rechtfertigen, bzw. können andere schneller eine getroffene Entscheidung nachvollziehen, helfen Werte auch hier Informationskosten zu senken, indem sie Komplexität vermindern (Maag, 1991, S. 22f).

Im folgenden Abschnitt wird ein kurzer Überblick über die ökonomische Theorie der Politik gegeben. Dabei wird deutlich, dass dort bereits vielfach mit einem soziologischen Ideologieverständnis gearbeitet wird, ohne dieses jedoch auf seine mikroökonomische Fundierung hin zu prüfen.

22 Reichart-Dreyer (1977, S. 19) spricht von funktionalem Zwangscharakter von Werten. Natürlich ist die Stabilisierung eines Systems oder eines Kollektivs auch nichts anderes als die Vermeidung von Verhandlungskosten zwischen den Konfliktpartnern, insofern ist die Stabilisierungsfunktion von Werten lediglich eine Konkretisierung der Sparfunktion von Transaktionskosten.

Lott (1990) sieht gerade in der Sozialisation, die in der Schule stattfindet die Begründung für ein öffentlich bereitgestelltes Schulsystem. Neben den privaten Gütern Bildung wird auch das nicht-marktfähige öffentliche Gut Sozialisation, das Transaktionskosten spart, produziert.

24 Manchmal findet man in der Literatur auch die Anpassungsfunktion in Konfliktfällen (vgl. Maag, 1991, S. 22f); dies könnte jedoch auch unter der Orientierungsfunktion subsumiert werden, da die Konfliktsituation lediglich eine spezielle Handlungssituation mit verschiedenen Alternativen darstellt. 


\section{2 Ökonomische Theorie der Politik}

\subsubsection{Der homo oeconomicus}

Üblicherweise geht man in der Ökonomie davon aus, dass das menschliche Verhalten durch drei zentrale Punkte beschreibbar ist. Erstens handeln ausschließlich Individuen, diese handeln zweitens rational und drittens eigennützig, wobei gerade hinsichtlich der letzten beiden Eigenschaften selbst in der ökonomischen Profession bis heute die kritischen Stimmen nicht verstummt sind. Es scheint jedoch so zu sein, dass in vielen Bereichen zumindest ,....im Durchschnitt und in der großen Zahl..."(Brennan und Buchanan, 1993, S. 68) die Annahmen des homo oeconomicus zu den besten Verhaltensprognosen menschlicher Handlungen führen. ${ }^{25}$

Zentrales und gleichzeitig innerhalb der ökonomischen Profession weitgehend unumstrittenes Element des homo oeconomicus ist die Annahme, dass ausschließlich Individuen in der Lage sind, Handlungen und Entscheidungen durchzuführen. Schumpeter (1908, S. 88) prägte für diesen Ansatz den Begriff des methodologischen Individualismus.

Die Existenz von Kollektiventscheidungen rührt also nicht daher, dass Kollektive als eigenständige Akteure Entscheidungen treffen, sondern daher, dass sich Individuen zuvor auf eine (für das Kollektiv) gültige Entscheidung einigen. Die nach außen wahrnehmbare Kollektiventscheidung ist dann lediglich Ausdruck der Interaktion der einzelnen Individualentscheidungen. ${ }^{26}$ Diese Vorgehensweise schließt natürlich nicht aus, dass eigenverantwortliche Individuen die Entscheidung treffen können, Agenten weitreichende Handlungsvollmachten zu übertragen. Hier werden dann - analog zum Markttausch - Spezialisierungsvorteile genutzt.

Der methodologische Individualismus schließt nicht aus, dass der Beitritt zu einem Kollektiv bei einem Individuum zu Verhaltensänderungen führen kann, da sich durch die Teilnahme an dem Kollektiv der Optionenraum für das Individuum gewandelt hat, denn es stehen neue Wahlmöglichkeiten offen und neue

Der Ausdruck des homo oeconomicus hat unterdessen weite Verbreitung zur Beschreibung des ökonomischen Verhaltensmodells gewonnen (vgl. u.a. Downs, 1957, S. 7, Tietzel, 1981, Frank, 1987). Für eine ausführliche Darstellung vgl. u.a. Woll (1994) und Kirchgässner (1991). Meckling (1976) und Brunner und Meckling (1977) führen ergänzend das Akronym REMM (resourceful evaluating maximizing man) zur Beschreibung ein.

Allerdings kann die individuelle Entscheidung auch dazu führen, dass gerade Vorbilder imitiert werden. Banerjee (1992) spricht dann von herd behaviour.8-3-631-75139-8 
Informationen werden verfügbar. Der methodologische Individualismus lässt also ein weites soziales Interaktionsnetz zu. Kirsch (1997, S. 17) fasst dies aphoristisch zusammen:

„....diese gesellschaftlichen Einflüsse [sind] nicht als Einflüsse der Gesellschaft zu interpretieren, sondern als Einflüsse von Individuen auf Individuen in der Gesellschaft."

Es wird in Abschnitt 2.4 gezeigt werden, dass damit eine viel weitreichendere Form der Einflussnahme gemeint ist, als dies üblicherweise in der Theorie der Externalitäten dargestellt wird.

Der Rationalitätsbegriff in der Ökonomie bedeutet, dass jedes Individuum in der Lage ist, aus den zur Verfügung stehenden Alternativen diejenige zu wählen, die gemäß seinem Kenntnisstand seinen Nutzen maximiert. Dies verlangt, dass es dem Individuum möglich ist, die präsentierten Alternativen gemäß seiner Präferenzen und innerhalb seiner Restriktionen in eine Rangordnung zu bringen, die erstens vollständig und zweitens transitiv ist (Arrow, 1963, S. 13). Downs (1957, S. 6) erweitert diesen Annahmenrahmen noch um die Annahmen, dass sich das Individuum bei Indifferenz zwischen zwei Alternativen trotzdem entscheiden kann und dass auch in Zukunft stets dieselbe Entscheidung getroffen würde. Beobachtbares Verhalten wäre dann ex definitione auch immer rational und ließe sich als Nettowohlstandsmaximierung unter Nebenbedingungen beschreiben. Dabei dient die Rationalitätsannahme v.a. der Reduktion von Komplexität bei der Modellierung, da das Zulassen von irrationalem Verhalten dazu führen muss, dass letztlich keine Verhaltensprognose möglich wäre (Sen, 1987, S. 11).

Dass die Wahlmöglichkeiten durch zumindest kurzfristig exogen vorgegebene monetäre und institutionelle Restriktionen begrenzt sind, ist unbestritten. Jedoch besteht weit weniger Konsens in der Wissenschaft, was als Präferenzen Eingang in die individuelle Nutzenfunktion findet und dadurch die Rangordnung der Entscheidung bestimmt. Üblicherweise gehen Ökonomen davon aus, dass Präferenzen intertemporal konstant sind. ${ }^{27}$ Die Präferenzen spiegeln die Bedürfnisse des Menschen wider sowie seine persönlichen Wertvorstellungen (Kirchgässner, 1991, S. 13f).

Rationalität im ökonomischen Sinn bedeutet indes nicht, dass alle Entscheidungen per se die bestmöglichen sind. Geht man nämlich davon aus, dass Informationen nicht kostenlos sind, gehen sowohl die Informationsbeschaffungs- als auch die Informationsverarbeitungskosten in das Entscheidungskalkül ein. Systematisch wird so ein unvollkommener Informationsstand erzielt, der in einer

27 So schreibt Fuchs (1994, S. 184) J. Mincer das Zitat zu: „...changes in taste is the economist's admission to defeat". Dennoch gibt es auch unter den Ökonomen zahlreiche Vertreter, die Präferenzveränderungen zulassen möchten; vgl. u.a. von Weizsäcker (1971), Sen (1973, S. 243), Engelhardt (1989, S. 40), Bar-Gill und Fershtman (2000) sowie Bowles (1998) und die dort angegebene Literatur. 
mit Unsicherheit behafteten Welt notwendigerweise die Gefahr von Fehlentscheidungen birgt. Der Informationsstand, der zu der „falschen“ Entscheidung geführt hat, ist allerdings das Ergebnis einer vorgeschalteten rationalen Entscheidung gewesen. ${ }^{28}$

Die dritte Grundannahme des Selbstinteresses als Triebfeder menschlichen Handelns geht bis auf Adam Smith zurück und der Satz

"It is not from the benevolence of the butcher, the brewer, or the baker, that we expect our dinner, but from their own interest. "(Smith, 1976a [1776], S. 26f)

gehört wohl zu den häufigst zitierten Sätzen in der Ökonomie. Individuen agieren ausschließlich aus dem Antrieb der Maximierung der eigenen Nutzenfunktion, gleichgültig in welcher Entscheidungssituation sie sich befinden. ${ }^{29}$ Seitdem ist diese Verhaltensannahme immer wieder zur Zielscheibe heftiger Kritik geworden, und die Kritiker verweisen regelmäßig ebenfalls auf Adam Smith. Allerdings zitieren sie sein erstes Buch The Theory of Moral Sentiments (Smith, 1976b [1759], S. 189), in dem er neben die Tugend der Eigenliebe, die vornehmlich dem Individuum dient, die Tugenden der Humanität, Gerechtigkeit, Großzügigkeit und des öffentlichen Interesses, welche hauptsächlich anderen dienlich sind, als Handlungsmotive stellt. ${ }^{30}$

Ausschließlich eigennutzorientiertes Verhalten könnte, so die Kritiker, viele beobachtbaren Verhaltensweisen wie z.B. Altruismus nicht erklären (Siebenhüner, 1997, S. 55), da der homo oeconomicus seinen Mitmenschen gegenüber neutral ist. Dies mag in einer statischen Betrachtung einer Entscheidung richtig sein, jedoch befindet sich jedes Individuum fortwährend in einer Sequenz von Entscheidungssituationen wohlwollendes Verhalten kann dann als eine Versicherung verstanden werden, dass man mit späterem Wohlwollen der anderen rechnen kann. Freilich muss gewährleistet sein, dass die gegenseitige Zusicherung zukünftigen Wohlverhaltens glaubwürdig ist.

Eine andere rein funktionale Begründung von „altruistischen Verhaltensweisen“ ist die Annahme, dass durch dieses Verhalten private Güter quasi als Kuppelprodukte für den Geber entstehen. Solche Privatgüter könnten dann z.B. soziale Anerkennung sein, wie sie Holländer (1990) modelliert oder gestärkte Selbst-

Vgl. zu den Informationskosten Stigler (1961), sowie zur Bedeutung der Informationskosten im politischen Wettbewerb Downs (1957, S. 207ff). Die Relevanz der Informationsverarbeitungskosten, die durch kognitive Begrenztheit entstehen, unterstreicht Simon (1955, 1995).

29 Brennan und Buchanan (1993, S. 67) bezeichnen dies als Symmetrieargument. Es ist illegitim, für Marktverhalten und Nichtmarktverhalten von unterschiedlichen Verhaltensannahmen auszugehen, da sonst die Wirkungen durch Änderungen der Rahmenbedingungen nicht mehr von den Wirkungen der Differenzen in den Annahmen zu unterscheiden wären. 
achtung, wie sie Kirsch (1997, S. 85ff) darstellt. Möchte man diese privaten Güter besitzen, muss man sich so verhalten, als wäre man altruistisch.

Sen (1977, S. 92) argumentiert allerdings, dass der Mensch unterscheiden kann zwischen seinen offenbarten Präferenzen und seinen gewünschten Präferenzen, da seine Handlungen nicht nur seine eigene Wohlfahrt berücksichtigen müssen. Auch Becker (1992, S. 335ff) argumentiert, dass der Mensch durchaus unzufrieden mit seinen Präferenzen sein kann, jedoch gewissermaßen Gefangener seiner Erfahrungen ist. Wenn dem so ist, bedarf es einer Erweiterung zumindest des Präferenzbegriffs, möchte man menschliches Verhalten exakter modellieren. Dies wird in Abschnitt 2.4 erfolgen.

Aufgrund der fundamentalen Symmetrieannahme, dass sich Menschen unabhängig von der Aufgabenstellung gemäß ihren Präferenzen und Restriktionen in vorhersagbarer Weise an Umweltänderungen anpassen, lässt sich die ökonomische Analysemethode auch auf politische Entscheidungsprozesse anwenden. Dafür muss man fragen, welche Akteure an diesem Entscheidungsprozess mitwirken, welchen Restriktionen diese Akteure unterworfen sind und inwiefern die institutionellen Anreizsysteme einzelner Positionen typische Präferenzen befriedigen. Es sollen im folgenden zunächst die zentralen Akteure gekennzeichnet werden, um daran anschließend die Frage zu erörtern, wann es zu einer Konvergenz und wann es zu einer Divergenz der angebotenen Politikprogramme - auch ohne Globalisierung - kommt.

\subsubsection{Die zentralen Akteure der Politik}

In den in dieser Arbeit untersuchten Staaten ist die (dominierende) Herrschaftsform die repräsentative Demokratie. Diese Form politischer Entscheidungsfindung ist dadurch gekennzeichnet, dass der Souverän per Wahl Handlungsbevollmächtigte bestimmt, deren Aufgabe es ist, den Mehrheitswillen durchzusetzen. Es handelt sich hierbei also um eine typische Principal-Agent-Situation, bei der der Principal (Wähler) Entscheidungsrechte an den Agenten (Politiker) überträgt. Solche Konstellationen sind insbesondere dadurch geprägt, dass positive Kontrollkosten eine vollständige Überwachung des Agenten ökonomisch verwehren. Dem Agenten bleibt also stets ein diskretionärer Handlungsspielraum, den er zur Verfolgung seiner privaten Interessen ausnutzen kann. Für den Wähler ist die Kontrolle nicht zuletzt deshalb schwer, weil die politische Entscheidung für ihre Umsetzung der Bürokratie bedarf. Dies bedeutet aber, dass 
ein zweites nachgelagertes Principal-Agent-Problem zwischen den Politikern und der Bürokratie besteht. ${ }^{31}$

Schließlich steht dem Wähler zur effizienteren Interessenäußerung und durchsetzung die Möglichkeit offen, Interessengruppen zu bilden, die v.a. der Politik Informationen bereitstellen und so politische Entscheidungen zugunsten dieser Interessengruppen (bzw. der durch diese Gruppe vertretenen Wähler) beeinflussen. ${ }^{32}$

\subsubsection{Die Wähler}

Beschränkt man die Rolle der Wähler innerhalb einer indirekten Demokratie auf den Wahlakt, ${ }^{33}$ ergibt sich für den homo oeconomicus ein zweistufiges Optimierungsproblem. Zunächst muss eine Entscheidung hinsichtlich der Wahlteilnahme und - falls dies bejaht wird - hinsichtlich der zu wählenden Partei getroffen werden. Für die konkrete Wahlentscheidung vergleicht der Wähler den auf den Wahltag abdiskontierten erwarteten (Netto-)Nutzenstrom, den die einzelnen zur Wahl stehenden Programme versprechen. Die Differenz der Nutzenwerte beschreibt nach Downs (1957, S. 39) das Parteidifferential. ${ }^{34}$ Ist das Parteidifferential positiv, hat der Wähler also eine Präferenz für die Regierungspartei, wird er für diese stimmen. Dabei gehen zahlreiche ökonomische und soziologische Faktoren in die Erwartungsbildung ein. Die ökonomischen Einflussfaktoren bestimmen die Wahlentscheidung mit, wenn der Wähler erstens die Änderung einer bestimmten ökonomischen Größe wahrnehmen kann, er zweitens diese

31 Die Rolle der Bürokratie ist für die weitere Analyse allerdings ohne Belang, daher wird sich hier auf die Akteure Politiker und Wähler beschränkt. Für eine umfassende ökonomische Analyse der Bürokratie vgl. u.a. Downs (1965) und Niskanen (1971, 1975).

32 Statt raumgreifender Darstellung der normativen und positiven Begründetheit von Interessengruppen wird auf die grundlegende Arbeit von Olson (1965) verwiesen.

Dies ist natürlich eine gewaltige Abstraktion der Realität, da eines der zentralen Merkmale liberaler Demokratien gerade die Möglichkeit ist, auch außerhalb des Wahlakts politisch aktiv zu werden. In Deutschland schützt Art. 5,I GG das Recht, der freien Meinungsäußerung und in Verbindung mit Art. 9,I GG das Recht Interessenvertretungen zu konstituieren. Die Beschränkung der politischen Teilnahme des Wählers auf den Wahlakt soll hier ausschließlich dazu dienen, zentrale Aspekte pointierter herauszuarbeiten.

34 Ist $\mathrm{U}_{\mathrm{t}}^{\mathrm{A}}$ der Nutzen, den das Individuum aus dem Programm der Regierungspartei zum Zeitpunkt $t$ erwartet und $U_{t}^{B}$ der erwartete Nutzen aus dem Programm der Oppositionspartei zum Zeitpunkt $t$, dann ergibt sich das Parteidifferential PD unter Berücksichtigung der individuellen Zeitpräferenzrate $\mathrm{r}$ aus:

$P D=\int_{1} U_{t}^{A} e^{-r t} d t-\int_{1} U_{t}^{B} e^{-r t} d t$ 
Änderung als relevant erachtet und er drittens diese Änderung mit einer konkreten Partei in Verbindung bringen kann (Stalder, 1995, S. 94ff). Hierbei ist hervorzuheben, dass sowohl die Wahrnehmung der Realität als auch die Entscheidung über die Relevanz der Größe und auch die Verbindung der Änderung mit einer bestimmten Partei subjektiv ist.

Die vorgeschaltete Entscheidung, tatsächlich zur Wahl zu gehen, trifft das Individuum dadurch, dass es die mit dem Wahlakt verbundenen Kosten mit den durch die Wahlteilnahme gesicherten Nutzen vergleicht. Nur wenn die Nutzen die Kosten übersteigen, geht ein rationales Individuum auch tatsächlich wählen. Bei der Wahl fallen Opportunitätskosten aufgrund des Zeitaufwands des Wahlakts und gegebenenfalls Informationskosten an. Dem stehen direkte Nutzen entgegen, die dann anfallen, wenn die eigene abgegebene Stimme die Wahl im Sinne des Individuums entscheidet - operationalisierbar als Produkt der Wahrscheinlichkeit, die entscheidende Stimme abgegeben zu haben und dem Betrag des Parteidifferentials. ${ }^{35}$ Auch wenn die Wahlkosten als sehr gering eingeschätzt werden, folgt für rationale Individuen aus diesem einfachen Kalkül, dass nur bei sehr hohen Parteidifferentialen und der Annahme eines sehr knappen Wahlergebnisses der Wahlakt rational ist.

Geht man z.B. davon aus, dass die Wahlkosten nur bei 20 DM liegen (entspricht etwa dem Nettostundenlohn eines wissenschaftlichen Mitarbeiters), dann zeigt Tabelle 2.2 für die Bundestagswahlen und die Bürgerschaftswahlen in Hamburg, wie hoch für ein repräsentatives Individuum das kritische Parteidifferential in DM sein muss, damit zumindest Indifferenz gegenüber dem Wahlakt besteht. Es wird eindrucksvoll deutlich, dass im Falle einer Bundestagswahl selbst dort, wo das Individuum nur von geringfügig unterschiedlich verteilten Gewinnchancen der teilnehmenden Parteien ausgeht $(q=0,501)$, ein Parteidifferential vorliegen müsste, das um ein Vielfaches das Weltsozialprodukt übersteigt. ${ }^{36}$

35 Da das Parteidifferential in enger Anlehnung an Downs (1957) als die Differenz zwischen dem erwarteten Nutzenstrom aus dem Regierungsprogramm und dem erwarteten Nutzenstrom aus dem Oppositionsprogramm definiert wurde, wird hier vom absoluten Betrag des Parteidifferentials gesprochen, um der Nutzengröße auch im Falle einer Oppositionspräferenz einen positiven Wert zuweisen zu können. Wenn im folgenden von Parteidifferential gesprochen wird, ist damit stets der absolute Betrag des Parteidifferentials gemeint.

Das kritische Parteidifferential PD* ist jene Größe, die bewertet mit der Wahrscheinlichkeit $\mathrm{p}$, wahlentscheidend zu sein, gerade den Wahlkosten $\mathrm{K}$ entspricht ( $\left.\mathrm{p} \cdot \mathrm{PD}^{*}=\mathrm{K}\right)$, und lässt sich wie folgt approximieren.

$\mathrm{PD}^{*}=\frac{K \cdot \sqrt{\pi \cdot \frac{N-1}{2}}}{\left[4 \cdot\left(q-q^{2}\right)\right]^{\frac{N-1}{2}}}$

Dabei bezeichnet $\mathbf{N}$ die (ungerade) Wählerzahl und qagibudie Wahrscheiln7iệheiteiner

Downloaded from PubFactory at 01/11/2019 07:52:01AM 
Tabelle 2.2 : Das kritische Parteidifferential PD*

Wahrscheinlichkeit, dass ein anderer für die andere Partei stimmt (q)

\begin{tabular}{lrrr} 
& $\mathrm{q}=0,5$ & $\mathrm{q}=0,501$ & $\mathrm{q}=0,51$ \\
\hline Bundesrepublik & $48.848,21$ & $1,1910^{58}$ & $\rightarrow \infty$ \\
Hamburg & $27.587,56$ & $315.576,32$ & $5,4410^{109}$ \\
\hline
\end{tabular}

Quelle: Bundeswahlleiter (1999a, 1999b); eigene Berechnungen gemäß der Formel für PD* aus Fußnote 36.

Es scheint also i.d.R. rational zu sein, sich nicht an Wahlen zu beteiligen. Die Beobachtung, dass dennoch die Mehrheit der wahlberechtigten Bevölkerung insbesondere an nationalen Wahlen teilnimmt, ging als Wahlparadoxon in die Literatur ein. Mittlerweile gibt es zahlreiche theoretische Ansätze, die versuchen, das Wahlparadoxon aufzulösen. Die Diskussion dieser Vorschläge soll in einem etwas breiteren Kontext in Abschnitt 2.3.2 erfolgen. Da die Struktur des Wahlparadoxons der beobachtbaren freiwilligen Bereitstellung öffentlicher Güter ähnlich ist, soll zunächst das Freifahrerproblem erläutert werden, um daran im Anschluss aus der Masse der empirischen Indizien abzuleiten, dass eine Integration des Wert- und Ideologiebegriffs in das Konzept des homo oeconomicus nötig erscheint.

\subsubsection{Die Politiker}

Der Großteil der polit-ökonomischen Literatur subsumiert unter dem Begriff Politiker alle diejenigen politischen Akteure, die über eine Wahl ein repräsentatives politisches Mandat erhalten haben, also alle Parlamentarier sowie alle Regierungsmitglieder (Stalder, 1995, S. 97).

Die traditionelle Theorie der Wirtschaftspolitik (welfare economics) modellierte Politiker als wohlwollende Planer, deren einziges Ziel es ist, eine gesamtgesellschaftliche Wohlfahrtsfunktion zu maximieren. In einer derartigen Welt wäre die Aufgabe der Politischen Ökonomie nur aufzuzeigen, wie die einzelnen Elemente der Wohlfahrtsfunktion zu spezifizieren sind, um dann unterschiedliche Instrumente hinsichtlich ihrer gesamtgesellschaftlichen Wohlfahrtswirkung zu diskutieren. ${ }^{37}$

abweichenden Stimmabgabe eines zufällig ,gezogenen“ anderen Wählers an. Die ausführliche Herleitung der Formel findet man z.B. bei Söllner (1998, S. 14).

37 Heute geht man im allgemeinen davon aus, dass es nicht möglich ist, eine gesamtwirtschaftliche Wohlfahrtsfunktion zu konstruieren. Erstens scheitert dies an der Unmöglichkeit, Präferenzen interpersonell zu vergleichen. Zweitens ist seit Arrow (1963) bekannt,

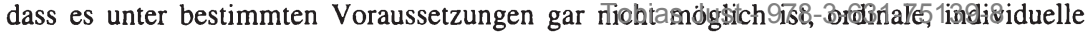


Ein solches Politikverständnis wäre aber nur durch argumentative Akrobatik mit dem Konzept des homo oeconomicus zu verbinden und muss daran scheitern, die Wirklichkeit zu erklären, da diese gerade dadurch gekennzeichnet ist, dass selbst leicht realisierbare Pareto-Verbesserungen oftmals nicht durchgeführt werden (Stiglitz, 1998, S. 5). Es scheint also nicht nur methodisch konsistent, sondern auch empirisch gerechtfertigt, von Individuen in der Politik auszugehen, die danach streben, ihren eigenen Nutzen zu maximieren.

Solche Nutzen sind in der Politik u.a. durch Ansehen, Macht und Einkommen erzielbar, ${ }^{38}$ deren Realisierbarkeit jeweils daran geknüpft ist, dass sich der Politiker auf dem politischen Markt durchsetzt. Da Wähler analog ihre Stimme eigennutzorientiert der Partei geben, deren Programm ihren Präferenzen am ehesten entspricht, wäre für den Politiker der Gewinn der nächsten Wahl oberstes Ziel, da ihn dies erst in die Lage versetzt, eigene Ziele zu verfolgen (Downs, 1957, S. 28). Ob dies jedoch zwangsläufig stimmenmaximierendes Verhalten bedeuten muss, wie es Downs (1957, S. 31) schlussfolgert, wurde in der Literatur nicht einheitlich beantwortet. Alternative Strategien könnten z.B. die Maximierung des Stimmenanteils, die Maximierung der Stimmenmehrheit oder das Erreichen einer minimalen Stimmenmehrheit sein. ${ }^{39}$

Politiker bieten also Programme an, die ihre Wiederwahl sichern helfen. Diese Grundannahme hat weitreichende Auswirkungen auf die Programmgestaltung und die Dynamik der Parteiprogramme im politischen Wettbewerb.

\subsubsection{Konvergenz oder Divergenz der Wahlprogramme}

\subsubsection{Die Medianwählerhypothese}

Aus der Charakterisierung von Wählern und Parteien kann Downs (1957, S. 114ff) die Dynamik des Parteienwettbewerbs herleiten. Innerhalb eines engen Annahmenrahmens führt das Downs'sche Modell des Parteienwettbewerbs in Anlehnung an die Überlegung von Hotelling (1929) zum Standortwettbewerb

Präferenzen widerspruchsfrei und unabhängig vom Entscheidungsverfahren zu aggregieren.

Barro (1973) unterscheidet den Begriff des Einkommens in eine Gehaltskomponente und eine Komponente, die dem Politiker durch die Befriedigung verschiedener Interessen zuflieBt.

39 Für eine ausführlichere Liste und eine Diskussion der einzelnen Strategien, vgl. Dinkel (1977, S. 43). 
dazu, dass stimmenmaximierende Parteien identische Wahlprogramme anbieten werden.

Innerhalb des einfachen Downs'schen Modells wird angenommen, Wähler besäßen vollständige Information, es fänden permanent Wahlen statt, es bestünde 100\%ige Wahlbeteiligung und die normalverteilten Präferenzen der Wähler sowie die Wahlprogramme zweier Parteien könnten eindeutig entlang eines gemeinsamen stetigen Trennkriteriums (Links-Rechts-Schema) angeordnet werden. Dass in einer solchen 2-Parteien-Welt stimmenmaximierendes Verhalten der Parteien zwangsläufig dazu führen muss, dass die Parteien ihr Programm an den Präferenzen des Medianwählers M orientieren, veranschaulicht Abbildung 2.2 .

\section{Abbildung 2.2 : Die Medianwählerhypothese}

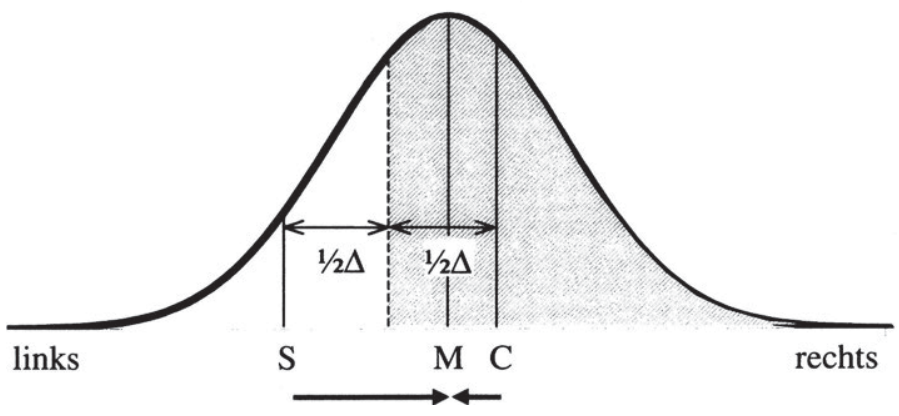

Quelle: In Anlehnung an Downs (1957, S. 118).

Würden die rechte Partei $\mathrm{C}$ und die linke Partei $\mathrm{S}$ ihre Programme wie in der Abbildung dargestellt mit einer ideologischen Differenz von $\Delta$ den Wählern präsentieren, würde C die Wahl gewinnen, da ihr die schraffierte Fläche an Wählerstimmen zufielen, wohingegen $S$ nur die verbleibende weiße Fläche an Wählern gewinnen könnte. Da der Wahlsieg als einziges Ziel der Parteien angenommen wurde, müsste sich $S$ ideologisch in Richtung des Medianwählers neu positionieren. Dies führt dann wiederum dazu, dass sich auch $C$ in Richtung $M$ bewegen muss, bis beide Parteiprogramme in $\mathrm{M}$ konvergieren und so ein Gleichgewicht erreicht wird.

Dieses Resultat ist natürlich eng an die restriktiven Annahmen gebunden, wie bereits Downs erkannt hat. So wurde die Medianwählerhypothese zwar in den folgenden Jahren Grundlage zahlreicher polit-ökonomischer Arbeiten, jedoch wuchs gleichzeitig eine umfangreiche Literatur, in der argumentiert wird, dass 
eine vollständige Konvergenz der Parteiprogramme unter realistischeren Annahmen nicht erfolgen wird.

\subsubsection{Langfristige Divergenz der Parteiprogramme}

Eine der auffälligsten Unzulänglichkeiten des einfachen Downs-Modells ist die Annahme, dass nur zwei Parteien konkurrieren. Zwar gibt es 2-Parteiensysteme, jedoch sind die meisten Parteiensysteme in Industriestaaten durch die Konkurrenz und Parlamentsrepräsentanz mehrerer Parteien charakterisiert. Downs (1957, S. 122ff) kommt zu dem Ergebnis, dass es stets ein langfristiges Gleichgewicht nach Neueintritt von Parteien gibt. Die Anzahl der Parteien richtet sich dann nach der Wählerverteilung - v.a. nach der Anzahl der Modi - und nach der Höhe der Markteintrittsbarrieren für neue Parteien. Selten (1971) hat diese Lösung durch die Aufstellung von sechs Gleichgewichtsbedingungen für eine beliebige Anzahl von Parteien geliefert. ${ }^{40}$ Können die beiden bereits etablierten Parteien den drohenden Markteintritt einer dritten Partei antizipieren, werden sie ihre ideologischen Programme so ausrichten, dass es erst gar nicht zu einem Eintritt kommt. Jedoch ist auch diese Prognose nur unter recht restriktiven Annahmen gültig (Bernholz und Breyer, 1994, S. 114).

Eine weitere Unzulänglichkeit des Downs-Modells, an der besonders Politikwissenschaftler Anstoß nahmen, ist die Reduktion der Trennlinien in einer Gesellschaft auf eine einzige Dimension. Dies bedeutet, dass man zum einen unterstellt, alle politischen Fragen seien ,räumlich“ exakt einzuordnen und dass man zum anderen nicht zulässt, dass bei einer Wahl immer mehrere Entscheidungsdimensionen relevant sein können. ${ }^{41}$ Lässt man mehrere Dimensionen zu, so wird das Gleichgewicht nicht mehr eindeutig sein können. Es kann zwar unter bestimmten Annahmen zu einer Mediankonvergenz in allen Dimensionen kommen, jedoch reagiert dieses Ergebnis nicht robust, falls man eine der Annahmen leicht modifiziert (Dinkel, 1977, S. 73ff; Mueller, 1989, S. 196ff). Diese Aussage kann sogar noch dahingehend erweitert werden, dass bisweilen Unsicherheit hinsichtlich der relevanten Dimensionen bestehen kann; es können neue Dimensionen entstehen, alte obsolet werden oder Dimensionen miteinander verschmelzen. In einer derart gekennzeichneten politischen Welt ist ein MedianwählerGleichgewicht in allen Dimensionen sehr unwahrscheinlich, da Politiker gege-

Seine Lösung gilt allerdings nicht für ein System mit drei Parteien. Im Falle dreier Parteien müsste man differenzieren. In einem Mehrheitswahlrecht käme es zu gar keiner Gleichgewichtslösung, in einem System mit proportionalem Wahlrecht würden sich alle drei Parteien im Median begegnen (Bernholz und Breyer, 1994, S. 121ff). 
benenfalls nicht einmal die relevante Dimension kennen (Kirsch, 1997, S. 249ff).

Aber selbst im einfachen Zwei-Parteien-Konkurrenzmodell mit nur einer relevanten Dimension ist eine Konvergenz der Parteiprogramme nicht sicher, falls zwei plausible Annahmen für den politischen Wettbewerb getroffen werden:

1. Es besteht Ungewissheit hinsichtlich der genauen Verortung der Wählerpräferenzen und folglich auch hinsichtlich der Präferenz des Medianwählers.

2. Die Einnahme der Medianwählerposition ist für die Partei mit Nutzenverzichten verbunden, die gegen einen möglichen Wahlgewinn abgewogen werden müssen. Solche Nutzenverzichte sind in dreierlei Form vorstellbar. Erstens könnte der Partei eine bestimmte Wählerschicht angehören, die einer Wahl fernbleibt, falls die bislang unterstützte Partei sich zu stark von der ideologischen Position entfernt. ${ }^{42}$ Dem Versuch, in der Mitte Wähler zu gewinnen, stehen Wählerverluste an den Rändern gegenüber. Dieser Wählerverlust fällt umso stärker aus, je geringer der Wähler die ideologische Neupositionierung einer Partei schätzt, also umso bedeutender die Tatsache ist, dass Wahlen nicht isolierte Ereignisse sind, sondern Einzelelemente innerhalb einer Sequenz von Wahlen.

Zweitens könnten „bedeutende“ Wähler damit drohen, z.B. finanzielle Unterstützung zurück zu halten, falls die bisher unterstützte Partei ihre Position zu sehr in Richtung Median korrigiert. Eine Berücksichtigung dieser Kosten muss dazu führen, dass die Partei nicht vollständig zum Median tendiert.

Schließlich könnten den Politikern Nutzen aus der Realisierung einer ganz konkreten Politik entstehen; sie wären ideologisch im Sinne einer Zielorientierung gebunden. Ein Verlassen ihrer ideologischen Grundposition, um eine Wahl zu gewinnen, muss dazu führen, dass sie Nutzenverzichte hinnehmen müssen, weil sie eine Politik verfolgen, die nicht ihren Präferenzen entspricht.

Ein verwandtes Argument ist, dass Spitzenpolitiker durch parteiinterne Konkurrenz sich zunächst am parteiinternen Median ausrichten müssen. Dieser fällt aber stets dann nicht mit dem gesellschaftlichen Median zusammen, wenn die Parteimitglieder eine eindeutige Politikpräferenz haben. Das Argument bedarf also der Annahme, dass Parteimitglieder ideologisch prädisponiert sind (Wittman, 1983; Calvert, 1985). Calvert (1985, S. 80) zeigt al-

42 Die Wähler, die sich der Wahl enthalten, folgen dem oben dargestellten Wählerkalkül. Da der Wahlakt selbst mit Kosten verbunden ist, ist es nicht rational, diese Kosten in Kauf zu nehmen, wenn das politische Programm zu weit von den eigenen Präferenzen entfernt ist. Im Falle der Medianwählerkonvergenz wäre jegliche Wahlteilnahme irrational, da die Parteiprogramme identisch sind und demnach für keinen Wähler ein positives Parteidifferential vorläge. Die Kosten würden dann stets die Nutzen überstegigen 
lerdings, dass die Ideologieorientierung allein noch keine Abweichung vom Medianwählerpostulat erzwingt. Erst wenn signifikante Unsicherheit hinsichtlich der Wählerverteilung besteht, werden die Programme divergieren.

Alle diese Punkte lassen sich einfach formalisieren. ${ }^{43} \mathrm{Zwei}$ Parteien $\mathrm{C}$ und $\mathrm{S}$ besitzen klare Politikpräferenzen, wobei die ideale Politik R der C-Partei rechts vom Median, die präferierte Politik L der S-Partei hingegen links vom Median liegen möge.

$$
\mathrm{L}<\text { Median }<\mathrm{R}
$$

Der realisierte Nutzen $U_{S}$ und $U_{C}$ der Parteien hängt davon $a b$, wie weit die später implementierte Politik x von ihrer idealen Vorstellung abweicht. Ihre jeweiligen Nutzenfunktionen seien strikt konkav und haben ihren Modus in $\mathrm{L}$ respektive R. Der konkrete Verlauf der Nutzenfunktion spiegelt die Intensität der ideologischen Position der Partei und deren Geldgeber wider. Je stärker die ideologische Bindung an die Position L bzw. R ist, umso größer sind die Nutzenverzichte im Falle eines $A b w e i c h e n s$ von $L$ bzw. $R$ und umso steiler verläuft die Nutzenfunktion. Der angestrebte direkte Nutzen aus dem Wahlsieg (Einkommen, Macht) wird mit Û bezeichnet und sei für beide Parteien gleich hoch. ${ }^{44}$

Gibt es nun Unsicherheit bezüglich der genauen Wählerverteilung, werden die Parteien Programme $\mathrm{x}_{\mathrm{C}}$ und $\mathrm{x}_{\mathrm{S}}$ anbieten, die nicht unbedingt mit ihren präferierten Positionen $\mathrm{L}$ und $\mathrm{R}$ übereinstimmen müssen. Die Wahrscheinlichkeit eines Wahlsiegs hängt dann von der Wählerverteilung und der relativen Lage von $\mathbf{x}_{C}$ und $\mathrm{x}_{S} \mathrm{ab}$. Die Wahrscheinlichkeit eines Sieges von $C$ lässt sich dann wie folgt formulieren: ${ }^{45}$

$$
\mathrm{P}=\mathrm{P}\left(\mathrm{x}_{\mathbf{S}} ; \mathrm{x}_{\mathrm{C}}\right)
$$

Es wird angenommen, dass, von identischen Median-Programmen ausgehend, sich die Siegchancen für die rechte Partei identisch verändern, wenn die linke Partei nach links oder die rechte Partei um denselben Abstand nach rechts rückt.

43 Vgl. Wittman (1983) und Calvert (1985) für ausführlichere Darstellungen und Bernholz und Breyer (1994, S. 115ff) für eine gute Lehrbuchdarstellung. Hier wird im wesentlichen der Präsentation von Alesina und Rosenthal (1995, S. 19ff) gefolgt.

44 Diese Annahme ist keineswegs zwingend, vereinfacht aber die Darstellung, ohne an den Aussagen etwas zu ändern.

45 Analog wäre dann die Wahrscheinlichkeit eines Wahlsiegs von $S$ durch: $\left[1-P\left(x_{S} ; x_{C}\right)\right]$ gegeben. In diese Wahrscheinlichkeitsfunktion kann auch die Wirkung von Nichtwählern integriert werden.

Tobias Just - 978-3-631-75139-8 
Fügt man alle diese Annahmen zusammen, lässt sich das Maximierungsproblem der Erwartungsnutzen $\left(E_{C}\right.$ und $\left.E_{S}\right)$ für beide Parteien wie folgt darstellen:

$$
\begin{aligned}
& \operatorname{Max}_{\mathrm{x}_{\mathrm{C}}} \mathrm{EU}_{\mathrm{C}}=\gamma\{\underbrace{\mathrm{P}\left(\mathrm{x}_{\mathrm{s}} ; \mathrm{x}_{\mathrm{C}}\right) \mathrm{U}_{\mathrm{C}}\left(\mathrm{x}_{\mathrm{C}}\right)}+[\underbrace{\left.1-\mathrm{P}\left(\mathrm{x}_{\mathrm{s}} ; \mathrm{x}_{\mathrm{C}}\right)\right] \mathrm{U}_{\mathrm{C}}\left(\mathrm{x}_{\mathrm{s}}\right)}\}+(1-\gamma) \mathrm{P}\left(\mathrm{x}_{\mathrm{S}} ; \mathrm{x}_{\mathrm{C}}\right) \hat{\mathrm{U}} \\
& \text { Ideolog. Nutzen Ideolog. Nutzen für } \\
& \text { für } \mathrm{C} \text {, falls } \mathrm{C} \quad \mathrm{C} \text {, falls } \mathrm{S} \text { gewinnt. } \\
& \text { gewinnt. }
\end{aligned}
$$

Der Parameter $\gamma$ (mit $0 \leq \gamma \leq 1$ ) legt das relative Gewicht des Partisanziels zum Eigenziel des Politikers fest. ${ }^{46}$ Reine Wahlsiegorientierung liegt dann vor, wenn $\gamma=0$ ist. Für $\mathrm{S}$ lässt sich der Erwartungsnutzen analog formulieren:

$$
\underset{\mathrm{x}_{\mathrm{S}}}{\mathrm{ax}_{\mathrm{S}}} \mathrm{EU}_{\mathrm{S}}=\gamma\left\{\mathrm{P}\left(\mathrm{x}_{\mathrm{S}} ; \mathrm{x}_{\mathrm{C}}\right) \mathrm{U}_{\mathrm{S}}\left(\mathrm{x}_{\mathrm{C}}\right)+\left[1-\mathrm{P}\left(\mathrm{x}_{\mathrm{S}} ; \mathrm{x}_{\mathrm{C}}\right)\right] \mathrm{U}_{\mathrm{S}}\left(\mathrm{x}_{\mathrm{S}}\right)\right\}+(1-\gamma)\left[1-\mathrm{P}\left(\mathrm{x}_{\mathrm{S}} ; \mathrm{x}_{\mathrm{C}}\right)\right] \hat{\mathrm{U}}
$$

Es lässt sich zeigen (Calvert, 1985), dass nur partielle Annäherung stattfindet und die Parteien gleichgewichtige Programme $\hat{\mathrm{x}}_{C}$ und $\hat{\mathrm{x}}_{S}$ mit folgender relativer Position anbieten werden:

$$
\mathrm{L}<\hat{\mathrm{x}}_{\mathrm{S}}<\operatorname{Median}<\hat{\mathrm{x}}_{\mathrm{C}}<\mathrm{R}
$$

Dass identische Programme kein Gleichgewicht darstellen können, lässt sich sehr einfach zeigen, indem man die ersten Ableitungen von (2.3) und (2.4) bildet, um das Maximierungsproblem zu lösen.$^{47}$ Wird im folgenden der Ausdruck $\mathrm{P}\left(\mathrm{x}_{\mathrm{S}} ; \mathrm{x}_{\mathrm{C}}\right)$ durch $\mathrm{P}(\bullet)$ ersetzt, um die Darstellung übersichtlicher zu gestalten, ergibt die erste Ableitung von $\mathrm{EU}_{\mathrm{C}}$ nach $\mathrm{x}_{\mathrm{C}}$ für die rechte Partei:

46 Natürlich kann dieser Gewichtungsparameter für beide Parteien unterschiedlich sein.

47 Die Argumentation erfolgt in Anlehnung an die Darstellung bei Bernholz und Breyer (1994, S. 115ff). 


$$
\begin{aligned}
\frac{\partial \mathrm{EU}_{\mathrm{C}}}{\partial \mathrm{x}_{\mathrm{C}}} & =\gamma\left[\mathrm{P}(\bullet) \frac{\partial \mathrm{U}_{\mathrm{C}}\left(\mathrm{x}_{\mathrm{C}}\right)}{\partial \mathrm{x}_{\mathrm{C}}}+\frac{\partial \mathrm{P}(\bullet)}{\partial \mathrm{x}_{\mathrm{C}}}\left(\mathrm{U}_{\mathrm{C}}\left(\mathrm{x}_{\mathrm{C}}\right)-\mathrm{U}_{\mathrm{C}}\left(\mathrm{x}_{\mathrm{S}}\right)\right)\right] \\
& +(1-\gamma)\left(\frac{\partial \mathrm{P}(\bullet)}{\partial \mathrm{x}_{\mathrm{C}}}\right) \cdot \hat{\mathrm{U}}=0
\end{aligned}
$$

In diesem Ausdruck werden bereits sehr gut die entgegengesetzten Kräfte deutlich. Zum einen führt eine marginale Bewegung der rechten Partei vom Median in Richtung ihrer präferierten Position zu einer Nutzensteigerung bewertet mit der Wahrscheinlichkeit des Wahlsiegs $\left(\mathrm{P}(\bullet) \cdot \frac{\partial \mathrm{U}\left(\mathrm{x}_{\mathrm{C}}\right)}{\partial \mathrm{x}_{\mathrm{C}}}>0\right)$. Zum anderen reduziert sich durch diese Bewegung die Wahrscheinlichkeit des Gewinns der Wahl, was wiederum zu Nutzeneinbußen führt, falls die Programme sich unterscheiden $\left(\frac{\partial \mathrm{P}(\bullet}{\partial \mathrm{x}_{\mathrm{C}}}\left(\mathrm{U}_{\mathrm{C}}\left(\mathrm{x}_{\mathrm{C}}\right)-\mathrm{U}_{\mathrm{C}}\left(\mathrm{x}_{\mathrm{S}}\right)\right)<0\right.$, da $\left.\frac{\partial \mathrm{P}(\bullet)}{\partial \mathrm{x}_{\mathrm{C}}}<0\right)$ und direkte Nutzen aus der Amtsübernahme entstehen $\left(\left(\frac{\partial \mathrm{P}(\bullet)}{\partial \mathrm{x}_{\mathrm{C}}}\right) \cdot \hat{\mathrm{U}}<0\right)$.

Für die linke Partei lautet die erste Ableitung analog:

$$
\begin{aligned}
\frac{\partial \mathrm{EU}_{\mathrm{S}}}{\partial \mathrm{x}_{\mathrm{S}}} & =\gamma\left[(1-\mathrm{P}(\bullet)) \cdot \frac{\partial \mathrm{U}_{\mathrm{S}}\left(\mathrm{x}_{\mathrm{S}}\right)}{\partial \mathrm{x}_{\mathrm{S}}}+\frac{\partial \mathrm{P}(\bullet)}{\partial \mathrm{x}_{\mathrm{S}}}\left(\mathrm{U}_{\mathrm{s}}\left(\mathrm{x}_{\mathrm{C}}\right)-\mathrm{U}_{\mathrm{S}}\left(\mathrm{x}_{\mathrm{S}}\right)\right)\right] \\
& -(1-\gamma)\left(\frac{\partial \mathrm{P}(\bullet)}{\partial \mathrm{x}_{\mathrm{S}}}\right) \cdot \hat{\mathrm{U}}=0
\end{aligned}
$$

Ein Gleichgewicht kann bei diesen beiden Bedingungen nur existieren, wenn sich die beiden Programme unterscheiden. Bei Identität der Programme (im Median) würden sich die Maximalbedingungen nämlich wie folgt verkürzen:

$$
\frac{\partial \mathrm{EU}_{\mathrm{C}}}{\partial \mathrm{x}_{\mathrm{C}}}=\gamma\left[\mathrm{P}(\bullet) \cdot \frac{\partial \mathrm{U}_{\mathrm{C}}\left(\mathrm{x}_{\mathrm{C}}\right)}{\partial \mathrm{x}_{\mathrm{C}}}\right]+(1-\gamma)\left(\frac{\partial \mathrm{P}(\bullet)}{\partial \mathrm{x}_{\mathrm{C}}}\right) \cdot \hat{\mathrm{U}}=0
$$

und 


$$
\frac{\partial \mathrm{EU}_{\mathrm{S}}}{\partial \mathrm{x}_{\mathrm{S}}}=\gamma\left[(1-\mathrm{P}(\bullet)) \cdot \frac{\partial \mathrm{U}_{\mathrm{S}}\left(\mathrm{x}_{\mathrm{S}}\right)}{\partial \mathrm{x}_{\mathrm{S}}}\right]-(1-\gamma)\left(\frac{\partial \mathrm{P}(\bullet)}{\partial \mathrm{x}_{\mathrm{S}}}\right) \cdot \hat{\mathrm{U}}=0
$$

Da der erste Summand von (2.8) auf jeden Fall ein positives Vorzeichen hat, kann (2.8) nur erfüllt sein, wenn der zweite Summand ein negatives Vorzeichen besitzt, also wenn sich die Siegchancen der konservativen Partei bei einem Rechtsruck reduzieren. Da dies aber gleichzeitig bedeutet, dass der zweite Summand von (2.9) dann (inklusive Minuszeichen) ebenfalls ein negatives Vorzeichen hätte, ${ }^{48}$ kann (2.9) nicht mehr erfüllt sein, da der erste Summand von (2.9) auf jeden Fall ein negatives Vorzeichen besitzt. Die Identität der Programme im Median kann also kein Gleichgewicht gewesen sein, da die Gleichgewichtsbedingungen (2.8) und (2.9) nicht simultan bei identischen Programmen gelten können.

\section{Abbildung 2.3 : Divergenz der Programme bei Parteiideologie und Unsicherheit}

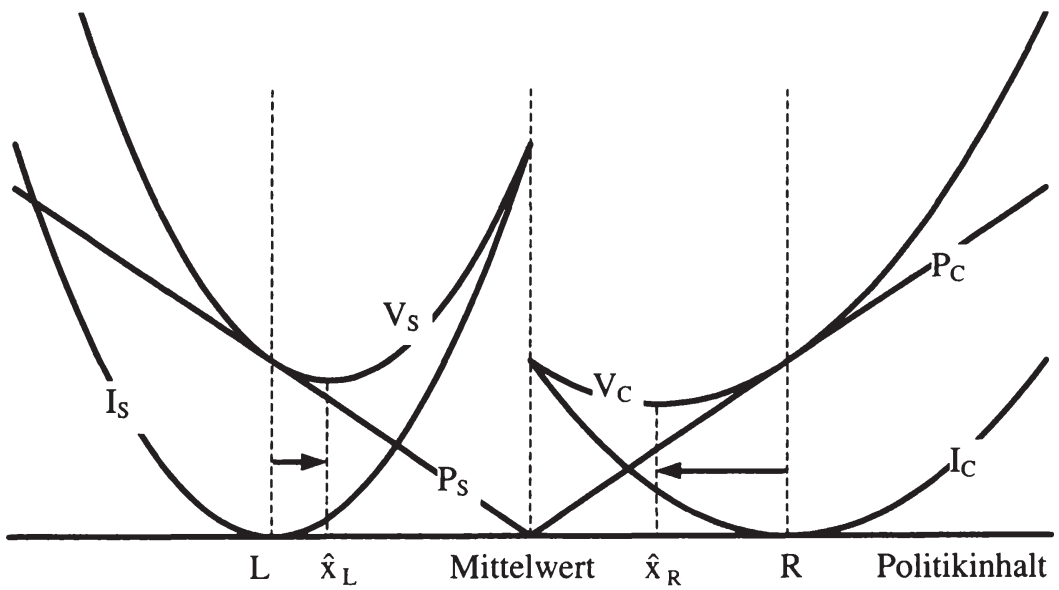

Quelle: Eigene Darstellung.

48

In der Medianposition reduziert ein Rechtsruck die Siegwahrscheinlichkeit der Rechtspartei in gleichem Maße, wie sie ein Rechtsruck der Konkurrenzpartei nach rechts erhöhen würde. Es muss also gelten:

$$
\frac{\partial \mathrm{P}(\bullet)}{\partial \mathrm{x}_{\mathrm{c}}}=-\frac{\partial \mathrm{P}(\bullet)}{\partial \mathrm{x}_{\mathrm{s}}}
$$


Abbildung 2.3 veranschaulicht die Überlegungen. Die Kurve $I_{S}\left(I_{C}\right)$ kennzeichnet die Nutzenverluste, die die S-Partei (C-Partei) tragen muss, wenn sie sich von ihrer ideologischen Präferenz L (R) in Richtung Mitte bewegt. Sie ist das Äquivalent zur oben erwähnten Nutzenfunktion. ${ }^{49}$ Die Kurve $\mathrm{P}_{\mathrm{S}}\left(\mathrm{P}_{\mathrm{C}}\right)$ stellt die Kosten eines möglichen Wahlverlustes dar, wenn S (C) nicht die Medianwählerstrategie wählt. Dieser Mittelpunkt sagt natürlich bei unsicherer Wählerverteilung nichts über den tatsächlichen Medianwähler aus, sondern lediglich über den erwarteten Medianwähler. Die Kurven $\mathrm{P}_{S}\left(\mathrm{P}_{\mathrm{C}}\right)$ drücken gerade diese Unsicherheit aus. Der Erwartungsnutzen der beiden Parteien wird also dort maximiert, wo die vertikale Summe V aus den Kurven I und P ihr Minimum erreicht.

Auch wenn diese Abbildung das Modell stark vereinfacht, ${ }^{50}$ können hieran die wichtigsten Implikationen sehr gut verdeutlicht werden.

- Je stärker der Partisaneffekt - entweder wegen bedeutender Geldgeber oder ideologischer Bindung -, umso steiler verläuft die I-Kurve und umso geringer ist der Konvergenzeffekt.

- Je eindeutiger die Informationen über den Medianwähler sind, umso teurer wird ein Abweichen von dieser erwarteten Position, umso steiler verläuft die P-Kurve und demnach wird die Konvergenz stärker ausfallen.

- Je ausgeprägter der eigentliche ideologische Standpunkt ist, umso weiter wird die eingenommene Wahlplattform vom vermeintlichen Medianwählerstandpunkt abweichen.

- Je stärker die Wähler ein Abrücken von der ursprünglichen Position mit Wahlabstinenz strafen, umso flacher verläuft die P-Kurve und umso eher werden die Parteien geneigt sein, auf ihren ideologischen Standpunkten zu verharren.

Zusammenfassend lässt sich also sagen, dass Parteiprogramme immer dann divergieren, wenn Unsicherheit hinsichtlich der genauen Verteilung der Wählerpräferenzen existiert und darüber hinaus der Bewegungsspielraum für Parteien durch ideologische Präferenzen bestimmter Wählergruppen, Geldgeber, der Parteibasis oder der Spitzenpolitiker eingeengt wird. Es scheint also, dass der Begriff der Ideologie für die mangelnde Konvergenz von Programmen entscheidende Bedeutung besitzt.

Da die Nutzenfunktion als strikt konkav definiert wurde, ist der Kurvenverlauf der Nutzenverluste strikt konvex.

50

In der Abbildung wird natürlich nicht die Interdependenz der beiden Policy-Plattformen deutlich. Die Wahrscheinlichkeit eines Wahlsieges hängt nämlich nicht nur von dem eigenen Programm, sondern auch vom Programm des Konkurrenten ab. Die dargestellten Kurven gelten nur für diese Gleichgewichtssituationias Just - 978-3-631-75139-8 
Allerdings wurde noch nicht geklärt, ob es ausreichend ist, Ideologie als Mittel zur Verfolgung materieller Interessen einzusetzen oder ob Ideologie ein eigenes Ziel im Sinne einer Werteorientierung darstellen muss. Wie im folgenden Kapitel gezeigt wird, gibt es gerade im Bereich der ökonomischen Analyse politischer Institutionen und menschlicher Interaktionen immer mehr Anzeichen dafür, dass eine breitere Definition des Ideologie- und Wertbegriffs auch für die ökonomische Theorie erforderlich ist.

\subsection{Notwendigkeit einer Berücksichtigung von Werten und Ideologien in der Ökonomie}

Handeln Individuen nun eigenverantwortlich und nutzenmaximierend, kommt es regelmäßig dann zu Friktionen, wenn Entscheidungen des einen Individuums den Entscheidungsraum oder direkt die Wohlfahrt eines anderen Wirtschaftssubjekts beeinflussen, ohne dass es für diese Einflussnahme eine marktliche Kompensation gäbe. Ökonomen sprechen dann von externen Effekten. Diese entstehen immer dann, wenn Eigentumsrechte ungenügend definiert wurden. Im Falle negativer Externalitäten bedeutet die Schädigung aufgrund fehlender Eigentumsrechte, dass Ressourcen fehlalloziiert werden und zwar zugunsten dessen, der den Schaden verursacht, da dieser für das Recht der Schädigung keinen Preis zu entrichten hat. Auch im Falle positiver Externalitäten kommt es zu Ineffizienzen, da der Produzent der Externalität die positive Wirkung nicht in sein Kalkül einbezieht und dementsprechend zu wenig von diesem externen Effekt zulässt.

\subsubsection{Individuelle Rationalität und kollektive Unvernunft}

Im Extremfall können Externalitäten bedeuten, dass das Ausschlussprinzip, das eine marktliche Preisbildung erst ermöglicht, nicht angewendet werden kann. Ein zusätzlicher Nutzer eines Gutes kann selbst bei Nichtleistung eines Kostenbeitrages nicht vom Konsum ausgeschlossen werden. ${ }^{51}$ In der finanzwissenschaftlichen Literatur werden öffentliche Güter darüber hinaus dadurch gekennzeichnet, dass Nichtrivalität im Konsum herrscht, dass also die Grenzkosten ei-

51 Eine etwas weiter gefasste Definition des Ausschlussprinzips besagt, dass der Ausschluss entweder nicht möglich ist - eine Internalisierung der externen Kosten ist auch durch Änderung des Systems der Eigentumsrechte nicht möglich - oder nicht gewollt ist (Blankart, 1998, S. 55). Fritsch, Wein und Ewers (1993, S. 255) resümieren allerdings, ,...dass es fast kein Gut gibt, für welches das Ausschlussprinzip grundsätzlich nicht angewandt werden kann." 
ner zusätzlichen Inanspruchnahme bei null liegen. ${ }^{52}$ Güter, die (zumindest zum Teil) durch diese Charakteristika beschrieben werden können, führen nun regelmäßig dazu, dass eine sich spontan bildende, marktliche Produktion nicht stattfindet, weil sich vollständig rationale Akteure in einer typischen Gefangenendilemmasituation befinden. Dieses Problem lässt sich einfach veranschaulichen.

Angenommen wird, dass zwei Individuen einen gleich großen Nutzen (U) aus der Realisation eines bestimmten öffentlichen Gutes ziehen können und die Produktionskosten (K) die Einzelnutzen, nicht aber die Summe der Einzelnutzen übersteigen ( $\mathrm{U}<\mathrm{K}<2 \mathrm{U}$ ). Geht man zunächst davon aus, dass die Transaktionskosten prohibitiv hoch sind, eine offene Kooperation also ausgeschlossen ist, so zeigt die Auszahlungsmatrix in Tabelle 2.3, dass für jedes rationale Individuum Nicht-Produktion die dominante Strategie ist, da jeder Akteur unabhängig von der Strategiewahl des anderen für die Freifahreroption (freerider) votieren wird. Die Einträge in den Feldern bezeichnen immer zuerst die Auszahlungen an Individuum $\mathrm{I}$ und dann die Auszahlungen an Individuum II. Es gilt: $\mathrm{U}>(\mathrm{U}-\mathrm{K} / 2)>0>(\mathrm{U}-\mathrm{K})$

Das Nash-Gleichgewicht wäre dann im Feld IV, das öffentliche Gut würde überhaupt nicht produziert, obwohl beide Akteure ein Interesse daran hätten, dass man gemeinschaftlich das Gut produziert und finanziert (Feld II). Man hätte das übliche Ergebnis eines Gefangenendilemmas, dass das Nash-Gleichgewicht nicht pareto-effizient ist.

Tabelle 2.3 : Auszahlungen in einem einfachen Gefangenendilemma

Individuum 2

\begin{tabular}{lccc}
\hline & Produktion & keine Produktion \\
\hline Produktion & $(\mathrm{U}-\mathrm{K} / 2) ;(\mathrm{U}-\mathrm{K} / 2)$ & $(\mathrm{U}-\mathrm{K}) ; \mathrm{U}$ & I \\
\hline $\begin{array}{l}\text { II } \\
\text { keine }\end{array}$ & & & \\
\hline
\end{tabular}

52 Es ist in der Literatur strittig, inwiefern beide Prinzipien zur Kennzeichnung eines öffentlichen Gutes vorliegen müssen. So sind beispielsweise Blümel, Pethig und von dem Hagen (1986) der Auffassung, dass lediglich das Kriterium der Nichtrivalität im Konsum konstitutives Merkmal öffentlicher Güter ist. Für eine detaillierte Diskussion der Kriterien vgl. u.a. Blankart (1998). 
Natürlich ist das oben dargestellte Dilemma bei zwei Spielern relativ einfach zu überwinden, wenn man keine prohibitiv hohen Transaktionskosten annimmt, denn dann würden beide Akteure miteinander kommunizieren und kooperieren, was dazu führen wird, dass eine vertragliche Verpflichtung zur Produktion Ergebnis einer solchen Kooperation wäre. ${ }^{53}$ Dies ist allerdings nur dann möglich, wenn das vertragliche Arrangement glaubhaft für alle Beteiligten ist, da ansonsten ein Problem öffentlicher Güter zweiter Ordnung entstünde (Coleman, 1991). Besteht keine Möglichkeit, vertragswidriges Verhalten zu sanktionieren, wäre vertragskonformes Handeln irrational. Die Einhaltung des Vertrages hätte selbst den Charakter eines öffentlichen Gutes. In kleinen Gruppen ist allerdings davon auszugehen, dass solche Sanktionsmechanismen durch positive oder negative selektive Anreize problemlos organisiert werden können (Coase, 1960, Demsetz, 1967).

Problematischer wird die Bereitstellung öffentlicher Güter in großen Kollektiven. Hier wird das Gefangenendilemma zum Sozialen Dilemma (Tullock, 1974), bzw. zur Tragödie der Allmende (Hardin, 1968). Jedes rationale Individuum hat einen starken Anreiz, seine wahren Präferenzen für ein öffentliches Gut zu verhüllen, in der Hoffnung, dass die anderen für die Bereitstellung Sorge tragen werden. Wenn dem so wäre, würde der eigene fehlende Beitrag an der Qualität des Gutes nur marginal etwas ändern. Gleichzeitig ist es dem Einzelnen nicht möglich, das gesamte Gut zu produzieren. Verhalten sich alle Akteure gemäß dieser Maxime, muss die individuelle Rationalität zu kollektiver Irrationalität führen. Obwohl alle an dem Gut interessiert sind, werden alle zu Trittbrettfahrern einer nicht stattfindenden Mitfahrgelegenheit.

Es ist angesichts dieser logischen Argumentation nun aber verwunderlich, dass die empirische Relevanz des strengen Trittbrettfahrerverhaltens zunehmend negiert wird. Der folgende Abschnitt fasst die wichtigsten Ergebnisse von Experimenten hinsichtlich des Freifahrerverhaltens zusammen, um daraus die Frage abzuleiten, was die Menschen dazu bewegt, entgegen dem prognostizierten Verhalten zu handeln. Dieser Überblick muss stark selektiv bleiben. Dawes (1980) berichtet, dass es bereits vor knapp 20 Jahren in der experimentellen Psychologie 1.000 Experimente zum Gefangenendilemma gab. Reine Gefangenendilemmaexperimente sind für diese Arbeit aber relativ uninteressant, da bei ihnen das geschilderte Freifahrerproblem unbedeutend ist. Folglich wird sich die folgende Darstellung auf Mehrpersonen-Experimente beschränken, bei denen die freerider-Problematik gravierender ist.

53 In obigen Beispiel kommt es immer dann zur Kooperation zwischen den beiden Spielern, wenn die individuellen Transaktionskosten $\mathrm{T}$ des Vertragsabschlusses und der Vertragsdurchsetzung kleiner sind als die Differenz der individuellen Nutzen und der halben Produktionskosten: $\mathrm{T}<\mathrm{U}-\mathrm{K} / 2$, denn dann ist gewährleistet, dass der Nettonutzen $(\mathrm{U}-\mathrm{K} / 2-\mathrm{T})$ positiv ist. 


\subsubsection{Ist der Mensch tatsächlich ein homo oeconomicus?}

Aus den Ausführungen in den Abschnitten 2.2 und 2.3.1 lässt sich eine Reihe empirisch überprüfbarer Hypothesen ableiten. Hier richtet sich der Fokus auf die drei folgenden Hypothesen:

1. Menschen werden sich nicht freiwillig bei der Produktion öffentlicher Güter beteiligen, sondern sich als Freifahrer betätigen.

2. Die Wahlbeteiligung wird bei rationalen Wählern sehr gering sein.

3. Politiker werden bemüht sein, diskretionäre Handlungsspielräume dahingehend auszunutzen, dass sie durch die Befriedigung spezieller Interessen ihr Einkommen und ihren Einfluss maximieren.

\subsubsection{Die Überprüfung der Freifahrerhypothese}

In der (Mikro-) Ökonomie hat sich die Methode des Laborexperiments, also der Versuch, Theorien durch weitreichende Kontrolle der relevanten Variablen innerhalb einer zwar nicht realen, jedoch realistischen Versuchsanordnung zu überprüfen, zwar seit den letzten dreißig Jahren sehr stark verbreitet, jedoch noch nicht die Bedeutung erlangt, die sie in anderen Sozialwissenschaften besitzt. Dies ist verwunderlich, da das Laborexperiment ermöglicht, einzelne Wirkungsmechanismen durch veränderte Experimentanordnungen genau zu extrahieren, also die ceteris paribus-Bedingung kontrolliert umzusetzen. ${ }^{54}$

Zur Überprüfung der Freifahrerhypothese wurden bereits zu Beginn der 70er Jahre von Psychologen Soziale-Dilemma-Situationen untersucht. In den folgenden Jahren wurden v.a. die Methoden verbessert, ohne die Ergebnisse radikal zu korrigieren. Eine sinnvolle Modellierung sollte dabei folgende Kriterien erfüllen:

- Die Gruppe muss hinreichend groß sein, und die Gruppenmitglieder müssen anonym sein.

- Die Auszahlungsmatrix muss genau definiert sein und sollte nicht auf Gütern, sondern monetären Auszahlungen basieren. So wird vermieden, dass

54 Die Überblicksartikel von Ledyard (1995), van Winden und Bosman (1996) und Weimann (1995) bieten eine anschauliche Diskussion der Vorteile von Laborexperimenten in der Ökonomie und zeigen übliche Kritikpunkte wie z.B. unzureichende Vereinfachung und Realitätsferne der Rahmenbedingungen der Experimente auf. Es sei angemerkt, dass sich der Anwendungsbereich ökonomischer Experimente stetig ausdehnt, sodass mittlerweile Experimente nicht nur innerhalb der Theorie kollektiven Handelns vorgenommen werden, sondern auch für die Risikotheorie, Theorie der Marktauktionen, Erwartungsbildung (Tietz, 1996, S. 124) sowie für die Theorie der Wahlentscheidungen und des Lobbying (van Winden und Bosman, 1996) an Bedeutung geyinnen 
unterschiedliches Verhalten nicht auf unterschiedliche Präferenzen hinsichtlich der produzierten Güter zurückzuführen ist. Außerdem sollte der Komplexitätsgrad des Versuchsaufbaus so gering wie möglich gehalten werden.

- Die ökonomischen Anreize müssen hinreichend groß sein. ${ }^{55}$

- Die Experimente dürfen kein one-shot-game sein, vielmehr müssen die Versuchspersonen die Möglichkeit zum Lernen besitzen.

Das einfache Modell zur Überprüfung der Freifahrerhypothese bildet die Struktur des Gefangenendilemmas ab (van Winden und Bosman, 1996, S. 56). Jeder Spieler erhält einen Geldbetrag, den er nach Belieben in eine private oder öffentliche Anlage investieren kann. Die Gesamtwohlfahrt wird dann maximiert, wenn alle Teilnehmer in das öffentliche Gut investieren; jedoch kann ein individueller Spieler seine Auszahlung maximieren, wenn er allein in die private Anlage investiert und alle anderen Spieler in die öffentliche. Ohne Kommunikation ist es für jedes Individuum demnach rational, nicht die pareto-optimale Strategie $\mathrm{zu}$ verfolgen.

Die grundlegende Erkenntnis aller Experimente ist, dass eben nicht grundsätzlich die dominante Strategie des strikten Freifahrerverhaltens gewählt wird, sondern dass zwischen $40 \%$ und $75 \%$ der Beiträge in die öffentliche Anlage investiert werden. ${ }^{56}$ Empirische Ergebnisse deuten darüber hinaus an, dass eine Gruppenvergrößerung nicht dazu führt, dass die durchschnittliche Zahlungsneigung sinkt. ${ }^{57}$ Wegbereitend waren hier die Studien von Marwell und Ames (1981) und Schneider und Pommerehne (1981), die beide schlussfolgerten, dass zwar Freifahrerverhalten beobachtbar sei, jedoch nur in geringem Umfang. Schließlich deuten die Experimente von Ockenfels und Weimann (1999) darauf hin, dass es

Dies zu operationalisieren, erweist sich erwartungsgemäß als äußerst schwierig. Hey (1991) machte bisher den plausibelsten Vorschlag, die Auszahlung in Höhe der Opportunitätskosten vorzunehmen, also in Höhe der ansonsten möglichen Lohneinkommen.

Weimann (1995, S. 232) fasst in seinem Überblicksartikel zusammen, dass zwischen $40 \%$ und $60 \%$ der Anfangsinvestitionen in die öffentliche Anlage fließen. Dies entspricht exakt dem Intervall, das Marwell und Ames (1981, S. 307) in ihrer Studie ermitteln, und in etwa dem Ergebnis von Schneider und Pommerehne (1981, S. 700), die Werte zwischen 53\% und $61 \%$ errechnen. Allerdings spannen van Winden und Bosman (1996, S. 56) ein weiteres Intervall - von 0\% bis $75 \%$ - auf, das v.a. auch die Ergebnisse der Studie von Kim und Walker (1984) berücksichtigt, welche eine durchschnittliche Beteiligung von lediglich 20\% ermittelten und bei der einige Personen die strenge Freifahreroption wählten.

Isaac, Walker und Williams (1994) kommen sogar zu dem Ergebnis, dass eine Vergrößerung der Gruppe zu einer Intensivierung der Zahlung führt. Hingegen sind die Resultate von Brunner (1998) und Smith, Kehoe und Cremer (1995) differenzierter. In beiden Analysen sinkt zwar die Zahl der freiwillig Zahlenden mit zunehmender Gruppengröße, deren durchschnittliche Zahlungshöhe bleibt aber weitgehend konstant. Mitglieder eines wachsenden Kollektivs werden also eher ab einer kritischen Gruppengröße sprunghaft freerider, als dass sie graduell weniger zahlen. Es gäbe demnach individuell unterschiedliche „Schmerzgrenzen“. 
müßig ist, das idealtypische Verhalten zu suchen, da Kooperationsneigung und solidarisches Verhalten in signifikantem Maße durch die Sozialisationsgeschichte der Individuen geprägt werden.

Die mangelnde Kongruenz zwischen theoretischer Prognose und empirischem Befund kann grundsätzlich durch vier Ursachen hervorgerufen worden sein:

1. Die Modelle sind unzureichend spezifiziert: eine oder mehrere der obigen Anforderungen werden nicht erfüllt. Dieses Argument stammt von Kim und Walker (1984), die zwar in ihrer Anordnung einzelne Spieler mit striktem Freifahrerverhalten isolieren konnten, jedoch bleiben auch bei ihnen die durchschnittlichen Beiträge zum öffentlichen Gut mit $20 \%$ relativ hoch.

2. Die Individuen sind nicht in der Lage, die Logik des Experiments zu durchschauen, es fehlt an analytischer Kapazität. Andreoni (1995) weist darauf hin, dass in seinem Experiment etwa die Hälfte derer, die Beiträge zum öffentlichen Gut leisteten, die Spielstruktur nicht verstanden hatten. Baron (1997, S. 330) konkretisiert die Fehleinschätzung der Experimentteilnehmer dahingehend, dass viele Versuchspersonen nicht erkennen, dass in Sozialen Dilemmasituationen ihre eigene Entscheidung das Gesamtergebnis nicht beeinflusst. ${ }^{58}$

3. Individuen haben eine Präferenz für gemeinschaftliche Aktivitäten. Andreoni (1995) erkennt hierin den Grund für die restlichen 50\% der Beitragszahlenden in seiner Studie. Zu einer ähnlichen Einschätzung kommen auch Brandts und Schram (1995).

4. Es wurde auch argumentiert, dass Individuen bemüht sind, das Gefangenendilemma dadurch zu durchbrechen, dass sie zunächst in goodwill investieren, um so Reputation aufzubauen und dadurch die Mitspieler zur Kooperation zu bewegen. Nachdem die Reputation in der Anfangsphase aufgebaut wurde, erodiert die Zahlungsbereitschaft in den Folgeperioden zunehmend. Dies könnte auch die tatsächliche Beobachtung erklären, dass die Beitragshöhe negativ von der Anzahl der Experimentrunden abhängt (Wilson und Sell, 1990).

Da es bisher noch nicht gelungen ist, ein Experiment so zu konstruieren, dass die strikte Freifahrerhypothese aufrecht gehalten werden konnte, kann das Argument von Kim und Walker (1984), bisherige Experimente wären nicht hinreichend realitätsnah konstruiert, bestenfalls Auftrag für Folgeuntersuchungen sein. $\mathrm{Ob}$ die hohe Anfangsinvestition in das öffentliche Gut Ausdruck strategischer Aktivität ist, untersuchte Andreoni (1988), der aber resümieren musste, dass

58 In der Studie von Baron (1997) antworteten $40 \%$ der Teilnehmer auf die Frage, warum sie nicht ihre Freifahreroption wählten, dass es in ihrem langfristigen Interesse wäre, eine begrenzte Ressource nicht aufzubrauchen. Da die Teilnehmer sich jedoch in einem großen Kollektiv befanden, ist ihr eigener, freiwilliger Beitrag nicht in der Lage, die Ressource langfristig zu retten. 
Spieler sogar eher mit ständig wechselnden Mitspielern als mit längerfristigen Partnern kooperieren. Auch die Beobachtung, dass es zwar einen Schlussrundeneffekt gibt, dieser jedoch keineswegs sehr dramatisch ausfällt, ist ein Anzeichen dafür, dass die These der strategischen Investition in goodwill nicht zu überzeugen vermag. ${ }^{59}$ Denn warum sollten Individuen, die ihre dominante Strategie zwar erkennen, diese jedoch zugunsten einer strategischen Investition zurückstellen, nicht wenigstens in der letzten Runde ihre strikte Freifahreroption wahrnehmen bzw. dies sogar in den Vorrunden antizipieren (Weimann, 1995, S. 233)?

Zusammenfassend lassen sich aus den ökonomischen Experimenten hinsichtlich der Freifahrerhypothese folgende Schlüsse ableiten: ${ }^{60}$

- Die strikte Freifahrerhypothese muss als falsifiziert gelten.

- Bei wiederholter Durchführung der Experimente sinken die Beiträge zum öffentlichen Gut, jedoch bleiben sie stets signifikant positiv.

- Es gibt einen Schlussrundeneffekt, d.h. die Beitragsleistung zum öffentlichen Gut wird in der letzten Runde im Durchschnitt ihr Minimum erreichen, jedoch weiterhin signifikant positiv bleiben.

Da die strikte Freifahrerhypothese jedoch Ausdruck des unterstellten Menschenbilds der Ökonomie ist, ist die Schlussfolgerung von Weimann (1995, S. 232) naheliegend:
„Wir sind nicht in der Lage, unter Verwendung des ökonomischen „model of man", die im Standard-Freifahrerexperiment gemachten Beobachtungen zu erklären."

Wenn die Hypothese rein rationalen Handelns also nicht in der Lage ist, tatsächliches menschliches Handeln adäquat abzubilden, dann ist es darüber hinaus nur folgerichtig, dass man bei dem Versuch menschliches Handeln angemessen zu modellieren, ein komplizierteres Menschenbild zu entwerfen hat, das neben dem ökonomischen Rationalkalkül ,verhaltenspsychologisch zu deutende Prädispositionen " (Weimann, 1995, S. 238) oder soziale Normen und Werte (van Winden und Bosman, 1996, S. 61) berücksichtigt. ${ }^{61}$

59 Der Schlussrundeneffekt besagt, dass in einem Spiel mit festgelegter Periodenzahl spätestens in der letzten Periode deutlich unterschiedliches Verhalten, d.h. im Sinne der strikten Freifahreroption, attestierbar sein muss. Einige Autoren können nicht einmal einen Schlussrundeneffekt ausmachen (Isaac, Walker und Thomas, 1984).

Weitere Erkenntnisse aus Freifahrerexperimenten finden sich in der Grundlagenliteratur aus Fußnote 54. Diese sind aber für diese Arbeit nicht relevant und sollen daher auch nicht dargestellt werden.

61 Frohlich und Oppenheimer (1984) weisen durch die Ergebnisse ihrer Studie darauf hin, dass solche Prädispositionen nicht notwendigerweise immer zum Wohl der anderen führen

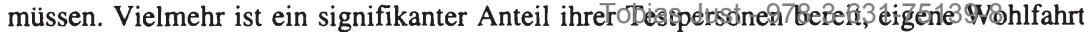


Auch die Diskussion, warum ein rationales Individuum doch zur Wahl geht, wird im folgenden deutlich machen, dass die Integration von Werten und Normen in das ökonomische Menschenbild für eine prognosesichere Erklärung des menschlichen Verhaltens in nicht-marktlich geregelten Gesellschaftsbereichen notwendig ist.

\subsubsection{Das Wahlparadoxon}

In Abschnitt 2.2.2.1 wurde argumentiert, dass (innerhalb des einfachen Modells, bei dem die Wahlteilnahme ausschließlich zu direkten, instrumentellen Nutzen führt) gerade in Kollektiven mit sehr vielen Beteiligten (Bundestagswahl) die Entscheidung, nicht wählen zu gehen, als rational erscheinen muss.

Tabelle 2.4 : Durchschnittliche Wahlbeteiligung in der EU-12

\begin{tabular}{|c|c|c|c|}
\hline $\begin{array}{l}\text { Belgien }^{\text {a) }} \\
(1946-1991) 16 \text { Wahlen }\end{array}$ & $92,8 \%$ & $\begin{array}{l}\text { Großbritannien } \\
(1945-1992) 14 \text { Wahlen }\end{array}$ & $76,6 \%$ \\
\hline $\begin{array}{l}\text { Dänemark } \\
\text { (1945-1990) } 20 \text { Wahlen }\end{array}$ & $85,6 \%$ & $\begin{array}{l}\text { Irland } \\
(1948-1992) 15 \text { Wahlen }\end{array}$ & $74,2 \%$ \\
\hline $\begin{array}{l}\text { BR Deutschland } \\
\text { (1949-1998) } 14 \text { Wahlen }\end{array}$ & $85,5 \%$ & $\begin{array}{l}\text { Italien }^{\text {a) }} \\
(1948-1992) 11 \text { Wahlen }\end{array}$ & $91,6 \%$ \\
\hline $\begin{array}{l}\text { Frankreich } \\
\text { (1958-1993) 10 Wahlen }\end{array}$ & $75,5 \%$ & $\begin{array}{l}\text { Niederlande } \\
\text { (1946-1989) } 14 \text { Wahlen }\end{array}$ & $89,1 \%$ \\
\hline $\begin{array}{l}\text { Griechenland }^{\text {a) }} \\
\text { (1974-1993) } 7 \text { Wahlen }\end{array}$ & $79,2 \%$ & $\begin{array}{l}\text { Portugal }^{\text {a) }} \\
(1976-1991) 7 \text { Wahlen }\end{array}$ & $77,5 \%$ \\
\hline $\begin{array}{l}\text { Luxemburg }^{\text {a) }} \\
\text { (1954-1989) } 8 \text { Wahlen }\end{array}$ & $89,8 \%$ & $\begin{array}{l}\text { Spanien } \\
(1977-1993) 6 \text { Wahlen }\end{array}$ & $74,2 \%$ \\
\hline
\end{tabular}

a) Wahlpflicht

Quelle: Eigene Berechnungen auf der Grundlage von Brettschneider et al (1994) und Bundeswahlleiter (1999b).

zu opfern, wenn dies dazu führt, dass sich ihre relative Wohlfahrtsposition (difference maximizing) verbessert - sie verzichten dann auf Einkommensteile, wenn dies damit einhergeht, dass andere Gesellschaftsmitglieder auf noch größere Einkommensteile verzichten müssen. 
Tabelle 2.4 zeigt für 12 Mitgliedstaaten der Europäischen Union (EU-12), dass es keine Demokratie gibt, in der die Wahlbeteiligung im Durchschnitt weit unterhalb von $75 \%$ liegt und dass selbst die Einführung der Wahlpflicht nur einen geringen Effekt auf die Wahlbeteiligung ausübt. Die durchschnittliche Wahlbeteiligung derjenigen Länder, welche die Wahlpflicht in der Verfassung verankert haben, liegt mit 87,9\% nur geringfügig oberhalb der Wahlbeteiligung der Länder, in denen der Wahlgang vom Gesetzgeber freigestellt wurde $(81,1 \%){ }^{62}$

Downs (1957, S. 265ff) versuchte das Wahlparadoxon zu lösen, indem er einen long-run participation value in die Nutzenfunktion des Wählers integrierte. Nach Downs erkennt der Wähler, dass der Verzicht auf den Wahlakt das Fundament der Demokratie unterhöhlt. Da der Wähler Interesse am Fortbestand der Demokratie hat, geht er wählen. Allerdings ist die Stabilität nur gewährleistet, wenn hinreichend viele Bürger von ihrem Wahlrecht Gebrauch machen, sodass Downs damit nur erreicht, dass aus dem Wahlparadoxon eine Soziale-DilemmaSituation entsteht, denn nun muss begründet werden, warum der rationale Wähler bei der Produktion des öffentlichen Gutes Demokratieerhalt nicht seine Freifahreroption wahrnimmt. An dieser Stelle wird die gedankliche Nähe des Freifahrerproblems und des Wahlparadoxons, wie sie in Abschnitt 2.2.2.1 angedeutet wurde, sichtbar.

Ferejohn und Fiorina (1974) machten den Lösungsvorschlag, dass Wähler gemäß der Minimax-Regret-Regel über den Wahlakt entscheiden würden. Danach entscheidet sich ein risikoaverser Wähler, der vor der Entscheidung steht, ob er wählen gehen soll oder nicht, für jene Alternative, bei der sein maximal möglicher Nutzenverlust unabhängig von der Eintrittswahrscheinlichkeit am geringsten ist. Bei sehr geringen Wahlkosten und genügend hohem Parteidifferential wird sich dann die Mehrzahl der Wahlberechtigten für den Wahlgang entscheiden. Das Argument von Ferejohn und Fiorina hat allerdings einige Schwachpunkte. So ist die Annahme, dass risikoaverse Individuen ohne Rekurs auf Eintrittswahrscheinlichkeiten entscheiden, nicht sehr überzeugend, da bei derart pathologischer Risikoaversion das Individuum erst gar nicht aus dem Bett aufstehen, geschweige denn auf die Straße gehen würde, um zu wählen (Kirchgässner, 1990, S. 449). Außerdem müsste dann die Argumentation geführt werden, warum rationale Individuen ausgerechnet in dieser Entscheidungssituation ohne Wahrscheinlichkeitsberücksichtigung entscheiden, nicht aber in allen übrigen (Mueller, 1989, S. 353).

62 Mit Hilfe eines Zweistichprobentests, der prüft, ob die Gesamtheit der Wahlen mit Wahlpflicht einen identischen Erwartungswert hat wie die Gesamtheit der Wahlen ohne Wahlpflicht, lässt sich zwar zeigen, dass die Hypothese der Erwartungswertidentität auf sehr hohem Signifikanzniveau (Irrtumswahrscheinlichkeit $<0,001$ ) abgelehnt werden muss, dennoch erstaunt die geringe Differenz der Mittelwerte der beiden d_ändergrappen.8 
Nach Riker und Ordeshook (1968) kann das einfache Wahlmodell deshalb nicht die gesamte Wahlbeteiligung erklären, weil neben dem bisher dargestellten direkten, aus dem Wahlausgang resultierenden Nutzen zusätzlich intrinsische, durch den Wahlgang entstehende Nutzen realisiert werden können. Diese intrinsischen, selektiven Anreize können unter dem Oberbegriff „Pflichterfüllung“ subsumiert werden. Der Bürger ist froh, seine Bürgerpflicht getan zu haben. ${ }^{63}$ Allerdings kann dem Modell zurecht vorgeworfen werden, dass es zwar in der Lage ist, die hohe Wahlbeteiligung zu erklären, jedoch neuen Erklärungsbedarf schafft.

„Thus, one needs only to explain why some citizens get a big bang out of pulling the lever while others don't. " (Ferejohn und Fiorina, 1974, S. 526)

Auch befürchten einige Autoren, dass bei konsequenter Anwendung des Arguments der Nutzenstiftung durch Pflichterfüllung auf alle politischen Akteure (Politiker, Bürokraten) die Public Choice Theorie ihre Prognosekraft verlöre (Mueller, 1989, S. 351; Brennan und Lomasky, 1993, S. 35f). Dieses Argument ist solange richtig, solange man keine Rational Choice Erklärung dafür hat, warum es für einen Menschen sinnvoll sein kann, sich pflichtgemäß zu verhalten. Ansonsten wäre der Umfang pflichtbewussten Verhaltens nicht zu prognostizieren. Hat man keinen Wahlzwang, kann das Gefühl der Pflichterfüllung nur durch intrinsische Sanktionsmechanismen durchgesetzt werden. Es müsste also begründet werden, wie und wann der homo oeconomicus zu seiner eigenen Sanktionsinstanz wird.

Es ist nicht uninteressant festzustellen, dass einige Autoren (vgl. Söllner, 1998, S. 19), die den Vorschlag von Riker und Ordeshook aufgrund der aufgezeigten Schwachstellen für ungeeignet innerhalb der Public Choice Theorie erachten, davon ausgehen, dass gesellschaftliche Sanktionen, ob positiv in Form von Anerkennung oder negativ in Form von Ermahnung oder Missachtung, ein Individuum dazu veranlassen können, zur Wahl zu gehen (oder zumindest zu der Behauptung, man hätte gewählt). Ein Individuum geht danach u.a. deshalb wählen, weil ihm daraus Nutzensteigerungen durch den Zuspruch Dritter entstehen oder Kosten vermieden werden, weil er möglichen sozialen Sanktionen ausweicht (Harbaugh, 1996).

Diese Verhaltensannahme könnte dann zwar erklären, warum einige Wahlberechtigte nur vorgeben, sie hätten gewählt, jedoch folgen daraus ähnliche Erklärungsnotstände wie aus der Argumentation von Riker und Ordeshook. Selbst wenn man die vereinfachende Annahme zulässt, dass die soziale Norm „man soll wählen" gewissermaßen vom Himmel fällt, schafft man so nur ein neues

63 Hinzu können auch noch extrinsische selektive Anreize gezählt werden, die dadurch entstehen, dass man Bekannte trifft, spazieren geht etc. (Kirsch, 1997, S. 225). Dieses Argument erscheint indes wenig überzeugend, da diese selektiven Anreize auch - oder gerade außerhalb des Wahllokals genossen werden könnenibias Just - 978-3-631-75139-8 
Freifahrerproblem, da diese Norm und die Normkontrolle öffentliche Güter darstellen. Man hätte wieder ein Problem öffentlicher Güter zweiter Ordnung (Coleman, 1991, S. 350). Zwar kann die soziale Norm erklären, warum das öffentliche Gut „hohe Wahlbeteiligung“ erzeugt werden kann, jedoch fehlt eine dezidierte Begründung, warum das zweite zentrale öffentliche Gut „Kontrolle und Sanktion der Nichtwähler" angesichts der Freifahreroption bereitgestellt würde. In Anlehnung an obiges Zitat von Ferejohn und Fiorina steht man dann vor dem Problem: One needs only to explain why some citizens get a big bang out of controlling their fellow citizens. Wenn man zudem unter den Kosten des Nichtwählens die intrinsische Sanktion „Entstehen von Schuldgefühlen“ subsumiert (Söllner, 1998, S. 18), entstehen zusätzliche Argumentationsschwierigkeiten, da hiermit dieselben individuumsinternen Sanktionsmechanismen angesprochen werden, die auch zum Befolgen der Pflicht führen würden. Möchte man das Argument erhalten, ist auch hier die Frage zu beantworten, warum Individuen intrinsische Sanktionsformen entwickeln. Dies entspricht aber der Frage nach der Bedeutung von individuellen Werten, wie sie in 2.1 gekennzeichnet wurden.

Schließlich soll ein Argument von Brennan und Lomasky (1993) dargestellt werden, von dem Söllner (1998, S. 17) sagt, es ist der "überzeugendste Ansatz“. Dieser Ansatz beruht auf der Annahme, dass ein Wähler durch den Wahlakt zwei unterschiedliche Nutzenkomponenten realisiert. Zum einen erhält der Wähler den Erwartungsnutzen aus dem mit der Eintrittswahrscheinlichkeit, der entscheidende Wähler zu sein, bewerteten Parteidifferential. Zum anderen kann er zusätzlich durch den Wahlakt selbst - durch das Ausdrücken einer konkreten Präferenz - so genannte expressive Nutzen realisieren. ${ }^{64}$ Diese Nutzen werden unabhängig vom Ausgang der Wahl realisiert. Diese expressiven Nutzen können in interne expressive Nutzen und externe, in der Interaktion mit anderen zustandekommende expressive Nutzen unterschieden werden. Der interne Nutzen entsteht dadurch, dass man sich selbst durch eine bestimmte Stimmabgabe besser fühlt, es geht also um eine Form der Selbstprojektion (Brennan und Lomasky, 1993, S. 40). ${ }^{65}$ Der externe expressive Nutzen entsteht dadurch, dass man ge-

64 Brennan und Lomasky (1993, S. 24) gehen davon aus, dass jede Entscheidung sowohl instrumentelle als auch expressive Nutzen generiert. Da jedoch in Marktentscheidungen der instrumentelle Nutzen in vollem Umfang durch die Entscheidung realisiert ist, in politischen Wahlentscheidungen dieser jedoch mit der Entrittswahrscheinlichkeit, der relevante Wähler zu sein, gewichtet werden muss, ist bei politischen Entscheidungen die relative Bedeutung der expressiven Nutzen nach Maßgabe dieser Wahrscheinlichkeit um ein Vielfaches höher als bei Marktentscheidungen. Ähnlich argumentierten auch Jones und Hudson (2000).

65 Brennan und Lomasky weisen zwar darauf hin, dass es interne und externe expressive Nutzen gibt, leiten daraus jedoch keine unterschiedlichen Fälle her. Da eine solche Systematik jedoch als Synopse der derzeitigen Diskussion um die Lösung des Wahlparadoxons interpretiert werden kann, werde ich sie ausführlich entwigkeln. 978-3-631-75139-8 
genüber anderen ein Bild von sich selber aufbaut. Hier könnte man analog von Fremdprojektion sprechen. Es kann, Harbaugh (1996) folgend, auch expressiver Nutzen allein durch den Wahlakt entstehen - man möchte dann vor sich selbst oder vor Mitbürgern als Wähler (egal welcher Couleur) bekannt sein. Unterscheidet man in dem Konzept der expressiven Nutzen in interne und externe expressive und darüber hinaus in positive und negative Nutzen, kann man vier Fälle unterscheiden. Negative Nutzen (Kosten) können dann entstehen, wenn eine konkrete Präferenz von anderen und/oder dem Individuum selbst gering geschätzt werden.

Dies soll an einem einfachen Beispiel illustriert werden: Es möge zwei Parteien $\mathrm{C}$ und $\mathrm{S}$ geben, die unterschiedlich weitreichende (glaubwürdige) Umverteilungsprojekte in ihren Programmen vorgesehen haben, wobei das Programm der Partei S die umfangreicheren Vorschläge enthält. Der Wähler A würde durch das Umverteilungsprogramm der S-Partei zum Nettozahler, hätte also ein pekuniäres Interesse an einem Wahlsieg der C-Partei. Allerdings ist A Mitglied eines großen Wählerkollektivs, sodass davon auszugehen ist, dass sein Parteidifferential für die Entscheidung, wählen zu gehen, keine Bedeutung hat. Die Tatsache, dass er durch den Wahlakt interne und externe expressive Nutzen verwirklichen kann, lässt ihn zur Wahlurne gehen. Im folgenden wird angenommen, dass dies stets der Fall ist.

Außerdem kann die Verteilung der beiden Nutzenkomponenten erklären, wann A eine bestimmte Partei wählt und wann er dies nur vorgibt, getan zu haben. ${ }^{66}$

1. A kann nun expressiven Nutzen daraus ziehen, dass er seine positive Einstellung gegenüber $\mathrm{C}$ zum Ausdruck bringt. Dieser expressive Nutzen ist dadurch realisierbar, dass er selber ein besseres Bild von sich hat und relevante Dritte seine Entscheidung befürworten und bereit sind, dies auch dem A zu

Seit der aufsehenerregenden Studie von Asch (1951), bei der gezeigt werden konnte, dass ein großer Prozentsatz von Individuen bereit ist, eine Falschaussage zu machen, nur um nicht eine Minderheitsmeinung vertreten zu müssen, ist die Bereitschaft zur Gruppenanpassung in zahlreichen Experimenten belegt worden. In dem Asch-Experiment soll ein Individuum die Länge einer Linie mit der Länge von drei anderen vergleichen, von denen eine dieselbe Länge hat wie die erste Linie, die anderen beiden aber beträchtlich davon abweichen. Einzeln befragt, wählen weniger als $1 \%$ der Befragten eine falsche Linie aus. Dies ändert sich jedoch, wenn der Befragte sich in einer Gruppe befindet, in der die anderen einstimmig eine objektive Falschaussage tätigen. Dann folgen 32\% der Befragten dieser falschen Gruppenmeinung. Auch für das Verhalten bei politischen Wahlen gibt es empirische Belege, dass Individuen ihre wahre Präferenz verbergen, um nicht der Mehrheitsmeinung in ihrem Umfeld zu widersprechen (Noelle-Neumann, 1980, S. 52ff). Kuran (1990, S. 11) spricht dann von preference falsification, wenn es für den einzelnen besser ist, seine wahre Präferenz zu verbergen, um Nutzen aus der Übereinstimmung der geäuBerten Präferenz mit der Kollektivmeinung zu ziebegnas Just - 978-3-631-75139-8 
sagen. Durch diese Bestätigung durch andere steigt der Nutzen des A. Im Ergebnis wird A für die C-Partei stimmen und dies auch zugeben.

2. In dem zweiten Fall zieht A wieder internen expressiven Nutzen daraus, dass er für die Partei stimmt, deren Programm seinen Präferenzen am nächsten kommt. Gleichzeitig soll in diesem Fall der externe expressive Nutzen nur dann realisiert werden, wenn A gegenüber Dritten ein Bild von sich zeichnet, das ihn als sozial engagierten Menschen beschreibt, wenn also die anderen der Meinung sind, A hätte für die S-Partei gestimmt. Die dominante Strategie des $A$ wäre dann, zwar für $C$ zu stimmen, jedoch zu behaupten, er hätte für $S$ gestimmt. Diese Lüge wird durch die Existenz sozialer Normen, die Anpassungsdruck in Form von externen Sanktionen (Tadel etc.), also Kosten bedeuten, erzwungen. Im Ergebnis wird $A$ also $C$ wählen, dies aber nicht zugeben, sondern behaupten, er hätte für die Umverteilungsmaßnahme gestimmt.

3. Der dritte Fall ist dadurch gekennzeichnet, dass die relevanten Akteure innerhalb des Umfeldes des A eine Stimmabgabe für die C-Partei begrüßen und dies auch sagen würden, A selber jedoch nur dadurch interne expressive Nutzen realisieren kann, wenn er für sich von sich selbst ein sozial engagiertes Bild entwirft; d.h., würde er seine Stimme nicht der S-Partei geben, müsste er mit internen Sanktionsmechanismen (Gewissensbisse, Selbstvorwürfe) rechnen. Um diesen auszuweichen, wird A entgegen seines pekuniären Interesses $\mathrm{S}$ wählen. Gleichzeitig wird er seine Wahlentscheidung verleugnen. ${ }^{67}$

4. Schließlich wäre eine Situation vorstellbar, in welcher der Wähler eine Präferenz für $\mathrm{C}$ hat, jedoch wie in Fall 3 den expressiven Nutzen nur dann realisiert, wenn er für S stimmt. Auch hier würden also interne Sanktionsmechanismen greifen. „Es kostet nicht viel, moralisch zu sein.“ (Söllner, 1998, S. 17). Gleichzeitig befindet sich der A in einem Umfeld, das die Unterstützung der S-Partei befürwortet. Auch in dieser Situation würde A immer S wählen. Dies würde A auch sagen, insgeheim jedoch auf einen Wahlsieg der C-Partei hoffen.

Tabelle 2.5 fasst diese vier Fälle in einer Matrix zusammen, ausgehend von der Annahme, dass A ein positives Parteidifferential zugunsten der C-Partei hat. ${ }^{68}$

67 Es ist an dieser Stelle besonders darauf hinzuweisen, dass sein Interesse hinsichtlich des Wahlausgangs unverändert zugunsten der C-Partei ausfällt. Da A aber kaum davon ausgehen kann, dass seine Stimme die entscheidende ist, maximiert er seinen Nutzen, indem er erstens zur Wahl geht, denn nur dadurch kann er überhaupt expressiven Nutzen realisieren, und indem er zweitens für S stimmt, denn nur dadurch vermeidet er internen expressiven Nutzenentgang.

68 Es ließen sich sehr leicht weitere Fälle konstruieren, in denen unterstellt wird, dass Lügen

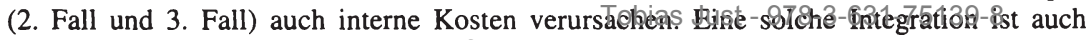


Es wird deutlich, dass die Existenz von expressiven Nutzenelementen nicht nur die hohe Wahlbeteiligung erklären kann, sondern darüber hinaus eine Reihe weiterer Schlussfolgerungen erlaubt. ${ }^{69}$ So führt die Drohung von sozialen Sanktionen (2. Fall) dazu, dass Individuen zwar gemäß ihren instrumentellen Nutzen abstimmen, dies jedoch nicht äußern.

\section{Tabelle 2.5 : Wahlentscheidung bei expressiven Nutzen}

\section{Externe positive Anreize}

(Lob, Anerkennung)
Externe negative Anreize

(Tadel)

\begin{tabular}{|c|c|c|c|c|}
\hline \multirow{3}{*}{$\begin{array}{l}\text { Interne positive } \\
\text { Anreize } \\
\text { (Freude über Erfüllung } \\
\text { eines bestimmten Bildes) }\end{array}$} & \multicolumn{2}{|l|}{ 1. Fall: } & \multicolumn{2}{|l|}{ 2. Fall: } \\
\hline & Wahl: & C-Partei & Wahl: & C-Partei \\
\hline & Äußerung: & C-Partei & Äußerung: & S-Partei \\
\hline \multirow{3}{*}{$\begin{array}{c}\text { Interne negative } \\
\text { Anreize } \\
\text { (Gewissensbisse) }\end{array}$} & 3. Fall: & & 4. Fall: & \\
\hline & Wahl: & S-Partei & Wahl: & S-Partei \\
\hline & Äußerung: & C-Partei & Äußerung: & S-Partei \\
\hline
\end{tabular}

Quelle: Eigene Darstellung

Außerdem kann ein interner Sanktionsmechanismus, der Wunsch nach der Erfüllung eines bestimmten Selbstbildes, dazu führen, dass Individuen entgegen ihrer instrumentellen Präferenz abstimmen. ${ }^{70}$ Dies ist immer dann gegeben, wenn der expressive Nutzen nicht mit dem instrumentellen Nutzen korreliert. Zwar gehen auch Brennan und Lomasky davon aus, dass in vielen Fällen Selektionsmechanismen dazu führen, dass Konsistenz zwischen instrumentellen und expressiven Nutzen besteht, z.B. weil jeder an der Definition seines sozialen

deswegen sinnvoll, da es widersprüchlich erscheint, dass der Wähler bei seiner Stimmabgabe ethische Prinzipien verfolgt, jedoch problemlos lügen kann. Das Tableau im Anhang A zeigt, inwiefern intrinsische Kosten durch Unaufrichtigkeit nicht nur das Kommunikationsverhalten, sondern sogar das Wahlverhalten beeinflussen können.

In diesem Modell wird sich ein rationales Individuum für den Wahlgang entscheiden, wenn die Summe aus externen und internen expressiven Nutzen die Kosten des Wahlaktes übersteigt.

70 Diese interne Sanktionierung entspräche der Annahme von Riker und Ordeshook, Bürger würden einer empfundenen Pflicht entsprechen. Dies wäre hier nicht nur die Befolgung der Wahl,,pflicht", sondern darüber hinaus noch die empfundene Pflicht, ein bestimmtes Verteilungsziel zu erreichen, bei dem der hier betrachtete Wähler allerdings Nettozahler würde. 
Umfelds mitwirkt, jedoch ist die Möglichkeit einer Abweichung von expressiven und instrumentellen Nutzen nicht nur akademische Fingerübung für ,logical purists" (Brennan und Lomasky, 1993, S. 37), wie die Ergebnisse von NoelleNeumann (1980, S. 52ff) zeigen konnten.

Es wurde argumentiert, dass jeder bisherige Versuch, mit Hilfe des Rational Choice Ansatzes das Wahlparadox zu lösen, neue Probleme kreierte. Es scheint nicht möglich zu sein, die hohe Wahlbeteiligung in westlichen Demokratien ohne die Zuhilfenahme von ,weichen“"Erklärungsgrößen zu begründen. Man mag diese Erklärungsgrößen Pflichtgefühl, soziale Normen, expressive Nutzen oder ethische Präferenzen nennen, stets wird die Frage nach der Entstehung dieser Werte und vor allem nach der Begründung, warum sich Menschen an diese Werte gebunden sehen, aufgeworfen.

Ein interessanter Gedanke wurde von Mueller $(1986,1989)$ in die Diskussion eingebracht. Danach wird selbst ein rein egoistischer Politiker, der ausschließlich an dem Wahlsieg interessiert ist, durch die Tatsache, dass Wähler (zumindest zum Teil) gesellschaftlich verantwortlich abstimmen, ein ureigenes Interesse verfolgen, wenn er ein am gesellschaftlichen Wohl orientiertes Programm anbietet, da dies seine Wahlaussichten steigert.

"The public interest conception of politics is pulled from the ashes by the selfish, but ethically conditioned voter's behaviour. "(Mueller, 1989, S. 369)

Außerdem scheint es in Anbetracht der grundlegenden Annahme der Verhaltenssymmetrie, dass Menschen in verschiedenen Situationen durch dasselbe Verhaltensmuster beschreibbar sind, naheliegend, dass auch auf der Angebotsseite des politischen Marktes - für die Politiker - gelten muss, dass Werte und Ideologien einen Einfluss auf ihre Politik haben. ${ }^{71}$

\subsubsection{Der Politiker als Ideologe}

Seit Stigler's (1971) wegbereitender Arbeit über Regulierung, in der er das bis dato auch in der wirtschaftswissenschaftlichen Fachliteratur vorherrschende idealistische Politik- und Staatsverständnis als unzureichend zurückweist und an dessen Stelle ein Modell entwirft, in dem das politische System als Instrument zur Durchsetzung privater Interessen von Interessengruppen ausgenutzt wird, entwickelte sich der Mainstream der Ökonomie in eine Richtung, die selbst

71 Allerdings heißt dies nicht, dass die Anreizsysteme für den Wähler und Politiker dieselben sind - so kann durchaus angenommen werden, dass der politische Bereich Anreize beinhaltet, die Politiker systematisch von einer wertegebundenen Politik abhalten oder dass es innerhalb des politischen Bereichs Selektionsmechanismen gibt, die weniger stark wertegebundene Politiker fördern. 
Stigler so zugespitzt nicht formulierte. ${ }^{72}$ So schreiben Kalt und Zupan (1984, S. 281):

"We suspect, however, that most economists would conclude that the pursuit of ideological objectives is not an important phenomenon."

Es stellt sich also die Frage: Ist das Abstimmverhalten der Repräsentanten maßgeblich durch Einzelinteressen relevanter Gruppen beschreibbar oder durch eine ideologische Position der Politiker?

Die wohl bedeutsamste Studie, die zu zeigen versuchte, dass nicht nur ökonomische Interessen das Abstimmverhalten der US-amerikanischen Kongressabgeordneten beeinflussen, sondern auch eine ideologische Restgröße sehr großen Einfluss ausübt, ist die Arbeit von Kau und Rubin (1979), ${ }^{73}$ die als erste das Abstimmverhalten der Abgeordneten bei einem größeren Set von Gesetzesvorlagen untersuchte. Die Autoren testen, ob das Abstimmergebnis der Abgeordneten durch sozio-ökonomische Determinanten ihrer Repräsentierten oder durch einen Ideologieindex bestimmt wird. Der Ideologieindex wurde auf der Grundlage eines Ratings der Abgeordneten durch die Interessengruppe Americans for Democratic Action (ADA) ermittelt. ADA weist jedem Abgeordneten relativ zu der Position der ADA einen Ideologiewert innerhalb eines Kontinuums zwischen links und rechts zu. ${ }^{74}$ Sie kommen zu dem Ergebnis, dass die Hypothese der Existenz ideologisch geleiteter Politik neben der sozio-ökonomischen Variablen als Erklärungsgröße für das beobachtete Abstimmungsverhalten, nicht verworfen werden kann. Allerdings sind sich sowohl Kau und Rubin, als auch nachfolgende Autoren (vgl. Kalt, 1981, 1983, Kalt und Zupan, 1984), die zu ähnlichen Ergebnissen kommen, bewusst, dass ihr Modell maßgeblich darauf aufbaut, dass alle potentiellen sozio-ökonomischen Bestimmungsfaktoren vollständig und korrekt in der Regressionsgleichung enthalten sind.

Ferner kritisiert Peltzman (1984), dass der Erklärungsgehalt der aus den Ratings von Interessengruppen abgeleiteten Ideologievariable zwar signifikant sein mag, jedoch nicht zwingend das misst, was sie messen soll, nämlich ideologische Prädisposition der Politiker jenseits der Interessen ihrer Wähler. Peltzman (1984, S. 183f) argumentiert, dass die Ideologievariable der Interessengruppe

Die Theorie von Stigler wurde von Peltzman (1976) formalisiert.

73

In ihrer Zusammenfassung schreiben Kau und Rubin, dass sie zu Beginn ihrer Studie eher davon ausgingen, dass sie die These von Stigler (1971) bestätigen würden. Für einen Überblick über Studien, die bei Einzelentscheidungen zu einem ähnlichem Ergebnis führten, siehe die angegebene Literatur bei Kau und Rubin (1979, S. 365f).

Dabei wurden die eigentlichen ADA-Werte um regionale Parteieffekte bereinigt, um Multikollinearität zwischen den ADA-Werten und den sozio-ökonomischen Variablen auszuschließen (Kau und Rubin, 1979, S. 370). López und Ramirez (2000) finden allerdings, dass die ADA-Werte auf konjunkturelle Schwankungen reagieren. Dies würde gegen eine Interpretation als Ideologieindikatoren sprechen.Tobias Just - 978-3-631-75139-8 
lediglich eine Proxy-Größe für materielle Interessen ist. Man kann viel ihres Erklärungsgehaltes dadurch reduzieren, dass man nicht nur sozio-ökonomische Mittelwerte aller Wahlberechtigten heranzieht, sondern darüber hinaus sozioökonomische Mittelwerte der finanziell Unterstützenden des Repräsentanten in die Regressionsgleichung mit aufnimmt, quasi als Bestimmungsfaktoren der Residualgröße Ideologie. Tatsächlich zeigt die empirische Untersuchung von Peltzman (1984, S. 193ff), dass die Ideologievariable keine nennenswerte Steigerung im Bestimmtheitsmaßes mehr erzeugt, nachdem die sozio-ökonomischen Variablen der Anhängerschaft integriert sind. Allerdings zeigt auch eine detailliertere Analyse isolierter issues bei Peltzman, dass in einzelnen Sachfragen (v.a. Sozialpolitik) ideologisches Abstimmen bedeutsam bleibt (S. 203).

Auch muss Peltzman (1984, S. 195) einräumen, dass - nicht ganz unerwartet zwischen seiner Ideologievariablen und den sozio-ökonomischen Faktoren der Anhänger so starke Multikollinearität besteht, dass eine zuverlässige Schlussfolgerung wohl nicht möglich ist. Darüber hinaus argumentieren Kalt und Zupan (1984, S. 280f), dass die Analyse von Peltzman die Gesamtheit des Abstimmverhaltens der Senatoren umfasst. Auf so hoher Aggregatebene müssen auf ideologische Überzeugung zurückzuführende Entscheidungen der Senatoren an Gewicht verlieren. Betrachtet man zentrale Einzelentscheidungen, so resümieren Kalt und Zupan (1984, S. 298), muss man die Schlussfolgerung zulassen, dass im Abstimmverhalten zumindest bei spezifischen Einzelentscheidungen der Abgeordneten Werte und Ideologien eine wichtige Rolle einnehmen. ${ }^{75}$

Bevor im nachfolgenden Abschnitt die Begriffe Wert und Ideologie in Rational Choice Modelle integriert werden, sei kurz darauf hingewiesen, dass es zunehmende empirische Bestätigung dafür gibt, dass selbst in wettbewerblichen Märkten Wertvorstellungen die Preissetzung beeinflussen können. Umfragen ergeben regelmäßig, dass Konsumenten bereit sind, signifikante Kosten zu tra-

75 Noll (1989) schlug in seiner ,sophisticated public interest theory“ ein Modell vor, in dem eine staatliche Regulierungsbehörde eingerichtet wird, falls prohibitiv hohe Transaktionskosten eine spontane Verhandlungslösung der Betroffenen nicht zustande kommen lassen. Ist die Behörde erst einmal institutionalisiert, formieren sich private Interessengruppen, um gemäß der Regulierungstheorie Stiglers Renten zu gewinnen. Dann ist das Ergebnis dieser staatlichen Intervention weder durch ein reines public interest, noch durch ein reines Interessengruppenmodell beschreibbar. Wichtig für die Argumentation ist hierbei, dass es innerhalb dieses Modells entweder intrinsische Motivation der Politiker für die Verfolgung eines ihrer Meinung entsprechenden public interest, also einer Ideologie, geben muss oder sie reagieren auf Drängen von Interessengruppen, deren Ziel die Verfolgung des öffentlichen Interesses ist. Gemäß der fundamentalen Annahme der Verhaltenssymmetrie des Menschen muss dann aber begründet werden, warum es Interessengruppen gibt, die sich für das öffentliche Interesse engagieren, dies jedoch gänzlich für den Politiker auszuklammern ist. Crone und Tschirhart (1998) finden dafür auch empirische Bestätigung, dass es Entscheidungen gibt, in denen die „sophisticated public interest theory“ neben der reinen Interessengruppentheorie zusätzliche Er Ekjlärungskraft_besitł5.139-8 
gen, um das öffentliche Gut „faire Preissetzung“ durchzusetzen (Kahnemann, Knetsch und Thaler, 1986a, 1986b).$^{76}$ Arrow (1990, S. 139) geht sogar noch einen Schritt weiter, wenn er feststellt, dass man eine Form kaufmännischer Ethik annehmen muss, möchte man die allgemeine Funktionsfähigkeit von Märkten verstehen. Ohne diese Wertebasierung müssten Sanktionsmechanismen mit zumindest extrem hohen, wenn nicht sogar prohibitiv hohen Transaktionskosten implementiert werden. ${ }^{77}$

Es gibt also zahlreiche Gründe dafür, weshalb es sinnvoll erscheint, das ökonomische Modell so zu erweitern, dass auch Werte Eingang finden - nicht nur, um menschliches Verhalten möglichst exakt abzubilden, sondern auch, um sichere Verhaltensprognosen abgeben zu können. Allerdings sieht sich die Ökonomie dabei in einem Dilemma. Zum einen gibt es wie oben gezeigt, gute Gründe, Werte und Ideologien zu berücksichtigen, zum anderen scheint die Integration im Widerspruch zu der strengen Rationalitätsannahme des homo oeconomicus zu stehen. Es nimmt also nicht wunder, dass Coleman (1991, S. 379) den Versuch, die Internalisierung von Werten in die Rational Choice Theorie einzubinden, ein "riskantes Unterfangen“ nennt. Ebenso spricht Kirsch (1997, S. 91) von einer "schockierenden Vorstellung“, innerhalb des Rational Choice Ansatzes zu argumentieren, dass der Einzelne ein Interesse daran haben könnte, Werte von außen aufoktroyiert zu bekommen, sich also den Vorstellungen anderer zu unterwerfen.

\subsection{Werte und Ideologien in Rational Choice Modellen}

Da die Existenz eines internen Sanktionssystems, das sich z.B. in Form von Gewissensbissen manifestiert, indes zu den Erfahrungen der meisten Menschen gehören sollte, gibt es für die Ökonomie zur Überwindung dieses Dilemmas des Rational Choice Ansatzes grundsätzlich drei Möglichkeiten:

1. Man vernachlässigt die Bedeutung menschlicher Werte und Ideologien, entweder weil man der Ansicht ist, dass gerade nicht die Interdisziplinarität der Wissenschaften, sondern ihre Arbeitsteilung bei der Abstraktion von Realität

$\mathrm{Zu}$ diesen Umfrageergebnissen muss einschränkend angemerkt werden, dass Umfrageergebnisse prinzipiell Laborexperimenten unterlegen sind, da es unplausibel erscheint, dass die Interviewantworten von der spezifischen Interviewsituation unabhängig sind. Die Antworten der Befragten können je nach Fragestellung und Fragesteller von der tatsächlich gewählten Handlung abweichen.

77 Diese Ansicht vertreten z.B. auch North (1988, S. 48), Held (1991, S. 31) und Haslinger (1997, S. 162f). Sie erkennen als Grund dafür die Unfähigkeit des Menschen, Verträge vollständig zu spezifizieren, bzw. das moral hazard-Problem bei asymmetrischer Information in einer Welt positiver Informationskosten zudiberwingen $978-3-631-75139-8$ 
bedeutsam ist, oder weil man davon ausgeht, dass menschliche Werte als Datum aufgefasst werden können und demnach nicht endogenisiert zu werden brauchen.

2. Man lässt den Rational Choice Ansatz vollständig fallen und sucht nach einem neuen Ansatz.

3. Man nimmt das „Risiko“ einer Integration von soziologischem Wissen hinsichtlich der Internalisierung von Werten in den Rational Choice Ansatz auf sich, und versucht zu erklären, inwiefern auch der homo oeconomicus sozial gebunden sein kann.

Da zum einen bereits in Abschnitt 2.3 argumentiert wurde, dass für zahlreiche wirtschaftliche Aktivitäten Werte und Ideologien relevant sind, und zum anderen eine völlige Neuentwicklung einer ökonomischen Theorie jenseits des methodologischen Individualismus weder möglich noch sinnvoll, vor allem aber auch nicht nötig erscheint, sollen im folgenden die bisher überzeugendsten Versuche dargestellt werden, wie Werte und Ideologien in das ökonomische Verhaltensmodell integriert werden können. ${ }^{78}$ Es wird hierbei nicht auf spieltheoretische Modelle eingegangen, da diese Modelle gerade darauf aufbauen, Werte und Ideologien rein funktionalistisch im Sinne einer für eine Kooperation zweckdienlichen Institution zu berücksichtigen. Dann bliebe die Ideologie wie bei Downs ein Mittel zur Eigennutzverfolgung. Hier soll aber gerade gezeigt werden, dass Werte und Ideologien im Rational Choice Ansatz Berücksichtigung finden können, ohne dass man den Zielcharakter einer Ideologie aufgeben muss. Außerdem „funktionieren“ die Modelle i.d.R. nur unter relativ restriktiven Bedingungen wie z.B. einem finiten Zeithorizont (Swank, 1998) oder der Annahme kleiner, homogener Gruppen (Holländer, 1990).

78 Exkursorisch sei auf einen berühmten Ansatz von Frank (1987) hingewiesen. Frank zeigt, dass es durchaus in bestimmten Situationen für ein nutzenmaximierendes Individuum sinnvoll sein kann, Werte derart internalisiert zu haben, dass ein Verstoß gegen dieses interne Wertefundament durch sichtbare Gefühlsregungen für andere bemerkbar wird. In Kooperationssituationen, in denen glaubhaft commitment gezeigt werden muss, ist die $\mathrm{Zu}$ sicherung über Gewissen und Gefühle transaktionskostenminimal, da durch seine unvermeidbare Gefühlsäußerung sein Verhalten glaubwürdig ist. In solchen Szenarien würde sich ein rationaler Akteur - hätte er die freie Wahl - für eine Wertebindung entscheiden. Allerdings ist der Ansatz begrenzt, da er nur für diejenigen Fälle sinnvoll Anwendung finden kann, in denen die Äußerung der Gefühle tatsächlich nutzensteigernd wirkt. Diese Modellierung entspricht aber eher der individuellen Entscheidung, ein Prinzip zu befolgen (vgl. dazu z.B. Kirsch, 1997, S. 86ff), als der internen Restriktion, gemäß einem Wert zu agieren, der nicht unbedingt nutzenfördernd sein muss as Just - 978-3-631-75139-8 


\title{
2.4.1 Das Selbstinteresse und das Interesse am eigenen Selbst
}

\author{
„l...J identity is fundamental to behavior, choice of identity may be \\ the most important „economic" decision people make. "Akerlof and \\ Kranton (2000, S. 717)
}

In 2.1.4 wurde die Argumentation Colemans dargestellt, nach der Sozialisationsagenten ein Interesse an der Internalisierung von Werten durch die Sozialisanden haben, solange die marginalen Internalisierungskosten noch unter den marginalen Kontroll- und Sanktionskosten liegen, die anfallen, wenn man gewünschtes Verhalten durch permanente Variation selektiver Anreize herzustellen versucht. Allerdings vernachlässigt diese Argumentation das Entscheidungskalkül des Sozialisanden, und man würde lediglich die Bildung eines völlig von außen determinierten homo soziologicus nach den ökonomischen Grundsätzen des Marginalkalküls modellieren. Innerhalb eines dem Rational Choice Ansatz verpflichteten ökonomischen Modells ist darüber hinaus aber eine zweite Frage relevant: unter welchen Bedingungen internalisiert der Sozialisand eine Wertvorstellung, oder wie Kirsch (1997, S. 91) es thematisiert, baut ein Gewissen auf, das ihn im Zweifel auch von seiner Eigennutzverfolgung abhalten kann.

Einige Autoren argumentieren, dass dieses Interesse an der Internalisierung von Werten nur erklärt werden kann, wenn man neben das Eigeninteresse als Handlungsmotivation (self-interest) ein grundsätzliches Interesse des Menschen an einer persönlichen Identität, eines Selbst, als zweite Handlungsmotivation stellt (interest in one's own self). ${ }^{79}$ Diese Selbstbildung ist nur gewährleistet, wenn das Individuum in der Gegenwart alle Handlungen der Vergangenheit und alle möglichen Handlungen der Zukunft akzeptiert und als mit seiner Identität vereinbar sieht (Kirsch, 1997, S. 92f). Die Internalisierung von Werten bzw. das Ausbilden eines Gewissens macht mit der eigenen Persönlichkeit nicht vereinbares Handeln in der Zukunft unwahrscheinlicher, da die internen Sanktionen den Preis von abweichendem Verhalten erhöhen.

Es wird davon ausgegangen, dass neben die materiellen Bedürfnisse, die der Mensch bemüht ist, zu befriedigen, das Bedürfnis nach der Schaffung und Aufrechterhaltung einer eigenständigen Identität tritt. Nach Taylor (1996, S. 55) ist diese Identitätsbildung jedoch nur möglich, wenn man für sich selber Wissen darüber erworben hat, ,[...]was für den Betreffenden von ausschlaggebender Bedeutung ist ". Der Erwerb dieses Wissens ist wiederum nur innerhalb eines bestimmten, für das Individuum exogen vorgegebenen Rahmens, eines moral

79 Hier wird v.a. der Darstellung von Kirsch (1994, 1997) gefolgt, da dessen Ausführungen in geeigneter Form später diagrammatisch dargestellt werden können. Die Überlegungen zu der Dualität von Selbstinteresse und Interesse am eigenen Selbst finden sich aber in vergleichbarer Art in den anderen Darstellungen. Vgl. u.a. auch Katterle (1991, S. 138), Taylor (1996) und Akerlof und Kranton (2000). 
space, möglich - nur innerhalb dieses moralischen Raumes ist es für das Individuum denkbar, ein Selbst zu konstituieren. Dabei kann der moralische Raum natürlich auch mehrdimensional sein, und die Dimensionen können auch interindividuell variieren. So können z.B. die in Abschnitt 3.1 anzusprechenden politisch relevanten cleavages ${ }^{80}$ als Dimensionen innerhalb des moralischen Raumes gelten. Innerhalb dieses moral space kann sich ein Individuum zwar zum Teil selbst positionieren, es kann jedoch nicht die Art des Rahmens frei manipulieren. Der Versuch, den Rahmen nicht gelten zu lassen, muss zwangsläufig dazu führen, dass man vor sich und für die Gesellschaft nichts mehr wert ist. ${ }^{81}$

Hat der Mensch also ein starkes Interesse an der Schaffung und Wahrung einer Identität und ist es richtig, dass diese Identität nur innerhalb gesellschaftlich vorformulierter Rahmen möglich ist, dann ist es für das Individuum nur rational, die Werte, die diesen Rahmen bilden, zu internalisieren, denn dies sichert die Selbstfindung transaktionskostenminimal, falls nicht sogar argumentiert werden kann, dass die Wertinternalisierung ein notwendiges Kriterium zur Identitätsbildung darstellt. Dies ist nicht unplausibel, da die alternative Strategie zur Identitätsbildung - die Befolgung einer vernunftbasierten selbst aufgestellten Regel eine geringere Handlungsrestriktion bedeutet als ein nicht notwendigerweise vernunftmäßig erfasster Wert und somit leichter zu zeitinkonsistentem Verhalten führt. Inwiefern ein Individuum bei der Wahl seiner Identität (seines Selbst) frei wählen kann, ist allerdings keineswegs klar. Auch scheint die Identität eines Menschen kein stabiles Konstrukt zu sein, sondern ist äußeren Einflüssen und damit Wandel unterworfen (Akerlof und Kranton, 2000).

Anhand einer möglichen Dimension des moralischen Raumes sollen die Überlegungen veranschaulicht werden. In Abbildung 2.4 wird die Dimension ,gleichmäßige Einkommensverteilung" entlang der Abszisse abgebildet. Umfangreiche staatliche Verteilungsbemühungen mögen als typisch linkes Ziel gelten unter der Annahme, dass diese Maßnahmen tatsächlich geeignet sind, die Einkommen zu nivellieren. ${ }^{82}$

Wenn der Ordinatenschnittpunkt 0 den gesellschaftlichen Konsens markiert (der z.B. durch das letzte Wahlergebnis ermittelt wurde), dann ist $0_{\mathrm{L}}$ eine in der öffentlichen Meinung als links empfundene Position, $0_{R}$ hingegen eine sehr marktliberale, rechte Position.

Als cleavage wird ein gesellschaftsinternes Trennkriterium verstanden, das ein größeres Kollektiv in kleinere, durch diese Konfliktlinie trennbare Teilkollektive scheidet.

81 Mit Hinblick auf die Gesellschaft drängt man sich selbst in die Asozialität (Kirsch, 1997, S. 101), mit Hinblick auf sich selbst erleidet man einen Verlust des Selbst. Dieser Verlust äußert sich in "[...] einem Gefühl der Leere, Fadheit, Vergeblichkeit, Zwecklosigkeit oder de[m] Verlust der Selbstachtung." (Taylor, 1996, S. 43). Die Berücksichtigung des Rahmens dient letztlich der Vermeidung dieser affektiven Kosten. 
Es wird deutlich, dass die Positionierung innerhalb des Raumes variieren kann, die unterschiedlichen 0-Punkte stellen den ideologischen Standort von Individuen dar und werden maßgeblich durch Wertvorstellungen und positives Wissen über Wirkungszusammenhänge determiniert (Kirsch, 1997, S. 106f), sodass verschiedene Subgesellschaften mit unterschiedlichen Weltsichten und Wissen auch zu anderen 0-Punkten gelangen können.

\section{Abbildung 2.4 : Verteilungsergebnis als Dimension im moral space}

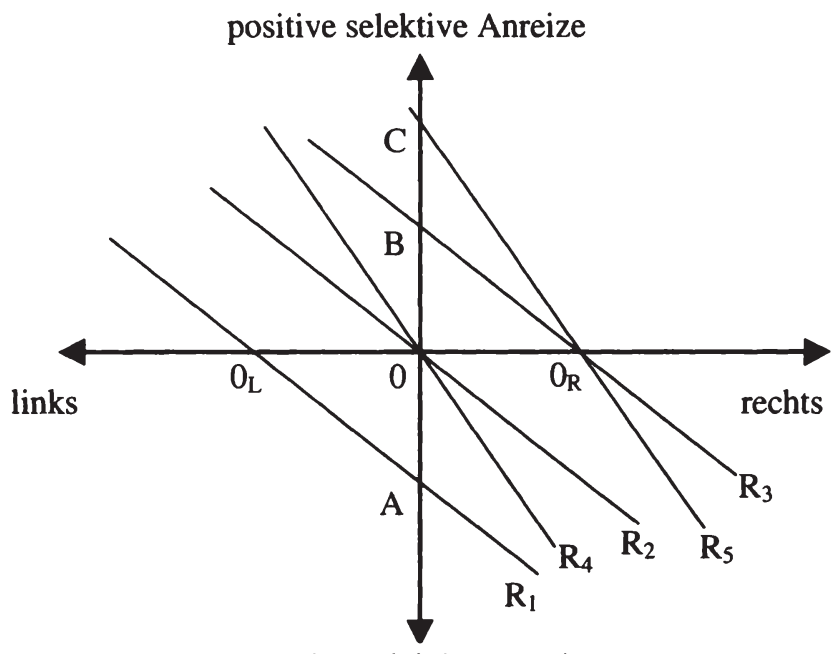

negative selektive Anreize

\section{Gleichmäßigere Einkommensverteilung}

Quelle: In Anlehnung an Kirsch (1991, S. 252).

Das Modell von Kirsch basiert nun auf der Grundannahme, dass sich Individuen zwar durch ihre Positionierung im Raum identifizieren, dies aber nicht gleich ihrem gezeigten Verhalten sein muss; viel mehr reagieren sie weiterhin auf Anreize.$^{83}$ Die Überlegung, dass die gelebte Moral in einem stetig funktionalen $\mathrm{Zu}$ -

83 Natürlich dient der unterstellte lineare Zusammenhang in der Graphik nur der Vereinfachung. Realiter ist eher davon auszugehen, dass auch für die Substitutionsbeziehung selfinterest und interest in one's own self eine abnehmende Grenzrate der Substitution vorliegt. Der Funktionsverlauf müsste dann strikt kenvex sein 
sammenhang mit den inhärenten Opportunitätskosten steht - Moral als substitutives Gut -, wurde erstmals von Hochman und Rodgers (1969) modelliert. ${ }^{84}$

Auch North (1992, S. 49) hält es für plausibel, dass die Bedeutung von Ideologie von der Höhe des Preises abhängt. Dies ist nichts anderes als das, was in der Reaktionskurve von Kirsch enthalten ist. So ist ein „Rechter“ mit $0_{\mathrm{R}}$ und der Reaktionskurve $\mathrm{R}_{3}$ dann bereit, den gesellschaftlichen Konsens (Punkt 0) zu tragen, wenn ihm zusätzlich positive selektive Anreize in Höhe von $0 \mathrm{~B}$ geboten werden. ${ }^{85}$ Seine Reaktionskurve $R_{3}$ beschreibt, welche zusätzlichen Transferleistungen sein Verhalten von seiner Grundhaltung $0_{R}$ aus beeinflussen können. $R_{5}$ ist die Reaktionskurve eines stärker ideologisch verfestigten Individuums; hier müssten Zahlungen stets deutlich höher ausfallen, um dessen Verhalten zu verändern. ${ }^{86}$ Analog würde ein „Linker" mit $0_{\mathrm{L}}$ als Moralstandard auch dann dem gesellschaftlichen Konsens zustimmen und nicht sein eigentliches Verteilungsziel befürworten, falls dieses bedeuten würde, dass er (zusätzlich zu seinem in $0_{\mathrm{L}}$ zu erwartenden Finanzierungsanteil) in Höhe von 0A Zahlungen zu leisten hätte. Eine drohende Zusatzzahlung macht natürlich nur für die wohlhabenden, paternalistischen Linken Sinn. Für die Mehrheit der weniger wohlhabenden Linken, die Umverteilungsgewinner sind, kann der negative Anreiz in Höhe von 0A als soziale Arbeitsleistung interpretiert werden. Droht bei steigenden Sozialtransfers ein Anstieg an gemeinnütziger Arbeit, wie es in Deutschland auch im Gespräch ist, dann wäre auch ein ,armer" Linker bereit, seine präferierte Position $0_{\mathrm{L}} \mathrm{zu}$ verlassen. Diese drohende (zusätzliche) Leistung ließe ihn für die geringere, gesellschaftlich konsensuale staatliche Intervention votieren.

Außerdem kann Kirsch aus seinem Versuch, die Erhaltung der menschlichen Identität als zweites Ziel mit der Wohlstandsmaximierung in einem Modell zu verbinden, plausible (aber noch weitgehend ungeprüfte) Hypothesen über die Wechselwirkung zwischen dem Streben nach materiellem Wert und der Suche nach personeller Integrität ableiten. Auch dem Modell von Kirsch liegt ein rational agierender und anreizreagibler Mensch zugrunde. Die Nutzenfunktion des

84 Dowell, Goldfarb und Griffith (1998) modellieren Moral als diskontinuierlichen trade-off zwischen anderen Gütern. In ihrem Modell gehen moralische Vorstellungen nicht nur als zusätzliches Element in die Nutzenfunktion ein, sondern auch als Lageparameter in die Budgetrestriktion.

Alternativ könnte man auch grundsätzlich positiv geneigte Reaktionsfunktionen modellieren. Dies würde bei einem Linken z.B. bedeuten, dass er seine Verteilungsziele preisgeben würde, wenn dies eine Nettozahlung für ihn bedeutet. Die Neigung der Reaktionsfunktion ist insofern egal, als sie lediglich angibt, dass der Mensch auch bei der Verfolgung von wertebasierten Zielen anreizreagibel ist. Durch den moral space beschriebenes, gewünschtes Verhalten kann dann durch positive oder negative Anreize motiviert werden. Um eine stärkere Vergleichbarkeit zum Kirsch-Modell zu gewährleisten, wurde hier die negative Steigung gewählt.

Ein völlig verhaltensfixierter Ideologe hat eine vollkgmmensunelagstischę Reaktignskurve. 
traditionellen homo oeconomicus wurde lediglich um eine neue Nutzenkomponente, die der persönlichen Identität, erweitert.

Allerdings lässt das Modell noch einige Fragen offen, die für die Fragestellung bedeutsam sind. So scheint die Theorie noch nicht so weit entwickelt zu sein, dass sie aus dem aufgezeigten Wechselverhältnis von Selbstinteresse zu Wohlstandsförderung und dem Interesse am eigenen Selbst zur Identitätsbildung eindeutige Hypothesen anböte. Einerseits postuliert Kirsch (1994, S. 268f), dass die individuellen 0-Punkte langfristig nicht (zu weit) vom gesellschaftlichen Konsens abweichen können, um die Bildung eines Selbst nicht zu gefährden. Andererseits sind die Individuen stets bereit, durch genügend hohe Zahlungen (entlang ihrer Reaktionskurve) von ihren Moralvorstellungen abzuweichen. Es bleibt indes offen, was nun ausschlaggebend für die Identitätsbildung des Individuums ist: die Kontinuität der eigenen Moralstandards (Kirsch, 1997, S. 92), dann dürfte umfangreiche Korrumpierung nicht möglich sein, oder die inhaltliche Nähe der eigenen Moralvorstellungen am gesellschaftlichen Konsens (Kirsch, 1994, S. 268), dann ist jedoch die Kontinuität des Ichs unter Umständen nicht mehr gegeben. ${ }^{87}$

Der gravierendste Mangel des Modells ist, dass nur vage angedeutet wird, welche Anreizmechanismen die 0-Punkte bestimmen. Insbesondere die Erklärung, dass die Lage der 0-Punkte über vorhandene Wertvorstellungen erreicht wird, verschiebt den Erklärungsbedarf nur eine Stufe weiter zurück, da nun offen bleibt, wie diese Wertvorstellungen internalisiert werden. Auch eine Begründung, wie es zur Verschiedenheit der Elastizitäten der einzelnen Reaktionskurven kommen kann, bietet das Modell nicht - sie sind exogen.

\subsubsection{Konkurrierende individuelle Präferenzordnungen}

Die Idee, dass der Mensch nicht nur seinem reinen Eigeninteresse folgt, sondern auch aus einer Motivation heraus, die man als soziale Verantwortung bezeichnen könnte und die in Konkurrenz zu seiner Handlungsmotivation aus Eigeninteresse steht, ist bereits sehr alt. So verfolgt Margolis (1982, S. 44) ihre Ursprünge bis zu Plato's Unterscheidung zwischen Privatmann und Bürger zurück. ${ }^{88}$ Spätestens seit Arrow (1963, S. 86ff) in seinem Buch Social Choice and

87 Auch die im Modell dargestellte substitutive Beziehung zwischen self-interest und interest in one's own self wird als nicht zwingend betrachtet (Kirsch, 1997, S. 110). Sicher ist die Argumentation plausibel, jedoch wird dadurch die empirische Hypothesenprüfung erschwert.

Dieser Gedanke wurde u.a. auch von Rousseau in seiner Gesellschaftsvertragslehre aufgenommen. Rousseau geht davon aus, dass der Mensch in sich gespalten ist. Er ist einerseits ein selbstinteressierter „Bourgeois“, der ausschließlich als Eigennutzmaximierer handelt

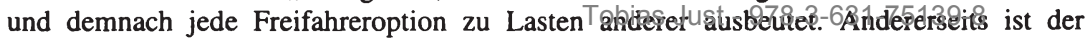


Individual Values die Unterscheidung zwischen Präferenzen (tastes) und zum Teil unbewussten letztendlichen Zielen (ultimate values) vornahm, werden in der Ökonomie auch Konzepte diskutiert, in denen der Mensch konkurrierende Sets von Nutzenfunktionen optimiert. Dabei beschreiben die tastes die konkreten Konsumpläne eines Individuums und die ultimate values die diesen Präferenzen übergeordneten eigentlich zentralen Ziele des Menschen. Harsanyi's Vorstellungen (1955, S. 314ff) sind noch weiter von dem traditionellen Bild des homo oeconomicus entfernt als diejenigen von Arrow. Während Arrows Konzept von selbstinteressierten, eigennutzmaximierenden Individuen ausgeht, die über ihre gezeigten Konsumpläne ihre teilweise im verborgenen allein eigenbezogenen Ziele zu verwirklichen suchen, unterstellt Harsanyi duale Nutzenfunktionen dergestalt, dass der Mensch sowohl eine subjektive Nutzenfunktion im neoklassischen Sinn besitzt, darüber hinaus jedoch (manchmal) in der Lage ist, das enge Korsett der Nutzenmaximierung zu verlassen, um seinen ethischen Präferenzen zu folgen. Diese sind völlig von der eigenen Person losgelöst und spiegeln seine Ansichten hinsichtlich des aus seiner Sicht gesellschaftlich Wünschenswerten wider. ${ }^{89}$

Zwanzig Jahre später greift Sen (1977) die Idee auf, indem er darauf hinweist, dass es drei Handlungsmotivationen gibt, einerseits die Eigennutzmaximierung im traditionellen Verständnis, zweitens eine Form, die sich am besten als egoistischer Altruismus (sympathy) kennzeichnen lässt, bei der ein Individuum deshalb gewillt ist, freiwillig Gesellschaftsbeiträge zu leisten, weil es ihm selbst ansonsten schlechter ginge. Diese Form des Altruismus ist also eigentlich nur eine Sonderform der Verfolgung des Eigeninteresses..$^{90}$ Darüber hinaus handelt der Mensch nach Sen (1977, S. 91ff) aber auch aus sozialer Verantwortung heraus (commitment). Dieses Handeln steht mitunter seinem Eigeninteresse entge-

Mensch aber auch „Citoyen“, was ihn in die Lage versetzt, ein höheres gesamtgesellschaftliches Ziel zu verfolgen, das seinem Streben als „Bourgeois“ entgegenstehen kann. Vgl. dazu Kirsch (1997, S. 184f) und für weitere frühe Hinweise auf den Menschen als geteiltes Selbst Kuran (1990, S. 16f). Vgl. vor allem auch die Ausführungen in 2.2.1 und dort die Schilderung der Sichtweise von Adam Smith.

89 Harsanyi (1955, S. 315) selbst geht davon aus, dass der Mensch zu dieser gesellschaftlich orientierten Betrachtungsweise wahrscheinlich nur selten befähigt, respektive gewillt ist. Die individuelle Wohlfahrtsfunktion, die seine ethischen Präferenzen abbildet, enthält dabei die einzelnen Nutzenfunktionen der anderen Gesellschaftsmitglieder als Elemente und gewichtet diese je nach Stand des Wissens eines Individuums (S. 321).

Der egoistische Altruismus umfasst z.B. die Investition in ein öffentliches Gut, um dadurch eine langfristig ausgerichtete Beziehung aufzubauen, die abdiskontiert höhere Nutzen stiftet, als wenn diese Kooperation nicht zustande kommen würde. Auch kann hierunter all jene Kooperationsbereitschaft subsumiert werden, die angestrengt wird, um eigenen intrinsischen Sanktionsmechanismen auszuweichen (Gewissensbisse) - man hilft anderen, um sich später keine Vorwürfe machen zu müssen und handelt also letztendlich aus Eigeninteresse. 
gen und ist nach Sen besonders bei der Produktion öffentlicher Güter relevant. Commitment kann als Wertebindung interpretiert werden, die zu unvorteilhaften Entscheidungen für das Individuum führen kann (Sen, 1987, S. 40ff).

Schließlich ist es das Verdienst von Margolis $(1981,1982)$, als erster versucht zu haben, die Berücksichtigung zweier Handlungsmotivationen zu formalisieren. Sein Konzept folgt ebenfalls eng der Vorstellung von Harsanyi, jedoch geht Margolis davon aus, dass das Gruppeninteresse immer Einfluss ausübt - nicht nur in den wenigen Momenten, in denen der Mensch sozusagen sich selbst verlassen kann. Bevor die Überlegungen von Margolis etwas detaillierter dargestellt werden, sollen die unterschiedlichen Konzepte schaubildartig in Tabelle 2.6 zusammengefasst werden.

Tabelle 2.6 : Die Modelle konkurrierender Nutzenfunktionen

\begin{tabular}{|c|c|c|c|}
\hline & \multicolumn{2}{|c|}{ Selbstbezogene Handlungsmotivation } & $\begin{array}{l}\text { Gesellschaftsbezogene } \\
\text { Handlungsmotivation }\end{array}$ \\
\hline $\begin{array}{l}\text { homo } \\
\text { oeconomicus }\end{array}$ & \multicolumn{2}{|c|}{ Eigennutzorientierung } & \\
\hline $\begin{array}{l}\text { Smith } \\
(1759 / 1776)\end{array}$ & \multicolumn{2}{|c|}{ Tugend der Eigenliebe } & $\begin{array}{l}\text { Tugenden der Huma- } \\
\text { nität, Gerechtigkeit, } \\
\text { Großzügigkeit und des } \\
\text { öffentlichen Interesses } \\
\end{array}$ \\
\hline Arrow (1951) & $\begin{array}{l}\text { Gezeigte Präferen- } \\
\text { zen gemäß tastes }\end{array}$ & $\begin{array}{l}\text { übergeordnete } \\
\text { values bestimmen } \\
\text { tastes }\end{array}$ & \\
\hline $\begin{array}{l}\text { Harsanyi } \\
(1955)\end{array}$ & \multicolumn{2}{|c|}{ subjektive Präferenzen } & $\begin{array}{l}\text { ethische Präferenzen } \\
\text { (selten relevant) }\end{array}$ \\
\hline $\operatorname{Sen}(1977)$ & $\begin{array}{l}\text { Eigennutzorientie- } \\
\text { rung }\end{array}$ & $\begin{array}{l}\text { egoistischer } \\
\text { Altruismus } \\
\text { (sympathy) }\end{array}$ & $\begin{array}{l}\text { uneigennütziger } \\
\text { Altruismus } \\
\text { (commitment) } \\
\end{array}$ \\
\hline $\begin{array}{l}\text { Margolis } \\
(1982)\end{array}$ & \multicolumn{2}{|c|}{ Eigennutzorientierung (S-Präferenzen) } & $\begin{array}{l}\text { Gruppeninteressen } \\
\text { (G-Präferenzen) }\end{array}$ \\
\hline $\begin{array}{l}\text { Kirsch } \\
(1991 ; 1994)\end{array}$ & Selbstinteresse & $\begin{array}{l}\text { Interesse } \\
\text { am eigenen Selbst }\end{array}$ & \\
\hline
\end{tabular}

Quelle: Eigene Darstellung.

Das Modell von Margolis basiert auf der Grundannahme, dass man menschliches Verhalten angemessen modellieren kann, indem man unterstellt, in jedem Menschen würden zu jeder Entscheidung zwei untersGhiedlighe Motizationen 
miteinander konkurrieren. Die konkrete Handlung ist dann das Ergebnis eines internen „Verhandlungsprozesses“ zwischen zwei Personen in dem jeweiligen Individuum. ${ }^{91}$ Auf der einen Seite steht die rein eigeninteressierte Person SSmith und auf der anderen Seite steht die rein gruppeninteressierte Person GSmith, welche allein das von G-Smith entworfene Gruppeninteresse verfolgt. Hierbei handelt es sich aber nicht um eine interindividuell konstante Größe, sondern G-Smith ist bemüht, das, was G-Smith als Gruppeninteresse perzipiert, zu maximieren.

Margolis führt zwei Begründungslinien aus, die diesen Ansatz rechtfertigen sollen. In seinem Evolutionsargument geht er davon aus, dass sowohl die Selbstals auch die Gruppenorientierung dominante Strategien im Überlebenskampf sein können und nebeneinander koexistieren können. Danach handelt der Mensch selbstorientiert, wenn es darum geht, sich in der eigenen Gruppe durchzusetzen, und er handelt gruppenorientiert, um die Gruppe gegenüber anderen Gruppen zu stärken (Margolis, 1982, S. 26ff). Seine Argumentation führt Margolis auf die Annahme von Charles Darwin zurück, der davon ausging, dass unterschiedliche Gruppen miteinander um knappe Ressourcen konkurrieren. Die Kooperation der Mitglieder innerhalb einer Gruppe erhöht die Chancen, sich gegenüber der anderen Gruppe durchzusetzen. Letztlich wäre die Gruppenorientierung aus der selbstbezogenen Überlebensorientierung abgeleitet und folglich eher vergleichbar mit Sen's egoistischem Altruismus.

Daher soll im folgenden v.a. der zweiten Argumentationslinie gefolgt werden. Diese geht von der Existenz einer allgemeingültigen internen „, Fair Share Allokationsregel" aus, die besagt, dass erstens die Neigung zur Befriedigung des reinen Selbstinteresses mit dem Umfang für soziale Zwecke verausgabter Mittel steigt, und dass zweitens der Umfang der für soziale Zwecke veranschlagten Ressourcen davon abhängt, wie sich der Grenznutzen aus Ressourcen für Gemeinschaftszwecke relativ zu dem Grenznutzen aus Ressourcen für Eigenzwecke verhält (Margolis, 1982, S. 36).

G möge die Nutzen beschreiben, die ein imaginärer gruppeninteressierter GSmith hat, wobei seine Nutzen nicht aus dem Akt des Gebens, sondern aus der Menge des insgesamt bereitgestellten Kollektivgutes resultieren. S hingegen möge die Nutzen des selbstinteressierten S-Smith darstellen. Die jeweiligen Grenznutzen werden durch $S^{\prime}$ und $\mathrm{G}^{\prime}$ gekennzeichnet, und g steht für die aufgewendeten Ressourcen zur Maximierung des Gruppeninteresses und $s$ für die aufgewendeten Ressourcen zur Befriedigung des Eigeninteresses. Dann lässt

91 Für die Organisation der „Verhandlung“ ist es nach Margolis nützlich, eine dritte, vermittelnde Person zu unterstellen, sodass das Modell von einer Dreiteilung der Person im Freud'schen Sinne ausgeht (Margolis, 1982, S. 53ff). Für eine neuere, spieltheoretische Darstellung vgl. die Modellierung als nicht-kooperatives Spiel zwischen dem egoistischen und altruistischen Selbst im Menschen bei Chirinkpo(1990) 
sich zeigen, dass es ein Gleichgewicht gibt, bei dem die Grenzneigung zur Befolgung des Selbstinteresses der Grenzneigung zur Befolgung des Gruppeninteresses entspricht und folglich eine intrapersonell stabile Ressourcenallokation vorgenommen wurde.

Es sei W eine subjektive Gewichtungsfunktion der konkurrierenden Interessen des Individuums, die festlegt, wie stark die Selbstinteressen im Vergleich zu den Gruppeninteressen bei der Entscheidung berücksichtigt werden. Die oben beschriebene Fair Share Allokationsregel besagt nun, dass W von dem Verhältnis der bisher verausgabten Mittel (g/s) abhängt, wobei gilt: $\frac{\partial W}{\partial(g / s)}>0$, d.h. je mehr

Ressourcen bereits für Gruppeninteressen ausgegeben wurden bei konstantem Einkommen, desto bedeutsamer wird annahmegemäß das Selbstinteresse.

Außerdem zeigt $\mathrm{G}^{\prime} / \mathrm{S}^{\prime}$ das Verhältnis zwischen den jeweiligen Grenznutzen an. Bei gegebenen Einkommen und steigenden Ausgaben für Gruppeninteressen $g$ sinkt dieses Verhältnis $\left(\frac{\partial\left(\mathrm{G}^{\prime} / \mathrm{S}^{\prime}\right)}{\partial \mathrm{g}}<0\right) .{ }^{92}$ Dann kann mit Hilfe von Teil A der Abbildung 2.5 veranschaulicht werden, dass im Punkt $\mathrm{GG}_{1}$ ein Gleichgewicht vorliegt, wenn die Bedingung $W=G^{\prime} / S^{\prime}$ erfüllt ist. Ist $W>G^{\prime} / S^{\prime}$ und geht man von abnehmenden Grenznutzen für $S$ aus, führt eine Reallokation von Ressourcen von gruppenbezogenen Ausgaben zu selbstbezogenen Ausgaben wieder zum Gleichgewicht zurück, da durch diese Umverteilung einerseits W sinkt und andererseits $\mathrm{G}^{\prime} / \mathrm{S}^{\prime}$ steigt. ${ }^{93}$

Eine interessante Implikation des Modells lässt sich durch den Teil B der Abbildung 2.5 darstellen. Hier ist $I_{1}$ die Budgetgerade und $G_{1}$ zeigt den Gleichgewichtspunkt, bei dem $\mathrm{W}=\mathrm{G}^{\prime} / \mathrm{S}^{\prime}$ gilt. Entlang einer Geraden aus dem Ursprung durch diesen Gleichgewichtspunkt bleibt $\mathrm{W}=\mathrm{g} / \mathrm{s}$ konstant; gleichzeitig steigt $G^{\prime} / S^{\prime}$ entlang dieser Geraden stetig an (unter der Annahme, dass $G^{\prime}$ durch zusätzliche Ausgaben eines Individuums unverändert bleibt und für $\mathrm{S}$ ab-

$\mathrm{G}^{\prime} / \mathrm{S}^{\prime}$ ist eine fallende Funktion, da bei konstantem Einkommen und steigendem $\mathrm{g}$ der Wert von S' steigt. Dies liegt daran, weil steigende Gruppenausgaben sinkende Individudausgaben bedeuten. Geht man von der üblichen Annahme sinkender Grenznutzen aus, folgt also aus der Zunahme von g auch eine Zunahme von S'. Gleichzeitig bleibt $\mathrm{G}^{\prime}$ konstant, da bei hinlänglich großer Gruppe kein Individuum davon ausgehen kann, dass die individuelle Mehrleistung auch für die Gruppe spürbar ist. Folglich sinkt der Wert des Bruches. W hingegen hat eine positive Steigung, da W die Bedeutung des Eigennutzmotivs darstellt, und diese Bedeutung nimmt annahmegemäß mit zunehmenden Gruppenausgaben zu.

93 Bei hinlänglich großen Gruppen kann davon ausgegangen werden, dass die individuelle Ressourcenverwendung keinen messbaren Einfluss auf $G^{\prime}$ hat, da $G^{\prime}$ nur von der Höhe der gesamt bereitgestellten Gutsmenge abhängt, nicht aber von den eigenen verausgabten Mitteln. 
nehmende Grenznutzen vorliegen). Nimmt dann das Einkommen von $I_{1}$ auf $I_{2}$ $\mathrm{zu}$, würde bei gleicher Ausgabenrelation die Gleichgewichtsbedingung verletzt werden, da im Punkt A dann $\mathrm{W}<\mathrm{G}^{\prime} / \mathrm{S}^{\prime}$ wäre. Ein neues Gleichgewicht $\mathrm{GG}_{2}$ könnte nur durch eine Umverteilung hin zu einer Stärkung von gruppenbezogenen Interessen erreicht werden. Der Einkommenspfad veranschaulicht, dass demnach gruppenbezogene Ausgaben in Margolis ${ }^{6}$ Modell superior sind.$^{94}$ Dies liegt natürlich daran, dass die Ausgaben zur Befriedigung des privaten Selbstinteresses zu abnehmenden Grenznutzen führen, die Ausgaben zur Befriedigung von Gruppeninteressen jedoch nicht.

Margolis kann mit seinem Modell gruppenorientiertes Verhalten beschreiben und ist damit auch in der Lage, z.B. das Wahlparadoxon aufzulösen (Margolis, 1982, S. 82ff), jedoch kann das Modell nicht in allen Punkten überzeugen. Zwar ist der naheliegende Kritikpunkt an der ungewohnten Darstellung dualer Nutzenfunktionen für ein Individuum gar nicht so bedeutsam, da Margolis dieses Vorgehen nur als erleichternden Kunstgriff wählt und dieselben Ergebnisse unter Verwendung einer einzigen Nutzenfunktion erzielt (Margolis, 1982, S. $147 \mathrm{ff})$.

\section{Abbildung 2.5 : Das Fair Share Gleichgewicht und der Einkommenspfad}

Teil A:

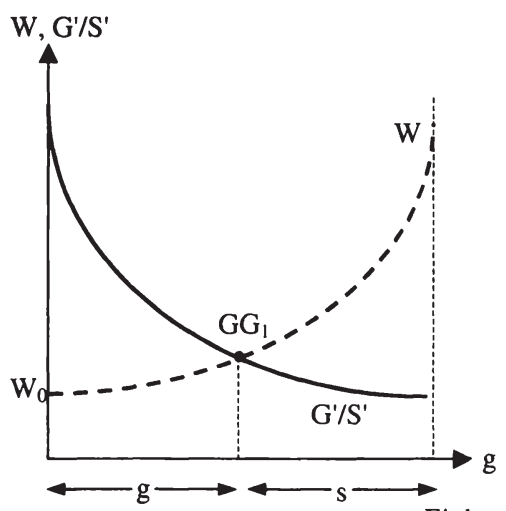

Einkommen
Teil B:

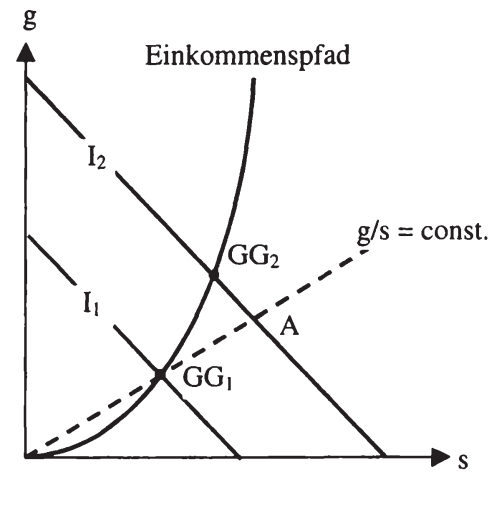

Quelle: Margolis (1982, S. 40).

94 Margolis (1982, S. 41) benutzt den Begriff superior in Abweichung zur gängigen Lehrbuchdefinition nicht für einen allgemein positiv einkommenselastischen Zusammenhang (vgl. z.B. Woll, 1990, S. 144), sondern für einen Zusammenhang mit einer positiven Einkommenselastizität größer eins. 
Allerdings ist seine Begründung für das Vorliegen einer solch zwiegespaltenen Persönlichkeit wenig überzeugend. Zum einen ist das Argument, dass Altruismus die dominante Strategie zur Gruppenselektion ist, nicht evolutionstheoretische Tatsache (vgl. Schotter, 1983, S. 556), und zum anderen übernimmt die Fair Share Allokationsregel dahingehend die Funktion eines Deus ex machina, dass Margolis nicht auf ihre eigene Begründetheit eingeht. Im Rahmen dieser Arbeit interessiert aber gerade die Konstituierung der Allokationsregel. Interpretiert man G-Smith als Person mit wertgebundenen Präferenzen und die Gewichtungsfunktion $\mathrm{W}$ als Maß für die Relevanz der Wertebindung, so interessiert, was G-Smith und v.a. W determiniert. Dies lässt die Theorie von Margolis jedoch nicht zu. Die Kritik von Taylor (1983, S. 152) zielt in eine ähnliche Richtung. Er wirft ein, dass die Möglichkeiten einer empirischen Prüfung der Theorie äußerst gering sind. Selbst wenn man die Modellierung zweier Nutzenfunktionen akzeptiert, bedarf es einer empirisch prüfbaren Theorie der Konstituierung von $\mathrm{W}$.

Es soll im folgenden Abschnitt gezeigt werden, dass dies in Anlehnung an das Konzept des Sozialkapitals möglich ist.

\subsubsection{Werte als Kapitalbestände des Menschen}

Die ersten beiden dargestellten Ansätze gingen jeweils von einer Dualität im Individuum aus. Zwar waren beide Modelle in der Lage, Wertorientierungen durch zusätzliche Handlungsmotivationen (entweder zur Sicherung des Selbst oder aufgrund einer unterstellten individuellen sozialen Nutzenfunktion) abzubilden, jedoch fehlte beiden Ansätzen eine klare Modellierung der Bestimmungsfaktoren dieser Wertebindung und -bildung, die zu empirisch prüfbaren Hypothesen auch hinsichtlich der Dynamik menschlicher Werte führen könnte.

Der folgende Ansatz überwindet diesen Mangel und ermöglicht, das soziologische Wertekonzept mit dem homo oeconomicus weitgehend auszusöhnen. Dies wird dadurch erreicht, dass zwei Grundannahmen neoklassischer Optimierungsmodelle leicht verändert werden:

1. Rationalität bedeutet nicht, dass der Mensch zu jeder Zeit Probleme vollständig beurteilen kann - seine Problemlösungsfähigkeit ist abhängig von seinen eigenen Erfahrungen und den Erfahrungen mit ihm im Kontakt stehender Dritter.

2. In die Haushaltsproduktionsfunktion im Sinne von Lancaster (1971) und v.a. Michael und Becker (1973) gehen neben Gütern, Dienstleistungen und der Zeit auch Kapitalbestände ein, von denen nur ein Teil unter der ausschließlichen Kontrolle des Individuums sind. 


\subsubsection{Begrenzte Problemlösungskompetenz}

Seit Simon $(1955,1957)$ das Konzept der begrenzten Rationalität (bounded rationality) in die Diskussion um ein geeignetes Menschenbild der Wirtschaftswissenschaften einführte, ${ }^{95}$ ist nicht nur über die allgemeine Sinnhaftigkeit des Konzepts gestritten worden, sondern auch bei denjenigen, die grundsätzlich dem Konzept folgen, gibt es starke Divergenzen bei der konkreten Definition (Beckmann, 1998, S. 321f). Allen Ansätzen ist zumindest gemeinsam, dass sie menschliches Verhalten nur als Ausdruck eines zwar intendierten, jedoch angesichts begrenzter Problemlösungsfähigkeit niemals vollständig gelingenden Optimierungskalküls begreifen. Für die folgenden Überlegungen ist das Schema von Heiner (1983, S. 562f) am geeignetsten. Dort wird begrenzte Rationalität als Lücke zwischen der Problemlösungskompetenz des Entscheiders und der Problemlösungsanforderung der Entscheidung (competence-difficulty-gap, C-D-Gap) erfasst. Dieses Modell ist deshalb besonders geeignet, da es v.a. durch die Konkretisierung von Denzau und North (1994, S. 8ff) eine klare Struktur aufweist. ${ }^{96}$

Die Problemlösungsanforderung ist weitgehend für den Entscheider exogen. Sie ist abhängig vom Umfang der benötigten Informationen, der Notwendigkeit, Einzelinformationen miteinander zu verknüpfen und der Verlässlichkeit von Informationsquellen, also der Höhe der Prüfkosten und der generellen Verfügbarkeit von Informationen. Je größer die Problemkomplexität ist, desto teurer wird es für den Entscheider, die für eine bestmögliche Entscheidung benötigte Informationsmenge zu sammeln und zu verarbeiten. Die C-D-Gap entsteht folglich dann, wenn die Informationskosten für eine first-best-Entscheidung für ein Individuum prohibitiv hoch sind. Wann dies für ein Individuum der Fall ist, hängt von dessen Problemlösungskompetenz - also von seiner Produktivität bei der Informationsverarbeitung - ab. ${ }^{97}$ Diese wiederum wird durch zahlreiche Faktoren bestimmt, die sich grob zwei Komponenten zuordnen lassen:

- Die kognitive Komponente beschreibt die Fähigkeit des Individuums, Informationen zu verarbeiten und wird zum einen durch die Zugriffsmöglichkeit auf eigene und fremde Erfahrungen bestimmt, welche wiederum von den

95 Simon (1955, S. 114) selber sprach zunächst von ,approximate rationality“. Seitdem ist eine Vielzahl von ähnlichen Begriffen entwickelt worden, von denen die Bezeichnung „,bounded rationality“ sich am ehesten durchsetzen konnte (vgl. z.B. Beckmann, 1998, S. 323ff). Daher soll sie auch hier Verwendung finden.

96 Ein ähnlicher Ansatz ist z.B. der von Furobotn und Richter (1994), die bounded rationality durch mangelnde Verfügbarkeit von Informationen und die Begrenztheit des menschlichen Verstandes begründen.

97 Es wird davon ausgegangen, dass grundsätzlich jedes Individuum jede Information erhalten und verarbeiten kann, dass aber nicht alle Menschen bei der Informationsbeschaffung und der Informationsverarbeitung gleich produktiv sind 
Faktoren Erziehung, Ausbildung, Einsatz von unterstützender Datenverarbeitungstechnik und Integration in soziale Netzwerke abhängen.$^{98}$ Zum anderen sind genetische Prädispositionen bedeutsam. Wie in jedem anderen Produktionsprozess führt auch hier eine unterschiedliche Faktorausstattung zu Divergenzen in der Produktivität, denn besser ausgebildete Personen können i.d.R. Informationen effektiver und schneller verarbeiten.

- Die eng mit der ersten verknüpfte motivationelle Komponente beinhaltet, inwiefern das Individuum durch institutionelle Anreize zu seinem Informationsstand motiviert wird. Neben den unter soziologischen oder psychologischen Aspekten interessanten Faktoren wie Erziehung und Erfahrungsgeschichte sind hierunter insbesondere institutionelle Arrangements wie z.B. die Ausgestaltung monetärer Anreizsysteme zu subsumieren.

Bereits an dieser Stelle ist es lohnenswert, innerhalb des Modells auf zwei Schlussfolgerungen hinzuweisen:

- In den ersten (formativen) Jahren wird die Problemlösungskompetenz fast ausschließlich durch Fremdentscheidungen bestimmt, und die ersten eigenen Erfahrungen werden i.d.R. innerhalb eines engen fremdbestimmten Rahmens gemacht. Dies könnte man gemäß der Rationalitätsannahme auch als Optimierungskalkül interpretieren. Solange jemand keine eigenen Erfahrungen gemacht hat, ist es transaktionskostensparend, sich auf "verlässliche“ Dritte zu berufen.

- Stellt man sich die kognitive Komponente als eine Informationsproduktionsfunktion mit den Faktoren Erziehung, Ausbildung, soziale Netzwerke und elektronische Datenverarbeitungskapazität vor, wird deutlich, dass der für das Individuum relative Preisverfall der Faktoren schulische Ausbildung und Datenverarbeitung in den letzten Jahrzehnten dazu führen musste, dass diese zur Produktion des Gutes Information relativ zu den anderen Input-Faktoren familiäre Erziehung und soziale Netzwerke stärkere Berücksichtigung finden mussten. Dies könnte dann als weiterer Grund für den beobachteten Bedeutungsverlust sozialer Netzwerke und die Individualisierungstendenz in modernen Industriegesellschaften gewertet werden.

Zusammenfassend ist festzuhalten, dass es rational ist, sich v.a. in den formativen Jahren durch andere leiten zu lassen. Außerdem ist bei Entscheidungen, in

Denzau und North (1994, S. 16ff) argumentieren, dass solche Kommunikationsnetze wiederum zu weiten Teilen auf gemeinsamen Werten und Ideologien aufbauen, um die Transaktionskosten der Kommunikation gering zu halten. Dies würde bedeuten, dass für Entscheidungen, bei denen soziale Netze zur Informationsgewinnung unumgänglich sind, vollständige Rationalität im neoklassischen Sinne nicht denkbar wäre. Es wird in 2.4.3.2 im Rahmen der Kennzeichnung der Elemente des menschlichen Humankapitals auf die sozialen Netzwerke zurück gegriffen. Die besondere Relevanz sozialer Netzwerke für die

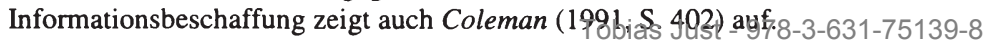


denen die C-D-Gap nicht geschlossen werden kann, ein Rekurs auf informationsverdichtende Entscheidungshilfen in Form von „mental models“ oder Ideologien unabdingbar. Gerade im Bereich öffentlicher Güter ist besonders häufig davon auszugehen, dass die Problemlösungsanforderung die Problemlösungskompetenz übersteigt. ${ }^{99}$ Daher ist es nicht verwunderlich, dass in diesem Bereich Ideologien eine wesentlich stärkere Rolle spielen als in marktwirtschaftlichen Kontexten. Mit Hilfe des erweiterten Humankapitalansatzes, der im folgenden erläutert wird, lässt sich zeigen, dass die Konsumpläne von Individuen intertemporal abhängig sind. Damit sind die beobachteten Konsumentscheidungen nicht nur Ausdruck von Präferenzen und relativen Preisen, sondern auch von vergangenen Entscheidungen und Entscheidungen Dritter.

\subsubsection{Theorie des Humankapitals}

In den meisten mikroökonomischen Lehrbuchdarstellungen werden die Präferenzen von Individuen hinsichtlich bestimmter Güter und Dienstleistungen durch Indifferenzkurvensysteme dargestellt. Diese Präferenzen sind üblicherweise exogen gegeben und sowohl unabhängig von der Zeit als auch von dem Verhalten anderer. Besonders auf der Grundlage der Arbeiten von Lancaster (1971) und von Becker ${ }^{100}$ ist es jedoch möglich, die mikroökonomische Haushaltstheorie dergestalt zu erweitern, dass einerseits die Bedeutung der Periodenkomplementarität, also der Pfadabhängigkeit des Konsums, und andererseits die Relevanz sozialer Interaktion Berücksichtigung bei der Modellierung der Konsumentscheidung finden können. In der „neuen“ mikroökonomischen Theorie maximieren Individuen eine Nutzenfunktion, in der nicht die Güter und Dienstleistungen direkt Eingang finden, sondern Waren (commodities), die mittels einer Haushaltsproduktionsfunktion erstellt werden. ${ }^{101}$ Für ein beliebiges Individuum lässt sich eine solche Nutzenfunktion $U$ wie folgt aufstellen:

$$
\mathrm{U}=\mathrm{U}\left(\mathrm{Z}_{1} ; \ldots ; \mathrm{Z}_{\mathrm{m}}\right)
$$

99 Wolf (1993, S. 79) nennt den Sachverhalt, dass bei vielen Entscheidungen der öffentlichen Hand nicht alle Wirkungskonsequenzen der Entscheidung bekannt sind, derived externalities. Hier ist es ex definitione nicht möglich, alle relevanten Informationen zu sammeln, da sie nicht vorliegen.

100 Vgl. u.a. Michael und Becker (1973), Becker (1974, 1992), Stigler und Becker (1977), sowie die Sammelbände Becker (1993) zu seinen Untersuchungen hinsichtlich des üblichen Begriffs des Humankapitals und Becker (1996) für einen Überblick über seine Arbeiten mit einem erweiterten Humankapitalbegriff.

${ }^{101}$ Lancaster (1971, S. 15ff) arbeitet mit einer Nutzenfunktion, in die direkt nur die Eigenschaften (characteristics) von Gütern eingehen. 
wobei $Z_{j}$ mit $j \in[1 ; m]$ die nutzenstiftenden commodities sind. Solche commodities sind z.B. Gesundheit, Reputation und Freude der Sinne (Becker, 1996, S. 5). Hier fußt die Theorie von Becker offenbar auf den Überlegungen von Arrow, dass der Mensch konkrete Konsumpläne (tastes) nur zur Verfolgung übergeordneter ultimate values umsetzt (vgl. S. 75f).

Diese Funktion ist zeitunabhängig. Die commodities werden durch eine Produktionsfunktion zu jedem Zeitpunkt $t$ durch (2.11) determiniert:

$$
Z_{t j}=Z_{t j}\left(X_{t j} ; T_{t j} ; P_{t} ; S_{t}\right)
$$

Hierbei ist $\mathrm{X}_{\mathrm{ij}}$ der Gütervektor, der zum Zeitpunkt $\mathrm{t}$ zur Erstellung der commodity $\mathrm{j}$ eingesetzt wird. $T_{t j}$ ist die für diese Produktion benötigte Zeit, $P_{t}$ ist der Bestand an persönlichem Kapital und $S_{t}$ ist der Bestand an Sozialkapital jeweils zum Zeitpunkt t. Während das Konzept des persönlichen Kapitals die Akkumulation von Erfahrungen durch vom Individuum selbst kontrollierte Entscheidungen erfasst, geht der Bestand an Sozialkapital auf Entscheidungen Dritter zurück, auf die das Individuum nur indirekt Einfluss nehmen kann.

Die Integration der Kapitalbestandsvektoren in die Produktionsfunktion erklärt, warum Präferenzen hinsichtlich der verwandten Güter und Dienstleistungen interpersonell und intertemporal selbst dann variieren können, wenn weder Preisnoch Einkommensänderungen stattgefunden haben. Die Kapitalbestände wirken in der Produktionsfunktion auf die Produktivität der eingesetzten Güter. Veränderungen in den Kapitalbeständen führen dann zu einer Reallokation der Güter $X_{i j}$ und der Zeiteinheiten $T_{t j}$. Zwar haben sich dann die durch den Kaufakt gezeigten Präferenzen hinsichtlich der Güter verändert, nicht jedoch die Präferenzen hinsichtlich der commodities $Z_{j}$, die in die Nutzenfunktion eingehen. So kann der Bestand an persönlichem Wissen hinsichtlich der Gesundheitsschädlichkeit eines konkreten Konsumplans (z.B. 20 Zigaretten am Tag) dazu führen, dass man von dem Gut Zigarette weniger nachfragt; dieses ändert jedoch nichts an der unverändert gebliebenen Präferenz bezüglich eines bestimmten Gesundheitsniveaus. Becker (1996, S. 17) spricht daher bei letzteren von MetaPräferenzen. $\mathrm{Da}$ in der Theorie Beckers diese unterschiedlichen Kapitalbestände zu großen Teilen das Verhalten von Menschen erklären, lohnt es, sie genauer zu charakterisieren.

Zu den Elementen des Vektors des persönlichen Kapitals $P_{t}$ zu jedem Zeitpunkt $t$ zählt u.a. jenes Wissen, das aus Bildungsinvestitionen generiert wurde und üblicherweise als Humankapital bezeichnet wird. Es ist aber sinnvoll, den Begriff weiter zu fassen und alle für die Produktion der commodities relevanten Erfah- 
rungen, also auch alle Konsumerfahrungen darunter zu subsumieren. ${ }^{102}$ Das Kapital wird in jeder Periode aus den Beständen der Vorperiode zuzüglich der in der Vorperiode angefallenen Nettoinvestitionen gebildet. Die Höhe der Nettoinvestition hängt von deren erwarteter Rendite, also von der erwarteten Produktivitätswirkung bei der Erstellung der commodities ab, und die Rendite hängt von der perzipierten Abschreibungsrate und der Zeitpräferenzrate bezüglich zukünftiger Perioden ab. Dies ist besonders dann interessant, wenn eine Erfahrung zwar in der Gegenwart positive Nutzen stiftet, jedoch zu einer Reduktion der Produktivität in der Zukunft führt. Schätzt z.B. ein Raucher seine Nutzenminderung in der Zukunft nur gering (er hat eine hohe Zeitpräferenzrate) und/oder überschätzt er die Abschreibungsrate (er unterschätzt die Suchtgefahr), wird er seinen Rauchkonsum in der Gegenwart erhöhen, damit jedoch über den Aufbau höheren „Rauchkapitals“ gleichzeitig den Konsum in der Zukunft festschreiben (Becker, 1992, S. 328ff). ${ }^{103}$ Dies führt dazu, dass seine Produktivität bei der Erstellung von commodities sinkt und so sein zukünftiger Nutzen aus diesen commodities ebenfalls zurückgeht. Es ist also die Unsicherheit bezüglich der Zeitpräferenzrate und der Abschreibungsrate, die dazu führt, dass v.a. in der Jugend Gewohnheiten und Abhängigkeiten entwickelt und Traditionen übernommen werden. ${ }^{104}$ Hinsichtlich der Bildung von Werten im soziologischen Verständnis ist jedoch der zweite Kapitalbestandteil, das Sozialkapital, bedeutsamer.

Der Begriff des Sozialkapitals geht auf Loury $(1977,1987)$ zurück, der damit die sozialen Strukturen, in die ein Individuum integriert ist, in ihrer Funktion als Ressourcen für ein Individuum erfassen wollte. ${ }^{105}$ Diese Ressourcen können

102 Die Vorstellung, dass man endogene Präferenzen über eine Pfadabhängigkeit des Konsums erfassen kann, geht nach von Weizsäcker (1971, S. 346) bis auf Marshalls Principles of Economics (1962) zurück.

Das heißt natürlich nicht, dass es unmöglich ist, vom Raucher zum Nichtraucher zu werden. Es impliziert aber die Möglichkeit, dass trotz Einsicht in die Gefahren des Nikotins der Konsum nicht eingeschränkt wird.

${ }^{104}$ Becker (1992, S. 328ff) definiert Gewohnheiten allgemein als solche intertemporalen Konsumbeziehungen, bei denen der Gegenwartskonsum positiv von dem Konsum der Vergangenheit abhängt. Eine Sucht läge dann vor, wenn früherer Konsum dazu führt, dass man in der Gegenwart oberhalb der Abschreibungsrate des Kapitals konsumiert. Eine Tradition nach Becker wäre dann gegeben, wenn die Abschreibungsrate so niedrig ist, dass Konsumentscheidungen der sehr weit rückliegenden Vergangenheit (sogar aus früheren Generationen) auf das momentane Konsumverhalten einwirken.

${ }^{105}$ In letzter Zeit wird zunehmend auch versucht, die Bedeutung von Sozialkapital als Aggregatgröße für makroökonomische Parameter zu isolieren. Die empirischen Ergebnisse sind aber nicht eindeutig. So argumentiert Fukuyama (1995) noch allein auf der Grundlage von Plausibilitätsüberlegungen. Die Untersuchungen von Putnam (1993) sowie Helliwell und Putnam (1995) für Italien, Putnam (1995) für die Vereinigten Staaten und die Analyse von

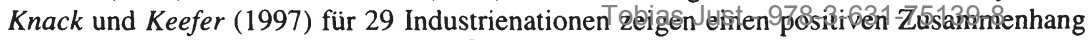


nach Coleman (1988) entweder als Verpflichtung und gegenseitige Erwartung, in der Form der bereits angesprochenen Informationsnetzwerke oder in der Gestalt gesellschaftlicher Normen vorliegen. ${ }^{106}$ Das Sozialkapital in dieser Definition beschreibt also den Integrationsgrad eines Individuums in soziale Netzwerke, und diese Integration begünstigt konkrete Handlungen, da sie deren Produktionskosten senkt. Dies bedeutet indes nicht, dass diese Handlungsbegünstigung per se auch für das Individuum nutzenfördernd sein muss (Coleman, 1991, S. 404 und Becker, 1996, S. 12).

Für die weiteren Überlegungen ist es sinnvoll, der Einschränkung von Becker (1996, S. 4) zu folgen. Becker definiert Sozialkapital in Gegenüberstellung zu seinem Begriff des persönlichen Kapitals als einen Vektor von Kapitalbeständen, der nicht direkt durch das Individuum selbst kontrolliert werden kann, sondern der durch Investitionen anderer in die Haushaltsproduktionsfunktion determiniert wird. ${ }^{107}$ Dies wird v.a. während der formativen Jahre geschehen, in denen quasi alle Erfahrungen aufgrund der extrem hohen C-D-Gap durch Dritte motiviert werden.

Ähnlich zum persönlichen Kapital bildet sich der Bestand an sozialem Kapital als Summe der Periodenanfangsbestände zuzüglich der Nettoinvestitionen der anderen Gesellschaftsmitglieder. Der Bestand an sozialem Kapital steigt also, wenn die Bruttoinvestitionen die Abschreibungen übersteigen. Analog zu der Wirkung von persönlichem Kapital können auch so Gewohnheiten, Abhängig-

zwischen dem Umfang von Vertrauen in einer Gesellschaft und dem wirtschaftlichen Wachstum bzw. der Investitionsleistung. Allerdings kommt Helliwell (1996a) für 13 asiatische Staaten und in einem Vergleich von Regionen der USA und Kanada (Helliwell, 1996b) zu dem Ergebnis, dass der Umfang an Sozialkapital - zumindest in der durch die verwandten Indikatoren operationalisierten Form - keine Erklärungskraft des Wirtschaftswachstums besitzt. Schließlich wird auf die kritischen Überblicksartikel von Woolcock (1998) und Fine (1999) verwiesen.

106

Die exakte Abgrenzung zwischen diesen drei Kategorien ist allerdings nicht immer möglich, da z.B. wechselseitige Erwartung in bezug auf ein bestimmtes Verhalten de facto einer Norm entspricht, die keiner expliziten externen Sanktion mehr bedarf, entweder weil Fehlverhalten keine Trittbrettfahrergewinne ermöglicht (Defektion führt sofort zur Beendigung der Produktion des Gemeinschaftsgutes) oder weil durch eine vorherige Internalisierung der Norm externe Sanktionsmechanismen obsolet werden. Ebenso kann man bei vielen Informationsnetzwerken davon ausgehen, dass es eine implizite Norm zumindest einer potentiellen Wechselseitigkeit gibt.

${ }^{107}$ Diese engere Definition vernachlässigt die Möglichkeit des Menschen, selber direkt seine Sozialstrukturen zu bestimmen. Diese Vernachlässigung ist dann aber unproblematisch, wenn der Einfluss des Einzelnen als sehr gering betrachtet werden kann, also in hinreichend großen Kollektiven. 
keiten und Traditionen generiert werden mit dem Unterschied, dass diese nicht direkt vom einzelnen Individuum kontrolliert werden können. ${ }^{108}$

\subsubsection{Werte und Humankapital}

Mit Hilfe einer graphischen Darstellung, die der Wohlfahrtsökonomik entliehen wurde, lässt sich das Phänomen „Werte“ im Rahmen des Humankapitalansatzes erläutern. Es können dann einige Hypothesen hinsichtlich der Wertebildung und des Wertewandels deduziert werden.

Beschrieben wird zunächst ein Haushalt, der Nutzen aus zwei produzierten commodities $Z_{3}$ und $Z_{4}$ zieht. Die Produktion von $Z_{3}$ und $Z_{4}$ erfolgt jeweils unter Einsatz der beiden Marktgüter $\mathrm{x}_{1}$ und $\mathrm{x}_{2}$ für beide Güter linear homogen. ${ }^{109}$ Fasst man zunächst $Z_{4}$ als rein privates Gut auf, dann ist es für Dritte nicht sinnvoll, in das dafür notwendige Humankapital zu investieren. Sozialkapital, das auch für die Produktion von $\mathrm{Z}_{4}$ eingesetzt werden kann, entsteht allein als Kuppelprodukt. Gibt es hingegen bei der Produktion von $Z_{3}$ starke positive Externalitäten, ist damit zu rechnen, dass dort eine gezielte Investition in Sozialkapital erfolgt, sodass $\mathrm{Z}_{3}$ in höherem Maße vom Sozialkapital $\mathrm{S}$ bestimmt wird. Beide Funktionen enthalten natürlich auch das persönliche Kapital als Argument. Dieser $\mathrm{Zu}$ sammenhang lässt sich wie folgt formalisieren: ${ }^{110}$

$$
\begin{gathered}
U=U\left(Z_{3} ; Z_{4}\right) \\
\text { mit } \\
Z_{3}=Z_{3}\left(x_{13} ; x_{23} ; \bar{S} ; \bar{P}\right) \text { bzw. } Z_{3}=x_{13} \cdot Z_{3}\left(1 ; \frac{x_{23}}{x_{13}} ; \frac{\bar{S}}{x_{13}} ; \frac{\bar{P}}{x_{13}}\right)
\end{gathered}
$$

${ }^{108}$ Hier wird ein Problem des Konzepts deutlich. Wenn das Sozialkapital eines Individuums eine Funktion der Investitionen aller Gesellschaftsmitglieder ist und diese Investitionen von rationalen Akteuren zumindest nur bei positiver Rendite gemacht werden, sie also ein grundsätzliches Interesse an der Gemeinschaftsinvestition haben, weist das Sozialkapital die üblichen Eigenschaften eines öffentlichen Gutes auf. Es ist folglich tendenziell mit einer Unterinvestition zu rechnen.

Die Annahme nicht-linear homogener Produktionsfunktionen ist problemlos möglich, führt aber zu keinen fundamental anderen Ergebnissen. Die Annahme abnehmender Grenzraten würde lediglich bedeuten, dass die Produktionsmöglichkeitenkurve im Quadranten IV stärker gekrümmt wäre.

$110 \overline{\mathrm{S}}$ und $\overline{\mathrm{P}}$ werden hier als Lageparameter exogenisiertbias Just - 978-3-631-75139-8 
und

$$
Z_{4}=Z_{4}\left(x_{14} ; x_{24} ; \bar{S} ; \bar{P}\right) \text { bzw. } Z_{4}=x_{24} \cdot Z_{4}\left(\frac{x_{14}}{x_{24}} ; 1 ; \frac{\bar{S}}{x_{24}} ; \frac{\bar{P}}{x_{24}}\right)
$$

Die Argumentationslinie kann am besten anhand der Abbildung 2.6 veranschaulicht werden. Diese ist analog zur wohlfahrtstheoretischen Herleitung der Transformationskurve aufgebaut (vgl. dazu z.B. Schumann, 1992, S. 262ff).

In Quadrant II ist ein Schachteldiagramm dargestellt. In Entsprechung zur wohlfahrtstheoretischen Darstellung ist hier die Kontraktkurve der geometrische Ort aller effizienten Inputkombinationen hinsichtlich der Produktionsmengen von $\mathrm{Z}_{3}$ und $\mathrm{Z}_{4}$. In keinem Punkt auf der Kontraktkurve kann gleichzeitig die Produktion beider commodities erhöht werden. ${ }^{111}$ Auf der Abszisse werden die Gutsmengen von $\mathrm{x}_{2}$, die für die commodity $Z_{3}$ eingesetzt werden $\left(\mathrm{x}_{23}\right)$, von $\mathrm{O}_{3}$ nach rechts abgetragen und die Gutsmengen, die von $x_{2}$ für $Z_{4}$ eingesetzt werden $\left(x_{24}\right)$, von $0_{4}$ nach links. Analog werden auf der Ordinate die Gutsmengen von $x_{1}$, die für $Z_{3}$ verwendet werden von $0_{3}$ nach oben gelesen $\left(x_{13}\right)$ und jene Gutsmengen von $x_{1}$, die für $\mathrm{Z}_{4}$ benötigt werden, von $\mathrm{O}_{4}$ nach unten gelesen. Im Quadranten III ist die lineare Ertragsfunktion für $Z_{4}$ dargestellt. Die Ertragsfunktion für $Z_{3}$ ist im Quadranten I abgebildet. Zunächst ist nur $Z_{3}^{1}$ relevant.

Die Produktionsmöglichkeitenkurve lässt sich nun im Quadranten IV durch einfaches Übertragen konstruieren. Dafür werden zunächst die Punkte auf der Kontraktkurve entlang der jeweiligen Isoquante auf die Gerade $\overline{0_{3} \mathrm{O}_{4}}$ projiziert. Da entlang dieser Geraden - anders als entlang der Kontraktkurve - ein konstantes Güterpreisverhältnis vorliegt, können die einzelnen Produktionsmengen über die beiden Ertragsfunktionen in den Quadranten I und III übertragen werden. Das Ergebnis ist die in IV dargestellte konkave Produktionsmöglichkeitenkurve, die gemeinsam mit der durch $U$ bestimmten Indifferenzkurvenschar das Optimum der Haushaltsproduktion festlegt - hier $\mathrm{H}_{1}$. Die Indifferenzkurven sind gemäß der Becker'schen Annahmen in der Zeit stabil, also unabhängig von Preisen, dem Einkommen und dem Kapitalbestand.

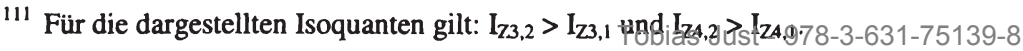




\section{Abbildung 2.6 : Eine Erhöhung des Sozialkapitals führt zu höherem Nutzenniveau}

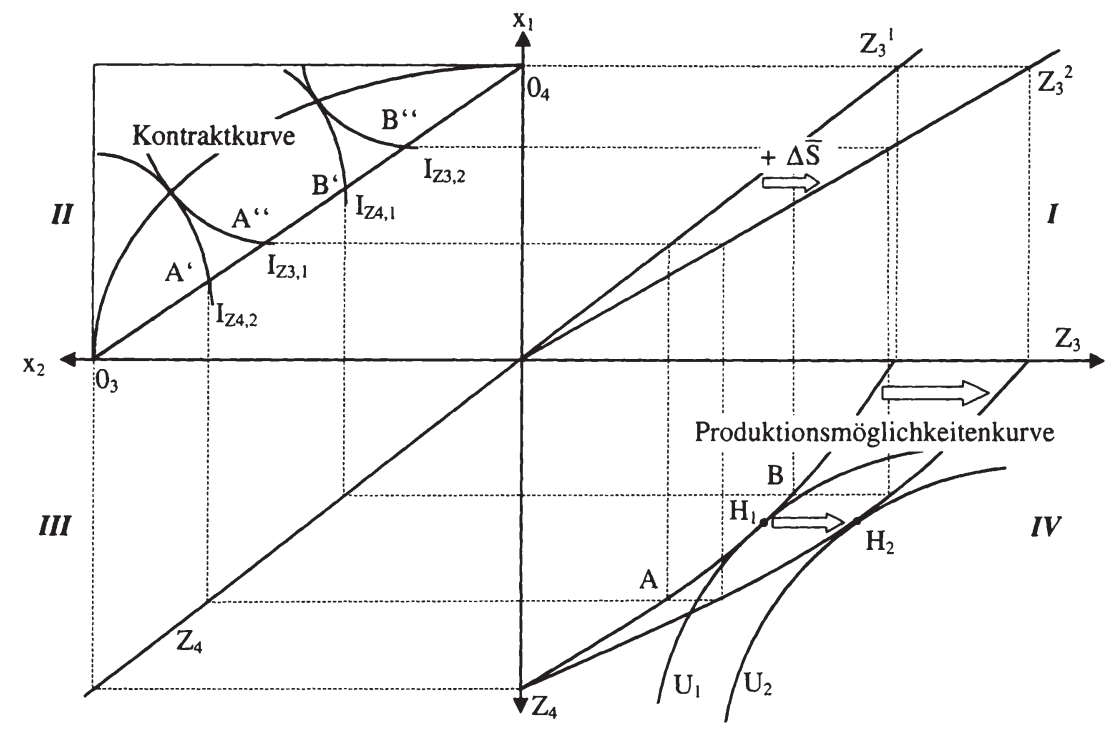

Quelle: Eigene Darstellung in Anlehnung an Schumann (1992, S. 264ff).

Eine Erhöhung des Sozialkapitals möge nun bedeuten, dass $\mathrm{Z}_{3}$ produktiver hergestellt werden kann. Dies ist darstellbar durch eine Rechtsdrehung der $\mathrm{Z}_{3^{-}}$ Kurve in Quadrant I von $Z_{3}^{1}$ auf $Z_{3}^{2}$, mit dem Ergebnis, dass sich die Produktionsmöglichkeitenkurve um den Schnittpunkt mit der $Z_{4}$-Achse nach außen dreht und so ein höheres Nutzenniveau $U_{2}$ erzielt werden kann. Durch die Fremdinvestition in Sozialkapital wählt das Individuum selber seine Inputs so (hier unverändert), dass nicht nur ein von anderen (gesellschaftlich?) erwünschtes Verhalten (mehr von $\mathrm{Z}_{3}$ ) auftritt, sondern auch das Individuum selber ein höheres Nutzenniveau erreichen kann.

Hier ist auch die commodity $Z_{4}$, an der die Gemeinschaft eigentlich kein Interesse hat, abhängig von S. Jedoch wirkt hier die Erhöhung des Sozialkapitals zum Beispiel durch einschränkende Verhaltensnormen wie eine negative Externalität auf das Individuum produktivitätsmindernd. Die Kurve $Z_{4}$ dreht sich von $\mathrm{Z}_{4}^{1}$ auf $\mathrm{Z}_{4}^{2}$ nach oben, sodass sich die Produktionsmöglichkeitenkurve nun an beiden Achsenabschnitten dreht. Je nachdem, ob der produktivitätssteigernde Effekt auf $Z_{3}$ oder die hemmende Wirkung auf $Z_{4}$ überwiegt, ist der Nettoeffekt auf den individuellen Nutzen positiv oder negativ (hier ist ein negativer Nettoeffekt dargestellt). 
Abbildung 2.7 : Eine Erhöhung des Sozialkapitals senkt das Nutzenniveau

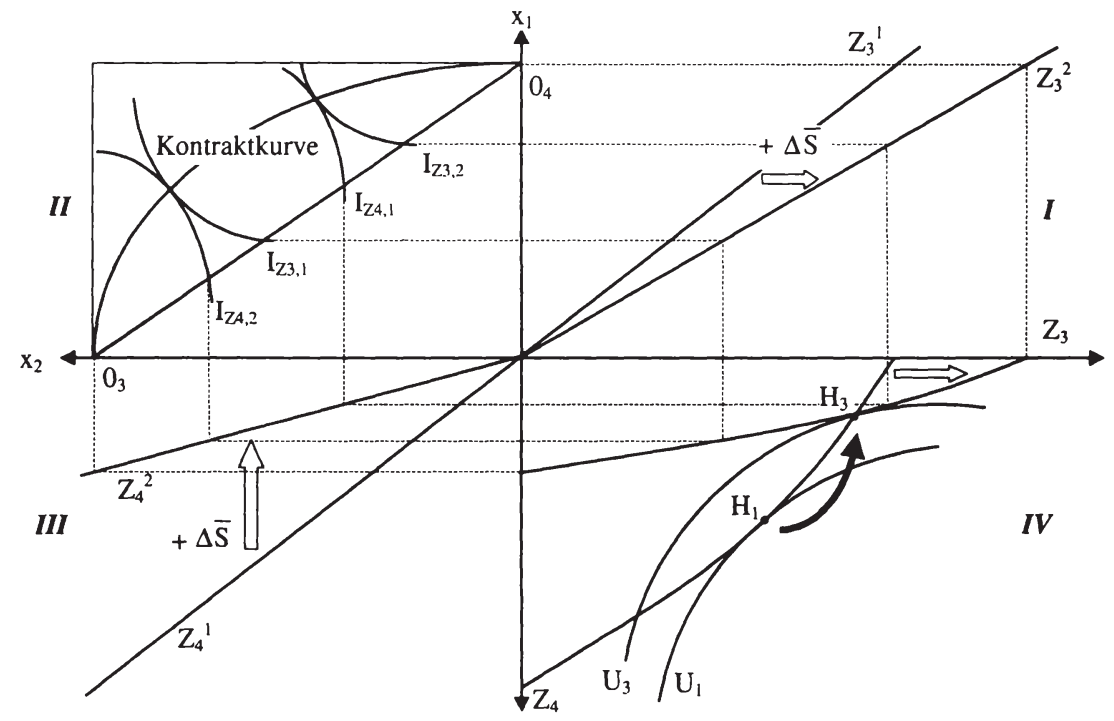

Quelle: Eigene Darstellung in Anlehnung an Schumann (1992, S. 264ff).

Dieses Modell ist nun in der Lage, Erkenntnisse der soziologischen Werteforschung abzubilden und darüber hinaus neue empirisch prüfbare Hypothesen zu generieren.

1. Werte sind Ausdruck einer Investition anderer in den Aufbau von Sozialkapital, die in erster Linie den Interessen der Investoren dienen. Die Allgemeinheit von Werten resultiert dann aus transaktionssparendem Investitionsverhalten - es wird dort investiert, wo eine Vielzahl positiver Verhaltensweisen induziert werden kann.

2. Aufgrund der sehr großen C-D-Gap während der formativen Jahre ist die Bedeutung des Sozialkapitals zu diesem Zeitpunkt besonders hoch. Da diese Anfangsbestände auch über die indirekte Beeinflussung der eigenen Erfahrungen in hohem Maße Konsumverhalten festschreiben können und dieses persönliche Kapital dann ebenfalls zu Gewohnheiten führen kann, ist die Bedeutung der formativen Jahre anhand dieses Modells erklärbar.

3. Darüber hinaus wird auch deutlich, dass die formativen Jahre zwar wichtig, aber nicht allein als Bestimmungsphase für Werte herangezogen werden können. Lebenszykluseffekte sind zu erwarten, da

- Sozialkapital sukzessive abgeschrieben wird und sein Fortbestand an permanente Reinvestition gebunden ist, 
- die Bedeutung des persönlichen Kapitals mit der Zeit zunimmt. Mit demselben Recht, wie in Abbildung 2.7 das Sozialkapital mit negativer Wirkung in die $\mathrm{Z}_{4}$-Funktion integriert wurde, kann angenommen werden, dass in vielen Fällen ein Zuwachs an persönlichem Kapital diese negative Wirkung reduziert und diese Reduktion vom Individuum zum Teil gesteuert werden kann,

- Bruttoinvestitionen in Sozialkapital an Rendite verlieren können. Dies wäre z.B. erklärlich, wenn Personen flexibler in ihren Sozialkontakten werden. Als Begründung könnte angeführt werden, dass junge Menschen früher das Elternhaus verlassen, dass sich die Berufswelt zu mehr regionaler und funktionaler Flexibilisierung verändert hat oder dass die Beendigung von Beziehungen (Scheidungen) mit geringeren Austrittskosten verbunden ist. Dann sinkt c.p. die Rendite aus einer langfristigen Investition und folglich auch das Investitionsvolumen. Auch kann die technische Entwicklung dazu führen, dass die relative Rendite aus Investitionen in Sozialkapital zurückgeht, weil andere Investitionen rentierlicher geworden sind. Diente z.B. ein Teil des Sozialkapitals als Instrument zur Informationsgewinnung, dann ist damit zu rechnen, dass diese Funktion im Zuge der Technisierung der Informationsverarbeitung zurückgedrängt wird. Beide Momente würden dann zu einem Abbau des Sozialkapitals und zu beobachtbarem Wertewandel, wenn nicht sogar zu Werteverlust führen.

4. Folglich ist Wertewandel dann wahrscheinlich, wenn es Änderungen in der durchschnittlichen Verweildauer in sozialen Netzen gibt (Familie, Arbeitsplatz, etc.) oder wenn sich der Informatisierungsgrad und die Informationsstruktur ändern (Einführung von Datenverarbeitung, Diversifizierung der Medien und Verbreiterung des Medienangebots). ${ }^{112}$ Wertewandel findet also immer dann statt, wenn sich die Beschränkungen für das Investitionsverhalten in Sozialkapital bzw. für das Desinvestitionsverhalten des Sozialisanden ändern.

5. Ist eine Desinvestition seitens des Sozialisanden in individuell negativ wirkendes, jedoch gesellschaftlich positiv erachtetes Sozialkapital zeit- also kostenintensiv, wird Zeitreichtum tendenziell zu einer Instabilität der Werte führen. Dies ist bemerkenswert mit Hinblick auf die rasanten Änderungen in der Arbeitswelt in den letzten Jahren (Arbeitszeitverkürzung, Entwicklung der Unterbeschäftigung, etc.) und würde bedeuten, dass der Weg in die Freizeitgesellschaft auch zu einer Erosion des Wertefundaments führt. Gleich-

112 Wichtig ist hierbei, dass nicht jeder Sozialkontakt gleichbedeutend mit einer Investition in Sozialkapital ist. Erst wenn die Sozialkontakte sehr langfristig sind, ist eine Investition in Wertebildung effizient. Zwar ist die moderne Berufswelt auch dadurch gekennzeichnet, dass man mit einer zunehmenden Zahl von Kontaktpersonen zusammenarbeitet, jedoch sind zahlreiche dieser Arbeitskontakte zu flüchtig, als dass der Aufbau einer gemeinsamen Wertebasis lohnenswert würde. 
zeitig hätten Zeitarme tendenziell stabilere Werte. Kommt Jennings (1990) in seiner Studie also zu dem Ergebnis, dass gut ausgebildete Arbeitnehmer tendenziell stabilere Wertestrukturen besitzen, kann dies daran liegen, dass sie zu den Zeitarmen gehören (niedrigere Akademikerarbeitslosigkeit, anspruchsvollere und zeitintensivere Tätigkeiten) und Desinvestitionen in Sozialkapital zu hohe Opportunitätskosten bedeuten.

6. In einer Gesellschaft, in der gesellschaftliche Schichten durchlässiger werden, ist es dann auch nicht verwunderlich, dass das Wahlverhalten nicht mehr angemessen durch die Zugehörigkeit zu einer gesellschaftlichen (Einkommens-)klasse dargestellt werden kann, da eigene Klassenzugehörigkeit und diejenige der relevanten Sozialisationsagenten auseinanderfallen können. Es gibt also eine zunehmende Zahl von Wählern, deren Werte maßgeblich durch Angehörige anderer Klassen mitgeprägt wurden. ${ }^{113}$

7. Werte und Ideologien sind insbesondere bei öffentlichen Gütern relevant, da durch die Existenz der positiven Externalitäten Dritte erst einen Anreiz zur Investition in Sozialkapital haben. Bei rein privaten Gütern, bei denen keine externen Effekte vorliegen, ist die Investition in Sozialkapital nicht sinnvoll. Daher lässt sich das Verhalten auf privaten Gütermärkten auch besonders gut durch das enge ökonomische Verhaltensmodell beschreiben.

Der Humankapitalansatz besitzt also die Vorzüge, dass er Werte und Ideologien innerhalb eines Konzepts erweiterter Nutzenfunktionen darstellen kann, ohne dass das Modell des homo oeconomicus fallen gelassen werden muss. Wenn der Bestand an Sozialkapital maßgeblich durch das bewusste und/oder unbewusste Investitionsverhalten anderer bestimmt wird, kann das wertegebundene Wohlverhalten des Menschen weiterhin adäquat als Eigennutzmaximierung unter $\mathrm{Ne}$ benbedingungen begriffen werden. Das Individuum ist dann durch seinen, von ihm nur marginal beeinflussbaren Bestand an Sozialkapital in seiner Handlungswahl zusätzlich zu den üblichen Zeit- und Budgetrestriktionen beschränkt. Werte und Ideologien werden hier als Restriktionen aufgefasst, wodurch sie sinnvoll von Ökonomen analysiert werden können. ${ }^{114}$ Gemäß der Theorie der revealed preferences bleibt die Aussage natürlich weiterhin richtig, dass Werte in die Präferenzen hinsichtlich der ausgewählten Güter und Dienstleistungen $\mathbf{x}_{\mathbf{i}}$ eingehen, wie dies in 2.2.1 ausgeführt wurde. Da dieser Teil der gezeigten Präferenzen jedoch in großem Umfang durch Dritte bestimmt wird, ist es sprachlich genauer, hier für das Individuum von einer Restriktion zu sprechen.

113 Vgl. zu der Erosion des Klassenwahlverhaltens in der Bundesrepublik u.a. Pappi (1987).

114 Zwar könnte man argumentieren, dass Werte und Ideologien zusätzlich direkt in die Nutzenfunktion U über die Grenzrate der Substitution zwischen den einzelnen Verbrauchsleistungen $\mathrm{Z}$ eingehen; da jedoch angenommen wurde, dass $\mathrm{U}$ intertemporal und sogar interpersonell konstant ist, kann dieser Zusammenhang als menschliche Konstante zumindest für Ökonomen unberücksichtigt bleiben.

Tobias Just - 978-3-631-75139-8 
Auch die Sozialisationsagenten handeln streng eigennutzorientiert, wenn sie in das Sozialkapital eines Individuums investieren, wobei ihr Investitionsverhalten wiederum zu Teilen durch ihren eigenen Bestand an Sozialkapital bestimmt werden mag. Sie richten ihr Investitionsverhalten an der erwarteten Rendite einer Investition in Sozialkapital und der erwarteten Rendite alternativer Anlagemöglichkeiten aus.

\subsubsection{Zusammenfassung und kritische Würdigung der Theorien}

Die ökonomische Theorie der Politik scheint in einigen Bereichen durch die Ergebnisse empirischer Forschung in Erklärungsnotstand geraten zu sein, dadurch dass Menschen in vielen öffentlichen Entscheidungen selbst dann Kooperationsbereitschaft zeigen, wenn dies ihrem Eigeninteresse entgegenläuft. Die drei dargestellten Ansätze zeigten, dass es grundsätzlich möglich ist, diesen scheinbaren Widerspruch zwischen ökonomischer Modellierung und beobachtetem Verhalten aufzulösen. In dem Modell von Kirsch wurde dafür eine zusätzliche Handlungsmotivation eingeführt. Menschen handeln wertgebunden, da sie eine Präferenz für die Schaffung und Erhaltung einer eigenen Identität haben. Diese Präferenz wird zum Teil durch die Vorgaben ihrer Umgebung geprägt. Sie agieren individuell und vollständig eigennutzorientiert, wobei diese Eigennutzorientierung durch den Zwang, die Identitätsdefinition über den gesellschaftlichen Rahmen herzustellen, dazu führt, dass man nicht zu weit von den akzeptierten Wertvorstellungen entfernt sein darf (Kirsch, 1997, S. 108). Allerdings fehlt eine klare ökonomische Begründung, wie es zu einer Differenz zwischen der eigenen und der gesellschaftlichen Ansicht (0-Punkt) kommt bzw. wie zeitliche Veränderungen entstehen können. ${ }^{115}$

Auch das Modell von Margolis modelliert gesellschaftlich relevante Werte in Form von Präferenzen. Bei Margolis handeln Menschen in Entscheidungssituationen nicht nur gemäß eines reinen Eigeninteresses, sondern berücksichtigen stets parallel das, was sie selber als Gemeinwohl begreifen. Ihre gemeinschaftsorientierte Handlung ist Ausdruck eines intrapersonellen Allokationsprozesses zwischen der reinen Eigennutzmotivation und einer Gemeinwohlorientierung. Anders als bei Kirsch wird hier allerdings eine der Grundannahmen des ökonomischen Menschenbildes aufgegeben. Gerade dieser Sachverhalt des MargolisModells macht die empirische Überprüfung unmöglich, da das Verhalten des

115 Die Hypothesen, die Kirsch (1997, S. 108) anbietet, bleiben noch vage. So formuliert Kirsch, dass positives Wissen und Wertvorstellungen in einem Wechselverhältnis stehen. Dies ist offensichtlich konform mit dem Sozialisationsverständnis in der Soziologie. Auch sein Hinweis auf unterschiedliche 0-Punkte je nach gesellschaftlicher Subgruppe deutet an, dass die Rolle der Sozialisationsagenten Berücksichtigung figden knann $_{-1}$ :75139-8 
Menschen dann immer von dem unerklärbaren Gewicht der beiden Personen in einem Menschen abhängt, und dieses Gewicht kann interpersonell verschieden sein.

Durch Zuhilfenahme des Humankapitalansatzes ist es möglich, sowohl die Bedeutung, die die Identitätsfindung für den Menschen besitzt, als auch die Gewichtungsfunktion W in dem Margolis-Modell zu endogenisieren. Demnach könnte das Humankapital als "missing link“ für die beiden anderen Theorien aufgefasst werden.

Für das Kirsch-Modell hieße das z.B., dass man die Identitätsfindung als eigene Verbrauchsleistung $\mathrm{Z}$ interpretiert, die grundsätzlich und für alle Menschen identisch in einem Substitutionsverhältnis gemäß der Grenzrate der Substitution der U(Z)-Funktion zu anderen Verbrauchsleistungen steht. Die konkrete Produktionsleistung eines Individuums hängt dann u.a. von seinen Kapitalbeständen $\mathrm{ab}$, sodass unterschiedliche soziale Umfelder mit jeweils verschiedenem Investitionsverhalten der Beteiligten auch zu differierendem Sozialbewusstsein, oder in der Terminologie des Kirsch-Modells, zu differierenden 0-Stellen und Elastizitäten führen.

Ähnlich ließe sich innerhalb des Margolis-Modells argumentieren, dass die Gewichtungsfunktion W des Menschen durch das Investitionsverhalten der Umwelt in Sozialkapital determiniert wird. Grundsätzlich scheint es möglich, empirisch prüfbare Hypothesen hinsichtlich der Bestimmungsfaktoren der Rendite einer solchen Investition in Sozialkapital aufzustellen. Da innerhalb dieser Arbeit allein die theoretische Möglichkeit einer Berücksichtigung von Werten und Ideologien im ökonomischen Modell interessiert, kann auf eine ausführliche Hypothesenprüfung verzichtet werden.

Auch der häufig geäußerte Einwand, dass Werte nicht nur rein funktionalen Charakter zur Überwindung von Sozialen-Dilemma-Situationen haben, wie dies in der Spieltheorie oft modelliert wird (vgl. Weise, 1995), sondern dass Werte auch zu sozial und individuell unerwünschtem Verhalten führen können $(\mathrm{Ku}$ bon-Gilke, 1995, S. 214; Sugden, 1989, S. 97), lässt sich mit Hilfe des Sozialkapitalansatzes begründen. Dies könnte nämlich darauf zurückzuführen sein, dass die Abschreibungsrate von Sozialkapital mitunter so gering ist, dass heute beobachtetes Verhalten noch durch lang zurückliegende Investitionen festgeschrieben wurde. Die damals rationale Investition bewirkt heute ein Verhalten, dass angesichts schnell veränderter Rahmenbedingungen als unsinnig erscheint. ${ }^{116}$

116 Allerdings muss man sich dabei im Klaren darüber sein, dass bei dieser Interpretation Erklärungsbedarf hinsichtlich der konkreten Höhe der jeweiligen Abschreibungsrate bleibt. Diese könnte z.B. vom Informationsstand des Individuums und seiner Bereitschaft, seine Werte einer rationalen Prüfung zu unterwerfen, abhängenust - 978-3-631-75139-8 
Zusammenfassend lässt sich also sagen, dass das ökonomische Verhaltensmodell offen genug ist, um selbst so komplizierte Sachverhalte wie Gemeinwohlorientierung und Selbstfindung zu berücksichtigen, solange man die zwei Annahmen akzeptiert, dass Menschen erstens nur beschränkt rational handeln können und dass ihr Konsumverhalten zweitens intertemporal durch ihr eigenes und das Verhalten anderer beeinflusst wird. Dass keine dieser beiden zusätzlichen Annahmen den homo oeconomicus in seinen Grundfesten erschüttert, steht auch für Kirchgässner (1991, S. 31f und S. 40ff) fest. Auch der wertgebundene Mensch reagiert auf Anreize systematisch und prognostizierbar und die internalisierte Wertestruktur ist Ausdruck dieses Sachverhalts. Folglich ist die Ökonomie keineswegs darauf angewiesen, Ideologie als reines Instrument zur kostengünstigen Wählergewinnung oder als exogene Restgröße zu verstehen.

In dem folgenden Kapitel sollen nun Ideologiedimensionen diskutiert werden, um daraus die für die Wirtschaftspolitik relevanten Dimensionen zu filtern, in einem Indikator zu verdichten und geeignete Hypothesen für die spätere empirische Prüfung zu entwickeln. 


\title{
Kapitel 3 \\ Konzeptionen ideologischer Wirtschaftspolitik
}

\begin{abstract}
„Les mots de droite et de gauche ne signifient plus rien. Ils ne signifient rien parlamentairement et rien non plus en dehors du Parlament. "(de Pressac, S. $34 f)$.
\end{abstract}

In diesem Kapitel soll gezeigt werden, wie in einem demokratischen Parteiensystem aus Ideologien unterschiedliche wirtschaftspolitische Ziele und Instrumenteinsätze entwickelt werden können. Dafür wird in einem ersten Schritt diskutiert, wie sich die Parteien ideologisch kategorisieren lassen. Insbesondere interessiert dabei das ubiquitäre Links-Rechts-Schema, das für den Vergleich politischer Ideologien Anwendung findet. Für dieses Schema wird in Abschnitt 3.3 ein Indikator vorgestellt, der in der späteren empirischen Analyse verwendet wird. Schließlich werden in Abschnitt 3.4 aus der Definition des Links-RechtsSchemas einzelne, fokale Elemente linker bzw. rechter Wirtschaftspolitik abgeleitet. Diese Elemente sind die Arbeitsmarktpolitik, die Preispolitik (Inflation) und zum dritten die zum Teil daraus resultierende Budgetpolitik.

\subsection{Politisch relevante Konfliktlinien}

Jede Gesellschaft ist durch unterschiedlich stark ausgeprägte Konflikte gekennzeichnet. Diese Konfliktlinien (cleavages ${ }^{117}$ ) wurden, folgt man Lipset und Rokkan (1967) durch historisch abfolgende gesellschaftliche Verwerfungen wie z.B. die Reformation, die Abkehr vom absolutistischen Feudalsystem im ausgehenden 18. Jahrhundert und zu Beginn des 19. Jahrhunderts und die Industrielle Revolution herausgebildet und sind zum einen durch Interessenheterogenität und zum anderen durch kulturelle Subsysteme innerhalb der Gesellschaft bestimmt. Konkret äußern sich cleavages z.B. in sozialen Klassen, Religionsgemeinschaften oder ethnischen Minderheiten in der Gesellschaft. Jedoch erst das Vorhan-

${ }^{117}$ Manche Autoren benutzen die Begriffe cleavage und Dimension unterschiedslos. Diesem

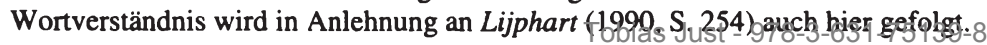


densein von Eliten innerhalb der einzelnen Gruppen lässt aus den zunächst nur latenten Interessengruppen feste Parteien entstehen. ${ }^{118}$

Da Parteien im wesentlichen durch ihre Funktion der Interessenmobilisierung und -vertretung bestimmt werden, bilden sich gesellschaftliche Konfliktlinien i.d.R. auch innerhalb des Parteiensystems ab (von Beyme, 1985, S. 13f). ${ }^{119}$ Nach Lijphart (1990, S. 254) müssen für politisch relevante Konfliktlinien folgende Kriterien zur Kennzeichnung von Parteien erfüllt sein: Erstens muss die ideologische Verortung der Partei, nicht der Wählerbasis, betrachtet werden, da es durchaus geschehen kann, dass ein in der Wählerbasis existentes cleavage keinen Eingang in die politische Diskussion findet. Zweitens sollte auf die ideologische Differenz zwischen Parteien und nicht innerhalb der Partei abgehoben werden. Drittens sollen nur die bedeutsamen Parteien, also diejenigen, die entweder Koalitionspotential oder genügend starken gesellschaftlichen Einfluss besitzen, als Grundlage für die Darstellung der Konfliktlinienstruktur des Parteiensystems dienen. Schließlich weist Lijphart (1990, S. 254) darauf hin, dass lediglich die persistenten cleavages herausgefiltert werden sollen, um politisch kurzfristige Moden unberücksichtigt zu lassen. ${ }^{120}$ Welche allgemeinen Konfliktlinien diese Kriterien erfüllen, wird in der Literatur nicht einheitlich beantwortet.

Tabelle 3.1 zeigt eine Übersicht der am häufigsten zitierten Studien. Sie veranschaulicht, dass generell zur Beschreibung von Parteiensystemen lediglich die sozio-ökonomische und die Konfliktlinie zwischen Kirche und Staat von allen Wissenschaftlern als existentes und vor allem persistentes cleavage anerkannt werden. Diese Einschätzung wird durch zahlreiche empirische Untersuchungen von Wahlergebnissen unterstrichen, in denen gezeigt werden konnte, dass das sozio-ökonomische cleavage gemeinsam mit der religiösen Konfliktlinie besonders starke Erklärungskraft der Wahlergebnisse besitzen. ${ }^{121}$

${ }^{118}$ Die folgende Darstellung folgt nicht sklavisch dem Konfliktlinienmodell von Lipset und Rokkan. Zentral ist nicht die ihrem Modell zur Entstehung von Parteien zugrunde liegende Theorie, sondern die naheliegende Überlegung, dass Parteien ideologische Spannungslinien in dem Wählerkollektiv abbilden. Für eine detaillierte kritische Würdigung des Konfliktlinienmodells vgl. z.B. Decker (2000).

${ }^{119}$ Die vier von $v$. Beyme genannten Funktionen der Parteien sind: erstens die Artikulation und Aggregation von Interessen, zweitens die Programmfunktion im Sinne einer ideologischen Übersetzung der Interessen in Ziele, drittens die Mobilisierung und Sozialisierung der Öffentlichkeit, da politische Ideologien der Massenunterstützung bedürfen, und viertens die Elitenrekrutierung und Regierungsbildung.

${ }^{120}$ Es wird im Abschnitt 3.2.3 noch ein weiteres nur für diese Untersuchung bedeutsames Kriterium herangezogen, nämlich das der wirtschaftspolitischen Relevanz hinsichtlich der hier untersuchten Teile der Wirtschaftspolitik.

${ }^{121} \mathrm{Vgl}$. dazu insbesondere die Studien von Fuchs und Klingemann (1990), Mayer und Perrineau (1992) und Falter, Klein und Schumann (1994)bias Just - 978-3-631-75139-8 
Tabelle 3.2 : Konfliktlinien in den Parteiensystemen der Industriestaaten

\begin{tabular}{|c|c|c|c|c|c|c|c|}
\hline $\begin{array}{l}\text { Konfliktlinien } \\
\text { Nationen }\end{array}$ & 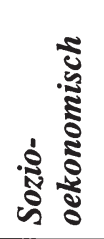 & 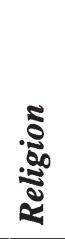 & 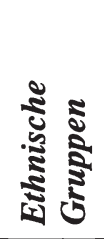 & $\frac{5}{80}$ & 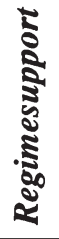 & 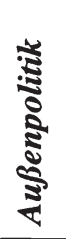 & 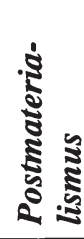 \\
\hline Österreich & $\mathrm{X}$ & $\mathrm{X}$ & & & & & \\
\hline Belgien & $X$ & $X$ & $X$ & & & & \\
\hline Dänemark & $X$ & & & $\mathrm{X}$ & & & \\
\hline Finnland & $X$ & & $X$ & $\mathrm{X}$ & $\mathrm{X}$ & & \\
\hline Frankreich & $\mathrm{X}$ & $X$ & & & $\mathrm{X}$ & $\mathrm{X}$ & \\
\hline Deutschland & $X$ & $X$ & & & & & \\
\hline Irland & $\mathrm{X}$ & & & & & $\mathrm{X}$ & \\
\hline Italien & $\mathrm{X}$ & $\mathrm{X}$ & & & $\mathrm{X}$ & $\mathrm{X}$ & \\
\hline Niederlande & $X$ & $X$ & & & & & $\mathrm{X}$ \\
\hline Norwegen & $X$ & $\mathrm{X}$ & & $\mathrm{X}$ & & & $\mathrm{X}$ \\
\hline Spanien & $\mathrm{X}$ & & & & $\mathrm{X}$ & $X$ & \\
\hline Schweden & $\mathrm{X}$ & & & $X$ & & & $\mathrm{X}$ \\
\hline Schweiz & $\mathrm{X}$ & $\mathrm{X}$ & & & & & \\
\hline Großbritannien & $\mathrm{X}$ & & & & & & \\
\hline$U S A^{(l)}$ & $\mathrm{X}$ & $\mathrm{X}$ & $\mathrm{X}$ & & & & \\
\hline Neuseeland $d^{(I)}$ & $\mathrm{X}$ & & $X$ & & & & \\
\hline Australien ${ }^{(1)}$ & $\mathrm{X}$ & & $X$ & & & & \\
\hline
\end{tabular}

(1) Eigene Einschätzung.

Quelle: In Anlehnung an Lijphart (1990, S. 255).

Es wäre jedoch zu stark vereinfacht, setzte man die sozio-ökonomische Konfliktlinie mit dem häufig verwandten Links-Rechts-Verortungskriterium gleich wie das z.B. Naßmacher (1994, S. 234) tut. Da die Ideologietrennung in links und rechts in der empirischen Forschung, insbesondere in der ökonomischen Literatur, das meist benutzte Trennungskriterium darstellt, erscheint es sinnvoll, etwas ausführlicher auf diese Begriffe einzugehen, bevor diskutiert wird, ob, 
und wenn ja, welche zusätzlichen Konfliktlinien zusätzliche Beachtung erhalten müssen.

\subsection{Links und rechts - un clivage dépassé?}

Die Verortung von Ideologien innerhalb eines einfachen Raumes ist gemäß den Erläuterungen zu den Funktionen von Ideologie in Abschnitt 2.1.4 rational und effizient. Das Schema, das sich insbesondere in Kontinentaleuropa seit über 200 Jahren als dafür dominant erwiesen hat, ist das Links-Rechts-Schema. Dass man auch heute noch sowohl in der Wissenschaft und den Medien als auch in der Politik von diesem Schema reichlich Gebrauch macht, mag erstaunen, da seit mindestens 70 Jahren von Politikern und Wissenschaftlern diskutiert wird, ob dieses Schema zur Darstellung von unterschiedlichen Politikinhalten überhaupt geeignet ist (vgl. de Pressac, 1931). 1963 einigten sich Gerhard Stoltenberg und Helmut Schmidt einvernehmlich, dass die Begriffe links und rechts überholt, wenngleich „unverwüstlich“" seien (zitiert nach Backes und Jesse, 1997, S. 13). Dennoch ist die Verortung von politischen Einstellungen und Programmen entlang dieses Maßstabes gängige Praxis. So spricht z.B. Dahrendorf (1998, S. 7) von der ,demokratischen Linken“ und der ,rechten Mitte“ und auch für Habermas (1998, S. 12) fällt es nicht schwer, eine „linke Stimmenmehrheit“ bei der Bundestagswahl 1998 auszumachen.

Allgemein erachten Fuchs und Klingemann (1990, S. 204) ein Verortungsschema dann als geeignet, wenn es eine überwiegende Mehrheit der Bevölkerung als Konzeptualisierungshilfe ihrer politischen Vorstellungen benutzen kann. ${ }^{122}$ In ihrer Untersuchung kommen sie zu dem Ergebnis, dass in den Niederlanden und Westdeutschland in den frühen 80er Jahren $90 \%$ der Befragten sich selber auf dem Links-Rechts-Kontinuum verorten konnten und dass sogar auf dem untersten Bildungsniveau mehr als 50\% der Befragten eine inhaltlich korrekte politische Assoziation hatten. ${ }^{123}$ Allerdings kommen nicht alle Studien zu einem so deutlichen Befund (Klingemann, 1979, S. 230).

122 Idealtypisch sollte ein Verortungsschema auf der einen Seite genügend Raum für generelle Symbolik besitzen, ohne auf der anderen Seite offen für alle Merkmale gesellschaftlicher Verschiedenheit zu sein. Außerdem sollten die abbildbaren Sachverhalte in der Hinsicht binär sein, dass es inhaltliche Gegensatzpaare gibt (Fuchs und Klingemann, 1990, S. 206). Auf letzteren Punkt weist auch Bobbio (1994, S. 60ff) in seiner Suche nach möglichen Trennkriterien von links und rechts hin.

${ }^{123}$ Für die USA sind diese Befragungsergebnisse signifikant niedriger, was an den unterschiedlichen Demokratisierungsbiographien der Staaten liegen wird. Auf diese Unterschiede wird im folgenden Abschnitt ausführlicher eingegangen 7 8-3-631-75139-8 
Umfragen in Frankreich zeigen, dass in den Jahren von 1981 bis 1991 der Anteil derer, die in der Lage sind, sich selbst in dem Schema einzuschätzen, immer zwischen $60 \%$ und $70 \%$ lag. Allerdings zeigen diese Umfragen auch, dass ein zunehmender Anteil der französischen Bevölkerung der Überzeugung ist, dass die Begriffe rechts und links nicht mehr in der Lage sind, die politische Realität abzubilden. ${ }^{124}$

Es stellt sich also zu Recht die Frage, ob das Verortungsschema Links-Rechts heute noch ausreichende Aussagekraft besitzt. Dies soll im folgenden diskutiert werden; dafür werden zunächst nach einer kurzen Darstellung der Entwicklung des räumlichen Schemas die in der Literatur häufig genannten Unterscheidungskriterien erörtert, um später mit Hilfe dieser Kriterien prüfbare Hypothesen aufstellen zu können.

\subsubsection{Geschichtliche Entwicklung der räumlichen Parteiverortung}

Die Herausbildung des Links-Rechts-Schemas als Erklärungsdichotomie politischer Wirklichkeit begann mit der ersten Nationalversammlung in Frankreich, die sich am 17. Juni 1789 aus der Generalständeversammlung bildete (Laponce, 1981, S. 47f). Durch die Aufhebung der tradierten vertikalen Ständeordnung waren die Abgeordneten zu einer Neuordnung gezwungen. Dies führte insbesondere im Zuge der Diskussion vor der Erklärung der Menschenrechte vom 26. August 1789 zu einer Zweiteilung der Nationalversammlung. Vom Rednerpult aus gesehen rechts formierten sich die Königstreuen des Ancien régime, die sich vornehmlich aus dem Stand der Aristokratie und des oberen Klerus rekrutierten. Auf der linken Seite gruppierten sich weite Teile des ehemaligen Dritten Standes und der niedere Klerus, wenngleich dies nicht allumfänglich galt (Laponce, 1981, S. 48). Bereits 1791 war es in der Presse nicht unüblich, Politikinhalte und Abgeordnete mit den Begriffen links und rechts zu beschreiben. Ferner wurden erste Differenzierungen zwischen den jeweiligen Polen gebräuchlich, sodass 1835 sieben Untergruppen entlang des Links-Rechts-Kontinuums identifiziert werden konnten (Laponce, 1981, S. 52). ${ }^{125}$

1241981 waren nur 33\% der Franzosen der Ansicht, das Verortungsschema sei nicht mehr angemessen, bis 1991 stieg dieser Prozentsatz stetig auf 55\% (Cayrol, 1992, S. 58f).

125

Decker (1998) sieht den eigentlichen Beginn des Schemas erst mit der Abgeordnetenkammer, die durch die Verfassung von 1814 legitimiert wurde. Diese Datierung ist deshalb gerechtfertigt, da der demokratische Pluralismus zwischen 1793 und 1794 durch die Herrschaft des Wohlfahrtsausschusses und später von 1799 bis 1814 durch die Regierung des Ersten Konsuls und späteren Kaisers Napoleons I. stark begrenzt wurde, sodass erst während der Restaurationsphase nach dem Wiener Kongress das Schema sinnvoll verwendet werden konnte (Backes und Jesse, 1997, S $\mathrm{S}_{\text {T }}$ Bilas Just - 978-3-631-75139-8 
In den Jahren nach dem Wiener Kongress 1814 breitete sich das Schema im Sprachgebrauch der jungen, sich demokratisierenden Staaten rasch aus; auch die 1848 in Deutschland formierten Parlamente organisierten die Sitzordnung gemäß der politischen Konnotation des Links-Rechts-Schemas, und gegen Ende des 19. Jahrhunderts wurden in ganz Europa Parteien gegründet, deren Namen direkt auf das Links-Rechts-Schema verwiesen (Laponce, 1981, S. 53ff). ${ }^{126}$ Die Tatsache, dass die kontinentaleuropäischen Demokratien zeitlich erst nach der Französischen Revolution konstituiert wurden und ihre Parlamente dem französischen Vorbild nachbildeten, erklärt, warum die Richtungsbegriffe in Kontinentaleuropa starke Anerkennung fanden und auch heute noch finden, nicht aber in jenen Staaten, die bereits vor der Französischen Revolution stabile Demokratievorläufer besaßen, nämlich Großbritannien und die Vereinigten Staaten. ${ }^{127}$ Dass jedoch gerade in den USA und Großbritannien Politiker zunehmend von diesem Schema Gebrauch machen, zeigt sich an der Zunahme des Verständnisses gerade in den gut ausgebildeten Schichten (Fuchs und Klingemann, 1990, S. 210). Dies könnte damit begründet werden, dass die in angelsächsischen Staaten vorherrschende Parteiendichotomie Liberal (respektive Labour) versus Konservativ nicht so deutlich die Polarität der Begriffe veranschaulicht und deshalb weniger zur Reduktion von Komplexität geeignet ist (vgl. Fuchs und Klingemann, 1990, S. 227). ${ }^{128}$

\subsubsection{Was ist links und was ist rechts?}

Was in der Nationalversammlung im Paris des ausgehenden 18. Jahrhunderts noch relativ problemlos mit den drei Schlagworten „Liberté, Égalité, Fraternité“ für links und dem Festhalten am Ancien régime für rechts festgemacht werden

${ }^{126}$ Dass heute kaum noch Parteien im Parteinamen eine Selbstverortung vornehmen, muss nicht als Indiz dafür interpretiert werden, dass sie nicht mehr als links oder rechts charakterisiert werden könnten, sondern ist wahrscheinlich eher wahltaktisch motiviert angesichts der Tatsache, dass den Begriffen auch semantische Konnotationen inhärent sind. Interessant ist, dass Fuchs und Klingemann (1990, S. 228) der Ansicht sind, dass rechts einen semantischen Vorteil gegenüber links hat, wohingegen Laponce (1981, S. 54) dies lediglich für den nicht-politischen Bereich zulässt und in der politischen Sphäre gerade das Gegenteil postuliert.

${ }^{127}$ So wird in Großbritannien die Sitzordnung unabhängig von der politischen Couleur durch die Zugehörigkeit zur Regierung respektive Opposition bestimmt. In den USA ist die Sitzordnung zwar auch parteigebunden, jedoch sitzen die politisch üblicherweise als rechts eingeschätzten Republikaner im Kongress auf der linken Seite des Sprechers (Decker, 1998, S. 35).

${ }^{128}$ Vgl. auch das Umfrageergebnis von Fuchs und Klingemann (1990, S. 213f), bei dem sowohl die Republikaner als auch die Demokraten von Teilen der Bevölkerung als links eingeschätzt wurden und von anderen Teilen als eherorechts. 
konnte, wurde in zwei Jahrhunderten durch die Verwendung desselben Begriffspaares für immer mehr Politikfelder zunehmend schwieriger. ${ }^{129}$ Erschwerend kommen zwei Dinge hinzu: Erstens sind die Begriffe links und rechts keine „absoluten Begriffe, sondern relative“ (Bobbio, 1994, S. 71) und können sich damit im politischen Raum entweder als Reflex auf die Aktion des politischen Gegners oder aus eigener Initiative verändern. Daher ist es wichtig, das stabile Element des Schemas zu finden. Zweitens ist es nicht zwingend, dass in allen Ländern dieselben Assoziationen mit den Begriffen einhergehen. Dies liegt an der Unterschiedlichkeit der geschichtlichen Ereignisse (Backes und Jesse, 1997, S. 29).

Dennoch bedeuten diese beiden Einschränkungen nicht, dass die „,babylonische Sprachverwirrung" (Backes und Jesse, 1997, S. 22) nicht aufzulösen wäre und auch nicht, dass das Schema nicht mehr erklärungskräftig wäre. Dies soll mit Hilfe des in der Wirtschaftspolitik üblicherweise verwandten Ziel-InstrumenteAkteure-Paradigmas veranschaulicht werden. Dabei dient das Paradigma lediglich dazu, die Vielfalt der Unterscheidungskriterien zu systematisieren und soll kein idealtypisches Konzept einer linken respektive rechten Politik abbilden.

Jede Form rationaler (Wirtschafts-)Politik lässt sich beschreiben, wenn man drei Fragen beantwortet: Was sind die übergeordneten Ziele? Mit Hilfe welcher Instrumente werden diese Ziele verfolgt? Wer sind die bei der Umsetzung der Instrumente relevanten politischen Akteure? Hinsichtlich der Ziele besteht in der Literatur weitgehender Konsens, den Lipset et al (1954, S. 1135) wie folgt darstellen: ${ }^{130}$

"By left we shall mean advocating social change in the direction of greater equality, political, economical, or social; by right we shall mean supporting a traditional, more or less hierarchical social order, and opposing change towards greater equality."

Linke Politik ist also stets darum bemüht, Ungleichheiten zu reduzieren, wohingegen rechte Politik dazu neigt, das als natürlich Empfundene und die damit verbundenen Ungleichheiten zu akzeptieren (Bobbio, 1994, S. 78). Dabei kann dieses Natürliche auf der einen Seite ein hohes Maß an Freiheit wie im Falle des Wirtschaftslebens implizieren, aber auch auf der anderen Seite eine gewisse Unfreiheit, wenn das Natürliche in religiösen oder ethnischen Tatbestandsmerkmalen Ausdruck findet. Analog ist eine Begrenzung der wirtschaftlichen Freiheit gerade dann ein typisch linkes Anliegen, wenn dadurch eine Erhöhung der wirtschaftlichen Gleichheit erzielt werden soll. Auf der anderen Seite setzt sich die

${ }^{129}$ Laponce (1981, S. 116ff) kommt bei einer Literaturdurchsicht auf nicht weniger als 29 verschiedene Trennkriterien in acht Kategorien, die dahingehend instabil sind, dass sie entweder von verschiedenen Autoren zu einem bestimmten Zeitpunkt unterschiedlich eingeschätzt wurden oder im Zeitablauf nicht eindeutig einem Pol des Kontinuums zugewiesen werden konnten. 
Linke für weitgehende Freiheiten ein, wenn dadurch eine Ungleichbehandlung aufgehoben werden kann. Für das cleavage Religion bedeutet dies, dass eine typisch rechte Position eine konkrete Religion als natürlich und einzig richtig empfindet und dementsprechend intolerant gegenüber Religionsfreiheit ist. Das oft herangezogene Ziel der Freiheit (u.a. Groys, 1993, S. 60) ist als mögliches Trennkriterium auf der Zielebene demnach ungeeignet, da es nicht eindeutig zugewiesen werden kann. ${ }^{131}$ Diese Überlegungen deuten an, dass die Frage nach links und rechts immer auch eine Spezifizierung der betrachteten Gleichheitskonzepte erfordert. Es muss gefragt werden, wer soll aufgrund welcher Begründung was erhalten? Gemäß der Konfliktlinien aus Abschnitt 3.1 lassen sich dann verschiedene Dimensionen von links und rechts auf der Zielebene differenzieren.

Die Konfliktlinie mit der größten wirtschaftspolitischen und wissenschaftlichen Relevanz ist das sozio-ökonomische cleavage. Hier stehen monetäre Größen (Einkommen, Vermögen) als Verteilungsobjekte im Vordergrund. Linke Parteien befürworten eine mehr oder weniger weitgehende Nivellierung der Einkommens- und Vermögensdifferenzen, rechte hingegen begrüßen ein aus dem Marktprozess entstehendes ,natürliches“ Verteilungsergebnis. Dies führt selbstverständlich dazu, dass eigeninteressierte Individuen der unteren Einkommensklassen tendenziell eher linken Parteien anhängig werden. Jedoch sollte aus den Darstellungen zum Wert- und Ideologiebegriff deutlich geworden sein, dass dies kein universelles Gesetz ist. Die Zugehörigkeit zu sozialen Klassen ist insbesondere in den Industrienationen der Nachkriegszeit kein hinreichender Indikator für die Selbstverortung auf dem Links-Rechts-Kontinuum, wie zahlreiche Studien belegen (Inglehart, 1990b, Falter, Klein und Schumann, 1994; Mayer und Perrineau, 1992).

Es wäre allerdings unzureichend, links und rechts ausschließlich innerhalb der sozio-ökonomischen Dimension festzumachen (Laponce, 1981, S. 136). Vielmehr ist der Gleichheitsbegriff auch für andere Konfliktlinien anwendbar. Wie oben angedeutet, würden rechte Parteien entlang der Konfliktlinie Religion stärker auf die Verschiedenheiten der Religionen und die Dominanz (Natürlichkeit) ihrer eigenen rekurrieren oder die Bedeutung der Religiosität gegenüber der A-

131 Grunberg und Schweisguth (1990) unterscheiden bei ihren Untersuchungen daher auch immer in "libéralisme culturel" und "libéralisme économique". Geht man jedoch davon aus, dass der marktliche Wettbewerb wenigstens in der Idealform nach Maßgabe des Leistungsprinzips zwar für formale Chancengleichheit, nicht aber für Ergebnisgleichheit sorgt, da Wettbewerb gerade durch die Suche nach kurzfristiger Marktmonopolisierung gekennzeichnet ist, dann muss der Versuch linker Egalisierungspolitik zu einer Reduktion der Wirtschaftsfreiheit führen; die Opposition zum „libéralisme économique“ folgt dann zwangsweise dem Versuch der wirtschaftlichen Gleichstellung. Das Prinzip der Freiheit wäre dem der Gleichheit subordiniert. 
religiosität hervorheben, während linke Parteien die Gleichheit der Menschen ungeachtet ihrer religiösen Überzeugung vertreten. ${ }^{132}$

Es hieße jedoch die religiöse Konfliktlinie übermäßig zu strapazieren, wenn man mit Laponce (1981, z.B. S. 127) schließt, dass der Gegensatz zwischen Profanität und Sakralität das bedeutsamere Trennkriterium zwischen links und rechts sei. Zwar lässt sich die Anwendbarkeit nicht bestreiten, jedoch ergibt sich daraus nicht, dass die religiöse Konfliktlinie die sozio-ökonomische dominiert. Es ist vielmehr davon auszugehen, dass die beiden Konfliktlinien in der politischen Realität zahlreiche Berührungspunkte haben und es so eine wechselseitige Beeinflussungsbeziehung zwischen ihnen gibt. ${ }^{133}$ Auch für die Konfliktlinien Ethnizität, Geographie und Außenpolitik lassen sich politische Parteien nach einem Gleichheitskonzept in rechts und links einteilen. ${ }^{134}$ Nationalistische Parteien akzentuieren die Ungleichheit der einzelnen Staaten, Völker oder Volksgruppen und wären demnach rechts einzuordnen. Linke Parteien betonen hingegen den Gleichheitsaspekt zwischen Staaten, Völkern und Gruppen.

Das Schwierige an der Zuordnung von Politikern, Parteien und Wählergruppen in das Schema, was im Endeffekt zu der „babylonischen Sprachverwirrung“ führt, ist, dass Parteien nicht in allen Punkten auf derselben Seite des LinksRechts-Schemas liegen müssen. Eine rechte nationalistische Partei könnte z.B. dahingehend links sein, dass sie sich für mehr vertikale Umverteilung einsetzt, also entlang des sozio-ökonomischen cleavage als links zu klassifizieren wäre (z.B. befürworten die nationalistischen Parteien NPD und DVU starke staatliche Intervention auf den Märkten). Komplizierend tritt hinzu, dass in der politischen und wissenschaftlichen Diskussion links und rechts nicht nur auf der Zielebene, sondern auch auf der Ebene der eingesetzten Instrumente festgemacht wird. Dabei ergibt sich aus dem Anspruch der Gleichstellung für die Linken und der Annahme, dass die als natürlich empfundenen Kräfte diese Egalisierung nicht bewirken können, die Forderung, dass eine rational planerische Instanz intervenieren muss. Allgemein kann diese Intervention auf drei Ebenen ablaufen:

132 Auch hier folgt die Forderung nach Religionsfreiheit dem Anspruch der Religionsgleichheit, respektive der Annahme der Gleichheit des Menschen jenseits der Religionen.

133 Vgl. dazu auch Decker (1998, S. 42).

134 In enger Anlehnung an Lipset und Rokkan (1967) müsste man außenpolitische Aspekte zwar eher zu den kurzfristigen Programminhalten (issues) zählen. In Anbetracht der Tatsache, dass zahlreiche Autoren auch in außenpolitischen Fragen Konfliktliniencharakter erkennen (vgl. Tabelle 3.1), wird auch hier der Begriff der Konfliktlinie für den Bereich der Außenpolitik verwendet. 


\section{1. materiell}

2. formal-rechtlich

3. symbolisch

Die materielle Ebene bedeutet z.B. für die Volkswirtschaft ein größeres Maß an Umverteilung von Einkommen und Vermögen. Sie bedeutet gegebenenfalls auch eine stärkere Intervention des Staates in den Produktionsprozess selbst, also Nationalisierung und Regulierung. ${ }^{135}$

Die formal-rechtliche Ebene bezieht sich auf Politikfelder, in denen eine gesetzliche Gleichstellung überhaupt noch erreicht werden müsste. ${ }^{136}$ Beispielhaft könnte hier die Diskussion um die doppelte Staatsangehörigkeit im Winter 1998/1999 in Deutschland genannt werden. Sie kann einerseits Grundbedingung späterer materieller Gleichheitsbemühungen sein, aber auch als Substitut für materielle Intervention relevant werden, wenn der Spielraum für Umverteilungspolitik erschöpft ist. Ähnlich können rein symbolisch-proklamatorische Instrumente als letzte Möglichkeit einer Positionierung der politischen Akteure betrachtet werden, wenn die zwei anderen genannten Instrumente als Handlungsalternativen nicht mehr zur Verfügung stehen.

Die Auswertungen von Parteiprogrammen und Regierungserklärungen zeigen, dass Proklamationen der Parteien in der Regel noch als guter Indikator ihrer Politik angesehen werden können. ${ }^{137}$ Allerdings flossen in diese Untersuchungen ausschließlich die schriftlichen Verlautbarungen der Parteien ein, die üblicherweise strategisch vage auch nur die ,small scale do-ables" (Rose, 1984, S. 67) abbilden, wohingegen der Eindruck der Wähler, dass Wahlversprechen nicht eingehalten werden, zu weiten Teilen auf mündliche Zusicherungen einzelner Politiker in der Wahlkampagne zurückzuführen sind, die nicht Eingang in die Analysen der Manifesto Research Group von Budge et al. finden. Diese Untersuchungen können dann zwar zeigen, dass sich Parteien an ihre Programme gebunden fühlen, jedoch ist dies kein Beweis gegen die mögliche These, dass die Alternative rhetorischer Profilierung durch die Macht der Sachzwänge an Bedeutung gewonnen hat.

Es wird in der Literatur darüber hinaus sehr oft davon ausgegangen, dass der Modus des Instrumenteneinsatzes, oder die „modi of social change“ (Fuchs und

135 Ziel wäre eine Reduktion der von Marshall bezeichneten quantitativen oder ökonomischen Ungleichheit (vgl. Marshall, 1950 sowie Dahrendorf, 1992).

${ }^{136}$ Hier spricht Marshall von einer Reduktion der qualitativen Ungleichheit (vgl. Marshall, 1950 sowie Dahrendorf, 1992).

137 Dies zeigen zahlreiche Studien der Manifesto Research Group, die die offiziellen Parteiprogramme und Regierungserklärungen auswerten, z.B. Budge und Hofferbert (1990), Hofferbert und Klingemann (1990), Hofferbert, Klingemann und yolkens_(1996):8 
Klingemann, 1990, S. 213) ein konstituierendes Merkmal der Unterscheidung zwischen rechts und links darstellen. ${ }^{138}$ Interpretiert man dies dergestalt, dass die Diskontinuität, gleichbedeutend mit Risikobereitschaft, ein Eigenwert darstellt, könnte dies ein stabiles Merkmal der Unterscheidung sein. Linke Parteien hätten dann unabhängig vom politischen Umfeld eine höhere Neigung, umfassende Reformen durchzuführen. Man kann den Modus des Instrumenteneinsatzes jedoch auch als notwendige Folge des Gleichheitsziels interpretieren, dann hätten linke Parteien nur deshalb (bisher) eine höhere Neigung zu weitgehenden Reformen und Strukturbrüchen gehabt, weil ein befriedigender Zustand noch nicht erreicht war. Ist hingegen ein als genügend empfundener Gleichheitszustand realisiert oder ist dieser unmöglich geworden, würde die Linke ihre Dynamik einbüßen und zur Bewahrerin des neuen Status quo werden, während die Rechte zum Motor von Reformen hin zum Status quo ante würde (Ritter, 1993, Dahrendorf, 1998).

Allgemein ist davon auszugehen, dass bestimmte Instrumente nur dann zum Einsatz kommen, wenn sie dem politischen Ziel dienlich sind. Instrumente können demnach nur dann als konstituierende Merkmale des Links-Rechts-Schemas gelten, wenn sie im Zeitablauf stabil sind. Ändert sich der Instrumentenmix einer bestimmten Partei, ist dies kein Indikator dafür, dass eine ideologische Neupositionierung erfolgt ist, sondern es ist womöglich lediglich Ausdruck eines politischen Lernprozesses in einem Land. ${ }^{139}$ Es könnte der (seltene?) Fall eingetreten sein, dass neue wissenschaftliche Erkenntnisse über die Wirksamkeit bestimmter Instrumente in die Politik Eingang fanden, oder es könnte eine neue Rahmensituation entstanden sein, die den alten Instrumenten die Wirksamkeit nahm. ${ }^{140}$

Hinsichtlich der Akteure scheint die traditionelle Einteilung, dass Rechte eher eine Führung durch Eliten bejahen, während Linke tendenziell eine weitgehende Selbstbestimmung aller befürworten (Valois, 1931), in den modernen freiheitlichen Demokratien an Bedeutung verloren zu haben. Gerade im sozio-

138 Valois (1931) attribuiert der Linken die Dynamik und der Rechten die Statik des Bewahrens. Ähnlich schreibt Laponce (1981), dass die Rechte für Kontinuität steht, wohingegen die Linke Diskontinuität befürwortet. Revelli (zit. nach Bobbio, 1994) unterscheidet ebenfalls in Fortschritt und Konservation. Groys (1993, S. 60) nennt es die „Alternative zum Status quo."

139 Der Ansatz von Inglehart (1990b) bei der Konstruktion eines Index für linke Politik aus den drei Einzelkriterien: Einkommensnivellierung, stärkere staatliche Intervention in die Wirtschaft und stärkere Nationalisierung der Industrie, erscheint daher unplausibel, da unterschiedliche Erfahrungen in einzelnen Ländern dazu geführt haben können, dass die beiden Instrumente nicht immer in der Lage sind, Einkommensnivellierung zu realisieren.

${ }^{140}$ Hinsichtlich der Schwierigkeit, ökonomische Grundideen in politische Entscheidungen einfließen zu lassen, vgl. u.a. Stigler (1976), Stiglitzo(1998) und Frey $y_{3}\left(2000{ }_{-1}{ }_{5139-8}\right.$ 
ökonomischen cleavage stünde dies auch im Widerspruch zu obigen Ausführungen, denn eine Eliten befürwortende Rechte stünde einer die freien Marktkräfte bejahenden Rechten entgegen. Analog impliziert das interventionistischplanerische Konzept einer Linken ein stark elitäres Grundverständnis, da es beinhaltet, dass die staatlichen Planer den individualistischen Marktkräften in der Bedürfnisbefriedigung überlegen sind.

Für die weitere Arbeit ist eine etwaige unterschiedliche Selektion der entscheidungsrelevanten Akteure nicht von Bedeutung und soll daher hier auch nicht ausführlich diskutiert werden.

\section{Abbildung 3.1 : Das Links-Rechts-Verortungsschema}

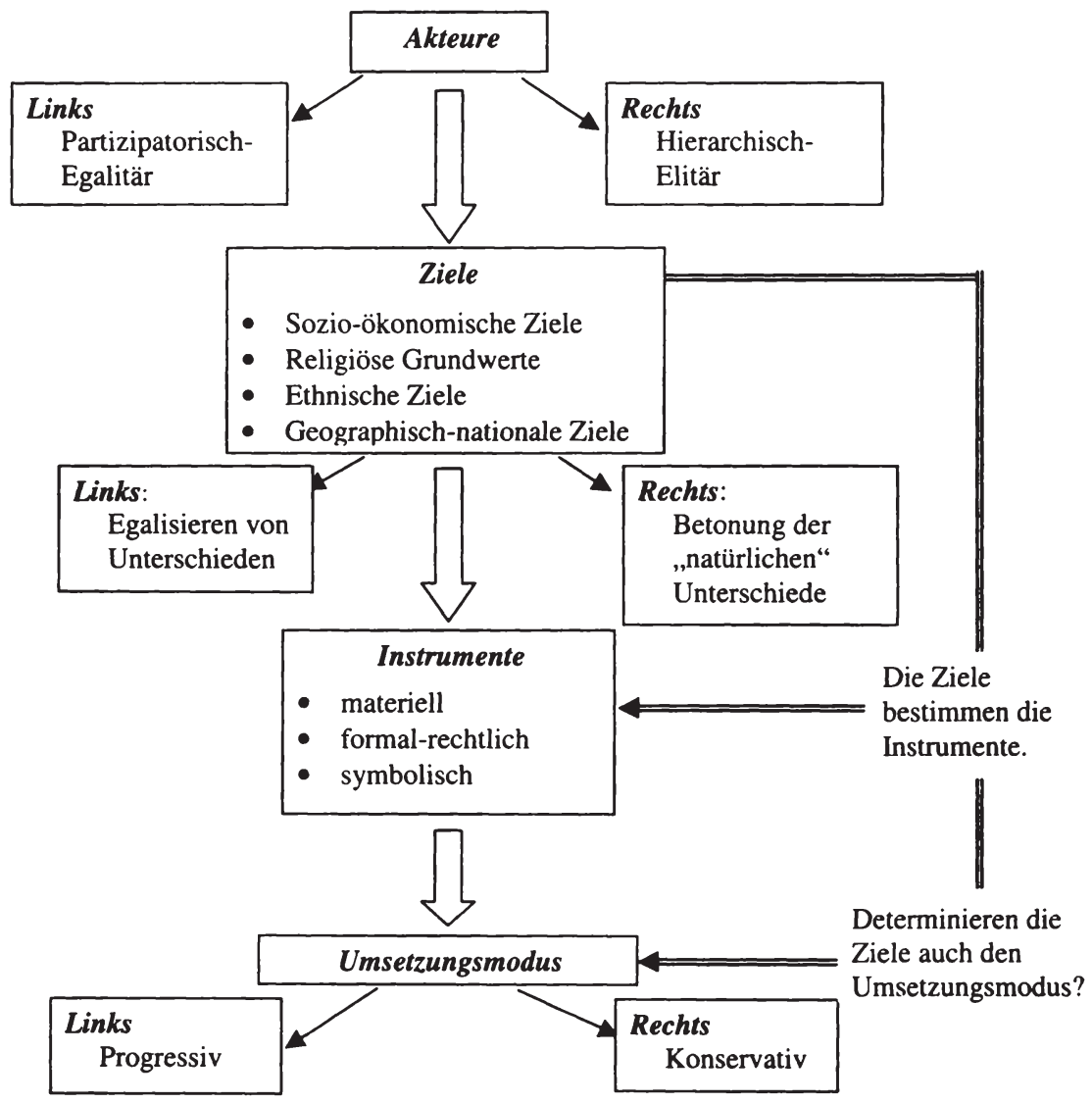

Quelle: Eigene Darstellung. 
Die Abbildung 3.1 fasst die Überlegungen zum Links-Rechts-Schema schaubildartig zusammen. Grundsätzlich müsste man zur Bewertung einer Partei oder eines Programmes alle aufgeführten Anwendungsbereiche prüfen und gegeneinander abwägen, da keine Partei in allen Bereichen zwangsläufig auf derselben Seite des Kontinuums einzugruppieren ist.

Da in dieser Arbeit v.a. auf die sozio-ökonomischen Ziele fokussiert wird, reduziert sich der Komplexitätsgrad erheblich. Falls es möglich ist, alle Parteien hinsichtlich des sozio-ökonomischen cleavage eindeutig auf dem Links-RechtsKontinuum zu positionieren, so hat dieses Trennkriterium keineswegs an Bedeutung verloren, selbst wenn durch die Pluralität der Gleichheitskonzepte eine generelle Verortung nicht möglich ist.

\subsubsection{Anzahl relevanter ideologischer Dimensionen}

Es stellt sich nun die Frage, welche der in Tabelle 3.1 und Tabelle 3.2 auf den Seiten 99ff präsentierten ,üblichen“ Konfliktlinien bei einer Untersuchung ideologischer Wirtschaftspolitik neben dem Links-Rechts-Schema herangezogen werden sollten? Wie in Abschnitt 3.2 diskutiert, ist zumindest die theoretische Verortung von Parteien innerhalb des Links-Rechts-Kontinuums für die sozioökonomische, die religiöse, die ethnische und die national-geographische Konfliktlinie durchaus sinnvoll. Zwar ist die Verortung von Parteien innerhalb des einfachen Links-Rechts-Schemas problematisch, da sie implizit davon ausgeht, dass alle vier einzelnen Konfliktlinien vollständig miteinander korrelieren, jedoch existiert derzeit keine Datenbasis, die einzelne Parteien entlang von vier untergeordneten Links-Rechts-Kontinua einteilt. Da der Schwerpunkt dieser Arbeit nicht eine grundlegende Klassifizierung von westlichen Parteien sein kann, muss auch hier mit der (stark vereinfachenden) Annahme gearbeitet werden, dass die Parteieinteilung entlang eines eindimensionalen Links-Rechts-Schemas ein hinreichend gutes aggregiertes Abbild dieser vier Konfliktlinien ist. Müssen jedoch in dieser Arbeit auch die anderen cleavages Regimeunterstützung, Außenpolitik und Postmaterialismus in diesem Indikator berücksichtigt werden, oder reicht die Berücksichtigung des sozio-ökonomischen cleavage für diese Problemstellung?

\subsubsection{Die Konfliktlinien Regimeunterstützung und Außenpolitik}

Es gibt gute Gründe dafür, die Konfliktlinie Regimeunterstützung nicht zu berücksichtigen. So werden in dieser Arbeit ausschließlich solche Länder untersucht, die während des Untersuchungszeitraums demokratisch stabil gewesen sind. Dies soll nicht heißen, dass es in diesen Staaten nicht auch systemablehnende Parteien gibt, jedoch ist davon auszugehen dass die Stabilität der Demo- 
kratien ein Anzeichen für die Bedeutungslosigkeit (insbesondere in wirtschaftspolitischen Fragen) dieser Parteien ist.

Für die Konfliktlinie Außenpolitik, falls sie nicht sowieso unter der nationalgeographischen Konfliktlinie subsumiert werden kann, lässt sich argumentieren, dass auch ihre Berücksichtigung nicht notwendig ist. So wurde in den letzten Jahren die Außenpolitik zunehmend durch inter- und supranationale Organisationen koordiniert (NATO, UN, EU), was den Handlungsspielraum einzelner nationaler Parteien stark einschränkte. Deutlicher Ausdruck dieser Koordinierung westlicher Außenpolitik ist nicht zuletzt der EG-Vertrag in der Fassung vom 2. Oktober 1997 (Amsterdamer Vertrag), in dem die Art. 11-28 die gemeinsame Außen- und Sicherheitspolitik regeln. So legt Art. 11 (1) fest:

„Die Union erarbeitet und verwirklicht eine Gemeinsame Außen- und Sicherheitspolitik, die sich auf alle Bereiche der Außen-und Sicherheitspolitik erstreckt [...].“

Es scheint also, dass die außenpolitische Konfliktlinie für diese Arbeit nicht sehr bedeutsam ist (zumindest nicht für den gesamten Untersuchungszeitraum), falls sie nicht sogar gänzlich als Dimension zur Parteiunterscheidung entfällt, da sie zumindest für die Mehrzahl der untersuchten Staaten nicht mehr im Sinne Lijpharts als persistentes cleavage bezeichnet werden kann. ${ }^{141}$

Weniger eindeutig, da in der Literatur sehr kontrovers diskutiert, ist die Bedeutung einer relativ jungen Konfliktlinie, der des Postmaterialismus. Aufgrund der seit 30 Jahren in der politikwissenschaftlichen und soziologischen Literatur andauernden Kontroverse soll auf dieses cleavage im folgenden ausführlicher eingegangen werden.

\subsubsection{Die Konfliktlinie Postmaterialismus}

Die Diskussion wurde von Inglehart (1971) initiiert, der zunächst auf der Maslow'schen Bedürfnispyramide aufbauend versuchte, die Veränderbarkeit menschlicher Werte in ein theoretisches Modell einzubinden. ${ }^{142}$ Für seine Analyse reduziert Inglehart das Konzept von Maslow (1981) darauf, dass der Mensch bemüht ist, hierarchisch geordnete Bedürfnisse nach ihrer relativen Dringlichkeit zu befriedigen, d.h. erst wenn den physiologischen Bedürfnissen Genüge getan wurde, kann der Mensch sich um die Befriedigung höherer Be-

${ }^{141}$ Dies wird exemplarisch durch die Beteiligung der Bundeswehr an dem ersten NATO Out of Area Einsatz im Rahmen des Kosovo-Krieges im Frühjahr 1999 deutlich, der durch die erste rot-grüne Bundesregierung verantwortet wird.

142 Neben den zahlreichen Veröffentlichungen von Inglehart (z.B. 1971, 1977, 1990a, 1990b, 1990c) sei hier nur auf die in Abramson und Inglehart (1995, S. 1-7) zitierte Literatur verwiesen. 
dürfnisse bemühen. Inglehart (1977, Ch. 2) nennt hier die Sicherheitsbedürfnisse und als höchste Stufe die nicht materiellen Selbstverwirklichungsbedürfnisse. ${ }^{143}$ In späteren Artikeln rekurriert Inglehart (1990a, S. 68; 1990c, S. 69) allerdings immer seltener auf Maslow, sondern formuliert eine allgemeine Knappheitshypothese, die besagt, dass analog zum Gesetz abnehmender Grenznutzen Menschen besonders knapp empfundenen Gütern den höchsten Wert zuweisen. Diese Knappheitshypothese gemeinsam mit einer Sozialisationshypothese, die von einem der Kristallisationsthese nachgebildeten Sozialisationsverständnis ausgeht und besagt, dass grundlegende Wertvorstellungen des Menschen durch die sozio-ökonomischen Umweltbedingungen während der formativen, also der jugendlichen Jahre geprägt werden und dann zwar nicht unabänderlich, jedoch weitgehend stabil bleiben (Inglehart, 1990a, S. 70), bildet das theoretische Fundament der Überlegungen Ingleharts.

Sind diese beiden Hypothesen korrekt, muss ein stetiger Wandel der sozioökonomischen Rahmenbedingungen dann auch immer in einem stetigen Wandel der menschlichen Werte widergespiegelt werden - es findet eine ,stille Revolution“ (Inglehart, 1971) statt. Seit Ende des 2. Weltkrieges, so Inglehart (1977), gab es fünf grundlegende Trends, die die Umweltbedingungen abfolgender Generationen stetig abänderten:

1. Steigendes Ausbildungsniveau,

2. Veränderung der Beschäftigungsstruktur,

3. Entwicklung von Massenkommunikationsmedien,

4. Stetiger wirtschaftlicher Aufschwung,

5. Abwesenheit eines tiefgreifenden Krieges.

Hierbei sind es insbesondere die letzten beiden Tatbestände, die dazu führten, dass weite Teile der Bevölkerung in zunehmendem Umfang mit materiellen Gütern versorgt werden konnten. Gemäß der Knappheitshypothese sollte dann beobachtbar sein, dass die Bedeutung der Befriedigung von physiologischen und Sicherheitsbedürfnissen stetig abnimmt.

Um diese Hypothese zu überprüfen, versuchte Inglehart zu zeigen, dass es eine Abnahme von so genannten Materialisten, also Menschen, deren formative Jahre in hohem Maß durch wirtschaftliche und physiologische Unsicherheit bestimmt waren und die dementsprechend heute diesen Bedürfnisse eine hohe Bedeutung

${ }^{143}$ In seinen empirischen Untersuchungen zieht Inglehart allerdings die physiologischen und Sicherheitsbedürfnisse zusammen und erfasst sie unter dem Begriff der materiellen Bedürfnisse. Maslow (1981, S. 62ff) selbst unterscheidet insgesamt fünf Hierarchieebenen; er differenziert die höchste Stufe Ingleharts in drei Einzelstufen, die in aufsteigender Reihung erstens das Bedürfnis nach Zugehörigkeit und Liebe, zweitens nach sozialer Achtung und Prestige und drittens nach Selbstverwirklichung sind. 
zuweisen, in der Bevölkerung gab und gibt. Gleichzeitig muss dann der Anteil so genannter Postmaterialisten, also Menschen, die in ihrer Jugend nicht wirtschaftliche oder physiologische Unsicherheit erfuhren und die dann stärker nicht-materielle Bedürfnisse zu befriedigen versuchen, ansteigen. ${ }^{144}$ Mittlerweile lässt sich die umfangreiche Literatur zum Wertewandel in zwei Gruppen einteilen. Die eine Gruppe benutzt das Konzept von Inglehart und wendet es auf zahlreiche Sachverhalte an, ohne grundlegend der Theorie Ingleharts zu widersprechen. Diese Autoren vertreten die Auffassung, dass durch die Berücksichtigung des Postmaterialismus-cleavage das Wählerverhalten und befürwortete Politikinhalte besser erklärt werden kann als durch eine eindimensionale Anwendung des Links-Rechts-Schemas. Es wäre dementsprechend notwendig, zwei Dimensionen zu untersuchen, eine sozio-ökonomische der so genannten ,Alten Politik“, die durch die Links-Rechts-Dimension abgebildet wird und eine ,Neue Politik"-Dimension, die durch das cleavage Postmaterialismus erfasst wird. ${ }^{145}$

Die zweite Gruppe steht dem Konzept von Inglehart sehr kritisch gegenüber. Ihre Kritikpunkte lassen sich im wesentlichen zu den folgenden vier Punkten verdichten:

- Soziologen werfen dem Modell Ingleharts vor, dass sein Menschenbild zu ,instinktoid“ sei, dass es zu wenig die soziale Konstitution des Menschen berücksichtige. So kritisiert z.B. Thome (1985, S. 28) sachlich und Klages (1992, S. 13f) leidenschaftlich, dass der Mensch bei Inglehart gemäß der Knappheitshypothese erst nach Befriedigung seiner egoistischen Bedürfnisse seiner zwar immanenten, jedoch unterdrückten Fähigkeit zu sozialer Interaktion außerhalb der individuellen Bedürfnisbefriedigung auch gewahr wird. Zwei Punkte sind diesem Argument von Thome und Klages entgegenzuhalten. Erstens ist es nicht erforderlich, dass jedes Modell des Menschen seine gesamte Komplexität erfasst, da ansonsten die Modellierbarkeit und die anschließende empirische Hypothesenprüfung an der Modellkonstruktion scheitern muss. Zweitens besagt die Knappheitshypothese eben nicht, dass es zu Sprungstellen innerhalb einer binären Entscheidungssituation kommen muss. Die Menschen sind auch bei Inglehart nicht nur entweder vollständige

144 Materialisten ordnen in Befragungen den Werten: Recht und Ordnung, wirtschaftliche Stabilität, Wirtschaftswachstum, Verteidigungsfähigkeit, Kriminalitätsbekämpfung eine höhere Bedeutung zu als den Werten: Mitspracherecht, Redefreiheit, Mitbestimmungsrecht in der Wirtschaft (z.B. Befugnisse des Betriebsrats und von Arbeitnehmervertretern in Aufsichtsräten), ästhetische Werte, Offenheit und Freundlichkeit, Rückdrängung der Dominanz monetärer Bewertung von Leistung. Postmaterialisten ordnen diese Werte idealtypisch genau umgekehrt.

145 Siehe Inglehart (1990a, S. 162ff, 1990c, S. 88) sowie Abramson und Inglehart (1995, S. 1f) und die dort angegebene Literatur. 
Materialisten oder hundertprozentige Postmaterialisten, sondern in der Mehrzahl ,Mischtypen“. ${ }^{46}$

- Ein weiterer oft genannter Kritikpunkt ist, dass Inglehart die Komplexität des Wertewandels auf eine einzige Dimension verdichtet und wesentliche andere Aspekte ausblendet (Thome, 1985, S. 59 und Bürklin, Klein und Ruß, 1994, S. 590f). Dies ist sicherlich richtig und Inglehart streitet auch gar nicht ab, dass der gesellschaftliche Wertewandel durch mehr als eine Dimension erfasst werden müsste (Inglehart und Klingemann, 1996, S. 320). Es ist jedoch auch hier anzumerken, dass es durchaus sinnvoll sein kann, eine einzige Dimension zu betrachten, um die Komplexität zu reduzieren und Einzeleffekte $\mathrm{zu}$ isolieren. Nur wenn der Anspruch des Inglehart'schen Modells gewesen wäre, den gesellschaftlichen Wertewandel vollständig abzubilden, könnte dieses Argument überzeugen. ${ }^{147}$ Ähnliches gilt für den Kritikpunkt, dass Inglehart das Konzept Maslows unzureichend vereinfacht hat (Thome, 1985, S. $12 \mathrm{ff}$ und Klages, 1992, S. 13f).

- Können die ersten beiden Punkte nur wenig überzeugen, so ist die Kritik, dass Inglehart wenig präzise Werte von Bedürfnissen trennt, durchaus gerechtfertigt (Klages, 1992, S. 14 und Murck, 1982, S. 137), denn eine solche Trennung ist notwendig, wenn man von dem Befriedigungsgrad physiologischer Bedürfnisse auf eine Veränderung gesellschaftlicher Werte rückfolgern möchte, so wie Inglehart dies bezweckt.

- Auch entspricht das einfache Sozialisationskonzept Ingleharts nicht (mehr) dem Stand der aktuellen Sozialisationsforschung. Wie in 2.1.2 dargestellt, geht man heute keineswegs mehr von einer überragenden Dominanz der formativen Jahre aus, sondern hat intragenerationale Verschiebungen als bedeutsam erkannt. Zwar kann auch hier grundsätzlich das Argument der Annahmenfreiheit bemüht werden, doch kann diese Annahmenfreiheit nur dann gerechtfertigt erscheinen, wenn die getroffenen Prämissen dominante Erklärungs- und Prognosekraft besitzen. Dies scheint in diesem Fall jedoch nicht so zu sein. So zeigt u.a. Klages (1992, S. 18f), dass es durchaus signifikante lebenszyklische Effekte in der Wertestruktur eines Menschen gibt.

${ }^{146}$ Allerdings machte bereits Lehner (1979, S. 320) auf eine interessante Inkonsistenz in den beiden Grundannahmen Ingleharts aufmerksam. Einerseits unterstellt die Knappheitsthese, dass der Mensch durch eine Veränderung relativer Preise zu Verhaltensänderungen motiviert wird, also sich rasch anpassen kann, gleichzeitig postuliert die Sozialisationshypothese, dass der Mensch nach Abschluss der formativen Jahre hinsichtlich seiner Normen und Werte für solch“ Anpassungsverhalten unempfänglich ist. Die Knappheitshypothese hätte demnach nur während der formativen Jahre Gültigkeit.

${ }^{147}$ Darüber hinaus ist der Vorzug des Inglehart-Konzepts, dass ihm ein theoretisches Modell als Grundlage dient und nicht nur Faktoren aus Zeitreihen extrahiert werden, denen anschließend die „Theorie“ folgt. 
Abgesehen von diesen Kritikpunkten ist für diese Arbeit einzig relevant, ob die potentielle neue Konfliktlinie Postmaterialismus zwingend berücksichtigt werden muss. Lijphart (1990, S. 264f) resümiert, dass die Bedeutung dieser Konfliktlinie noch zu gering ist, als dass sie zur Charakterisierung von Parteiensystemen in allen Industrienationen herangezogen werden könnte. Darüber hinaus stellt er fest, dass Postmaterialismus in hohem Maße mit der Verortung (von Parteien oder Personen) als links korreliert. ${ }^{148}$ Schmitt und Hofrichter (1992, S. 198f) kommen zwar zu dem Ergebnis, dass die PostmaterialismusSelbstverortung von Befragten unabhängig ist von ihrer Links-RechtsOrientierung. Sie betonen jedoch gleichzeitig, dass die postmateriellen Werte wie Mitspracherechte und Mitgestaltungsmöglichkeiten sowohl für rechte als auch linke Parteien instrumentellen Charakter zur Verfolgung ihrer eigentlichen ideologischen Ziele annehmen. Dann wäre eine Berücksichtigung dieses cleavage in dieser Arbeit jedoch wenig ergiebig, da die postmateriellen „Werte“ eigentlich nur instrumentelle Ableitungen aus den bedeutenderen ideologischen Zielen wären.

Schließlich zeigen Untersuchungen, dass ein eindimensionales Wahlmodell erstaunlich hohe Erklärungskraft besitzt. So schätzen Poole und Daniels (1985, S. 378 ), dass $81 \%$ der Varianz des Wahlverhaltens von amerikanischen Kongressabgeordneten durch ein einfaches Links-Rechts-Schema erklärt werden können. Ein zusätzlicher Faktor bringt lediglich ein um 6\%-Punkte höheres Bestimmtheitsmaß. Auch Poole und Rosenthal (1991) kommen zu dem Ergebnis, dass zumindest für die USA bis auf eine kurze Zeit vor dem Sezessionskrieg über 90\% der Entscheidungen im Kongress durch eine Skalierung der Repräsentanten entlang einer einzigen Dimension abgebildet werden können.

Angesichts der aufgezeigten analytischen Mängel, der noch dünnen empirischen Bestätigung innerhalb einer nur kurzen Periode einerseits und der Erkenntnis, dass man mit einer Dimension das politische Spektrum zwar nicht vollständig, jedoch weitgehend erklären kann, wird in der empirischen Untersuchung nur das Links-Rechts-Schema zugrunde gelegt. Dafür soll im folgenden ein geeigneter Indikator zur Verortung von Parteien innerhalb dieses Schemas konstruiert werden.

\subsection{Operationalisierung des Links-Rechts-Schemas}

Möchte man den Handlungsspielraum ideologischer Wirtschaftspolitik abschätzen, benötigt man einen Indikator, der Regierungen, insbesondere auch Koaliti-

${ }^{148}$ Dies stellte auch Inglehart (1977, S. 60ff) bereits fest. Siehe auch Falter, Klein und Schumann (1994, S. 212).

Tobias Just - 978-3-631-75139-8 
onsregierungen entlang einer Ideologieskala eindeutig zuordnen kann. Aus den obigen Ausführungen wird deutlich, dass dies v.a. ein Indikator sein muss, der das Links-Rechts-Schema angemessen darstellt.

In Anbetracht der Multidimensionalität des Begriffspaars links und rechts ist davon auszugehen, dass die Schwerpunkte linker und rechter Parteien in unterschiedlichen Ländern und zu unterschiedlichen Zeitpunkten in wechselnden Dimensionen lagen. Keiner der Indikatoren, die bisher in vorliegenden empirischen Untersuchungen verwandt wurde, konnte diese Komplexität abbilden. Im folgenden werden zunächst Anforderungen an einen geeigneten Indikator dargestellt, um im Anschluss daran für diese Studie einen geeigneten Indikator abzuleiten.

\subsubsection{Anforderungen an einen Links-Rechts-Indikator}

Mittlerweile sind in der empirischen politikwissenschaftlichen Literatur zahlreiche Vorschläge gemacht worden, Parteien und Regierungen entlang eines Kontinuums zu bestimmen. Dass viele dieser Vorschläge eher auf einer ad hoc Basis gemacht wurden, wäre nur dann ein rein methodisches Problem, wenn die unterschiedlichen Indizes stark miteinander korrelierten. Dann hätte die konkrete Wahl des Index nur unmaßgeblichen Einfluss auf das Forschungsergebnis. Dies ist jedoch gerade nicht der Fall, wie Schmidt (1996, S. 160f) zeigen konnte. Schmidt untersuchte sechs unterschiedliche, in der Literatur verwandte Indikatoren für die Beschreibung einer Regierungskoalition und kam zu dem Ergebnis, dass von 15 Korrelationskoeffizienten ${ }^{149}$ lediglich sieben zumindest auf dem 5\%-Niveau signifikant sind und nur ein einziger einen Wert des PearsonKorrelationskoeffizienten über 0,80 annimmt. Schmidt (1996, S. 162) folgert daraus, dass eine eindimensionale Klassifikation offenbar unzureichend ist. Dies ist richtig, falls unterschiedliche Indikatoren auf unterschiedliche Dimensionen des Links-Rechts-Schemas abheben. Andererseits leitet sich daraus das Erfordernis ab, dass die konkrete Auswahl des verwandten Indikators auf die Aufgabenstellung angepasste Anforderungen möglichst umfangreich erfüllen sollte, um so Verzerrungen auszuschließen. Dabei sind die folgenden Anforderungen für die vorliegende Arbeit aufgestellt worden. ${ }^{150}$

${ }^{149}$ Für sechs einzelne Variable ergeben sich insgesamt 15 paarweise Korrelationen; die erste Variable ist mit den fünf anderen zu korrelieren, die zweite mit vier anderen, die dritte mit drei, die vierte mit zwei und die fünfte nur noch mit einer weiteren Variable. Insgesamt sind also $(5+4+3+2+1=15)$ Korrelationen erstellbar.

${ }^{150}$ Für eine andere Fragestellung könnten andere Anforderungen gelten und demnach auch ein anderer Indikator Vorzug genießen. 
- Der Indikator sollte die gesamte Komplexität der Regierung abbilden. Zwar mag es in den meisten Regierungen eine dominante Partei geben, würde man deren Position jedoch ausschließlich zur Kennzeichnung der gesamten Regierung heranziehen, muss dies zu Ungenauigkeiten führen, da so unterstellt wird, dass die restlichen Parteien keinen Einfluss besitzen. Es sollten also alle Regierungsparteien Eingang in den Indikator finden.

- Der Indikator sollte ferner als Kontinuum konzipiert werden, innerhalb dessen sich Parteien positionieren können. Vorlage kann z.B. die Parteienklassifikation gemäß Expertenaussagen von Castles und Mair (1984) sein, die ein Kontinuum zwischen dem Wert 0 (extreme Linke) und dem Wert 10 (extreme Rechte) aufspannt. Sicherlich ist die erzielte Exaktheit nur scheinbar, da in der Praxis ein Indikatorwert von 2,5 und 2,7 kaum inhaltlich abgrenzbar sein wird. Die Alternative wäre jedoch, dass man eine diskrete Anzahl von Klassen bildet, innerhalb derer größere Unterschiede existieren können als zwischen Punkten angrenzender Klassen. Abbildung 3.2 kann dies veranschaulichen:

\section{Abbildung 3.2 : Parteienverortung in Klassen}

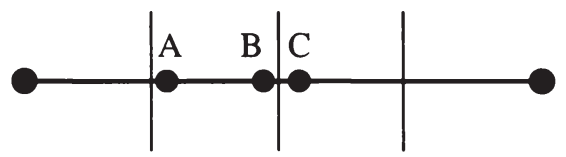

Links Mitte Mitte Rechts

Links Rechts

Die Partei B ist entlang einer kontinuierlichen Darstellung eher mit der Partei $\mathrm{C}$ zu vergleichen, eine Klassenbildung würde sie aber mit der Partei $\mathrm{A}$ identisch erfassen. Graduelle Unterschiede würden so fortdefiniert. Einzig in reinen Zweiparteiensystemen ist es nicht erforderlich, mit einem Kontinuum zu arbeiten. Da jedoch die meisten Parteiensysteme in den OECD-Ländern Mehrparteiensysteme sind, ist es sinnvoll, eine stetige Variation zuzulassen. Darüber hinaus können in unterschiedlichen Ländern unter Parteilabels (Sozialdemokratie, Christdemokraten) stark differierende Inhalte zusammengefasst sein. ${ }^{151}$ Gerade in cross-section-Studien sollte daher ein Kontinuum den Vorzug erhalten.

${ }^{151}$ Es finden sich mehrere Studien, die Parteien nach Maßgabe ihrer Mitgliedschaft in internationalen Dachorganisationen (z.B. der Sozialistischen Internationale) in linke oder rechte Parteien unterteilen. Vgl. zu einem kurzen Überblick über diese Arbeiten Schmidt (1996, S. 160). 
- Angesichts der Vielschichtigkeit des Begriffspaars links und rechts muss die Grundlage für die vorgenommene Verortung eindeutig sein. Nach Castles und Mair (1984, S. 74) sind die einzig zulässigen Verfahren zum einen eine Expertenbefragung, wobei möglichst aus den Befragungsergebnissen mehrerer Experten ein Mittelwert gebildet werden sollte. ${ }^{152}$ Zum anderen könnten Massenbefragungen der Wähler über deren Einschätzung herangezogen werden. Insbesondere die Expertenbefragungen sollten jedoch berücksichtigen, dass die Einschätzung gemäß den programmatischen Inhalten der Parteien erfolgen und nicht gemäß den bereits umgesetzten Politikinhalten, da sonst die empirische Analyse tautologisch würde. Werden linke Parteien als solche definiert, die typisch linke Politikinhalte umgesetzt haben, ist es nicht verwunderlich, dass die empirische Analyse dies auch zeigt.

- Idealerweise sollte der Indikator programmatische Neuverortungen einzelner Parteien zulassen. Er sollte also nicht über den gesamten Untersuchungszeitraum konstant bleiben müssen. Fand in den letzten Jahren eine ideologische Annäherung der Parteien statt, ist die Abnahme der Politikunterschiede im Zweifel nicht auf die restringierende Wirkung globaler Märkte zurückzuführen, sondern Ausdruck geänderter Politikpräferenzen.

- Zur Beurteilung einer Periode sind alle Informationen über diese Periode heranzuziehen. Carlsen (1997, S. 147) nimmt für seinen Links-Rechts-Indikator eine Beurteilung des gesamten Jahres nach Maßgabe der Regierungszusammensetzung zu Beginn des Jahres vor. Zur Erklärung eines Gesamtjahresdurchschnittswertes führt das aber regelmäßig dann zu Verzerrungen, wenn es in diesem Jahr einen Regierungswechsel gab. Vorzugsweise ist daher mit einer Einschätzung zu arbeiten, die alle Regierungswechsel und -umbildungen eines Jahres berücksichtigt.

Im folgenden wird der Indikator beschrieben, der am besten die obigen Anforderungen erfüllt und daher in der empirischen Analyse verwendet wird.

\subsubsection{Indikatorbildung}

\subsubsection{Theoretische Konzeption}

Gross und Sigelman (1984) entwickelten zur Beschreibung und Klassifikation von Parteiensystemen vier zusammenfassende Indikatoren, von denen für diese

152 In ihrer Expertenbefragung wurden Parteien auf ihrer Skala von 0-10 von Experten z.T. sehr unterschiedlich eingeschätzt. Die italienische PSDI wurde z.B. einmal mit einem Wert von 3 als gemäßigte Linke und ein anderes Mal mit einem Wert von 8 als relativ weit rechts eingeschätzt. Solch starke Abweichungen sind allerdings_eher die Aưnghahme. 


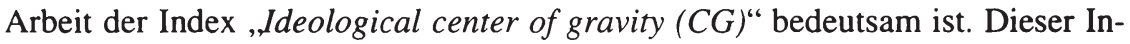
dikator bewertet das relative Gewicht jeder Partei innerhalb einer Regierungskoalition mit ihrer ideologischen Position auf einem Links-Rechts-Kontinuum. So lässt sich ein konkreter Wert zur Beschreibung der Regierung ermitteln. In der empirischen Analyse ist die kürzeste Zeiteinheit ein Quartal. Man benötigt also für jedes Quartal des gesamten Untersuchungszeitraums einen CG-Wert. Zur Beschreibung einer Regierungskoalition zu jedem beliebigen Zeitpunkt lässt sich nach Gross und Sigelman (1984, S. 467) die Variable CG im Quartal t und Jahr $\mathrm{j}$ wie folgt bilden:

$$
\mathrm{CG}_{\mathrm{tj}}=\sum_{\mathrm{i}=1}^{\mathrm{n}} \mathrm{T}_{\mathrm{itj}} \mathrm{C}_{\mathrm{i}}
$$

Dabei ist $T_{i t j}$ der prozentuale Anteil der Sitze, den jede Partei i der insgesamt $n$ Parteien im Kabinett im Quartal $t$ des Jahres $j$ inne hat und $C_{i}$ entspricht der Position der Partei i auf einem Links-Rechts-Kontinuum. Wünschenswert wäre es, wenn man in jedem Quartal einen neuen Wert zur Einschätzung einer Partei hätte. Diese Daten liegen allerdings nicht in dieser Detailliertheit vor. Für jede Partei gibt es in der Regel nur eine Einschätzung nach derselben Vorgehensweise. In den Fällen, wo in der empirischen Analyse Jahresdaten verwendet wurden, wurde ein Wert für das center of gravity als Durchschnittswert der vier Quartalswerte errechnet.

$$
\mathrm{CG}_{\mathrm{j}}=\frac{1}{4} \sum_{\mathrm{i}=1}^{4} \sum_{\mathrm{i}=1}^{\mathrm{n}} \mathrm{T}_{\mathrm{itj}} \mathrm{C}_{\mathrm{i}}
$$

Diese Indikatoren vernachlässigen allerdings, dass in keinem Land die Regierungsparteien uneingeschränkt die Politik eines Landes bestimmen. So kann es insbesondere in stark föderativen Staaten zu einer Machtteilung kommen, wenn sich die Mehrzahl der Landesregierungen aus anderen Parteien rekrutiert als die Bundesregierung. Da in der empirischen Analyse indes Makrodaten im Vordergrund stehen, scheint diese Vereinfachung gerechtfertigt, wenn man davon ausgeht, dass dafür in erster Linie die Regierung der höchsten föderativen Ebene verantwortlich zeichnet.

\subsubsection{Praktische Umsetzung}

Für die quartalsweise Bestimmung von CG war es erforderlich, für jeden der 19 OECD-Staaten alle Kabinette des Untersuchungszeitraums innerhalb des LinksRechts-Kontinuums zu verorten. Daten über die Parteizugehörigkeit einzelner Kabinettsmitglieder in den untersuchten Stakiten U urden 3-âs- Teersethedenen 
Jahrgängen des Political Data Yearbook des European Journal of Political Research entnommen. Dort sind alle Kabinettsmitglieder und deren Parteizugehörigkeit seit 1945 in einer taggenauen Zuordnung zu finden. Für die Regierungsdaten der USA wurden Mackie und Rose $(1974,1997)$ herangezogen.

Zur Bewertung der einzelnen Parteien wurde der Expertenbefragung von Castles und Mair (1984) gefolgt. In ihrer Studie werden Parteien auf einem Kontinuum von 0 (links) bis 10 (echts) von mehreren Experten pro Land positioniert. Der Mittelwert aus den einzelnen Expertenmeinungen bezüglich einer Partei wurde als Maßgabe für deren Ideologiewert genommen. ${ }^{153}$ Jene Parteien, die nicht von der Castles/Mair-Studie erfasst wurden, weil entweder die Länder nicht Berücksichtigung fanden (Japan und Schweiz) oder weil sich die Parteien erst nach 1984 gründeten, wurden anhand der Parteibeschreibungen bei Gorvin (1989) und Mackie und Rose $(1974,1997)$ in dem Kontinuum verortet. Dies war v.a. dann unproblematisch, wenn in den Quellen vermerkt war, dass die neuen Parteien Absplitterungen von vorher existierenden Parteien waren. Für die Bewertung der japanischen und schweizerischen Parteien wurde zusätzlich die Einschätzungen von Cusack (1995) herangezogen. Parteilose Kabinettsmitglieder bekamen jeweils den Medianwert 5 zugewiesen. Eine vollständige, tabellarische Übersicht der Parteipositionen ist im Anhang B zu finden. Insgesamt wurden alle Kabinette im Zeitraum von 1960 bis 1997 für insgesamt 148 Parteien in 19 OECD-Staaten aufgenommen. Die Abbildung 3.3 fasst die Ergebnisse der Parteienverortung schaubildartig zusammen. ${ }^{154}$ Was obige Indikatoren noch nicht berücksichtigt haben, ist eine Neuverortung der Parteien. Es wird hier also von der Annahme ausgegangen, daß Parteien ihre ideologische Position nicht anpassen. Dies ist sicherlich ein nicht ungravierender methodischer Defekt, da so die Downs'sche Konvergenzdynamik quasi fortdefiniert wurde. Da es jedoch keine kontinuierlichen Parteiverortungen gibt, ist im Rahmen einer empirischen Analyse kein anderer Weg gangbar. Cusack (1997a, S. 382) sieht dieses Problem ebenfalls, argumentiert jedoch, dass Parteien, die glaubwürdig bleiben möchten, radikale Kurswechsel zu vermeiden haben. Ist dies richtig, würde die Annahme der Konstanz nur zu geringen Verzerrungen führen.

${ }^{153}$ Dass diese Einschätzung auch nicht unangreifbar ist, wird z.B. durch den Positionsvergleich von CDU und FDP deutlich. Bei Castles und Mair steht die CDU wesentlich weiter rechts als die FDP, obwohl man argumentieren könnte, dass die FDP eine deutlich marktliberalere - also traditionell als weiter rechts empfundene - Stellung einnimmt. Die Experteneinschätzungen scheinen also mehrere Links-Rechts-Dimensionen parallel zu berücksichtigen. Dies verzerrt die Daten. Eine Bereinigung kann aber nicht erfolgen, da es keine getrennte Experteneinschätzung für die potentiell herangezogenen cleavages gibt.

154 Eine ähnliche Abbildung liefern auch die Daten von Laver und Hunt (1992). In ihrer Studie aggregierten sie die aus Interviews gewonnenen Einschätzungen von 355 Experten, die eine Links-Rechts-Lozierung von insgesamt 109 Parteien in 21 Industrienationen vornahmen. Für eine schaubildartige Darstellung ihrer Ergebnisseuvgl. Wagschgl (1996. S9 47). 
Abbildung 3.3 : Positionierung der Parteien von 19 OECD-Staaten im Links-Rechts-Kontinuum

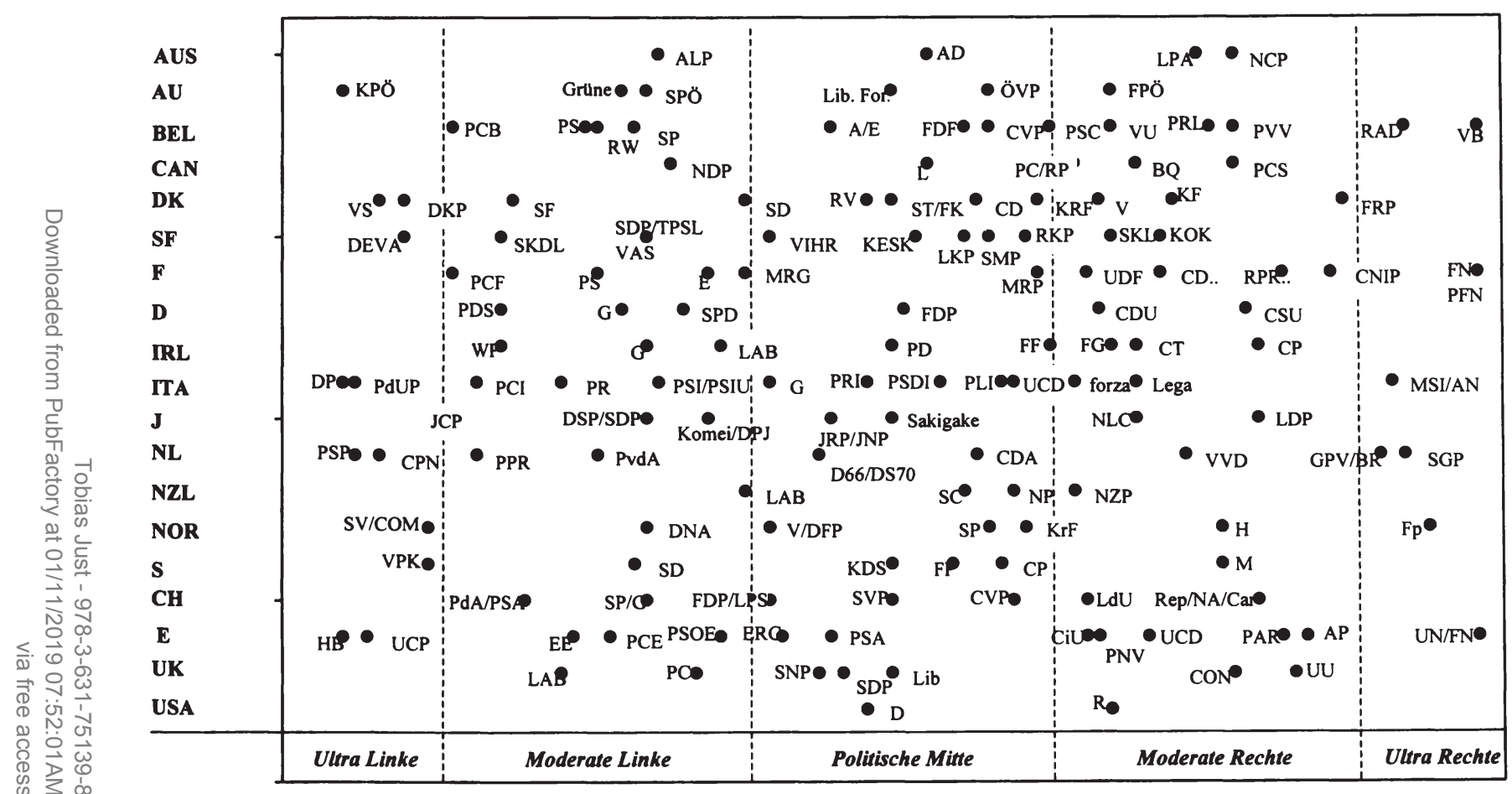

Quelle: Eigene Darstellung in Anlehnung an die Abbildung bei Castles und Mair (1984, S. 84). 
Es wird im folgenden außerdem argumentiert, dass es empirische Hinweise für eine weitgehende Konstanz der ideologischen Programmatik von Parteien gibt.

\subsubsection{Bedarf es einer dynamischen Flexibilisierung des Index?}

Um zu testen, ob eine Flexibilisierung notwendig ist, wurde auf die Befragungsergebnisse der Eurobarometer EB37 und EB38 zurückgegriffen. ${ }^{155}$ Dort wurden Wähler 1992 gefragt, sich selber einer von fünf Gruppen von links bis rechts zuzuordnen und anschließend anzugeben, welche Partei sie bevorzugen. Dadurch dass zu jeder Partei eine Vielzahl von Wählern befragt wurde, die keine einheitliche Einstufung vornahmen, lässt sich ein stetiger Indikator bilden in Gestalt eines arithmetischen Mittels aller Wählermeinungen. ${ }^{156}$ Die so erhaltenen Eurobarometer-Indikatoren wurden dann mit den Parteipositionierungen nach Castles und Mair korreliert.

\section{Abbildung 3.4 : Eurobarometer-Befragung und Castles-Mair-Studie im Vergleich}

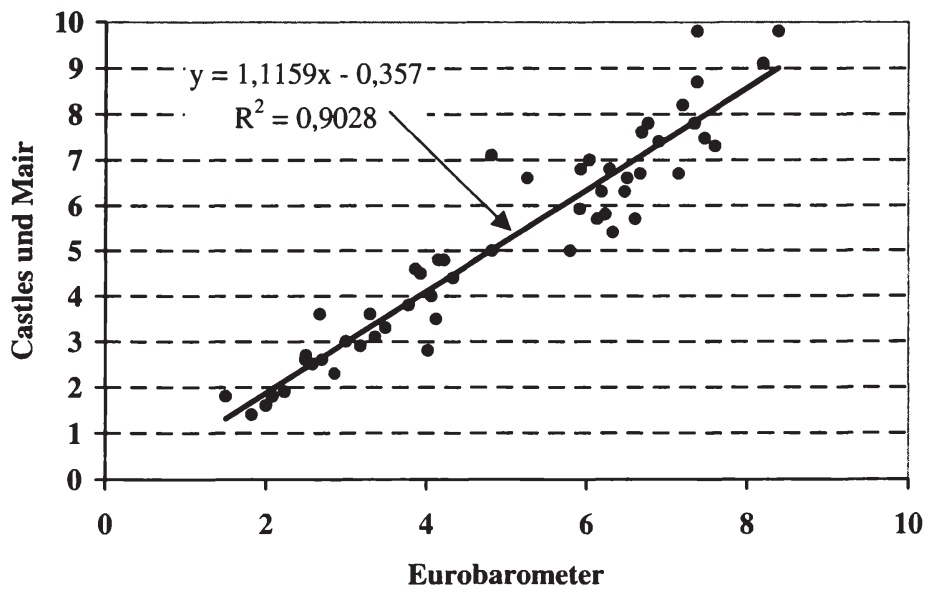

Quelle: Brettschneider et al. (1994), Castles und Mair (1984) und eigene Berechnungen.

${ }^{155}$ Die Daten stammen von Brettschneider et al. (1994, S. 614f).

${ }^{156}$ Interessant ist auch hier die teilweise stark abweichende Einschätzung hinsichtlich einzelner Parteien. Beispielsweise wurde die SPD von 46 Prozent der Befragten, die sich als extrem links einstuften, aber gleichzeitig von 6 Prozent derer, die sich als äußerst rechts verorten, gewählt. Dies kann natürlich durch gruppensoziologische Prozesse bedingt sein, wie in Kapitel 2 gezeigt wurde. 
Das Ergebnis veranschaulicht Abbildung 3.4. Das Bestimmtheitsmaß $\mathrm{R}^{2}$ ist mit 0,9028 erstaunlich hoch. ${ }^{157}$ Dies entspricht einem Pearson-Korrelationskoeffizienten von 0,95 und ist eine bemerkenswert gute Übereinstimmung, da die beiden Befragungen nicht nur acht Jahre auseinander liegen, sondern auch die Gruppen der Befragten jeweils unterschiedlich konstituiert waren. Sowohl die Expertenbefragung, als auch eine hinreichend große Befragung der Wähler führen zu einer nahezu identischen Parteieinschätzung. Für diese Arbeit ist die zentrale Aussage dieser Korrelation jedoch, dass zumindest für diesen Zeitraum nicht von gravierenden Neuverortungen ausgegangen $\mathrm{zu}$ werden braucht. Die Annahme konstanter Parteipositionen ist demnach zumindest nicht widerlegt.

\subsection{Idealtypische linke und rechte Wirtschaftspolitik}

In Kapitel 3.2.2 wurde argumentiert, dass linke bzw. rechte Politik insbesondere durch eine unterschiedliche Konzeption hinsichtlich der Bewertung von verschiedenen Ungleichheitskontexten gekennzeichnet werden kann. Wie oben begründet, soll von den vier Konfliktlinien, bei denen langfristig unterschiedliche Gleichheitskonzeptionen benannt werden konnten (sozio-ökonomische, religiöse, ethnische und geographische Konfliktlinien) im folgenden nur noch die sozio-ökonomische Konfliktlinie Berücksichtigung finden.

Im Zentrum steht dabei eine Betrachtung der personellen Einkommensverteilung. Eine idealtypisch linke Wirtschaftspolitik müsste also darauf gerichtet sein, Einkommensdifferenzen zu egalisieren, wohingegen rechte Wirtschaftspolitik diese Unterschiede solange als gerechtfertigt erachtet, wie diese Einkommensunterschiede Ausdruck eines „natürlichen“ Verteilungsprozesses sind. Es wird zunächst kurz dargestellt, inwiefern ein natürlicher Verteilungsprozess zu einer starken Ungleichverteilung der Einkommen führt, um daraus den Möglichkeitenraum staatlicher Egalisierungspolitik aufzuzeigen bzw. die Inzidenz einzelner wirtschaftspolitischer Maßnahmen auf das Verteilungsziel darzustellen. Diese Verteilungswirkungen können dann nämlich erklären, warum es starke Politikpräferenzen linker und rechter Parteien gibt.

\subsubsection{Determinanten der personellen Einkommensverteilung}

In allen marktwirtschaftlichen Gesellschaften ist die empirische Beobachtung gemacht worden, dass die Einkommen logarithmisch normal-, also rechtsschief

${ }^{157}$ Dies ändert sich auch nicht, wenn man den Ordinatenabschnitt auf den Ursprung festlegt. Dies ist sinnvoll, da der Ordinatenabschnitt hier wenig Aussagekraft besitzt. Dann sinkt $\mathbf{R}^{2}$ lediglich auf einen Wert von 0,8996.

Tobias Just - 978-3-631-75139-8 
verteilt sind (Siebke, 1992, S. 391ff). ${ }^{158}$ Tabelle 3.3 zeigt für die Bundesrepublik Deutschland für den Zeitraum von 1950 bis 1988 die durchschnittlichen Einkommensanteile der einzelnen Einkommensquintile in \%.

Tabelle 3.3 : Durchschnittliche Einkommensverteilung in Deutschland von 1950-1988

\begin{tabular}{|c|c|c|c|c|c|}
\hline & 1. Quintil & 2. Quintil & 3. Quintil & 4. Quintil & 5. Quintil \\
\hline $\begin{array}{l}\text { Arithmetischer } \\
\text { Mittelwert }\end{array}$ & 6,70 & 11,25 & 15,94 & 22,21 & 43,90 \\
\hline $\begin{array}{l}\text { Standard- } \\
\text { abweichung }\end{array}$ & 0,68 & 0,73 & 0,51 & 0,63 & 1,41 \\
\hline $\begin{array}{l}\text { Variations- } \\
\text { koeffizient }\end{array}$ & 0,10 & 0,06 & 0,03 & 0,03 & 0,03 \\
\hline
\end{tabular}

Quelle: Eigene Berechnungen auf der Grundlage von DIW-Wochenbericht (verschiedene Jahrgänge). ${ }^{159}$

Dabei können die dargestellten Werte für die Bundesrepublik als gute Näherung auch für andere Marktwirtschaften angenommen werden (vgl. Li, Squire und Zou, 1998). Die Rechtsschiefe der Verteilung ist offensichtlich, die ärmsten $20 \%$ der westdeutschen Haushalte verfügten im Durchschnitt nur über 6,7\% der Einkommen, wohingegen die reichsten $20 \%$ insgesamt $43,9 \%$ des deutschen Einkommens verdienten. Des weiteren zeigen sowohl die verhältnismäßig niedrige Standardabweichung als auch der niedrige Variationskoeffizient, dass die Einkommensverteilung in Deutschland in diesem Zeitraum nur geringen Veränderungen unterworfen war. ${ }^{160}$

158 Allerdings bezeichnet Külp (1994, S. 166) den Verlauf der lognormalen Verteilung als linksschief. Folgt man jedoch der gängigen Definition für Schiefe, nach der eine linksschiefe Verteilung dadurch gekennzeichnet ist, dass der Modus rechts vom Median liegt (Schlittgen, 1993, S. 156), dann kann die personelle Einkommensverteilung keine linksschiefe Funktion sein; das hieße nämlich, es gibt mehr wohlhabende als arme Mitbürger.

159 Ich danke Thomas $R$. Cusack vom Wissenschaftszentrum Berlin, der mir Zugang zu diesem Datensatz ermöglichte. Die Werte beruhen auf einer Umrechnung von Cusack, da die Zuordnung in den Wochenberichten nicht für alle Jahrgänge einheitlich erfolgte.

${ }^{160} \mathrm{Li}$, Squire und Zou (1998) kommen in einer umfangreichen Zeitreihenanalyse von 49 Staaten zu dem Ergebnis, dass es zwar starke Unterschiede in den Einkommensverteilungen der Staaten gibt, dass jedoch innerhalb eines Landes die Einkommensverteilung weitgehend stabil ist. 
Seit Pigou werden als Gründe für die Rechtsschiefe der Verteilung zahlreiche Ursachen diskutiert. ${ }^{161}$ Pigou selbst begründete die Konzentration der Einkommen mit der Heterogenität der Startbedingungen der Marktteilnehmer, die auf den Erfolg bzw. Misserfolg der früheren Generationen zurückgeht (Külp, 1994, S. 167). Dies bildete die Grundlage für die Überlegungen u.a. von Becker (1967), der v.a. unterschiedliches Investitionsverhalten in Humankapital als Begründung für unterschiedliche Einkommen annahm. Dieses Investitionsverhalten ist zum einen durch unterschiedliche Präferenzen der Individuen, einen unterschiedlichen Informationsstand hinsichtlich der Rentierlichkeit der Bildungsinvestition und zum letzten durch budgetäre oder andere Restriktionen bezüglich des Erwerbs dieses Humankapitals determiniert. Menschen verdienen demnach deshalb verschieden hohe Einkommen, weil sie unterschiedlichen Zugriff oder Präferenzen hinsichtlich des Erwerbs von Bildung besitzen.

Friedman (1953) unterstreicht diesen Punkt der Präferenzheterogenität als Determinante für die Einkommensverteilung, indem er darauf hinweist, dass sich Menschen auch in ihrer Risikoneigung voneinander unterscheiden. Zwar bedeutet eine höhere Risikoneigung nicht per se auch höheres Einkommen, da das höhere Risiko auch die Möglichkeit höherer Verlust impliziert. Jedoch führen Unterschiede in der Risikoneigung zwangsläufig auch zu einer größeren Streuung der Einkommen, da sowohl die Gewinne als auch die Verluste zunehmen. Schließlich entscheidet auch die freiwillige Arbeit-Freizeit-Allokation des einzelnen über seine Einkommenshöhe; also nicht nur die intertemporale Zeitallokation (Bildung versus Arbeit) bestimmt die Höhe des Einkommens, sondern natürlich auch die intratemporale Zeitallokation (Arbeit versus Freizeit).

Diese Effekte unterstützen in der Wirkungsrichtung einen zentralen Konzentrationsmechanismus, auf den bereits Gibrat (1931) aufmerksam machte. Gibrat argumentiert, dass normalverteilte proportionale Einkommenserhöhungen zu einer Konzentration der Einkommen, also einer rechtsschiefen Einkommensverteilung führen müssen. Dieser Mechanismus ist in Abbildung 3.5 veranschaulicht. In dieser Simulation wird als Ausgangspunkt eine vollständige Gleichverteilung der Einkommen (von je 100 Geldeinheiten) auf die 100 Mitglieder einer Modellgesellschaft zum Anfangszeitpunkt angenommen. Untersucht wurde die Veränderung der Einkommensverteilung in der Gesellschaft, wenn die Einkommensentwicklung jedes einzelnen Mitglieds um ein multiplikativ verknüpftes, normalverteiltes Zufallselement $\mathrm{X}$ mit dem Erwartungswert $\mu$ und der Varianz $\sigma^{2}\left(X \sim N\left(\mu ; \sigma^{2}\right)\right)$ und ihren jeweiligen Ausprägungen $X_{n}$ ergänzt ist. Das heißt, das Einkommen in der k-ten Periode mit $k \in[1 ; 100]$ für jede Person i mit $i \in[1 ; 100]$ ergibt sich gemäß der Formel:

${ }^{161}$ Für eine detaillierte Darstellung und Bewertung der diskutierten Einflüsse vgl. Grüske (1985). 


$$
\begin{gathered}
y_{i, k}=y_{i, 1} \cdot \prod_{n=1}^{k}\left(1+\frac{X_{n}}{100}\right) \\
\text { mit } \\
X \sim N\left(\mu ; \sigma^{2}\right)
\end{gathered}
$$

Startet z.B. eine Person i mit einem Einkommen von 100 in Periode 1 und wurde die standardnormalverteilte Zufallszahl $\mathrm{X}_{\mathrm{i}, 1}=0,98$ generiert, so beträgt das Einkommen dieser Person zu Beginn der zweiten Periode:

$\mathrm{y}_{2}=100 \cdot\left(1+\frac{0,98 / 100}{}\right)=100,98$ usw.

Abbildung 3.5 zeigt deutlich, dass selbst bei einer standardnormalverteilten $\mathrm{Zu}$ fallsvariable nach 100 Perioden ein signifikantes Abweichen von der Ausgangsverteilung realisiert ist. ${ }^{162}$ Das Ausmaß der Ungleichverteilung wird umso gröBer, je stärker die Varianz der Normalverteilung ist. Hier kommt die Friedman'sche Überlegung deutlich hervor: Stärkere Streuung der Risikoneigung erhöht auch die Streuung der Einkommensverteilung. Natürlich ist die Entwicklung noch stärker ausgeprägt, wenn man von einer Gleichverteilung der Zufallsvariable innerhalb hinreichend großer Grenzen (hier zwischen -20 und +20 ; $\mathrm{X} \sim \mathrm{GV}[-20 ;+20])$ ausgeht. ${ }^{163}$

Das bedeutet, dass selbst ohne Berücksichtigung der eingangs erwähnten Theorien ein „natürlicher“, stochastischer Entwicklungsprozess zu einer lognormalverteilten Einkommensverteilung führt.

162 Tatsächlich zeigt sich ein monotones Sinken des Verhältnisses des untersten Quintils zum Gesamteinkommen über alle 100 Perioden. Im Durchschnitt fällt der Anteil des untersten Quintils um 0,0002\%-Punkte pro Periode. Das Bestimmtheitsmaß $\mathrm{R}^{2}$ dieser Trendanpassung liegt bei 0,9494. Eine Zufälligkeit des Ergebnisses ist also auszuschließen.

${ }^{163}$ Die empirischen Werte dieser Simulation bei 100 Perioden und 100 Gesellschaftsmitgliedern lauten:

\begin{tabular}{lrrc}
\hline & $\boldsymbol{N}(\mathbf{0} ; \mathbf{1})$ & $\boldsymbol{N}(\mathbf{0} ; \mathbf{1 6})$ & $\boldsymbol{G V}[-\mathbf{2 0} ;+\mathbf{2 0}]$ \\
\hline Summe der Einkommen & $10.071,77$ & $10.822,67$ & $8.721,04$ \\
Durchschnittseinkommen & 100,78 & 107,67 & 78,32 \\
Standardabweichung der Einkommen & 9,70 & 38,53 & 83,35 \\
Maximalwert & 120,77 & 207,54 & 320,56 \\
Minimalwert & 78,90 & 36,61 & 2,037 \\
\hline
\end{tabular}




\section{Abbildung 3.5 : 100 Perioden-Simulation der Entwicklung der Einkommensverteilung}

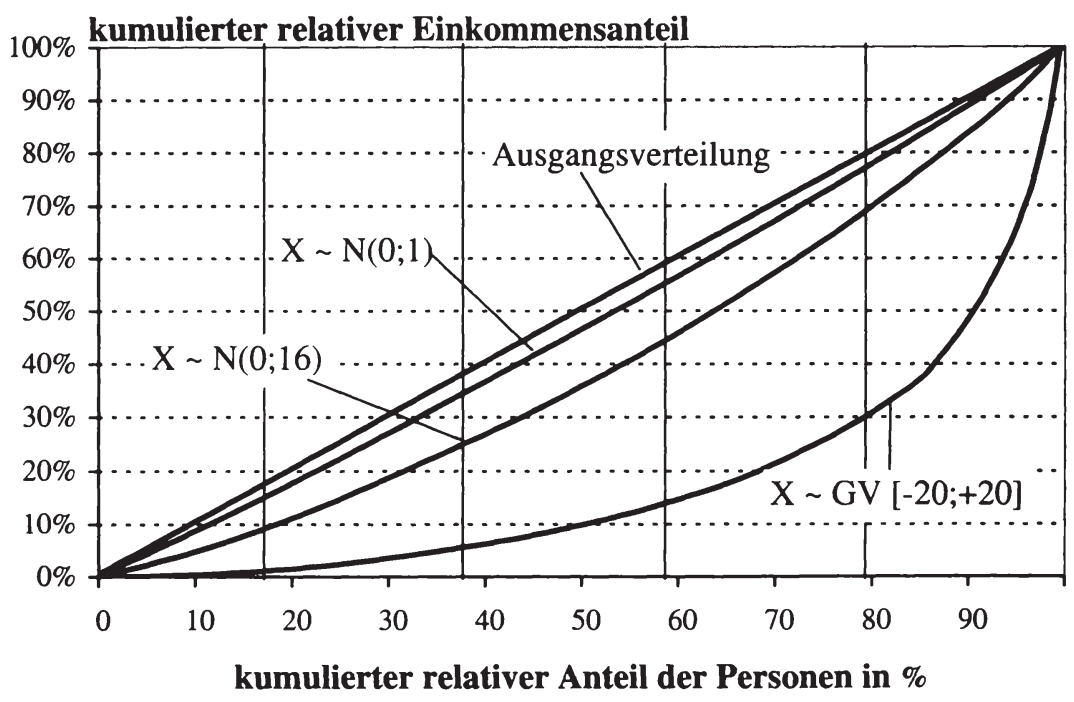

Quelle: Eigene Berechnungen.

Als idealtypisch rechte Politikrichtlinie würde gemäß der Ausführungen in Abschnitt 3.2 eine Politik gelten, die diesen ,natürlichen“ Konzentrationsprozess zulässt. Die häufig gemachte Aussage, dass typisch rechte Politik eine Politik der Reichen ist, ist nach dieser Interpretation ungenau. Zwar bevorteilt der oben gekennzeichnete Verteilungsprozess immer diejenigen, die zu Beginn (im Modell zufallsbedingt) zu höheren Einkommen kamen, jedoch steht innerhalb einer idealtypisch rechten Konzeption nicht das Umverteilungsergebnis im Vordergrund, sondern der Umverteilungsprozess.

Dies ist bei einer idealtypisch linken Politik anders. Hier ist gerade das gewünschte Verteilungsergebnis der Einkommensegalisierung die Begründung für die Ablehnung des natürlichen Verteilungsprozesses.

Möchte man nun rechte und linke Wirtschaftspolitik genauer bestimmen, muss man untersuchen, welche Politikinhalte zu welchen Verteilungsergebnissen führen. Dafür ist es zunächst nötig, die unterschiedlichen Dimensionen der Wirtschaftspolitik näher zu bestimmen. Das soll im folgenden Kapitel erfolgen. 


\subsubsection{Das wirtschaftspolitische Instrumentarium}

Üblicherweise unterscheidet man in der Theorie der Wirtschaftspolitik in Ordnungs- und Prozesspolitik, wobei ordnungspolitische Maßnahmen langfristig den geltenden Rahmen festlegen, in dem sich gesellschaftliches Handeln vollzieht, wohingegen Prozesspolitik direkt in die Abläufe der gesellschaftlichen Handlungen innerhalb des zuvor bestimmten ordnungspolitischen Rahmens eingreift. Tabelle 3.4 zeigt eine Übersicht der einzelnen Aspekte der Ordnungs- und Prozesspolitik.

\section{Tabelle 3.4 : Ordnungs-und Prozesspolitik als Komponenten der Wirtschaftspolitik}

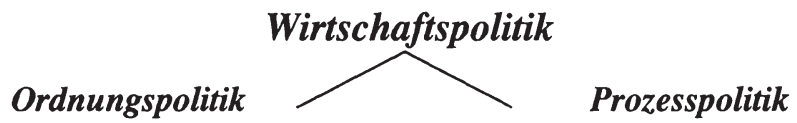

Gestaltung der Rahmenbedingungen Steuerung der Abläufe

Gestaltungsbereiche

- Eigentumsverfassung

- Koordinationsmechanismus (Markt versus Staat)

- Marktverfassung: Konkretisierung der Eingriffsbereiche (Sozialverfassung)

- Finanzverfassung

- Geldverfassung

- Budgetierungsregeln

- Außenwirtschaftsverfassung
Steuerungsbereiche

- Produktion und Beschäftigung (De-) Regulierung von Güter- und Faktormärkten

- Einkommensverteilung

- Preispolitik

- Einkommensverwendung

- Zahlungsbilanzpolitik

- Wechselkurspolitik

- Strukturpolitik

\section{Politikmerkmale}

- Hauptträger: Legislative

- Zeithorizont: langfristig

- Primärziel: Wirtschaftssystem (qualitative Größen)

- Sekundärziel: Prozessergebnis (quantitative Größen)

\section{Politikmerkmale}

- Hauptträger: Exekutive, Notenbank

- Zeithorizont: kurz- und mittelfristig

- Primärziel: Prozessergebnis (quantitative Größen)

- Sekundärziel: Wirtschaftssystem (qualitative Größen)

Quelle: In Anlehnung an Cassel (1988, S. 156). 
Da Ordnungspolitik den Systemrahmen vorgibt, ist sie naturgemäß langfristiger angelegt als die Prozesspolitik. Deutliche partisanpolitische Effekte sind daher eher auf der Prozessebene zu isolieren. Dies soll nicht heißen, dass nicht auch auf der Ordnungsebene parteipolitische Diskrepanzen existieren und auch ideologisch motivierte Konzepte umgesetzt werden können (z.B. Mitbestimmungsrecht), jedoch unterliegt der Ordnungsrahmen viel stärkeren Änderungsanforderungen als der Eingriff in laufende Prozesse. So ist die Eigentumsordnung (Art. 14GG) sowie die Sozialstaatlichkẹit (Art. 20GG in Verbindung mit Art. 79 Abs. 3GG) unabänderbar. ${ }^{164}$ Auch wurden Teile der Ordnungspolitik an supranationale Organisationen delegiert, sodass auch dort geringere Handlungsmöglichkeiten bestehen. Beispielsweise ist die Außenwirtschaftspolitik maßgeblich durch die Vollendung des Binnenmarktes (v.a. Art. 1-3 und Art. 131-135 des EG-Vertrags in der Fassung vom 2. Oktober 1997) sowie durch die Verträge der Uruguay-Runde des GATT (General Agreement on Tariffs and Trade) im April 1994 bestimmt. Das Marktwirtschaftlichkeitsprinzip ist ebenfalls im EG-Vertrag enthalten (Art. 4 Abs. 1 sowie die wettbewerbspolitischen Artikel 81-89 des EGVertrags in der Fassung vom 2. Oktober 1997), und auch die Geldverfassung ist mittlerweile durch europäische Normen bestimmt. ${ }^{165}$

Zwar gibt es auch auf der Prozessebene Tendenzen, den nationalstaatlichen Freiraum durch supranationale Instanzen einzugrenzen, diese sind jedoch noch sehr vage und lassen größere Handlungsfreiräume. ${ }^{166}$ Außerdem vollzogen sich die zentralen internationalen Abschlüsse sehr langsam. Auch wenn einschneidende Abkommen eindeutigen Zeitpunkten zuzuordnen sind, so sind sie doch Ausdruck eines langen Entstehungsprozesses. Beispielsweise wurde der Grundstein für die Unterzeichnung des Amsterdamer Vertrages bereits durch die Unterzeichnung des EGKS-Vertrags (Vertrag über die Europäische Gemeinschaft für Kohle und Stahl) am 18. April 1951 gelegt. Ebenso ist die Konstituierung der WTO (World Trade Organisation) am 1. Januar 1995 lediglich der letzte Schritt eines globalen Zollsenkungsweges, der mit der ersten Zollsenkungsrunde nach der Genfer-Runde am 1. Januar 1948 begann. Diesem ersten Schritt folgten sieben weitere, bevor die WTO gegründet wurde. Den Effekt einer einzigen nationalstaatlichen Regierung im Zusammenspiel mit allen anderen Regierungen während dieses Prozesses abzuschätzen, ist wohl kaum zu bewerkstelligen.

164 Allerdings besteht durch den Sozialisierungsartikel (Art. 15GG) Spielraum, das private Eigentum in den Dienst der Allgemeinheit zu stellen.

165 Die grundsätzliche Norm ist Art. 4 Abs. 2 EG-Vertrag in der Fassung vom 2. Oktober 1997. Die konkrete Ausgestaltung steht in Art. 105-124 sowie in zahlreichen Protokollen, die dem Vertragswerk von Amsterdam als rechtsverbindliche Anlagen angefügt sind.

Vgl. dazu den neu verfassten Titel VIII (Art. 125-130) im Amsterdamer Vertragswerk von 1997. 
Ein dritter Grund für eine Fokussierung auf die Prozesspolitik ist rein pragmatischer Art. Ordnungspolitik ist seines Wesens nach qualitativ und entzieht sich daher zwar nicht vollständig, aber weitgehend einer ökonometrischen Untersuchung. Demgegenüber sind viele Ergebnis- und Instrumentvariable der Prozesspolitik verhältnismäßig leicht quantitativ zu erheben und daher besser für eine empirische Untersuchung geeignet.

Es ist natürlich darauf zu verweisen, dass jede Änderung der Ordnungsparameter starke Rückwirkungen auf die Prozessvariablen hat. Dies ist ja gerade der Untersuchungsgegenstand der Arbeit: inwiefern hat die Änderung des Ordnungsrahmens hin zu stärkerer internationaler Verflechtung die Möglichkeit prozesspolitischer Steuerung eingeengt? Der empirische Teil geht dabei allerdings rekursiv vor: Durch die Analyse der Prozessparameter wird versucht, auf den restringierenden Einfluss der Ordnungspolitik zu schließen.

Für die Bestimmung idealtypischer linker und rechter Prozesspolitik ist es erforderlich, die Verteilungswirkung von einzelnen Instrumenten zu analysieren. Da sich die empirische Untersuchung auf der Instrumentenebene auf die Analyse der Budgetstruktur, insbesondere der Ausgabenstruktur gesamtstaatlicher Haushalte, und auf der Zielebene auf das Beschäftigungs- und auf das Preisniveauziel beschränkt, wird im folgenden auch nur auf die verteilungswirksamen Effekte dieser Bereiche eingegangen.

\subsubsection{Verteilungswirkungen einzelner prozesspolitischer Elemente}

In diesem Kapitel sollen die Verteilungswirkungen von Änderungen makroökonomischer Ziel- und Instrumentvariable untersucht werden. Zunächst wird erläutert, welche Bevölkerungsgruppe besonders die Kosten der Arbeitslosigkeit trägt, anschließend werden die Kosten und Verteilungseffekte von Inflation präsentiert, um zum Schluss die theoretischen und empirischen Ergebnisse von Untersuchungen hinsichtlich der Inzidenz von staatlichen Ausgaben zusammenzufassen.

\subsubsection{Verteilungswirkungen der Arbeitslosigkeit}

\subsection{Die individuellen Kosten der Arbeitslosigkeit}

Die Kosten, die jedem Arbeitslosen aus seiner Nichtbeschäftigung entstehen, werden maßgeblich durch seine Verdienstausfälle bestimmt. Die Kosten jedoch allein durch diese Einkommensausfälle zu schätzen, greift zu kurz, da zum einen die mögliche Verdienstentwicklung nicht berücksichtigt wird: Durch die Teil- 
nahme am Arbeitsprozess ist eine permanente Investition in betriebsspezifisches Humankapital gewährleistet, wodurch die Produktivität und damit auch die Verdienstaussicht in der Zeit zunimmt. Arbeitslosigkeit führt dazu, dass man nicht mehr die Möglichkeit hat, in betriebsspezifisches Humankapital zu investieren. Zum anderen werden die Kosten der Arbeitslosigkeit durch staatliche Transferleistungen (auch Ausbildungsleistungen) aufgefangen, und die meisten Menschen können der neu gewonnenen Freizeit ebenfalls einen Wert zuweisen (der jedoch im Falle unfreiwilliger Arbeitslosigkeit natürlich hinter dem Wert der Arbeit zurückbleibt).

Beschreibt $Y_{0}$ das Einkommen zum Zeitpunkt des Arbeitsverlusts, $T_{t}$ die staatlichen Transfers in jeder Periode $t$ mit $t \in[1 ; n]$, in der eine Person arbeitslos ist, $F_{t}$ den Wert der Freizeit, ${ }^{167} \mathrm{r}_{\mathrm{h}}$ die durchschnittliche prozentuale Einkommenssteigerung, die aufgrund der permanenten Investition in Humankapital gewährleistet ist, und $\mathrm{r}$ den Marktzins, dann kennzeichnet (3.4) den Verlust L, der einem Individuum während der Nicht-Beschäftigung entsteht: ${ }^{168}$

$$
L=\sum_{t=1}^{n}\left[\frac{Y_{0}\left(1+r_{h}\right)^{t}-T_{t}-F_{t}}{(1+r)^{t}}\right]
$$

Geht man im weiteren davon aus, dass durch die Beschäftigungslosigkeit nicht nur die Verdienstentwicklung aussetzt, sondern marktfähiges Humankapital mit der Rate $d$ abgeschrieben wird, so sinkt das Einkommen bei Wiedereinstellung $Y_{n}$ im Vergleich zu fortlaufender Weiterbeschäftigung in Höhe von (3.5):

$$
Y_{n}=\left[Y_{0}\left(1+r_{h}\right)^{n}-Y_{0}(1+d)^{-n}\right]
$$

Folgen nach der Arbeitslosigkeit noch m Perioden der Beschäftigung, so ist der gesamte Verlust (als Barwert zum Zeitpunkt $t_{0}$ ), der durch die Arbeitslosigkeit für das Individuum entsteht, durch (3.6) definiert:

$$
L=\sum_{t=1}^{n}\left[\frac{Y_{0}\left(1+r_{h}\right)^{t}-T_{t}-F_{t}}{(1+r)^{t}}\right]+\frac{1}{(1+r)^{n}} \cdot \sum_{s=n+1}^{m}\left[\frac{Y_{n}\left(1+r_{h}\right)^{s}}{(1+r)^{s}}\right]
$$

${ }^{167}$ Die Höhe des Wertes F richtet sich dabei nicht nur nach der Präferenz für Freizeitaktivitäten, sondern auch nach der Möglichkeit, Nichtmarkteinkommen zu erzielen. 
Dieser Verlust L entsteht also im Falle von Arbeitslosigkeit. Für jedes Individuum ist dieser Verlust mit Risiko behaftet. Aus diesem persönlichen Risiko lässt sich die individuelle Eintrittswahrscheinlichkeit für das Ereignis Arbeitslosigkeit $\mathrm{P}(\mathrm{U})$ ableiten. Der potentielle (abdiskontierte) Lebensverdienst $\mathrm{G}$ ist durch (3.7) beschrieben:

$$
G=\sum_{u=1}^{m+n} \frac{Y_{0}\left(1+r_{h}\right)^{u}}{(1+r)^{u}}
$$

Dann kann man aus (3.6) und (3.7) unter Beachtung des individuellen Risikos das abdiskontierte Lebenseinkommen EU darstellen. Je größer das Risiko der Beschäftigungslosigkeit c.p., desto geringer ist dann auch der Erwartungswert des Lebenseinkommens. ${ }^{169}$

$$
E U=P(U) \cdot(G-L)+(1-P(U)) \cdot G
$$

Es ist also grundsätzlich zu erwarten, dass jene Bevölkerungsgruppen im besonderen Maße die Kosten der Arbeitslosigkeit zu tragen haben, die

- ein überproportionales Risiko des Beschäftigungsverlustes und eine reduzierte Wahrscheinlichkeit der Wiedereinstellung besitzen, die also nur geringes und/oder stark branchenspezifisches Humankapital besitzen und/oder in Branchen mit hohem Rationalisierungspotential arbeiten,

- wenig Möglichkeiten besitzen, nichtmarktliche Einkommen zu realisieren,

- in Branchen arbeiten, in denen eine starke Einkommensdynamik besteht, da hier ein Einkommensverlust starke Einkommensverzichte bedeutet.

Bevor die Verteilungseffekte skizziert werden, soll kurz auf die gesamtwirtschaftlichen Kosten von Unterbeschäftigung eingegangen werden.

169 Diese Darstellung reduziert die Realität sehr stark, da angenommen wird, dass man im Leben nur einmal über einen bestimmten Zeitraum arbeitslos werden kann und dieser Zeitraum zu Beginn der Arbeitsperiode liegt. Eine exaktere Ermittlung des Erwartungswertes müsste berücksichtigen, dass man mehrmals beschäftigungslos werden kann. Der Erwartungswert des Lebenseinkommens wäre dann der Erwartungswert einer Sequenz von $(n+m)$ Erwartungswerten aus den Einzelperioden. Auch müsste berücksichtigt werden, dass die Eintrittswahrscheinlichkeit wohl intertemporal nicht konstant ist. Realistischere Annahmen würden an den Kernaussagen des Modells jedoch nichts ändern, daher scheint die Vorgehensweise gerechtfertigt. 


\subsection{Die gesamtwirtschaftlichen Kosten der Arbeitslosigkeit}

Dafür ist es zunächst notwendig, den Begriff der Unterbeschäftigung näher zu bestimmen. In Deutschland gelten ausschließlich diejenigen als erwerbslos, die bei den Arbeitsämtern registriert wurden. Diese Zahl in Relation zu der Anzahl der Erwerbspersonen ergibt die Arbeitslosenquote, die als Indikator für das Ausmaß an volkswirtschaftlicher Unterbeschäftigung gilt. Diese Zahl ist jedoch in vielerlei Hinsicht ungenau. Zum einen werden u.a. diejenigen, die Kurzarbeit aufgenommen haben und jene, die an Arbeitsbeschaffungsmaßnahmen teilnehmen, nicht in der Arbeitslosenquote berücksichtigt. Der Sachverständigenrat zur Begutachtung der gesamtwirtschaftlichen Lage (SVR) gibt daher zusätzlich Auskunft über den Umfang der verdeckten Arbeitslosigkeit (SVR, 1998, S. 340). ${ }^{170}$ Für die weitere Analyse ist die Wahl der ,richtigen“ Arbeitslosenquote dann von geringer Relevanz, wenn alle Maße stark miteinander korreliert sind. Davon ist im allgemeinen auszugehen (Hibbs, 1987, S. 46f), sodass für die hier vorgelegte Untersuchung die exakte absolute Größenordnung des Unterbeschäftigungsproblems keine Bedeutung hat.

Als zweites muss gefragt werden, welches überhaupt die Größenordnung der volkswirtschaftlichen Kosten der Arbeitslosigkeit ist, um anschließend die Frage beantworten zu können, auf wen diese Kosten entfallen. Seit Okun (1962) das Diktum aufstellte, dass ein 1\%-iger Anstieg der Arbeitslosenquote zu einem Output-Verlust von 2-3\% führt, lassen sich die volkswirtschaftlichen Kosten der Unterbeschäftigung relativ exakt berechnen. ${ }^{171}$ Hibbs (1987, S. 51) errechnet, dass allein 1984 ein 1-prozentiger Anstieg der Arbeitslosenquote für die USA zu entgangener Produktion von 75 Mrd. US-\$ führte. Blinder (1987, S. 35) rechnet unter der Annahme, dass die natürliche Arbeitslosenquote in den USA bei 5,8\% liegt, sogar einen volkswirtschaftlichen Verlust von $154 \mathrm{Mrd}$. US-\$ für denselben Zeitraum aus.

Für Deutschland zeigt eine einfache Regression der Veränderungsrate des realen Bruttoinlandsprodukts (Y) auf die Veränderung der Arbeitslosenquote (U), dass für Deutschland im wesentlichen derselbe Zusammenhang gilt: ${ }^{172}$

${ }^{170}$ Für eine exakte Abgrenzung des Begriffs der verdeckten Arbeitslosigkeit, vgl. SVR (1998, S. 80, Tabelle 27, Fußnote 5). Für eine ausführliche Diskussion verschiedener Konstruktionen von Arbeitslosenquoten, vgl. Hibbs (1987, S. 43ff) sowie Litz (1999) für eine Diskussion unterschiedlicher Konzepte im internationalen Vergleich.

${ }^{171}$ Es ist darauf hinzuweisen, dass diese Relation von Okun für Abweichungen vom Gleichgewicht, also für konjunkturelle Schwankungen gilt. Das Problem der strukturellen Arbeitslosigkeit, also quasi ein Verschieben des Gleichgewichtspunktes, ist hierin nicht abgebildet.

172 Es wurde der Untersuchungszeitraum von 1961-1997 gewählt, wobei ab 1992 Daten für das wiedervereinigte Deutschland zugrunde lagehiazwast gibt de 63 sachversiändigenrat 


$$
\left(\ln Y_{t}-\ln Y_{t-1}\right)=0,34-0,0199\left(U_{t}-U_{t-1}\right)
$$

$$
\mathrm{R}^{2}=0,57
$$

$\mathrm{DW}=1,833$

Insgesamt werden also immerhin 57\% der Varianz der Wachstumsrate durch die Veränderung der Arbeitslosenquote erklärt. Auch hat der Regressionskoeffizient das erwartete Vorzeichen und ist auf dem 1\%-Signifikanzniveau von null verschieden. Außerdem kann angesichts des Durbin-Watson-Wertes mindestens auf dem 5\%-Niveau Autokorrelation der ersten Ordnung ausgeschlossen werden. ${ }^{173}$ Die Zahlen bedeuten, dass allein der Anstieg der Arbeitslosigkeit um 1,4\%Punkte im Jahr 1997 zu einem zusätzlich entgangenen Output von 86,4 Mrd. DM geführt hat. ${ }^{174}$

Der SVR (1998, S. 348) schätzt die gesamte Produktionslücke 1997 als Differenz zwischen Produktionspotential und realisiertem Bruttoinlandsprodukt auf 229,6 Mrd. DM. ${ }^{175}$

\subsection{Verteilungseffekte}

Um die Verteilungswirkung eines Anstiegs der Arbeitslosenquote abzuschätzen, hat sich in der Literatur der Ansatz von Blinder und Esaki (1978) durchgesetzt. In einem OLS-Schätzmodell (ordinary least squares) regressieren sie die Anteile der einzelnen Einkommensquintile auf die Höhe der Arbeitslosenquote, der Inflation sowie eine Trendvariable. ${ }^{176}$ In einer späteren Schätzung desselben Modells auf der Basis einer längeren Zeitreihe kommt Hibbs (1987, S. 79ff) für die USA und den Zeitraum von 1947-1980 zu dem Ergebnis, dass ein Anstieg der

(1998, S. 348) auch schon für 1991 Daten für Gesamtdeutschland an. Der Datenpunkt für 1991 zeigt sich im Scatterplot der Residuen jedoch eindeutig als Ausreißer. Daher wurde auch für 1991 noch auf eine Berücksichtigung ostdeutscher Daten verzichtet. Die Untersuchung kürzerer Teilperioden brachte keine maßgeblichen Abweichungen von dem dargestellten Ergebnis. Daher wird auf deren Präsentation verzichtet. In Klammern ist der tWert angegeben.

173 Allerdings besagt der traditionelle Ansatz von Okun, dass Fluktuationen im Output zu Fluktuationen in der Arbeitslosenquote führen. In dem Regressionsansatz, der im wesentlichen dem Ansatz von Hibbs (1987, S. 50) folgt, wurde die Darstellung gedreht. Die Aussage bleibt natürlich erhalten, dass Arbeitslosigkeit mit Produktionsverlusten einhergeht.

174 Das reale Bruttoinlandsprodukt in Preisen von 1991 belief sich auf 3.101,4 Mrd. DM.

${ }^{175}$ Für eine ausführliche Darstellung der Errechnung des gesamtwirtschaftlichen Produktionspotentials, vgl. SVR (1998, S. 284f).

${ }^{176}$ Sie verwenden zusätzlich eine Dummy-Variable, um die Statistikumstellung im Jahr 1958 zu berücksichtigen sowie einen Interaktionsterm aus der Dummy- und der Trendyariable. 
Arbeitslosenquote um 1\%-Punkt die Einkommensanteile der beiden unteren Quintile um jeweils 0,1\%-Punkte senkt, wohingegen der Einkommensanteil des höchsten Quintils um 0,2\%-Punkte ansteigt. Die Ergebnisse für die USA sind statistisch signifikant auf dem 1\%-Niveau. Ein Anstieg der Arbeitslosenquote hat demnach für die USA stark regressive Verteilungseffekte. ${ }^{177}$ Auch Gramlich (1974) sowie Gramlich und Laren (1984) schließen aus ihren Untersuchungen, dass eine Verschlechterung der Arbeitsmarktperformance insbesondere die Angehörigen unterer Einkommensklassen schlechter stellt.

\section{Tabelle 3.5 : Arbeitslosigkeit nach der Berufsausbildung in Deutschland}

\begin{tabular}{|c|c|c|c|c|c|}
\hline & \multicolumn{2}{|c|}{$\begin{array}{l}\text { Erwerbspersonen } \\
\text { (älter als } 15 \text { Jahre) }\end{array}$} & \multicolumn{2}{|c|}{$\begin{array}{l}\text { Registrierte } \\
\text { Arbeitslose }\end{array}$} & \multirow{2}{*}{$\begin{array}{l}\text { Arbeitslo } \\
\text { senquote } \\
(2) /(1)^{*} 100\end{array}$} \\
\hline & $\begin{array}{l}\text { abs. in Tsd. } \\
\text { (1) }\end{array}$ & $\begin{array}{c}\text { in \% von } \\
\text { Gesamt }\end{array}$ & $\begin{array}{l}\text { abs. in Tsd. } \\
\text { (2) }\end{array}$ & $\begin{array}{c}\text { in \% von } \\
\text { Gesamt }\end{array}$ & \\
\hline ohne Ausbildung ${ }^{a}$ & 8.449 & 20,98 & 1.531 & 38,62 & 18,12 \\
\hline Betriebl. Ausbildung ${ }^{\text {b) }}$ & 22.465 & 55,80 & 2.080 & 52,46 & 9,26 \\
\hline Fachschule & 3.632 & 9,02 & 156 & 3,93 & 4,30 \\
\hline Fachhochschule & 2.129 & 5,29 & 56 & 1,41 & 2,63 \\
\hline Universität & 3.588 & 8,91 & 142 & 3,58 & 3,96 \\
\hline GESAMT & 40.263 & 100,00 & 3.965 & 100,00 & 9,85 \\
\hline
\end{tabular}

Anmerkungen: a) Die Daten über die Berufsausbildung der Bevölkerung beruhen auf freiwilligen Angaben der Befragten innerhalb des Mikrozensus. Da bei diesen Befragungsergebnissen lediglich 213.000 keine Angaben machten, wurden sie vollständig der Gruppe „ohne Berufsausbildung“ zugeordnet. Die Arbeitslosenquote dieser Gruppe ist dadurch gegebenenfalls leicht unterzeichnet.

b) Diese Gruppe umfasst sowohl betriebliche Ausbildungen als auch Ausbildungen an Berufsfachschulen.

Quelle: Statistisches Bundesamt (1999, S. 118 und S. 369) sowie eigene Berechnungen, Daten für Ende September 1998.

177 Allerdings konnte dieses Ergebnis für Deutschland und den Zeitraum von 1960-1988 nicht bestätigt werden. Außer der Trendvariable war kein einziger Parameter signifikant, teilweise waren sogar die Vorzeichen der Koeffizienten unkorrekt. Das Schätzergebnis wurde auch nicht besser, als die Höhe der staatlichen Transferausgaben und die beiden Ölschocks als erklärende Variable berücksichtigt wurden. Inwiefern die geringe Anzahl der Datensätze (es lagen nur für 17 Jahre Verteilungsdaten vor) bzw. die Güte dieser Verteilungsdaten das Ergebnis verzerrt oder die Intensität der Arbeitsmarktregulierung in Deutschland zu diesem Resultat führt, kann hier nicht abschließend beantwortet werden. Die benutzten Verteilungsdaten stammen ebenfalls von Thoma B $_{\text {oi CS usack }}$ - 978-3-631-75139-8 
Eine Aufschlüsselung der Arbeitsmarktstatistik nach dem Ausbildungsgrad in Deutschland gibt zumindest einige Hinweise, dass auch für Deutschland - trotz der angesprochenen schlechten Regressionsergebnisse - ein regressiver Zusammenhang zwischen Arbeitslosenquote und Einkommensverteilung vermutet werden kann. Geht man davon aus, dass im Durchschnitt eine längere und höher qualifizierte Ausbildung auch zu höheren Einkommen führt, so zeigt Tabelle 3.5 deutlich, dass besonders die schlechter Qualifizierten und demnach jene mit niedrigerem Einkommen ein deutlich höheres Arbeitsmarktrisiko zu tragen haben. ${ }^{178}$

Da oben gezeigt wurde, dass das Arbeitsplatzrisiko maßgeblichen Anteil an der Bestimmung des Einkommensverlusts durch Arbeitslosigkeit besitzt, kann hieraus gefolgert werden, dass ein allgemeiner Anstieg der Arbeitslosenzahlen stark regressiv wirkt.

\subsubsection{Preispolitik}

\subsection{Die Kosten der Inflation}

Die Kosten der Inflation werden in der ökonomischen Literatur weniger übereinstimmend diskutiert als die Kosten der Unterbeschäftigung. Werden die Erwartungen rational gebildet, muss man zwischen den Kosten, die aus einer erwarteten Preissteigerung resultieren und jenen, die aus unerwarteter Inflation entstehen, unterscheiden.

Bei erwarteter Inflation entstehen v.a. zusätzliche Transaktionskosten, die den Wirtschaftsablauf verteuern. Erstens steigen die Opportunitätskosten der realen Kassenhaltung. Diese gesunkene Kassenhaltung impliziert zum einen direkte Transaktionskosten dadurch, dass man häufiger zur Bank muss (,shoeleather costs", Mankiw, 1999, S. 177), zum anderen indirekte Transaktionskosten dadurch, dass den Individuen Nutzenverzichte entstehen, weil sie nicht ihre erstbeste Wahl bei Preisstabilität treffen können, sondern ihr Portfolio umbilden müssen (Cassel, 1992, S. 316). Werden die shoeleather costs regelmäßig als gering eingeschätzt, herrscht hinsichtlich der Nutzenverzichte aus geringerer Kassenhaltung Uneinigkeit. Tobin (1972, S. 15) misst diesen Kosten kein Gewicht bei, wohingegen Alexander und Loef (1975) die Kosten bei mäßiger Inflation für Deutschland bereits auf 1,7 Mrd. DM pro Jahr schätzen.

178 Vgl. auch Freeman und Schettkat (1999, S. 103) für einen ähnlichen Überblick für die USA und Drèze und Sneessens (1997) für alle OECD-Staaten. Bis auf Italien gilt dort jeweils derselbe Zusammenhang. 
Zusätzliche Wohlfahrtsverluste entstehen dann, wenn die Preisanpassung selber Kosten, die „menu costs“, verursacht (Mankiw, 1999, S. 177). Dann führen unregelmäßige und unkoordinierte Preisanpassungen an das gestiegene Preisniveau zu starken Verzerrungen der relativen Preise und damit zu Ineffizienzen, da die ausgewiesenen Preise nicht die zugrunde liegenden Knappheitsrelationen widerspiegeln. Drittens entstehen Transaktionskosten, da die intertemporale Vergleichbarkeit von Preisrelationen erschwert wird.

Bei einer unerwarteten Inflation entstehen für risikoaverse Individuen Kosten aus der Unsicherheit über die Preisentwicklung per se und zudem für die Vertragspartei, die die tatsächliche Preisentwicklung schlechter stellt, also z.B. den Kreditgeber, falls die Inflationsrate höher ausfällt als zuvor angenommen. ${ }^{179} \mathrm{Da}$ die Höhe der Inflationsrate mit der Variabilität der Inflationsrate korreliert ist, steigen mit zunehmender Inflationsrate auch die Kosten aus unerwarteter Inflation (Blinder, 1987, S. 50).

Inwiefern diese Kosten Rückwirkungen auf die Wachstumsrate einer Volkswirtschaft haben, ist noch nicht abschließend verstanden (Cassel, 1992, S. 314). Allerdings deutet die Mehrzahl der Studien, die in den 90er Jahren durchgeführt wurden, eher in Richtung eines negativen Zusammenhangs zwischen der Höhe der Inflationsrate und der Höhe der Wachstumsrate. Barro (1995) schätzt die Reduktion der Wachstumsrate, die aus einem Anstieg der Inflationsrate von 5\% auf $50 \%$ resultiert auf 1-1,5\%-Punkte. Gylfason und Herbertsson (1996) kommen zu ähnlichen Ergebnissen; ein Anstieg der Inflationsrate um 45\%-Punkte senkt die Wachstumsrate um 0,6-1,3\%-Punkte. ${ }^{180}$ Angesichts dieser eher geringen Effekte bei mäßiger Inflation resümiert Blinder (1987, S. 51):

„As rational individuals, we do not volunteer for a lobotomy to cure a head cold. Yet, as a collectivity, we routinely prescribe the economy equivalent of lobotomy (high unemployment) as a cure for the inflationary cold. "

Ähnlich sieht Hibbs (1987, S. 124) die wahren Kosten der Inflation in den Konsequenzen, die aus der Inflationsbekämpfung resultieren. Dies wirft natürlich die Frage auf, warum werden diese hohen Kosten eingegangen, wenn moderate Inflationsraten für die Wähler gar nicht zu dramatischen Wohlfahrtsverlusten füh-

179 Dies gilt natürlich nur, falls die Verträge keinen Inflationsausgleich berücksichtigen. Mankiw (1999, S. 179) wundert sich, dass bei vorherrschender Risikoaversion die meisten Verträge nicht preisniveauindexiert sind.

180

Allerdings kommen andere Studien zu durchaus ökonomisch signifikanten Größen. So schätzt z.B. Feldstein (1996), dass in den USA ein Absenken der Inflationsrate um 2\%Punkte zu einem permanenten positiven Wohlfahrtseffekt in der Größenordnung von 1\% des Bruttoinlandsprodukts führen wird. Bakshi, Haldane und Hatch (1997) kommen für Großbritannien zu dem Ergebnis, dass eine Reduktion des Preisauftriebes um 2\%-Punkte immerhin dauerhafte Wohlfahrtssteigerungen in der Höhe von $0,2 \%$ vom Bruttoinlandsprodukt zur Folge hätte. 
ren? Hibbs (1987, S. 129ff) argumentiert, dass die Wahlbürger Inflationsbekämpfung durch die geäußerten Befürchtungen über zu starke Preissteigerung nachfragen und Politiker diese Nachfrage lediglich bedienen. Dies beantwortet die Frage allerdings nur unbefriedigend, da ungeklärt bleibt, wieso rationale Individuen die aus der Inflationsbekämpfung resultierenden hohen Kosten, die zur Beseitigung nur geringer Kosten erforderlich sind, nicht erkennen. ${ }^{181}$

Blinder's Erklärung (1987, S. 51ff), dass dieses Phänomen darauf zurückzuführen ist, dass Individuen unter Inflationsillusion leiden, scheint Blinder selbst nicht wirklich zu befriedigen, weist er doch wiederholt darauf hin, dass dieses nur eine spekulative Hypothese ist. ${ }^{182}$ Ähnlich vorsichtig formuliert Mankiw (1999, S. 176): „The public's distaste for inflation may be partly psychological.“

Das Interesse an Inflationsbekämpfung könnte immerhin auch dadurch gerechtfertigt werden, falls sich die (geringen) Kosten der Inflation sehr ungleichmäßig auf die einzelnen Bevölkerungsgruppen verteilten. Daher sollen nun die Verteilungswirkungen der Inflation untersucht werden.

\subsection{Inflation und die personelle Einkommensverteilung}

Allgemein können jene als Inflationsverlierer bezeichnet werden, deren durchschnittlich nachgefragter Warenkorb einer stärkeren Preissteigerung unterworfen ist als jener Warenkorb, der der Ermittlung der gesamtwirtschaftlichen Preissteigerung zugrunde gelegt wird. ${ }^{183}$

Hibbs (1987, S. 79f) konstruiert ein Schätzmodell, bei dem er für jedes Einkommensquintil den Einkommensanteil als Regressand und die Preissteigerungsrate als Regressor verwendet, um den Einfluss der Inflation auf die relative

181 Es darf indes nicht argumentiert werden, dass die Inflationskosten so gering sind, dass Stabilitätspolitik unsinnig ist. Die Kosten der Inflation sind hoch genug, dass die Vermeidung der Inflation dominante Strategie sein sollte.

182 Diese Inflationsillusion entsteht dadurch, dass die Mehrzahl der Arbeitnehmer nicht versteht, dass Lohnsteigerungen nicht allein durch gestiegene Produktivität, sondern eben auch durch einen Anstieg des allgemeinen Preisniveaus wachsen. Geht man davon aus, dass der Lohnzuwachs „,verdient“ ist, dann reduziert Inflation natürlich den Einkommenszuwachs. Bei einem Produktivitätsanstieg um $2 \%$ und einer Inflationsrate von $10 \%$ ist mit Lohnzuwächsen von $12 \%$ zu rechnen. Schreibt der Arbeitnehmer seinen nominalen Lohnzuwachs allein seiner besseren Leistung zu, reduziert die Inflation seine Lohnsteigerung um $83,3 \%$. Dieser ,robbery coefficient“ nimmt bei konstantem Produktivitätsanstieg mit zunehmender Inflationsrate degressiv zu.

${ }^{183}$ Es geht in diesem Abschnitt allein um die Wirkung der Inflation auf die personelle Einkommensverteilung. Dass Inflation vielfältige Verteilungswirkungen hat, ist selbstverständlich. Stets verteilt Inflation bei nicht indexierten Verträgen Einkommen von Gläubigern zu Schuldnern und vom privaten Sektor zum Ptgat Just - 978-3-631-75139-8 
Position der Einkommensbezieher abzuschätzen. Dabei konnte er für kein einziges Quintil einen signifikanten Zusammenhang feststellen. Auch Blinder (1987, S. 54) fasst seine Literaturübersicht so zusammen, dass Inflation nicht die unteren Einkommensquintile schlechter stellt. Tabelle 3.6 zeigt eine ähnliche multiple OLS-Regression für die einzelnen Einkommensquintile in Deutschland als abhängige Variable für den Zeitraum von 1960-1988. ${ }^{184}$ Zusätzlich wurde der Anteil der gesamten Staatsausgaben am Bruttoinlandsprodukt als erklärende Variable aufgenommen, sodass insgesamt fünf Schätzgleichungen mit jeweils dem folgenden Aufbau geschätzt wurden:

$$
\text { Quintil }_{\mathrm{it}}=\alpha_{\mathrm{i}}+\beta_{1 \mathrm{i}} \text { Inflation }_{\mathrm{t}}+\beta_{2 \mathrm{i}} \frac{\text { Staatsausgaben }_{\mathrm{t}}}{\text { BIP }_{\mathrm{t}}}+\varepsilon_{\mathrm{it}}
$$

Tabelle 3.6 : Der Einfluss der Inflation auf die Einkommensquintile in Deutschland

\begin{tabular}{lccccc}
\hline & 1. Quintil & 2. Quintil & 3. Quintil & 4. Quintil & 5. Quintil \\
\hline Konstante $\boldsymbol{a}_{i}$ & 2,705 & 6,692 & 14,628 & 25,221 & 50,730 \\
& $-0,075$ & $-0,259^{* *}$ & $-0,177^{*}$ & 0,071 & $0,431^{*}$ \\
Inflation & $(-1,360)$ & $(-4,456)$ & $(-2,856)$ & $(1,001)$ & $(2,856)$ \\
Staatsausgaben $_{\mathbf{t}}$ & $0,102^{* *}$ & $0,128^{* *}$ & $0,045+$ & $-0,078^{*}$ & $-0,197^{* *}$ \\
$\frac{\text { BIP }_{t}}{\boldsymbol{R}^{2}}$ & $(4,667)$ & $(5,781)$ & $(1,834)$ & $(-2,768)$ & $(-3,276)$ \\
\hline Durbin-Watson & 0,55 & 0,71 & 0,31 & 0,26 & 0,44 \\
\hline
\end{tabular}

Anmerkungen: t-Werte in Klammern, $+(\alpha<0,1),{ }^{*}(\alpha<0,05),{ }^{* *}(\alpha<0,01)$.

Quelle: Eigene Berechnungen. Die Daten für die Staatsausgaben stammen aus Cusack (1991), die Inflationsdaten aus $S V R$ (1998) und die Daten zur Einkommensverteilung aus DIW-Wochenbericht (verschiedene Jahrgänge, vgl. auch Fußnote 159).

Die Analyse für Deutschland ist trotz der begrenzten Interpretierbarkeit angesichts des schlechten Datenmaterials aufschlussreich. Die Regressionskoeffizienten für die Staatsausgabenvariable haben weitgehend das erwartete Vorzeichen und sind mindestens auf dem 10\%-Signifikanzniveau von null verschieden. Ein Anstieg der ausgabenwirksamen Staatstätigkeit in Relation zum Bruttoinlandsprodukt wirkt eindeutig regressiv.

${ }^{184}$ Es lagen allerdings nicht zu allen Jahren Daten zur Einkommensverteilung vor, daher konnten lediglich 17 Datensätze untersucht werden. Die Tabelle sollte daher vorsichtig interpretiert werden. 
An dieser Stelle sind allerdings die Inflationskoeffizienten von besonderem Interesse. Zwar ist die Analyse übereinstimmend mit der Aussage von Blinder, der Koeffizient des untersten Quintils ist nicht statistisch signifikant. Jedoch besitzen die Koeffizienten für das zweite und dritte Einkommensquintil negative Vorzeichen und sind signifikant von null verschieden. Dies ließe sich dahingehend als Bestätigung für das Medianwählertheorem interpretieren, da der Medianwähler i.d.R. im dritten Quintil sein dürfte.

Dieses Ergebnis ist jedoch dahingehend zu relativieren, dass sich die Ergebnisse für die Inflationskoeffizienten als wenig robust erweisen, falls man zusätzliche Variable (z.B. die Arbeitslosenquote wie oben bereits kurz angesprochen) einführt. ${ }^{185}$ Auch lässt sich lediglich für das erste Quintil die Hypothese von Autokorrelation sicher ablehnen. Die Durbin-Watson-Werte für das zweite, dritte und vierte Quintil liegen im Unschärfebereich, und für das fünfte Quintil muss sogar eindeutig Autokorrelation der ersten Ordnung vermutet werden.

Auch greift diese Untersuchung gegebenenfalls zu kurz, da sie ausschließlich auf die Einkommensverteilung abhebt und die Vermögensverteilung außen vor lässt. Moore (1989) kam zu dem Ergebnis, dass Inflation als Vermögensvernichter überproportional die Position der oberen Quintile erodiert. Dann hätten die oberen Einkommensgruppen, die i.d.R. auch die oberen Vermögensgruppen sind, ein stärkeres Interesse an einer Begrenzung der Inflation.

Aus diesen Ergebnissen lässt sich also ableiten, dass eine idealtypisch linke Wirtschaftspolitik kein besonderes Augenmerk auf die Preispolitik richten wird. Besonders deutlich kommt dies in dem berühmt gewordenen Zitat von Helmut Schmidt zum Ausdruck: „Lieber 5\% Inflation als 5\% Arbeitslosigkeit“ (zit. nach Scherf, 1986, S. 34). ${ }^{186}$ Auf der anderen Seite wird eine idealtypische rechte Politik dann eine stabilere Preispolitik vertreten, wenn Inflation den natürlichen Preisfindungsprozess behindert. Eine rechte Wirtschaftspolitik, die sich als Klientelpolitik für die „Besserverdienenden“ versteht, müsste ebenfalls darum bemüht sein, die Vermögenspositionen zu erhalten und würde eine stabile Preispolitik anstreben.

${ }^{185}$ Dies könnte angesichts des Phillipskurvenzusammenhangs natürlich auch aufgrund von Multikollinearität zwischen den Variablen Arbeitslosigkeit und Inflation bewirkt worden sein.

186 Allerdings hat Thorbecke (1995) für die Volcker-Rezession in den USA 1981-1982 ermittelt, dass die kontraktive Geldpolitik dazu führte, dass sich Vermögensbesitzer dadurch besser stellten, dass die nominelle Verzinsung in Schuldverträgen nicht schnell genug angepasst wurde. Ihre reale Verzinsung stieg demnach an und folglich führte die kontraktive Geldpolitik zu einer eher ungleichmäßigen Einkommensverteilung. Dies ist natürlich im Einklang mit den Ausfuihrungen in Abschnitt 3.4.3.1.3. Jas Just - 978-3-631-75139-8 


\subsubsection{Budgetpolitik}

Es scheint naheliegend zu sein, dass man Unterschiede politischer Konzeptionen insbesondere in den öffentlichen Haushalten sucht, falls den einzelnen Positionen der Budgets tatsächlich eine ,politische Programmfunktion“ (Hansmeyer und Rürup, 1975, S. 8) zukommt. Dann ließen sich unterschiedliche Konzeptionen anhand systematisch abweichender Größe und Gewicht einzelner Budgetpositionen ablesen.

Als normatives Grundgebot gilt dabei, dass der Staat regelmäßig jene Aufgaben zu erfüllen hat, die der Markt (noch) nicht bereitzustellen in der Lage ist. Hierbei klingt bereits an, dass öffentliche Budgets bestenfalls als Indikator für die staatliche Aufgabenerfüllung gelten können, nicht jedoch damit gleichzusetzen sind. ${ }^{187}$

Seit Musgrave (1959) wird bei der Analyse staatlicher Finanzpolitik zwischen den Zielbereichen Allokation, Distribution und Stabilisierung unterschieden. Dabei versteht sich Allokationspolitik als logischer Versuch, gewünschte Güter effizient, also ohne Verschwendung volkswirtschaftlicher Ressourcen, bereitzustellen. Staatliche Intervention ist dann notwendig, falls Märkte bei der Güterversorgung vollständig versagen oder zu volkswirtschaftlich überhöhten Kosten führen. ${ }^{188}$ Gerade am Beispiel reiner öffentlicher Güter wird deutlich, dass in diesem Bereich staatlicher Aktivität auf der Ausgabenseite verhältnismäßig wenig Spielraum für ideologische Akzentsetzung besteht, da diese Güter gerade dann effizient bereitgestellt werden, wenn die Nutzungsmöglichkeit möglichst umfassend ist. ${ }^{189}$

Distributionspolitik hat insbesondere als Ziel, die marktmäßig zustandekommende Einkommens- und Vermögensverteilung zu verändern. Da diese Ziele

${ }^{187}$ Eine Vernachlässigung der nichtbudgetären Elemente der staatlichen Aufgabenerfüllung ist im Rahmen dieser Arbeit dann unproblematisch, wenn keine systematische interideologische Diskrepanz zwischen der gesamten Aufgabenerfüllung und den staatlichen Ausgaben existiert. Da diesbezüglich keine Hypothese bekannt ist, wird im folgenden davon ausgegangen, dass es derartige Unterschiede in der staatlichen Aufgabenerfüllung nicht gibt. Eine detaillierte Übersicht aller Eingriffsmöglichkeiten des Staates in die personelle Einkommensverteilung zeigt Grüske (1985, S. 419).

Übliche Gründe für Marktversagen werden in der Existenz externer Effekte, Unteilbarkeiten in der Produktion und Asymmetrien der Informationsverteilung erkannt (Fritsch, Wein und Ewers, 1993, Brümmerhoff, 1992, S. 49ff und Wolf, 1993). Als Extremfall externer Effekte werden in der Finanzwissenschaft insbesondere so genannte öffentliche Güter thematisiert, die dann vorliegen, wenn vollständige Nichtrivalität im Konsum und NichtausschlieBbarkeit vorliegen (vgl. u.a. Brümmerhoff, 1992, S. 79).

189 Allerdings sagt dies noch nichts über die Finanzierung der Ausgaben in der Allokationsabteilung aus. Hier kann es durchaus zu werturteilsbehafteten Entschegidungenkgommen. 
maßgeblich von dem zugrunde liegenden Gerechtigkeitsbegriff abhängen, sind hier abweichende Zielvorgaben dann zwangsläufig, wenn es in einer Gesellschaft heterogene Gerechtigkeitsvorstellungen gibt. Davon ist in allen Industrienationen auszugehen, wie in Abschnitt 3.2 argumentiert wurde. Linke Parteien werden jene Ausgabenbereiche stärken, die überproportional unteren Einkommensgruppen Nutzen stiften, wohingegen rechte Parteien versuchen werden, distributive Ausgaben auf ihren öffentlichen Gutsbestandteil zurückzudrängen. ${ }^{190}$

Schließlich dienen stabilisierungspolitisch motivierte Ausgaben dazu, konjunkturell bedingte Schwankungen der Wirtschaftsentwicklung zu glätten, um durch die so gewährleistete Verstetigung der Produktion Reibungsverluste und mit den Schwankungen verbundene Wohlfahrtseinbußen (Abneigung gegen Inflation und Unterbeschäftigung) zu reduzieren. Die durch den Einsatz von Stabilitätspolitik eingesparten Transaktionskosten und Wohlfahrtseinbußen zeigen an, dass es konsistenter ist, die Stabilitätspolitik als Sonderfall der Allokationspolitik zu verstehen (Brümmerhoff, 1992, S. 6 und Wagner, 1996, S. 10f).

Es gilt im folgenden zu fragen, welche Ausgabengruppen starke Verteilungswirkungen haben. Eine idealtypische linke Regierung wird dann im Rahmen ihrer Möglichkeiten bemüht sein, besonders diese Ausgabengruppen absolut und/oder in Relation zu anderen Ausgabengruppen zu stärken. Aus einer strengen Rational Choice Sicht müsste allerdings die Frage etwas anders formuliert werden: Stimmenmaximierende Politiker würden nämlich weniger bemüht sein, jene Budgetpositionen mit den ,wahren“, materiellen Verteilungswirkungen zu stärken, wenn dies in der Bevölkerung nicht gewürdigt wird. Linke Politik wäre dann eher dadurch gekennzeichnet, dass jene Ausgabengruppen gestärkt werden, die nach populärer Auffassung redistributive Wirkung besitzen. Weicht diese populäre Auffassung jedoch von der realen ökonomischen Inzidenz ab, würden stimmenmaximierende Politiker entgegen der Interessen ihrer Klientel vorgehen (Wilensky, 1975, S. 67). Bei der Hypothesenprüfung wird diese Annahme einer systematischen Unfähigkeit der Wahlbürger, die Verteilungswirkungen von Budgetpositionen abzuschätzen, nicht berücksichtigt. Zwar ist eine Uninformiertheit kurzfristig angesichts der Komplexität des Wirkungszusammenhangs durchaus im Bereich des Möglichen. Es ist jedoch davon auszugehen, dass mittel- bis langfristig dieses Informationsdefizit der Wähler durch Aufklärungsarbeit der Opposition und/oder von Interessengruppen reduziert wird. Diese zu

190 Thurow (1971) argumentierte als einer der ersten, dass Umverteilung dann als öffentliches Gut interpretierbar ist, wenn es eine Nachfrage danach gäbe. Eine marktliche Organisation wird dann regelmäßig das Gut Umverteilung in zu geringem Umfang bereitstellen, da es zum üblichen Freifahrer-Verhalten kommen wird. In diesem Fall wäre staatliche Verteilungspolitik aus allokativen Gründen geboten. Allerdings schätzen Ireland und Johnson (1970) die Nachfrage nach Umverteilung im Durchschnitt auf lediglich 1\% des Einkommens in den USA. 
erwartende Reaktion der politischen Gegner zwingt dann ex ante jede Regierungspartei, die ein langfristiges Interesse an der eigenen Glaubwürdigkeit hat, zu ex ante ,richtiger" Budgetpolitik. Zweitens wurde gerade in dieser Arbeit darauf abgehoben, dass eine ideologische Prädisposition der Politiker gerade mit dem Rational Choice Ansatz vereinbar ist und realiter politisches Verhalten prägt. Schließlich gibt es methodische Schwierigkeiten, die Kenntnis der Wirkungszusammenhänge seitens der Bürger angemessen zu operationalisieren. Daher wird sich bei der Hypothesenprüfung darauf beschränkt, zentrale Erkenntnisse der Forschung zur Ausgabeninzidenz als Ausgangspunkte zu nehmen.

Allgemein erweisen sich Analysen zur Inzidenz von staatlicher Aktivität als recht schwierig, was bereits darin zum Ausdruck kommt, dass zwischen formaler Inzidenz, also der gesetzesmäßig niedergelegten, gewünschten Inzidenz, und der materiellen, also der tatsächlichen Inzidenz unterschieden wird (Brümmerhoff, 1992, S. 439). So führt beispielsweise eine einseitige Faktorsubvention zu zahlreichen Output- und Faktorsubstitutionseffekten in den direkt betroffenen Wirtschaftsbereichen, aber auch in den indirekt verflochtenen Bereichen. ${ }^{191}$

Auch darf nicht übersehen werden, dass die materielle Budgetinzidenz einer Staatsausgabe nicht zuletzt von ihrer Finanzierung abhängt. So hängt die materielle Inzidenz des gesamten öffentlichen Haushalts natürlich auch von der Ausgestaltung des Steuersystems ab (Blankart, 1998, S. 518ff). Der Untersuchungsschwerpunkt liegt hier jedoch auf der Ausgabenseite, daher wird sich auch auf die Studien zur Ausgabeninzidenz beschränkt.

Wartenberg (1979, S. 226) kommt in seiner Analyse der Inzidenz der gesamten Ausgaben für Deutschland zu dem Ergebnis, dass der Nettoeffekt staatlicher Aktivität in absoluten Werten progressiv wirkt, dass dieser absolute Verteilungseffekt jedoch nicht sehr stark ausgeprägt ist, sodass hinsichtlich der relativen Verteilungswirkung staatlicher Aktivität eine klare Umverteilung nach unten attestiert werden kann. Dies ist im Einklang mit den Regressionsergebnissen der Tabelle 3.6, bei denen ebenfalls ein deutlich regressiver Effekt geschätzt wurde:

191 Wird beispielsweise ein Produktivitätsanstieg im Wirtschaftsbereich A nicht über höhere Lohnzahlungen weitergegeben, sondern setzen Lohnsubventionen ein, um in diesem Bereich Arbeitsplätze zu schaffen, so weitet sich natürlich die Arbeitsnachfrage in diesem Sektor aus. Dies führt aber gleichzeitig zu Faktorreallokation aus dem Wirtschaftsbereich B. Hier wird der Faktor Arbeit relativ verknappt und damit verteuert. Dies führt mittelfristig zu einer Faktorsubstitution im Bereich B hin zu stärkerem Kapitaleinsatz. Gleichzeitig ändern sich natürlich die relativen Preise auf den Gütermärkten zugunsten von A. Je nachdem wie kapitalintensiv/arbeitsintensiv die einzelnen Wirtschaftsbereiche produzieren und wie mobil die Produktionsfaktoren sind, können die tatsächlichen Mengenwirkungen stark streuen (Harberger, 1962; eine detaillierte flussdiagrammatische Darstellung zeigt Stolz, 1983, S. 35ff). 
Im Schriftum wird allgemein den beiden Ausgabengruppen Soziale Sicherung und Bildung die höchste Distributionswirkung zugeschrieben. Wie oben gesehen, tragen untere Einkommensgruppen ein signifikant höheres Risiko der Erwerbslosigkeit. Eine soziale Absicherung dieses Risikos muss also stark regressiv wirken (Cameron, 1978). Auch hinsichtlich der öffentlichen Bildungsausgaben wird üblicherweise von einer regressiven Nutzenwirkung ausgegangen. Schmidt (1980, S. 42) sieht in Bildungsausgaben einen ,zentralen Hebel“, um über die Qualifizierung zukünftiger Arbeitnehmer deren Erwerbslosigkeitsrisiko $\mathrm{zu}$ reduzieren und dadurch Ungleichheit zu verringern. ${ }^{192}$ Dies betont auch Castles (1989, S. 431) und widerspricht damit einer auf Wilensky (1975) zurückgehenden Hypothese, dass wohlfahrtsstaatliche Bildungssysteme tendenziell eher der Mittelschicht förderlich sind. Hier lohnt eine Differenzierung, die beide Hypothesen verbinden kann. Wilensky (1975, S. 4f) weist nämlich explizit darauf hin, dass es bei seinem Argument ausschließlich um höhere, universitäre Ausbildung geht. Diese Trennung der Verteilungswirkung von Ausgaben für höhere Ausbildung und Forschung von Ausgaben für Grundbildung macht auch Hanusch (1976, S. 103). ${ }^{193}$

Auch bezüglich Investitionen in Infrastruktur (Hanusch, 1976, S. 104 und Devarajan und Hossain, 1995), Ausgaben für öffentliche Verwaltung im Sinne eines öffentlichen ,employers of last resort", der bei Versagen der privatwirtschaftlichen Arbeitsnachfrage stabilisierend eingreift (Schmidt, 1980, S. 48), und beschäftigungserhaltender Subventionen (Cameron, 1978) deuten bisherige empirische Studien auf eine regressive Wirkung hin.

Dem stehen jene Ausgaben gegenüber, die eher als allokationspolitische Ausgaben bezeichnet werden können. Deren regressive Verteilungswirkung der Kosten wird üblicherweise als sehr gering erachtet. Dazu gehören Ausgaben für die innere Sicherheit, für auswärtige Angelegenheiten und nationale Verteidigung (Hanusch, 1976, S. 103) aber auch Umweltmaßnahmen (Zimmermann, 1985). ${ }^{194}$

192 In Deutschland erhöht jedes zusätzliche Bildungsjahr den Lohn um etwa 5\%; in den USA sogar um 7\% (Freeman und Schettkat, 1999, S. 99).

${ }^{193} \mathrm{Li}$, Squire und Zou (1998, S. 35) gehen davon aus, dass die rechtsschiefe Einkommensverteilung u.a. auch dadurch zu begründen ist, dass Mitglieder der oberen Einkommensgruppen mehr Ressourcen aufbringen können, die sie in rent-seeking Aktivitäten investieren können. Dem könnte man hinzufügen, dass sie nach der Olson'schen Logik der Kollektivbildung (1965) aufgrund der geringeren Zahl auch besser organisierbar sind. Der Umfang der Ausbildung der ärmeren Majorität kann diesen Ausbeutungsspielraum begrenzen, da das durch die Ausbildung gestiegene Verständnis der politischen Prozesse seitens der Mehrheit für Politiker das Risiko erhöht, durch Korrumpierbarkeit in Missgunst bei der Mehrheit zu geraten.

194 Zimmermann (1985, S. 276) unterscheidet sechs Teilsektoren, von denen vier KostengröBen und zwei Nutzengrößen umfassen. Von diesen sechs Teilsektoren wirken fünf zu

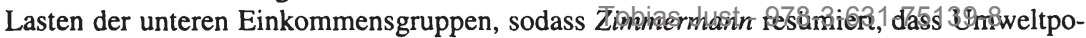




\subsubsection{Zusammenfassung}

Ziel dies Abschnitts ist es gewesen, aus dem Trennkriterium für rechts und links (Gleichheitspostulat) wirtschaftspolitische Typen zu skizzieren.

Es wurde gezeigt, dass sowohl auf der Ebene makroökonomischer AggregatgröBen als auch auf der Ebene der budgetären Instrumente sinnvoll zwischen linken (einkommensegalisierenden) und rechten (einkommensstreuenden) Zielen unterschieden werden kann. Die Ergebnisse werden in Tabelle 3.7 zusammengefasst und dienen später als Grundlage für die Hypothesenbildung innerhalb des empirischen Teils.

Tabelle 3.7 : Linke und rechte Wirtschaftspolitik im Vergleich

\begin{tabular}{lcc}
\hline & $\begin{array}{c}\text { Linke } \\
\text { Wirtschaftspolitik }\end{array}$ & $\begin{array}{c}\text { Rechte } \\
\text { Wirtschaftspolitik }\end{array}$ \\
\hline Makroökonomische Ziele & & \\
Beschäftigungsziel & ++ & \pm 0 \\
Preisstabilität & \pm 0 & + \\
\hline Budgetäre Zwischenziele & & \\
Außenpolitik & \pm 0 & + \\
Verteidigung & \pm 0 & + \\
Umwelt & \pm 0 & + \\
Innere Sicherheit & \pm 0 & + \\
Wissenschaft/Hochschule & \pm 0 & + \\
Verkehr/Infrastruktur & + & \pm 0 \\
Erziehung/Bildung & + & \pm 0 \\
Soziale Sicherung & ++ & - \\
Subventionen & ++ & - \\
Gesamt & + & \pm 0 \\
\hline
\end{tabular}

Anmerkung: ++: sehr hohe Bedeutung; +: hohe Bedeutung; \pm 0 : mittlere Bedeutung; - geringe Bedeutung

Quelle: Eigene Darstellung.

Es soll im folgenden diskutiert werden, ob diese unterschiedlichen ideologischen Ziele auch tatsächlich umgesetzt werden können, oder ob der Politik dafür grundsätzlich die Möglichkeiten fehlen. Falls Politik tatsächlich gravierende

litik im Aggregat eher zugunsten der Wohlhabenden wirkt. Dies gilt umso mehr, als dass keine oder nur geringe Maßnahmen ergriffen worden sind, diese regressive Wirkung der

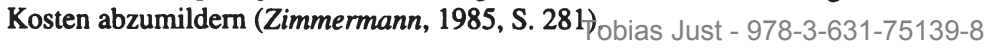


Bedeutung für wirtschaftliche Aggregate hat, dann ist zu erwarten, dass der Konjunkturverlauf die Bewegungen auf dem politischen Markt widerspiegelt. Es würde dann zu Konjunkturzyklen kommen, die durch die politischen Akteure induziert wurden.

\subsection{Politische Konjunkturzyklen}

Die Anzahl der unterschiedlichen Erklärungsansätze des Konjunkturphänomens ist gewaltig. Nach Woll (1990, S. 526) konnten bereits in den zwanziger Jahren über 200 Ursachenhypothesen unterschieden werden. Seitdem haben sich v.a. die formalen Konjunkturmodelle stark weiterentwickelt, ohne jedoch bislang befriedigenden Konsens in der ökonomischen Profession gebracht zu haben, sodass Blomberg (1997, S. 25) resigniert feststellt: ${ }^{195}$ „The truth is, we don't really know why we have business cycles." Unter Konjunkturen werden dabei üblicherweise mehrjährige Schwankungen der makroökonomischen Aggregate verstanden, die trotz jeweiliger Besonderheiten einer regelmäßigen Struktur folgen (z.B. Vosgerau, 1978, S. 428).

Seit Mitte der 70er Jahre wurde zur Erklärung dieser regelmäßigen Schwankungen neben ökonomischen Faktoren auch eine Vielzahl politischer Einflussfaktoren diskutiert. ${ }^{196}$ Nach einem kurzen Überblick über den Stand der Konjunkturtheorie werden im folgenden die Modelle politischer Konjunkturzyklen systematisiert und sowohl ihre theoretische als auch empirische Aussagekraft diskutiert.

\subsection{1 Überblick über den Stand der Konjunkturtheorie}

Die große Zahl der unterschiedlichen Konjunkturtheorien macht eine trennscharfe Systematik äußerst schwer. Ein in der Literatur häufig verwendeter Systema-

195 Im Original ist der Ausspruch in Großbuchstaben hervorgehoben.

${ }^{196}$ Bereits Kalecki (1943) hatte einen politisch initiierten Konjunkturzyklus modelliert, der auf der Wirkungskette beruhte, dass Politiker in der Lage, aber nicht immer gewillt sind, durch einen geeigneten Instrumenteneinsatz auch Vollbeschäftigung zu realisieren. Nach Kalecki reagieren die politischen Akteure auf Wunsch der Industriellen nur unzureichend, um über den Druck des Arbeitsmarktes Arbeitsdisziplin und ein geringeres Drohpotential der Gewerkschaften zu erzwingen. Darüber hinaus hat auch Åkerman (1947) ,political economic cycles" feststellen können. Åkerman untersuchte den Zusammenhang zwischen dem Industrieaktienindex und dem Stimmenanteil, den die Republikaner bei den Präsidentschaftswahlen erringen konnten, und kam zu dem Ergebnis, dass die Politik kurzfristig die Wirtschaft beeinflussen kann. Vgl. zu weiteren frühen Arbeiten die Literatur bei Paldam (1997, S. 343f). 
tisierungsansatz (Heubes, 1991; Wagner, 1996) unterscheidet die zentralen Konjunkturtheorien nach drei Kriterien: ${ }^{197}$

1. Keynesianische versus Neoklassische Modelle,

2. Annahmen bezüglich der Erwartungsbildung,

3. Exogene versus endogene Konjunkturerklärungen.

ad (1):

Theorien, die auf der keynesianischen Grundannahme der Möglichkeit stabiler Gleichgewichte bei Unterbeschäftigung aufbauen und eine Anpassung zum Gleichgewicht über eine Mengenanpassung modellieren, kommen regelmäßig zu dem Ergebnis, dass staatliche Stabilisierungspolitik eine sinnvolle wirtschaftspolitische Option ist, da Schwankungen um den allgemeinen Wachstumstrend als nachfragebestimmte Ungleichgewichte aufgefasst werden können (Wagner, 1996, S. 27). Die Modelle, die der monetaristischen Kritik folgten, gingen hingegen von der neoklassischen Grundannahme aus, dass markträumende Preise vorherrschen. Diese führen zumindest langfristig zu stabilen Vollbeschäftigungs-Gleichgewichten, woraus konsequent gefolgert werden kann, dass staatliche Tätigkeit keine langfristigen realen Wirkungen erbringt und daher regelgebunden werden sollte. Friedman und Schwartz (1963, S. 69) sahen in der Persistenz zufälliger Schwankungen der Wachstumsrate des Geldes die Ursache für die Persistenz der Konjunkturschwankungen.

ad (2):

Für die Entwicklung der Konjunkturtheorie von entscheidender Bedeutung war die Berücksichtigung dynamischer Prozesse über die Erwartungsbildung der Individuen hinsichtlich relevanter Parameter. Diese Unterscheidung ist für die makroökonomische Theorie so bedeutsam, dass sie für Heubes (1991) das Kriterium zur Kennzeichnung älterer oder neuerer Konjunkturtheorie darstellt - sowohl für die Modelle keynesianischer als auch für jene neoklassischer Provenienz. Insbesondere durch Arbeiten von Lucas (1973) und Sargent und Wallace (1975) wurde das Konzept der rationalen Erwartungsbildung dem bislang in der makroökonomsichen Theorie dominierenden Konzept der adaptiven Erwartungsbildung gegenübergestellt. ${ }^{198} \mathrm{Da}$ diese Unterscheidung auch für die Syste-

${ }^{197}$ Diese Dreiteilung wird nicht den umfangreichen Darstellungen der beiden Lehrbücher gerecht, genügt aber für diese kurze Einführung. Für andere Systematiken vgl. z.B. Teichmann (1976, S. 18ff), dessen Überblick noch nicht die neuklassische Revolution der rationalen Erwartungen berücksichtigen konnte und Woll (1990, S. 526ff).

198 Als Vater der Theorie rationaler Erwartungsbildung gilt allerdings Muth (1966) b 9 -8 
matik der politischen Konjunkturzyklenmodelle bedeutsam sein wird, lohnt es, diesen Unterschied etwas genauer zu kennzeichnen.

Bei adaptiver Erwartungsbildung korrigieren Individuen ihre Erwartungen hinsichtlich eines beliebigen ökonomischen Parameters in jeder Periode nach Maßgabe der Abweichung der letzten Erwartung vom tatsächlich eingetretenen Wert. So wird ein einmaliger Erwartungsfehler zwar stetig reduziert, jedoch niemals völlig ausgeglichen. Dies lässt sich wie folgt formal zeigen. Sei ${ }_{t-1} X_{t}^{e}$ die Erwartung hinsichtlich einer beliebigen Variable X zum Zeitpunkt t-1 für die folgende Periode $t$, dann gilt für adaptive Erwartungsbildung:

$$
\begin{gathered}
{ }_{t-1} X_{t}^{e}={ }_{t-2} X_{t-1}^{e}+\lambda \cdot\left(X_{t-1}-{ }_{t-2} X_{t-1}^{e}\right) \\
\text { mit } \\
0 \leq \lambda \leq 1
\end{gathered}
$$

Der Parameter $\lambda$ stellt dabei die Unfähigkeit der Individuen dar, aus Fehlern zu lernen, und der Klammerausdruck zeigt den Erwartungsirrtum der Individuen. ${ }^{199}$

Da bei adaptiver Erwartungsbildung ausschließlich Daten der Vergangenheit in das Kalkül einfließen, könnte man argumentieren, dass dies nicht dem ökonomischen Menschenbild entspricht, da zu jedem Zeitpunkt auch Informationen über zukünftige Entwicklungen verfügbar sind und demnach von rationalen Individuen auch genutzt werden. Die Theorie rationaler Erwartungen geht davon aus, dass Individuen alle erhältlichen Informationen über die zukünftige und vergangene Entwicklung ökonomischer Parameter berücksichtigen (Wagner, 1996, S. 32). So werden systematische Erwartungsirrtümer ausgeschlossen, d.h. der Erwartungswert des Vorhersageirrtums ist gleich null. Allerdings bedeutet dies nicht, dass es nicht doch zu Abweichungen zwischen gebildeter Erwartung und dem tatsächlich eingetretenen Wert kommen kann. Zum einen können Schocks auftreten, über die zuvor keine Informationen vorlagen, zum anderen ist es unzulässig, rationale Erwartungsbildung mit vollkommener Information gleichzusetzen, da der Informationsgrad natürlich dem üblichen Rationalitätskalkül folgt, bei dem Grenzkosten mit den Grenznutzen der Information übereinstimmen

199 Da der Wert für ${ }_{t-2} \mathrm{X}_{\mathrm{t}-1}^{\mathrm{e}}$ und weiter zurückliegende Perioden analog gebildet wurden, kann adaptive Erwartungsbildung auch wie folgt dargestellt werden:

$$
{ }_{t-1} X_{t}^{e}=\lambda \cdot\left[{ }_{t-2} X_{t-1}^{e}+\sum_{i=1}^{\infty}\left((1-\lambda)^{i}{ }_{t-2-i} X_{t-1-i}^{e}\right)\right] \text {. }
$$

Hier wird die Abhängigkeit der aktuellen Erwartung von allen vorherigen Erwartungswerten besonders deutlich. Vgl. dazu Blomberg (1997brss. 36) - 978-3-631-75139-8 
(Paldam, 1997, S. 350). Vollständige Informationen wären demnach unökonomisch.

Die Einführung rationaler Erwartungen führte zu einer Fülle neuer Konjunkturmodelle. Die von Sargent und Wallace (1975) vorgebrachte allgemeine PolitikIneffektivitäts-Hypothese wurde von Neukeynesianern ${ }^{200}$ durch Modelle zu entkräften versucht, mit denen gezeigt werden sollte, dass bei langfristigerer Stabilität der Lohnentscheidung im Vergleich zu politischen Entscheidungen auftretende Schwankungen durchaus mit Hilfe politischer Steuerung ausgeglichen werden können (Fischer, 1977). Spätere Modelle versuchten eine Mikrofundierung für Lohn- und Preisrigiditäten herzuleiten (Wagner, 1996, S. 45ff). ${ }^{201}$

Parallel musste das Lucas'sche Konzept, dass nur nicht-antizipierte Geldmengenänderungen zu realen Outputänderungen führen, aufgrund fehlender empirischer Fundierung fallen gelassen werden. Auch antizipierte Änderungen in der Geldmenge führen zu Schwankungen in realen Aggregatgrößen. Stattdessen wurde das Konzept der real business cycles populär, bei dem alle Konjunkturschwankungen durch eine serielle Abfolge von realen Schocks (v.a. Produktivitätsschocks) erklärt werden, die dazu führen, dass es zu einer intertemporalen Substitution von Arbeit und Freizeit kommt (Kydland und Prescott, 1982). Alle beobachtete Arbeitslosigkeit wäre demnach Ausdruck dieser intertemporalen Allokationsentscheidung zwischen Arbeit und Freizeit und folglich ex definitione freiwillig.

ad (3):

Schließlich lassen sich Modelle danach unterscheiden, ob sie Konjunkturschwankungen dauerhaft erklären können, ob sich die Abweichungen vom Trend also endogen aus den ökonomischen Variablen erklären lassen oder ob man die Persistenz von Wirtschaftsschwankungen nur durch fortwährende exogene Störungen erklären kann. Solche exogenen Störungen können dann die bereits angesprochenen Produktivitätsschocks sein, aber auch Präferenzschocks auf der Nachfrageseite oder Wirkungen politischer Entscheidungen (Kriege, Revolutionen, institutioneller Wandel).

200 Die Verwendung der Begriffe Neu-, Post-, und Neokeynesianer ist in der Literatur nicht einheitlich. Hier wird der Begriff Neukeynesianer in Anlehnung an die Unterscheidung bei Felderer und Homburg (1994, S. 28) für Autoren verwendet, die bemüht sind, das Konzept der rationalen Erwartungen in keynesianische Modelle zu integrieren.

201 Lohnrigiditäten werden dabei v.a. durch die Theorie impliziter Kontrakte, den insideroutsider-Ansatz und die These der Effizienzlöhne erklärt. Im Mittelpunkt der Erklärung von Preisrigiditäten stehen Modelle monopolistischer Konkurrenz sowie Transaktionskostenmodelle. 
Man kann einen politischen Konjunkturzyklus als eine Konjunkturschwankung definieren, die durch das politische System bewirkt wurde (Paldam, 1997, S. 342). Sie stellen also eine exogene Störung des ökonomischen Systems dar. Diese Definition ist allgemein genug, um darunter sowohl durch politische Entscheidungen intendierte als auch nicht beabsichtigte Schwankungen zu subsumieren. Bei letzteren werden allerdings regelmäßig solche Schwankungen, die durch Unkenntnis der Zeitverzögerungen der Wirtschaftspolitik entstehen, nicht unter dem Begriff politischer Konjunkturzyklen analysiert, sondern ausschließlich jene, die von Politikern bewusst verursacht wurden oder bewusst in Kauf genommen werden. Frey (1976, S. 96) bezeichnet diese als politische Konjunkturzyklen im engeren Sinne.

In der Literatur werden vier unterschiedliche Modelltypen politischer Konjunkturzyklen unterschieden. Zum einen wird nach den Zielen der politischen Akteure differenziert. Es wird also in Modelle mit wiederwahlorientierten Politikern, die ihre gesamte Wirtschaftspolitik unter das Diktat der Stimmenmaximierung stellen, und in Modelle mit ideologisch prädisponierten Politikern, die v.a. eine ganz bestimmte Wirtschaftspolitik umsetzen wollen, unterschieden. Zum anderen werden die Modelle gemäß der unterstellten Erwartungsbildung in solche Modelle mit adaptiven Erwartungen und in solche mit rationalen Erwartungen unterteilt. ${ }^{202}$

\section{Tabelle 3.8 : Übersicht der verschiedenen Modelle politischer Konjunkturzyklen}

\begin{tabular}{ccc}
\hline & $\begin{array}{c}\text { Adaptive } \\
\text { Erwartungsbildung }\end{array}$ & $\begin{array}{c}\text { Rationale } \\
\text { Erwartungsbildung }\end{array}$ \\
\hline $\begin{array}{c}\text { Wiederwahlorientierte } \\
\text { Politiker }\end{array}$ & $\begin{array}{l}\text { Nordhaus (1975), } \\
\text { MacRae (1977) }\end{array}$ & $\begin{array}{l}\text { Rogoff und Sibert (1988) } \\
\text { Persson und Tabellini (1990) }\end{array}$ \\
\hline $\begin{array}{c}\text { Ideologiemaximierende } \\
\text { Politiker }\end{array}$ & Hibbs (1977) & Alesina (1987) \\
\hline
\end{tabular}

Ideologie- und wieder- Frey und Lau (1968),

wahlorientierte Politiker Frey und Schneider (1978a,b)

Quelle: Erweiterte Darstellung der Abbildung bei Alesina, Roubini und Cohen (1997, S. 2).

${ }^{202}$ Blomberg (1997, S. 26) spricht statt von adaptiven von irrationalen Erwartungen und Alesina, Roubini und Cohen (1997, S. 2) bezeichnen solche Modelle als Modelle mit einer ausbeutbaren Phillips-Kurve. Dies ist zwar dahingehend genauer, da nicht immer explizit die Erwartungsbildung erörtert wurde. Im folgenden wird jedoch auch weiterhin von adaptiven Erwartungen gesprochen, da diese Erwartungsbildung zumindest implizit diesen Modellen zugrunde liegt. 
Tabelle 3.8 bietet einen Überblick über die in den folgenden Abschnitten zu diskutierenden Modelle: ${ }^{203}$ Zunächst werden jene Modelle dargestellt, denen „opportunistische", wiederwahlorientierte Politiker zugrunde liegen. Zwar wurde in Kapitel 2.3 ausführlich erörtert, dass eine solche Modellierung vollkommen opportunistischer Politiker zu kurz greift, auch wurde in Kapitel 3.3 anhand der Experteneinschätzung der wichtigsten Parteien in ausgewählten OECD-Staaten verdeutlicht, dass in den meisten Ländern keine Konvergenz der politischen Programme stattgefunden hat, dennoch ist auch eine Berücksichtigung der rein wiederwahlorientierten Ansätze sinnvoll: Zum einen wurde die Parteienverortung anhand der erklärten Ziele der Parteien vorgenommen; diese Erklärungen müssen aber keineswegs tatsächlich der realisierten Politik entsprechen. Es könnte selbst bei Divergenz der Programme auch langfristig immer dann zu einer Konvergenz der Wirtschaftspolitik kommen können, wenn es dem Wähler nicht möglich ist, die realisierte Politik angesichts starker Informationsasymmetrien zu beurteilen. Zum anderen dienen diese Ansätze als Referenzmodelle zu den später entwickelten Partisanmodellen.

\subsubsection{Wiederwahlorientierte politische Akteure}

\subsubsection{Das Nordhaus / MacRae-Modell}

Das traditionelle Modell allein wiederwahlorientierter Politiker stammt von Nordhaus (1975). ${ }^{204}$ Unabhängig von Nordhaus entwickelte MacRae (1977) ein Modell politischer Konjunkturzyklen, ging jedoch - anders als Nordhaus - davon aus, dass Politiker bemüht sind, die Gesamtstimmenverluste innerhalb einer Legislaturperiode zu minimieren. Wiederwahlorientierte Politiker bei Nordhaus hingegen versuchen ausschließlich, ihre Stimmengewinne in der Wahlperiode zu maximieren unter Berücksichtigung der wirtschaftlichen Restriktionen. Diese Modellannahme scheint dem Ansatz rein wiederwahlorientierter Politiker angemessener, daher wird ausschließlich das Nordhaus-Modell dargestellt. Die Ergebnisse bezüglich des induzierten Konjunkturverlaufs in beiden Modellen unterscheiden sich indes nicht. Das Nordhaus-Modell geht von den folgenden Annahmen aus: ${ }^{205}$

Dieses Schaubild ist sehr schematisch und kann nicht die gesamte Vielfalt politischer Konjunkturmodelle erfassen. Es fehlt der hier nicht wiedergegebene election-promisecycle, der von Paldam (1997) aus den Daten heraus gelesen wurde.

${ }^{204}$ Für eine gute Übersicht der frühen Modelle politischer Konjunkturzyklen vgl. Dinkel (1977).

${ }^{205}$ Diese Zusammenstellung basiert auf Alesina, Roubini, Cohen $\left(19973-\right.$ S $\left._{3} 20\right)$ 
A.1 Die Wirtschaft ist durch den grundsätzlichen trade-off zwischen Arbeitslosigkeit und Inflation gekennzeichnet (modifizierte PhillipsKurve). Durch die Integration von Erwartungen hinsichtlich der zukünftigen Preisentwicklung ist der Verlauf der kurzfristigen PhillipsKurve flacher als derjenige der langfristigen Kurve. Das bedeutet, es gibt zusätzlich einen intertemporalen trade-off zwischen gegenwärtiger und zukünftiger Wirtschaftslage.

A.2 Die Erwartungsbildung erfolgt dabei adaptiv.

A.3 Politiker sind in der Wahl der Instrumente unterschiedslos. Ihr einziges Ziel ist der Gewinn der Wahl. Außerdem kennen sie die Präferenzen der Wähler.

A.4 Ferner stehen nur zwei Parteien zur Wahl, eine Regierungspartei und eine Oppositionspartei.

A.5 Wähler bewerten die Regierungsleistung (v.a.) mit Hilfe der Indikatoren Inflationsrate und Arbeitslosenquote. Beide Indikatoren gehen negativ in ihre Wahlfunktion ein, wobei die Arbeitslosenquote im Quadrat, die Inflationsrate hingegen nur linear in der Wahlfunktion Eingang findet, d.h., die Regierung wird voll für die Wirtschaftslage verantwortlich gemacht. Darüber hinaus diskontieren die Wähler frühere Ereignisse sehr stark ab, sodass wahlbestimmend nur die Wirtschaftslage im Wahljahr selbst ist. Dies bedeutet auch, dass sie allein retrospektiv wählen, also anders als dies im Modell von Downs angenommen wurde. Schließlich verstehen die Wähler nur unzureichend den trade-off zwischen Inflation und Arbeitslosigkeit und sind nicht in der Lage, aus ihren Erwartungsfehlern zu lernen.

A.6 Die Politiker können zielgenau Nachfragepolitik dosieren und dadurch direkt den Umfang der Arbeitslosigkeit bestimmen. Sie können also exakt einen Punkt auf der kurzfristigen Phillips-Kurve nach ihren Wünschen wählen.

Die grundlegende Annahme, dass die Entwicklung makroökonomischer Daten als recht guter Indikator für die Wiederwahlaussichten einer Regierung dienen kann, gilt seit langem als empirisch belegte Erkenntnis und bietet die Motivation dafür, dass Politiker die Entwicklung dieser Daten manipulieren wollen. ${ }^{206} \mathrm{Be}$ reits ̊kerman (1947, S. 108) hat darauf hingewiesen, dass in Demokratien die meisten Stimmen durch die wirtschaftliche Lage determiniert werden. Zahlreiche Forscher haben diesen Zusammenhang bekräftigt. Insbesondere die Studie

${ }^{206}$ Zwar ist für jedes einzelne Individuum ausschließlich die Entwicklung der eigenen wirtschaftlichen Situation relevant, doch ist eine Konzentration auf die makroökonomischen Daten dann rational, wenn diese Informationen leichter zugänglich sind und mit der individuellen Einkommensentwicklung stark korrelieren bias Just - 978-3-631-75139-8 
von Fair (1978) zeigte, dass aus der Entwicklung der Arbeitslosenzahlen und der Inflationsrate bereits der Ausgang der amerikanischen Präsidentschaftswahlen weitgehend richtig prognostiziert werden kann. Nannestad und Paldam (1994, S. 237) resümieren, dass nach heutigem Stand der Forschung in etwa ein Drittel der Stimmenwanderung auf Änderungen der ökonomischen Konditionen zurückzuführen ist. Es ist also davon auszugehen, dass wiederwahlorientierte Politiker einen starken Anreiz haben, makroökonomische Daten wahltaktisch zu beeinflussen, sofern sie dazu in der Lage sind und nicht vom Wähler in späteren Wahlen abgestraft werden.

Aus diesen Annahmen ergibt sich der Ablauf eines politischen Konjunkturzyklus, der durch wiederwahlorientierte Politiker induziert wurde, direkt. Kurz vor den Wahlen macht die Regierung expansive Fiskalpolitik, um dadurch das Wirtschaftswachstum anzuregen und so die Arbeitslosigkeit zu senken. Eine Senkung der Arbeitslosenquote führt zu höherer Wählerzufriedenheit und steigert damit die Wiederwahlchancen der Regierung. Allerdings führt die expansive Politik zu einer Akzeleration der Inflation. Da dies nach der Erwartungsbildungsannahme jedoch nur adaptiv geschieht, fallen die Kosten der Inflation erst nach der Wahl an, und aufgrund der Annahme kurzsichtiger Wähler, die diesen Zusammenhang nicht verstehen, brauchen Regierungspolitiker keine Stimmenverluste wegen drohender zukünftiger Kosten zu fürchten. Selbst wenn die Inflation bereits vor der Wahl anzieht, muss die Regierung nicht um ihre Wiederwahl bangen, da der Outputeffekt erhalten bleibt, solange die Inflationserwartungen adaptiv gebildet werden.

Nach der Wahl wird die Preisentwicklung beschleunigt und dementsprechend passen sich schrittweise auch die Inflationserwartungen der Individuen an. Möchte sich die Regierung auch für die folgenden Wahlen die Möglichkeit expansiver Politik offen halten, wird sie versuchen, durch kontraktive Politik die Inflationsraten und damit die Inflationserwartungen zu senken. Dies geht aber einher mit sinkenden Wachstums- und steigenden Arbeitslosenraten. Die so generierte Rezession nach der Wahl wird annahmegemäß bei vergesslichen Wählern den nächsten Wahlausgang dann nicht mehr beeinträchtigen, wenn der Kontraktionsprozess rechtzeitig vor der nächsten Wahl abgeschlossen wurde.

Regierungswechsel sind in diesem Modell also nur dann möglich, wenn es exogene Schocks oder zusätzliche, hier nicht berücksichtigte Entscheidungsparameter gibt, welche die Bemühungen der Regierungspartei überkompensieren, oder falls es Kosten des Regierens gibt. Diese Kosten des Regierens beschreiben eher ein empirisches als ein theoretisches Gesetz, das besagt, dass im Durch- 
schnitt eine Regierung pro Legislaturperiode mit einem Stimmenverlust von 1,7\%-Punkten rechnen muss (Paldam, 1997, S. 346). ${ }^{207}$

An dem Modell von Nordhaus wurde reichhaltig Kritik geübt. Zum einen wurde die Erwartungsbildung kritisiert, die in der Unmöglichkeit der Wähler, zukunftsorientierte Informationen zu berücksichtigen und damit letztlich auch im verwandten Phillips-Kurven-Modell, ihren Ausdruck findet. Zum anderen wurde beanstandet, dass der allein wiederwahlorientierte Politiker kein angemessenes Modell für die Untersuchung politischer Prozesse sei. Der bedeutsamste Einwand gegen das Modell ist aber der Mangel an empirischer Validität. Die Mehrzahl der empirischen Untersuchungen konnte keine politischen Konjunkturzyklen im Sinne Nordhaus ' finden. Die empirischen Ergebnisse werden im Detail in 3.5.5 präsentiert. Es ist also nötig, die beiden zentralen Kritikpunkte ernst zu nehmen und das Modell weiter zu entwickeln. Zunächst soll eine Gruppe von Modellen vorgestellt werden, in denen rationale Erwartungsbildung modelliert wird, die ansonsten aber im wesentlichen den Annahmerahmen des NordhausModells unangetastet lassen. ${ }^{208}$

\subsubsection{Rationale Erwartungen und wiederwahlorientierte Politiker}

Es dauerte über ein Jahrzehnt, bis gezeigt werden konnte, dass auch durch eine Zulassung rationaler Erwartungsbildung zumindest die theoretische Möglichkeit politischer Konjunkturzyklen, die durch wiederwahlorientierte Politiker induziert werden, Bestand hat.

Cukierman und Meltzer (1986) und Rogoff und Sibert (1988) schlugen unabhängig voneinander Signaling-Modelle vor, bei denen die amtierende Regierung mit Hilfe der Höhe der Transferleistungen respektive der Struktur der Staatseinnahmen den Wählern ihre Kompetenz signalisieren kann. ${ }^{209}$ Kompetente Regierungen sind in der Lage, einen gewünschten Umfang öffentlicher Güter zu geringe-

${ }^{207}$ Der Versuch, dieser Beobachtung ein theoretisches Konzept zu unterlegen, hat v.a. zwei Elemente. Zum einen mag es in einer Gesellschaft eine Nachfrage nach politischem Wechsel geben, z.B. um die langfristige Stabilität einer Demokratie zu sichern, die ja gerade davon lebt, dass der Wechsel gewaltlos möglich ist. Zum anderen wird argumentiert, dass der Opposition inkonsistente Versprechen leichter fallen als einer Regierungspartei (Paldam, 1997, S. 346).

${ }^{208}$ Nordhaus selbst hat diesen Mangel seines Modells wohl bereits erkannt, verweist er doch in Fußnote 2 auf Seite 182 darauf, dass auch zukunftsgerichtete Informationen in die Erwartungsbildung einfließen können.

${ }^{209}$ Rogoff und Sibert (1988) gehen davon aus, dass der Regierung zwei Finanzierungskanäle offen stehen; zum einen können sie eine allokationsneutrale (lump-sum) Steuer verwenden oder sie finanzieren ihre Ausgaben durch verzerrende Seigniorage, also mit Hilfe einer Inflationssteuer. 
ren Kosten zu produzieren als inkompetente Regierungen. Rogoff (1990) ergänzt dieses Modell durch die besondere Berücksichtigung staatlichen Investitionsverhaltens. Kurz vor der Wahl benutzt die Regierung die Höhe des Investitionsvolumens als Signal für ihre Regierungskompetenz.

Persson und Tabellini (1990, Kapitel 5) zeigten, dass man diese Modellstruktur auch auf den Phillips-Kurven-Zusammenhang anwenden kann. Der Kompetenzgrad einer Regierung drückt sich bei ihnen darin aus, dass eine kompetente Regierung in der Lage ist, bei gegebenen Inflationserwartungen und gegebener Inflation in größerem Umfang Arbeitslosigkeit kurzfristig abzubauen. ${ }^{210}$ Da dieses Modell leichter dem Modell von Nordhaus gegenübergestellt werden kann, wird sich hier auf die Darstellung dieses Modells beschränkt. Im Detail lässt sich das Modell von Persson und Tabellini durch die folgenden Annahmen beschreiben::211

A.1* Die Wirtschaft ist gekennzeichnet durch die Gültigkeit der PhillipsKurve, die um einen Kompetenzterm erweitert wurde. ${ }^{212}$ Dieser Term zeigt die Fähigkeit der Regierung an, exogene Schocks aufzufangen oder Arbeitsmarktbedingungen effizient auszugestalten. Er ist als gleitender Durchschnitt der Kompetenzeinschätzungen der aktuellen und der letzten Periode definiert, d.h., der exakte Kompetenzwert einer Regierung ist für die Wähler nur mit einem 1-Perioden-Lag ermittelbar.

A.2* Die Inflationserwartungen werden rational gebildet.

A.3* Wie im Nordhaus-Modell sind die Politiker in ihren Präferenzen homogen und hauptsächlich wiederwahlorientiert. Allerdings geht in ihre Nutzenfunktion auch die Wirkung ihrer Politik auf das Gemeinwohl der Wähler ein. Diese Integration sozialer Verantwortung in die Nutzenfunktion des Politikers stellt für diesen Opportunitätskosten wahltaktischer Intervention dar. Eine Manipulation ist dann also für die inkompetentere Partei teurer zu bewerkstelligen. Diese Ergänzung ist für das Zustandekommen des Gleichgewichts von zentraler Bedeutung (Rogoff und Sibert, 1988, S. 5). Die Parteien unterscheiden sich also lediglich in ihrer Kompetenz.

${ }^{210}$ Modellhaft geschieht das so, dass in den Term der Phillips-Kurve ein Kompetenzparameter additiv hinzugefügt wird.

211 Die Annahmen, die mit einem Stern gekennzeichnet sind, weichen von denen des Nordhaus-Modells ab.

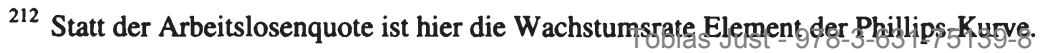


A.4 Es gibt nur zwei Kandidaten, die Regierungspartei und die Oppositionspartei. $^{213}$

A.5* Wähler entscheiden sich für den Kandidaten, der den Erwartungswert ihres Nutzens maximiert. Dabei geht auch hier die Wachstumsrate positiv im Quadrat und die Inflationsrate negativ linear in die Nutzenfunktion ein. Die Präferenzen der Wähler sind homogen. Die Wähler leiten aus der wirtschaftlichen Istlage die Kompetenz der Politiker ab, wobei sie sofort die Daten für das Wirtschaftswachstum erhalten können. Die Informationen bezüglich der Inflationsraten stehen ihnen aber nur eine Periode später zur Verfügung.

A.6* Die Inflation kann direkt durch die Politiker kontrolliert und manipuliert werden.

Auch in diesem Modell haben wiederwahlorientierte Politiker einen Anreiz, die makroökonomischen Daten wahlentscheidend zu beeinflussen. Da die Wähler bemüht sind, den kompetentesten Politiker zu wählen, sie jedoch die Kompetenz nicht direkt, sondern nur indirekt aus der wirtschaftspolitischen performance ableiten können, sind alle Politiker bestrebt, durch opportunistische Manipulationen so kompetent wie möglich zu erscheinen. ${ }^{214}$ Allerdings sind sie unterschiedlich erfolgreich.

Persson und Tabellini zeigen, dass sowohl ein separating als auch ein pooling equilibrium möglich sind. ${ }^{215}$ Welches Gleichgewicht entsteht, hängt von der Kompetenzeinschätzung der Wähler ab. Das separating equilibrium ist der interessantere Fall, da hier die Akteure rational ihre Kompetenz offenbaren.

Da ein Politiker mit einem hohen Kompetenzniveau dies dem Wähler auch zeigen möchte, wird er Wirtschaftswachstum induzieren, das für einen weniger kompetenten Gegner unerreichbar wäre. Er steigert also die Inflationsrate der Folgeperiode über das Maß der erwarteten Höhe. Diesen Wert kann der inkompetente Politiker nie erzielen. Dies liegt daran, dass in Annahme A.3* Gemeinwohlorientierung berücksichtigt wurde. Diese restringiert jeden Politiker in seinen Manipulationsanstrengungen. Gäbe es diese Restriktion nicht, würde es zu

${ }^{213}$ Rogoff und Sibert (1988) modellieren allerdings ein Kontinuum von Alternativen.

${ }^{214}$ Hieran wird die Bedeutung der Annahmen, dass einerseits Kompetenz nicht direkt beobachtbar ist und nur die Wachstums-, nicht aber die Inflationsrate sofort verfügbar ist, deutlich. Wären sowohl Wirtschaftswachstum als auch die Inflationsrate für jede Periode erkennbar, gäbe es keinen Signalspielraum für die Politiker und damit auch keinen politischen Konjunkturzyklus (Persson und Tabellini, 1990, S. 82).

${ }^{215}$ Man spricht bei Spielen, in denen Akteure bei asymmetrischer Informationsverteilung Signale setzen können von einem separating equilibrium, wenn beide Spieler unterschiedliche Strategien verfolgen. Pooling equilibria sind dadurch gekennzeichnet, dass die Spieler dieselbe Strategie wählen (Fudenberg und Tirole big9 JuSt 33778-3-631-75139-8 
einer infiniten Manipulationsspirale kommen. Da die Wähler ex ante unsicher bezüglich des exakten Kompetenzniveaus der Regierung sind, bilden sie ihre Erwartungen als einen Mittelwert aus beiden, sodass im Falle des kompetenten Regierenden die Inflationserwartungen hinter der tatsächlichen Inflation zurück bleiben, wohingegen im Falle eines inkompetenten Regierenden die Erwartungen die tatsächliche Inflation übersteigen. Je nach Kompetenzgrad kommt es also vor einer Wahl zu einem Aufschwung oder Abschwung. ${ }^{216}$ Es kommt allerdings nicht zu einem gegengerichteten Schwung nach der Wahl.

Im Falle des pooling equilibrium generieren beide Politikertypen vor der Wahl dieselbe Beschäftigungswirkung. Da der inkompetentere Politikertyp dies jedoch nur durch stärkere Expansionspolitik erreichen kann, kommt es bei ihm nach der Wahl zu stärkerer Preissteigerung, wohingegen im Falle einer kompetenteren Regierung nach der Wahl die Inflationsraten sinken werden. Da diese Preiseffekte jedoch für den Wähler vor der Wahl nicht erkennbar sind, er seine Kompetenzeinschätzung also nur anhand des Beschäftigungsziels treffen kann, stehen die Wahlchancen für beide Politikertypen bei $50 \%$.

Insgesamt sind die Schwankungen sowohl im Fall des separating sowie im pooling equilibrium aufgrund der Rationalitätsannahme kurzlebiger und weniger stark ausgeprägt und v.a., da die Art des Gleichgewichts durch die Kompetenzeinschätzung des Wählers bestimmt wird, auch äußerst schwer zu prognostizieren (Berthold und Fehn, 1994, S. 171).

Die Kritik an diesen Modellen setzt an vier Punkten an: Erstens wird kritisiert, das Ergebnis, dass besonders kompetente Politiker auch besonders stark in die Wirtschaft eingreifen, stelle ein ,unpleasant feature“ dar (Alesina, Roubini und Cohen, 1997, S. 32). Dies ist allerdings angesichts der Annahme der Wiederwahlorientierung nicht besonders verwunderlich. Es scheint vielmehr der folgerichtige Ausdruck der Opportunismusannahme zu sein, dass der kompetentere Politiker diese Kompetenz zur Verfolgung der eigenen Ziele einsetzt. Außerdem gilt diese Aussage nur kurz vor der Wahl. In Nicht-Wahlperioden hat der kompetente Politiker keinen Anreiz, das wirtschaftspolitische Instrumentarium zu missbrauchen. Damit ist bereits der zweite viel gravierendere Einwand angedeutet: Es scheint realitätsfern, dass sich Politiker in bezug auf ihre Werte- und Ideologiebindung in keiner Weise unterscheiden.

Außerdem mag man es in einer Welt rationaler Erwartungen für ungerechtfertigt erachten, davon auszugehen, dass die Wähler zwar sofort Wachstumsdaten besitzen können, nicht jedoch die Inflationsentwicklung überblicken. Schließlich erklären diese Modelle keine echten Zyklen, sondern nur unsystematische Schocks um den Wahltermin. Diese Schocks sind deshalb unsystematisch, da sie

${ }^{216}$ Dies ist ein weiterer Unterschied zum Rogoff und Sibert-Modell. Hier ist die Wirkungsrichtung immer gleich in Richtung eines Aufschwypgs Just - 978-3-631-75139-8 
von dem nicht ex ante ermittelbaren Kompetenzniveau der Regierung abhängen. Da die zentrale Größe der Kompetenz nur äußerst schwer operationalisierbar ist, entziehen sich diese Modelle auch weitgehend einer empirischen Prüfung.

Im folgenden sollen nun den rein wiederwahlorientierten Ansätzen die ideologiebestimmten Modelle gegenübergestellt werden. Zunächst wird dabei wieder die wegbereitende Arbeit, die auf adaptiver Erwartungsbildung basierte, dargestellt, um im Anschluss daran ein Modell mit rationaler Erwartungsbildung zu beschreiben.

\subsubsection{Ideologieorientierte politische Akteure}

Die zentrale Kritik an den rein wiederwahlorientierten Ansätzen war die Annahme, dass Politiker unterschiedslos dieselbe Politik verfolgen. Geht man jedoch zum einen davon aus, dass es innerhalb der Wahlbevölkerung unterschiedliche Gruppen und Interessen gibt, so ist es plausibler, dass Parteien als die politischen Interessenvertreter einzelner Gruppen deren Interessen vertreten. Parteien bieten also eine konkrete Ideologie an, entweder weil sie damit bestimmte Wählergruppen ansprechen möchten oder weil sie aufgrund ihrer internalisierten Wertestruktur dies möchten.

Die Partisantheorie wurde nahezu parallel zu der Theorie der wiederwahlorientierten politischen Konjunkturzyklen entwickelt. Ende der 70er Jahre legte Hibbs (1977) den Grundstein mit einer rein empirischen Arbeit. Obwohl bereits Hibbs zeigen konnte, dass die empirische Relevanz von Partisanmodellen wesentlich größer ist als diejenige der rein wiederwahlorientierten Modelle des Nordhaus/MacRae-Zuschnitts, wurden Partisanmodelle in den 80er Jahren in der Ökonomie nahezu vollständig ausgeblendet. ${ }^{217}$ Eine Renaissance erlebte die Partisantheorie Ende der 80er Jahre, als es Alesina (1987) gelang, das Konzept der rationalen Erwartungsbildung in die Theorie zu integrieren. Seitdem ist sowohl die theoretische als auch die empirische Literatur zu Partisanmodellen stark angewachsen.

\subsubsection{Die Partisantheorie von Hibbs}

Das Interesse von Hibbs (1977) war weniger die Konstruktion eines theoretischen Modells, als vielmehr die empirische Untersuchung von makroökonomi-

${ }^{217}$ Ein Faktum, das Paldam (1997, S. 357) augenzwinkernd, doch deswegen nicht zwingend wahrheitsuntreu, wie folgt kommentiert: „Economists are more easily convinced by theory than by empirical observation. Furthermore, economists probably have a bias for liking theories that argue that politics do not matter. "

Tobias Just - 978-3-631-75139-8 
schen Politikergebnissen in Demokratien mit wechselnden Regierungen. Zwar fehlt die Einbindung in ein theoretisches Modell, jedoch ist dies leicht aus seiner empirischen Studie nachzuzeichnen: ${ }^{218}$

A.1 Die Wirtschaft ist durch den Phillips-Kurven trade-off gekennzeichnet.

A.2 Inflationserwartungen werden adaptiv gebildet. Dabei ist der Anpassungsprozess scheinbar sehr langsam, nahezu statisch. In dem Schätzmodell für die USA wird die Konvergenz zum Gleichgewicht erst in 25 Jahren erreicht (Hibbs, 1977, S. 1485). In dem Modell für Großbritannien dauert der Anpassungsprozeß immerhin 4 Jahre.

A.3** Parteien sind unterschiedlich. Wie in 3.4 diskutiert, sind Politiker linker Parteien eher dazu bereit, für einen hohen Beschäftigungsstand eine höhere Inflationsrate in Kauf zu nehmen als Politiker rechter Parteien. Nach Hibbs lässt sich die Ursache für die unterschiedliche Präferenz der Parteien in der Verteilungswirkung von Beschäftigungs- und Preispolitik hinsichtlich ihrer Wählerklientel finden (vgl. die Ausführungen in 3.4.3).

A.4 Es gibt nur zwei Kandidaten, eine Regierungs- und eine Oppositionspartei.

A.5** Analog zu den Ausführungen in A.3** haben auch Wähler unterschiedliche Präferenzen hinsichtlich Arbeitslosigkeit und Inflation. Es lassen sich zwei Gruppen differenzieren, welche die Wählerschaft der linken respektive der rechten Partei konstituieren.

A.6 Die Regierung ist durch den Einsatz geeigneter Instrumente in der Lage, eine beliebige Position auf der Phillips-Kurve mit vollständiger Zielgenauigkeit zu realisieren.

Aus diesem Modell ergibt sich der politische Konjunkturzyklus nach Hibbs sehr direkt. Gewinnt eine linke Partei die Wahl, betreibt sie expansive Politik und reduziert so einerseits die Arbeitslosigkeit, erhöht jedoch andererseits die Inflationsrate. Kommt eine rechte Partei hingegen an die Regierung, verringert sie den Preisauftrieb und induziert durch die kontraktive Politik Arbeitslosigkeit. Da in dem Modell der Anpassungsproze $B$ der Erwartungen sehr langsam verläuft, kommt es zu keiner Verlagerung der kurzfristigen Phillips-Kurve, sodass sowohl die Beschäftigungsveränderung als auch die Inflationswirkung dauerhaft sind. Geht man davon aus, dass es keine umfangreichen Wählerwanderungen gibt die innerhalb des Modells nur dadurch zustande kommen können, dass Wähler aus der unteren in die obere Einkommensklasse aufsteigen - so kann ein Regie-

${ }^{218}$ Vgl. für die Liste der Annahmen Alesina, Roubini und Cohen (1997, S. 47) sowie für eine mathematische Formulierung des zugrunde liegenden Modells Blomberg (1997, S. $41 \mathrm{ff}$ ). Der Doppelstern kennzeichnet Abweichungen zum|Nordhays-M9dello31-75139-8 
rungswechsel und damit erst ein Konjunkturzyklus entstehen, wenn zusätzlich außerökonomische issues Relevanz besitzen und die bereits angesprochenen Kosten des Regierens bestehen.

Die Kritik an dem Modell von Hibbs lässt sich in zwei Gruppen unterteilen, Kritik an dem zugrunde liegenden theoretischen Modell und Kritik an der gewählten Untersuchungsmethode. Der Schwerpunkt während der ersten Jahre nach Erscheinen des Artikels lag eindeutig auf letzterem (Payne, 1979 und Beck, 1982) und konnte entweder von Hibbs (1979) sofort entkräftet werden oder verstand sich sowieso nur als ergänzender Beitrag zu dessen Ausführungen. Gravierender waren die theoretischen Kritikpunkte, die erst Ende der achtziger Jahre aufkamen.

Von zahlreichen Autoren wurde bemängelt, dass die Erwartungsbildung, auf der das Modell basiert, dahingehend irrational ist, dass zukunftsgerichtete Informationen systematisch unterschlagen zu werden scheinen. ${ }^{219}$ Darüber hinaus wurde Hibbs vorgeworfen, dass in seinem Modell die beobachtbaren Politikergebnisse (Arbeitslosigkeit und Inflation) durch die Regierung in dieser Periode ohne Zeitverzögerung und punktgenau dosiert erzielt werden können (Chappell und Keech, 1986, S. 71).

Der Kritikpunkt, den darüber hinaus Stalder (1995, S. 201) anführt, dass Hibbs Ideologie ausschließlich instrumental zur Befriedigung von Umverteilungspräferenzen der Parteiklientel auffasst, erscheint indes ungerechtfertigt. Wie oben bereits ausgeführt, ist es durchaus sinnvoll, politischen Ideologien auch Zielcharakter für Politiker zuzuschreiben. Da dies jedoch mühelos und v.a. hinsichtlich seiner Schlussfolgerungen wirkungslos in die Annahmen von Hibbs integriert werden könnte, stellt sich dieser Defekt als eher untergeordnet dar. Alesina (1987, S. 651) verweist explizit darauf, dass politische Ideologie beispielsweise als Ausdruck spezieller Klientelpolitik interpretiert werden kann.

\subsubsection{Die rationale Partisantheorie von Alesina}

Zwar war Alesina (1987) nicht der erste, der versuchte, rationale Erwartungen in die Partisantheorie zu integrieren (vgl. Fußnote 219), jedoch derjenige mit der nachhaltigsten Wirkung sowohl auf die theoretische Weiterentwicklung als auch auf die empirische Überprüfung.

Sein Modell basiert im wesentlichen auf den Konjunkturtheorien der Neukeynesianischen Schule nach Fischer (1977) und lässt sich durch die folgenden An-

219 Die ersten, die dies beanstandeten und das Modell von Hibbs auch um rationale Erwartungen ergänzen konnten, waren Minford und Peel (1982), Lächler (1984), Kirchgässner (1986), Chappel und Keech (1986, S. 72), und Alesing (1987 St S952) 
nahmen beschreiben, die eine Zusammenführung der Annahmenrahmen des Persson und Tabellini-Modells und des Hibbs-Modells darstellen: ${ }^{220}$

A.1 Die Wirtschaft ist durch den üblichen Phillips-Kurven trade-off beschreibbar, d.h., das Wirtschaftswachstum hängt von der mit der natürlichen Arbeitslosenquote $\mathrm{zu}$ vereinbarenden Wachstumsrate und der Abweichung der Inflationsrate vom Anstieg der Nominallöhne ab. Dabei richtet sich die Wachstumsrate der Nominallöhne nach der Inflationserwartung der Individuen (Alesina, 1987, S. 654).

A.2* Die Inflationserwartungen werden rational gebildet.

A.3** Parteien unterscheiden sich in ihren präferierten Politikergebnissen und demnach auch in ihren präferierten Politikinhalten. Linke Parteien sind stärker bemüht, Arbeitslosigkeit zu beseitigen und sind dafür stärker geneigt, Inflation zu akzeptieren. ${ }^{221}$ Auf jeden Fall bedeuten Inflation und Arbeitslosigkeit für beide Parteien ein Übel, lediglich in relativ verschiedener Gewichtung.

A.4 In jeder Wahl kann nur zwischen zwei Parteien gewählt werden.

A.5*** Wähler unterscheiden sich in ihren Präferenzen hinsichtlich Arbeitslosigkeit und Inflation und kennen die Präferenzen der Parteien. Anhand dieser Kenntnis geben sie ihre Stimme derjenigen Partei, die ihren Erwartungsnutzen maximiert. Wie sich die Präferenzen auf die Wahlbevölkerung verteilen, ist in jeder Wahl unsicher. In dem Modell wird angenommen, dass zuerst die Lohnabschlüsse verhandelt werden und anschließend Wahlen stattfinden. Diese Annahme ist analog zu dem Ansatz von Fischer (1977) von entscheidender Bedeutung, da auf diese Weise kurzfristig Lohnrigiditäten möglich werden. ${ }^{222}$

A.6* Politiker können Inflation direkt beeinflussen.

Ähnlich wie in dem Modell von Persson und Tabellini werden politische Konjunkturzyklen vornehmlich dadurch induziert, dass Unsicherheit hinsichtlich des

${ }^{220}$ Der dreifache Stern zeigt an, wo das Alesina-Modell von allen zuvor besprochenen Modellen abweicht.

221

Dies ist entweder so modellierbar, dass es unterschiedliche Zielwerte für Inflation und Wirtschaftswachstum gibt (Alesina, 1987, S. 655) oder dass identische Zielwerte mit unterschiedlichem Gewicht in die jeweilige Zielfunktion der Parteien eingehen (Alesina und Sachs, 1988, S. 66).

222 An anderer Stelle (Alesina und Rosenthal, 1995, S. 186) wird deutlich gemacht, dass dies realistischerweise heißen muss, dass zumindest ein Teil der Tarifverhandlungen den Wahltermin überlappen. Eine Verallgemeinerung mit zeitlich unbestimmten Tarifabschlüssen ändert nichts an dem Ergebnis, nur an der Größe der induzierten Konjunkturschwankung. 
Wahlausgangs besteht und vor der Wahl bindende Lohnabschlüsse ausgehandelt werden. Alesina und Sachs (1988, S. 64) machen deutlich, dass in ihrer Modellwelt im Sinne von Sargent und Wallace (1975) jenseits von unerwarteten Schocks vollständige Politikneutralität besteht.

Da die Wähler einerseits wissen, dass die Parteien unterschiedliche Inflationsziele haben (bzw. Inflation und Arbeitslosigkeit in ihren Zielfunktionen unterschiedlich gewichten), andererseits jedoch ex ante Unsicherheit über das Wahlergebnis herrscht, werden sie ihre Inflationserwartungen, die innerhalb der Lohnverhandlungen berücksichtigt werden, aus einem gewichteten Mittel aus den Präferenzen der zur Wahl stehenden Parteien bilden. ${ }^{223}$ Das Gewicht für diese Erwartungswertermittlung wird dabei durch die Wahrscheinlichkeit des Sieges der jeweiligen Partei determiniert, d.h. vor der Wahl liegt die Inflationserwartung der Wähler zwischen dem Optimalwert der linken Partei und dem Optimalwert der rechten Partei.

Nach der Wahl bestimmt die gewählte Partei sofort ihre gewünschte Inflationsrate und induziert so eine Änderung der wirtschaftlichen Entwicklung. Gewinnt nämlich die rechte Partei, liegt deren präferierte Inflationsrate unterhalb der Inflationserwartungen der Wähler. Gemäß der unterstellten Phillips-KurvenRelation wird durch diese Abweichung nach unten eine Rezession induziert, die Arbeitslosigkeit steigt über das $\mathrm{Ma} ß$ der natürlichen Arbeitslosenquote an. Gleichzeitig sinkt die Inflationsrate. In der Folgeperiode passen sich die Inflationserwartungen und damit die Nominallöhne nach unten an und die Wachstumsrate konvergiert wieder zu der natürlichen Rate.

Gewinnt jedoch die linke Partei, liegt die Inflationsrate nach der Wahl oberhalb der erwarteten Inflationsrate und führt dadurch zu einer Expansion der Wirtschaft. Am Ende der ersten Periode können die Wähler allerdings ihre Nominallöhne an die neue Inflationserwartung anpassen und senken dadurch das Wirtschaftswachstum in der zweiten Periode nach der Wahl auf die natürliche Rate. Die Inflationsrate hingegen bleibt während einer linken Regierung dauerhaft höher.

Der Konjunkturzyklus der rationalen Partisantheorie ist folglich dadurch gekennzeichnet, dass Wachstumsraten linker und rechter Parteien lediglich zu Beginn ihrer Legislaturperiode voneinander abweichen können und dass die Inflationsrate linker Regierungen permanent höher ist als diejenige rechter Regierungen. Die Amplitude der Schwankung wird dabei zum einen durch die Größe des Überraschungsmoments des Wahlerfolgs bestimmt und zum anderen durch die Ideologiedifferenz der beiden Parteien. Die Länge der Schwankung richtet sich hingegen nach der Flexibilität der Nominallohnanpassung. Werden beispiels-

${ }^{223}$ Wichtig ist also nicht, dass Unsicherheit über den Wahlausgang am Wahltag herrscht, sondern am Tag der Tarifabschlüsse (Alesina und Rosenthal 1 1995 S $_{3}$ - 1896).75139-8 
weise zweijährige Lohnverträge abgeschlossen, dauert die vorübergehende $\mathrm{Ab}$ weichung zwei Perioden.

Die Kritikpunkte an der rationalen Partisantheorie lassen sich im wesentlichen zwei Gruppen zuordnen. Die erste Gruppe der Kritik könnte als Annahmenkritik beschrieben werden. Die Einwände, die dieser Gruppe angehören, sind entweder mittlerweile durch verfeinerte Modellierung obsolet geworden oder leicht auszuräumen. Neumann (1989, S. 91) schreibt beispielsweise, dass es nicht unbedingt ein Zeichen von Rationalität ist, wenn der Wähler seine Stimme einer Partei gibt, die nur aufgrund einer Wahlüberraschung reale Effekte auslösen kann. Dem wurde in späteren Modellen dadurch Rechnung getragen, dass die strenge Rationalitätsannahme aufgegeben wurde. Stattdessen wird davon ausgegangen, dass Informationen effizient genutzt werden (Alesina, 1991). ${ }^{224}$ Auf ähnliche Annahmenkritik hinsichtlich der direkten Steuerbarkeit der Inflationsrate und der vermeintlichen Irrationalität zu früher und zu inflexibler Tarifverträge gehen Alesina und Rosenthal (1995, S. 186) ausführlich ein. In der Realität sind Tarifverträge nicht zentral und einfach zu verhandeln, sondern sie überlappen und die Verhandlung nimmt Zeit in Anspruch, eine punktgenaue Vertragsunterzeichnung zum Tag nach der Wahl ist organisatorisch unmöglich. Eine gewisse Anzahl überlappender Tarifverträge liegt also stets vor. ${ }^{225}$ Inwiefern allerdings direkt Inflationspolitik betrieben werden kann, ist ausgesprochen fraglich und sicherlich nicht unabhängig von den institutionellen Ausgestaltungen der nationalen Zentralbanken.

Wesentlich gravierender als diese Punkte sind die Vorwürfe, dass die Theoriebildung zu empirischen Implikationen führt, die im Widerspruch zu den stilisierten Fakten des Konjunkturverlaufs stehen. So scheint ein Modell, das auf der Gültigkeit der natürlichen Arbeitslosenquote aufbaut angesichts weitgehender Persistenz der Arbeitslosigkeit jenseits dieser Rate in vielen Industriestaaten unangemessen. Berücksichtigt man die Möglichkeit persistenter Arbeitslosigkeit, so sind längerfristige Partisaneffekte durchaus vorstellbar, da dann unerwartete Inflation auch in zukünftigen Perioden die Arbeitslosigkeit vermindert. Dann entsteht aber ein empirisches Identifikationsproblem, da die rationale Partisantheorie mit Hysteresiseffekten dieselbe Wirkung prognostiziert wie die traditionelle Partisantheorie (Alogoskoufis, Lockwood und Philippopoulos, 1992, Fehn, 1993, Belke, 1996, 2000 und Berlemann, 1999).

224 Allerdings entkräftet dies nicht wirklich das Argument von Neumann. Es lieBe sich dem Neumann-Argument indes entgegenhalten, dass für den Akt der Stimmabgabe sowieso andere Gründe vorliegen als reine instrumentelle Nutzen gemäß dem Parteidifferential (vgl. die Diskussion zum Wahlparadoxon in Abschnitt 2.3.2.2).

${ }^{225}$ Die Tatsache nicht inflationsindexierter Verträge muss aber auch hier verwundern (Mankiw, 1999). 
Darüber hinaus ist dem Modell von Alesina die Folgerung inhärent, dass sich die Realeinkommen antizyklisch verhalten. ${ }^{226}$ Dies ist aber nicht im Einklang mit der Datenlage (Hibbs, 1992, S. 366 und Snowdon und Vane, 1997, S. 224). Schließlich gilt auch hier wie für alle Modelle, die auf der Politikineffektivitätshypothese aufbauen, dass die Mehrzahl der empirischen Studien zu dem Ergebnis kommt, dass nicht nur unerwartete monetäre Schocks reale OutputWirkungen zeitigen, sondern auch erwartete (Sheffrin, 1989, S. 257 und Gärtner, 1994, S. 106).

Was den bisherigen Modellen übereinstimmend zugrunde lag, war die Annahme, dass Politikerpräferenzen unveränderlich sind. Entweder sie sind wiederwahlorientiert und verfolgen alle dieselben Ziele, wenn sie im Amt sind, oder sie sind ideologieorientiert und haben typische Präferenzen hinsichtlich bestimmter Makrovariablen. Es ist interessant, dass gerade jene Theorien, die versuchen, diese Beschränktheit zu überwinden, vergleichsweise geringe Beachtung fanden. Für das Modell von Hibbs (1994), in dem er lernfähige Politiker modelliert, die unter Unsicherheit bereit sind, ihre Zielfunktion anzupassen, ist dies noch dadurch zu begründen, dass der Ansatz noch zu „neu“ ist. Dies kann allerdings nicht für den Ansatz von Frey und Schneider (1978a, 1978b, 1979) gelten, der bereits vor über 20 Jahren zeigen konnte, dass Wiederwahlorientierung und Ideologieorientierung prinzipiell vereinbar sind.

\subsubsection{Ideologie- und wiederwahlorientierte Politiker}

Ende der 70er Jahre präsentierten Frey und Schneider sowohl eine Reihe theoretischer als auch zahlreiche empirische Studien, die ihr Konzept der Interdependenz zwischen Politik und Wirtschaft darstellten. ${ }^{227}$ Ihre Modellierung beruht dabei auf der grundlegenden Überlegung, die erstmals von Frey und Lau (1968) vorgestellt wurde, dass Nutzen sowohl aus einer möglichen Amtsübernahme als auch aus der Realisierung einer ideologiespezifischen Wirtschaftspolitik entsteht. Parteien verfolgen also im wesentlichen Partisanziele unter der Nebenbedingung der Wiederwahl. Dies lässt sich in die Annahmenstruktur übertragen: ${ }^{228}$

A.1 Die Wirtschaft ist durch den grundsätzlichen trade-off zwischen Arbeitslosigkeit und Inflation gekennzeichnet (modifizierte Phillips-

${ }^{226}$ Nach einem Regierungswechsel nach links kommt es zum Aufschwung und zu einem beschleunigten Preisauftrieb. Da der Anstieg des Bruttoinlandsprodukts nur kurzfristig ist, der Anstieg der Inflation jedoch dauerhaft, müssten in der Folge des politisch induzierten Aufschwungs die Realeinkommen zurückgehen.

227 Vgl. u.a. Frey $(1976,1978)$ und Frey und Schneider (1978a, 1978b, 1979).

Die neuen Annahmen sind mit vier Sternen gekennzeichnet rst - 978-3-631-75139-8 
Kurve). ${ }^{229}$ Diese Annahme ist für die Kernaussagen des Modells weniger relevant und daher nur implizit enthalten (z.B. Frey, 1981, S. 15).

A.2 Die Erwartungsbildung erfolgt dabei adaptiv. Auch dies ist nur aus den Beschreibungen des Ablaufs zu deduzieren.

A.3**** Politiker maximieren ihren Erwartungsnutzen. Dies gelingt ihnen am besten, wenn sie innerhalb der politischen, ökonomischen, legalen und administrativen Beschränkungen ihr Partisanziel verfolgen. Die größte Handlungsbeschränkung geht dabei von dem politischen Wiederwahlerfordernis aus, da nach einer Wahlniederlage definitionsgemäß auch nicht die präferierte Politik verfolgt werden kann. ${ }^{230}$

A.4**** Die Anzahl der zur Wahl stehenden Parteien ist für das Modell unerheblich.

A.5 Wähler bewerten die Politiker u.a. anhand zentraler ökonomischer Variablen, z.B. der Arbeitslosenquote und Inflationsrate. Angesichts ideologisch prädisponierter Politiker ist die Schlussfolgerung, dass auch die Wählerpräferenzen heterogen sind, naheliegend. Darüber hinaus diskontieren Wähler Ereignisse aus der Vergangenheit ab.

A.6**** Die Politiker sind in der Lage, die Wirtschaft zu beeinflussen, besitzen jedoch keine vollständige Information über die exakten Wirkungszusammenhänge, sodass eine punktgenaue Dosierung nicht möglich ist (Frey, 1978, S. 211).

Aus diesen Annahmen bilden Frey und Schneider nun keinen formalen Optimierungsansatz, sondern stellen Verhaltensregeln auf, nach denen Politiker innerhalb der durch Unsicherheit gekennzeichneten Umgebung agieren. Zentrale Bedeutung für diese Verhaltensregeln fällt dabei der Wiederwahlaussicht zu, für die regelmäßig bereitstehende Umfrageergebnisse als Indikator dienen. Ob Politiker die Wiederwahl als gefährdet erachten, hängt dann von zwei Parametern ab. Bedeutsam für ihre Entscheidung ist die Höhe der aktuellen Popularität $\left(\mathrm{POP}_{\mathrm{t}}\right.$ ) relativ zu einem Referenzwert der Popularität (POP*). Dieser Referenzwert ist sozusagen der Interventionspunkt, ab dem die Regierungspartei bereit

${ }^{229}$ Dies wird allerdings keineswegs überall deutlich. So schreibt Frey (1976, S. 115) z.B.: „Das Ziel besteht in diesem Falle darin, als Folge einer bewusst herbeigeführten Senkung der Arbeitslosenquote und/oder der Inflationsrate [...] einen Popularitätsgewinn zu erzielen."

${ }^{230}$ Eine ähnliche Abwägung zwischen Ideologieziel und Wiederwahlziel wurde in Abschnitt

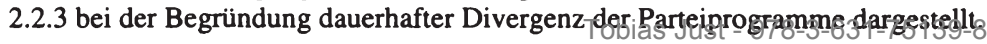


ist, von ihrem Partisanziel abzuweichen und mehrheitsfördernde Maßnahmen zu ergreifen. ${ }^{231}$

Dies ist allerdings nur in Wahljahren bedeutsam, da in Nicht-Wahljahren die Regierungspartei aufgrund der „Vergesslichkeit“ der Wähler nicht auf hohe Popularitätswerte angewiesen ist. Entscheidende Unterschiede ergeben sich jedoch im Wahljahr:

1. Ist $P P_{t} \geq P O P^{*}$, dann besteht für die Regierung kein Anlass zur Kurskorrektur, da ihre ideologisch motivierte Politik scheinbar nicht den Wahlerfolg gefährdet.

2. Ist $\mathrm{POP}_{\mathrm{t}} \leq \mathrm{POP}^{*}$, ist die Wiederwahl nach Einschätzung der Partei hinreichend gefährdet, sodass wirtschaftspolitische Instrumente zur Wahlsicherung eingesetzt werden. Dies wird dann üblicherweise unabhängig von der politischen Couleur im Nordhaus-Sinn expansive Fiskal- und/oder Geldpolitik sein.

Dies bedeutet, dass hier politische Konjunkturzyklen nicht in derselben Regelmäßigkeit auftreten, wie dies z.B. im Nordhaus-Modell der Fall ist. Vielmehr kommt es nur dann zu einem politischen Konjunkturzyklus à la Nordhaus, falls im Wahljahr die Popularität der Regierungspartei hinreichend klein ist. Darüber hinaus ist mit einem Konjunkturzyklus à la Hibbs zu rechnen, falls es trotzdem zu einem Regierungswechsel kommt, da angenommen wurde, dass Parteien die Möglichkeit besitzen, wirtschaftliche Zielvariablen gemäß ihrer Partisanziele zu manipulieren. Die Kritikpunkte richten sich erstens darauf, dass die Theorie noch nicht in ein Modell rationaler Erwartungen integriert worden ist (Alesina, Roubini und Cohen, 1997, S. 64). Zweitens wird die Annahme kritisiert, dass bei einem Popularitätsüberschuss Wiederwahlbemühungen im Sinne expansiver Wirtschaftspolitik unterbleiben. Nach Schenk (1994, S. 74) hätte die Regierungspartei dann gegebenenfalls keine Sicherheit, falls ein exogener Schock die Popularitätsdaten unerwartet erodierte. Dieser Einwand ist allerdings wenig haltbar, da in POP* durchaus bereits eine Risikoprämie enthalten sein kann. Im Rahmen der Annahmen, dass jedes Verlassen der eigenen ideologischen Position für die Politiker nutzenmindernd ist, richtet sich POP* also einerseits nach der Wiederwahlaussicht, andererseits nach den Kosten der verlassenen Position. Eine Steigerung der Popularität jenseits von POP* wäre dann irrational. Tabelle 3.9 fasst die zentralen Aussagen der Modelle zusammen.

${ }^{231}$ Frey und Schneider (1978a, S. 179) arbeiten mit konstanten POP*-Werten in der Nähe von $58 \%$. Es ließe sich sicherlich auch mit variablen POP*-Werten arbeiten, dahingehend, dass POP* z.B. zunimmt, je näher eine Wahl rückt oder dass POP* umso größer wird, je größer das Parteidifferential zwischen der Regierungs- und der Oppositionspartei ist. Da eine solche Funktion an der grundsätzlichen Modellstruktur jedoch nichts ändert, ist es durchaus angemessen, von konstanten POP*-Werten auszugehen-8-3-631-75139-8 
Tabelle 3.9 : Synopse der wichtigsten Modelle politischer Konjunkturzyklen

\begin{tabular}{|c|c|c|c|c|c|c|}
\hline \multirow{2}{*}{$\begin{array}{l}\text { Er- } \\
\text { wartungen }\end{array}$} & \multicolumn{3}{|c|}{ Wiederwahlorientierte Politiker } & \multicolumn{2}{|c|}{ Ideologieorientierte Politiker } & \multirow{2}{*}{$\begin{array}{c}\begin{array}{c}\text { Ideologie und } \\
\text { Wahlorientierung }\end{array} \\
\text { Adaptiv (5) }\end{array}$} \\
\hline & Adaptiv (1) & Ratio & nal (2) & Adaptiv (3) & Rational (4) & \\
\hline 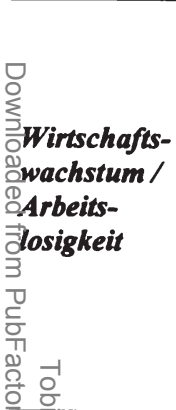 & $\begin{array}{l}\text { Vor der Wahl: } \\
\text { Durch expansive } \\
\text { Politik sinkt die Ar- } \\
\text { beitslosigkeit. } \\
\text { Nach der Wahl: } \\
\text { Durch kontraktive } \\
\text { Politik wird Wirt- } \\
\text { schaftswachstum } \\
\text { gesenkt, die Ar- } \\
\text { beitslosigkeit steigt. }\end{array}$ & $\begin{array}{l}\text { Separating } \\
\text { Inkompetente } \\
\text { Regierung } \\
\text { bewirkt } \\
\text { Rückgang des } \\
\text { Wachstums, } \\
\text { kompetente } \\
\text { Regierung } \\
\text { leistet Be- } \\
\text { schäfti- } \\
\text { gungssteige- } \\
\text { rung. }\end{array}$ & $\begin{array}{l}\text { Pooling } \\
\text { Keine Wir- } \\
\text { kung. Beide } \\
\text { Typen Politi- } \\
\text { ker haben } \\
\text { dieselbe Be- } \\
\text { schäftigungs- } \\
\text { wirkung. }\end{array}$ & $\begin{array}{l}\text { Die linke Partei } \\
\text { hat im Durch- } \\
\text { schnitt in der ge- } \\
\text { samten Legisla- } \\
\text { turperiode höhere } \\
\text { Wachstumsraten } \\
\text { und niedrigere } \\
\text { Arbeitslosenzah- } \\
\text { len als rechte } \\
\text { Parteien. }\end{array}$ & $\begin{array}{l}\text { Vor der Wahl: } \\
\text { Natürliche Wachstumsrate. } \\
\text { Nach der Wahl: } \\
\text { Gewinnt eine linke Partei, führt } \\
\text { dies kurzfristig zu höherem } \\
\text { Wachstum. Gewinnt eine rechte } \\
\text { Partei, sinkt die Wachstumsrate } \\
\text { kurzfristig unter die natürliche } \\
\text { Rate. }\end{array}$ & $\begin{array}{l}\text { Ist in Wahljahren } \\
\text { POP }_{\mathrm{t}} \leq \mathrm{POP}^{*} \text {, } \\
\text { wird ein Kon- } \\
\text { junkturzyklus wie } \\
\text { in (1) induziert. } \\
\text { Ansonsten sind } \\
\text { Ideologieunter- } \\
\text { schiede wie in (3) } \\
\text { zu beobachten. }\end{array}$ \\
\hline 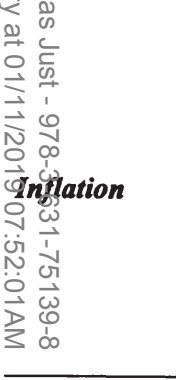 & $\begin{array}{l}\text { Vor der Wahl: } \\
\text { Expansive Politik } \\
\text { lässt Inflation verzö- } \\
\text { gert steigen. } \\
\text { Nach der Wahl: } \\
\text { Kontraktive Politik } \\
\text { senkt die Inflations- } \\
\text { raten wieder. }\end{array}$ & $\begin{array}{l}\text { Vor und/oder } \\
\text { nach der Wahl } \\
\text { führen In- } \\
\text { kompetente } \\
\text { Deflation her- } \\
\text { bei und Kom- } \\
\text { petente Infla- } \\
\text { tion. }\end{array}$ & $\begin{array}{l}\text { Inkompetente } \\
\text { Politiker müs- } \\
\text { sen inflatio- } \\
\text { nieren, um } \\
\text { dieselbe Be- } \\
\text { schäftigungs- } \\
\text { wirkung zu } \\
\text { erzielen wie } \\
\text { die kompe- } \\
\text { tenten Politi- } \\
\text { ker. }\end{array}$ & $\begin{array}{l}\text { Analog haben } \\
\text { linke Parteien } \\
\text { während der ge- } \\
\text { samten Legisla- } \\
\text { turperiode höhere } \\
\text { Inflationsraten zu } \\
\text { verzeichnen als } \\
\text { rechte Parteien. }\end{array}$ & $\begin{array}{l}\text { Vor der Wahl: } \\
\text { Ist eine rechte Partei an der } \\
\text { Macht, so ist die Inflationsrate } \\
\text { tendenziell geringer als bei einer } \\
\text { linken Partei. } \\
\text { Nach der Wahl: } \\
\text { Gewinnt eine linke Partei, steigt } \\
\text { die Inflationsrate an; gewinnen } \\
\text { die Rechten, sinkt die Inflation. }\end{array}$ & \\
\hline
\end{tabular}

Quelle: Eigene Darstellung. 


\subsubsection{Empirischer Befund politischer Konjunkturzyklen}

Die Theorie politischer Konjunkturzyklen wurde schon sehr früh auf ihre empirische Validität geprüft. Bereits Åkerman (1947) beschränkte seine Ausführungen nicht nur auf theoretische Überlegungen, sondern versuchte, seine Thesen mit Hilfe graphischer Zeitreihendarstellungen zu stützen. Mittlerweile ist die Anzahl der empirischen Arbeiten nahezu unübersehbar geworden, wobei eine systematische Zusammenfassung nicht nur durch die reine Zahl erschwert wird, sondern v.a. durch die Verschiedenheit der jeweils gewählten Vorgehensweisen. Gemäß der obigen Klassifikation von Modellen lassen sich zumindest fünf verschiedene Theorien differenzieren mit jeweils unterschiedlichen inhärenten Hypothesentests. ${ }^{232}$

Darüber hinaus unterscheiden sich die Analysen stark hinsichtlich der gewählten empirischen Methodik (deskriptive Statistik, Zeitreihenanalyse, Querschnittsanalyse, Panelregressionen) sowie der verwendeten Datenreihen (Untersuchungszeiträume, Erhebungshäufigkeit einzelner Variablen) und Auswahl/ Anzahl der berücksichtigten Länder, sodass die Vergleichbarkeit der einzelnen Studien nicht uneingeschränkt gewährleistet ist (Berlemann, 1999, S. 250f).

Die folgende Zusammenfassung ist bewusst knapp gehalten und erhebt keineswegs den Anspruch, die gesamte empirische Literatur zur Thematik politischer Konjunkturzyklen zu berücksichtigen. Da jedoch bereits einige überzeugende Überblicksdarstellungen in der Literatur vorliegen, ${ }^{233}$ spiegelt diese selektive Darstellung durchaus den momentanen Stand der empirischen Forschung wider. In Analogie zur Darstellung der zugrunde liegenden Theorien werden die Ergebnisse getrennt nach der Handlungsmotivation der politischen Akteure in solche Modelle, die von rein wiederwahlorientierten (opportunistischen) Politikern ausgehen, und solche Modelle, die ideologische Handlungsbindungen zulassen, unterschieden.

\footnotetext{
${ }^{232}$ Wie erwähnt, ist dabei jedoch immer deutlicher festzustellen, dass die verwendete Klassifikation zunehmend erodiert. Dies muss dann zwangläufig auch die Unterschiede im empirischen Forschungsdesign zunehmend verwischen.

Besonders lesenswert ist der Artikel von Paldam (1997) sowie das Buch von Alesina, Cohen und Roubini (1997). Die Entwicklung der empirischen Überprüfung der Partisantheorie ist sehr gut in Hibbs (1992) sowie Belke (1996) nachgezeichnet. Einen_stärkeren Ak-

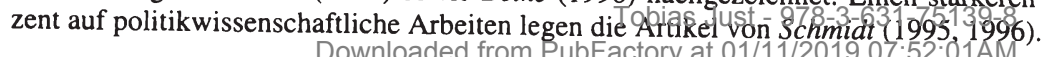




\subsubsection{Wiederwahlorientierte Politiker}

In seinem wegweisenden Aufsatz versuchte Nordhaus (1975), seine Theorie politischer Konjunkturzyklen mit Hilfe eines Binomialtests der Arbeitslosenquoten vor und nach einer Wahl in neun OECD-Staaten zu überprüfen, konnte jedoch lediglich für die USA, Neuseeland und Deutschland einen signifikanten Unterschied zwischen den beiden Teilperioden feststellen, die mit seiner Theorie konform geht. Die anderen Länder (Australien, Kanada, Frankreich, Japan, Schweden und Großbritannien) weisen keinen signifikanten Unterschied zwischen der Entwicklung der Arbeitslosenquote vor und nach einer Wahl auf. Dinkel (1977, S. 179f) weist darauf hin, dass selbst diese „dünne empirische Begründung“ weiter einzuschränken ist, da das Modell von Nordhaus ein paralleles Sinken der Arbeitslosenquote und Steigen der Inflationsrate vor der Wahl prognostiziert. Nordhaus beschränkt sich jedoch nur auf die Überprüfung der Entwicklung der Mengengröße. McCallum (1978) lehnt in seiner Studie sogar für die USA die Hypothese der Existenz eines politischen Konjunkturzyklus ab. ${ }^{234}$

Paldam (1979) findet zwar in seiner Analyse von 17 OECD-Staaten einen schwachen Konjunkturzyklus, der auf politische Intervention zurückgeführt werden kann, kommt jedoch zu dem Ergebnis, dass entgegen der Prognose von Nordhaus nicht das Wahljahr das expansivste Jahr ist, sondern das zweite Jahr nach der Wahl. In einer groß angelegten Studie, die den Zeitraum von 1960 bis 1987 für 18 OECD-Staaten umfasst, kommen Alesina, Cohen und Roubini $(1992,1993)$ zu dem Ergebnis, dass zwar in Vorwahljahren die Geld- und Fiskalpolitik theoriekonform expansiv sind, dies jedoch keine messbaren Rückwirkungen auf die makroökonomischen Aggregate Wirtschaftswachstum, Arbeitslosenquoten und Inflation hat, sodass auch sie die Existenz eines politischen Konjunkturzyklus des Nordhaus-Zuschnitts ablehnen. ${ }^{235}$

In ihren Untersuchungen können Alesina, Cohen und Roubini $(1992,1993)$ jedoch nicht die Hypothese der kurzfristigen Budgetzyklen - so wie sie das Modell rationaler Individuen bei wiederwahlorientierten Politikern von Rogoff und Sibert (1988) nahe legt - verwerfen. Auch der erweiterte Ansatz Rogoffs (1990) findet durch die Arbeit von Crosby, Brown und Malady (1995) empirische Be-

${ }^{234}$ Die Synopse von Paldam (1997) zeigt, dass für die USA die empirischen Ergebnisse alles andere als eindeutig sind.

235 Dies gilt auch nur für den gesamten Datensatz innerhalb der Panelregressionen. In einzelnen Länderanalysen kamen die Autoren zu leicht unterschiedlichen Ergebnissen. Für Deutschland und Neuseeland konnten reale Effekte gemäß der Nordhaus-Theorie gefunden werden. Darüber hinaus konnte die Theorie nur für Neuseeland und Australien hinsichtlich eines politischen Konjunkturzyklus im Geldmengenaggregat $\mathbf{M}_{1}$ auf dem 5\%Signifikanzniveau gestützt werden. Dieses Ergebnis bestätigen Alesina, Cohen und Roubi-

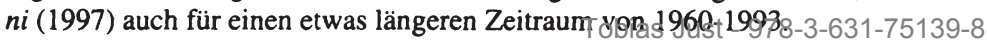


stätigung. Zu ähnlichen Ergebnissen kommt auch Schuknecht (1996) in seiner Analyse von 35 Entwicklungsländern. Es sind v.a. bei geschlossenen Volkswirtschaften deutliche, kurzfristige Veränderungen der staatlichen Ausgabenströme vor Wahlen feststellbar. Diese Budgetzyklen können jedoch nicht in ähnlich starke Schwankungen des Outputs übersetzt werden. Wie oben bereits kurz erwähnt, stellt sich die empirische Überprüfung der Modellaussagen von Persson und Tabellini (1990) sehr schwer dar, da es bisher nicht gelungen ist, die zentrale Kompetenz-Variable der Regierung in Relation zur Opposition zum jeweiligen Wahlzeitpunkt angemessen zu operationalisieren. Außerdem müsste bei einer empirischen Prüfung zusätzlich begründet werden, ob etwaige Schwankungen der Arbeitslosenquote und der Inflationsrate um den Wahlzeitpunkt eher Ausdruck des separating equilibrium oder des pooling equilibrium sind. Dies ist deswegen notwendig, da die Inflationswirkung der kompetenten (und inkompetenten) Regierung in beiden Gleichgewichtstypen entgegengesetzt zueinander ist. Ist es jedoch nicht möglich, sinnvoll zwischen diesen Gleichgewichten zu unterscheiden, kann selbst der beste Kompetenzindikator keine eindeutige Schätzung ergeben.

\subsubsection{Ideologisch gebundene Politiker}

Nach der Partisantheorie von Hibbs $(1977,1987)$ mit adaptiver Erwartungsbildung unterscheiden sich die Politikergebnisse linker und rechter Wirtschaftspolitik dauerhaft. Eine linke Regierung hätte im Durchschnitt über die gesamte Legislaturperiode niedrige Arbeitslosenquoten und höhere Inflationsraten zu verzeichnen als eine rechte Regierung. Für Großbritannien ermittelte Hibbs (1977, S. 1481) eine Differenz der steady-state Werte zwischen der Arbeitslosenquote unter einer linken Regierung und der Arbeitslosenquote unter einer rechten Regierung von $0,62 \%$-Punkte, wobei der steady-state-Wert in seiner Simulation nach ungefähr vier Jahren erreicht wird. Für die USA ermittelt Hibbs (1977, S. 1485) einen langfristigen Unterschied in den steady-state Werten von 5,46\%Punkten, wobei dieses steady-state jedoch erst nach 25 Jahren erreicht wird. Nach acht Jahren sind die Wirkungsunterschiede verschiedener Regierungen mit 2,36\%-Punkten immerhin noch beachtlich.

Beck (1982) kommt für die USA zwar auch zu dem Ergebnis, dass die Parteipolitik einen nachweisbaren Einfluss auf die Arbeitslosenquote hat, schätzt jedoch die Wirkungsdifferenz nur auf 1\%-Punkt in acht Jahren. Beck begründet sein schwächeres Ergebnis damit, dass es gravierende innerparteiliche Unterschiede zwischen einzelnen Regierungen gibt - nicht jede Regierung, die unter dem „linken“ Parteinamen agiert, macht in demselben Maße typisch „linke“ Politik. 
Paldam (1989a) findet in seiner 17-Länder-Studie - mit Ausnahme der USA lediglich schwach signifikante Bestätigung des Hibbs-Zyklus. ${ }^{236}$

Alesina, Cohen und Roubini (1997, S. 163ff) können in einer 18-LänderAnalyse die Hypothese dauerhaft unterschiedlicher Arbeitslosigkeit zunächst nicht verwerfen, argumentieren jedoch, dass dieses Ergebnis lediglich Ausdruck starker Unterschiede zu Beginn der Legislaturperioden ist. Sie konnten nämlich zuvor zeigen, dass es gemäß der rationalen Partisantheorie deutliche, transitorische Effekte in der Beschäftigung nach Regierungswechseln gibt. Diese verschwinden jedoch nach einer Anpassungszeit. Die Unterschiede in den Durchschnittswerten der gesamten Legislaturperiode sind dann nur durch den starken transitorischen Effekt zu Beginn begründet. Dieselbe Aussage treffen auch Alesina und Rosenthal (1995, S. 182ff). Sie finden klare Bestätigung für die rationale Partisantheorie sowohl für die Output-Variable als auch für die Inflationsvariable..$^{237}$

Übereinstimmend damit kann Berlemann (1999) für ein 15-Länder umfassendes Sample keine permanenten Partisaneffekte hinsichtlich der Arbeitslosenquote finden. Allerdings findet Berlemann Bestätigung für dauerhaft unterschiedliche Niveaus der Inflationsrate in einer 20 Länder-Studie. Auch diese Ergebnisse scheinen darauf hinzudeuten, dass die rationale Partisantheorie das bessere Modell der wirtschaftlichen Bedeutung von Regierungswechseln darstellt. ${ }^{238}$

Bereits Alesina und Sachs (1988) fanden für die USA kurzfristige Schwankungen im Output nach einer Wahl. Da die regierten Individuen zügig ihre Erwartungen an die neue (oder die alte) Regierungspolitik anpassen, ist dieser Schock in den realen Größen nur temporär. Zwar realisieren demokratische Regierungen durchschnittlich um 2,0\%-Punkte höhere Wachstumsraten über den gesamten Legislatur-Zeitraum als republikanische Regierungen. Dies liegt jedoch nur daran, dass in der ersten Hälfte die durchschnittliche Differenz der Wachstumsraten 3,4\%-Punkte beträgt; in der zweiten Hälfte sind indes kaum Unterschiede zu verzeichnen. ${ }^{239} \mathrm{Zu}$ ähnlichen Ergebnissen kommen auch Chappell und Keech (1986). Bei ihnen sind noch im dritten Jahr messbare Unterschiede nachweisbar, nicht aber im vierten Regierungsjahr. Auch Paldam (1989b, S. 20) findet „...an animal in the data looking a lot like the Alesina RE [rational expectation] Parti-

${ }^{236}$ Allerdings charakterisiert Hibbs (1992, S. 368) die methodische Vorgehensweise von Paldam als ,...rather esoteric parametric test procedure“, begründet diese Einschätzung jedoch nicht.

${ }^{237}$ Eine ausführliche Übersicht über die Testverfahren der einzelnen Studien zur rationalen Partisantheorie bietet Belke (1996, S. 202-216).

${ }^{238}$ Wobei auffällt, dass jene Studien, die am eindeutigsten die rationale Partisantheorie stützen, aus dem Arbeitskreis um Alberto Alesina - dem Begründer dieser Theorie - kommen.

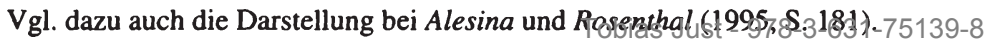


san Cycle." Zwar kann Paldam (1989a) auch die Hypothese von dauerhaften Partisaneffekten nicht verwerfen, jedoch sind seine Ergebnisse hinsichtlich der rationalen Partisantheorie für mehr Wahlen nachweisbar und auf einem höheren Signifikanzniveau von null verschieden. Auch Belke (1996) kommt in seiner sehr detaillierten empirischen Untersuchung der Entwicklung der Arbeitslosenquote sowie der Inflationsrate - auch unter Berücksichtigung von HysteresisEffekten - zu dem Ergebnis, dass sowohl für die USA als auch für Deutschland die Hypothese der Existenz von rationalen Partisaneffekten nicht verworfen werden konnte. Die Ergebnisse deuten in die Richtung, dass diese Effekte zwar schwach, jedoch hoch signifikant sind. Auch in einer jüngeren Veröffentlichung unterstreicht Belke (2000) mit Hilfe eines Error Correction-Ansatzes, dass temporäre Partisaneffekte langfristige Wirkungen auf dem deutschen Arbeitsmarkt im Sinne der um Hysteresis erweiterten rationalen Partisantheorie haben.

Allerdings kommen nicht alle Autoren zu einem so eindeutigen Bescheid für die Theorie von Alesina. Sheffrin (1989) findet innerhalb seines 15-Länder Samples lediglich fünf Länder, für die die Existenz rationaler Partisaneffekte nicht verworfen werden konnte. Interessanterweise findet Sheffrin jedoch für drei Länder (Australien, Österreich und Frankreich) Konjunkturverläufe, die der Theorie von Alesina diametral entgegen stehen. Auch die Ergebnisse von Haynes und Stone (1995) können die Theorie von Alesina nicht stützen.

Die rationale Partisantheorie enthält darüber hinaus noch eine weitere empirisch prüfbare Implikation. Der reale Schock entsteht ja gerade dadurch, dass die Wähler unsicher hinsichtlich des Wahlausgangs sind und ihre Erwartungen gemäß eines mit der Wahrscheinlichkeit des Wahlsiegs gewichteten Mittelwertes der Politikpräferenzen der Parteien bilden. Es ist also zu vermuten, dass der reale Effekt umso größer ist, je stärker der tatsächliche Wahlausgang den vorherigen Umfrageergebnissen widerspricht. So ist es doch verwunderlich, dass Hibbs (1992, S. 366) in seinem Überblicksartikel feststellen muss:

„Oddly, the RPT's [Rational Partisan Theory] central theoretical innovation - partisan effects depend on ex ante electoral uncertainty - never has been tested."

Bis 1992 lag lediglich eine einzige Studie vor (Chappell und Keech, 1988), die Umfragedaten berücksichtigte und dabei keine Bestätigung für die Hypothese liefern konnte. Alesina, Cohen und Roubini (1997, Kapitel 5) konstruieren einen etwas anderen Indikator, der zwar auf Umfragedaten basiert, diese jedoch erweitert. Nach ihrer Formel wird neben dem Umfrageergebnis zum Zeitpunkt des Tarifvertrages noch die erwartete Veränderung bis zum Wahltermin berücksichtigt (durchschnittliche Veränderung der Umfrageergebnisse multipliziert mit der Anzahl der Monate bis zum Wahltag). Mit Hilfe des so gebildeten Unsicherheitsparameters können sie für die USA die Hypothese, dass eine größere Wahlüberraschung auch zu heftigeren, kurzfristigen realen Schocks (Wirtschaftswachstum und Arbeitslosenquote) führt, nicht verwerfen Berlemann $_{1}(1999, \mathrm{~S}$. 
281ff) untersucht mit Hilfe eines etwas einfacheren Indikators für die Wahlüberraschung dieselbe Fragestellung und findet die These bestätigt, dass größere Überraschungsmomente auch zu stärkeren Effekten in der Arbeitslosenquote führen. ${ }^{240}$ Es scheint also durchaus gerechtfertigt zu sein, auch in diesem Punkt der rationalen Partisantheorie Erklärungskraft zuzuschreiben.

Die bisher genannten Studien untersuchten makroökonomische Zielgrößen (Wirtschaftswachstum, Arbeitslosenquote und Inflationsrate) hinsichtlich einer Struktur, die die eine oder andere Partisantheorie bestätigen konnte. Ähnliche Untersuchungen wurden für Instrumentvariable (Verschuldungsneigung, Geldmengenaggregate) durchgeführt. Auch hier deuten die meisten Ergebnisse in die Richtung, dass klare Partisaneffekte isolierbar sind (u.a. Cusack, 1997a und 1997b, Carlsen, 1997, Schmidt, 1996, Alesina, Cohen und Roubini, 1997).

Schließlich soll nicht vernachlässigt werden, dass auch jene Modelle, die versucht haben, die Annahmen allein wiederwahl- bzw. ausschließlich ideologieorientierten Politiker miteinander in einem Ansatz zu verbinden, empirische Bestätigung fanden (z.B. Frey, 1978, Frey und Schneider, 1978a, 1978b).

Zusammenfassend lässt sich festhalten, dass sowohl in den Zielvariablen als auch in den Instrumentvariablen die empirischen Ergebnisse durchaus dafür sprechen, dass politische Parteien unterschiedliche Präferenzen bezüglich wirtschaftlicher Eckdaten besitzen. Diese Präferenzen führen in der Regel zu unterschiedlichem Instrumenteneinsatz, der dann wiederum zur Induktion von Konjunkturschwankungen führt.

Da im weiteren Verlauf der Untersuchung abzuschätzen ist, inwiefern diese Differenz durch die mögliche Verengung politischer Handlungsspielräume angesichts zunehmender internationaler wirtschaftlicher Verflechtung gegebenenfalls verändert wurde, wird im folgenden Kapitel zunächst die historische Entwicklung sowie die momentane Bedeutung internationaler Wirtschaftsintegration skizziert, um dann Ländergruppen sowie Zeitphasen unterschiedlich starker wirtschaftlicher Integration zu bilden.

Berlemann (1999, S. 284) konstruiert seine Variable, indem er die kumulierten Stimmenanteile einer Partei in allen Umfragen des Jahres vor einer Wahl zu der Summe der kumulierten Stimmenanteile aller Umfrageergebnisse der teilnehmenden Parteien ins Verhältnis setzt. Dies vernachlässigt jedoch mögliche Trends in den Umfragedaten, die von rationalen Individuen in die Zukunft fortgeschrieben werden können und bewertet alle Umfrageergebnisse gleich stark. 
Tobias Just - 978-3-631-75139-8

Downloaded from PubFactory at 01/11/2019 07:52:01AM

via free access 


\title{
Kapitel 4 \\ Definition und Messung des Grads der Globalisierung
}

\begin{abstract}
„Globalisierung ist sicher das am meisten gebrauchte - missbrauchte - und am seltensten definierte, wahrscheinlich missverständlichste, nebulöseste und politisch wirkungsvollste (Schlag- und Streit-) Wort der letzten, aber auch der kommenden Jahre. “ Beck (1997, S. 42)
\end{abstract}

Der Begriff „Globalisierung“ hat einen Siegeszug in die wissenschaftliche und politische Sprachwelt vollzogen wie kaum ein anderes Schlagwort der letzten Jahre. Bereits 1996 wurde der Begriff von der Gesellschaft für deutsche Sprache $(G f d S)$ zu einem der Wörter des Jahres gewählt $(G f d S, 2000)$. In der (sozial-) wissenschaftlichen Diskussion lässt sich dieser Siegeszug am eindrucksvollsten anhand der Eintragshäufigkeit in die Literaturdatenbank WISO festmachen; 1989 konnten von 520.000 Einträgen gerade 60 unter dem Begriff Globalisierung gefasst werden, 1996 waren es bereits ca. 1.200 Treffer bei 1,1 Mio. Eintragungen (Dörrenbacher, 1999, S. 28ff) - der Anteil der Globalisierungstitel stieg also in etwa um den Faktor 10. Dabei ist bemerkenswert, dass der Begriff je nach Gegenstand der Untersuchung sehr verschiedene Definitionen und Abgrenzungen zuzulassen scheint. Sell (1999, S. 70ff) unterscheidet allein in der ökonomischen Profession 12 „Annäherungsversuche“. ${ }^{241}$ Diese Unterschiedlichkeit im Begriffsverständnis findet ihren deutlichsten Ausdruck darin, dass in der politischen Diskussion das Stichwort Globalisierung gleichermaßen als Projektionsfläche für nahezu jede ökonomische, politische oder gesellschaftliche Hoffnung oder Befürchtung instrumentalisiert wird (vgl. Landmann, 1999, S. 133).

Es sollen in diesem Kapitel sowohl Ländergruppen als auch Zeitphasen des Globalisierungsprozesses herausgearbeitet werden. Dies wird im darauf folgenden Kapitel zentraler Bestandteil der empirischen Tests sein, da dort untersucht wird, inwiefern Ländergruppen unterschiedlicher internationaler Öffnung auch unterschiedlich starke Partisaneffekte aufzeigen.

Angesichts der Unklarheit des Begriffs Globalisierung ist es dafür zunächst erforderlich, eine Definition des Begriffs Globalisierung zu geben. Dabei wird nicht der Anspruch erhoben, dass die gewählte Definition die umfassendste ist;

241 Und dabei ist der Begriff „Globalisierung“ keineswegs auf die Wirtschaftswissenschaften beschränkt (vgl. für eine Sammlung der Bereiche-zBBaBeck, 19978_S.39)-75139-8 
sie erscheint lediglich mit Blick auf die hier interessierende Frage als besonders zweckmäßig. Darauf aufbauend wird kurz die Diskussion zusammengefasst, ob - und wenn ja - inwiefern das Globalisierungsphänomen eine grundlegend neue Erscheinung ist. Diese Diskussion hilft abzuschätzen, welche Konsequenzen daraus für die Politik resultieren und ob tatsächlich mit einem race to the bottom der sozialstaatlichen Elemente zu rechnen ist, wie vielfach behauptet wird (z.B. Sinn, 1990; Scharpf, 1997), denn dann würden auch sämtliche nationalen Partisaneffekte nivelliert werden. Aus dieser Diskussion leitet sich dann direkt die Frage ab, warum rationale Politiker bereit sind, durch die Ratifizierung von internationalen Liberalisierungsabkommen den Globalisierungsprozess zu beschleunigen und dadurch (gegebenenfalls) Handlungsspielräume $\mathrm{zu}$ verlieren. Daher soll anschließend ein polit-ökonomisches Modell entwickelt werden, mit dem gezeigt werden kann, dass die Marktöffnung durchaus eine sinnvolle Strategie für wiederwahlorientierte Politiker sein kann.

Im nächsten Abschnitt wird im Rahmen einer Clusteranalyse gezeigt, dass der Globalisierungsprozess keineswegs gleichmäßig verlief, sondern in einzelne Phasen unterteilt werden kann. Außerdem kann mit Hilfe einer Clusteranalyse das gesamte Ländersample in verschiedene Globalisierungscluster unterteilt werden. Diese Ländercluster machen deutlich, dass nicht alle Staaten gleich intensiv in die internationale Arbeitsteilung eingebunden sind. Folglich kann auch nicht erwartet werden, dass die Globalisierung gleich bedeutsam für die unterschiedlichen Ländergruppen ist. Sowohl die Zeit- als auch die Ländercluster dienen im folgenden Kapitel dann als Grundlage für die Argumentation, ob stärkere internationale Integration einer Volkswirtschaft auch tatsächlich deren Handlungsspielräume reduziert oder nicht.

\subsection{Was ist Globalisierung?}

In dieser Untersuchung wird Globalisierung durch die zunehmende Verflechtung von Akteuren (Unternehmen, Konsumenten, Politikern, Interessengruppen etc.) und Aktivitäten (Handel, Kapitaltransfer, Migration) in größer werdenden geographischen und organisatorischen Einheiten gekennzeichnet (vgl. u.a. auch Hübner, 1998, S. 17). Damit wird deutlich, dass es sich um einen Prozeß handelt, von dem angenommen wird, dass er noch nicht abgeschlossen ist (Dörrenbacher, 1999, S. 22). ${ }^{242}$

${ }^{242}$ Auch Hirst und Thompson (1996, S. 10) nennen die vollständig globalisierte Welt einen idealisierten Zustand, den sie als Referenzmaßstab setzen. Beck (1997, S. 29) verwendet für den Zustand eines abgeschlossenen Globalisierungsprozesses den Begriff der Globalität. 
Nach Hirst und Thompson (1996, S. 10ff) ist eine internationalisierte Welt eine Welt aus einzelnen Nationalstaaten, die miteinander in Kooperationsbeziehungen stehen, jedoch weiterhin die maßgeblichen politischen und wirtschaftlichen Akteure bleiben. Eine globalisierte Welt hingegen bedeutet, dass die Kooperationsbeziehung eine neue Qualität erreicht hat, sodass nationalstaatliche Politik unzureichend geworden ist. Das neue System lässt sich aufgrund der neuen Qualität nicht mehr durch nationalstaatliche, dezentralisierte Politik steuern, sondern nur durch eine Form von global governance kontrollieren. Eine solche Trennung ist für diese Untersuchung ungeeignet, weil sie das Forschungsziel quasi per Definition vorwegnimmt. In dieser Arbeit werden daher die Begriffe Globalisierung und Internationalisierung sowie deren Teilmengen Regionalisierung, Triadisierung und Multinationalisierung als Formen einer generellen Verflechtungsdynamik verstanden. Der Begriff der Globalisierung dient hier lediglich als Platzhalter für jede Form von internationaler wirtschaftlicher Integration. Dies heißt auch, dass ausschließlich solche Aspekte berücksichtigt werden, die das Erkenntnisobjekt Wirtschaft betreffen. ${ }^{243}$ In diesem Kontext steht Globalisierung in erster Linie für die zunehmende internationale Integration der Güterund Faktormärkte aber gegebenenfalls auch, als Antwort darauf, für die zunehmende internationale Abstimmung der Wirtschaftspolitik.

\subsection{Begründung des Globalisierungsprozesses}

Der Globalisierungsprozess kann am besten verstanden werden als das Ergebnis der Wirkungstrias Ökonomie, Technologie und Politik. Dabei stellt das ökonomische Element den theoretischen, quasi immer gültigen Regelrahmen dar. Dieser ökonomische Regelrahmen beschreibt die Grundannahme, dass alle Akteure rationale Nutzenmaximierer innerhalb der jeweiligen Restriktionen sind. Beobachtbare Handelsverflechtung, Kapital- und Arbeitsmobilität sind folglich Ausdruck einer Vielzahl von rationalen nutzenmaximierenden Einzelentscheidungen. Globalisierung ist dann die notwendige Folge, wenn sich maßgebliche Rahmenbedingungen auf signifikante Weise ändern und dazu führen, dass rationale Individuen häufiger die Option einer internationalen Strategie verfolgen. Diese Änderungen von maßgeblichen Rahmenbedingungen können nun in technologischen sowie politischen Entwicklungen gefunden werden. Die im folgenden zuerst dargestellten wirtschaftlichen Komponenten sind dann lediglich der Gradmesser, wie stark die anderen beiden Komponenten wirkten.

${ }^{243}$ Nicht-wirtschaftliche Aspekte der Globalisierung, die ebenfalls Bedeutung für die Ausgestaltung nationaler Wirtschaftspolitik besitzen, werden in dieser Arbeit nicht berücksichtigt; für soziologische und kulturelle Komponenten vgl. z.B. Beck (1997) oder den Sammelband von Scott (1997). 


\subsubsection{Wirtschaftliche Komponenten der Globalisierung}

Folgt man der $O E C D$ (1996, S. 20), lässt sich Internationalisierung durch die folgenden Kategorien des Unternehmerverhaltens erklären:

- strategische Aktionen: als first-mover oder Imitator von erfolgversprechenden Strategien.

- Entwicklung eines Wettbewerbsvorteils (Technologie, Ressourcen, Humankapital etc.).

- Konsolidierung eines/dieses Wettbewerbsvorteils (Zugang zu Technologien, Ressourcen, Humankapital etc.).

- Organisatorischer Wandel (internationales outsourcing).

Diese vier Formen unternehmerischen Handelns wirken sowohl auf den Gütermärkten als auch auf den Faktormärkten. Ströme von Gütern- und Dienstleistungen folgen dabei im wesentlichen den Gesetzmäßigkeiten, wie sie die moderne Außenwirtschaftstheorie beschreibt: Existenz von komparativen Kostenvorteilen zwischen Nationen, Ausnutzung von Größenvorteilen bei heterogenen Präferenzen der Konsumenten und unterschiedlich stark ausgenutzte Lernkurveneffekte. ${ }^{244}$ Dabei richtet sich der Umfang des Außenhandels immer nach den rechtlichen und technischen Restriktionen.

Kapitalbewegungen zwischen Ländern ergeben sich v.a. durch Ausnutzung von Zinsdifferenzen als Arbitragegeschäfte, in Form von Direktinvestitionen zur Begleitung oder Substitution von Exportgeschäften, zur Reduktion des Anlagerisikos durch Diversifizierung des Anlageportfolios oder zur Fristentransformation (Obstfeld, 1998, S. 10f). Auch die internationalen Kapitaltransfers sind stets Ausdruck der herrschenden institutionellen und technischen Umwelt. Möchte man also Globalisierung, abzulesen anhand der wirtschaftlichen Verflechtungsmaßzahlen, erklären, muss man die Veränderung dieser technologischen und institutionellen Gegebenheiten untersuchen.

\subsubsection{Technologische Komponenten der Globalisierung}

Technische Entwicklungen wirkten in zweierlei Hinsicht in Richtung fortschreitender Integration. Erstens führten diverse technische Neuerungen dazu, dass die Transportkosten von Gütern drastisch sanken (vgl. Abbildung 4.1). Dies impliziert, dass sich der Handel besonders bei jenen Gütern verstärkt, bei denen die Transportkosten einen bedeutenden Anteil an den Gesamtkosten einnehmen.

${ }^{244}$ Vgl. z.B. Maennig und Wilfling (1998) oder Krugmaniund QSbstfeld (20999):75139-8 


\section{Abbildung 4.1 : Entwicklung der Transport- und Kommunikationskosten}

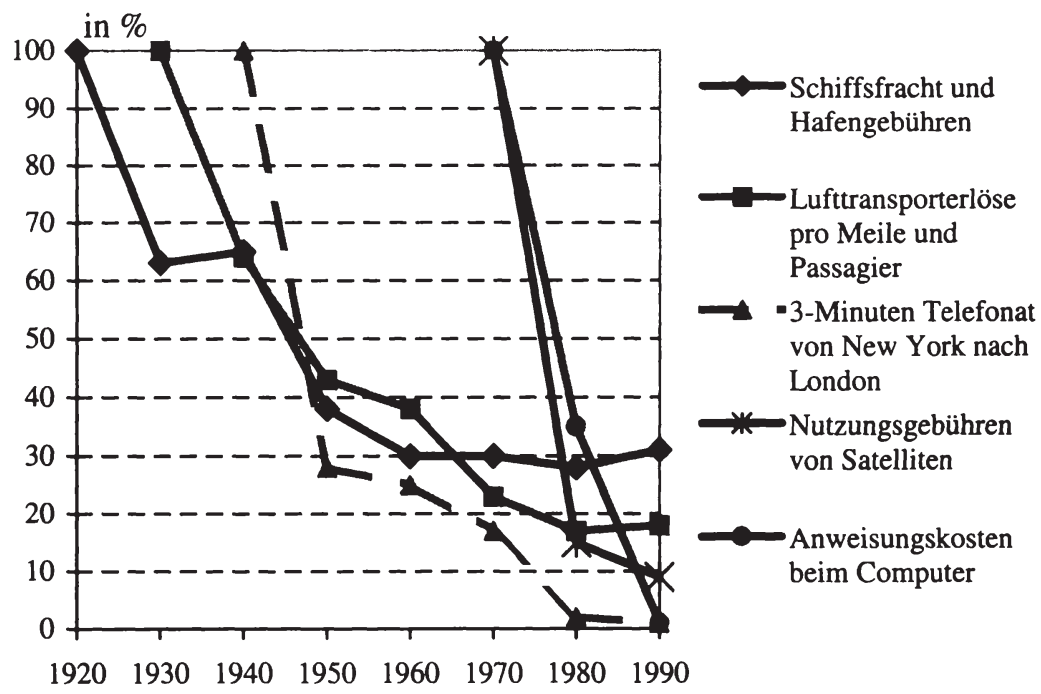

Quelle: Weltbank (1995, S. 62), Cairncross, (1997, S. 10).

Zweitens sorgten technische Entwicklungen für eine drastische Reduktion der Informationsverarbeitungskosten. Es ist gerade dieser Aspekt der Entwicklungen im Bereich der Informations- und Kommunikationstechnologie, der für die Integration der Weltwirtschaft noch bedeutsamer gewesen ist. Um dies zu zeigen, ist es nötig, auf die Transaktionstheorie Williamsons (1990, S. 59ff) einzugehen. Nach Williamson lassen sich Transaktionen durch drei Kriterien kennzeichnen: ihre Faktorspezifität, die Unsicherheit der Transaktion und die Häufigkeit der Transaktion. Dabei beschreibt der Grad der Faktorspezifität das Ausmaß der Abhängigkeit einer Transaktion von einem bestimmten Faktor (Sachkapital, Humankapital, Standort). Es ist unmittelbar einsichtig, dass zunehmende Faktorspezifität dazu führt, dass eine Unternehmung bei Annahme von opportunistischem Verhalten ${ }^{245}$ durch Anbieter des relevanten Faktors ausgenutzt werden kann. Eine Integration des Faktors in unternehmensinterne Koordinationsstrukturen wird wahrscheinlicher, da dadurch das Unternehmen der Abhängigkeit und

245 Williamson (1990, S. 54) definiert Opportunismus als „...die Verfolgung des Eigeninteresses unter Zuhilfenahme von List." Damit opportunistisches Verhalten transaktionsgefährdend wirken kann, muss allerdings zusätzlich angenommen werden, dass es keine unbegrenzte Rationalität im Sinne vollständiger Information gibt (Williamson, 1990, S. 75), da ansonsten möglicher Opportunismus vollständig antizipiert würde und demnach stets die unterlegene Strategie wäre. 
folglich hohen Faktorentgelten entgehen kann. Der Aspekt der Unsicherheit lässt sich in parametrische und Verhaltensunsicherheit unterteilen, wobei die parametrische Unsicherheit die Möglichkeit einer Änderung exogener Rahmendaten beschreibt und die Verhaltensunsicherheit das Potential strategischer Aktion seitens des möglichen Vertragspartners darstellt (Williamson, 1990, S. 64ff). Auch hier wird sofort deutlich, dass ein Anstieg der Verhaltensunsicherheit (v.a. bei spezifischen Transaktionen) eher eine vertikale Integration der Transaktion bewirkt. Schließlich ist ebenfalls offensichtlich, dass Unsicherheit hinsichtlich des Verhaltens der möglichen Vertragspartner dann geringere Bedeutung besitzt, wenn eine Transaktion sehr häufig anfällt, der Transaktionspartner folglich ein Interesse daran hat, durch korrektes Verhalten eine dauerhafte Vertragsbeziehung zu erhalten. Die Revolution der Informations- und Kommunikationskosten (vgl. Abbildung 4.1) führt nun dazu, dass die Koordination und Kontrolle von Produktionsprozessen des Vertragspartners kostengünstiger werden. ${ }^{246}$ Dadurch sinkt die Anzahl der beabsichtigten und unbeabsichtigten Vertragsverletzungen (Missverständnisse und Opportunismus), und letztlich wird die Verhaltensunsicherheit reduziert. Dies hat zur Folge, dass es für Unternehmen zunehmend sinnvoll wird, Produktionsprozesse zu desintegrieren.

Werden zusätzlich die Güter- und Faktormärkte international geöffnet (s. 4.2.3), findet diese vertikale Desintegration natürlich auch zunehmend international statt; das Volumen des Güter- und Kapitalverkehrs nimmt zu. Dieser Effekt wird dadurch verstärkt, dass durch die Grenzöffnungen für jeden Vertragspartner größere Märkte entstehen und die Anzahl der potentiellen Vertragspartner steigt; ersteres erhöht die Häufigkeit der Transaktion, letzteres reduziert die Faktorspezifität der Transaktion. Beide Effekte resultieren ebenfalls in einer zunehmenden Aufspaltung der Produktionsprozesse.

\subsubsection{Politische und institutionelle Komponenten der Globalisierung}

Seit Ende des zweiten Weltkrieges befinden sich die westlichen Industrienationen in einem kontinuierlichen Integrationsprozess. Dieser Prozess lässt sich auf drei Handlungsebenen beobachten. Auf der untersten Ebene stehen unilaterale Öffnungsschritte, die einzelne Nationalstaaten in eigener Regie vorgenommen haben. Dies betrifft oftmals die Öffnung eines Landes für ausländischen Kapitalzufluss, teilweise auch ausländische Arbeitskräftezuwanderung. Hinsichtlich der Liberalisierung der Gütermärkte gibt es sowohl regional begrenzte Integrationsabkommen (Europäische Union [EU], European Free Trade Area [EFTA],

${ }^{246}$ Der Preis für $10^{6}$ Arbeitsschritte im Computer pro Sekunde (MIPS - million instructions per second) ist von 1991 bis 1997 von 230 US-\$ auf 3,42 US-\$ gesunken (US-Department

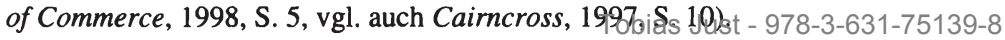


North American Free Trade Area [NAFTA], Australian New Zealand Free Trade Area [ANZCERTA]), als auch überregional quasi global verhandelte Integrationsabkommen (die Verhandlungen des General Agreement on Tariffs and Trade [GATT], die letztlich zur Gründung der World Trade Organization [WTO] führten). Tabelle 4.1 zeigt für 19 OECD-Staaten die wesentlichen internationalen Integrationsabkommen der letzten 40 Jahre. ${ }^{247}$ Es wird deutlich, dass alle untersuchten OECD-Staaten seit 1960 ihre Gütermärkte stärker in den Weltmarkt eingebunden haben. Allerdings zeigen sich dabei interessante Unterschiede. Die globalen Handelsliberalisierungsabkommen innerhalb des GATT wurden nahezu ausnahmslos von den OECD-Staaten unterschrieben. ${ }^{248}$ Hingegen gab es auf regionaler Ebene deutlich unterschiedliche Integrationstiefen und -geschwindigkeiten.

Grundsätzlich kann man die regionalen Integrationsstufen Freihandelszone, Zollunion und Binnenmarkt unterscheiden. Bei einer Freihandelszone werden zwar die Binnenzölle innerhalb des Gebiets beseitigt, jedoch sind die Außenzölle der einzelnen teilnehmenden Staaten noch nicht vereinheitlicht. Dieser Harmonisierungsschritt ist erst durch die Gründung einer Zollunion vollzogen. Ein Binnenmarkt schließlich beschreibt ein länderübergreifendes Wirtschaftssystem, bei dem alle Güter- und Faktormärkte de jure vollständig integriert sind.

Die Integrationstiefe der EG- (EU-) Staaten ist deutlich am umfangreichsten. Seit 1970 lag der Anteil der intraregionalen Exporte der EG (EU) am Gesamtexport der EG (EU) stets über 50\% mit zunehmender Tendenz. ${ }^{249}$ Für die CUSTA lag dieses Verhältnis seit 1970 immer um 35\%, für die EFTA um 30\% und für die ANZCERTA um 7\% (de la Torre und Kelly, 1992, S. 20). ${ }^{250}$

${ }^{247}$ Es wurden nur jene GATT-Verhandlungen berücksichtigt, die seit 1960 zum Abschluss kamen. Ferner wurde von einer Darstellung solcher regionaler Abkommen abgesehen, von denen kein großer Einfluss für die OECD-Staaten angenommen werden kann. Dies betrifft z.B. die von 1975-2000 unterzeichneten vier Lomé-Abkommen der EU oder das seit 1975 bestehende Freihandelsabkommen der USA mit Israel (de la Torre und Kelly, 1992).

Lediglich an der verhältnismäßig unbedeutenden Dillon-Runde, die 1961 abgeschlossen wurde, nahmen Irland, die Schweiz und Spanien nicht teil.

249 Intraregionaler Handel wird derjenige Teil des Handels genannt, der innerhalb einer Wirtschaftsregion, z.B. der EU, abläuft. Interregionaler Handel ist dann jener Handel, der zwischen Staaten stattfindet, die nicht derselben Wirtschaftsunion angehören.

${ }^{250}$ Hierbei blieb allerdings noch unberücksichtigt, dass die 1972 abgeschlossenen bilateralen Verträge zwischen der EG und EFTA für das Jahr 1977 eine de facto Freihandelszone aller EG- und EFTA-Staaten generierten. 
Tabelle 4.1 : Wichtige Integrationsabkommen der führenden OECD-Staaten

\begin{tabular}{|c|c|c|c|c|c|c|c|c|}
\hline & \multicolumn{4}{|c|}{ regionale Abkommen } & \multicolumn{4}{|c|}{ (globale) GATT-Runden } \\
\hline & $\begin{array}{l}\text { Freihandels- } \\
\text { zone }\end{array}$ & Zollunion & Binnenmarkt & EWS $^{1)}$ & $\begin{array}{l}\text { Dillon } \\
(1961)\end{array}$ & $\begin{array}{l}\text { Kennedy } \\
\text { (1967) }\end{array}$ & $\begin{array}{l}\text { Tokio } \\
\text { (1979) }\end{array}$ & $\begin{array}{l}\text { Uruguay } \\
\text { (1993) }\end{array}$ \\
\hline Australien & $\begin{array}{l}\text { ANZCERTA } \\
(1965)\end{array}$ & - & - & - & $x$ & $x$ & $x$ & $x$ \\
\hline Österreich & $\begin{array}{l}\text { EFTA (1960) } \\
\text { EFTA/EG }\end{array}$ & EU (1995) & EU (1995) & EWS (1995) & $x$ & $x$ & $x$ & $x$ \\
\hline Belgien & EG (1957) & EG (1968) & EU (1993) & EWS (1979) & $x$ & $x$ & $x$ & $x$ \\
\hline Kanada & $\begin{array}{l}\text { CUSTA } \\
(1988)^{3)}\end{array}$ & - & - & - & $x$ & $x$ & $x$ & $x$ \\
\hline Dänemark & $\begin{array}{l}\text { EFTA (1960) } \\
\text { EG (1973) }\end{array}$ & EG (1973) & EU (1993) & EWS (1979) & $x$ & $x$ & $x$ & $x$ \\
\hline Finnland & $\begin{array}{l}\text { EFTA (1961) } \\
\text { EFTA/EG }\end{array}$ & EU (1995) & EU (1995) & - & $x$ & $x$ & $x$ & $x$ \\
\hline Frankreich & EG (1957) & EG (1968) & EU (1993) & EWS (1979) & $x$ & $x$ & $x$ & $x$ \\
\hline Deutschland & EG (1957) & EG (1968) & EU (1993) & EWS (1979) & $x$ & $x$ & $x$ & $x$ \\
\hline Irland & EG (1973) & EG (1973) & EU (1993) & EWS (1979) & - & $x$ & $x$ & $x$ \\
\hline Italien & EG (1957) & EG (1968) & EU (1993) & EWS $(1979)^{4)}$ & $x$ & $x$ & $x$ & $x$ \\
\hline 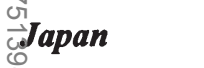 & 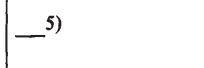 & - & - & - & $x$ & $x$ & $x$ & $x$ \\
\hline
\end{tabular}


Tabelle 4.1 (Fortsetzung)

\begin{tabular}{|c|c|c|c|c|c|c|c|c|}
\hline & \multicolumn{4}{|c|}{ regionale Abkommen } & \multicolumn{4}{|c|}{ (globale) GATT-Runden } \\
\hline & $\begin{array}{l}\text { Freihandels- } \\
\text { zone }\end{array}$ & Zollunion & Binnenmarkt & EWS $^{1)}$ & $\begin{array}{l}\text { Dillon } \\
(1961)\end{array}$ & $\begin{array}{l}\text { Kennedy } \\
(1967)\end{array}$ & $\begin{array}{l}\text { Tokio } \\
(1979)\end{array}$ & $\begin{array}{l}\text { Uruguay } \\
\text { (1993) }\end{array}$ \\
\hline Niederlande & EG (1957) & EG (1968) & EU (1993) & EWS (1979) & $x$ & $x$ & $x$ & $x$ \\
\hline Neuseeland & $\begin{array}{l}\text { ANZCERTA } \\
(1965)\end{array}$ & - & - & - & $x$ & $x$ & $x$ & $x$ \\
\hline Norwegen & $\begin{array}{l}\text { EFTA (1960) } \\
\text { EFTA/EG }\end{array}$ & - & - & - & $x$ & $x$ & $x$ & $x$ \\
\hline Schweden & $\begin{array}{l}\text { EFTA (1960) } \\
\text { EFTA/EG }\end{array}$ & EU (1995) & EU (1995) & - & $x$ & $x$ & $x$ & $x$ \\
\hline Schweiz & $\begin{array}{l}\text { EFTA (1960) } \\
\text { EFTA/EG }\end{array}$ & - & - & - & - & $x$ & $x$ & $x$ \\
\hline Spanien & EG (1986) & EG (1986) & EU (1993) & EWS (1989) & 一 & $x$ & $x$ & $x$ \\
\hline$\underset{C}{E} K$ & $\begin{array}{l}\text { EFTA (1960) } \\
\text { EG (1973) }\end{array}$ & EG (1973) & EU (1993) & EWS $(1990)^{4)}$ & $x$ & $x$ & $x$ & $x$ \\
\hline $\mathbb{U}_{0}^{\bar{n}} S A$ & $\begin{array}{l}\text { CUSTA (1988) } \\
\text { NAFTA (1995) }\end{array}$ & - & - & - & $x$ & $x$ & $x$ & $x$ \\
\hline
\end{tabular}

Alnmerkungen:

${ }^{1}$ EWS steht für Europäisches Wăhrungssystem, das am 1. Januar 1979 in Kraft trat.

2) EFTA/EG: Freihandelskooperation zwischen der EFTA und der EG (1972 beschlossen und 1977 weitgehend realisiert).

3) CUSTA steht für Canada-United States Free Trade Area.

4) Italien und Großbritannien scheiden im September 1992 kurzzeitig aus dem EWS aus.

5) Japan ist zwar Mitglied in einigen asiatischen Organisationen wie z.B. der ASEAN [Association of Southeast Asian Nations]; der Umfang der Handelsliberalisierung ist aber äußerst gering.

Quellen: $\quad$ Eigene Zusammenstellung auf der Grundlage von de la Torre und Kelly (1992), IMF (1993), Kohler-Koch und Woyke (1996), Maennig und Wilfling (1998), WTO (2000). 
Dass die Integrationstiefe der EG (EU) im Gegensatz zu den anderen Freihandelszonen stetig zunahm, liegt zum einen daran, dass der Club der EGMitglieder stetig ausgeweitet wurde, und zum anderen darin begründet, dass die EG nicht nur eine Freihandelszone blieb, sondern auch die Faktormärkte integrierte. Im Abschnitt 4.6 wird auf den Vergleich der unterschiedlichen Integrationsregimes rekurriert.

Da oftmals (z.B. Zevin, 1992 und Bairoch, 1996) das Argument geäußert wurde, dass die Integrationsschritte nach dem zweiten Weltkrieg lediglich die Protektionspolitik der Zwischenkriegsjahre rückgängig machten und dass folglich die Weltwirtschaft nicht stärker integriert sei als vor dem 1. Weltkrieg, soll im folgenden Abschnitt dieses Argument kurz auf seine Stichhaltigkeit geprüft werden. ${ }^{251}$

\subsection{Globalisierung gestern und heute}

Es ist fraglos ungenau zu sagen, erst seit einigen Jahren wären Globalisierungstendenzen festzustellen. So spricht von Weizsäcker (1999, S. 47f) denn auch lediglich von einer ,neuen Etappe " eines viel umfassenderen Globalisierungstrends, der seit einigen Jahrhunderten immer wieder Phasen der wirtschaftlichen Öffnung sowie des Protektionismus aufwies. ${ }^{252}$

Einige Autoren gehen sogar so weit zu sagen, dass die letzte Globalisierungsphase, die mit dem Ende des 1. Weltkrieges endete, umfassender war als die heutige (Zevin, 1992; Bairoch, 1996) ${ }^{253} \mathrm{Ihr}$ Argument lässt sich anhand von einigen Daten veranschaulichen; Tabelle 4.2 zeigt den Anteil, den die Exporte am Bruttoinlandsprodukt (BIP) zu unterschiedlichen Zeitpunkten der letzten 100 Jahre für ausgewählte Länder ausmachten. Für die USA lag die Relation erst in den 90er Jahren des 20. Jahrhunderts über dem entsprechenden Verhältnis von 1890. In Japan sind sogar 1997 noch nicht die Werte der 20er Jahre erreicht. Ähnliches gilt für die Länder der heutigen Europäischen Union; 1992 war deren Relation in etwa so hoch wie 1913. Allerdings ist bemerkenswert, und diese Aussage entgeht Bairoch (1996) aufgrund seiner kürzeren Datenreihe, dass der

251 Vgl. dazu auch die Diskussion des „law of constant protection“ in Fußnote 294.

${ }^{252}$ Wann indes dieser Trend einsetzte, wird in der Literatur alles andere als einheitlich beantwortet. Karl Marx legte den Beginn bereits in das 15. Jahrhundert, da sich zu diesem Zeitpunkt erste kapitalistische Systeme bildeten. Andere Autoren datieren den Beginn jedoch erst auf das Ende des Ost-West-Konflikts am Ende des 20. Jahrhunderts (vgl. dazu Beck, 1997, S. 44).

${ }^{253}$ Die umfassendste Analyse der Globalisierungsphase vor dem 1. Weltkrieg ist wahrscheinlich die Arbeit von O'Rourke und Williamson (1999)oias Just - 978-3-631-75139-8 
Außenhandel seit der Vollendung des europäischen Binnenmarktes 1992 wieder deutlich stärker gewachsen ist als das BIP.

Tabelle 4.2 : Exporte von Handelswaren als Prozentanteil vom BIP

\begin{tabular}{cccc}
\hline & USA & West-Europa & Japan \\
\hline 1890 & 6,7 & 14,9 & 5,1 \\
1913 & 5,4 & 18,3 & 12,6 \\
1929 & 5,0 & 14,5 & 13,6 \\
1938 & 3,7 & 7,1 & 13,0 \\
1950 & 3,8 & 13,4 & 6,8 \\
1970 & 4,0 & 17,4 & 9,7 \\
1992 & 7,5 & 21,7 & 8,8 \\
1997 & 12,1 & 31,4 & 11,1 \\
\hline
\end{tabular}

Anmerkungen: Bairoch gibt nicht an, welche Staaten in der Gruppe West-Europa zusammengefasst wurden. Bis auf 1950 und 1997 beruhen die Werte auf 3Jahresdurchschnittswerten. Für 1997 wurden die 15 EU-Staaten zu WestEuropa gezählt.

Quelle: Für die Jahre 1890-1992 vgl. Bairoch (1996), S. 179, für 1997 vgl. OECD (1999).

Eine ähnliche Aussage lässt sich mit Blick auf die Kapitalströme (hier ausdrücklich Direkt- und Portfolioinvestitionen) im intertemporalen Vergleich machen. Tabelle 4.3 zeigt, dass die Relation aus der Höhe der Leistungsbilanz zum BIP lediglich für Deutschland einen historischen Höchstwert im letzten Jahrzehnt erreicht hat. Für alle anderen (hier dargestellten) Länder waren zu Beginn des Jahrhunderts teilweise deutlich höhere Werte verbucht. Die naheliegende Schlussfolgerung könnte sein, dass auch die Kapitalmärkte vor 100 Jahre zumindest einen ähnlich hohen Integrationsgrad erreicht hatten wie heute. ${ }^{254}$

Hinsichtlich der Mobilität des Faktors Arbeit war die Welt Ende des 19. Jahrhunderts deutlich globaler, als sie es heute ist; durchschnittlich emigrierten 2-5\% der westeuropäischen Bevölkerung innerhalb eines Jahrzehnts (Baldwin und Martin, 1999, S. 37).

254 Allerdings ist dieser abgeleitete Kapitalmarktindikator nicht sehr zuverlässig. Zwar ist bei relativ konstanten Devisenreserven der Saldo der Leistungsbilanz immer gleich dem Saldo der Kapitalverkehrsbilanz, jedoch können bei identischen Salden ungleich große Summanden vorliegen. Auch können geringe Kapitalströme gerade dann ein Zeichen hoher Kapitalmarktintegration sein, wenn der internatienale Zinsasusglejich nerfekt funytioniert. 
Jedoch besitzt die aktuelle Globalisierungsphase einige Kennzeichen, die sie deutlich von früheren Phasen unterscheidet und die den Schluss zulassen, dass die Weltwirtschaft am Ende des 20. Jahrhunderts stärker integriert ist als vor dem 1 . Weltkrieg.

Tabelle 4.3 : Absolute Höhe der Leistungsbilanz relativ zum BIP von 1970-1996

\begin{tabular}{lccccc}
\hline & UK & USA & Frankreich & Deutschl. & Japan \\
\hline $1870-1889$ & 4,6 & 0,7 & 2,4 & 1,7 & 0,6 \\
$1890-1913$ & 4,6 & 1,0 & 1,3 & 1,5 & 2,4 \\
$1919-1926$ & 2,7 & 1,7 & 2,8 & 2,4 & 2,1 \\
$1927-1931$ & 1,9 & 0,7 & 1,4 & 2,0 & 0,6 \\
$1932-1939$ & 1,1 & 0,4 & 1,0 & 0,6 & 1,0 \\
$1947-1959$ & 1,2 & 0,6 & 1,5 & 2,0 & 1,3 \\
$1960-1973$ & 0,8 & 0,5 & 0,6 & 1,0 & 1,0 \\
$1974-1989$ & 1,5 & 1,4 & 0,8 & 2,1 & 1,8 \\
$1989-1996$ & 2,6 & 1,2 & 0,7 & 2,7 & 2,1 \\
\hline
\end{tabular}

Quelle: Baldwin und Martin (1999, S. 17).

Baldwin und Martin (1999, S. 30) und Bordo, Eichengreen und Irwin (1999 S. 7) weisen darauf hin, dass nicht zuletzt durch einen starken Anstieg der Staatsquote (Staatsausgaben in Relation zum BIP) innerhalb der letzten 100 Jahre der Anteil der non-tradeables am gesamten Bruttoinlandsprodukt stark gestiegen ist. Bei relativ konstantem Verhältnis der Exporte zum BIP bedeutet das, dass dieselbe Quote von immer weniger Industrien getragen wird. Diese Industrien haben demnach eine deutliche Öffnung erfahren und folglich auch einen starken Anstieg der internationalen Konkurrenz aufzuweisen. Nehmen wir für WestEuropa beispielsweise an, dass die durchschnittliche Staatsquote um die Jahrhundertwende bei $10 \%$ lag und heute ungefähr mit $50 \%$ zu beziffern ist, dann lässt sich mit Hilfe der Werte aus Tabelle 4.2 ermitteln, dass der Anteil der Exporte an der privaten Produktion im Jahre 1913 nicht die ausgewiesenen 18,3\%, sondern 20,3\% ausmacht. Gegen Ende des 20. Jahrhunderts machten die Exporte in Europa indes nicht 31,4\%, sondern 62,8\% der privaten Produktion aus. Selbst wenn man zugesteht, dass diese Überschlagsrechnung sehr ungenau ist, so wird doch deutlich, dass obige Zahlen die Entwicklung des Integrationsgrad des Warenhandels stark unterzeichnen.

Ein weiteres Merkmal des modernen Globalisierungsprozesses im Vergleich zu früher ist das bereits angesprochene Aufbrechen der Wertschöpfungskette. Dies 
bedeutet, dass die Warenströme wesentlich komplexer geworden sind; Unternehmen sind an mehreren Punkten der Wertschöpfungskette in die internationale Arbeitsteilung eingebunden. Auch lässt sich heute gerechtfertigterweise von Globalisierungsentwicklungen sprechen, da die Zahl der in die Weltwirtschaft integrierten Länder stetig zugenommen hat; Entwicklungs- und Schwellenländer sind heute stärker in die internationale Arbeitsteilung eingebunden als vor 100 Jahren (Freytag, Meier und Weiß, 1998, S. 10).

Hinsichtlich der Investitionsströme weisen Bordo, Eichengreen und Irwin (1999, S. 28ff) darauf hin, dass sich Direktinvestitionsströme vor der Jahrhundertwende auf sehr wenige Sektoren konzentrierten - allein der Eisenbahnsektor nahm 40\% der britischen Direktinvestitionen auf. ${ }^{25}$ In der heutigen Globalisierungsphase sind wesentlich mehr Sektoren in internationale Kapitalströme integriert, was gleichbedeutend damit ist, dass auch wesentlich mehr Sektoren sensibel auf Entwicklungen in anderen Ländern reagieren.

Einen der zentralen Unterschiede der beiden Globalisierungsphasen sehen Bordo, Eichengreen und Irwin (1999) - ähnlich wie Bairoch (1996) - allerdings in der Entwicklung von Institutionen. Dazu zählen sie nicht nur internationale Organisationen, sondern v.a. jene Institutionen, die den Informationsfluss erleichtern, wie z.B. Kommunikationsmedien oder vergleichbare Rechnungssysteme. Beide Errungenschaften führten dazu, dass das Vertrags- und Kontrollrisiko einer ausländischen Aktivität gesenkt wurde. Dies ist für sie auch ein Grund dafür, dass trotz zugenommener Integration die Krisenanfälligkeit des globalen Wirtschaftssystems eher ab- als zugenommen hat. Dies bedeutet nicht, dass Krisen völlig ausgeschlossen sind (z.B. Asien-Krise 1996/1997 oder die Mexiko-Krise 1994), sondern dass die Krisen in Relation zu der Größe der Kapitalströme geringer ausfallen und dass sich die betroffenen Länder rascher wieder erholen können als früher.

Alle diese Wesensmerkmale weisen in dieselbe Richtung. Die Weltwirtschaft ist heute stärker integriert als zu jedem anderen Zeitpunkt der Geschichte. Dies beeinflusst dann auch zwangsläufig die Wirkungsverbundenheit nationaler Wirtschaftspolitik mit anderen Staaten. Wheeler und Pozo (1997) können z.B. zeigen, dass Schocks in Großbritannien zunehmend die makroökonomischen Geld-, Einkommens- und Preisparameter in den USA in Folgeperioden beeinflussen. Für die erste Globalisierungsepisode können Wheeler und Pozo jedoch keinen signifikanten Zusammenhang feststellen.

255 Baldwin und Martin (1999, S. 35) kommen zwar grundsätzlich zu demselben Urteil; sie schätzen den Anteil, den die Eisenbahn als Investitionsobjekt für Direktinvestitionen vor 1914 ausmachte, auf 20\%. Allerdings konzentrieren sie sich dabei nicht auf GroBbritannien, sondern berücksichtigen alle großen Investitionsländer ingihgen_Rechnengens 
Ist es also richtig, dass die aktuelle Globalisierungsphase in Art und Umfang grundsätzlich neu ist, reicht es offensichtlich nicht, mögliche Konsequenzen mit Verweis auf die historische Entwicklung abzutun, sondern man muss die Diskussion ganz neu führen. Innerhalb der aktuellen Diskussion wird dabei am heftigsten gestritten, inwiefern der Globalisierungstrend staatlichen Interventionismus und dabei insbesondere staatliche Umverteilungspolitik überhaupt noch zulässt.

\subsection{Race to the Bottom oder Race to the Top?}

Besonders in der politischen Rhetorik und in populärwissenschaftlichen Veröffentlichungen (z.B. Martin und Schumann, 1997) wird immer wieder betont, dass die logische Konsequenz zunehmender Globalisierung sein muss, dass mobile Produktionsfaktoren (Human- und Realkapital) an jene Standorte abwandern, in denen die Abgabenbelastung besonders niedrig ist. Innerhalb dieses Argumentationsstrangs ist die logische Konsequenz die zwischenstaatliche Konkurrenz der Systeme um diese knappen Ressourcen mit der Folge, dass die mobilen Faktoren von der Steuerbelastung befreit werden und die Abgabenlast auf die immobilen Produktionsfaktoren fällt. Da der Grad der Mobilität heute mit dem Ausbildungsniveau korreliert (Straubhaar, 1998), ${ }^{256}$ müsste die Abgabenbelastung gerade der gering Qualifizierten ansteigen. Da ferner nach dem Stolper-Samuelson-Theorem gerade diese Gruppe in Industrieländern relative Entlohnungseinbußen hinzunehmen hat, falls sich eine kapitalintensiv produzierende Volkswirtschaft dem Weltmarkt öffnet, wäre die direkte Folgerung, dass in dieser Gruppe die Nettolöhne sinken müssen oder dass die Abgabenlast solange gesenkt wird, bis sich die Staaten auf dem Mindeststandard treffen ${ }^{257}$ Es käme zu dem viel zitierten race to the bottom insbesondere im Bereich der Sozialausgaben.

Diese Argumentation erhält ernstzunehmenden Flankenschutz auch von wichtigen Vertretern der ökonomischen Profession. Sinn (1990) argumentiert, dass Harmonisierungsbedarf der staatlichen Ausgaben- und Einnahmenpolitik be-

${ }^{256}$ Hier ist darauf hinzuweisen, dass tatsächliche Migration immer eine Teilmenge von potentieller Migration darstellt. Zwar sind gering Qualifizierte angesichts hoher Einkommensdifferentiale oftmals potentielle Migranten, jedoch wird die Migration entsprechend der Einwanderungspolitik des Einwanderungslandes oft nicht zugelassen, wohingegen Hochqualifizierte gern gesehene Gäste sind.

${ }^{257}$ Es ist beachtenswert, dass dieses Verteilungsszenario keineswegs zwangsläufig ist. Es sind durchaus Fälle innerhalb einer Stolper-Samuelson-Modellwelt konstruierbar, in der alle beteiligten Produktionsfaktoren positive Einkommensentwicklungen nach der Grenzöffnung für Güterhandel verzeichnen (Bhagwati, 1999-2S.J225f)978-3-631-75139-8 
steht, da der zentrale Aspekt der Sozialgesetzgebung die Absicherung gegen nicht am Markt versicherbare Risiken ist. Eine staatliche Intervention wäre folglich dann effizienzsteigernd, wenn eine grundsätzliche Nachfrage nach einer Risikoabsicherung auch der nicht marktlich versicherbaren Risiken bestünde. Dies anzunehmen, erscheint Sinn plausibel. Auf der Grundlage des Rawls'schen (1971) Konzepts des veil of ignorance argumentiert Sinn, dass sich Menschen zu Beginn ihres Lebens, wenn sie noch nicht wissen können, ob sie im Leben zu den Gewinnern oder den Verlierern gehören werden, immer für Umverteilungsmechanismen entscheiden, um die Kosten, die entstehen, wenn man Verlierer wird, zu reduzieren. Unterschiede in nationalen Sozialversicherungssystemen offerieren die Möglichkeit, dass diejenigen, die sich ex post als Gewinner erweisen, jene Versicherungsgesellschaft mit hohen Versicherungsbeiträgen verlassen können. Dies muss nach Sinn zum Zusammenbruch des eigentlich effizienzsteigernden Verteilungsmechanismus führen. ${ }^{258}$ Dieser Zusammenbruch des Sozialsystems liegt folglich in einem Zeitinkonsistenzproblem begründet - die potentiellen maßgeblichen Träger eines Umverteilungsmechanismus zur Reduktion nicht versicherbarer Risiken sehen sich diesen Risiken nicht mehr ausgesetzt und haben keinen Anreiz, diese Versicherung für andere zu leisten.

Andere Autoren betonen, dass es zahlreiche Argumente dafür gibt, dass das Szenario des race to the bottom unzutreffend ist. So argumentieren z.B. Siebert und Koop (1990, S. 5ff) und Schäfer (1999, S. 12), dass Wettbewerb zwischen den Systemen nur in jenen Bereichen der Wirtschaftspolitik zu einer Harmonisierung auf unterem Niveau führen wird, in denen es kein Innovationspotential gibt. Internationale Systemharmonisierung muss jedoch in innovationsfähigen Bereichen scheitern, da selbst wohlmeinende Politiker ex ante nicht das bestmögliche System bestimmen können. Wettbewerb ist im Hayek'schen Sinne (1968) zur Entdeckung der besten Lösung erforderlich.

So kommen denn auch Siebert und Koop (1990), Berthold (1997) und von Weizsäcker (1999) zu ganz anderen Szenarien. Zwar gehen sie davon aus, dass die Besteuerung von mobilen Faktoren erschwert und folglich zurückgehen wird. Dies bedeutet aber keineswegs das Ende des Sozialstaats und den Zwang zur Harmonisierung. ${ }^{259}$ Streng im Sinne des klassischen Tiebout-Modells (1956) des Wettbewerbs zwischen lokalen Anbietern wird argumentiert, dass unterschiedliche Präferenzen nach öffentlichen Gütern in verschiedenen Ländern auch unterschiedliche Sozialregime parallel existieren lassen können. ${ }^{260}$ Dies illustriert $v$.

${ }^{258}$ Vgl. auch Scharpf (1997).

${ }^{259}$ Es geht im folgenden ausschließlich um die Umverteilungskomponente staatlicher Intervention. Sozialversicherungen z.B. haben immer auch eine allokative Komponente. Dieses effizienzsteigernde Element bleibt auf jeden Fall erhalten (Krueger, 2000).

${ }^{260}$ Dabei ist sicherlich der auffäligste Unterschied, dass Tiebout in seinem idealtypischen

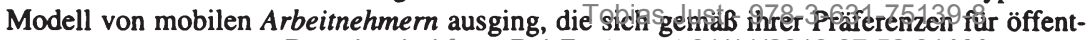


Weizsäcker (1999, S. 59ff) mit Hilfe des Konzepts des Wettbewerbslohns. Dieser ist die Summe aus dem Individuallohn, den der Arbeitnehmer erhält und dem Kollektivlohn, der den Umfang des fiskalischen Staatseingriffs darstellt. Der Wettbewerbsprozess gleicht lediglich die Wettbewerbslöhne aus. Gibt es aber in einzelnen Ländern unterschiedliche Auffassungen hinsichtlich der Aufteilung dieses Lohnes auf Individual- und Kollektivlohn, so folgt, dass verschiedene soziale Standards koexistieren können. Ähnlich ist auch die Schlussfolgerung von Feld (2000a, S. 195), der betont, dass Einkommensumverteilung durchaus möglich ist und auch in Zukunft sein wird, solange die Steuerpolitik von den Steuerzahlern akzeptiert wird. Dabei ist bemerkenswert, dass die Steuermoral zunimmt, wenn die Stimmbürger stärkeres Mitspracherecht bei der Steuergesetzgebung über direktdemokratische Institutionen bekommen (Pommerehne und Weck-Hannemann, 1996). ${ }^{261}$ Dies führt dann wiederum dazu, dass in Regionen, in denen die Bürger über Budgetfragen mitentscheiden können, die Höhe der Einkommensteuer an Bedeutung bei der Wahl des Wohnorts verliert. Feld (1997) spricht davon, dass in Jurisdiktionen, in denen die Bürger eine VoiceOption besitzen, die Exit-Option seltener Anwendung findet. Für die Schweizer Kantone findet er dafür auch empirische Bestätigung. ${ }^{262}$

Auch mit Bezug auf die mobilen Faktoren ist es keineswegs eindeutig, dass sie überhaupt keinen Anteil an der Finanzierung öffentlicher Leistungen haben werden. Sie werden sich dann beteiligen, wenn den Finanzierungslasten höhere Nutzen aus dem öffentlichen Gut gegenüberstehen.

So ist die empirische Evidenz zur Überprüfung des Kapitalsteuerwettbewerbs keineswegs eindeutig. Feld (2000b, S. 97-158) kommt in seiner Synopse der vorliegenden empirischen Studien zum lokalen, regionalen und internationalen Steuerwettbewerb zu einem sehr gemischten Fazit. Lediglich bei multinationa-

liche Güter die optimale Gebietskörperschaft aussuchen. In der heutigen Diskussion ist der mobile Faktor indes Kapital. Tiebout selber räumt ein, dass realiter der Faktor Arbeit nicht so mobil ist, dass der Wettbewerb zwischen lokalen Systemen vollkommen sein kann. Da der Faktor Kapital heute vergleichsweise mobil geworden ist, ist der moderne Systemwettbewerb eher mit der Tiebout'schen Idealwelt vereinbar. Gleichwohl müssen, damit das Tiebout-Ergebnis erzielt werden kann, weitere zentrale Annahmen erfüllt sein (vgl. Tiebout, 1956, S. 450): die Kapitalanleger müssen vollständige Informationen haben, es gibt genügend viele konkurrierende Anbieter, und es gibt keine Externalitäten zwischen den Gemeinschaften. Das schließt dann auch intertemporale Externalitäten im Sinne des oben angesprochenen Zeitinkonsistenzproblems ein.

${ }^{261}$ Zunehmend gibt es theoretischen und empirischen Befund, dass eine Stärkung direktdemokratischer Elemente in einer Verfassung effizienzsteigernd sein kann. Vgl. neben vielen Kirchgässner (2000), Feld und Matsusaka (2000) und Zimmermann und Just (2000).

${ }^{262}$ Feld (1997) testet die original Tiebout-Hypothese mobiler Arbeitskräfte. Es wird nicht geprüft, ob in Jurisdiktionen, in denen die Stimmbürger budgetäre Kompetenzen besitzen, auch der fiskalische Wettbewerb um mobiles Kapitaleingeschränkt ist_631-75139-8 
len Unternehmen wird die internationale Standortwahl durch Steuergesetzgebung (Kapitalbesteuerung) signifikant dergestalt beeinflusst, dass niedrigere Steuersätze (höhere öffentliche Subventionen) auch zu einer Zuwanderung an Kapital von multinationalen Unternehmen führten. ${ }^{263}$ Für die Gesamtheit aller Unternehmen gilt ein ähnlicher Zusammenhang zwar auf der regionalen, nicht aber auf der lokalen Ebene. Dass sie sich dann auch tatsächlich an der Finanzierung beteiligen, erfordert allerdings eine strengere Anwendung des Äquivalenzprinzips. Es ist sogar nicht auszuschließen, dass dies für Teile der Umverteilungspolitik gelten kann. Sichert z.B. die Existenz einer funktionierenden Sozialversicherung einen geringen Krankheitsstand, höhere Arbeitsmotivation und verhaltenere Arbeitskämpfe (sozialer Frieden), dann ist es auch für gewinnorientierte Unternehmen rational, diese Umverteilungsmechanismen zu erhalten (Siebert und Koop, 1990, S. 12). ${ }^{264}$

Berthold (1997, S. 29f) macht auf einen weiteren Sachverhalt aufmerksam: Wenn die Öffnung der Märkte für alle beteiligten Nationen zu Einkommenszugewinnen führt und man davon ausgeht, dass die Güter „Gerechtigkeit" und „Sicherheit" superiore Güter sind, dann wird die Nachfrage nach diesen Gütern im Zuge der Globalisierung auch überproportional zunehmen. ${ }^{265}$

Schäfer (2000) weist sogar darauf hin, dass Globalisierung auch bedeutet, dass potentielle politische Renten dadurch verhindert werden, dass Politiker durch den Handlungsdruck zu effektiver Politik gedrängt werden. Globalisierung zwingt verstärkt zur Innovation, um mögliche Pioniergewinne zu realisieren. Selbst wenn man zulässt, dass nicht jedes Sozialexperiment erfolgreich sein muss, wird nach Schäfer der Systemwettbewerb insgesamt zu einer höheren allokativen Effizienz führen. Dieser Mechanismus spielt sich vornehmlich auf der Ordnungs- und weniger auf der Prozessebene ab.

Es besteht allerdings weitgehend Konsens darüber, dass die Globalisierung ineffizient bereitgestellte öffentliche Güter (v.a. Umverteilung) nicht mehr zulässt.

${ }^{263}$ Aber selbst bei der Gewinnverlagerung über Transferpreise in Gebietskörperschaften mit niedrigeren Gewinnsteuersätzen sind die bislang durchgeführten Untersuchungen nicht eindeutig (Feld, 2000b, S. 153f).

Dass das Argument des sozialen Friedens keine akademische Gedankenspielerei darstellt, belegen Entwicklungen in zahlreichen unterentwickelten Ländern, in denen fehlende soziale Sicherungssysteme zu steigenden Kriminalitätszahlen führten, die wiederum die wohlhabenden Bürger zu steigenden privaten Sicherungsausgaben veranlassten (Grill, 2000).

${ }^{265}$ Freilich sind bei diesem Argument die Hauptprobleme der Bereitstellung der Güter „Sicherheit" und „Gerechtigkeit“ ausgeklammert. Da es sich hierbei nämlich um öffentliche Güter handelt, muss zum einen das Trittbrettfahrerproblem und zum anderen das oben dargestellte Zeitinkonsistenzproblem gelöst werden. Interessanterweise gibt es empirischen Befund, dass bislang ein positiver Zusammenhang zwischen der Offenheit eines

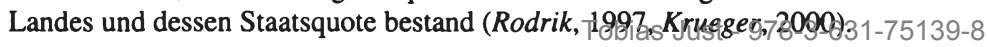


Der Wettbewerb zwischen den Systemen führt dazu, dass der Umfang der staatlichen Interventionstiefe um den Betrag der Ineffizienzen abnehmen wird. ${ }^{266}$

Es wurde in Kapitel zwei gezeigt, dass grundsätzlich ideologische Politikunterschiede innerhalb eines demokratischen Systems erhalten bleiben können. Es wurde darüber hinaus in Kapitel drei argumentiert, dass ideologisch gefärbte Wirtschaftspolitik, die weitgehend auf Werturteilen beruht, sowohl in der Arbeitsmarktpolitik, der Preispolitik als auch in der Haushaltspolitik zu sehr verschiedenen Politikergebnissen führen kann. Inwiefern diese Unterschiede auch angesichts international integrierter Märkte bestehen bleiben, kann allein eine empirische Analyse zeigen. Hinsichtlich der Persistenz ideologischer Ausrichtung von Wirtschaftspolitik sind grundsätzlich drei Entwicklungen möglich:

1. Es kommt tatsächlich zu dem race to the bottom. Dann müssten die Staatsausgaben und Staatseinnahmen dramatisch abnehmen und zwar unabhängig von der ideologischen Ausrichtung der amtierenden Partei. Bei Ländern, die weniger stark international integriert sind, sollte dieser Effekt geringer ausfallen.

2. Es werden lediglich die ineffizienten Regulierungen durch Arbitragegeschäfte von abwandernden Human- und Realkapitaleignern reduziert. Die Reduzierung der Ausgaben und Einnahmen des Staates würde zwar stattfinden, aber der Staatseingriff bliebe in den Ländern signifikant verschieden. Innerhalb eines Landes hätten alle Parteien nur noch die Möglichkeiten, den Präferenzen der Wahlbürger zu entsprechen. Unterschiede zwischen Parteien könnten nicht identifiziert werden.

3. Es gibt weiterhin signifikanten Staatseingriff und es besteht Spielraum der Parteien, ideologische Akzente zu setzen. Es kommt dann nicht nur zu Unterschieden zwischen verschieden stark integrierten Wirtschaften, sondern auch zwischen ideologisch unterschiedlich ausgerichteten Regierungen.

Der gemeinsame Nenner aller drei Hypothesen ist allerdings, dass Handlungsspielräume insgesamt reduziert werden. Es soll daher im folgenden Abschnitt der Frage nachgegangen werden, warum rational handelnde Politiker sukzessiv durch Öffnung der Märkte eigene Handlungsmacht abgegeben haben.

266 Neben Siebert und Koop (1990), Berthold (1997) und von Weizsäcker (1999) vgl. auch Willgerodt (1998), Eekhoff (1998) Schäfer (1999, 2900) und Krugeger $(2000)_{5139-8}$ 


\subsection{Politische Ökonomie der Gütermarktliberalisierung}

Dies soll in einem polit-ökonomischen Modell geschehen, in dem wiederwahlorientierte Politiker die Zollpolitik als Instrument nutzen, um ihre Wiederwahlwahrscheinlichkeit zu erhöhen.

In polit-ökonomischen Modellen der Protektion wird davon ausgegangen, dass es einen Markt für Protektion gibt, auf dem Politiker als Anbieter und einzelne Interessenvertretungen v.a. der Industrie als Nachfrager von unterschiedlicher Zollschutzintensität auftreten (Baldwin, 1982, Magee und Brock, 1983, Frey, 1985 und Amelung, 1989).

Um die Wahlchancen zu erhöhen, mögen Politiker Stimmen aus zwei unterschiedlichen Wählergruppen benötigen. Die erste Gruppe stellt die Mehrzahl der Bevölkerung dar, deren Wohlfahrtsposition durch vergrößerte Konsummöglichkeiten mit zunehmender Handelsliberalisierung zunimmt. Allerdings sind diese Nutzengewinne pro Kopf verhältnismäßig gering, sodass ein Abweichen von der für diese Gruppe optimalen Freihandelsoption nur zu geringen Verlusten an Wählerstimmen führen wird. Dies wird dadurch verstärkt, dass die Gruppe der Freihandelsbefürworter sehr groß und heterogen ist (Frey, 1985, S. 24) und dass somit nur mit geringer organisierter Lobby-Aktivität zu rechnen ist (Olson, $1965)^{267}$

Auf der anderen Seite befindet sich eine kleine, homogene Gruppe von Zollbefürwortern, deren Nutzenniveau stark sinkt, falls ihr optimales Zollniveau verlassen würde. Wähler aus dieser Gruppe strafen die Regierung durch Stimmenentzug sehr schnell ab, da Zollschutz für ihren Wirtschaftssektor von großer Bedeutung bei ihrer Wahlentscheidung ist. ${ }^{268}$ Geht man davon aus, dass es keine Konfliktbeziehungen der Zollpolitik zu anderen Politikfeldern gibt, dann könnte als Ziel der Regierung postuliert werden, die Stimmenverluste aufgrund der Zollpolitik zu minimieren. Wichtig ist, dass die Zollpolitik lediglich ein Parameter im gesamten Parteiprogramm ist. Solange keine Aussagen über die anderen wahlrelevanten Programminhalte gemacht werden, ist also auch keine Aussage hinsichtlich des Wahlausgangs möglich. Daher ist es für die zur Wahl ste-

Es kann sogar davon ausgegangen werden, dass es in der Wahlbevölkerung ein Informationsdefizit hinsichtlich der Vorteilhaftigkeit der Freihandelsposition bzw. hinsichtlich der exakten Höhe der Vorteile gibt (Frey, 1985, S. 24).

Sowohl die Gruppe der Protektionsbefürworter als auch die Gruppe der Freihandelsbefürworter ist natürlich in der Realität heterogener, als es dieses einfache Modell annimmt. Beispielsweise vertritt jeder Konsument nicht nur seine Interessen als Konsument, sondern immer auch als Einkommensbezieher die Interessen seiner Branche. AuBerdem gibt es mitunter unterschiedliche Interessenlagen innerhalb einer Branche (Kapitaleigner, Arbeiter, Humankapitaleigner). Allerdings weist Magee (1980) darauf hin, dass die Interessen von Arbeit und Kapital in den meisten Branchen kongruieren. 978-3-631-75139-8 
henden Politiker mit Hinblick auf diesen einen Parameter rational, mögliche Stimmenverluste in der Zollpolitik zu minimieren.

Die Verlustfunktionen der beiden Wählergruppen $V_{G 1}$ und $V_{G 2}$ zeigen an, wieviele Stimmen einer Partei verloren gehen, wenn sie von der Idealposition der jeweiligen Gruppe abweicht. Diese Funktionen sind streng konvex mit einem einzigen Minimum auf der gruppenspezifischen Präferenzposition $\mathrm{z}_{\mathrm{Gi}}$ mit $i \in\{1 ; 2\}$ :

$$
\mathrm{V}_{\mathrm{Gi}}=\lambda_{\mathrm{i}} \cdot\left(\mathrm{z}-\mathrm{z}_{\mathrm{Gi}}\right)^{2}
$$

Dabei steht $\mathrm{z}_{\mathrm{G} 1}$ für die optimale Zollstrategie der großen Gruppe der Konsumenten, $\mathrm{z}_{\mathrm{G} 2}$ für das optimal erachtete Zollniveau aus Sicht der protektionsnachfragenden Gruppe und $\mathrm{z}$ für das tatsächlich realisierte Zollniveau, $\lambda_{i}$ mit $\mathrm{i} \in$ $\{1 ; 2\}$ und $\lambda_{2}>\lambda_{1}>0$ repräsentieren die Bedeutung der Zollpolitik für die Wahlentscheidung der beiden Gruppen. ${ }^{269}$

Wiederwahlorientierte Politiker minimieren die Stimmenverlustfunktion V(z) in (4.2), wobei $\theta$ der Wähleranteil der ersten Gruppe ist:

$$
\begin{gathered}
\min _{\mathrm{z}} \mathrm{V}(\mathrm{z})=\theta \cdot \lambda_{1} \cdot\left(\mathrm{z}-\mathrm{z}_{\mathrm{G} 1}\right)^{2}+(1-\theta) \cdot \lambda_{2} \cdot\left(\mathrm{z}-\mathrm{z}_{\mathrm{G} 2}\right)^{2} \\
\text { mit } \\
0,5<\theta<1
\end{gathered}
$$

Differenziert man (4.2) nach $\mathrm{z}$ und setzt das Ergebnis gleich null, folgt daraus die notwendige Bedingung für ein lokales Extremum: $:^{270}$

$$
\mathrm{z}^{*}=\frac{\theta \cdot \lambda_{1} \cdot \mathrm{z}_{\mathrm{G} 1}+(1-\theta) \cdot \lambda_{2} \cdot \mathrm{z}_{\mathrm{G} 2}}{\theta \cdot \lambda_{1}+(1-\theta) \cdot \lambda_{2}}
$$

${ }^{269}$ Der streng konvexe Verlauf der Verlustfunktion der Gruppe 2 bedeutet, dass bei einem Zollsatz größer als $\mathrm{z}_{\mathrm{G} 2}$ auch Stimmen der Gruppe 2 verloren gehen. Dies mag zunächst überraschen, da das Schutzniveau der Gruppe doch bei jeder Zollsatzerhöhung steigt. Da die Gruppe 2 jedoch immer auch Teile der Finanzierungslasten zu tragen hat, ist die Modellierung einer eindeutigen Optimalsituation gerechtfertigt.

Allgemein minimieren Politiker die Zielfunktion: $\mathrm{V}(\mathrm{z})=\theta \cdot \mathrm{V}_{\mathrm{Gl}}(\mathrm{z})+(1-\theta) \cdot \mathrm{V}_{\mathrm{G} 2}$.

Daraus ergibt sich die Optimalbedingung für den stimmenmaximierenden Zollsatz:

$\theta \cdot \frac{\partial \mathrm{V}_{\mathrm{G} 1}}{\partial \mathrm{z}}=-(1-\theta) \cdot \frac{\partial \mathrm{V}_{\mathrm{G} 2}}{\partial \mathrm{z}}$. 
Dies ist ein Minimum, da $\frac{\partial^{2} \mathrm{~V}}{\partial \mathrm{z}^{2}}=2 \cdot\left(\theta \cdot \lambda_{1}+(1-\theta) \cdot \lambda_{2}\right)>0$ ist.

Der Sachverhalt sei an einem Beispiel mit den folgenden Parameterwerten illustriert:

$$
\begin{array}{lll}
\lambda_{1}=4 & \mathrm{z}_{\mathrm{G} 1}=0 & \theta=0,8 \\
\lambda_{2}=40 & \mathrm{z}_{\mathrm{G} 2}=0,8 &
\end{array}
$$

Aus (4.3) folgt für das Beispiel $z^{*}=57 \%$. Abbildung 4.2 zeigt dies graphisch. Dabei werden die prozentualen Stimmenverluste der zweiten Gruppe auf der linken Ordinate und die prozentualen Stimmenverluste der ersten Gruppe sowie die prozentualen Verluste der gesamten Wählerschaft auf der rechten Ordinate abgetragen. Bei Freihandel werden 25,6\% der Wähler aus der Gruppe 2 nicht für die Partei stimmen (linke Ordinate). Dies entspricht einem gesamten Stimmenverlust von $5,12 \%$ aller Wähler. Im Verlustminimum bei einem Zollsatz von $57 \%$ werden aus der ersten Gruppe 1,12\% der Wähler und aus der zweiten Gruppe 2,12\% aufgrund des jeweils falschen Protektionsniveaus nicht für diese Partei stimmen. Auf die gesamte Bevölkerung gerechnet werden 1,46\% der Wähler aufgrund der Zollpolitik nicht für diese Partei stimmen.

\section{Abbildung 4.2 : Bestimmung der stimmenmaximierenden Zollsätze}

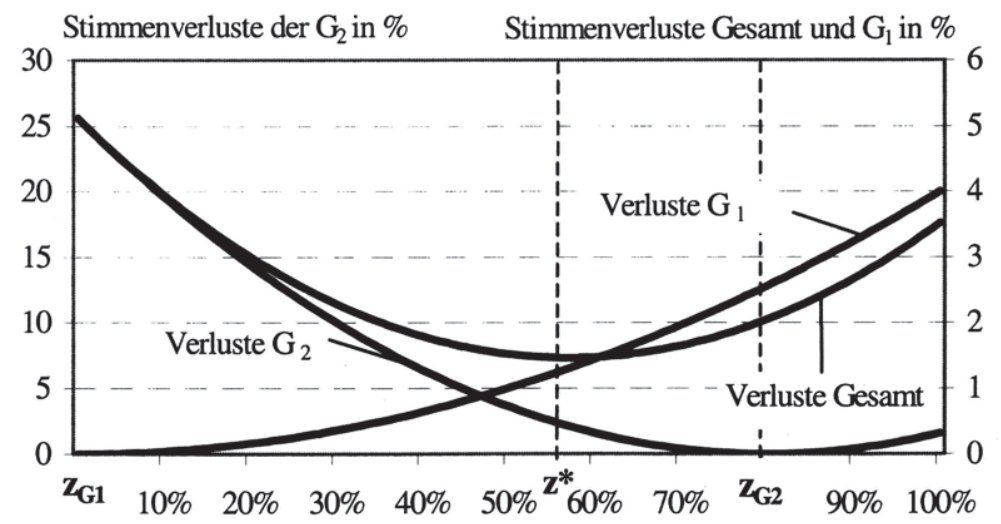

Zollsatz

Um zu prüfen, inwiefern eine Parameteränderung auch zu einer Veränderung des politisch optimalen Zollsatzes führt, ist das totale Differential von (4.3) zu bilden: 


$$
\mathrm{dz} * \frac{\partial \mathrm{z}^{*}}{\partial \lambda_{1}} \mathrm{~d} \lambda_{1}+\frac{\partial \mathrm{z}^{*}}{\partial \lambda_{2}} \mathrm{~d} \lambda_{2}+\frac{\partial \mathrm{z}^{*}}{\partial \theta} \mathrm{d} \theta+\frac{\partial \mathrm{z}^{*}}{\partial \mathrm{z}_{\mathrm{G} 1}} \mathrm{dz}_{\mathrm{G} 1}+\frac{\partial \mathrm{z}^{*}}{\partial \mathrm{z}_{\mathrm{G} 2}} \mathrm{dz} \mathrm{G}_{\mathrm{G}}
$$

Dabei gilt für die einzelnen partiellen Ableitungen:

$$
\frac{\partial \mathrm{z}^{*}}{\partial \lambda_{1}}=\frac{\lambda_{2} \cdot \theta \cdot(\theta-1) \cdot\left(\mathrm{z}_{\mathrm{G} 2}-\mathrm{z}_{\mathrm{G} 1}\right)}{\left(\theta \cdot \lambda_{1}+(1-\theta) \cdot \lambda_{2}\right)^{2}}<0
$$

Der Nenner von (4.5) ist stets positiv und wegen $0,5<\theta<1$ und $\mathrm{z}_{\mathrm{G} 2}>\mathrm{z}_{\mathrm{G} 1}$ ist der Zähler immer kleiner null.

$$
\frac{\partial \mathrm{z}^{*}}{\partial \theta}=\frac{\lambda_{1} \cdot \lambda_{2}\left(\mathrm{z}_{\mathrm{G} 1}-\mathrm{z}_{\mathrm{G} 2}\right)}{\left(\theta \cdot \lambda_{1}+(1-\theta) \cdot \lambda_{2}\right)^{2}}<0
$$

Wegen $\mathrm{z}_{\mathrm{G} 1}<\mathrm{z}_{\mathrm{G} 2}$ und $\lambda_{1}, \lambda_{2}>0$ ist der Zähler von (4.6) immer negativ; der Nenner ist wie zuvor positiv.

$$
\frac{\partial \mathrm{z}^{*}}{\partial \lambda_{2}}=\frac{\lambda_{1} \cdot \theta \cdot(\theta-1) \cdot\left(\mathrm{z}_{\mathrm{G} 1}-\mathrm{z}_{\mathrm{G} 2}\right)}{\left(\theta \cdot \lambda_{1}+(1-\theta) \cdot \lambda_{2}\right)^{2}}>0
$$

$\mathrm{Da} 0,5<\theta<1$ und $\mathrm{z}_{\mathrm{G} 1}<\mathrm{z}_{\mathrm{G} 2}$ wird der Zähler von (4.7) positiv, der Nenner ist immer positiv.

$$
\frac{\partial z^{*}}{\partial z_{G 2}}=\frac{(1-\theta) \cdot \lambda_{2}}{\theta \cdot \lambda_{1}+(1-\theta) \cdot \lambda_{2}}>0
$$

Da $0,5<\theta<1$ und $\lambda_{2}>0$, sind sowohl Zähler als auch Nenner von (4.8) immer größer null. Folglich gilt für das Vorzeichen des totalen Differentials aus $(4.4):^{271}$

${ }^{271}$ Die Betrachtung einer Änderung von $\mathrm{z}_{\mathrm{G} 1}$ ist nicht nötig, da die Freihandelsstrategie immer die optimale Strategie der ersten Gruppe ist, wohingegen $\mathrm{z}_{\mathrm{G} 2}$ durchaus variabel ist, da je nach Konkurrenzsituation der Industrie ein anderes Protektionsniveau nachgefragt werden kann. 


$$
\begin{gathered}
\mathrm{dz}^{*}<0 \text {, wenn }\left|\frac{\partial \mathrm{z}^{*}}{\partial \lambda_{1}} \mathrm{~d} \lambda_{1}\right|+\left|\frac{\partial \mathrm{z}^{*}}{\partial \theta} \mathrm{d} \theta\right|>\left|\frac{\partial \mathrm{z}^{*}}{\partial \lambda_{2}} \mathrm{~d} \lambda_{2}\right|+\left|\frac{\partial \mathrm{z}^{*}}{\partial \mathrm{z}_{\mathrm{G} 2}} \mathrm{dz} \mathrm{G}_{\mathrm{G} 2}\right| \\
\text { und } \\
\mathrm{dz}^{*}>0 \text {, wenn }\left|\frac{\partial \mathrm{z}^{*}}{\partial \lambda_{1}} \mathrm{~d} \lambda_{1}\right|+\left|\frac{\partial \mathrm{z}^{*}}{\partial \theta} \mathrm{d} \theta\right|<\left|\frac{\partial \mathrm{z}^{*}}{\partial \lambda_{2}} \mathrm{~d} \lambda_{2}\right|+\left|\frac{\partial \mathrm{z}^{*}}{\partial \mathrm{z}_{\mathrm{G} 2}} \mathrm{dz}_{\mathrm{G} 2}\right|
\end{gathered}
$$

Diese entgegengesetzt wirkenden Zusammenhänge lassen eine eindeutige Entwicklung von $\mathrm{z}^{*}$ in der Zeit nur sehr schwer prognostizieren. Allerdings lassen sich einige Plausibilitätsüberlegungen anstellen. Es wäre demnach wahrscheinlicher, dass in westlichen Demokratien die wirtschaftliche und technische Entwicklung der letzten Jahrzehnte dazu führte, dass die zollsenkenden Effekte auf $\lambda_{1}$ und $\theta$ überwogen und dass es folglich für wahlsiegorientierte Politiker rational war und ist, die Zölle zu senken.

- Die Außenhandelsbeziehungen moderner Industriestaaten sind aufgrund von steigenden Skalenerträgen in der Produktion sowie heterogener Präferenzen der Nachfrager zunehmend durch intraindustrielle Handelsbeziehungen gekennzeichnet (Krugman und Obstfeld, 2000 und Siebert und Rauscher, 1991, S. 504ff): ${ }^{272}$ Beispielsweise machten für Deutschland 1999 die Exporte von chemischen Erzeugnissen 12,7\% der gesamten Exporte aus und gleichzeitig waren $9,1 \%$ aller Importe chemische Erzeugnisse. Ähnliches gilt für elektrotechnische Erzeugnisse; 13,3\% aller Exporte und 11,9\% aller Importe kamen aus diesem Segment (Deutsche Bundesbank, 2000, S. 504). Dieser Trend lässt sich auch allgemein für Industriestaaten zeigen: So stieg der GrubelLloyd-Index, ${ }^{273}$ der den Umfang des intraindustriellen Handels abbildet, von einem Durchschnittswert für 22 OECD-Staaten von 44,1 im Jahre 1970 auf einen Wert von 57,7 im Jahre 1990 (OECD, 1996, S. 30). Dabei war von den 22 Staaten Norwegen das einzige Land, dessen Wert des Grubel-Lloyd-Index in der Periode sank. Die norwegische Sonderstellung erklärt sich durch den hohen Anteil der Erdölexporte an den Gesamtexporten Norwegens. Da es im

${ }^{272}$ Intraindustrieller Handel bezeichnet den Austausch ähnlicher Produkte des gleichen Sektors (Maennig und Wilfling, 1998, S. 89).

${ }^{273}$ Der Grubel-Lloyd-Index GL errechnet sich dabei wie folgt (Grubel und Lloyd, 1975): $\mathrm{X}_{\mathrm{i}}$ sind die Exporte der Branche i eines Landes und $\mathrm{Q}_{i}$ die Importe eines Landes aus derselben Branche i mit $i \in[1 ; n]$.

$$
G L=\frac{\sum_{i=1}^{n}\left(X_{i}+Q_{i}\right)-\sum_{i=1}^{n}\left|X_{i}-Q_{i}\right|}{\sum_{i=1}^{n}\left(X_{i}+Q_{i}\right)}=1-\frac{\sum_{i=1}^{n}\left|X_{i}-Q_{i}\right|}{\sum_{i=1}^{n}\left|X_{i}+Q_{i}\right|} \text { Tobia }
$$


Zuge der Ölkrisen in den siebziger Jahre erst lohnend wurde, das norwegische Erdöl zu fördern und den Ölexporten keine Ölimporte entgegen standen, sank der Grubel-Lloyd-Index für Norwegen.

Steigt der Anteil intraindustrieller Handelsbeziehungen, wird die Anzahl der Protektion nachfragenden Wirtschaftsbereiche sinken, da bei glaubhafter Drohung von Retorsionszöllen durch das Ausland ansonsten Exportpotential verloren ginge. ${ }^{274}$ Zudem ist bei hinlänglich spezialisierten Anbietern (monopolistische Konkurrenz) Protektion sowieso nicht mehr erforderlich. Letztendlich führten also der technische Fortschritt und die Möglichkeit der Konsumenten, ihre Präferenzen zu offenbaren, dazu, dass der Anteil der Protektionsnachfrager sinkt ( $\theta$ steigt). Des weiteren führt dieser Wandel in der Anbieterstruktur dazu, dass innerhalb der Gruppe der Befürworter der Freihandelsposition die Zahl derer zunimmt, deren Nutzen stark von dem realisierten Protektionsniveau abhängt, d.h. $\lambda_{1}$ steigt ebenfalls.

- Die Revolution der Informations- und Kommunikationstechnik führt dazu, dass die Koordination auch heterogener Präferenzen einfacher wird. Konsumenten sind wesentlich billiger über Zollwirkungen zu informieren und können Wissen und Einstellungen einfacher (weil billiger) verbreiten. Auch dies führt letztlich zu einem Anstieg von $\lambda_{1}$.

Hingegen ist es höchst fraglich, ob für Protektionsbefürworter die Höhe des Zollsatzes eine größere Bedeutung erlangt hat, da sich an der grundlegenden Situation der Importkonkurrenz nichts geändert hat. Zwar hat sich in Westeuropa die Arbeitsmarktsituation drastisch verändert, so dass ein möglicher Arbeitsplatzverlust eine geringere Wiederbeschäftigungswahrscheinlichkeit bedeutet. Gleichzeitig haben sich aber auch die sozialen Sicherungsinstitutionen stark ausgeweitet. Die Kosten des Arbeitsplatzverlustes wurden dadurch vermindert.

Hinsichtlich der Entwicklung von $\mathrm{z}_{\mathrm{G} 2}$ ist angesichts gesunkener Transportkosten mit höherer Auslandskonkurrenz v.a. in den Sektoren zu rechnen, bei denen die Transportkosten einen substanziellen Teil des Warenwertes ausmachen. Dies gilt sicherlich nur für eine sehr kleine Gruppe von Sektoren in hochentwickelten Industriestaaten.

274 Eine Ausnahme liegt dann vor, wenn der Grubel-Lloyd-Index nur deswegen angestiegen ist, weil sich ein Land einseitig geöffnet hat. Dann könnte eine Branche i jetzt zwar im Inland stärkere Konkurrenz durch ausländische Unternehmen erfahren, ohne jedoch zusätzliche Absatzchancen im Ausland gewonnen zu haben. Die Folge wäre dann, dass diese Branche Retorsionszölle seitens des Inlands nachfragen würde. Dieses Szenario könnte zwar im Einzelfall zutreffen, jedoch spricht sowohl das symmetrische Ansteigen der Import- und Exportströme aller Länder als auch die stetigen Liberalisierungsanstrengungen der maßgeblichen OECD-Staaten (vgl. Tabelle 4.1) dafür, dass der skizzierte Fall eines Absinkens der Zollnachfrage wohl insgesamt der empixisch, bedeqtsamere Fall $/$ sejngsollte. 
Fasst man diese Plausibilitätsüberlegungen zusammen, ist es für die meisten demokratischen Staaten der westlichen Welt wahrscheinlicher, dass die Veränderung der Struktur der Wählergruppen eher in Richtung einer Senkung der Zölle geführt hat bzw. führen wird. Demnach legt dieses polit-ökonomische Modell die Schlussfolgerung nahe, dass die schrittweise Öffnung der Märkte nicht unbedingt als Ausdruck größerer Einsicht der Politiker in die positive Wohlfahrtswirkung offener Gütermärkte gewertet werden muss, sondern auch verursacht wurde durch technische Änderungen der Produktions- und den damit induzierten Änderungen in den Wählerstrukturen. Da sich dieser Strukturwandel stetig vollzogen hat, ließe sich so auch erklären, warum das realisierte Zollniveau als Ausdruck eines langsam sinkenden politisch optimalen Zollniveaus nicht in einer einzigen großen Verhandlungsrunde auf null gesenkt wurde, sondern schrittweise in mehreren Runden reduziert wurde.

In Anlehnung an die Ausführungen in 2.2.3.2, wo gezeigt wurde, dass eine vollständige Konvergenz der Parteiprogramme dann unwahrscheinlich ist, wenn unterschiedliche Parteien auch verschiedene ideologische Prädispositionen haben, lässt sich argumentieren, dass die Zollpolitik neben der reinen wahltaktischen Zollsetzung auch eine ideologische Nutzenkomponente besitzt. Geht man ebenfalls in Anlehnung an die Notation in 2.2.3.2 davon aus, dass die rechte Partei C eher eine freihändlerische Position verfolgt und die linke Partei S eher eine protektionistische Position einnimmt, dann ist die Zielfunktion der Politiker in Gleichung (4.2) zu erweitern. Wenn die ideale rechte Zollpolitik mit der Freihandelsoption $\mathrm{z}_{\mathrm{G} 1}$ zusammenfällt und die ideale linke Politik mit der protektionistischen Politik der Gruppe $2 \mathrm{z}_{\mathrm{G} 2}$, dann werden die Parteien die Programme $\mathrm{z}_{\mathrm{C}}$ und $z_{S}$ anbieten, die ihren Erwartungsnutzen EU maximieren. $U_{i}\left(z_{i}\right)$ mit $i \in\{S, C\}$ sei der ideologische Nutzen der jeweiligen Partei aus dem jeweiligen Zollniveau und $\hat{U}$ ist der Nutzen, der aus dem Wahlsieg direkt erfolgt. Dabei gilt, dass die Wahrscheinlichkeit eines Sieges von $\mathrm{C}$ definiert ist als:

$$
\mathrm{P}=\mathrm{P}\left(\mathrm{z}_{\mathrm{S}} ; \mathrm{z}_{\mathrm{C}}\right)
$$

Die Zielfunktion der C-Partei ist folglich definiert als:

$$
\begin{aligned}
\max _{\mathrm{z}_{\mathrm{C}}} \mathrm{EU}_{\mathrm{C}} & =\gamma \cdot\left\{\mathrm{P}\left(\mathrm{z}_{\mathrm{S}} ; \mathrm{z}_{\mathrm{C}}\right) \cdot \mathrm{U}_{\mathrm{C}}\left(\mathrm{z}_{\mathrm{C}}\right)+\left[1-\mathrm{P}\left(\mathrm{z}_{\mathrm{S}} ; \mathrm{z}_{\mathrm{C}}\right)\right] \cdot \mathrm{U}_{\mathrm{C}}\left(\mathrm{z}_{\mathrm{S}}\right)\right\} \\
& +(1-\gamma) \cdot \mathrm{P}\left(\mathrm{z}_{\mathrm{S}} ; \mathrm{z}_{\mathrm{C}}\right) \hat{\mathrm{U}}
\end{aligned}
$$

Der Parameter $\gamma$ ist ein individuelles Gewicht der beiden Nutzenkomponenten. Für die Partei S lässt sich die Zielfunktion völlig analog formulieren: 


$$
\begin{aligned}
\max _{\mathrm{z}_{\mathrm{S}}} \mathrm{EU}_{\mathrm{S}} & =\gamma \cdot\left\{\mathrm{P}\left(\mathrm{z}_{\mathrm{S}} ; \mathrm{z}_{\mathrm{C}}\right) \cdot \mathrm{U}_{\mathrm{S}}\left(\mathrm{z}_{\mathrm{C}}\right)+\left[1-\mathrm{P}\left(\mathrm{z}_{\mathrm{S}} ; \mathrm{z}_{\mathrm{C}}\right)\right] \cdot \mathrm{U}_{\mathrm{S}}\left(\mathrm{z}_{\mathrm{S}}\right)\right\} \\
& +(1-\gamma) \cdot\left[1-\mathrm{P}\left(\mathrm{z}_{\mathrm{S}} ; \mathrm{z}_{\mathrm{C}}\right)\right] \hat{\mathrm{U}}
\end{aligned}
$$

Gemäß den Ausführungen in 2.2.3.2 lässt sich in Anlehnung an Calvert (1985) zeigen, dass es eine gleichgewichtige Zollstrategie der beiden Parteien gibt, für die gilt:

$$
\mathrm{z}_{\mathrm{G} 1}<\mathrm{z}_{\mathrm{C}}<\mathrm{z}^{*}<\mathrm{z}_{\mathrm{S}}<\mathrm{z}_{\mathrm{G} 2}
$$

Neben der zuvor gemachten Aussage, dass durch Strukturwandel bewirkte Verschiebungen in der Wählerschaft eine fortschreitende Marktöffnung rationale Strategie wiederwahlorientierter Politiker sein kann, lässt sich jetzt zusätzlich feststellen, dass die Marktöffnung umso wahrscheinlicher ist, je stärker der ideologische Aspekt in den Nutzenfunktionen insbesondere der rechten Parteien verankert ist (und diese auch die Möglichkeit einer Politikumsetzung hatten).

\section{Tabelle 4.4 : CG-Werte während der GATT-Runden und in den Zwischenphasen}

\begin{tabular}{l|l|c|c}
\hline \multicolumn{2}{l|}{} & $\begin{array}{l}\text { Mittlerer CG-Wert } \\
\text { von 18 OECD- } \\
\text { Staaten }\end{array}$ & $\begin{array}{l}\text { Mittlerer CG-Wert } \\
\text { der 5 ,großen“ } \\
\text { OECD-Staaten }\end{array}$ \\
\hline $1960-1961$ & Dillon-Runde & 5,65 & 7,00 \\
$1962-1963$ & Interimsphase & 5,63 & 6,86 \\
$1964-1967$ & Kennedy-Runde & 5,28 & 5,92 \\
$1968-1972$ & Interimsphase & 5,41 & 6,15 \\
$1973-1979$ & Tokio-Runde & 4,94 & 5,65 \\
$1980-1985$ & Interimsphase & 5,48 & 6,29 \\
$1986-1993$ & Uruguay-Runde & 5,35 & 6,57 \\
$1994-1997$ & „Interimsphase“ & 5,10 & 6,34 \\
\hline \multicolumn{2}{l|}{ ungew. Durchschnitt GATT-Runden } & 5,31 & 6,28 \\
\multicolumn{1}{l}{ ungew. Durchschnitt Interimsphasen } & 5,41 & 6,41 \\
\hline
\end{tabular}

T) Diese Länder umfassen: Australien, Österreich, Belgien, Kanada, Dänemark, Finnland, Frankreich, Deutschland, Irland, Italien, Japan, Niederlande, Neuseeland, Norwegen, Schweden, Schweiz, Großbritannien, USA.

2) Diese Länder umfassen: Frankreich, Deutschland, Japan, Großbritannien, USA.

Quelle: Für die CG-Werte vgl. die Quellenangaben im Anhang B. Die Zeitangaben der

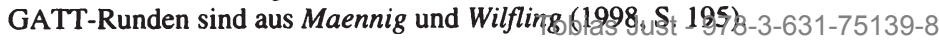


Allerdings erweist sich eine empirische Überprüfung dieser Ideologiehypothese auf internationaler Ebene als äußerst schwierig, da sich die Zusammensetzung der Länderregierungen während der einzelnen Verhandlungszeiträume permanent geändert hat, sodass der konkrete Einfluss einer einzelnen Regierung auf das Endergebnis nur sehr schwer einzuschätzen ist. Tabelle 4.4 zeigt die durchschnittlichen Werte des center of gravity $(C G)^{275}$ von $18 \mathrm{bzw}$. von 5 OECD Staaten für die jeweiligen GATT-Verhandlungszeiträume bzw. die dazwischen liegenden Perioden (Interimsphasen).

Abgesehen davon, dass die Anzahl der GATT-Runden bzw. der Interimsphasen viel zu klein ist, um gesicherte statistische Signifikanzaussagen treffen zu können, zeigen die Daten nicht, dass es zwischen GATT-Runden und Interimsphasen systematische Abweichungen der durchschnittlichen Ideologieausrichtung der bedeutsamsten Verhandlungspartner gibt. ${ }^{276}$

Es soll nun untersucht werden, ob der Globalisierungsprozess noch genauer in einzelne Phasen eingeteilt werden kann. Da die Öffnung eines Landes nicht nur auf den Gütermärkten stattfindet und nicht nur auf globaler (GATT-) Ebene, muss man zur besseren Einschätzung der Öffnungsprozesse eine multivariate Analyse durchführen. Dies wird im folgenden Abschnitt getan.

\subsection{Globalisierungscluster}

Neben der Suche nach Globalisierungsphasen soll in diesem Abschnitt gefragt werden, ob es unterschiedliche Gruppen von Ländern gibt, die verschieden intensiv international eingebunden sind.

In vielen Arbeiten zur Globalisierung finden sich Indikatoren, die den Umfang und die Entwicklung des Internationalisierungsprozesses abbilden sollen (z.B. Berthold, 1997 und Freytag, Meier und Weiß, 1998). Dies sind zumeist ökonomische Relationen (z.B. Exporte in Relation zum Bruttoinlandsprodukt). Diese Kategorie von Indikatoren werden im folgenden als ökonomische Indikatoren bezeichnet und kennzeichnen die tatsächlich am Markt stattfindenden Transaktionen. Sie werden im ersten Abschnitt untersucht.

275 Das center of gravity beschreibt auf einer Skala von 0 (links) bis 10 (rechts) die Regierungen der einzelnen Länder. Zu einer ausführlichen Beschreibung der theoretischen Konzeption und der Umsetzung dieses Indikators vgl. Kapitel 3.3.

${ }^{276}$ Diese Aussage hat auch dann Bestand, wenn man die Perioden in Teilperioden unterteilt. GATT-Runden werden nicht grundsätzlich von ,rechteren“ Regierungen angestrengt oder schneller beendigt. Dies gilt natürlich nur auf diesem sehr hohen Abstraktionsgrad. Eine detailliertere Analyse kann an dieser Stelle allerdings nichtserfolgen-3-631-75139-8 
Daneben ist es jedoch auch lohnend, einen Blick auf die Entwicklung der Liberalisierungspolitik, z.B. abzulesen an der Beteiligung an Freihandelsabkommen, zu werfen, da so das Handlungspotential von ökonomischen Agenten und nicht nur ihre tatsächlichen Handlungen ermittelt werden können. Die dafür verwendeten Indikatoren werden als politische Indikatoren bezeichnet und stehen im Zentrum des darauf folgenden Abschnitts. Es ist durchaus vorstellbar, dass beide Clusterungen zu unterschiedlichen Ergebnissen führen. So ist es möglich, dass Länder, die ihren Außenhandel sehr stark liberalisiert haben, de facto wenig $\mathrm{Au}$ Benhandel betreiben, da sie weitgehend autark sind. Gleichzeitig mag es Länder geben, die ihre Märkte weniger stark liberalisiert haben und sich nur gegenüber wenigen Partnern geöffnet haben, von denen sie stark abhängen, sodass ihr ökonomischer Öffnungsgrad hoch ist, obwohl ihre politischen Indikatoren das Gegenteil vermuten lassen.

\subsection{1 Ökonomische Globalisierungsindikatoren}

Ökonomische Indikatoren erfassen üblicherweise die Handelsströme, die Kapitalströme und die Migrationsströme und setzen diese zu einer Bezugsgröße (z.B. BIP oder Bruttoinlandsinvestitionen) in Relation. Hier werden die Migrationsströme vernachlässigt, und es wird allein auf drei Maßzahlen zurück gegriffen, die den Umfang des Warenhandels, den Umfang der Direktinvestitionsströme und der Portfolioinvestitionsströme abbilden.

\subsubsection{Umfang von Handels- und Kapitalströmen als Globalisierungsin- dikatoren}

In der Literatur hat sich zur Erfassung des Handelsvolumens der Offenheitsgrad als Maßzahl durchgesetzt. Dieser ist definiert als die Summe der Exporte und Importe dividiert durch das Bruttoinlandsprodukt (z.B. Berthold, 1997, S. 26). Möchte man jedoch eine Aussage hinsichtlich der Verflechtung von tatsächlich international tätigen Unternehmen treffen, dann birgt der so definierte Offenheitsgrad dann ein konzeptionelles Problem, wenn sich die Wirtschaftsstruktur im Zeitablauf stark verändert hat. Da in den meisten Industriestaaten der Anteil des Staatssektors am BIP stark gestiegen ist und da dieser Sektor in der Regel sehr geringe Außenhandelsbeziehungen aufweist, ist es sinnvoll, den Offenheitsgrad leicht anzupassen. Im folgenden wird der Offenheitsgrad definiert als: ${ }^{277}$

${ }^{277}$ Genauer wäre es, vom BIP die Summe der non-tradeables abzuziehen. Dies ist allerdings besonders in einer längeren Zeitreihe nur sehr schwer zu schätzen. Der Staatskonsum dient hier als einfache Proxy-Größe. 


$$
\text { Offenheitsgrad }=\frac{\text { Exporte }+ \text { Importe }}{\text { BIP-Staatskonsum }}
$$

Tabelle 4.5 zeigt für die 19 untersuchten OECD-Staaten die Entwicklung dieses Indikators. Zwar wird deutlich, dass in den letzten 35 Jahren der Außenhandel in (fast) allen Industriestaaten stärker gewachsen ist als das BIP, es erstaunt aber, dass gemäß dieser Maßzahl die 90er Jahre gar nicht die Jahre der stärksten $\mathrm{Zu}$ wachsraten dieses Indikators darstellen, sondern dass dies die 70er Jahre sind. Seit Anfang der 80er Jahre bleibt der Außenhandelsindikator für die Industriestaaten fast konstant. ${ }^{278}$

Tabelle 4.5 : Entwicklung des Offenheitsgrads von 1963-1997

\begin{tabular}{l|ccccc|c}
\hline & $\mathbf{1 9 6 3}$ & $\mathbf{1 9 7 0}$ & $\mathbf{1 9 8 0}$ & $\mathbf{1 9 9 0}$ & $\mathbf{1 9 9 7}$ & $\mathbf{1 9 9 7 / 1 9 6 3}$ \\
\hline Australien & 0,29 & 0,27 & 0,33 & 0,32 & 0,38 & 1,31 \\
Österreich & 0,43 & 0,52 & 0,65 & 0,69 & 0,74 & 1,71 \\
Belgien & 0,82 & 1,03 & 1,38 & 1,52 & 1,71 & 2,08 \\
Kanada & 0,33 & 0,42 & 0,58 & 0,53 & 0,82 & 2,45 \\
Dänemark & 0,60 & 0,61 & 0,74 & 0,71 & 0,74 & 1,23 \\
Finnland & 0,41 & 0,53 & 0,71 & 0,50 & 0,76 & 1,86 \\
Frankreich & 0,24 & 0,30 & 0,45 & 0,45 & 0,49 & 2,08 \\
Deutschland & 0,34 & 0,41 & 0,59 & 0,61 & 0,56 & 1,65 \\
Irland & 0,73 & 0,83 & 1,27 & 1,16 & 1,47 & 2,03 \\
Italien & 0,28 & 0,30 & 0,46 & 0,39 & 0,46 & 1,66 \\
Japan & 0,19 & 0,20 & 0,28 & 0,19 & 0,20 & 1,05 \\
Niederlande & 0,91 & 0,89 & 1,09 & 1,06 & 1,19 & 1,32 \\
Neuseeland & 0,46 & 0,44 & 0,59 & 0,53 & 0,52 & 1,14 \\
Norwegen & 0,58 & 0,66 & 0,68 & 0,67 & 0,69 & 1,19 \\
Schweden & 0,45 & 0,53 & 0,73 & 0,67 & 0,88 & 1,94 \\
Schweiz & 0,53 & 0,62 & 0,69 & 0,68 & 0,70 & 1,31 \\
Spanien & 0,18 & 0,21 & 0,30 & 0,34 & 0,51 & 2,78 \\
UK & 0,37 & 0,40 & 0,53 & 0,52 & 0,58 & 1,56 \\
USA & 0,08 & 0,10 & 0,21 & 0,19 & $\mathbf{0 , 2 3}$ & 2,83 \\
\hline ungew. Mittelwert & $\mathbf{0 , 4 3}$ & $\mathbf{0 , 4 9}$ & $\mathbf{0 , 6 5}$ & $\mathbf{0 , 6 2}$ & $\mathbf{0 , 7 2}$ & $\mathbf{1 , 6 6}$ \\
\hline
\end{tabular}

Quelle: Eigene Berechnungen auf der Grundlage vom $I M F$, International Financial Statistics (div. Jahrgänge).

${ }^{278}$ Es kann eingeräumt werden, dass diese Entwicklung bei diesem Indikator auch daran liegen kann, dass seit den 80 er Jahren einige Staaten den Staatsanteil zurückdrängten. Dem ist allerdings entgegenzuhalten, dass sich diese Entwicklung auch für den traditionellen Offenheitsgrad ergibt (Berthold, 1997, S. 26). Für den Fall Deutschland lässt sich das Sinken der Maßzahl in den 90er Jahren auch durch die Wiedervereinigung erklären, da dadurch Teile des Außenhandels zu Binnenhandel wurden.Just - 978-3-631-75139-8 
Diese Entwicklung beruht allerdings zu Teilen nicht auf einem Mengeneffekt, sondern auf einem Preiseffekt, der durch die beiden Ölpreisschocks verursacht wurde. Da die Nachfrage nach Öl sehr unelastisch reagiert, stieg der Wert des importierten respektive exportierten Öls drastisch an (vgl. Endres und Querner, 2000 , S. 154). Dies ist auch der Grund dafür, warum der Offenheitsgrad von Norwegen in den 70er Jahren am wenigsten anstieg: In den meisten OECDStaaten bedeutete eine Verteuerung des Ölimports zum Teil ein Verdrängen anderer inländischer Nachfrage, somit stieg nur der Zähler in 4.14. In Norwegen bedeutete der Preisanstieg indes eine Erhöhung der Ölexporte und dadurch auch des BIP. Dies bedeutet, dass sowohl der Zähler als auch der Nenner in 4.14 gleichgerichtet betroffen waren.

In den 80er Jahren verringerte sich die Veränderungsdynamik des Offenheitsgrads, weil dem positiven Mengeneffekt zusätzlicher Außenhandelstätigkeit der negative Preiseffekt der Ölpreissenkung entgegenstand. Auch dieser Sachverhalt macht deutlich, warum es sinnvoll ist, zwischen der ökonomischen und der politischen Clusterung zu unterscheiden.

Die Entwicklung der Kapitalströme soll durch zwei Kennzahlen erfasst werden. Zum ersten wird die Summe der ab- und zufließenden Direktinvestitionen zu den inländischen Bruttoinvestitionen (gross capital formation) ins Verhältnis gesetzt. $^{279}$ Von ausländischen Direktinvestitionen wird dann gesprochen, wenn davon ausgegangen werden kann, dass der Investor ein langfristiges Investitionsinteresse verfolgt und/oder weitreichende Kontrolle über das Investitionsobjekt gewinnen möchte. Dieser vagen Definition ist inhärent, dass die Abgrenzung von Direktinvestitionen nicht immer einheitlich erfolgt. So wird die Grenze zwischen Direktinvestitionen in internationalen Statistiken bei einer 10\%igen Beteiligung gezogen, in Deutschland hingegen wurde bis 1995 noch eine Mindestbeteiligung von $25 \%$ als Kriterium für Direktinvestitionen genommen (Hübner, 1998, S. 152). Im folgenden wird mit Daten des IMF Balance of Payments Yearbook (diverse Jahrgänge), also mit der weitergehenden Abgrenzung gearbeitet.

Bei Investitionen mit einer geringeren Beteiligung des Investors als $10 \%$ wird davon ausgegangen, dass entweder keine maßgebliche Kontrolle über das ausländische Unternehmen gewünscht wird und/oder dass das Investitionsinteresse nur kurzfristiger Art ist. Man spricht dann von Portfolioinvestitionen. Bei der Erfassung erweisen sich Portfolioinvestitionen als sehr schwierig, da sie erst seit 1973 im IMF Balance of Payments Yearbook ausdrücklich ausgewiesen werden. Die Kennzahl für die Portfolioinvestitionen wird ebenfalls aus dem Quotienten

${ }^{279}$ Die Daten für die Inlandsinvestitionen sind dem IMF International Financial Statistics (diverse Jahrgänge) entnommen. 
der ab- und zufließenden Portfolioinvestitionen einerseits und den inländischen Bruttoinvestionen andererseits gebildet.

Der Trend ist eindeutig. Sowohl der Umfang der Direktinvestitionen als auch der Umfang der Portfolioinvestitionen hat sich in Relation zu den inländischen Bruttoinvestionen sehr stark ausgeweitet. ${ }^{280}$ Hierbei ist die Entwicklung bei den kurzfristigen internationalen Kapitalbewegungen noch ausgeprägter als bei den langfristigen Kapitalbewegungen. Es wird auch deutlich, dass die Direktinvestitionen in den 80er Jahren den relativ stärksten Zuwachs erlebten, die Portfolioinvestitionen hingegen erst in den 90er Jahren.

Tabelle 4.6 : Entwicklung von Direkt- und Portfolioinvestitionen in 19 OECD-Staaten

\begin{tabular}{|c|c|c|c|c|c|c|c|c|c|c|}
\hline & \multicolumn{5}{|c|}{$\frac{\text { Direktinvestitionen }}{\text { inl. Bruttoinvestitionen }}$} & \multicolumn{5}{|c|}{$\frac{\text { Portfolioinvestitionen }}{\text { inl. Bruttoinvestitionen }}$} \\
\hline & 1963 & 1970 & 1980 & 1990 & 1997 & 1963 & 1974 & 1980 & 1990 & 1997 \\
\hline Australien & 0,09 & 0,09 & 0,05 & 0,12 & 0,18 & n.v. & 0,00 & 0,04 & 0,12 & 0,15 \\
\hline Österreich & n.v. & 0,03 & 0,02 & 0,06 & 0,10 & n.v. & 0,04 & 0,11 & 0,06 & 0,48 \\
\hline Belgien & n.v. & 0,08 & 0,07 & 0,29 & 0,40 & n.v. & 0,07 & 0,11 & 0,29 & 2,25 \\
\hline Kanada & 0,04 & 0,06 & 0,06 & 0,11 & 0,18 & n.v. & 0,06 & 0,08 & 0,11 & 0,19 \\
\hline Dänemark & 0,03 & 0,03 & 0,03 & 0,09 & 0,20 & n.v. & n.v. & 0,03 & 0,09 & 0,38 \\
\hline Finnland & 0,00 & 0,02 & 0,01 & 0,09 & 0,34 & n.v. & 0,01 & 0,03 & 0,09 & 0,45 \\
\hline Frankreich & 0,02 & 0,03 & 0,04 & 0,18 & 0,25 & n.v. & 0,00 & 0,03 & 0,18 & 0,50 \\
\hline Deutschland & 0,02 & 0,03 & 0,03 & 0,01 & 0,08 & n.v. & 0,01 & 0,03 & 0,01 & 0,44 \\
\hline Irland & n.v. & n.v. & n.v. & 0,11 & 0,28 & n.v. & 0,05 & 0,05 & 0,11 & 0,24 \\
\hline Italien & 0,04 & 0,03 & 0,01 & 0,06 & 0,08 & n.v. & 0,01 & 0,01 & 0,06 & 0,71 \\
\hline Japan & 0,01 & 0,01 & 0,01 & 0,05 & 0,03 & n.v. & 0,01 & 0,04 & 0,05 & 0,15 \\
\hline Niederlande & 0,06 & 0,12 & 0,13 & 0,43 & 0,41 & n.v. & 0,07 & 0,11 & 0,43 & 0,82 \\
\hline Neuseeland & 0,00 & 0,02 & 0,09 & 0,41 & 0,10 & n.v. & n.v. & n.v. & 0,41 & 0,04 \\
\hline Norwegen & 0,01 & 0,03 & 0,02 & 0,09 & 0,22 & n.v. & 0,00 & 0,04 & 0,09 & 0,58 \\
\hline Schweden & 0,04 & 0,04 & 0,04 & 0,32 & 0,71 & n.v. & 0,01 & 0,12 & 0,32 & 1,87 \\
\hline Schweiz & n.v. & n.v. & n.v. & 0,17 & n.v. & n.v. & 0,11 & 0,32 & 0,17 & n.v. \\
\hline Spanien & 0,01 & 0,03 & 0,04 & 0,14 & 0,15 & n.v. & 0,01 & 0,00 & 0,14 & 0,26 \\
\hline UK & 0,08 & 0,09 & 0,14 & 0,25 & 0,50 & n.v. & 0,09 & 0,11 & 0,25 & 0,67 \\
\hline USA & 0,04 & 0,05 & 0,06 & 0,08 & 0,15 & n.v. & 0,02 & 0,03 & 0,08 & 0,34 \\
\hline $\begin{array}{l}\text { ungewichteter } \\
\text { Mittelwert }\end{array}$ & $\mathbf{0 , 0 3}$ & 0,05 & 0,05 & 0,16 & 0,24 & n.v. & 0,03 & 0,07 & 0,16 & $\mathbf{0 , 5 8}$ \\
\hline
\end{tabular}

Quelle: Eigene Berechnungen auf der Basis der IMF Balance of Payments Yearbooks (div. Jahrgänge) und den IMF International Financial Statistics (div. Jahrgänge).

${ }^{280}$ Es darf allerdings nicht übersehen werden, dass insbesondere Direktinvestitionsströme sehr starken Zufallsschwankungen unterworfen sind. So kann eine einzige vollzogene, internationale Großfusion erhebliche Wirkung in der Gesamtstatistik besitzen. Solche Schwankungen sind auch in den $90 \mathrm{er}$ Jahren sehr ausgeprägt, der Trend ist jedoch eindeutig. 
In den folgenden beiden Abschnitten sollen mit Hilfe der Clusteranalyse zum einen die zeitliche Entwicklung in einzelne Phasen unterteilt und zum anderen aus dem gesamten Ländersample Gruppen mit ähnlicher Entwicklung isoliert werden.

\subsubsection{Phasen der Globalisierung}

In einem ersten Schritt wird versucht, mit Hilfe aller drei Indikatoren (Außenhandel, Direktinvestitionen und Portfolioinvestionen) sinnvolle Zeitabschnitte abzugrenzen. Leider lässt die unzureichende Datenlage für alle drei Indikatoren nur eine Berücksichtigung von 14 Ländern und den Jahren von 1974 bis 1997 $\mathrm{zu}^{281}$ Die zu clusternden Objekte stellen hier die Jahre dar, und die Variablen sind die drei Indikatoren für die 14 Länder. Es gibt also bei 42 Variablen und 24 Objekten insgesamt 1008 Einzelbeobachtungen. Die Clusteranalyse wurde mit Hilfe des Programms SPSS 9.0 durchgeführt.

Zwar unterscheiden sich die Wertebereiche der drei Indikatoren nicht sehr stark, dennoch wurden die Daten zunächst standardisiert, um etwaige Verzerrungen, die durch unterschiedliche Mittelwerte und/oder Streuungen der einzelnen Variablen entstehen können, zu vermeiden. ${ }^{282}$ Als Proximitätsmaß wurde die quadrierte Euklidische Distanz gewählt, um größeren Abweichungen auch stärkeres Gewicht $\mathrm{zu}$ geben. Als Fusionierungsalgorithmus wurde auf das WardVerfahren zurückgegriffen ${ }^{283}$ In Tabelle 4.7 ist dargestellt, welche zusammen-

Die Länder Dänemark, Neuseeland, Schweiz, Belgien und Irland wurden aufgrund fehlender Daten nicht berücksichtigt. Außerdem waren für die Jahre vor 1974 keine Portfolioinvestitionsströme in den IMF Balance of Payments Yearbooks ausgewiesen. Zwar wurden auch kurzfristige Ströme dargestellt, da jedoch nicht ersichtlich war, welche dieser Einzelpositionen in den Folgejahren als Portfolioinvestitionen bezeichnet werden, wird darauf verzichtet, vor 1974 kurzfristige Investitionsströme zu erfassen. Möchte man alle Länder berücksichtigen, muss man die Clusteranalyse auf den Außenhandelsindikator beschränken. In dieser Clusteranalyse wäre eine 2-Clusterlösung am naheliegendsten, jedoch ergibt die 4-Clusterlösung die unten vorgeschlagene Phaseneinteilung. Da die Berücksichtigung der Kapitalströme notwendig erscheint, wird daher im folgenden der 4-Clusterlösung der Vorzug gegeben (darauf wird später noch detailliert eingegangen).

${ }^{282}$ In SPSS 9.0 wird dafür das Verfahren z-scores verwendet. Dieses Verfahren entspricht der üblichen Standardisierungsprozedur, bei dem von jeder Merkmalsausprägung der Mittelwert des Merkmals subtrahiert und das Ergebnis durch die Standardabweichung des Merkmals dividiert wird.

283 Der Ward-Algorithmus wurde gewählt, da er nach Backhaus, Erichson, Plinke und Weiber (1996, S. 298) als "...sehr guter Fusionierungsalgorithmus angesehen werden..." kann. Das Ward-Verfahren prüft mit Hilfe des Fehlerquadratsummenkriteriums (Varianzkriteriums), welche Objekte (hier Jahre) zusammengefasst werden sollen, sodass der Zuwachs der Fehlerquadratsumme minimiert wird. Dies führt dazu, dass die Varianz in der jeweiligen Gruppe möglichst gering ist. 
gehörenden Zeitabschnitte bei unterschiedlicher Clusteranzahl gefunden wurden. Die angegebenen Werte der Fehlerquadratsumme geben ein erstes Anzeichen, welche Clusteranzahl zu bevorzugen ist. Das Elbow-Kriterium ${ }^{284}$ legt die Unterteilung in drei Cluster nahe. Zwar wäre auch eine 2-Clusterlösung durch die Werte vertretbar, die graphische Darstellung in Tabelle 4.7 zeigt jedoch, dass an der Stelle der 3-Clusterlösung ein größerer „Knick“ vorliegt; daher wird diese Lösung bevorzugt.

\section{Tabelle 4.7 : Clusteranalyse der ökonomischen Indikatoren für den Zeitraum 1974-1997 (nach Jahren)}

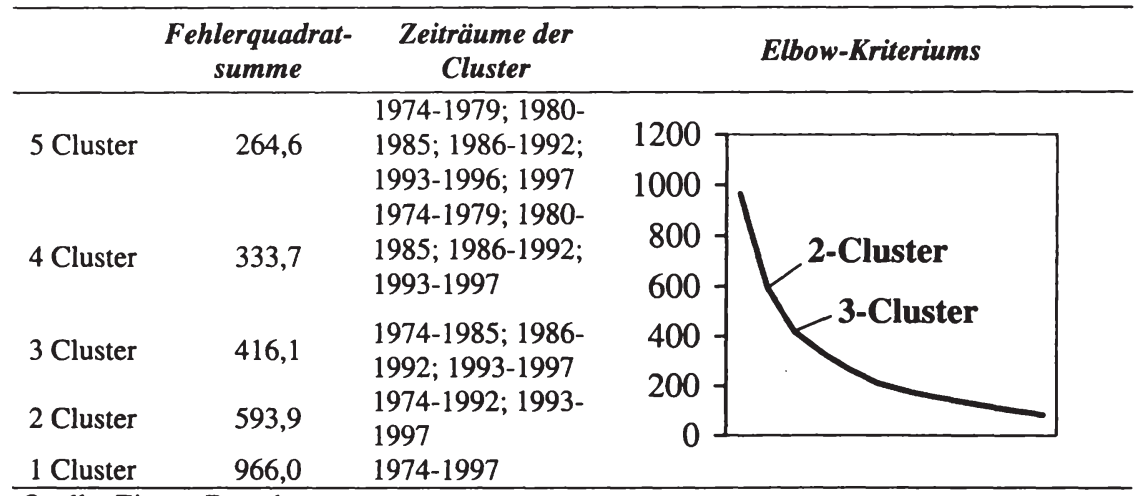

Quelle: Eigene Berechnungen.

Die Besonderheit der 90er Jahre wird noch deutlicher, wenn man statt des WardVerfahrens das Single-Linkage-Verfahren (in SPSS: nearest neighbor Verfahren) als Fusionierungsalgorithmus wählt. Dieser Algorithmus ist besonders geeignet, Ausreißer zu identifizieren (Backhaus, Erichson, Plinke und Weiber, 1996, S. 292). Für jede Anzahl an Clustern (2 bis 5) werden die Jahre 1974 bis $1992 \mathrm{zu}$ einem Cluster zusammengefasst. Dies ist ein deutliches Zeichen dafür, dass bis 1992 der Globalisierungsprozess recht stetig verlief, sich danach allerdings sprunghaft entwickelte. In diesem Analysekontext bedeutet die Isolierung von Außenseitern, dass die Veränderung in den Indikatorwerten zu dem folgenden Jahr stärker ausfällt als im Jahr zuvor.

${ }^{284}$ Das Elbow-Kriterium gibt an, welcher Übergang von einer Clusteranzahl zu der nächsten Clusteranzahl zu dem größten (relativen) Heterogenitätszuwachs, gemessen durch den Anstieg der Fehlerquadratsumme beim Ward-Verfahren, führt. Dieser Heterogenitätszuwachs lässt sich dann in einer graphischen Darstellung, bei der die Fehlerquadratsummen gegen die Anzahl der Cluster abgetragen werden, als Ellbogen, also als „Knickstelle“, veranschaulichen (Backhaus, Erichson, Plinke und Weiber 1996 s. S.9310 3-631-75139-8 
Um zumindest einen fundierten Hinweis zu bekommen, inwiefern die Jahre vor 1974 in diesen Prozess eingeordnet werden können, wird der zugrunde liegende Datensatz abgeändert. Jetzt gehen 11 Länder $^{285}$ und nur zwei Indikatoren (AuBenhandel und Direktinvestitionen) in die Clusteranalyse ein. Für diese 22 Variablen liegen Daten für die Periode 1963 bis 1997 vor; somit gehen bei 35 Jahren (Objekten) und 22 Variablen insgesamt 770 Einzelwerte in die Analyse ein. Die Clusterung entsprechend des Ward-Verfahrens zeigt eine deutliche Dominanz einer 3-Clusterlösung nach dem Elbow-Kriterium. Dabei umfasst der erste Cluster die Jahre 1963 bis 1973, der zweite Cluster die Jahre 1974 bis 1992 und der dritte Cluster die Jahre 1993-1997. ${ }^{286}$ Auch dieser Analyse wurde eine Überprüfung mit Hilfe des Single-Linkgage nachgeschaltet, und auch hier zeigten sich die Jahre nach 1992 jeweils als Ausreißer. Die Berücksichtigung der Portfolioinvestitionen führt also v.a. dazu, den Zeitraum von 1974 bis 1992 noch einmal zu unterteilen.

Nach diesen Ausführungen lässt sich der Globalisierungsprozess folglich in vier Phasen unterteilen:
1. Phase: 1963 bis $1973^{287}$
System von Bretton Woods
2. Phase: 1974 bis 1985
3. Phase: 1986 bis 1992
EG-Erweiterung, Ölkrisen.
v.a. Anstieg der Direktinvestitionen.
4. Phase: 1993 bis 1997
Effekte durch EU-Gründung, Uruguay-Runde.

Im folgenden Kapital wird eine ähnliche Clusterung für die untersuchten Länder durchgeführt.

\subsubsection{Ländercluster im Globalisierungsprozess}

Mit dem zur Verfügung stehenden Datenmaterial erweist sich die Clusterung der Länder als wesentlich schwieriger als die Gruppierung der Jahre, da für einige Länder (Dänemark, Irland, Neuseeland) erst für die 90er Jahre vollständige Investitionsdaten vorliegen. Zudem sind die Kapitalströme von Belgien über große Zeiträume gemeinsam mit den Kapitalströmen Luxemburgs ausgewiesen. Zwei gleichermaßen unbefriedigende Wege sind gangbar, möchte man nicht die An-

${ }^{285}$ Die in der Analyse berücksichtigten Länder sind: Australien, Kanada, Finnland, Frankreich, Deutschland, Italien, Niederlande, Schweden, Spanien, UK, USA.

${ }^{286}$ Auch eine Clusterung der Jahre nur nach dem Außenhandelskriterium zeigt deutlich, dass die Jahre von 1963 bis 1973 zu einem Cluster gehören.

${ }^{287}$ In der folgenden Analyse werden die Jahre 1960, 1961 und 1962 ebenfalls dem ersten Cluster zugerechnet. Dies ergibt sich allerdings aufgrund unzureichender Daten bei den Kapitalströmen einzig aus einer Clusteranalyse der Außenhandelsvariable für den Zeitraum 1960 bis 1997. 
zahl der untersuchten Länder verringern. Zum einen kann man die Clusterung nur auf der Basis des Außenhandelskriteriums vornehmen. Dies ist jedoch problematisch, da zuvor gezeigt wurde, dass insbesondere die Integration der Kapitalmärkte als maßgebliches Kennzeichen der Globalisierung betrachtet werden muss. Zum anderen kann man die Clusteranalyse auf die Jahre beschränken, für die Werte existieren, also auf die Jahre seit 1990. Dadurch gruppiert man aber eben nur die Länder, die in den letzten Jahren ähnliche Entwicklungen hatten und muss unterstellen, dass dies auch eine gute Annäherung für die davor liegenden 30 Jahre ist. Beide Alternativen haben nicht unbedeutende theoretische Defekte und liefern stark von einander abweichende Clusterungen. Aufgrund der großen Bedeutung der Kapitalmarktintegration wird im folgenden der zweite Weg gewählt.

Die Ländernamen wurden als zu clusternde Objekte definiert, und die Indikatoren für den Außenhandel, für Direktinvestitionen und Portfolioinvestitionen für die Jahre 1990 bis 1997 wurden als Variable bestimmt. Bevor mit Hilfe der quadrierten Euklidischen Distanz die Distanzmatrix erstellt wurde, wurden auch hier die Daten standardisiert. Die Clusterung erfolgte erneut mit Hilfe des WardAlgorithmus. ${ }^{288}$ Die Ergebnisse sind in Tabelle 4.8 dargestellt.

Tabelle 4.8 : Clusteranalyse der ökonomischen Indikatoren für den Zeitraum 1990-1997 (nach Ländern)

\begin{tabular}{|c|c|c|}
\hline & Fehlerquadratsumme & Clusterergebnisse \\
\hline 5 Cluster & 86,9 & $\begin{array}{l}\text { 1. BEL } \\
\text { 2. NL, NZL, S, UK } \\
\text { 3. CAN, DK, SF, NOR, CH } \\
\text { 4. IRL } \\
\text { 5. AUS, AU, F, D, ITA, J, E, USA }\end{array}$ \\
\hline 4 Cluster & 110,9 & $\begin{array}{l}\text { 1. BEL } \\
\text { 2. NL, NZL, S, UK } \\
\text { 3. CAN, DK, SF, IRL, NOR, CH } \\
\text { 4. AUS, AU, F, D, ITA, J, E, USA }\end{array}$ \\
\hline 3 Cluster & 151,7 & $\begin{array}{l}\text { 1. BEL } \\
\text { 2. NL, NZL, S, UK } \\
\text { 3. alle übrigen Länder }\end{array}$ \\
\hline 2 Cluster & 230,6 & $\begin{array}{l}\text { 1. BEL } \\
\text { 2. alle übrigen Länder }\end{array}$ \\
\hline 1 Cluster & 378,0 & alle Länder \\
\hline
\end{tabular}

Quelle: Eigene Berechnungen.

${ }^{288}$ Die Prüfung auf Ausreißer mit Hilfe des Single-Linkage Algorithmus brachte kein funda-

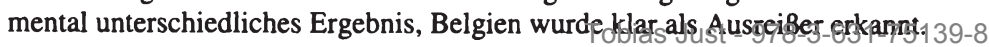


Die Bestimmung der optimalen Clusteranzahl ist nicht eindeutig, da das ElbowKriterium kein klares Ergebnis anbietet. Dies wird auch an dem progressiven Anstieg der Fehlerquadratsumme in Tabelle 4.8 deutlich. Es gibt mehrere starke Heterogenitätszuwächse. Da die 2-Clusterlösung, bei der ein Cluster aus einem Land und der zweite Cluster aus allen übrigen Ländern besteht, nicht sinnvoll ist, und die 5-Clusterlösung mit zwei Clustern, die nur ein einziges Land enthalten ebenfalls problematisch ist, wird eine Anpassung der zwei anderen Clusterlösungen gewählt. Nach der 4-Clusterlösung würde Belgien ein eigener Cluster zugewiesen. Fasst man die ersten beiden (offensten) Ländergruppen zusammen, folgt die so erhaltene (3-Gruppen) Lösung einem einfachen hochmittel-niedrig Prinzip. ${ }^{289}$

Diese Clusterlösung ist auch aufgrund einer zweiten Clusteranalyse gerechtfertigt: Dabei wurde der gesamte Untersuchungszeitraum herangezogen, jedoch nur der Außenhandelsindikator als Variable benutzt. Die Distanzmatrix wurde wieder mit der quadrierten Euklidischen Distanz aufgebaut, und die Fusionierung erfolgte mit dem Ward-Algorithmus Es zeigte sich eine deutliche 3Clusterlösung. Allerdings waren diese Cluster teilweise unterschiedlich zusammengesetzt. Dies kann nicht überraschen und zeigt, dass die Kapitalmarktindikatoren gerechtfertigterweise berücksichtigt werden müssen. Ergäbe sich nämlich dieselbe Ländereinteilung, bräuchte man die Kapitalmarktindatoren zur Beschreibung nicht. ${ }^{290}$ Hier wird die oben dargestellte methodische Dilemmasituation deutlich: Entweder man vernachlässigt die wichtigen Kapitalmarktindikatoren und rekurriert allein auf den Außenhandel, dann nimmt man die Clustereinteilung aus Fußnote 290, oder man berücksichtigt für den verkürzten Zeitraum alle drei Indikatoren, dann ist die oben skizzierte Clusterlösung zu wählen. Die Ländergruppierung, mit der im Kapitel 5 weiter gearbeitet werden soll, lautet dann wie folgt.

1. überdurchschnittlicher Globalisierungsgrad: BEL, NL, NZL, S, UK

2. durchschnittlicher Globalisierungsgrad: $\quad$ CAN, DK, SF, IRL, NOR, CH

3. unterdurchschnittlicher Globalisierungsgrad: AUS, AU, F, D, ITA, J, E, USA.

Aufgrund der Bedeutung der Kapitalmärkte (siehe oben) wird dieser Einteilung der Vorzug gegeben. Diese Clustereinteilung lässt sich relativ gut durch die

${ }^{289}$ Es muss bei der Außenseiterrolle von Belgien bedacht werden, dass der Kapitalverkehr nur gemeinsam mit Luxemburg ausgewiesen wurde. Dies kann dazu geführt haben, dass gerade die kurzfristigen Kapitalströme die belgische Position stark überzeichnen. Eine Zuordnung zum zweiten Cluster wäre gerade dann zu rechtfertigen.

${ }^{290}$ Die Clusterung nach dem Außenhandelskriterium führt zu folgender Clustereinteilung:

1. Cluster: BEL, NL, IRL

2. Cluster: S, CAN, SF, AU, NZL, UK, D, CH, NOR, DK

3. Cluster: F, ITA, AUS, E, J, USA. 
Konstruktion von drei abgeleiteten Indikatoren veranschaulichen. Ist Off ${ }_{i t}$ der oben beschriebene Offenheitsgrad für jedes Land i mit $i \in[1 ; n]$ und jedes Jahr $j$ mit $j \in[1990 ; 1997]$, dann ist der abgeleitete Offenheitsgradindikator Off_ $I_{i}$ für jedes Land definiert als:

$$
\text { Off_I } I_{i}=\frac{1}{t} \sum_{F=1}^{t} \frac{O_{i j}}{\frac{1}{n} \sum_{i=1}^{n} O f f_{i j}}
$$

Ein Land, für das Off_ $I_{i}>1$ gilt, ist folglich ein Land, das im Durchschnitt über alle Jahre überdurchschnittlich stark Außenhandel betrieben hat. Analog gilt für ein Land mit Off_ $I_{i}<1$, dass dieses Land im Durchschnitt über alle Jahre unterdurchschnittlich stark in die internationale Arbeitsteilung integriert war. In ähnlicher Weise lässt sich ein abgeleiteter Indikator für Direktinvestitionen FDI_I $\mathbf{I}_{\mathbf{i}}$ und ein Indikator für Portfolioinvestitionen $P_{-} I_{i}$ für jedes Land $i$ konstruieren. Mit $\mathrm{FDI}_{\mathrm{ij}}$ als die Direktinvestitionsströme eines Landes zu jedem Zeitpunkt $\mathrm{t}$ ist FDI_I $I_{i}$ definiert als:

$$
\text { FDI_I } I_{i}=\frac{1}{t} \sum_{=1}^{l} \frac{F_{i j}}{\frac{1}{n} \sum_{i=1}^{n} F D I_{i j}}
$$

Kennzeichnet $P_{i j}$ schließlich die Portfolioinvestitionsströme eines Landes i zu jedem Zeitpunkt $t$, so ist $P_{-} I_{i}$ definiert als:

$$
P_{-} I_{i}=\frac{1}{t} \sum_{j=1}^{t} \frac{P_{i j}}{\frac{1}{n} \sum_{i=1}^{n} P_{i j}}
$$

Ein Land mit FDI_ $I_{i}>1$ gilt natürlich dann als überdurchschnittlich stark durch Direktinvesitionsströme charakterisiert und ein Land mit FDI_ $I_{i}<1$ als ein unterdurchschnittlich in Direktinvestitionsströme eingebundes Land. Die Argumentation für $P_{-} I_{i}$ ist dabei natürlich vollkommen analog zu den anderen beiden Indikatoren Off_I $I_{i}$ und FDI_ $I_{i}$. In Tabelle 4.9 sind die Werte für die drei abgeleiteten Indikatoren zusammengestellt und nach ihrem ungewichteten Mittelwert aufsteigend sortiert.

Insgesamt wird die gefundene Ländergruppierung relativ gut durch den ungewichteten Mittelwert abgebildet, wennglich auch hier die Schwierigkeiten bei der Bestimmung der optimalen Clusteranzahl offensichtlich ist. 
Tabelle 4.9 : Kennzeichnung der ökonomischen Cluster

\begin{tabular}{|c|c|c|c|c|}
\hline & Off_I $I_{i}$ & FDI_I & $\mathbf{P}_{-} \mathbf{I}_{\mathbf{i}}$ & ungew. Mittel \\
\hline Japan (3) & 0,274 & 0,131 & 0,364 & 0,256 \\
\hline USA (3) & 0,323 & 0,553 & 0,595 & 0,490 \\
\hline Deutschland (3) & 0,837 & 0,308 & 0,665 & 0,603 \\
\hline Australien (3) & 0,547 & 0,722 & 0,594 & 0,621 \\
\hline Italien (3) & 0,650 & 0,318 & 0,955 & 0,641 \\
\hline Spanien (3) & 0,622 & 0,693 & 0,646 & 0,654 \\
\hline Österreich (3) & 1,048 & 0,404 & 0,748 & 0,733 \\
\hline Norwegen (2) & 1,015 & 0,726 & 0,525 & 0,755 \\
\hline Frankreich (3) & 0,695 & 1,001 & 0,715 & 0,804 \\
\hline Kanada (2) & 1,040 & 0,731 & 0,861 & 0,877 \\
\hline Finnland (2) & 0,987 & 0,905 & 1,034 & 0,975 \\
\hline Schweiz (2) & 0,933 & 1,124 & 0,936 & 0,997 \\
\hline Dänemark (2) & 1,109 & 0,866 & 1,083 & 1,019 \\
\hline Neuseeland (1) & 0,840 & 1,862 & 0,464 & 1,055 \\
\hline Irland (2) & 2,042 & 1,051 & 0,653 & 1,249 \\
\hline Schweden (1) & 1,141 & 1,905 & 1,296 & 1,447 \\
\hline UK (1) & 0,847 & 1,618 & 1,901 & 1,456 \\
\hline Niederlande (1) & 1,672 & 2,084 & 0,854 & 1,537 \\
\hline Belgien (1) & 2,376 & 2,000 & 4,112 & 2,829 \\
\hline
\end{tabular}

Anmerkungen: Hinter dem Ländernamen steht die Nummer des Clusters.

Quelle: Eigene Berechnungen.

\subsubsection{Politische Globalisierungscluster}

Nun lässt sich argumentieren, dass die Handlungsspielräume der Politik nicht nur durch die tatsächlich stattgefundene wirtschaftliche Verflechtung in Form von Waren- und Kapitalströmen begrenzt wird. Vielmehr stellt gerade der nicht realisierte, wohl aber potentiell mögliche Kapitalexport ein enormes Drohpotential der Unternehmen dar, um eine unternehmensfreundliche Politik durchzusetzen. Möchte man also Unterschiede in den Handlungsspielräumen der Politik analysieren, muss man diese Facette mitberücksichtigen. Im folgenden sollen daher ergänzend die 19 OECD-Staaten mit Hilfe von zwei Liberalisierungsindikatoren in Gruppen unterschiedlicher Internationalisierungsgrade unterteilt werden. Gleichzeitig soll versucht werden, den Untersuchungszeitraum in einzelne Globalisierungsphasen zu unterteilen. 


\subsubsection{Indikatoren für Kapitalmarkt- und Handelsliberalisierung}

Analog zu dem vorherigen Abschnitt soll jetzt ein Indikator für die Liberalisierung der Gütermärkte und ein Indikator für die Öffnung der Kapitalmärkte gebildet werden. Der erste Indikator bildet die Liberalisierung der Kapitalmärkte ab und wird der Arbeit von Quinn und Inclan (1997) verdankt. ${ }^{291}$ Quinn und Inclan bilden einen Offenheitsindikator auf der Grundlage von Einschätzungen des IMF, wie sie im Annual Report on Exchange Restrictions seit 1950 erfasst werden. Sie unterscheiden in Restriktionen hinsichtlich der Leistungsbilanz sowie hinsichtlich der Kapitalverkehrsbilanz und fassen die qualitativen Aussagen in den $I M F$-Reports in sechs einzelnen Intervallskalen von 0 bis 2 zusammen, wobei die Intervalllänge 0,5 beträgt. ${ }^{292}$

Tabelle 4.10 : Kapitalmarktliberalisierung

\begin{tabular}{l|rrrrr}
\hline & $\mathbf{1 9 6 0}$ & $\mathbf{1 9 7 0}$ & $\mathbf{1 9 8 0}$ & $\mathbf{1 9 9 0}$ & $\mathbf{1 9 9 3}$ \\
\hline Australien & 2,5 & 2 & 2 & 3 & 3 \\
Österreich & 1,5 & 2,5 & 3 & 3,5 & 3,5 \\
Belgien & 3 & 3 & 3 & 4 & 4 \\
Kanada & 4 & 3,5 & 3 & 4 & 4 \\
Dänemark & 2,5 & 3 & 3 & 4 & 4 \\
Finnland & 0,5 & 1,5 & 2 & 3,5 & 3,5 \\
Frankreich & 3 & 3 & 3 & 3,5 & 4 \\
Deutschland & 4 & 4 & 3,5 & 4 & 4 \\
Irland & 2 & 2 & 3 & 2,5 & 4 \\
Italien & 2,5 & 3 & 3 & 3,5 & 4 \\
Japan & 2 & 2 & 2,5 & 2,5 & 3 \\
Niederlande & 3 & 3 & 3 & 4 & 4 \\
Neuseeland & 1,5 & 1,5 & 2,5 & 3,5 & 3,5 \\
Norwegen & 1,5 & 1,5 & 2 & 4 & 4 \\
Schweden & 2,5 & 2,5 & 3 & 3,5 & 4 \\
Schweiz & 4 & 4 & 4 & 4 & 4 \\
Spanien & 1,5 & 2 & 2 & 3 & 3 \\
UK & 2 & 2 & 4 & 4 & 4 \\
USA & 4 & 3,5 & 4 & 4 & 4 \\
\hline ungew. Mittelwert & $\mathbf{2 , 5 0}$ & $\mathbf{2 , 6 1}$ & $\mathbf{2 , 9 2}$ & $\mathbf{3 , 5 8}$ & $\mathbf{3 , 7 6}$ \\
\hline Qulle Dan
\end{tabular}

Quelle: Datensatz bereitgestellt von Dennis P. Quinn und gemäß Quinn und Inclan (1997).

${ }^{291}$ Insbesondere bin ich Dennis P. Quinn von der Georgetown University dankbar, der mir seinen gesamten Datensatz zugänglich gemacht hat. Der bereitgestellte Datensatz umfasst die Jahre 1950 bis 1993 und ist damit um fünf Jahre umfangreicher als der Datensatz, der in der Studie Quinn und Inclan (1997) verwendet wurde.

292 Natürlich ist die Übertragung von qualitativen Merkmalen in eine Intervallskala problematisch, da implizit von einer konstanten Intervalllänge ausgegangen wird. Zur Durchführung der weiteren Datenanalyse scheint diese Vereinfachung abyerdings ye 
Im folgenden werden lediglich die zwei Einzelskalen bezüglich der Kapitalverkehrsbilanz benutzt, um eine Doppelerfassung der Gütermarktbeschränkungen durch die Restriktionen hinsichtlich der Leistungsbilanz und dem zweiten Indikator zu vermeiden. Der Indikator zur Liberalisierung des Kapitalverkehrs bewegt sich also in den Grenzen von 0 bis 4 in Schritten von 0,5. Tabelle 4.10 zeigt einen Ausschnitt des Datensatzes für die 19 OECD-Staaten und ausgewählte Jahre. Es ist ein deutlicher Trend zu höheren Werten des Indikators und folglich auch zu größerer Liberalisierung der Kapitalmärkte zu verzeichnen; dabei ist die deutlichste Liberalisierungsdynamik für die 80er Jahre offensichtlich.

Der zweite Indikator erfasst die Liberalisierung der internationalen Güterströme. Dafür wurde eine einfache Intervallskala entwickelt, die maßgeblich auf den Daten der Tabelle 4.1 aufbaut und die Einbindung der Staaten in regionale und überregionale Handelsabkommen abbildet.

Die Skalierung fußt dabei auf folgender Punktewertung, die offensichtlich die Werte 0 bis 13 annehmen kann: ${ }^{293}$

- Einbindung in eine Freihandelszone

- $\quad$ klein (ANZCERTA)

$+1$

- mittel (EFTA/CUSTA/NAFTA)

- $\quad$ groß (EG/EU und EG/EFTA)

- Einbindung in eine Zollunion

- Einbindung in einen Binnenmarkt

- Europäisches Währungssystem

- GATT-Abkommen

- $\quad$ Dillon-Runde 1961

- Kennedy-Runde 1967

- $\quad$ Tokio-Runde 1979

- $\quad$ Uruguay-Runde 1993
$+2$

$+3$

$+1$

$+1$

$+1$

$+1$

$+2$

$+2$

$+2$

${ }^{293}$ Die Unterscheidung in kleine, mittlere und große Freihandelszonen erfolgte nach dem Verhältnis des intraregionalen Handels in Bezug zum Gesamthandelsvolumen einer Region (siehe dazu die Angabe in 4.2.3). Die Berücksichtigung des Europäischen Währungssystems (EWS) mag zunächst überraschen. Da das EWS allerdings bedeutete, dass nationale Zentralbanken zur Sicherung einer festgelegten Wechselkursschwankungsbreite massive Wechselkurspolitik betreiben mussten, führte dies zu umfangreichen Eingriffen in die Handelsströme. Nationale Außenwirtschaftspolitik wurde dadurch dem Diktat der europäischen Wechselkursziele unterworfen. Schließlich wurde die Punktevergabe der GATTRunden gemäß den Angaben hinsichtlich der durchschnittlichen Zollsatzsenkung der einzelnen Runden bei Maennig und Wilfling (1998, S. 295) vorgenommen. Danach führte die Dillon-Runde 1961 nur zu einer Senkung des durchschnittlichen Zollsatzes von 7\%, die anderen Runden hingegen zu Zollsatzsenkungen von über $30 \%$, die Uruguay-Runde sogar von $50 \%$. Schließlich muss auch hier darauf hingewiesen werden, dass das Erstellen einer Intervallskala eine starke Vereinfachung der Tatbestände bedeutet. Der Gewinn an Analysemöglichkeiten mag jedoch den Verlust an theoretisgher Ekgaktbeit reshtfertigemg-8 
Für ausgewählte Jahre und für die 19 OECD-Staaten werden die Werte für diesen Indikator in Tabelle 4.11 präsentiert. Es wird eindrucksvoll gezeigt, dass alle OECD-Staaten zumindest in regional begrenzten Räumen in den letzten $40 \mathrm{Jah}$ ren zunehmend Handelsbeschränkungen abgebaut haben.

Mit Hilfe dieser beiden Indikatoren soll nun versucht werden, Ländercluster zu bilden und einzelne Globalisierungsphasen zu isolieren. ${ }^{294}$

Tabelle 4.11 : Gütermarktliberalisierung

\begin{tabular}{l|ccrrr}
\hline & 1960 & 1970 & 1980 & 1990 & 1997 \\
\hline Australien & 0 & 4 & 6 & 6 & 8 \\
Österreich & 2 & 5 & 8 & 8 & 13 \\
Belgien & 3 & 7 & 10 & 10 & 13 \\
Kanada & 0 & 3 & 5 & 7 & 9 \\
Dänemark & 2 & 5 & 10 & 10 & 13 \\
Finnland & 0 & 5 & 8 & 8 & 12 \\
Frankreich & 3 & 7 & 10 & 10 & 13 \\
Deutschland & 3 & 7 & 10 & 10 & 13 \\
Irland & 0 & 2 & 9 & 9 & 12 \\
Italien & 3 & 7 & 10 & 10 & 13 \\
Japan & 1 & 3 & 5 & 5 & 7 \\
Niederlande & 3 & 7 & 10 & 10 & 13 \\
Neuseeland & 0 & 4 & 6 & 6 & 8 \\
Norwegen & 2 & 5 & 8 & 8 & 10 \\
Schweden & 2 & 5 & 8 & 8 & 12 \\
Schweiz & 2 & 4 & 8 & 8 & 10 \\
Spanien & 0 & 2 & 4 & 9 & 12 \\
UK & 2 & 5 & 9 & 10 & 13 \\
USA & 0 & 3 & 5 & 7 & 9 \\
\hline ungew. Mittelwert & $\mathbf{1 , 4 7}$ & $\mathbf{4 , 7 3}$ & $\mathbf{7 , 8 4}$ & $\mathbf{8 , 3 7}$ & $\mathbf{1 1 , 2 1}$ \\
\hline
\end{tabular}

Quelle: Eigene Zusammenstellung gemäß der Angaben in Tabelle 4.1.

${ }^{294}$ Es könnte eingeräumt werden, dass die Senkung der tarifären Handelshemmnisse durch eine Stärkung der nicht-tarifären Handelshemmnisse substituiert wurde. Tatsächlich gibt es schwache empirische Bestätigung für die These, dass der Umfang der nicht-tarifären Handelshemmnisse seit den 80er Jahren zunahm (Hasse, 1995, S. 9). Dennoch verwirft die Mehrzahl der Außenhandelsökonomen die These eines "law of constant protection“ (Bhagwati, 1988, S. 53). Dieser Ansicht wird hier gefolgt. Zudem gibt es bei den nichttarifären Handelshemmnissen ein gravierendes Datenerfassungsproblem, da die Hemmnisse definitionsgemäß schwierig zu quantifizieren sind. Daher wird auf die - methodisch sicherlich gerechtfertigte - Berücksichtigung weicher Formen des Protektionismus verzichtet. 


\subsubsection{Phasen im Globalisierungsprozess}

Der Untersuchungszeitraum beläuft sich auf die Jahre von 1960 bis 1993, da für diesen Zeitraum für die beiden oben dargestellten Indikatoren Daten vorliegen. Im folgenden soll mit Hilfe der Clusteranalyse versucht werden, Brüche in der Entwicklung aufzuzeigen.

Dafür werden die beiden oben ermittelten Indikatoren (Kapitalmarkt- und Gütermarktliberalisierung) für alle 19 OECD-Staaten als Variable definiert. Die zu clusternden Objekte sind die Jahre. Somit erhält man für zwei Indikatoren, 19 Staaten und 34 Jahre insgesamt 1.292 Einzelbeobachtungen. Da die Indikatoren unterschiedliche Definitionsbereiche aufweisen, folglich auch wahrscheinlich unterschiedliche Mittelwerte und Varianzen besitzen, werden die Daten zunächst standardisiert.

Tabelle 4.12 : Clusteranalyse der politischen Indikatoren für den Zeitraum 1960 bis 1993 (nach Jahren)

\begin{tabular}{lcl}
\hline & $\begin{array}{c}\text { Fehlerquadrat- } \\
\text { summe }\end{array}$ & \multicolumn{1}{c}{ Zeiträume der Cluster } \\
\hline 6 Cluster & 168,0 & $\begin{array}{l}1960-1966 ; 1967-1973 ; 1974-1978 ; \\
1979-1980 ; 1981-1987 ; 1988-1993\end{array}$ \\
& & $\begin{array}{l}1960-1966 ; 1967-1973 ; 1974-1978 ; \\
5 \text { Cluster }\end{array}$ \\
& 197,3 & $\begin{array}{l}1979-1987 ; 1988-1993 \\
\text { 4 Cluster }\end{array}$ \\
3 Cluster & 246,6 & $1960-1966 ; 1967-1978 ; 1979-1987 ;$ \\
2 Cluster & 383,7 & $1988-1993$ \\
1 Cluster & 533,0 & $1960-1966 ; 1967-1978 ; 1979-1993$ \\
\hline
\end{tabular}

Quelle: Eigene Berechnungen.

Als Proximitätsmaß wurde erneut die quadrierte Euklidische Distanz verwendet, um größeren Abweichungen auch ein überproportionales Gewicht zuzuweisen. Als Fusionierungsalgorithmus findet das Ward-Verfahren Anwendung. ${ }^{295}$ Tabelle 4.12 zeigt die letzten sechs gebildeten Cluster und die dazugehörigen Fehlerquadratsummen. Nach dem Elbow-Kriterium wären offensichtlich zwei

295 Zuvor wurde auch mit Hilfe des Single-Linkage-Algorithmus geprüft, ob sich Ausreißer in dem Datenset befinden. Es zeigte sich, dass das Jahr 1993 als ein solcher Außenseiter verstanden werden kann. Später wird auf diesen Sachverhalt zurüickgegriffent-75139-8 
Clusterungen denkbar, die Einteilung in zwei Cluster oder die Einteilung in vier Cluster. ${ }^{296}$

Durch eine zusätzliche Clusteranalyse allein des Handelsliberalisierungsindikators für den Zeitraum von 1960-1997 lässt sich zeigen, dass der Zeitraum 19931997 ein eigenes Cluster zugewiesen bekommt. ${ }^{297}$ Auch hier wurde mit der quadrierten Euklidischen Distanz und dem Ward-Fusionierungsalgorithmus gearbeitet. Die Analyse der Fehlerquadratsummen ergab dabei die deutlichste Knickstelle (Ellbogen) für die 4-Clusterlösung.

In der weiteren Arbeit soll daher von einer Einteilung des Globalisierungsprozesses in fünf Phasen ausgegangen werden. Dies sind die vier Phasen, die die erste Analyse zeigte, zuzüglich der fünften Phase von 1993-1997, die die zweite Analyse ergab:

1. Phase 1960-1966 EG, EFTA, Dillon-Runde, wenig liberale Kapitalmärkte

2. Phase 1967-1978 Kennedy-Runde, EG, mäßig liberale Kapitalmärkte

3. Phase 1979-1987 Tokio-Runde, EWS, starke Kapitalmarktliberalisierung

4. Phase 1988-1992 CUSTA, starke Kapitalmarktliberalisierung

5. Phase 1993-1997 Uruguay-Runde, EU, NAFTA

Im folgenden Abschnitt soll nun die Frage untersucht werden, ob sich verschiedene Ländergruppen abgrenzen lassen.

\subsubsection{Ländercluster im Globalisierungsprozess}

Das Vorgehen bei der Bildung von Ländergruppen ist analog zu obiger Prozedur. Die beiden Indikatoren für Handels- und Kapitalmarktliberalisierung wurden für jedes Land und jedes Jahr gebildet. Dieses Mal wurden jedoch die Jahreszahlen als Variable und die Länder als zu clusternde Objekte deklariert. Es gab folglich bei insgesamt 19 Objekten und 68 Variablen 1.292 Einzelbeobachtungen. Diese wurden zunächst standardisiert, um auf der Grundlage der standardisierten Variablenwerte die quadrierte Euklidische Distanz als Proximitäts-

296 Eine Homogenitätsprüfung der gefundenen Cluster mittels eines F-Wertes ist nicht erforderlich, da das Ergebnis durch die Art des Datensatzes vorbestimmt ist. Beide Indikatoren haben einen stetigen Trend in der Zeit, sodass für jeden Indikator in jedem gefundenen Cluster die Varianz innerhalb des Clusters geringer ausfallen muss als die Varianz für die Grundgesamtheit einer Variable.

${ }^{297}$ Dies erklärt auch, warum zuvor das Single-Linkage-Verfahren bei der Clusteranalyse der beiden Kriterien das Jahr 1993 als Ausreißer isolient hast.Just - 978-3-631-75139-8 
maß für alle Objektpaare zu bilden. Anschließend wurde die eigentliche Clusterung mit Hilfe des Ward-Verfahrens durchgeführt. ${ }^{298}$

\section{Tabelle 4.13 : Clusteranalyse der politischen Indikatoren für den Zeitraum 1960 bis 1993 (nach Ländern)}

\begin{tabular}{|c|c|c|}
\hline & $\begin{array}{c}\text { Fehlerquadrat- } \\
\text { summe }\end{array}$ & Clusterergebnisse \\
\hline 5 Cluster & 293,5 & $\begin{array}{l}\text { 1. AUS, J, NZL, E } \\
\text { 2. AU, IRL, S, UK } \\
\text { 3. BEL, DK, F, D, ITA, NL } \\
\text { 4. CAN, CH, USA } \\
\text { 5. NOR, SF }\end{array}$ \\
\hline 4 Cluster & 371,1 & $\begin{array}{l}\text { 1. BEL, DK, F, D, ITA, NL (Cluster I) } \\
\text { 2. AU, SF, IRL, NOR, S, UK (Cluster II) } \\
\text { 3. CAN, CH, USA (Cluster III) } \\
\text { 4. AUS, J, NZL, E (Cluster IV) }\end{array}$ \\
\hline 3 Cluster & 530,3 & $\begin{array}{l}\text { 1. AUS, AU, SF, IRL, J, NZL, NOR, S, E, UK } \\
\text { 2. BEL, DK, F, D, ITA, NL } \\
\text { 3. CAN, CH, USA }\end{array}$ \\
\hline 2 Cluster & 807,0 & $\begin{array}{l}\text { 1. BEL, DK, F, D, NL } \\
\text { 2. AUS, AU, CAN, SF, IRL, ITA, J, NZL, NOR, S, CH, } \\
\text { E, UK, USA }\end{array}$ \\
\hline 1 Cluster & $1.224,0$ & 1. alle Länder \\
\hline
\end{tabular}

Quelle: Eigene Berechnungen.

Die Prüfung des Elbow-Kriteriums zeigt, dass in dieser Analyse die Einteilung in vier Cluster gerechtfertigt erscheint. Zwar weist auch der Übergang von der 2-Clusterlösung zur 1-Clusterlösung eine deutliche "Knickstelle" auf, doch ist diese weniger stark ausgeprägt als der Knick für die 4-Clusterlösung. Bei der 4Clusterlösung wurde in Klammern noch die in der Abbildung 4.3 verwandte Clusternummerierung angegeben (römische Ziffern).

Die vier gefundenen Cluster verteilen sich nahezu idealtypisch auf die beiden zugrunde liegenden Indikatoren. Dies soll mit Hilfe von abgeleiteten Indikatoren veranschaulicht werden. Ist $\mathrm{H}_{\mathrm{ij}}$ der oben beschriebene Indikator der Handelsliberalisierung für jedes Land i mit $i \in[1 ; n]$ und jedes Jahr $j$ mit $j \in[1 ; t]$, dann ist der abgeleitete Handelsindikator $\mathrm{HI}_{\mathrm{i}}$ für jedes Land definiert als:

298 Auch hier wurde zunächst mit dem Single-Linkage-Verfahren auf Ausreißer geprüft. Dies zeigte, dass gegebenenfalls Irland als Ausreißer betrachtet werden kann. Da das Herauslassen Irlands aus der Clusterung mit dem Ward-Verfahren keine anderen Gruppierungen ergab, wurde Irland in dem Datensatz belassen. Tobias Just - 978-3-631-75139-8 


$$
\mathrm{HI}_{\mathrm{i}}=\frac{1}{\mathrm{t}} \sum_{\mathrm{j}=1}^{\mathrm{t}} \frac{\mathrm{H}_{\mathrm{ij}}}{\frac{1}{\mathrm{n}} \sum_{\mathrm{i}=1}^{\mathrm{n}} \mathrm{H}_{\mathrm{ij}}}
$$

Ein Land, für das $\mathrm{HI}_{\mathrm{i}}>1$ gilt, ist folglich ein Land, das im Mittel über alle Jahre überdurchschnittlich stark seinen Außenhandel liberalisiert hat. Analog gilt für ein Land $\mathrm{mit}_{\mathrm{HI}}<1$, dass dieses Land im Durchschnitt über alle Jahre unterdurchschnittlich stark seinen Außenhandel geöffnet hat.

In ähnlicher Weise lässt sich ein abgeleiteter Kapitalmarktindikator $\mathrm{KI}_{\mathrm{i}}$ für jedes Land $\mathrm{i}$ konstruieren. Mit $\mathrm{K}_{\mathrm{ij}}$ als dem oben beschriebenen Indikator für die Liberalisierung der Kapitalmärkte nach Quinn und Inclan, ist $\mathrm{KI}_{\mathrm{i}}$ definiert als:

$$
\mathrm{KI}_{\mathrm{i}}=\frac{1}{\mathrm{t}} \sum_{\mathrm{j}=1}^{\mathrm{t}} \frac{\mathrm{K}_{\mathrm{ij}}}{\frac{1}{\mathrm{n}} \sum_{\mathrm{i}=1}^{\mathrm{n}} \mathrm{K}_{\mathrm{ij}}}
$$

Auch hier gilt ein Land mit $\mathrm{KI}_{\mathrm{i}}>1$ als überdurchschnittlich offen hinsichtlich seiner Kapitalmärkte und ein Land mit $\mathrm{KI}_{\mathrm{i}}<1$ als ein unterdurchschnittlich kapitaloffenes Land. Trägt man beide Indikatoren $\mathrm{HI}_{i}$ und $\mathrm{KI}_{i}$ gegen einander $\mathrm{ab}$, zeigt sich deutlich die oben ermittelte Clusterung.

Abbildung 4.3 : Ländercluster der politischen Indikatoren

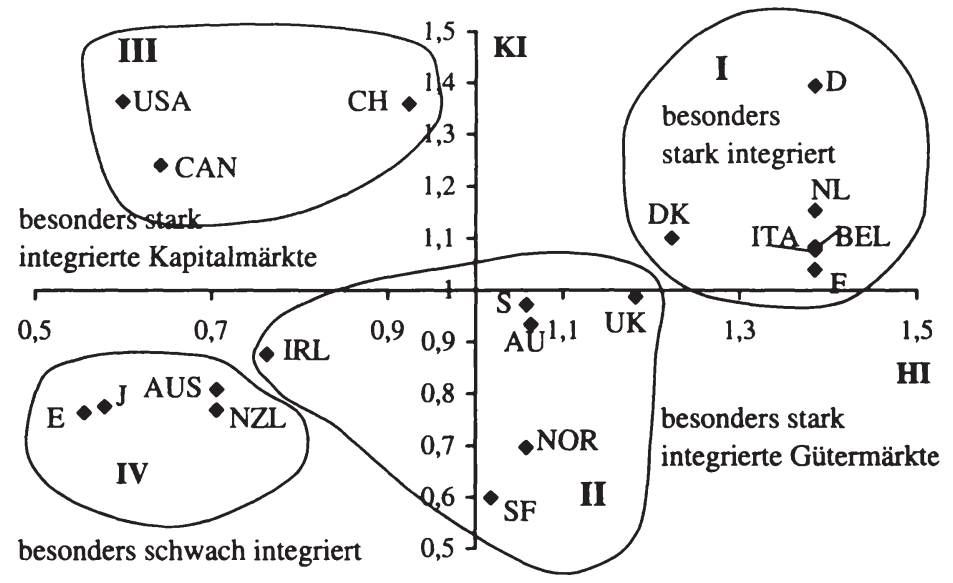

In dieser Darstellung wird die Ausreißerrolle von Irland, wie sie die nach dem Single-Linkage-Verfahren durchgeführte Clusterung ergab, besonders gut deutlich, denn ohne Irland ließen sich alle Cluster deutlich einem der vier 
Quadranten zuordnen. Angesichts dieser Tatsache mag es erstaunen, dass Irland dem Cluster II zugeordnet wurde. Dies liegt daran, dass Irland relativ spät dem GATT beigetreten ist (erst in der Kennedy-Runde) und erst spät ein Freihandelsabkommen unterzeichnete (1973 den EG-Vertrag). Dies führt dazu, dass Irland in den letzten 25 Jahren eine ähnliche Entwicklung hatte wie die Länder des Clusters II, aufgrund des sehr niedrigen Anfangswertes ist der Durchschnittswert für den $\mathrm{HI}$ jedoch unterdurchschnittlich.

Es ist auch darauf hinzuweisen, dass diese Clusterung auf der Basis aller 34 Jahre durchgeführt wurde und dass Clusterungen der Länder auf der Grundlage von einzelnen Jahren teilweise zu verschiedenen Clusterergebnissen sowohl hinsichtlich der optimalen Anzahl der Cluster als auch hinsichtlich der Gruppenzusammensetzung führen. Da die hier vorgenommene Clusterung die Aussage zuläßt, welche Länder über den gesamten Zeitraum eine ähnliche Entwicklung durchliefen, erscheint sie besonders geeignet, Ländergruppen zu bilden. ${ }^{299}$ Im folgenden wird mit der oben dargestellten 4-Cluster-Lösung gearbeitet.

\subsubsection{Vergleich der politischen und ökonomischen Cluster- ergebnisse}

Wie eingangs des Kapitels vermutet, konnten die Clusteranalysen zeigen, dass es einen Unterschied macht, ob man die Offenheit eines Landes durch seine tatsächliche Einbindung in die Wirtschaft, abzulesen an ökonomischen Fundamentaldaten, oder durch seine Liberalisierungs- und Integrationspolitik operationalisiert. Sowohl hinsichtlich der Clusteranzahl als auch hinsichtlich der Grenzziehung und bei den Länderclustern auch hinsichtlich der Clusterinhalte ergeben sich zum Teil gravierende Abweichungen.

Besonders deutlich wird die Unterscheidung dieser beiden Clusterungen bei den Ländern Deutschland, Frankreich und Italien. Durch die Integration in den europäischen Binnenmarkt zählen sie zu den politisch am stärksten liberalisierten Länder - gleichzeitig sind sie bei der ökonomischen Clusterung in dem Cluster mit den niedrigsten Integrationsmaßen. Dies könnte natürlich auch daran liegen, dass diese relativ großen Länder weitgehend autark sind und eine stärker diversifizierte Wirtschaftsstruktur aufweisen als andere (oftmals kleinere) Länder, die ebenfalls in der Europäischen Union sind, jedoch wesentlich höhere Offenheitsgrade aufweisen. Es scheint demnach sinnvoll zu sein, die empirischen Tests des

299 Dies gilt v.a. auch deshalb, da bei einer 1-Jahresbetrachtung der Fusionierungsalgorithmus sehr sensibel auf kleine Änderungen in den Variablenwerten reagiert. Selbst kleine (zufällig) in diesem Jahr stattfindende Änderungen der Variablenwerte führen dann zu starken Verschiebungen in den Clusterergebnissen. 
folgenden Kapitels zum Vergleich mit beiden Clusterergebnissen durchzuführen.

Tabelle 4.14 : Vergleich der Clusterergebnisse

\begin{tabular}{llll}
\hline \multicolumn{1}{c}{ Ökonomische Cluster } & \multicolumn{2}{c}{ Politische Liberalisierungscluster } \\
\hline Zeitliche Entwicklung & Zeitliche Entwicklung \\
1. Phase: & $1960-1973$ & 1. Phase: & $1960-1966$ \\
2. Phase: & $1974-1985$ & 2. Phase: & $1967-1978$ \\
3. Phase: & $1986-1992$ & 3. Phase: & $1979-1987$ \\
4. Phase: & $1993-1997$ & 4. Phase: & $1988-1992$ \\
& 5. Phase: & $1993-1997$ \\
\hline Ländercluster & Ländercluster \\
1. Cluster: BEL, NL, NZL, S, UK & 1. Cluster: D, DK, ITA, NL, BEL, F \\
2. Cluster: CAN, DK, SF, IRL, NOR, CH & 2. Cluster: UK, S, AU, NOR, SF, IRL \\
3. Cluster: AUS, AU, F, D, ITA, J, E, USA & 3. Cluster: USA, CAN, CH \\
& 4. Cluster: E, J, AUS, NZL \\
\hline
\end{tabular}

Quelle: Eigene Zusammenstellung gemäß den obigen Berechnungen. 
Tobias Just - 978-3-631-75139-8

Downloaded from PubFactory at 01/11/2019 07:52:01AM

via free access 


\section{Kapitel 5 \\ Globalisierung und nationale Wirtschaftspolitik - Empirie}

"Econometricians have found their Philosophers" Stone; it is called regression analysis and is used for transforming data into "significant " results!" Hendry (1980, S. 390)

Nachdem im letzten Kapitel die Diskussion nachgezeichnet wurde, inwiefern die Globalisierung der Märkte die nationale Politik zur Teilnahme an einem race to the bottom zwingt oder nicht, erscheint es angesichts dieser kontroversen und keineswegs abgeschlossenen Debatte notwendig, empirisch die Fragestellung zu prüfen, ob die Öffnung der Märkte zu einer Vereinheitlichung der nationalen Wirtschaftspolitik geführt hat. Dies ist Inhalt dieses Kapitels.

Es werden drei Aspekte der Wirtschaftspolitik untersucht. Zum ersten wird geprüft, in welchem Umfang ideologisch unterschiedlich prädisponierte Parteien auf dem Arbeitsmarkt verschiedene Ergebnisse erzielten und ob diese Unterschiedlichkeit durch die Marktöffnung reduziert wurde. Dafür werden sowohl Modelle, die die traditionelle Partisantheorie nach Hibbs abbilden, als auch rationale Partisanmodelle des Alesina-Typs geschätzt. ${ }^{300}$ Im Anschluss daran wird das zweite Element der Partisanmodelle, die Preisentwicklung als Reflex unterschiedlicher Regierungsparteien, untersucht.

Zum dritten wird schließlich analysiert, ob verschiedene ideologische Positionen von Regierungen aus den Budgetstrukturen bzw. deren Veränderungen herausgelesen werden können. Dabei wird ausschließlich die Ausgabenseite der Budgets betrachtet. Es wird geprüft, inwiefern linke Regierungen tatsächlich jenen Ausgabenkategorien stärkeres Gewicht gegeben haben, die größere Verteilungswirkungen besitzen, wie die Ausführungen in Abschnitt 3.4.3.3 erwarten lassen. Dies ist die Grundlage für die Überprüfung der Hypothese, dass im Zuge der internationalen Marktöffnung die Handlungsspielräume nationaler Politik verschwinden müssen. Gilt dies, dann dürften die Differenzen in den Budgetstrukturen nicht mehr (so deutlich) zu finden sein.

Außerdem wird versucht, eine Antwort auf die Frage zu geben, inwiefern linke Parteien nicht nur Verteilungsziele verfolgen, sondern grundsätzlich reformfreudiger (reformfähiger) sind. Dies müsste dann tendenziell anhand einer höheren

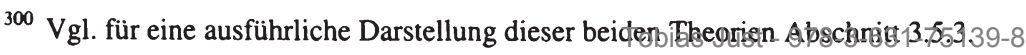


Variabilität der Budgetstruktur zu erkennen sein, wenn man die Annahme gelten lässt, dass zumindest Teile von Reformvorhaben immer ausgabenwirksam sind.

\subsection{Handlungsspielräume ideologisch motivierter Arbeits marktpolitik}

In diesem Abschnitt werden mögliche Partisaneffekte auf dem Arbeitsmarkt hinsichtlich ihrer Existenz und Persistenz untersucht. Es wurde in Kapitel 3 argumentiert, dass eine linke Regierung stärkeres Interesse an einer Reduktion der Arbeitslosigkeit hat als eine rechte Regierung, da die Verteilungswirkungen von Arbeitslosigkeit regressiv sind. Dementsprechend legt die Partisantheorie von Hibbs nahe, dass in Regierungsphasen, in denen eine linke Regierung amtierte, auch tendenziell geringere Arbeitslosenquoten zu verzeichnen sein sollten als in jenen Phasen, in denen eher rechts gerichtete Parteien in der Regierungsverantwortung standen. Auch die rationale Partisantheorie von Alesina lässt zumindest für die erste Anpassungszeit nach einem Regierungswechsel unterschiedliche Arbeitsmarktperformance erwarten.

Wie in Abschnitt 3.5.2.2 gezeigt wurde, haben beide Theorie zumindest zum Teil empirischen Gehalt. Hier wird deshalb der Schwerpunkt auf die Beantwortung von zwei Fragen gelegt. Zum einen wird eine Antwort auf die Frage gesucht, ob besonders offene Länder besonders geringen ideologischen Spielraum haben. Zum zweiten wird untersucht, ob durch die internationale wirtschaftliche Öffnung in den letzten Jahren die ideologischen Prägeräume der Parteien verengt wurden. Es gilt also die Hypothese zu prüfen, ob die Partisantheorien, falls sie überhaupt Gültigkeit besitzen, diese insbesondere für geschlossene Volkswirtschaften haben.

\subsubsection{Beschreibung der Daten}

Es werden Quartalsdaten der nationalen Arbeitslosenquoten für 19 OECDStaaten verwendet. Der Untersuchungszeitraum umfasst die Jahre von 1960 bis 1997, wobei allerdings nicht für alle Staaten für den gesamten Zeitraum Daten verfügbar waren. Tabelle 5.1 gibt einen Überblick über die Länder, die Eingang in die Untersuchung finden sowie den Zeitraum, für den die OECD Quartalsdaten bereitstellt.

Man könnte argumentieren, dass bei Panelstudien das Problem der Vergleichbarkeit der Arbeitsmarktdaten die Validität der Schätzung verringert. Dieser Einwand hat auch hier angesichts der verwandten nationalen Arbeitslosenquoten seine Berechtigung, und dies nicht nur, weil die Definitionen voneinander ab- 
weichen, sondern auch, weil die nationalen Konzepte im Zeitablauf zahlreiche Anpassungen erfahren haben (Litz, 1999, S. 176).

\section{Tabelle 5.1 : Stützzeiträume und Erhebungsmodi der nationalen Arbeits- losenquoten}

\begin{tabular}{|c|c|c|}
\hline Staat & Stützzeitraum & Datenerhebungsmodus (saisonbereinigt) \\
\hline Australien & $3 / 1966-4 / 1997$ & $\begin{array}{l}\text { Arbeitslose in Relation zu den zivilen Erwerbspersonen } \\
\text { (umfragebasiert) }\end{array}$ \\
\hline Österreich & $1 / 1960-4 / 1997$ & reg. Arbeitslose in Relation zu den abhängig Beschäftigten \\
\hline Belgien & $1 / 1979-4 / 1997$ & reg. Arbeitslose in Relation zu den abhängig Beschäftigten \\
\hline Kanada & $1 / 1960-4 / 1997$ & $\begin{array}{l}\text { Arbeitslose in Relation zu den zivilen Erwerbspersonen } \\
\text { (umfragebasiert) }\end{array}$ \\
\hline Dänemark & $1 / 1970-4 / 1997$ & $\begin{array}{l}\text { reg. Arbeitslose in Relation zu den gesamten Erwerbsper- } \\
\text { sonen }\end{array}$ \\
\hline Finnland & $1 / 1960-4 / 1997$ & $\begin{array}{l}\text { Arbeitslose in Relation zu den zivilen Erwerbspersonen } \\
\text { (umfragebasiert) }\end{array}$ \\
\hline Frankreich & $1 / 1970-4 / 1997$ & $\begin{array}{l}\text { Arbeitslose in Relation zu den gesamten Erwerbspersonen } \\
\text { (umfragebasiert) }\end{array}$ \\
\hline Deutschland & $1 / 1962-4 / 1997$ & $\begin{array}{l}\text { reg. Arbeitslose in Relation zu den abhängig Beschäftigten } \\
\text { (Westdeutschland) }\end{array}$ \\
\hline Irland & $1 / 1983-4 / 1997$ & $\begin{array}{l}\text { Arbeitslose in Relation zu den gesamten Erwerbspersonen } \\
\text { (umfragebasiert) }\end{array}$ \\
\hline Italien & $1 / 1960-4 /$ & $\begin{array}{l}\text { reg. Arbeitslose in Relation zu den gesamten Erwerbsper- } \\
\text { sonen }\end{array}$ \\
\hline Japan & $1 / 1960-4 / 1997$ & $\begin{array}{l}\text { Arbeitslose in Relation zu den gesamten Erwerbspersonen } \\
\text { (umfragebasiert) }\end{array}$ \\
\hline Niederlande & $1 / 1982-4 / 1997$ & $\begin{array}{l}\text { Arbeitslose in Relation zu den gesamten Erwerbspersonen } \\
\text { (umfragebasiert) }\end{array}$ \\
\hline Neuseeland & $1 / 1981-4 / 1997$ & $\begin{array}{l}\text { Arbeitslose in Relation zu den zivilen Erwerbspersonen } \\
\text { (umfragebasiert) }\end{array}$ \\
\hline Norwegen & $1 / 1972-4 / 1997$ & $\begin{array}{l}\text { Arbeitslose in Relation zu den zivilen Erwerbspersonen } \\
\text { (umfragebasiert) }\end{array}$ \\
\hline Schweden & $1 / 1970-4 / 1997$ & $\begin{array}{l}\text { Arbeitslose in Relation zu den zivilen Erwerbspersonen } \\
\text { (umfragebasiert) }\end{array}$ \\
\hline Schweiz & $1 / 1983-4 / 1997$ & $\begin{array}{l}\text { reg. Arbeitslose in Relation zu den gesamten Erwerbsper- } \\
\text { sonen }\end{array}$ \\
\hline Spanien & $3 / 1976-4 / 1997$ & $\begin{array}{l}\text { Arbeitslose in Relation zu den gesamten Erwerbspersonen } \\
\text { (umfragebasiert) }\end{array}$ \\
\hline UK & $1 / 1960-4 / 1997$ & $\begin{array}{l}\text { Arbeitslose in Relation zu den gesamten Erwerbspersonen } \\
\text { (umfragebasiert) }\end{array}$ \\
\hline USA & $1 / 1960-4 / 1997$ & $\begin{array}{l}\text { Arbeitslose in Relation zu den gesamten Erwerbspersonen } \\
\text { (umfragebasiert) }\end{array}$ \\
\hline
\end{tabular}

Quelle: $O E C D$, Main Economic Indicators, Paris, diverse Jahrgänge.

Dennoch werden nicht die standardisierten OECD Daten herangezogen, da zum ersten die Vergleichbarkeit selbst dieser Datenınicht, yollständig gesvähtrleistet ist 
(Litz, 1999), und weil zum zweiten diese standardisierten Daten nur für einen sehr kurzen Zeitraum und ein begrenztes Ländersample vorliegen, wodurch letztlich die Validität der Schätzer ebenfalls stark reduziert würde. ${ }^{301}$ Drittens sollte davon auszugehen sein, dass die Partisantheorien unabhängig vom Erfassungskonzept gelten, falls es keine systematischen, ideologisch motivierten Anpassungen der nationalen Arbeitslosenquoten gibt. Es werden im folgenden also die oben dargestellten nationalen Arbeitslosigkeitskonzepte verwendet. Zur Kennzeichnung der Ideologieausrichtung der einzelnen Regierungen wird das in Abschnitt 3.3.2.1 eingeführte center of gravity (CG) verwendet. ${ }^{302}$

\subsubsection{Traditionelle Partisantheorie}

Das Hibbs-Modell basiert auf der Annahme, dass Individuen ihre Erwartungen adaptiv anpassen, sodass es längerfristige Abweichungen von der natürlichen Arbeitslosenquote geben kann. Es sollen insgesamt drei Hypothesen geprüft werden. Erstens wird getestet, ob linke Parteien tatsächlich eine bessere Arbeitsmarktperformance realisieren. Zweitens wird daran anschließend geprüft, ob es durch die Globalisierungsphasen erklärbare Brüche in diesem Schema gibt, und drittens wird getestet, ob durch unterschiedlich starke internationale Integration der Staaten erklärbare Unterschiede der untersuchten Länder existieren.

Das verwandte Analyseinstrument ist die Panelregression mit fixed effects. ${ }^{303}$ Bei der Verwendung solcher LSDV-Schätzer (least squares dummy variable) für dynamische Paneldaten stellt sich mitunter das Problem, dass die Koeffizienten mit einem Bias geschätzt werden. Dieser Bias ist v.a. bei sehr kurzen Zeitreihen nicht zu vernachlässigen, und es wäre ein GMM-Modell (generalized method of moments) vorzuziehen. Allerdings können Judson und Owen (1999) zeigen, dass für längere Zeitreihen ( $>30$ Perioden) der LSDV-Schätzer nicht schlechter ist als ein GMM-Schätzer. Daher wird dieser methodisch einfachere Weg gewählt. ${ }^{304}$

Ein weiteres Problem bei diesem Verfahren ist, dass wahrscheinlich die für OLS-Schätzer wichtige Annahme, dass die Zeitreihe stationär ist, verletzt wird,

301 Außerdem werden die folgenden Modelle jeweils als fixed effects Modelle geschätzt, um gerade Länderspezifika auffangen zu können, die aufgrund unterschiedlicher Erfassungskonzepte verzerrend wirken.

302 Dieser Index bewertet das relative Gewicht jeder Partei innerhalb einer Regierungskoalition mit ihrer ideologischen Position auf einem Links-Rechts-Kontinuum.

303 Alle folgenden Regressionen wurden mit Hilfe von EViews3 durchgeführt.

304 Vollständig verschwindet der Bias allerdings erst bei einer unendlich großen Anzahl von Perioden (Nickell, 1981). 
da Arbeitslosenquoten zumindest für zahlreiche europäische Staaten eine starke Persistenzneigung aufweisen. ${ }^{305}$ Dieses Problem lässt sich allerdings begrenzen, indem man nicht die originären Arbeitslosigkeitsdaten regressiert, sondern deren Abweichungen von einem Mittelwert (Ramanathan, 1992, S. 493). Dieses Verfahren wurde auch von anderen Autoren bei ähnlicher Fragestellung angewandt (Alesina, Cohen und Roubini, 1992 und 1997, Berlemann, 1999) und soll auch hier benutzt werden. Außerdem werden dadurch die Einflüsse der Weltkonjunktur auf die inländische Arbeitslosenquote erfasst. ${ }^{306}$ Es wird also ein autoregressives Modell der folgenden Form geschätzt:

$$
\begin{gathered}
U_{i, t}^{\text {Diff }}=\alpha_{i}+\beta_{1} \cdot U_{i, t-1}^{\text {Diff }}+\ldots+\beta_{n} \cdot U_{i, t-n}^{\text {Diff }}+\gamma \cdot C_{i, t}+\varepsilon_{i, t} \\
\text { mit } \\
U_{i, t}^{\text {Diff }}=U_{i, t}-U_{t}^{w} \\
\text { und } \\
U_{t}^{w}=\sum_{=}^{J} \frac{U_{j, t} \cdot B_{j} P_{j, t}}{\sum_{F=1}^{j} B I P_{j, t}}
\end{gathered}
$$

Der Index $\mathrm{i}$ ist der Länderindex und geht von 1 bis 19, der Index $t$ ist der Zeitindex für die Quartale 1/1960 bis 4/1997. Der Index n gibt an, wie viele Lags berücksichtigt werden müssen, $\varepsilon_{i, t}$ sind die jeweiligen Residuen. Der Regressand $\mathrm{U}_{\mathrm{i}, \mathrm{t}}^{\text {Diff }}$ ist also die Differenz der nationalen Arbeitslosenquote $\left(\mathrm{U}_{\mathrm{i}, \mathrm{t}}\right)$ zum Zeitpunkt $t$ von der durchschnittlichen Arbeitslosenquote der OECD-Staaten $U_{t}^{w}$ zum Zeitpunkt t. Dieser Mittelwert errechnet sich per Periode aus den Daten für die Arbeitslosenquoten der in dieser Periode vorliegenden $\mathrm{J}$ Länder (mit $\mathrm{J} \leq 19$, da nicht in jeder Periode alle Länder berücksichtigt werden konnten), gewichtet mit ihren Bruttoinlandsproduktanteilen an der Summe des Bruttoinlandspro-

305 Stationarität ist dann gegeben, wenn für die Störterme $\varepsilon_{\mathrm{t}}$ und $\varepsilon_{\mathrm{t}-\mathrm{s}}$ mit $\left.\left.s \in\right] 0 ; \mathrm{t}-1\right]$ gilt: $\operatorname{Var}\left(\varepsilon_{\mathrm{l}}\right)$ $=\operatorname{Var}\left(\varepsilon_{\mathrm{t}-\mathrm{s}}\right)$, vgl. z.B. Ramanathan (1992, S. 495). Bei persistenter Arbeitslosigkeit (mit hinlänglich langer Verzögerung der Anpassung) ist diese Grundannahme offenbar verletzt, da bei persistenter Arbeitslosigkeit die mögliche Variation nach unten definitionsgemäB nicht möglich ist. Arbeitslosenquote als Regressor in die Schätzgleichung integrierte, wie dies z.B. Sheffrin (1989) für die Wachstumsraten der OECD-Staatem machtust - 978-3-631-75139-8 
dukts aller Länder, die in diesem Jahr Arbeitslosenquoten verfügbar hatten. ${ }^{307}$ Außerdem wird natürlich die besonders interessierende politische Variable $\mathrm{CG}_{\mathrm{i}, \mathrm{t}}$ für das center of gravity eingeführt. Das Modell wird jeweils als fixed effects Modell geschätzt, um Länderspezifika $\left(\alpha_{\mathrm{i}}\right)$ zu berücksichtigen. Da der CG-Wert für rechte Regierungen höhere Werte annimmt als für linke Regierungen, ist das theoriekonforme Vorzeichen von $\gamma$ positiv. Im folgenden werden die Koeffizienten für die Länderdummys nicht dargestellt, da sie für die Argumentation ohne Bedeutung sind. ${ }^{308}$

In einem ersten Schritt wird das gesamte Panel von 19 Ländern über den Zeitraum von $1 / 1960$ bis $4 / 1997$ geschätzt. Sukzessive wurden zusätzliche Lags hinsichtlich der Differenz der Arbeitslosenquoten berücksichtigt, um das Modell mit der besten Erklärungskraft zu finden. Es wurden nur jene Lags herangezogen, deren Einfluss auf dem 5\%-Signifikanzniveau nicht verworfen werden konnte. Für das gesamte Panel ergab sich so eine optimale Lagstruktur von 5 Perioden. In Tabelle 5.2 sind drei unterschiedliche Modelle abgebildet mit jeweils unterschiedlich unterstellter wirtschaftspolitischer Wirkungsverzögerung. In Modell (1) wird angenommen, die Wirtschaftspolitik wirkt quasi sofort, in Modell (2) wird eine 3-monatige Verzögerung (Lag von einer Periode, $\mathrm{CG}_{\mathrm{t}-1}$ ) und in Modell (3) eine 6-monatige Wirkungsverzögerung (Lag von zwei Perioden, $\mathrm{CG}_{\mathrm{t}-2}$ ) modelliert. ${ }^{309}$

Nach der Theorie von Hibbs sollte bei einer linken Regierung mit niedrigeren Arbeitslosenquoten zu rechnen sein als bei einer rechten Regierung; je weiter links eine Regierung in dem Ideologiespektrum steht, desto eher wird sie geringere Werte als der OECD-Mittelwert erreichen (sofern nicht andere Schocks diesen Zusammenhang überlagern). Zwar haben die CG-Variablen in allen drei Modellen das ,richtige“ Vorzeichen, jedoch ist der Koeffizient nur im Modell

${ }^{307}$ Einige Autoren (z.B. Berlemann, 1999) benutzen zeitinvariate Gewichte, die auf der Grundlage einer einzigen Periode und eines reduzierten Ländersamples (dort die G7Staaten) beruhen. Dieser Ansatz wird hier ausdrücklich nicht verfolgt. Zwar liegt der Anteil, den die G7-Staaten am gesamten BIP der 19 Länder ausmachen, relativ konstant zwischen $84 \%$ und $89 \%$, jedoch gibt es innerhalb der G7 teilweise gravierende Anteilsverschiebungen. So lag der Anteil, den das US-amerikanische BIP am gesamten BIP einnahm 1960 bei $55,14 \%, 1995$ jedoch nur noch bei 32,95\%. Gleichzeitig stieg der Anteil des japanischen BIP von 4,66\% (1960) auf einen Anteil von 23,3\% (1994) an. Diese Veränderungen würden bei invariaten Gewichtungsfaktoren nicht berücksichtigt werden können.

${ }^{308}$ Zudem ist ein t-Test auf einzelne Länderdummies in einer Panelregression nicht aussagekräftig (Judge et al., 1988, S. 476).

309 Es wurden auch Modelle mit noch längerer Wirkungsverzögerung geschätzt. Dabei wurden jedoch keine Verbesserungen des Erklärungsgehalts hinsichtlich der relevanten CGVariable erzielt. Daher wird auf ihre Darstellung verbiehtetust - 978-3-631-75139-8 
ohne Wirkungsverzögerung auf dem $10 \%$-Niveau von Null verschieden. ${ }^{310}$ Auch deutet die absolute Höhe der Koeffizientenwerte von $\mathrm{CG}_{\mathrm{t}}$ an, dass der Einfluss der Ideologievariable nicht sehr groß zu sein scheint. ${ }^{311}$

\section{Tabelle 5.2 : Partisaneffekte auf dem Arbeitsmarkt im Gesamtpanel 1960-1997}

\begin{tabular}{lccc}
\hline & \multicolumn{2}{c}{ abhängige Variable: $\mathrm{U}_{\mathrm{t}}^{\text {Diff }}$} \\
unabhängige Variable & $(1)$ & $(2)$ & $(3)$ \\
\hline $\mathrm{U}_{\mathrm{t}-1}^{\text {Diff }}$ & $1,355^{* *}$ & $1,355^{* *}$ & $1,356^{* *}$ \\
$\mathrm{U}_{\mathrm{t}-2}^{\text {Diff }}$ & $(62,044)$ & $(62,013)$ & $(62,039)$ \\
& $-0,218^{* *}$ & $-0,218^{* *}$ & $-0,219 * *$ \\
$\mathrm{U}_{\mathrm{t}-3}^{\text {Diff }}$ & $(-5,948)$ & $(-5,954)$ & $(-5,970)$ \\
$\mathrm{U}_{\mathrm{t}-4}^{\text {Diff }}$ & $-0,075^{*}$ & $-0,074^{*}$ & $-0,074^{*}$ \\
$\mathrm{U}_{\mathrm{t}-5}^{\text {Diff }}$ & $(-2,040)$ & $(-2,015)$ & $(-2,019)$ \\
& $-0,152^{* *}$ & $-0,152^{* *}$ & $-0,152^{* *}$ \\
$\mathrm{CG}_{\mathrm{t}}$ & $(-4,166)$ & $(-4,172)$ & $(-4,149)$ \\
& $0,066^{* *}$ & $0,066^{* *}$ & $0,065^{* *}$ \\
$\mathrm{CG}_{\mathrm{t}-1}$ & $(3,046)$ & $(3,032)$ & $(3,005)$ \\
$\mathrm{CG}_{\mathrm{t}-2}$ & $0,008+$ & - & - \\
\hline $\mathrm{R}^{2}$ & $(1,845)$ & 0,007 & - \\
\hline $\mathrm{Anm}^{2}$ & - & $(1,512)$ & 0,004 \\
& & - & $(0,925)$ \\
\hline
\end{tabular}

Anmerkungen: $\quad \mathrm{t}$-Werte in Klammern, $+(\alpha<0,1),{ }^{*}(\alpha<0,05),{ }^{* *}(\alpha<0,01)$.

Im folgenden soll nun geprüft werden, ob für Subsamples, die auf der Basis der besprochenen Globalisierungsindikatoren gebildet wurden, differenziertere Aussagen möglich sind.

${ }^{310}$ Dieses Ergebnis scheint relativ robust, denn Berlemann (1999, S. 279) kommt bei ähnlicher Modellspezifikation für ein kleineres Ländersample und kürzere Zeitreihen sowie einer anders spezifizierten Ideologievariable zu demselben Ergebnis.

311 Allerdings ist hier darauf hinzuweisen, dass z.B. die beiden großen deutschen Volksparteien bei dem verwandten Indikator 3,4 Punkte trempen; (siehs Anhagg(6)31-75139-8 


\subsubsection{Cluster gemäß den ökonomischen Globalisierungsindikatoren}

In Kapitel 4 wurden jene Globalisierungscluster als „ökonomisch“ bezeichnet, die mit Hilfe der Variablen Handelsvolumen, Direktinvestitionsströme und Portfolioinvestitionsströme gewonnen wurden. Es ließen sich insgesamt vier Zeitphasen und drei Ländergruppen (relativ gut) voneinander trennen. Es sollen nun zwei Hypothesen geprüft werden:

1. Partisaneffekte auf dem Arbeitsmarkt waren ausgeprägter in den ersten Jahrzehnten nach dem Zweiten Weltkrieg und wurden im Zuge der Globalisierung zunehmend eingeschränkt.

2. Partisaneffekte lassen sich vornehmlich in relativ geschlossenen Staaten finden, da in diesen die Weltmärkte ein geringeres Gewicht besitzen.

Der Schätzansatz ist vollkommen analog zu dem obigen Modell. Lediglich das Ländersample und der Stützzeitraum wurden variiert. Dadurch ergab sich zum Teil eine etwas kürzere optimale Lagstruktur des Modells bezüglich der Variablen $U^{\text {Diff }}$. Es werden zu jedem Zeitraum und Ländersample zwei Schätzgleichungen angegeben, zum einen eine Gleichung mit der ideologischen Variable ohne Wirkungsverzögerung (1) und zum anderen eine Gleichung mit einer Wirkungsverzögerung in der CG-Variable um eine Periode (2). ${ }^{312}$

In Tabelle 5.3 sind die Regressionsergebnisse für die drei Ländercluster jeweils für den gesamten Untersuchungszeitraum dargestellt. Bei Gültigkeit der Globalisierungsthese sollte ein deutlicher Unterschied zwischen dem besonders offenen ersten Cluster und dem besonders geschlossenen dritten Cluster zu erkennen sein. Dies ist offensichtlich nicht der Fall. Zwar haben die meisten Koeffizienten für CG das erwartete Vorzeichen, jedoch ist nur für den zweiten Cluster hinreichende statistische Signifikanz gewährleistet, um einen Einfluss gerechtfertigterweise anzunehmen.

In Tabelle 5.4 sind dann Regressionen für die einzelnen Globalisierungsphasen für alle Länder aufgeführt. Auch hier sind die meisten Vorzeichen korrekt, jedoch ausnahmslos nicht signifikant auf einem der üblichen Signifikanzniveaus. Interessanterweise ist sogar gerade für den ersten Zeitcluster ein negatives Vorzeichen bei $\mathrm{CG}_{\mathrm{t}}$ und $\mathrm{CG}_{\mathrm{t}-1}$ ausgewiesen.

Dieser schlechte Befund für die Partisantheorie wird durch detailliertere Regressionen für die drei Ländercluster und die vier Zeitphasen zum Teil untermauert. ${ }^{313}$ In der Gruppe der besonders offenen Länder (1. Cluster) gibt es zumindest für die Phase von 1974 bis 1992 recht gute Bestätigung für die traditionelle Par-

${ }^{312}$ Es wurden auch Modell mit $\mathrm{CG}_{\mathrm{t}-2}$ geschätzt. Diese erzielten jedoch keine besseren Schätzergebnisse und werden daher nicht dargestellt.

${ }^{313}$ Die Regressionsergebnisse sind im Anhang C im Detailsdargestellt:-3-631-75139-8 
tisantheorie mit zum Teil hoch signifikanten Koeffizienten. Auch ist hier von 1974-1997 ein deutliches Abschwächen des Partisaneffektes zu erkennen. Dies ließe sich dahingehend interpretieren, dass für den ersten Cluster sowohl die Partisanthese als auch die Globalisierungsthese für den Zeitraum von 1974 bis 1997 einige Bestätigung erhält. Vor 1974 gilt dieser Zusammenhang indes nicht.

Tabelle 5.3 : Arbeitsmarkteffekte für die ökonomischen Cluster 1960-1997

\begin{tabular}{|c|c|c|c|c|c|c|}
\hline \multicolumn{7}{|c|}{ abhängige Variable: $U_{1}^{\text {Diff }}$} \\
\hline \multirow[b]{3}{*}{$\begin{array}{l}\text { unabhängige } \\
\text { Variable }\end{array}$} & \multirow{2}{*}{\multicolumn{2}{|c|}{$\begin{array}{c}\text { 1. Cluster } \\
(\mathrm{BEL}, \mathrm{NL}, \mathrm{NZL}, \mathrm{S}, \mathrm{UK})\end{array}$}} & \multirow{2}{*}{\multicolumn{2}{|c|}{$\begin{array}{c}\text { 2. Cluster } \\
\text { (CAN, DK, SF, IRL, } \\
\text { NOR, CH) }\end{array}$}} & \multirow{2}{*}{\multicolumn{2}{|c|}{$\begin{array}{c}\text { 3. Cluster } \\
\text { (AUS, AU, F, D, ITA, } \\
\text { J, E, USA) }\end{array}$}} \\
\hline & & & & & & \\
\hline & (1) & (2) & (1) & (2) & (1) & (2) \\
\hline $\mathrm{U}_{\mathrm{t}-1}^{\mathrm{Diff}}$ & $\begin{array}{c}1,414^{* *} \\
(33,503)\end{array}$ & $\begin{array}{c}1,415^{* *} \\
(33,494)\end{array}$ & $\begin{array}{c}1,431^{* *} \\
(35,918)\end{array}$ & $\begin{array}{c}1,428 * * \\
(35,848)\end{array}$ & $\begin{array}{c}1,340 * * \\
(43,964)\end{array}$ & $\begin{array}{c}1,340^{* *} \\
(43,972)\end{array}$ \\
\hline $\mathrm{U}_{\mathrm{t}-2}^{\text {Diff }}$ & $\begin{array}{c}-0,437 * * \\
(-10,323)\end{array}$ & $\begin{array}{c}-0,436^{* *} \\
(-10,291)\end{array}$ & $\begin{array}{c}-0,297 * * \\
(-4,290)\end{array}$ & $\begin{array}{l}-0,295^{* *} \\
(-4,266)\end{array}$ & $\begin{array}{c}-0,185^{* *} \\
(-3,611)\end{array}$ & $\begin{array}{c}-0,185^{* *} \\
(-3,613)\end{array}$ \\
\hline $\mathrm{U}_{\mathrm{t}-3}^{\text {Diff }}$ & - & - & $\begin{array}{c}-0,156^{* *} \\
(-3,912)\end{array}$ & $\begin{array}{l}-0,156^{* *} \\
(-3,911)\end{array}$ & $\begin{array}{c}-0,177 * * \\
(-5,884)\end{array}$ & $\begin{array}{l}-0,178^{* *} \\
(-5,886)\end{array}$ \\
\hline $\mathrm{CG}_{\mathrm{t}}$ & $\begin{array}{c}0,005 \\
(0,619)\end{array}$ & - & $\begin{array}{c}0,023+ \\
(1,946)\end{array}$ & - & $\begin{array}{c}0,002 \\
(0,337)\end{array}$ & - \\
\hline $\mathrm{CG}_{1-1}$ & - & $\begin{array}{c}-0,000 \\
(-0,046)\end{array}$ & - & $\begin{array}{c}0,026^{*} \\
(2,212)\end{array}$ & - & $\begin{array}{c}0,000 \\
(0,025)\end{array}$ \\
\hline$\overline{R^{2}}$ & 0,99 & 0,99 & 0,99 & 0,99 & 0,99 & 0,99 \\
\hline
\end{tabular}

Im zweiten Ländercluster ist lediglich für die letzte Zeitphase ein signifikanter Koeffizient ausgewiesen und in der Ländergruppe, die als verhältnismäßig geschlossen anzusehen ist, haben die einzigen Koeffizienten, die signifikant von null verschieden sind, sogar das falsche Vorzeichen. Zusammenfassend bieten die ökonomischen Cluster für die Partisantheorie nach Hibbs und folglich auch für die Globalisierungsthese nur sehr unzureichende empirische Belege.

Es gilt im folgenden zu prüfen, ob die mangelnde Erklärungskraft der CGVariable nur an falscher Clusterbildung oder eher an dem grundsätzlich unzureichenden Erklärungsgehalt der Thesen lag. Daher werden nun dieselben Schätzungen auf der Grundlage der politischen Cluster vorgenommen. 
Tabelle 5.4 : Arbeitsmarkteffekte in der Clusterung gemäß ökonomischen Globalisierungsindikatoren (alle Ländercluster)

\section{abhängige Variable: $U_{t}^{\text {Diff }}$}

unabh. $1961-1973$ (1. Zeitcluster) $1974-1985$ (2. Zeitcluster) $1986-1992$ (3. Zeitcluster) $1993-1997$ (4. Zeitcluster)

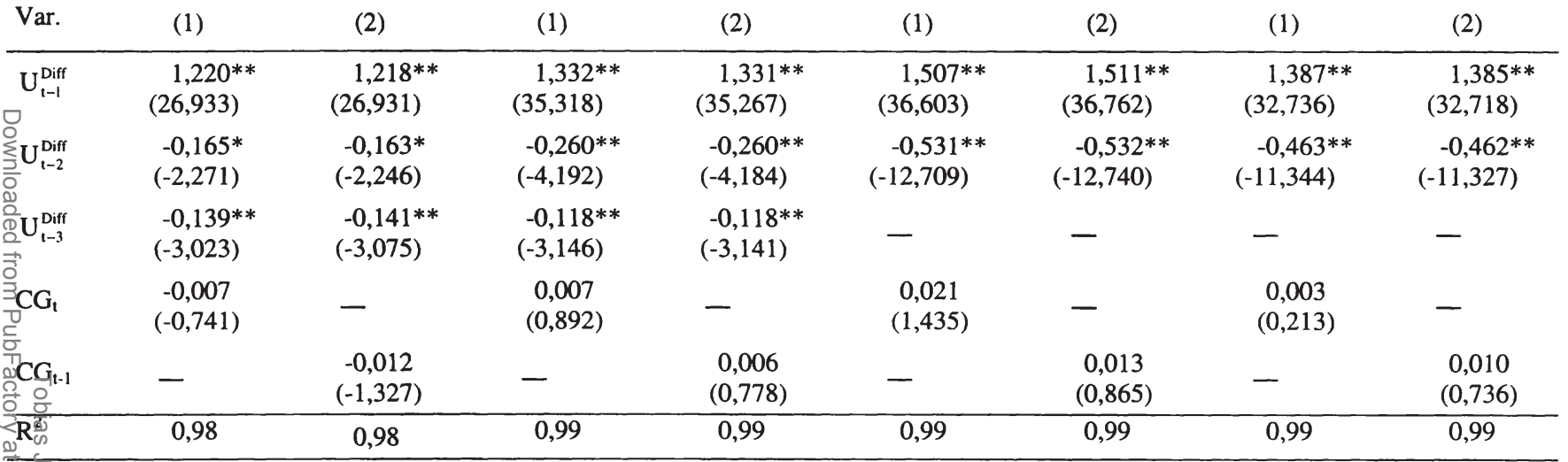

A: $\quad \mathrm{t}$-Werte in Klammern, $+(\alpha<0,1),{ }^{*}(\alpha<0,05),{ }^{* *}(\alpha<0,01)$. 


\subsubsection{Cluster gemäß den politischen Globalisierungsindikatoren}

Die politischen Variablen für Liberalisierung auf den Kapitalmärkten von Quinn und Inclan sowie der innerhalb dieser Arbeit vorgestellte Indikator für die Liberalisierung der Gütermärkte bildeten die Grundlage für die zweite Clusteranalyse der Länder und Zeit. Es wurde gezeigt, dass gerade in bezug auf die Ländergruppierung starke Abweichungen zwischen den beiden Clusterungen bestehen. Das wirft die Frage auf, ob der Umfang der tatsächlichen Liberalisierung ein besserer Maßstab für die Restriktionen der Globalisierung sein könnte als der Umfang der Markttransaktionen. Es sollen daher im folgenden die beiden Thesen aus dem vorherigen Abschnitt mit anderen Subsamples geprüft werden. Dabei unterscheidet sich die Schätzmethode nicht von dem oben gewählten autoregressiven Ansatz.

Da bei der Clusterung nach politischen Liberalisierungsvariablen sowohl mehr Zeitphasen als auch mehr Ländergruppen isoliert wurden, sinkt in den meisten Regressionen der Stichprobenumfang. Dies kann dazu führen, dass nur noch ein einziges Land in der Regression Eingang findet, wie z.B. Japan im 4. Ländercluster für den Zeitraum 1960-1966. Insbesondere die Einzelergebnisse im Anhang $\mathrm{C}$ sind also mitunter sehr vorsichtig zu interpretieren. ${ }^{314}$ Auch in dieser Clusterung gibt es für die traditionelle Partisantheorie keine eindeutigen Ergebnisse, sondern nur wenige Hinweise, dass sie für einzelne Subsamples Gültigkeit haben könnte. Auch der Einfluss der Globalisierung ist nicht sehr deutlich aus den Regressionen herauszulesen.

Tabelle 5.5 zeigt Regressionsergebnisse für den gesamten Untersuchungszeitraum 1960 - 1997 und für die vier politischen Cluster. Bei acht geschätzten Koeffizienten für $\mathrm{CG}_{\mathrm{t}}$ und $\mathrm{CG}_{\mathrm{t}-1}$ deuten zwar sechs in die richtige Richtung, allerdings ist von den beiden Koeffizienten, die signifikant von null verschieden sind, ein Koeffizient mit dem nicht theoriekonformen negativen Vorzeichen versehen. Dies ist darüber hinaus gerade in dem vierten Cluster der Fall, in dem die besonders gering integrierten Länder zusammengefasst sind. Nach der Globalisierungsthese sollte aber gerade dort der deutlichste Partisaneffekt erkennbar sein.

\footnotetext{
${ }^{314}$ Nicht zuletzt auch deshalb, weil durch die kürzeren Stützzeiträume der Bias der Koeffizienten steigt. 
Tabelle 5.5 : Arbeitsmarkteffekte in der Clusterung gemäß politischen Globalisierungsindikatoren 1960-1997

abhängige Variable: $U_{1}^{\text {Diff }}$

\section{Cluster}

unabh.

Var. (D, DK, ITA, NL, BEL, F)

2. Cluster

(UK, S, AU, NOR, SF, IRL)

(2)

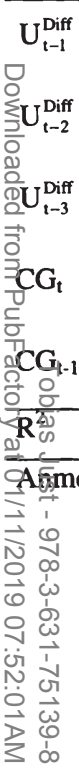

3. Cluster (USA, CAN, CH)
4. Cluster

(E, J, AUS, NZL)

\begin{tabular}{|c|c|c|c|c|c|c|c|}
\hline (1) & (2) & (1) & (2) & (1) & (2) & (1) & (2) \\
\hline $\begin{array}{c}1,306^{* *} \\
(34,982)\end{array}$ & $\begin{array}{c}1,306^{* *} \\
(34,992)\end{array}$ & $\begin{array}{c}1,491^{* *} \\
(40,005)\end{array}$ & $\begin{array}{c}1,491 * * \\
(39,943)\end{array}$ & $\begin{array}{c}1,414 * * \\
(29,715)\end{array}$ & $\begin{array}{c}1,413 * * \\
(29,668)\end{array}$ & $\begin{array}{c}1,463 * * \\
(34,663)\end{array}$ & $\begin{array}{c}1,463 * * \\
(34,737)\end{array}$ \\
\hline $\begin{array}{c}-0,324^{* *} \\
(-8,638)\end{array}$ & $\begin{array}{c}-0,324^{* *} \\
(-8,645)\end{array}$ & $\begin{array}{c}-0,384^{* *} \\
(-5,832)\end{array}$ & $\begin{array}{c}-0,383^{* *} \\
(-5,804)\end{array}$ & $\begin{array}{c}-0,440 * * \\
(-9,178)\end{array}$ & $\begin{array}{l}-0,439 * * \\
(-9,157)\end{array}$ & $\begin{array}{c}-0,491 * * \\
(-11,924)\end{array}$ & $\begin{array}{c}-0,492 * * \\
(-11,960)\end{array}$ \\
\hline- & - & $\begin{array}{l}-0,127^{* *} \\
(-3,386)\end{array}$ & $\begin{array}{c}-0,128 * * \\
(-3,416)\end{array}$ & - & - & - & - \\
\hline $\begin{array}{c}0,012 \\
(1,366)\end{array}$ & - & $\begin{array}{c}0,012+ \\
(1,694)\end{array}$ & - & $\begin{array}{c}0,015 \\
(1,019)\end{array}$ & - & $\begin{array}{c}-0,014 \\
(-1,536)\end{array}$ & - \\
\hline- & $\begin{array}{c}0,013 \\
(1,401)\end{array}$ & - & $\begin{array}{c}0,009 \\
(1,349)\end{array}$ & - & $\begin{array}{c}0,014 \\
(0,905)\end{array}$ & - & $\begin{array}{c}-0,018+ \\
(-1,881)\end{array}$ \\
\hline 0,99 & 0,99 & 0,99 & 0,99 & 0,99 & 0,99 & 0,99 & 0,99 \\
\hline
\end{tabular}


Tabelle 5.6 : Arbeitsmarkteffekte in der Clusterung gemäß politischen Globalisierungsindikatoren (alle Länder)

\begin{tabular}{|c|c|c|c|c|c|c|c|c|c|c|}
\hline \multirow{3}{*}{$\begin{array}{l}\text { unabh. } \\
\text { Var. }\end{array}$} & \multicolumn{8}{|c|}{ abhängige Variable: $U_{1}^{\text {Diff }}$} & & \\
\hline & \multicolumn{2}{|c|}{$1960-1966$} & \multicolumn{2}{|c|}{1967 - 1978} & \multicolumn{2}{|c|}{1979 - 1987} & \multicolumn{2}{|c|}{1988 - 1992} & \multicolumn{2}{|c|}{1993 - 1997} \\
\hline & (1) & (2) & (1) & (2) & (1) & (2) & (1) & (2) & (1) & (2) \\
\hline $\mathbf{U}_{t-2}^{\text {Diff }}$ & $\begin{array}{l}-0,431^{* *} \\
(-6,341)\end{array}$ & $\begin{array}{l}-0,430 * * \\
(-6,342)\end{array}$ & $\begin{array}{l}-0,395 * * \\
(-9,936)\end{array}$ & $\begin{array}{l}-0,394^{* *} \\
(-9,913)\end{array}$ & $\begin{array}{c}-0,144^{*} \\
(-2,193)\end{array}$ & $\begin{array}{c}-0,144^{*} \\
(-2,202)\end{array}$ & $\begin{array}{c}-0,584^{* *} \\
(-11,642)\end{array}$ & $\begin{array}{c}-0,585^{* *} \\
(-11,637)\end{array}$ & $\begin{array}{c}-0,463 * * \\
(-11,344)\end{array}$ & $\begin{array}{c}-0,462 * * \\
(-11,327)\end{array}$ \\
\hline$\frac{2}{\frac{2}{3}} \mathrm{CG}_{\mathrm{t}}$ & $\begin{array}{c}-0,026 \\
(-1,424)\end{array}$ & - & $\begin{array}{c}0,013 \\
(1,311)\end{array}$ & - & $\begin{array}{c}-0,004 \\
(-0,470)\end{array}$ & - & $\begin{array}{c}0,022 \\
(0,977)\end{array}$ & - & $\begin{array}{c}0,003 \\
(0,213)\end{array}$ & - \\
\hline 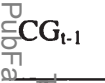 & - & $\begin{array}{c}-0,031+ \\
(-1,686)\end{array}$ & - & $\begin{array}{c}0,010 \\
(0,967) \\
\end{array}$ & - & $\begin{array}{c}-0,004 \\
(-0,467)\end{array}$ & - & $\begin{array}{c}0,010 \\
(0,456) \\
\end{array}$ & - & $\begin{array}{c}0,010 \\
(0,736) \\
\end{array}$ \\
\hline
\end{tabular}

An̈merkungen: $\quad \mathrm{t}$-Werte in Klammern, ${ }^{+}(\alpha<0,1),{ }^{*}(\alpha<0,05),{ }^{* *}(\alpha<0,01)$.

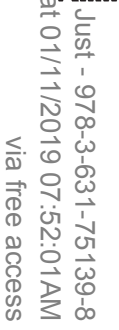


Ein ähnliches Bild wird durch die Regressionsergebnisse in Tabelle 5.6 gezeichnet. Dort werden für alle Länder und die einzelnen Zeitabschnitte CGKoeffizienten geschätzt. Auch hier findet sich weder eine Bestätigung der Partisan- noch der Globalisierungsthese. Von den acht geschätzten Koeffizienten haben gerade die Hälfte das korrekte Vorzeichen und kein einziger Koeffizient ist wenigstens auf dem 5\%-Signifikanzniveau von null verschieden.

Schließlich wurden für jeden Ländercluster Einzelregressionen für die fünf Zeitabschnitte geschätzt. ${ }^{315}$ Auch diese Ergebnisse sind uneindeutig, können aber ein etwas differenzierteres Bild malen. Im ersten Cluster der besonders stark integrierten Länder ist kein einziger Regressionskoeffizient für CG signifikant von null verschieden, jedoch weisen sieben der acht Koeffizienten das richtige Vorzeichen auf. Im zweiten Ländercluster sind ebenfalls sieben Koeffizienten mit dem erwarteten Vorzeichen versehen und zwei Koeffizienten sind mindestens auf dem 10\%-Signifikanzniveau von null verschieden.

Ein Zeittrend ist indes nicht zu beobachten. Im dritten Cluster sind sechs der acht Koeffizienten theoriekonform, von denen sogar fünf auf dem 5\%-Niveau signifikant sind. Betrachtet man nur die ersten drei Cluster, könnte man schließen, dass die Partisantheorie eine umso bessere Bestätigung findet, je weniger die Länder international integriert sind. Dies könnte zumindest als Indiz für die Richtigkeit der Globalisierungsthese gewertet werden, würde jedoch die Resultate stark überzeichnen, da der vierte Cluster, der nach der Globalisierungsthese eigentlich die beste Bestätigung der Partisantheorie zeigen sollte, nur drei theoriekonforme Koeffizienten aufweisen kann, von denen kein einziger signifikant ist. ${ }^{316}$ Außerdem ist zu berücksichtigen, dass in die Detailregressionen zum Teil nur sehr kleine Stichprobengrößen eingingen, wodurch die Aussagekraft der geschätzten Koeffizienten deutlich eingeschränkt ist.

Immerhin lässt sich feststellen, dass es schwache Hinweise gibt, dass die traditionelle Partisantheorie zumindest für einzelne Länder(gruppen) und Zeiträume Erklärungskraft besitzt.

315 Die detaillierten Ergebnisse finden sich im Anhang C.

${ }^{316}$ Dass der vierte Cluster in dieses Schema nicht hineinpasst, könnte daran liegen, dass die dort zusammengefassten Länder in vielerlei Hinsicht nicht wirklich eine homogene Gruppe darstellen. So war Spanien relativ lange weitgehend geschlossen, öffnete sich aber durch den EG-Beitritt 1986 radikal. Dass Spanien dem vierten Cluster zugerechnet wurde, liegt daran, dass es für den gesamten Untersuchungszeitraum eher geschlossen als offen war. Gerade für den Zeitraum, als Spanien geschlossen war, liegen aber nicht ausreichend Daten vor. Als zweite Besonderheit im vierten Cluster hat Japan in dem Zeitraum von 1960 bis 1993 überhaupt keinen Regierungswechsel gekannt. Die konservative japanische LDP, die in dieser Zeit die Regierung stellte, hatte in der gesamten Regierungsphase zu keinem Zeitpunkt ein gravierendes Arbeitsmarktproblem. Es kann also durchaus argumentiert werden, dass der vierte Cluster lediglich ein statistisches Artefakt der Clusteranalyse ist. 


\subsubsection{Zusammenfassung}

Weder die Clusterung nach ökonomischen Variablen, noch die Gruppierung nach politischen Variablen können mehr als vereinzelte Bestätigung der Partisantheorie mit dauerhaften Unterschieden in den Arbeitslosenquoten liefern. In beiden Clusterungen sind zwar die meisten Vorzeichen theoriekonform, jedoch sind nur eine kleine Zahl der Koeffizienten signifikant von null verschieden, sodass eine statistisch gesicherte Aussage nicht möglich ist. Dann lässt sich aber auch die Globalisierungsthese, dass der Einfluss ideologisch motivierter Arbeitsmarktpolitik in der Zeit zurückgeht, und/oder dass zwischen unterschiedlich offenen Ländern auch unterschiedlich stark ausgeprägte wirtschaftspolitische Prägung existiert anhand der verwandten Daten nicht hinreichend prüfen.

Allerdings ist bei der politischen Clusterung zwischen den ersten drei Ländergruppen eine deutliche Abstufung auffällig. Dies würde andeuten, dass die politische Clusterung geeigneter ist, als Maßstab der Handlungsrestriktion aufgrund von Marktöffnung zu dienen. Allerdings fällt der vierte Ländercluster aus dieser Deutung heraus.

Dass die obigen Schätzansätze keine eindeutigen Ergebnisse lieferten, könnte natürlich auch daran liegen, dass die traditionelle Partisantheorie von Hibbs nur ein unzureichendes Bild der Realität ist und dass die rationale Partisantheorie nach Alesina besser geeignet ist, ideologische Politikelemente darzustellen. Dies zu prüfen, ist Inhalt des folgenden Abschnitts.

\subsubsection{Rationale Partisantheorie}

Gemäß der Theorie rationaler Partisaneffekte nach Alesina gibt es zwar deutliche Unterschiede in der Arbeitsmarktperformance einer rechten respektive einer linken Regierung. Diese kommt aber nicht wie bei Hibbs aufgrund anderer stabilisierungspolitischer Akzentsetzung während der gesamten Periode zustande, sondern beruht allein darauf, dass die Inflationserwartungen der Bürger nach einem Regierungswechsel im Durchschnitt nicht erfüllt werden. Diese Erwartungsenttäuschung führt bei einem überraschenden Sieg der linksgerichteten Regierung zu einem expansiven Effekt auf dem Arbeitsmarkt. Umgekehrt führt der Sieg einer rechten Regierung dazu, dass die Preiserwartungen der Bürger über den tatsächlichen Werten liegen und damit letztlich zu einer Kontraktion. Beide Effekte sind aber nach Maßgabe der Möglichkeiten der Erwartungsanpassung nur transitorische Effekte. Das heißt, die Ideologievariablen in dem Schätzmodell müssen leicht angepasst werden, um diesen transitorischen Effekt abzubilden. Für die anderen Variablen dieses autoregressiven Modells gelten dieselben Aussagen wie zuvor im Partisanmodell mit adaptiven Erwartungen. 


$$
\begin{gathered}
\mathrm{U}_{\mathrm{i}, \mathrm{t}}^{\text {Diff }}=\alpha_{\mathrm{i}}+\beta_{1} \cdot \mathrm{U}_{\mathrm{i}, \mathrm{t}-1}^{\text {Diff }}+\ldots+\beta_{\mathrm{n}} \cdot \mathrm{U}_{\mathrm{i}, \mathrm{t}-\mathrm{n}}^{\mathrm{Diff}}+\gamma \cdot \mathrm{RCG}_{\mathrm{i}, \mathrm{t}}+\varepsilon_{\mathrm{i}, \mathrm{t}} \\
\mathrm{mit} \\
\mathrm{RCG6}_{\mathrm{i}, \mathrm{t}}=\mathrm{CG}_{\mathrm{i}, \mathrm{t}}-\mathrm{CG}_{\mathrm{i}, \mathrm{t}-6}
\end{gathered}
$$

Hier ist die transitorische Ideologievariable als die Differenz des Wertes für das center of gravity der Periode $\mathrm{t}$ und dem CG-Wert vor sechs Quartalen definiert. Die Konzeption von RCG6 ${ }_{i, t}$ bedeutet, dass ein Regierungswechsel nach rechts zu einem positiven Wert führt, ein Wechsel nach links hingegen zu einem Wert kleiner null. Es wird unterstellt, dass nach 18 Monaten der transitorische Effekt abgeklungen ist, sodass nach sechs Perioden ohne Regierungswechsel RCG6 $6_{i, t}$ den Wert Null zugewiesen bekommt, egal welche Partei an der Regierung war. ${ }^{317}$ Damit ist die Ideologievariable RCG6 ${ }_{\mathrm{i}, \mathrm{t}}$ im Modell mit rationalen Erwartungen etwas anders spezifiziert als in den Arbeiten von Alesina (z.B. Alesina, Cohen und Roubini, 1997, S. 83ff). Bei ihnen wird lediglich eine DummyVariable verwendet, die entweder einen Ruck nach links oder rechts nach einer Wahl erfasst. Dies kann aber zum einen die gegebenenfalls wichtigen Zwischentöne gerade der Koalitionsregierungen nicht angemessen berücksichtigen und zum anderen bleiben mögliche Änderungen der Kabinette während einer Legislaturperioden außerhalb der Analyse.

Die Tabelle 5.7 fasst die Regressionsergebnisse für das Gesamtsample aller 19 OECD-Staaten für den Untersuchungszeitraum von 1960-1997 zusammen. In den ersten beiden Modellen wurde die Variable RCG4 ${ }_{i, t}$ verwandt, jeweils einmal ohne Wirkungsverzögerung (1) und einmal mit einer Wirkungsverzögerung von einem Quartal (2). Bei den folgenden zwei Modellen fand die Variable RCG6 $_{\mathrm{i}, \mathrm{t}}$ Verwendung, wieder einmal ohne Wirkungsverzögerung (3) und einmal mit einem Lag von einer Periode (4). ${ }^{318}$

${ }^{317}$ Da die rationale Partisantheorie keine detaillierte Aussage über die exakte Dauer des transitorischen Effekts zulässt, wird zusätzlich die Variable $R C G 4_{i, t}=C_{i, t}-C G_{i, t-4}$ verwendet. Diese unterscheidet sich nur dadurch, dass davon ausgegangen wird, dass die Erwartungsanpassungen schon nach 12 Monaten vollzogen sind. Außerdem wurde mit noch längeren transitorischen Phasen der Erwartungsanpassung gearbeitet, jedoch waren die Ergebnisse bereits bei RCG $_{i, t}$ nicht mehr so eindeutig wie die dargestellten Zusammenhänge. Da durch diese Änderungen der Modelle keine Verbesserung der Ergebnisse erzielt werden konnte, wird auf die Darstellung der Schätzresultate ebenfalls verzichtet.

${ }^{318}$ Es wurden auch Modelle mit einem Wirkungslag von 2 Perioden geschätzt. Bei RCG4 $4_{i, t}$ ergaben sich in etwa dieselben Koeffizienten als auch Signifikanzniveaus. Bei RCG6 ${ }_{i, \imath}$ wurde jedoch ein kleinerer Koeffizientenwert geschätzt, der auch nicht signifikant von null verschieden war. Auf die Darstellung der Ergebbisse swird-daherêrerzichtet39-8 
Tabelle 5.7 : Rationale Arbeitsmarkteffekte im Gesamtpanel 1960-1997

\begin{tabular}{|c|c|c|c|c|}
\hline \multicolumn{5}{|c|}{ abhängige Variable: $U_{t}^{\text {Diff }}$} \\
\hline $\begin{array}{l}\text { unabhängige } \\
\text { Variable }\end{array}$ & (1) & (2) & (3) & (4) \\
\hline$U_{t-1}^{\text {Diff }}$ & $\begin{array}{c}1,355^{* *} \\
(62,056)\end{array}$ & $\begin{array}{c}1,354 * * \\
(61,965)\end{array}$ & $\begin{array}{c}1,349 * * \\
(61,333)\end{array}$ & $\begin{array}{c}1,349 * * \\
(60,978)\end{array}$ \\
\hline$U_{t-2}^{\text {Diff }}$ & $\begin{array}{l}-0,217 * * \\
(-5,933)\end{array}$ & $\begin{array}{l}-0,217^{* *} \\
(-5,932)\end{array}$ & $\begin{array}{c}-0,225 * * \\
(-6,086)\end{array}$ & $\begin{array}{l}-0,225^{* *} \\
(-6,063)\end{array}$ \\
\hline$U_{t-3}^{\text {Diff }}$ & $\begin{array}{c}-0,075^{*} \\
(-2,033)\end{array}$ & $\begin{array}{c}-0,073^{*} \\
(-1,993)\end{array}$ & $\begin{array}{c}-0,087^{*} \\
(-2,368)\end{array}$ & $\begin{array}{c}-0,084^{*} \\
(-2,276)\end{array}$ \\
\hline$U_{t-4}^{\text {Diff }}$ & $\begin{array}{c}-0,152 * * \\
(-4,160)\end{array}$ & $\begin{array}{c}-0,152 * * \\
(-4,166)\end{array}$ & $\begin{array}{l}-0,064 * * \\
(-2,959)\end{array}$ & $\begin{array}{l}-0,066^{* *} \\
(-3,017)\end{array}$ \\
\hline$U_{t-5}^{\text {Diff }}$ & $\begin{array}{l}0,065^{* *} \\
(3,004)\end{array}$ & $\begin{array}{c}0,064 * * \\
(2,983)\end{array}$ & - & - \\
\hline $\mathrm{RCG}_{4}$ & $\begin{array}{c}0,012^{*} \\
(2,147)\end{array}$ & - & - & - \\
\hline $\mathrm{RCG}_{1-1}$ & - & $\begin{array}{c}0,012^{*} \\
(2,152)\end{array}$ & - & - \\
\hline RCG6, & - & - & $\begin{array}{l}0,012 * * \\
(2,596)\end{array}$ & 一 \\
\hline $\mathrm{RCG6}_{\mathrm{l-1}}$ & - & 一 & - & $\begin{array}{c}0,012^{*} \\
(2,523)\end{array}$ \\
\hline $\mathrm{R}^{2}$ & 0,99 & 0,99 & 0,99 & 0,99 \\
\hline
\end{tabular}

Anmerkungen: $\quad \mathrm{t}$-Werte in Klammern, $+(\alpha<0,1),{ }^{*}(\alpha<0,05),{ }^{* *}(\alpha<0,01)$.

In jeder der gewählten Modellspezifikationen haben die besonders interessierenden Koeffizienten der Ideologievariablen das richtige Vorzeichen und sind mindestens auf dem 5\%-Signifikanzniveau von null verschieden. Zumindest in den ersten 18 Monaten nach einem Regierungswechsel scheint es also deutliche Partisaneffekte auf dem Arbeitsmarkt zu geben. Im untersuchten Zeitraum stieg die Arbeitslosenquote bei einem Regierungswechsel zu einer rechteren Partei kurzfristig an, wohingegen der Wechsel zu einer Linksregierung ein kurzfristiges Absinken der Arbeitslosenquote nach sich zog. Da die Ergebnisse der RCG6Variablen im Vergleich zu den Ergebnissen der RCG4-Variablen etwas deutlicher ausfallen, wird im folgenden auch nur noch mit RCG6 gearbeitet.

Es soll jetzt geprüft werden, inwiefern diese Ergebnisse hinsichtlich der Wahl der Zeitperiode bzw. hinsichtlich der Ländergruppe robust sind. 


\subsubsection{Cluster gemäß den ökonomischen Indikatoren}

Um die Globalisierungsthese zu überprüfen, werden die drei Ländergruppen auf rationale Partisaneffekte untersucht. Bei Richtigkeit der Globalisierungsthese sollten die geschlosseneren Volkswirtschaften deutlich stärkere Partisaneffekte aufweisen als die offenen Volkswirtschaften.

In Tabelle 5.8 sind die Regressionsergebnisse für die drei ökonomischen Cluster für die RCG6-Variable jeweils mit einer einperiodigen Wirkungsverzögerung und ohne eine Wirkungsverzögerung dargestellt. Die Vorzeichen sind vollständig theoriekonform und zum Teil auch auf dem 5\%-Niveau signifikant. Vor allem für den ersten, besonders offenen Cluster lässt sich die Hypothese einer Unterschiedslosigkeit der Arbeitslosenquote in den ersten 18 Monaten nach einem Regierungswechsel in beiden Modellspezifikationen deutlich ablehnen. In den beiden anderen Clustern ist dieser Zusammenhang nicht so eindeutig. Im zweiten Cluster lässt sich die rationale Partisanhypothese nicht bestätigen und im dritten Cluster lediglich für die Modellvariante ohne Wirkungsverzögerung der Politik. Dies bedeutet, dass hieraus nicht auf die Richtigkeit der Globalisierungsthese geschlossen werden kann; es scheint sogar eher das Gegenteil zu gelten: In den besonders offenen Ländern des ersten Clusters gibt es die deutlichsten Partisaneffekte.

Bei der Betrachtung der Regressionsergebnisse für alle Länder und die einzelnen Zeitphasen, erhält man ein etwas anderes Ergebnis (Tabelle 5.9). Hier ist zu erkennen, dass für das Gesamtländersample lediglich für die Zeit von 1974-1985 signifikante rationale Partisaneffekte gefunden werden können. Sowohl für die Zeit vor 1974 als auch für die Zeit nach 1985 lassen sich zwar überwiegend theoriekonforme Koeffizienten erkennen, von denen jedoch keiner signifikant von null verschieden ist. Man könnte dieses Ergebnis auch so interpretieren: Die Globalisierungsthese findet nur in der Zeit nach 1973, also nach dem Zusammenbruch von Bretton Woods, Bestätigung, da in den drei Folgeperioden der Partisaneffekt stetig abnimmt.

Dieses Ergebnis lässt sich weiter differenzieren, wenn man Einzelregressionen für jeden der drei Ländercluster und dort jeweils für die vier Zeitphasen vornimmt. ${ }^{319}$ Es zeigt sich ein recht heterogenes Bild. Für den ersten Cluster, für den die beste Bestätigung der Partisantheorie für den gesamten Zeitraum gefunden wurde, wird sichtbar, dass diese Bestätigung wider Erwarten v.a. durch die Jahre nach 1985 erreicht wird. Damit wäre für diesen Cluster die Globalisierungsthese widerlegt.

${ }^{319}$ Die detaillierten Ergebnisse finden sich wiederumTint ishlarg C.78-3-631-75139-8 
Tabelle 5.8 : Arbeitsmarkteffekte für die ökonomischen Cluster 1960-1997

\begin{tabular}{|c|c|c|c|c|c|c|}
\hline \multicolumn{7}{|c|}{ abhängige Variable: $U_{t}^{\text {Diff }}$} \\
\hline \multirow[b]{3}{*}{$\begin{array}{l}\text { unabhängige } \\
\text { Variable }\end{array}$} & \multirow{2}{*}{\multicolumn{2}{|c|}{$\begin{array}{c}\text { 1. Cluster } \\
(\mathrm{BEL}, \mathrm{NL}, \mathrm{NZL}, \mathrm{S}, \mathrm{UK})\end{array}$}} & \multirow{2}{*}{\multicolumn{2}{|c|}{$\begin{array}{c}\text { 2. Cluster } \\
\text { (CAN, DK, SF, IRL, } \\
\text { NOR, CH) }\end{array}$}} & \multirow{2}{*}{\multicolumn{2}{|c|}{$\begin{array}{c}\text { 3. Cluster } \\
\text { (AUS, AU, F, D, ITA, } \\
\text { J, E, USA) }\end{array}$}} \\
\hline & & & & & & \\
\hline & (1) & (2) & (1) & (2) & (1) & (2) \\
\hline$U_{t-1}^{\text {Diff }}$ & $\begin{array}{l}1,310 * * \\
(28,077)\end{array}$ & $\begin{array}{c}1,304^{* *} \\
(27,538)\end{array}$ & $\begin{array}{c}1,428 * * \\
(35,190)\end{array}$ & $\begin{array}{c}1,427 * * \\
(35,022)\end{array}$ & $\begin{array}{c}1,328 * * \\
(43,107)\end{array}$ & $\begin{array}{l}1,332 * * \\
(43,006)\end{array}$ \\
\hline$U_{t-2}^{\text {Diff }}$ & $\begin{array}{c}-0,154^{*} \\
(-1,981)\end{array}$ & $\begin{array}{c}-0,144+ \\
(-1,839)\end{array}$ & $\begin{array}{l}-0,290 * * \\
(-4,129)\end{array}$ & $\begin{array}{l}-0,289 * * \\
(-4,089)\end{array}$ & $\begin{array}{l}-0,180 * * \\
(-3,502)\end{array}$ & $\begin{array}{l}-0,184 * * \\
(-3,568)\end{array}$ \\
\hline $\mathrm{U}_{t-3}^{\text {Diff }}$ & $\begin{array}{l}-0,188 * * \\
(-4,072)\end{array}$ & $\begin{array}{l}-0,192 * * \\
(-4,129)\end{array}$ & $\begin{array}{l}-0,159 * * \\
(-3,920)\end{array}$ & $\begin{array}{l}-0,160 * * \\
(-3,893)\end{array}$ & $\begin{array}{l}-0,174 * * \\
(-5,725)\end{array}$ & $\begin{array}{l}-0,172 * * \\
(-5,637)\end{array}$ \\
\hline RCG6, & $\begin{array}{l}0,018^{*} \\
(2,161)\end{array}$ & - & $\begin{array}{c}0,004 \\
(0,321)\end{array}$ & - & $\begin{array}{c}0,013 * \\
(2,064)\end{array}$ & - \\
\hline RCG6 $_{t-1}$ & - & $\begin{array}{l}0,018 * \\
(2,171)\end{array}$ & - & $\begin{array}{c}0,011 \\
(0,978)\end{array}$ & - & $\begin{array}{c}0,009 \\
(1,335)\end{array}$ \\
\hline $\mathrm{R}^{2}$ & 0,99 & 0,99 & 0,99 & 0,99 & 0,99 & 0,99 \\
\hline
\end{tabular}

Anmerkungen: $\quad \mathrm{t}$-Werte in Klammern, $+(\alpha<0,1),{ }^{*}(\alpha<0,05),{ }^{* *}(\alpha<0,01)$.

Im zweiten Cluster lässt sich überhaupt keine Bestätigung für die rationale Partisantheorie finden; die einzigen signifikant von null verschiedenen Koeffizienten besitzen das verkehrte Vorzeichen. Für den dritten Ländercluster, der die besonders autarken Länder zusammenfasst, wird die rationale Partisantheorie tatsächlich nur in der ersten Zeitphase bestätigt. In den darauf folgenden Perioden sinken die geschätzten Koeffizienten stetig und demnach bei relativ konstanter Standardabweichung auch die entsprechenden $t$-Werte. Für den besonders autarken Cluster ließe sich folglich ein schwacher Hinweis für die Richtigkeit der Globalisierungsthese finden. Dies ist jedoch angesichts der Tatsache, dass im besonders offenen ersten Cluster gerade das Gegenteil gefunden wurde, nicht als sehr gute Bestätigung zu werten. Außerdem muss auch hier darauf hingewiesen werden, dass bei den Einzelregressionen nur wenige Regierungswechsel in die Schätzungen eingehen konnten. Dies reduziert fraglos die Aussagekraft der Ergebnisse der Einzelregressionen, da bei geringerem Stichprobenumfang AusreiBer stärker ins Gewicht fallen und schwerer als solche identifiziert werden können. 
Tabelle 5.9 : Arbeitsmarkteffekte in der Clusterung gemäß ökonomischen Globalisierungsindikatoren (alle Ländercluster)

abhängige Variable: $U_{t}^{\text {Diff }}$

unabh. $1961-1973$ (1. Zeitcluster) $1974-1985$ (2. Zeitcluster) $\quad 1986-1992$ (3. Zeitcluster) $1993-1997$ (4. Zeitcluster)

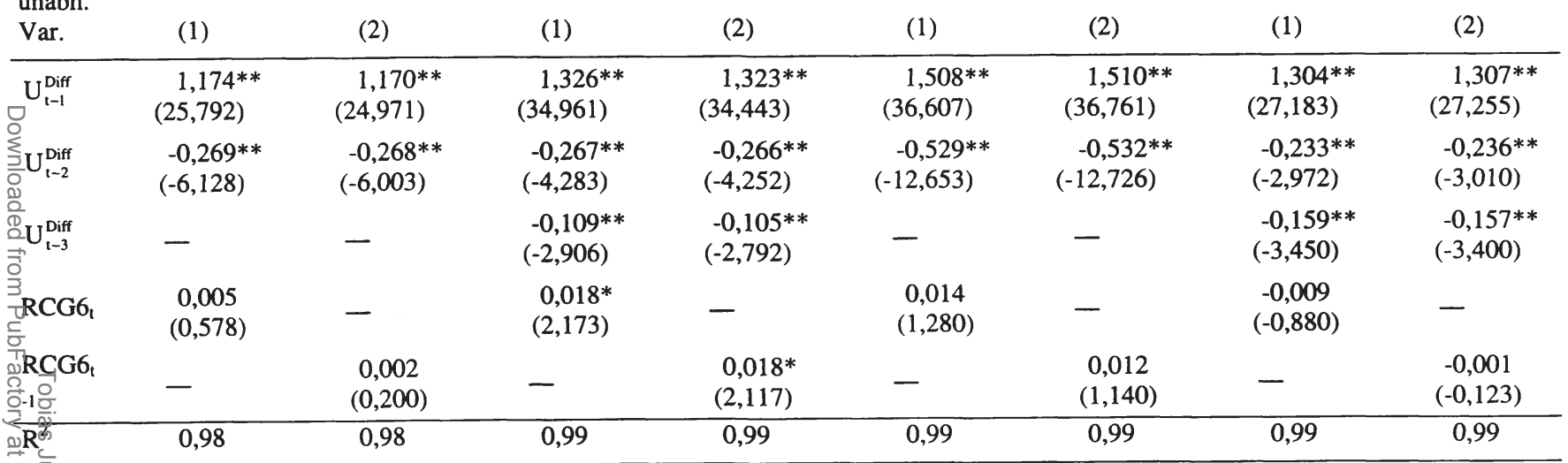

Afnmerkungen: $\quad \mathrm{t}$-Werte in Klammern, $+(\alpha<0,1),{ }^{*}(\alpha<0,05),{ }^{* *}(\alpha<0,01)$. 
Es wird immerhin deutlich, dass die Gültigkeit der rationalen Partisantheorie stark von Länderspezifika abhängt, wobei der ökonomische Offenheitsgrad kein wichtiges erklärendes Länderspezifikum zu sein scheint, welches Land besonders gut der rationalen Partisantheorie folgt und welches nicht. Es bleibt zu prüfen, ob gegebenenfalls die Clusterbildung der Länder nach den politischen Indikatoren zu anderen Resultaten führt.

\subsubsection{Cluster gemäß den politischen Indikatoren}

Auch wenn die Ländercluster gemäß den politischen Indikatoren zu relativ starken Verschiebungen in der Ländergruppenstruktur führt, so ändert sich das oben skizzierte Bild in den aggregierten Betrachtungen nur sehr wenig. Tabelle 5.10 zeigt zunächst die Regressionsergebnisse für die vier Ländercluster und den gesamten Stützzeitraum. Auf sehr hohem Signifikanzniveau kann für den ersten Cluster die Hypothese, dass für die ersten 18 Monate die ideologische Ausprägung der Regierung keinen Einfluss auf das Arbeitsmarktergebnis hat, widerlegt werden. Ebenfalls recht gute Bestätigung für die rationale Partisantheorie lässt sich im dritten Cluster finden.

Für die anderen beiden Ländergruppen werden indes keine signifikanten Koeffizienten ermittelt, sodass es daher nicht möglich ist, mit Hilfe dieser Ergebnisse Rückschlüsse auf Globalisierungseinflüsse zu ziehen. Sowohl gemäß den ökonomischen als auch gemäß den politischen Clustern finden sich in den besonders offenen Volkswirtschaften die beste Bestätigung für die rationale Partisantheorie. Demnach scheint die Öffnung eines Landes den ideologischen Profilierungsspielraum einer Regierung auf dem Arbeitsmarkt nicht einzuengen. Auch bei der Analyse der einzelnen Zeitphasen über alle Länder (Tabelle 5.11) finden sich ähnliche Ergebnisse wie zuvor. Lediglich für die Phase von 1967 bis 1978 finden sich hoch signifikante theoriekonforme Koeffizienten.

Es bleibt zu prüfen, ob die einzelnen Ländergruppen für die fünf Zeitphasen unterschiedliche Entwicklungen durchlaufen haben. ${ }^{320}$ Wie bereits aus der Analyse der Ländergruppen für den gesamten Zeitraum zu erwarten war, ist für den zweiten und vierten Cluster für keine der Zeitphasen ein eindeutiger Zusammenhang erkennbar. Interessant sind allerdings die Ergebnisse für den ersten und dritten Cluster. 
Tabelle 5.10 : Arbeitsmarkteffekte in der Clusterung gemäß politischen Globalisierungsindikatoren 1960-1997

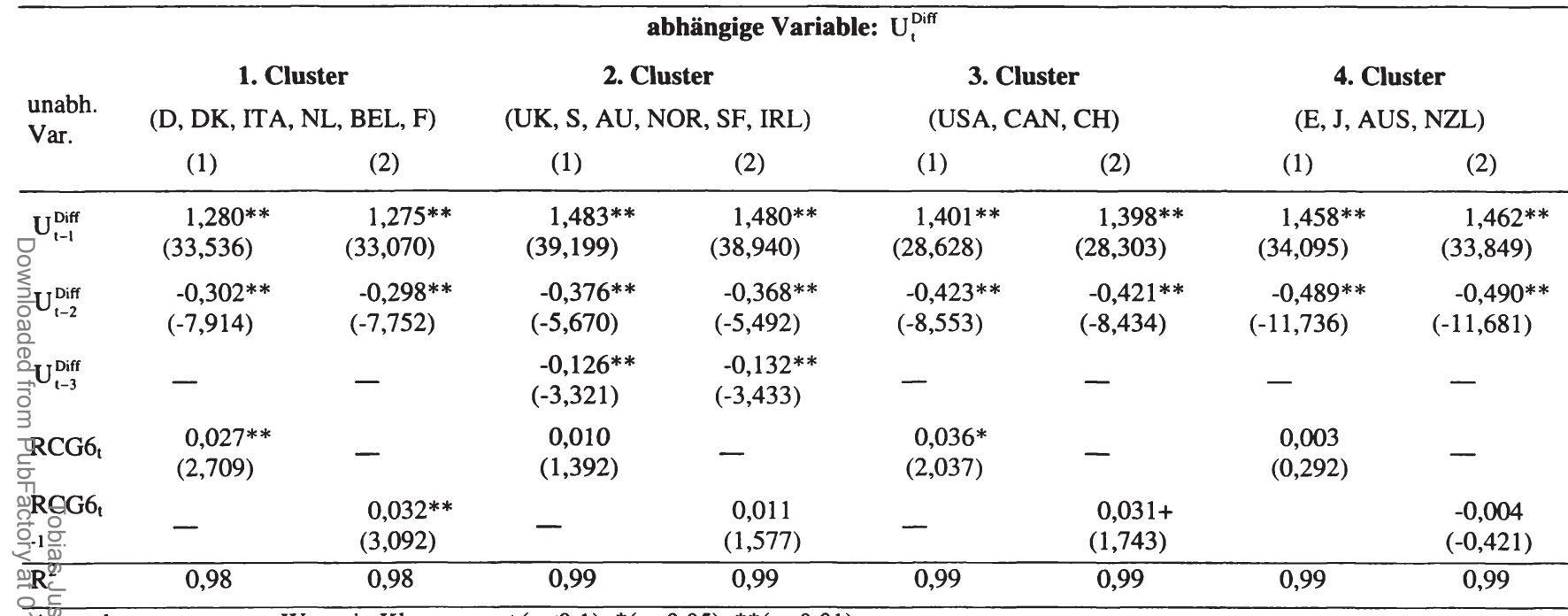

Añmerkungen: $\quad \mathrm{t}$-Werte in Klammern, $+(\alpha<0,1),{ }^{*}(\alpha<0,05),{ }^{* *}(\alpha<0,01)$. 
Tabelle 5.11 : Arbeitsmarkteffekte in der Clusterung gemäß politischen Globalisierungsindikatoren (alle Länder)

\begin{tabular}{|c|c|c|c|c|c|c|c|c|c|c|}
\hline \multicolumn{11}{|c|}{ abhängige Variable: $U_{t}^{\text {Diff }}$} \\
\hline \multirow{2}{*}{$\begin{array}{l}\text { unabh. } \\
\text { Var. }\end{array}$} & \multicolumn{2}{|c|}{$1960-1966$} & \multicolumn{2}{|c|}{1967 - 1978} & \multicolumn{2}{|c|}{1979 - 1987} & \multicolumn{2}{|c|}{$1988-1992$} & \multicolumn{2}{|c|}{1993 - 1997} \\
\hline & (1) & (2) & (1) & $(2)$ & (1) & (2) & (1) & (2) & (1) & (2) \\
\hline$\underbrace{U_{t-2}^{\text {Diff }}}_{S}$ & - & 一 & $\begin{array}{l}-0,385^{* *} \\
(-9,506)\end{array}$ & $\begin{array}{l}-0,386^{* *} \\
(-9,471)\end{array}$ & $\begin{array}{c}-0,151^{*} \\
(-2,287)\end{array}$ & $\begin{array}{c}-0,147 * \\
(-2,222)\end{array}$ & $\begin{array}{c}-0,582 * * \\
(-11,566)\end{array}$ & $\begin{array}{c}-0,584^{* *} \\
(-11,620)\end{array}$ & $\begin{array}{c}-0,233 * * \\
(-2,972)\end{array}$ & $\begin{array}{l}-0,236 * * \\
(-3,010)\end{array}$ \\
\hline RCG6, & $\begin{array}{c}0,004 \\
(0,232)\end{array}$ & 一 & $\begin{array}{l}0,025 * * \\
(2,803)\end{array}$ & 一 & $\begin{array}{c}0,006 \\
(0,661)\end{array}$ & - & $\begin{array}{c}0,011 \\
(0,724)\end{array}$ & - & $\begin{array}{c}-0,009 \\
(-0,880)\end{array}$ & - \\
\hline 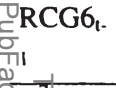 & 一 & $\begin{array}{c}-0,011 \\
(-0,583) \\
\end{array}$ & - & $\begin{array}{c}0,024 * * \\
(2,693) \\
\end{array}$ & - & $\begin{array}{c}0,008 \\
(0,885) \\
\end{array}$ & - & $\begin{array}{c}0,007 \\
(0,484)\end{array}$ & - & $\begin{array}{c}-0,001 \\
(-0,123)\end{array}$ \\
\hline
\end{tabular}

Anmerkungen: $\quad \mathrm{t}$-Werte in Klammern, $+(\alpha<0,1),{ }^{*}(\alpha<0,05),{ }^{* *}(\alpha<0,01)$.

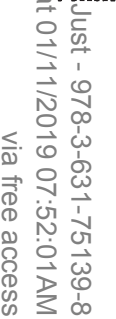


Im ersten Cluster nimmt der Erklärungsgehalt der RCG6-Variable von 1967 bis 1992 stetig ab, in der letzten Zeitphase ist das Vorzeichen sogar umgekehrt. Argumentiert man eng im Alesina-Modell, so scheint es, dass für diese sechs sehr stark zusammenwachsenden europäischen Staaten der Handlungsspielraum so stark verengt wird, dass der Wähler immer seltener durch politikinduzierte Inflation überrascht wird und folglich der transitorische Output-Effekt nach einem Regierungswechsel zunehmend ausbleibt. ${ }^{321}$ Dies ist im dritten Cluster anders. Hier lässt sich zwar ein Zusammenhang gemäß der rationalen Partisantheorie seit 1967 vermuten, wenngleich diese Vermutung nicht durch statistische Signifikanz gestützt wird. Die einzige Zeitphase, in der das $10 \%$-Signifikanzniveau erreicht wird, ist aber gerade die letzte Zeitphase. ${ }^{322}$

Hier scheint die Globalisierungsthese also eine (schwache) Bestätigung zu finden. Für jene Ländergruppen, bei denen rationale Partisaneffekte isoliert werden konnten (Cluster 1 und 3), verebben diese in den besonders offenen Ländern, bleiben jedoch in den relativ geschlossenen Ländern erhalten oder verstärken sich sogar. Da aber für den zweiten und vierten Cluster keine theoriekonformen Ergebnisse gefunden werden konnten, sollte dies mit ausgesprochener Vorsicht interpretiert werden. Die Regressionen zeigen v.a., dass weder die rationale Partisantheorie noch die Globalisierungsthese für alle OECD-Staaten Allgemeingültigkeit besitzen.

\subsubsection{Zusammenfassung}

Die vorangehenden Abschnitte konnten eine Reihe teilweise unerwarteter Ergebnisse erbringen. Erstens wurde für die traditionelle Partisantheorie nur sehr wenig Bestätigung gefunden, folglich konnte auch die Globalisierungsthese hier nicht wirklich geprüft werden. Zweitens wurde die rationale Partisantheorie gerade in den Regressionen mit sehr großen Samples recht eindrucksvoll bestätigt. Allerdings wurde auch gezeigt, dass die rationale Partisantheorie keineswegs generelle und länderübergreifende Bedeutung besitzt, sondern dass es Ländergruppen gibt, für die diese Theorie eher Gültigkeit zu haben scheint als für andere. Zwar erbrachten die Schätzungen, dass über den gesamten Stützzeitraum gerade die besonders offenen Länder gut die rationale Partisantheorie abbilden, jedoch konnte auch verdeutlicht werden, dass diese Bestätigung v.a. für die Jah-

${ }^{321}$ Inwiefern dieses Ergebnis auf die Kräfte der Globalisierung oder die zunehmende Unabhängigkeit der Zentralbanken zurückzuführen ist, lässt sich hier allerdings nicht sagen. Im Rahmen der Analyse der Budgetstrukturunterschiede wird noch eine weitere Argumentationslinie aufgebaut.

${ }^{322}$ Die neu gewählte Periodenlänge von 1988 bis 1997 ist dadurch begründet, dass von 1988 bis 1992 in keinem der drei Staaten des dritten Clusters ein Regierungswechsel stattfand. Folglich wäre die Analyse der RCG6-Variablen nīchtimöglish: 978-3-631-75139-8 
re vor 1980 gilt. Es lässt sich also hinsichtlich des Arbeitsmarktes zusammenfassen:

1. Die rationale Partisantheorie scheint Berechtigung zu haben, nicht jedoch die traditionelle Partisantheorie.

2. Dies gilt allerdings nicht für alle hier untersuchten Ländergruppen gleichermaßen.

3. Der Offenheitsgrad ist ein eher unzureichender Indikator zur Unterscheidung der Länder, bei denen die Partisantheorien gelten, von jenen Ländern, bei denen dies nicht der Fall ist.

4. Es gibt in der politischen Clusterung und bei den Ländern, bei denen die rationale Partisantheorie gilt, Anhaltspunkte dafür, dass durch zunehmende Integration in supranationale Institutionen der Partisaneffekt nach einem Regierungswechsel abnimmt.

5. Es ist keine Aussage möglich, ob die gefundenen transitorischen Partisaneffekte auf dem Arbeitsmarkt tatsächlich durch die Überraschungsinflation im Sinne der rationalen Partisantheorie verursacht wurden. Es ist auch möglich, dass besonders nach einem Regierungswechsel Parteien bemüht sind, ,ihre Politikpräferenz" durchzusetzen. Dies könnte dann bei einer linken Regierung zu höheren Staatsausgaben führen. Es ist möglich, dass in einer Welt mit vollkommenem Kapitalverkehr und flexiblem Wechselkurs solch expansive Fiskalpolitik nur nach einer Anpassungsfrist wirkungslos bliebe, während des transitorischen Anpassungsprozesses hätte diese Fiskalpolitik kurzfristig positive Outputeffekte. Der transitorische Arbeitsmarkteffekt wäre dann nicht das Ergebnis von enttäuschten Erwartungen hinsichtlich der Inflation, sondern Ausdruck einer nur temporär wirkenden expansiven Fiskalpolitik der Linken. Darauf wird in Abschnitt 5.3 noch zurückzukommen sein.

Da sowohl bei der rationalen als auch bei der traditionellen Partisantheorie die Arbeitsmarktperformance und die Inflationsentwicklung über das Konzept der Phillipskurve zusammengehören, soll nun ergänzend geprüft werden, ob es Partisaneffekte bei der Preisentwicklung gibt.

\subsection{Partisanpolitik, Inflation und Globalisierung}

Sowohl in der traditionellen Partisantheorie von Hibbs als auch in der rationalen Partisantheorie von Alesina weichen die Inflationsraten zwischen linken und rechten Regierungen während der gesamten Legislaturperiode aufgrund des Phillipskurvenzusammenhangs voneinander $a b$. Linke Regierungen hätten danach stets höhere Inflationsraten während ihrer Legislaturperiode zu verzeichnen als rechte Regierungen. 
Auch in diesem Kapitel liegt der Schwerpunkt nicht nur in der Untersuchung der generellen Existenz von Partisaneffekten; dies wurde in anderen Studien bereits getan (z.B. Alesina, Cohen, Roubini, 1997, Berlemann, 1999), sondern in der Untersuchung der Erklärungskraft des Offenheitsgrads von Ländern für die Gültigkeit der Partisantheorie.

\subsubsection{Datenbeschreibung und Modellspezifikation}

Es werden die bereits zuvor genannten 19 OECD-Staaten untersucht. Der Stützzeitraum ist von $1 / 1960$ bis 4/1997. ${ }^{323}$ Als Indikator für die Preisentwicklung im Jahr $\mathrm{t}$ des Landes i dienen Quartalsdaten der Änderungsrate des nationalen Konsumentenpreisindex $\pi_{\mathrm{i}, \mathrm{t}}{ }^{324}$ Ähnlich wie bei der Erfassung der Arbeitslosenquoten ist die Erfassung der Preisentwicklung kein einfaches Unterfangen. Da Preisindizes immer auf Warenkörben beruhen, hängt die Güte der Erfassung der Preisentwicklung davon ab, inwiefern man in den zugrunde liegenden Warenkörben die relevanten Güter und deren relevante Qualität erfasst hat.

Alesina und Roubini (1990) folgend, wird auch hier ein autoregressives crosssection time-series Panel-Modell mit fixed effects geschätzt, bei dem die Weltinflationsrate $\pi_{t}^{w}$ und das center of gravity CG als erklärende Variablen Eingang finden. Die Weltinflationsrate soll die äußeren Bestimmungsfaktoren für das inländische Preisniveau im Sinne eines Inflationsimports abbilden. Explizit wird die Weltinflationsrate durch den periodenbezogenen Mittelwert der mit den jeweiligen Inlandsprodukten gewichteten nationalen Inflationsraten aller untersuchten Staaten approximiert, ${ }^{325}$ sodass das folgende Modell zu schätzen ist:

${ }^{323}$ Die einzige Ausnahme bildet hier Spanien. Zwar liegen auch für Spanien Inflationsdaten für den gesamten Zeitraum vor, jedoch ist Spanien erst seit 3/1977 sinnvoll in die demokratische Parteienlandschaft einzubeziehen. Für die Errechnung der Weltinflationsrate wurde Spanien allerdings mitberücksichtigt, da Spanien auch vor 1977 am internationalen Wirtschaftsgeschehen teilnahm.

${ }^{324}$ Es werden ausschließlich Inflationsdaten aus den IMF Financial Statistics Yearbooks benutzt.

325 Andere Studien (Alesina, Cohen und Roubini, 1992, 1997, Berlemann, 1999) arbeiten mit dem gewichteten Mittelwert der G7 Staaten und einem festen Gewichtungsfaktor. Analog zur Begründung der Ermittlung der durchschnittlichen Arbeitslosenquote in den OECDStaaten wird auch hier die umfassendere und flexiblerie Sewaichpingsmethode vorgezogen. 


$$
\begin{gathered}
\pi_{i, t}=\alpha_{i}+\beta_{1} \cdot \pi_{i, t-1}+\ldots+\beta_{n} \cdot \pi_{i, t-n}+\beta_{n+1} \cdot \pi_{t}^{w}+\gamma \cdot C_{i, t}+\varepsilon_{i, t} \\
\text { mit } \\
\pi_{t}^{w}=\sum_{i=1}^{n} \frac{\pi_{i, t} \cdot B_{i} P_{i, t}}{\sum_{i=1}^{n} B I P_{i, t}}
\end{gathered}
$$

Der hier besonders interessierende Koeffizient ist natürlich $\gamma$.

Alle im weiteren diskutierten Modelle wurden erneut mit fixed effects für die 19 Länder geschätzt, um mögliche nationale Besonderheiten, wie z.B. den Unabhängigkeitsgrad der jeweiligen Nationalbank, zu erfassen. Auch hier wurden die Koeffizienten dieser Länderdummies nicht dargestellt, da sie bei dieser Untersuchung nicht von Interesse sind.

\subsubsection{Schätzergebnisse}

Zunächst wird das gesamte Panel auf Partisaneffekte bei der Inflationsperformance geschätzt.

Dafür werden drei unterschiedliche Modelle nebeneinander dargestellt. Im Modell (1) wird angenommen, es gäbe keine Wirkungsverzögerung, d.h. $C_{t}$ geht ohne Wirkungslag in die Regressionsgleichung ein. In Modell (2) wird ein dreimonatiger Lag unterstellt $\left(\mathrm{CG}_{\mathrm{t}-1}\right)$ und schließlich wird in Modell (3) von einem sechsmonatigen Wirkungslag $\left(\mathrm{CG}_{\mathrm{t}-2}\right)$ ausgegangen. ${ }^{326}$

Die Ermittlung der optimalen Lagstruktur der Inflationsraten erfolgte analog zu der Vorgehensweise bei der Lagstruktur der Arbeitslosenraten. Es wurden alle jene Lags mit aufgenommen, die mindestens auf dem 5\%-Signifikanzniveau Einfluss auf die aktuelle Inflationsrate zeigten.

Zwar sind die Koeffizienten in allen drei Modellspezifikationen im Einklang mit der Partisantheorie; das negative Vorzeichen zeigt an, dass bei einem hohen CGWert (einer rechten Partei) die Inflationsrate niedriger ausfällt. Jedoch ist keiner der Koeffizienten signifikant von null verschieden. Die Hypothese, dass linke

${ }^{326}$ Es wurden auch längere Wirkungsverzögerungen geschätzt, jedoch waren die Schätzergebnisse nicht signifikant, daher wird auf die Darstellung verzichtet. Es wurden darüber hinaus Modelle geschätzt, bei denen zusätzlich eine Dummy-Variable OIL für die Ölpreisschocks der Jahre 1973-1975 und 1979-1981 als exogene Variable berücksichtigt wurde. Deren Koeffizient hatte erwartungsgemäß ein positives Vorzeichen, das auf dem $1 \%$ Signifikanzniveau von null verschieden war. $\mathrm{Da}$ in diesen Modellen die Koeffizienten der CG-Variablen nahezu unverändert blieben, werden auch diese Modellspezifikationen hier nicht dargestellt. 
und rechte Regierungen dieselbe Inflationsperformance aufweisen, kann zumindest auf dieser Aggregationsebene nicht verworfen werden. Es soll im folgenden untersucht werden, ob dieses Ergebnis mit Hilfe einer detaillierten Untersuchung der einzelnen Ländergruppen konkretisiert werden kann.

Tabelle 5.12 : Schätzergebnisse für die Inflationsperformance im Gesamtpanel 1960-1997

\begin{tabular}{|c|c|c|c|}
\hline \multicolumn{4}{|c|}{ abhängige Variable: $\pi_{t}$} \\
\hline unabhängige Variable & (1) & (2) & (3) \\
\hline$\pi_{t-1}$ & $\begin{array}{c}1,114^{* *} \\
(58,431)\end{array}$ & $\begin{array}{c}1,118^{* *} \\
(59,162)\end{array}$ & $\begin{array}{c}1,116^{* *} \\
(58,420)\end{array}$ \\
\hline$\pi_{t-2}$ & $\begin{array}{c}-0,145^{* *} \\
(-5,138)\end{array}$ & $\begin{array}{l}-0,157 * * \\
(-5,611)\end{array}$ & $\begin{array}{l}-0,155^{* *} \\
(-5,501)\end{array}$ \\
\hline$\pi_{t-3}$ & $\begin{array}{l}-0,106^{* *} \\
(-5,852)\end{array}$ & $\begin{array}{l}-0,099 * * \\
(-5,542)\end{array}$ & $\begin{array}{l}-0,100 * * \\
(-5,578)\end{array}$ \\
\hline$\pi_{t}^{w}$ & $\begin{array}{c}0,147 * * \\
(15,478)\end{array}$ & $\begin{array}{c}0,148 * * \\
(15,798)\end{array}$ & $\begin{array}{c}0,149 * * \\
(15,804)\end{array}$ \\
\hline $\mathrm{CG}_{\mathrm{t}}$ & $\begin{array}{c}-0,020 \\
(-1,330)\end{array}$ & - & - \\
\hline $\mathrm{CG}_{\mathrm{t}-1}$ & - & $\begin{array}{c}-0,018 \\
(-1,254)\end{array}$ & - \\
\hline $\mathrm{CG}_{\mathrm{t}-2}$ & - & - & $\begin{array}{c}-0,016 \\
(-1,063)\end{array}$ \\
\hline $\mathrm{R}^{2}$ & 0,94 & 0,95 & 0,95 \\
\hline
\end{tabular}

Anmerkungen: $\quad \mathrm{t}$-Werte in Klammern, $+(\alpha<0,1),{ }^{*}(\alpha<0,05),{ }^{* *}(\alpha<0,01)$.

\subsubsection{Cluster gemäß den ökonomischen Indikatoren}

Es wird geprüft, ob die Preisentwicklung in den einzelnen Ländern zumindest teilweise durch die herrschende Regierungsideologie erklärt werden kann und wenn ja, ob dieser Einfluss je nach Offenheitsgrad, hier operationalisiert durch ökonomische Indikatoren, schwankt.

In Tabelle 5.13 werden zunächst für den gesamten Stützzeitraum und die drei unterschiedlichen offenen Ländergruppen separate Regressionsergebnisse präsentiert. Zwar mag es gemäß der Globalisierungsthese überraschen, dass ausschließlich im ersten Cluster, der die besonders offenen Länder beinhaltet, signifikante Bestätigung der Partisantheorie vorliegt. Dies ist jedoch gleichzeitig im Einklang mit den Schätzergebnissen der rationalen Partisantheorie, da auf dem Arbeitsmarkt auch nur für den ersten Cluster signifikant theoriekonforme Schätzergebnisse gefunden wurden. Bei der Analyse der einzelnen Zeitphasen 
unter Berücksichtigung des vollständigen Ländersamples (Tabelle 5.14) lassen sich zwar durchgängig korrekte Vorzeichen finden, jedoch nur drei der acht Koeffizienten sind signifikant von null verschieden. Da diese Koeffizienten zudem in den letzten zwei Zeitphasen vorgefunden werden, lässt sich die These, dass die fortschreitende Öffnung der Märkte keinen Einfluss auf das parteipolitische makroökonomische Profilierungspotential hat, nicht verwerfen. Die parteipolitischen Ausrichtungen erklären heute eher mehr als früher die Schwankungen in den realisierten Inflationsraten.

Bei einer detaillierteren Betrachtung wird dies noch deutlicher: Rechnet man für jede der drei Ländergruppen und die vier Zeitphasen getrennte Regressionen, so zeigt sich nämlich ein sehr uneinheitliches Bild. ${ }^{327}$ Die Koeffizienten der CGVariablen haben in etwa gleich häufig das theoriekonforme wie das entgegengesetzte Vorzeichen. Allerdings haben die signifikant von null verschiedenen Koeffizienten eher das durch die Partisantheorie prognostizierte negative Vorzeichen. Dies ist aber nicht sehr häufig und nicht regelmäßig der Fall.

\section{Tabelle 5.13 : Inflationsperformance für die ökonomischen Cluster} 1960-1997

\begin{tabular}{|c|c|c|c|c|c|c|}
\hline \multicolumn{7}{|c|}{ abhängige Variable: $\pi_{\mathrm{t}}$} \\
\hline \multirow[b]{2}{*}{$\begin{array}{l}\text { unabhängige } \\
\text { Variable }\end{array}$} & \multicolumn{2}{|c|}{$\begin{array}{c}\text { 1. Cluster } \\
(\mathrm{BEL}, \mathrm{NL}, \mathrm{NZL}, \mathrm{S}, \mathrm{UK})\end{array}$} & \multicolumn{2}{|c|}{$\begin{array}{c}\text { 2. Cluster } \\
\text { (CAN, DK, SF, IRL, } \\
\text { NOR, CH) }\end{array}$} & \multicolumn{2}{|c|}{$\begin{array}{c}\text { 3. Cluster } \\
\text { (AUS, AU, F, D, ITA, } \\
\text { J, E, USA) }\end{array}$} \\
\hline & (1) & (2) & (1) & (2) & (1) & (2) \\
\hline$\pi_{t-1}$ & $\begin{array}{l}1,173^{* *} \\
(32,115)\end{array}$ & $\begin{array}{l}1,174 * * \\
(32,177)\end{array}$ & $\begin{array}{l}1,056^{* *} \\
(32,076)\end{array}$ & $\begin{array}{l}1,055^{* *} \\
(32,022)\end{array}$ & $\begin{array}{l}1,150^{* *} \\
(38,135)\end{array}$ & $\begin{array}{l}1,160 * * \\
(39,594)\end{array}$ \\
\hline$\pi_{t-2}$ & $\begin{array}{l}-0,246 * * \\
(-4,459)\end{array}$ & $\begin{array}{l}-0,247 * * \\
(-4,482)\end{array}$ & $\begin{array}{l}-0,205^{* *} \\
(-6,565)\end{array}$ & $\begin{array}{l}-0,205 * * \\
(-6,555)\end{array}$ & $\begin{array}{l}-0,155^{* *} \\
(-3,413)\end{array}$ & $\begin{array}{l}-0,192 * * \\
(-4,342)\end{array}$ \\
\hline$\pi_{t-3}$ & $\begin{array}{l}-0,077^{*} \\
(-2,223)\end{array}$ & $\begin{array}{l}-0,077^{*} \\
(-2,227)\end{array}$ & - & - & $\begin{array}{l}-0,107 * * \\
(-3,717)\end{array}$ & $\begin{array}{l}-0,086 * * \\
(-3,062)\end{array}$ \\
\hline$\pi_{t}^{w}$ & $\begin{array}{l}0,163 * * \\
(8,934)\end{array}$ & $\begin{array}{l}0,163^{* *} \\
(8,942)\end{array}$ & $\begin{array}{l}0,165^{* *} \\
(9,020)\end{array}$ & $\begin{array}{l}0,166^{* *} \\
(9,067)\end{array}$ & $\begin{array}{l}0,117 * * \\
(8,560)\end{array}$ & $\begin{array}{l}0,121^{* *} \\
(9,119)\end{array}$ \\
\hline $\mathrm{CG}_{\mathrm{t}}$ & $\begin{array}{l}-0,049+ \\
(-1,908)\end{array}$ & - & $\begin{array}{l}-0,051 \\
(-1,381)\end{array}$ & - & $\begin{array}{c}0,012 \\
(0,611)\end{array}$ & - \\
\hline $\mathrm{CG}_{1-1}$ & - & $\begin{array}{l}-0,052 * \\
(-2,024)\end{array}$ & - & $\begin{array}{l}-0,037 \\
(-1,008)\end{array}$ & - & $\begin{array}{c}0,011 \\
(0,594)\end{array}$ \\
\hline $\mathrm{R}^{2}$ & 0,94 & 0,94 & 0,93 & 0,93 & 0,96 & 0,96 \\
\hline
\end{tabular}

Anmerkungen: $\quad \mathrm{t}$-Werte in Klammern, $+(\alpha<0,1),{ }^{*}(\alpha<0,05),{ }^{* *}(\alpha<0,01)$.

${ }^{327}$ Die Regressionsergebnisse für die Detailregressiōnenafinden sighsebeafalls im3Anhang C. 
Tabelle 5.14 : Inflationsperformance in der Clusterung gemäß ökonomischen Globalisierungsindikatoren (alle Ländercluster)

\begin{tabular}{|c|c|c|c|c|c|c|c|c|}
\hline \multirow{3}{*}{$\begin{array}{l}\text { unabh. } \\
\text { Var. }\end{array}$} & \multicolumn{7}{|c|}{ abhängige Variable: $\pi_{\mathrm{t}}$} & \\
\hline & \multicolumn{2}{|c|}{$1961-1973$ (1. Zeitcluster) } & \multicolumn{2}{|c|}{1974 - 1985 (2. Zeitcluster) } & \multicolumn{2}{|c|}{$1986-1992$ (3. Zeitcluster) } & \multicolumn{2}{|c|}{1993 - 1997 (4. Zeitcluster) } \\
\hline & (1) & (2) & (1) & (2) & (1) & (2) & (1) & $(2)$ \\
\hline$\pi_{t-1}$ & $\begin{array}{c}0,973 * * \\
(30,099)\end{array}$ & $\begin{array}{c}0,973 * * \\
(30,055)\end{array}$ & $\begin{array}{c}1,073 * * \\
(32,125)\end{array}$ & $\begin{array}{c}1,080^{* *} \\
(33,049)\end{array}$ & $\begin{array}{c}1,167 * * \\
(27,228)\end{array}$ & $\begin{array}{c}1,174 * * \\
(27,446)\end{array}$ & $\begin{array}{c}1,034 * * \\
(20,619)\end{array}$ & $\begin{array}{c}1,034 * * \\
(20,565)\end{array}$ \\
\hline 2 & $\begin{array}{c}-0,104 * \\
(-2,305)\end{array}$ & $\begin{array}{c}-0,104^{*} \\
(-2,310)\end{array}$ & $\begin{array}{l}-0,134 * * \\
(-2,747)\end{array}$ & $\begin{array}{c}-0,159 * * \\
(-3,320)\end{array}$ & $\begin{array}{l}-0,303 * * \\
(-7,124)\end{array}$ & $\begin{array}{l}-0,307 * * \\
(-7,213)\end{array}$ & $\begin{array}{l}-0,259 * * \\
(-5,142)\end{array}$ & $\begin{array}{l}-0,261 * * \\
(-5,170)\end{array}$ \\
\hline$\stackrel{\circ}{\unrhd} \pi$ & $\begin{array}{l}-0,124 * * \\
(-3,867)\end{array}$ & $\begin{array}{l}-0,123^{* *} \\
(-3,846)\end{array}$ & $\begin{array}{l}-0,144 * * \\
(-4,608)\end{array}$ & $\begin{array}{l}-0,129 * * \\
(-4,236)\end{array}$ & - & 一 & - & - \\
\hline$\frac{0}{3}$ & $\begin{array}{c}0,274^{* *} \\
(11,311)\end{array}$ & $\begin{array}{c}0,275^{* *} \\
(11,361)\end{array}$ & $\begin{array}{c}0,193 * * \\
(10,881)\end{array}$ & $\begin{array}{c}0,194 * * \\
(11,237)\end{array}$ & $\begin{array}{c}0,082^{*} \\
(2,146)\end{array}$ & $\begin{array}{c}0,083^{*} \\
(2,153)\end{array}$ & $\begin{array}{l}0,357 * * \\
(3,704)\end{array}$ & $\begin{array}{l}0,364 * * \\
(3,766)\end{array}$ \\
\hline 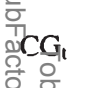 & $\begin{array}{c}-0,043 \\
(-1,421)\end{array}$ & - & $\begin{array}{c}-0,010 \\
(-0,343)\end{array}$ & - & $\begin{array}{l}-0,114^{*} \\
(-2,507)\end{array}$ & - & $\begin{array}{c}-0,038+ \\
(-1,651)\end{array}$ & - \\
\hline 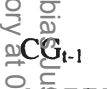 & - & $\begin{array}{c}-0,036 \\
(-1,180)\end{array}$ & - & $\begin{array}{c}-0,025 \\
(-0,834) \\
\end{array}$ & - & $\begin{array}{c}-0,079+ \\
(-1,731) \\
\end{array}$ & - & $\begin{array}{c}-0,034 \\
(-1,387) \\
\end{array}$ \\
\hline $\mathbf{R}^{2}$ & 0,84 & 0,84 & 0,94 & 0,94 & 0,91 & 0,91 & 0,84 & 0,84 \\
\hline
\end{tabular}


Ferner ist zwischen den einzelnen Ländergruppen kein großer Unterschied hinsichtlich der Koeffizienten festzustellen, weder in bezug auf deren Höhe noch in bezug auf deren Signifikanzniveau. Es ist keineswegs so, dass die besonders geschlossenen Länder überdurchschnittlich deutlich der Partisantheorie folgen.

Interessant ist darüber hinaus, dass insbesondere die letzten Zeitphasen überdurchschnittlich häufig einen signifikant von null verschiedenen Koeffizienten (mit korrektem Vorzeichen) aufweisen. Dies lässt sich vorsichtig als Widerlegung der Globalisierungsthese interpretieren, da es offenbar gerade in den besonders globalisierten Zeitphasen so etwas wie Partisaneffekte bei den Inflationsraten zu geben scheint. Dies ist aber nicht zuletzt aufgrund der jeweils relativ kleinen Stichproben der Einzelcluster nur ausgesprochen vorsichtig zu interpretieren.

Die empirischen Schätzungen zeigen, dass es bei der ökonomischen Clusterung klare Hinweise dafür gibt, dass zumindest für einige Staaten die rationale Partisantheorie angemessen zur Modellierung der Interaktion von Wirtschaft und Politik ist, dass dies aber keineswegs für alle Länder in gleichem Maße zutrifft. Auch wurde gezeigt, dass in dieser Clusterung keine Anhaltspunkte gefunden werden konnten, dass die Globalisierungsthese korrekt ist. Die Öffnung der Staaten führt demnach keineswegs dazu, dass die Existenz von Partisaneffekten zurückgeht.

\subsubsection{Cluster gemäß den politischen Indikatoren}

Da es grundsätzlich möglich ist, dass die eher unbefriedigenden Schätzergebnisse des vorherigen Abschnittes nur aufgrund der "falschen“ Clusterung zustande kamen, sollen analoge Schätzungen auf der Grundlage der politischen Clusterung erfolgen. Das Bild, das dadurch gezeichnet wird, ist ein etwas, jedoch nicht grundsätzlich, anderes. Bei der Analyse der vier Ländercluster für den gesamten Zeitraum (Tabelle 5.15) wurde zwar überwiegend das korrekte Koeffizientenvorzeichen für die CG-Variable geschätzt, jedoch kein einziges Mal auf signifikantem Niveau.

Hinsichtlich der Globalisierungsthese wird ebenfalls kein theoriebestätigendes Bild entworfen, da die absoluten t-Werte von Cluster 1 bis Cluster 4 stetig ansteigen. Dies würde bedeuten, dass die Partisaneffekte mit zunehmender Globalisierung eher an Erklärungskraft gewinnen als verlieren. Ähnlich zeigt auch die Untersuchung des Gesamtländersamples für einzelne Zeitperioden (Tabelle 5.16), dass die Zeitphasen zunehmender wirtschaftlicher Integration eher mit stärkeren als mit schwächeren Partisaneffekten in den Inflationsraten einhergehen. Auch dies ist deutlich im Widerspruch zur Globalisierungsthese. 
Tabelle 5.15 : Inflationsperformance in der Clusterung gemäß politischen Globalisierungsindikatoren 1960-1997

abhängige Variable: $\pi_{t}$

$\begin{array}{lllll} & \text { 1. Cluster } & \text { 2. Cluster } & \text { 3. Cluster } & \text { 4. Cluster }\end{array}$

Var.

(D, DK, ITA, NL, BEL, F) (UK, S, AU, NOR, SF, IRL)

(USA, CAN, CH)

(E, J, AUS, NZL)

\begin{tabular}{|c|c|c|c|c|c|c|c|c|}
\hline & (1) & (2) & (1) & (2) & (1) & (2) & (1) & (2) \\
\hline$\pi_{t-1}$ & $\begin{array}{c}1,119^{* *} \\
(34,179)\end{array}$ & $\begin{array}{c}1,119 * * \\
(34,188)\end{array}$ & $\begin{array}{c}1,072 * * \\
(33,020)\end{array}$ & $\begin{array}{c}1,073 * * \\
(33,037)\end{array}$ & $\begin{array}{c}1,308 * * \\
(29,891)\end{array}$ & $\begin{array}{c}1,309 * * \\
(29,922)\end{array}$ & $\begin{array}{c}1,158^{* * *} \\
(26,400)\end{array}$ & $\begin{array}{c}1,170^{* * *} \\
(26,461)\end{array}$ \\
\hline 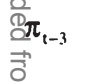 & - & - & - & - & - & - & $\begin{array}{l}-0,122 * * \\
(-2,909)\end{array}$ & $\begin{array}{c}-0,099 * \\
(-2,392)\end{array}$ \\
\hline 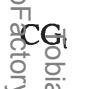 & $\begin{array}{c}0,009 \\
(0,352)\end{array}$ & - & $\begin{array}{c}-0,013 \\
(-0,516)\end{array}$ & - & $\begin{array}{c}-0,062 \\
(-1,415)\end{array}$ & - & $\begin{array}{c}-0,052 \\
(-1,479)\end{array}$ & - \\
\hline $\begin{array}{ll}0 G_{1-2} \\
\circ \\
\infty\end{array}$ & - & $\begin{array}{c}0,011 \\
(0,447)\end{array}$ & - & $\begin{array}{c}-0,006 \\
(-0,247)\end{array}$ & - & $\begin{array}{c}-0,056 \\
(-1,281)\end{array}$ & - & $\begin{array}{c}-0,048 \\
(-1,396)\end{array}$ \\
\hline$R_{0}^{2}$ & 0,95 & 0,95 & 0,94 & 0,94 & 0,96 & 0,96 & 0,95 & 0,95 \\
\hline
\end{tabular}


Tabelle 5.16 : Inflationsperformance in der Clusterung gemäß politischen Globalisierungsindikatoren (alle Länder)

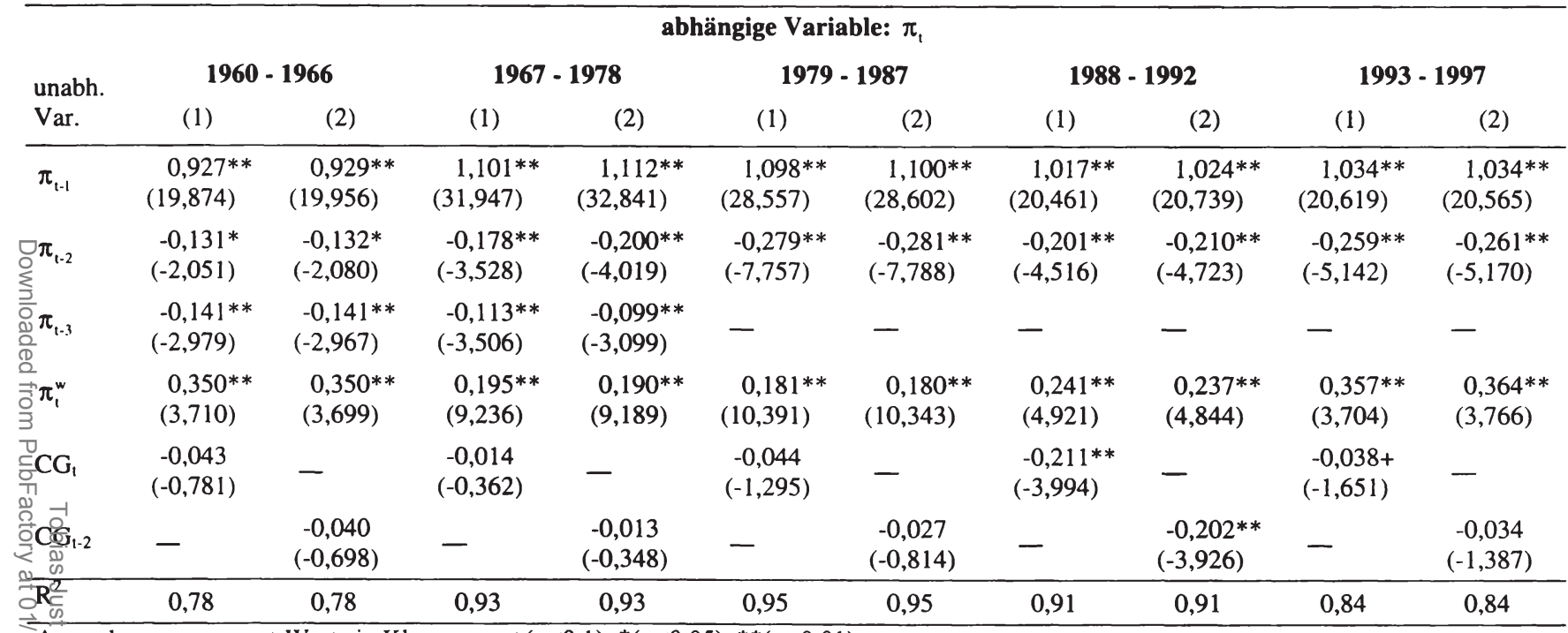

Anmerkungen: $\quad \mathrm{t}$-Werte in Klammern, $+(\alpha<0,1),{ }^{*}(\alpha<0,05),{ }^{* *}(\alpha<0,01)$. 
Interessant ist schließlich, dass der Zeitraum mit der besten statistischen Absicherung der rationalen Partisantheorie hinsichtlich der Inflation (1988-1997) nicht jenem Zeitraum der besten statistischen Absicherung hinsichtlich des Arbeitsmarktes (1967-1978) entspricht. Bei strenger Gültigkeit der rationalen Partisantheorie müsste aber erwartet werden, dass in diesem Punkte Kongruenz herrscht - gilt die rationale Partisantheorie auf dem Arbeitsmarkt, so muss sie es auch hinsichtlich der Inflationsraten tun. Ist dem nicht so, wie in diesem Fall, so kann dies entweder als Defekt der Theorie rationaler Partisaneffekte gewertet werden, oder durch starke, überlagernde Schocks verursacht worden sein. Immerhin wurden die OECD-Staaten im Zeitraum von 1967 bis 1978, in dem starke Partisaneffekte auf dem Arbeitsmarkt gefunden wurden, durch zwei Ölkrisen aus dem Gleichgewicht gebracht. Diese Ölkrisen stellten alle Regierungen, egal welcher Couleur, in hohem Maße vor das Problem steigender Preise.

Zusätzliche Details lassen sich bei der Betrachtung der Ergebnisse der Einzelregressionen sowohl nach Ländergruppen als auch nach Zeitphasen getrennt finden. ${ }^{328}$ Insgesamt überwiegt die Zahl der Koeffizienten mit einem theoriekonformen Vorzeichen. Jedoch sind erstens nicht einmal ein Drittel der Koeffizienten signifikant, und zweitens haben fast $40 \%$ der Koeffizienten das „falsche“ Vorzeichen.

Tendenziell sind die Resultate eher geeignet, einen strukturellen Unterschied der Ländercluster eins und drei aufzuzeigen. So findet die Partisantheorie im dritten Cluster mit relativ autarken Staaten die beste Bestätigung, wohingegen in dem besonders offenen ersten Cluster quasi jede Interpretation möglich ist. Zwar besteht kein linearer Zusammenhang zwischen dem Offenheitsgrad und der Güte der Schätzergebnisse (Cluster zwei und vier sind in etwa ähnlich hinsichtlich der Prüfung der Partisantheorie), mit Hinblick auf die deutlichen Ergebnisse des ersten und dritten Clusters lässt sich aber vage formulieren, dass diese Analyse eher den Schluss zulässt, dass stärker liberalisierte Länder auch besonders wenig Spielraum bei der politischen Gestaltung des Preisniveaus haben. ${ }^{329}$ Mit Hinblick auf die Ergebnisse, die bei den Gesamtsampleregressionen erzielt wurden, kann dies aber nur heißen, dass die Ergebnisse der Partisantheorie, v.a. aber bezüglich der Globalisierungsthese, offenbar nicht robust hinsichtlich der Auswahl der Länder und Zeitperioden zu sein scheinen.

Die Globalisierungsthese kann folglich durch die hier zugrunde liegenden Daten nicht hinreichend belegt werden.

${ }^{328}$ Die Details der Regressionsanalysen finden sich im Anhang C der Arbeit.

${ }^{329}$ Dies ist nicht zuletzt deswegen ausgesprochen vorsichtig zu behardeln, da ein ähnlicher Zusammenhang für den Zeitablauf nicht möglich ist; besonders in den Phasen, in denen die Märkte sich öffneten, finden sich jene Koeffizienten, die am ehesten im Einklang mit der Partisantheorie stehen. 


\subsection{Zusammenfassung der Ergebnisse bezüglich Inflation und Arbeitsmarkt}

In beiden (recht verschiedenen) Clusterungen findet die Partisantheorie der Inflationsraten nur schwache empirische Bestätigung. Dies ist weitgehend im Einklang mit den Ergebnissen von Alesina, Cohen und Roubini (1997, S. 156ff), die je nach Definition des Ideologieindikators bestenfalls auf dem 10\%-Niveau für das gesamte Ländersample Hinweise auf Partisaneffekte finden können. Berlemann (1999, S. 270ff) findet indes für ein Sample mit 20 Ländern und etwas anderem Stützzeitraum sogar auf dem 5\%-Signifikanzniveau Bestätigung für die Theorie. ${ }^{330}$ Die Ergebnisse scheinen folglich nicht sehr robust bezüglich der Länder- und Zeitraumauswahl sowie der Variablendefinition.

Auf dem Arbeitsmarkt lässt sich eher ein transitorischer als ein permanenter Effekt feststellen. Zwar gibt es auch Hinweise auf die Existenz eines traditionellen Partisanzyklus, jedoch ist es angesichts der Tatsache, dass der transitorische Effekt stärker ausgeprägt ist, plausibel, dass der permanente Effekt allein aufgrund des transitorischen Arbeitsmarkteffekts geschätzt wurde. Insbesondere bei Regressionen, die auf einem größeren Ländersample und längeren Zeitperioden beruhen, also statistisch stärker abgesichert sind, scheint die rationale Partisantheorie das bessere Modell zu sein. In Tabelle 5.17 werden alle geschätzten Koeffizienten der Ideologievariablen noch einmal schematisch verdichtet dargestellt.

Die rationale Partisantheorie impliziert, dass sowohl ein kurzfristiger Partisaneffekt auf dem Arbeitsmarkt als auch ein permanenter Preiseffekt simultan vorliegen müssen. Tabelle 5.18 zeigt daher einen Vergleich der Koeffizienten in den Regressionen zur rationalen Partisantheorie in bezug zum Arbeitsmarkt und zur Inflation. Es werden tatsächlich sehr häufig simultan theoriekonforme Koeffizienten für dieselben Sample (Art der Cluster, Ländergruppe, Zeitraum) geschätzt. Dies mag nicht überraschen, da der Zusammenhang der Phillipskurve ja gerade eine gegenläufige Entwicklung der Arbeitslosenquote und der Inflation erwarten lässt. Wenn also die rationale Partisantheorie hinsichtlich der Arbeitslosenquote und der Inflation einzeln bestätigt werden konnte, sollte daraus aufgrund des Phillipskurvenzusammenhangs auch die simultane Gültigkeit resultieren.

${ }^{330}$ Dessen Ideologievariable weicht ebenfalls von den hier verwandten Variablen leicht ab. Auch berücksichtigt Berlemann einen zusätzlichen Dummy für die zeitliche Nähe der Euro-Einführung. Dies ist in dieser Arbeit nicht notwendig, da ein Hauptaugenmerk gerade auf der Analyse der unterschiedlichen Zeitphasen lag. Wie oben gesehen, konnte dabei aber gerade nicht gezeigt werden, dass die Partisaneffekte in jenen Jahren, die besonders weit von der europäischen Währungsunion liegen.augl beşnders stask ausgepgägt sind. 
Tabelle 5.17 : Übersicht über die Koeffizienten der Ideologievariablen

\begin{tabular}{|c|c|c|c|c|c|}
\hline & & \multicolumn{2}{|c|}{ Theoriekonform } & \multicolumn{2}{|c|}{ Nicht theoriekonform } \\
\hline & & signif. $^{a)}$ & nicht signif. & nicht signif. & signif. \\
\hline \multirow{3}{*}{$\begin{array}{l}\text { Traditionelle } \\
\text { Partisantheorie } \\
\text { Arbeitsmarkt }\end{array}$} & ök. Cluster & $2(4)^{b)}$ & $9(10)$ & $3(7)$ & $-(3)$ \\
\hline & pol. Cluster & $1(8)$ & $11(15)$ & $4(6)$ & $2(3)$ \\
\hline & Gesamt & 1 & 2 & - & - \\
\hline \multirow{3}{*}{$\begin{array}{l}\text { Rationale } \\
\text { Partisantheorie } \\
\text { Arbeitsmarkt }\end{array}$} & ök. Cluster & $4(7)$ & $8(10)$ & $2(4)$ & $-(3)$ \\
\hline & pol. Cluster & $6(6)$ & $8(14)$ & $4(12)$ & $-(3)$ \\
\hline & Gesamt & 4 & - & - & - \\
\hline \multirow{3}{*}{$\begin{array}{l}\text { Partisantheorie } \\
\text { Inflation }\end{array}$} & ök. Cluster & $5(7)$ & $7(5)$ & $2(11)$ & $-(-)$ \\
\hline & pol. Cluster & $3(10)$ & $13(13)$ & $2(13)$ & $-(2)$ \\
\hline & Gesamt & - & 3 & - & - \\
\hline Anmerkungen: & $\begin{array}{l}\text { a) signifikar } \\
\text { b) Die erste } \\
\text { bellen im } \\
\text { auf die D }\end{array}$ & $\begin{array}{l}\text { ndestens } \\
\text { bezieht } \\
\text { angeg } \\
\text { ergebnis }\end{array}$ & $\begin{array}{l}\text { dem } 10 \%-\mathrm{Ni} \\
\text { auf die } \mathrm{Regr} \\
\text { sind, die } \mathrm{Za} \\
\text { Anhang C. }\end{array}$ & $\begin{array}{l}\text { onsergebniss } \\
\text { in Klamme }\end{array}$ & $\begin{array}{l}\text { in den } \\
\text { ziehen }\end{array}$ \\
\hline
\end{tabular}

Interessant ist allerdings, dass in einigen Fällen ein Zusammenhang geschätzt wurde, der sich entgegen dem Phillipskurvenverlauf verhält (mittlere Spalte). Dies könnte natürlich ein rein statistischer Zufallseffekt sein, da in der Übersicht nicht nur die signifikant von null verschiedenen Koeffizienten berücksichtigt wurden. Dann ist das konkrete Vorzeichen aber selber als ein Zufallsereignis zu interpretieren. Es ist aber auch möglich, dass expansive „linke“ Politik durch kontraktive Geldpolitik einer unabhängigen und stabilitätsorientierten Zentralbank zügig konterkariert wurde. Dann gab es zwar einen Rückgang der Arbeitslosenquote, jedoch keinen dauerhaften Anstieg der Inflation.
Tabelle 5.18 : Simultane Gültigkeit der rationalen Partisantheorie in bezug auf den Arbeitsmarkt und die Inflation

\begin{tabular}{lccc}
\hline & $\begin{array}{c}\text { Beide Koeffizienten } \\
\text { theoriekonform }\end{array}$ & $\begin{array}{c}\text { Unterschiedlicher } \\
\text { Befund }\end{array}$ & $\begin{array}{c}\text { Beide Koeffizienten } \\
\text { nicht theoriekonform }\end{array}$ \\
\hline ökonomische Cluster & $10(7)$ & $4(15)$ & $-(2)$ \\
politische Cluster & $12(14)$ & $6(14)$ & $-(8)$ \\
Gesamt & 3 & - & - \\
\hline
\end{tabular}

Anmerkungen: Es wurde nicht auf Signifikanz geachtet, sondern nur auf die Richtigkeit des geschätzten Vorzeichens.

Die Zahlen vor der Klammer beziehen sich erneut auf die Regressionen im Haupttext, die Zahlen in der Klammer nehmen Bezug auf die Regressionen im Anhang C. 
Hinsichtlich der Globalisierungshypothese konnten keineswegs so eindeutige Ergebnisse wie hinsichtlich der Partisantheorie gefunden werden. Es konnten sogar eher Hinweise für die Gültigkeit einer entgegengesetzten Globalisierungsthese isoliert werden. Insbesondere bei den Regressionen mit den politischen Clustern lässt sich eher schlussfolgern, dass in den letzten Jahren der ideologische Prägeraum hinsichtlich des Preisniveaus größer als kleiner geworden ist. Dieses Ergebnis findet sich auch bei Alesina, Cohen und Roubini (1997, S. 159ff) angedeutet. Diese setzen das Ende des Bretton Woods Systems 1973 als Scheidelinie an und zeigen, dass in der Periode von 1960-1973 keine Bestätigung für die Partisantheorie gefunden werden kann, wohl aber für den Zeitraum von 1960-1987. Ihre Interpretation ist, dass offenbar das System fester Wechselkurse wesentlich stärker restringierenden Einfluss ausgeübt hat als die internationale Marktöffnung der Folgejahre. In der Tat ist ja gerade in einem System fester Wechselkurse Geldpolitik wirkungslos. Insbesondere die Regressionsergebnisse der ökonomischen Clusterung, die ja auch im Jahr 1973 einen Schnitt machen, können diese Ergebnisse allerdings nur zum Teil bestätigen und zeigen, dass es zu einfach ist, das Jahr 1973 als Strukturbruch zu kennzeichnen, denn auch nach 1973 gab es nicht durchgängig theoriekonforme Koeffizienten.

\section{Tabelle 5.19 : Zusammenfassung der Ergebnisse hinsichtlich der Globalisierungsthese}

\begin{tabular}{|c|c|c|c|}
\hline & $\begin{array}{l}\text { Globalisierungsthese } \\
\text { tendenziell bestätigt }^{\text {a) }}\end{array}$ & $\begin{array}{l}\text { Eher Befund für entge- } \\
\text { gengerichtete These }\end{array}$ & $\begin{array}{l}\text { Keine Trendaussage } \\
\text { möglich }\end{array}$ \\
\hline $\begin{array}{l}\text { Trad. Partisantheo- } \\
\text { rie (Arbeitsmarkt) }\end{array}$ & $-(2)^{c)}$ & $5(3)$ & $1(7)$ \\
\hline $\begin{array}{l}\text { Rat. Partisantheorie } \\
\text { (Arbeitsmarkt) }\end{array}$ & $4(4)$ & $4(4)$ & $-(6)$ \\
\hline $\begin{array}{l}\text { Partisantheorie } \\
\text { (Inflation) }\end{array}$ & $2(-)$ & $6(7)$ & $-(7)$ \\
\hline $\begin{array}{ll}\text { Anmerkungen: } & \text { I } \\
& \mathrm{d} \\
& \mathrm{t} \\
& \mathrm{n} \\
& \text { b) } \mathrm{I} \\
& \mathrm{O} \\
& \mathrm{P} \\
& \text { c) } \\
& \mathrm{D} \\
& \mathrm{h}\end{array}$ & $\begin{array}{l}\text { In diese Gruppe wurden } \\
\text { dercluster oder einen Ze } \\
\text { ten erkennen ließen (sin } \\
\text { niveau, Umkehrung des } \\
\text { In diese Gruppe fallen je } \\
\text { offenen Länder oder di } \\
\text { Partisaneffekte zeigten. } \\
\text { Die Zahlen vor der Kl } \\
\text { Haupttext, die Zahlen in } \\
\text { hang C. }\end{array}$ & $\begin{array}{l}\text { ene Ergebnisse aufgenom } \\
\text { tcluster einen Trend zu g } \\
\text { ende Koeffizientenwerte } \\
\text { uvor theoriekonformen V } \\
\text { ne Ergebnisse, bei denen } \\
\text { besonders offenen Zeit } \\
\text { ammer beziehen sich au } \\
\text { den Klammern auf die De }\end{array}$ & $\begin{array}{l}\text { men, die für einen Län- } \\
\text { ringeren Partisaneffek- } \\
\text { sinkendes Signifikanz- } \\
\text { orzeichens). } \\
\text { entweder die besonders } \\
\text { hasen die deutlichsten } \\
\text { die Regressionen im } \\
\text { tailregressionen im An- }\end{array}$ \\
\hline
\end{tabular}

Würde sich diese hier nur vage zum Ausdruck kommende Vermutung in folgenden Arbeiten bestätigen, dass der Prägeraum makroökonomischer Partisanpolitik in den letzten Jahren eher größer als kleiner geworden ist, könnte man darin eine 
weitere Antwort auf die in Kapitel 4 aufgeworfene Frage finden, warum rational agierende Politiker dazu bereit sind, die globale Integration der Märkte zu forcieren.

$\mathrm{Da}$ in einigen Regressionen auch für die 90er Jahre signifikant theoriekonforme Koeffizienten geschätzt wurden, wäre es auch unzureichend, von einem grundsätzlichen Lernprozess auszugehen, der dann zu vereinheitlichter Politik geführt hätte. In Tabelle 5.19 werden die Ergebnisse hinsichtlich der Globalisierungsthese zusammengefasst. Eine klare Bestätigung für die Globalisierungsthese lässt sich nicht finden, insbesondere bei den Inflationsergebnissen scheint sogar die entgegengesetzte Hypothese eher durch die Daten gedeckt zu sein.

Zusammenfassend lässt sich sagen, dass die benutzten Indikatoren für Globalisierung die Aussage, die Integration der Güter- und Kapitalmärkte vereinheitlicht jede nationale Wirtschaftspolitik zumindest für die Aggregate Arbeitslosigkeit und Inflation nicht zulassen. Es bleibt zu prüfen, ob zumindest der budgetäre Handlungsspielraum signifikante Einschränkung durch die Marktöffnung erfuhr. Dies soll im folgenden Abschnitt geschehen.

\subsection{Budgetäre Handlungsspielräume}

In Kapitel 3 wurde argumentiert, dass man linke und rechte Politik sowohl in ihren Zielen als auch hinsichtlich des Umsetzungsmodus von Programmen unterscheiden kann. Danach sind linke Parteien nicht nur eher bereit, Umverteilungspolitik in die Richtung stärkerer Einkommens- und Vermögensegalisierung vorzunehmen, sie sind in der Vorgehensweise auch progressiver, das heißt, sie sind eher zu Veränderungen des Status quo bereit. Sind rechte Parteien grundsätzlich weniger wandlungsbereit, dann werden sie diese Änderungen der Linken nicht so schnell rückgängig machen. Dementsprechend gibt es zwei zu prüfende Hypothesen:

1. Linke Parteien stärken eher regressiv wirkende Staatsausgaben, um dadurch ihr Verteilungsziel zu erreichen (Verteilungshypothese).

2. Linke Parteien sind grundsätzlich eher zu stärkeren Strukturbrüchen innerhalb der Staatsausgaben bereit bzw. in der Lage (Umsetzungshypothese).

Im folgenden wird das verwendete Datenmaterial beschrieben. Im Anschluss daran werden die beiden Hypothesen getestet. Begonnen wird dabei mit der Prüfung der Verteilungshypothese, die eine stärkere Beachtung in der Literatur gefunden hat. Schließlich wird die Umsetzungshypothese untersucht, die bisher nicht empirisch getestet wurde. 


\subsubsection{Methodische Vorbemerkungen und Beschreibung der Daten}

Es werden die Budgetstrukturen von 18 OECD-Staaten für den Zeitraum von 1960 bis 1996 analysiert. Dabei werden die Budgetdaten der in den vorherigen Kapiteln untersuchten Staaten berücksichtigt. Der verwandte Datensatz stammt von Thomas $R$. Cusack und wurde auch schon an anderer Stelle mit einem etwas kürzeren Zeitraum benutzt (Cusack, 1991, 1992). Für Neuseeland waren keine Daten verfügbar. Der zugrunde liegende Datensatz basiert auf Jahresdaten und beinhaltet Angaben zu den folgenden Ausgabengruppen, jeweils als relative Größe zum Bruttoinlandsprodukt. ${ }^{331}$

1. Verteidigungsausgaben

2. Externe Transferausgaben (an das Ausland)

3. Interne Transferausgaben (an inländische Haushalte)

4. Zivile Konsumausgaben des Staates

5. Zinsausgaben

6. Subventionen

7. Brutto-Investitionsausgaben

Die Summe dieser Budgetpositionen wird im folgenden als gesamte Staatsausgaben bezeichnet. Als flexibler Teil der Staatsausgaben werden die Gesamtausgaben abzüglich der Verteidigungs- und der Zinsausgaben bezeichnet.

Da die Untersuchung auf cross-section Budgetdaten beruht, stellt sich das Problem, dass Vereinfachungen und Aggregationen des Datenmaterials unerlässlich werden. Erstens gilt es, ein möglichst umfassendes Haushaltskonzept für den Staat festzulegen, um zu berücksichtigen, dass in einzelnen Ländern ähnliche Güter unterschiedlich budgetiert werden. Das heißt, es müssen gegebenenfalls Parafisci berücksichtigt werden wie z.B. die Sozialversicherungen.

Zweitens ist zu beachten, dass die untersuchten Staaten unterschiedlich stark föderativ organisiert sind, ${ }^{332}$ d.h. öffentliche Güter werden in den einzelnen Ländern nicht per se auch auf derselben Haushaltsebene erfasst. Um etwaige Verzerrungen zu vermeiden, die ausschließlich aufgrund einer unterschiedlichen

${ }^{331}$ Für eine detaillierte Beschreibung des Datensatzes vgl. Cusack (1991). Die aktualisierten Daten sind unter der Homepage von T.R. Cusack auf der Internetadresse http://www.wzberlin.de/wb/ herunterladbar (Oktober 2000).

$332 \mathrm{Vgl}$. Lijphart (1984) für einen Vergleich des föderativen,Elęments.in_QE,CB-Stgaten. 
Erfassung von Ausgaben entstehen, ist es nötig, das Aggregat aller staatlichen Haushalte zu betrachten. ${ }^{333}$

Drittens muss berücksichtigt werden, dass es über den gesamten Untersuchungszeitraum keine international gültige Haushaltssystematik gibt, sodass die einzelnen Ausgabenpositionen uneinheitlich bezeichnet sind und zum Teil unterschiedliche Einzelausgaben umfassen. Diese Schwierigkeiten der Vergleichbarkeit einzelner Ausgabenpositionen zwingt zu einer höheren Aggregationsebene in Form eines kleinsten gemeinsamen Nenners.

Möchte man, wie dies hier der Fall ist, zudem eine Untersuchung in Zeitdimensionen durchführen, werden die Probleme der Vergleichbarkeit noch größer, da selbst auf nationaler Ebene starke Brüche in der Verbuchung von Ausgaben existieren.

\subsubsection{Verteilungsziele als ideologisches Trennkriterium}

Die Synopse der Inzidenzanalysen einzelner Budgetpositionen in Kapitel 3.4.3 hat gezeigt, dass eine regressiv verteilungsorientierte Haushaltspolitik v.a. in den Ausgabengruppen soziale Sicherung und Subventionen sichtbar werden sollte, da diese Positionen die deutlichsten Verteilungswirkungen haben. Übersetzt in die Ausgabenkategorien des hier verwendeten Datensatzes bedeutet dies, dass insbesondere die Kategorien Transfers und Subventionen unter linken Regierungen stärker wachsen müssten als bei rechten Regierungen.

Bei den staatlichen Konsumausgaben (hier zivile Konsumausgaben) ist eine grundsätzliche Tendenzvermutung schwierig. Einerseits ist zu erwarten, dass rechte Parteien jene Konsumausgaben, die in die Gruppen Außenpolitik, innere Sicherheit, Umwelt, Wissenschaft und Hochschule fallen, zumindest nicht weniger stark ausbauen als linke Parteien. Andererseits werden idealtypische linke Parteien die Konsumausgaben in den Ausgabengruppen Verkehr und Erzie-

${ }^{333}$ Dies ist indes auch kein Königsweg, da in stark föderativ organisierten Staaten durchaus die Situation entstehen kann, dass einer linken Regierung auf der Bundesebene überwiegend rechte Regierungen auf der Ebene der Bundesländer gegenüberstehen. Haben beide Ebenen unterschiedliche Präferenzen hinsichtlich der Ausdehnung des Staatsanteils, wird der Nettoeffekt davon abhängen, welches Gewicht das föderative Element hat. Da die Ideologievariable in dieser Arbeit aber allein aus der Regierungskoalition auf Bundesebene errechnet wird, bleibt der föderative Ideologieaspekt unberücksichtigt. Um diese Verzerrung zu vermeiden, wären detaillierte Informationen nötig, welches Gewicht die föderativen Ebenen am Gesamthaushalt einnehmen, und wie die ideologische Ausrichtung der unteren Körperschaften verteilt sind. Die verwendeten Budgetdaten bieten leider keine Information über die genaue Aufteilung der einzelnen Ausgabenpositionen, sodass es unmöglich ist, die Verzerrung aufgrund der Nichtbeachtung der ideologischen Tendenz der Bundesländer zu vermeiden. 
hung/Bildung stärken. Der Nettoeffekt auf das Aggregat „zivile Konsumausgaben" ist also ex ante offen.

Insgesamt werden staatlichen Ausgaben insgesamt eher regressive Verteilungswirkungen attribuiert, sodass zu erwarten ist, dass Zuwächse im staatlichen Gesamtbudget eher in die Legislaturperioden linker Regierungen fallen werden. Anhand des oben beschriebenen Datensatzes sollen diese Einzelhypothesen eingehend untersucht werden.

\subsubsection{Das Modell}

In den folgenden Modellen werden insgesamt fünf endogene Variablen untersucht:

- Veränderung des Anteils der staatlichen Gesamtausgaben am Bruttoinlandsprodukt einer Periode (D_GESAUS).

- Veränderung des Anteils der flexiblen Gesamtausgaben am Bruttoinlandsprodukt einer Periode; dabei sind die flexiblen Gesamtausgaben durch die Gesamtausgaben abzüglich der Militär- und Zinsausgaben operationalisiert. Diese werden herausgerechnet, da zum einen die Militärausgaben zu großen Teilen die internationale Krisenanfälligkeit widerspiegeln und daher für die Regierungen exogene Größen darstellen. Zum anderen hängen die Zinsausgaben maßgeblich vom jeweiligen Zinssatz und dem Schuldenstand ab. Da der Schuldenstand Ausdruck vergangener Verschuldungsneigung ist und der Zinssatz gerade bei offenen Kapitalmärkten kaum durch Regierungspolitik verändert werden kann, haben auch die Zinsausgaben zu jedem Zeitpunkt weitgehend Datumscharakter (D_FLEX). ${ }^{334}$

- Veränderung des Anteils der Transferausgaben am Bruttoinlandsprodukt einer Periode (D_TRANS).

- Veränderung des Anteils der Subventionsausgaben am Bruttoinlandsprodukt einer Periode (D_SUBVENT).

- Veränderung des Anteils der Ausgaben für zivilen Staatskonsum am Bruttoinlandsprodukt einer Periode (D_ZIV).

Als erklärende Variablen werden in der Literatur einige wichtige Prädiktoren diskutiert. Ausgehend von Wagners Gesetz des wachsenden Staatsanteils (Wag-

${ }^{334}$ Natürlich ließe sich die tatsächliche Variationsmasse bei einer detaillierten Analyse von Einzelhaushalten noch besser in kurzfristig disponible und kurzfristig nicht-disponible Größen trennen. Für eine gleichzeitige cross-section und time-series Analyse diesen Umfangs scheitert dies aber an der mangelnden Datenyerfügharkei.78-3-631-75139-8 
ner, 1893) lässt sich argumentieren, dass wirtschaftliches Wachstum $\left(W_{I W A_{t}}\right)$ bei einer positiven Einkommenselastizität der Nachfrage nach staatlich bereitgestellten Gütern größer eins zu überproportionalem Wachstum der Gesamtausgaben des Staates führt. Andererseits bedeutet Wirtschaftswachstum auch, dass die Höhe der Transferzahlungen zurückgeht. Beide entgegengerichteten Faktoren lassen die Nettowirkung des Wirtschaftswachstums auf die Höhe der Änderung des Budgets ungewiss werden.

Ferner wird eine Variable der Überraschung $\left(\mathrm{UEB}_{\mathrm{t}}\right)$ verwendet. Geht man davon aus, dass der Staatshaushalt in der Erwartung einer bestimmten wirtschaftlichen Zukunftslage der Wirtschaft aufgestellt wird, so muss jede unerwartete Änderung dieser Zukunftslage Rückwirkungen auf die Änderung des Anteils der Staatsausgaben haben. In Anlehnung an Roubini und Sachs (1989) sowie $\mathrm{Cu}$ sack (1997a) wird die Variable für Überraschungswachstum als die Differenz aus der tatsächlichen Wachstumsrate und dem arithmetischen Mittelwert der letzten drei Perioden errechnet. ${ }^{335}$ Ist $\mathrm{UEB}_{\mathfrak{t}}$ positiv, wächst die Wirtschaft schneller als bei der Budgetplanung erwartet wurde, daher sollte das Vorzeichen von $\mathrm{UEB}_{\mathrm{t}}$ negativ sein.

Des weiteren wird die Veränderung der Arbeitslosenquote $\left(D_{-} U_{t}\right)$ als Variable benutzt, um jene Ausgabenänderungen zu erklären, die nicht auf unterschiedliche Generosität der Regierung zurückzuführen sind, sondern durch die Konjunkturlage erzwungen werden. ${ }^{336}$

Außerdem findet eine Maastricht-Dummyvariable (MAAST ${ }_{t}$ ) Verwendung. Sie nimmt für jene Länder, die den Maastrichter Vertrag unterzeichneten, für die Jahre nach 1992 den Wert eins an, ansonsten den Wert null. Dies soll den Einfluss der Marktöffnung vom fiskalischen Konvergenzprozess der Euro-Länder trennen. Es ist anzunehmen, dass dieser Konvergenzprozess eher zu Ausgabenkürzungen führte, um v.a. die Haushaltskriterien zu erfüllen.

Als erste zentrale Variable wird das center of gravity $\left(\mathrm{CG}_{\mathrm{t}}\right)$ als Ideologievariable benutzt. Diese Variable impliziert, dass in jedem Jahr einer Regierungszeit

335 Da negative Wachstumsraten im verwendeten Zeitraum der OECD-Länder keine Seltenheit waren, wurde statt des eigentlich theoretisch korrekten geometrischen Mittelwertes das arithmetische Mittel verwendet. Außerdem ist zur direkten Vergleichbarkeit mit den anderen Studien darauf hinzuweisen, dass die Überraschungsvariable hier umgekehrt definiert wurde: Cusack (1997a) subtrahiert die aktuelle Wachstumsrate vom Mittelwert der letzten drei Jahre. Seine geschätzten Koeffizienten haben daher ein umgekehrtes Vorzeichen.

${ }^{336}$ Es lässt sich argumentieren, dass in den Fällen, in denen die Partisantheorie für den Arbeitsmarkt Bestätigung findet, hier natürlich leichte Multikollinearität zwischen den beiden Variablen Ideologie und Veränderung der Arbeitslosenquote besteht. Da der Partisanzusammenhang aber nicht sehr ausgeprägt ist und bestenfalls einen kleinen Teil der Ände-

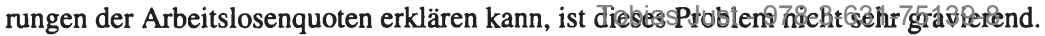


(selbst nach der Wiederwahl) eine vergleichbar intensive ideologische Ausgabenpolitik betrieben wird. Es ist aber auch plausibel, dass v.a. nach einem Regierungswechsel Budgetanpassungen solange stattfinden, bis die neue, ideologisch motivierte Ausgabenstruktur und -höhe erreicht ist, und dass danach deutlich geringere Budgetanpassungen stattfinden. Daher wird eine zweite Ideologievariable RCG4, parallel getestet. Diese ist analog zu der Variable zur Prüfung der rationalen Partisantheorie konzipiert: $R C G 4_{t}=C_{t}-C G_{t-4}$. Ein Regierungswechsel nach links führt dann in den ersten vier Perioden zu einem negativen Wert für RCG4 einem positiven Wert. ${ }^{337}$ Ein negatives Koeffizientenvorzeichen bedeutet folglich, dass linke Regierungen tendenziell höhere Änderungen des Ausgabenanteils in den ersten (vier) Jahren ihrer Regierungszeit realisieren als rechte Regierungen.

Schließlich wird eine Trendvariable (TREND) berücksichtigt. Geht man davon aus, dass es auch in der Summe aller öffentlichen Güter abnehmende Grenznutzen gibt, so ist damit zu rechnen, dass die Ausgabenzuwächse tendenziell abnehmend sind. Das Vorzeichen der TREND-Variable wäre dann negativ.

Die Schätzmethode ist erneut eine Panelregression mit fixed effects, die eventuelle Länderspezifika auffangen sollen. Dabei sind folgende Modelle zu schätzen: ${ }^{338}$

$$
\begin{aligned}
\text { D_GESAUS }_{i, t}= & \alpha_{i}+\beta_{1} \cdot \text { TREND }+\beta_{2} \cdot \text { UEB }_{i, t}+\beta_{3} \cdot D_{-} U_{i, t}+\beta_{4} \cdot \text { MAAST }_{i, t} \\
& +\beta_{5} \cdot \text { WIWA }_{i, t}+\gamma_{1} \cdot \text { CG }_{i, t}+\varepsilon_{i, t} \\
& \text { bzw. }
\end{aligned}
$$

$$
\begin{aligned}
\text { D_GESAUS }_{i, \mathrm{t}} & =\alpha_{\mathrm{i}}+\beta_{1} \cdot \text { TREND } \beta_{2} \cdot \mathrm{UEB}_{\mathrm{i}, \mathrm{t}}+\beta_{3} \cdot \mathrm{D}_{-} \mathrm{U}_{\mathrm{i}, \mathrm{t}}+\beta_{4} \cdot \mathrm{MAAST}_{\mathrm{i}, \mathrm{t}} \\
& +\beta_{5} \cdot \mathrm{WIWA}_{\mathrm{i}, \mathrm{t}}+\gamma_{2} \cdot \mathrm{RCG}_{\mathrm{i}, \mathrm{t}}+\varepsilon_{\mathrm{i}, \mathrm{t}}
\end{aligned}
$$

${ }^{337}$ Es wird im folgenden oftmals auch die Variable RCG2, verwendet. Diese bedeutet, dass der Anpassungsproze $B$ schneller abläuft und ist definiert als: $R C G 2_{t}=C G_{t}-C_{t-2}$. RCGVariablen mit längeren Wirkungsintervallen führten i.d.R. zu schlechteren Schätzergebnissen.

338 Statt GES_AUS ${ }_{\mathrm{i}, \mathrm{t}}$ können natürlich alle anderen endogenen Variablen in Gleichung (5.4) eingesetzt werden. In jenen Fällen, in denen einzelne Regressoren keine oder vernachlässigbar geringe Erklärungskraft besaßen, wurden diese Variablen, falls sie nicht für die Hypothesenprüfung zentral waren, aus der Regressionsgleichung entfemnt.75139-8 
Für die verwendeten Regressoren gelten dabei die folgenden Definitionen:

\begin{tabular}{|c|c|c|}
\hline Name & Definition & $\begin{array}{l}\text { Vorzeichen- } \\
\text { erwartung }\end{array}$ \\
\hline TREND & Trendvariable & $\beta_{1}<0$ \\
\hline WIWA $_{\mathrm{i}, \mathrm{t}}$ & $\begin{array}{l}\text { Wachstumsrate des realen Bruttoinlands- } \\
\text { produkts }\end{array}$ & $\beta_{2}=?$ \\
\hline $\mathrm{UEB}_{\mathrm{i}, \mathrm{t}}$ & $\mathrm{UEB}_{\mathrm{i}, \mathrm{t}}=\mathrm{WIWA}_{\mathrm{i}, \mathrm{t}}-\frac{1}{3} \sum_{\mathrm{k}=1}^{3} \mathrm{WIWA}_{\mathrm{i}, \mathrm{t}-\mathrm{k}}$ & $\beta_{3}<0$ \\
\hline D_U $U_{i, t}$ & Erste Veränderung der Arbeitslosenquote & $\beta_{4}>0$ \\
\hline MAAST $_{i, t}$ & Maastricht-Dummyvariable & $\beta_{5}<0$ \\
\hline $\mathrm{CG}_{\mathrm{i}, \mathrm{t}}$ & Center of gravity & $\gamma_{1}<0$ \\
\hline $\mathrm{RCG}_{\mathrm{i}, \mathrm{t}}$ & $\mathrm{RCG}_{\mathrm{i}, \mathrm{t}}=\mathrm{CG}_{\mathrm{i}, \mathrm{t}}-\mathrm{CG}_{\mathrm{i}, \mathrm{t}-4}$ & \\
\hline $\mathrm{RCG} 2_{\mathrm{i}, \mathrm{t}}$ & $R C G 2_{i, t}=C G_{i, t}-C G_{i, t-2}$ & $\gamma_{2}<0$ \\
\hline
\end{tabular}

\subsubsection{Schätzergebnisse}

Zunächst werden die Veränderungen der Aggregatgrößen D_GESAUS und D_FLEX für das gesamte Ländersample und den gesamten Untersuchungszeitraum von 1960 bis 1996 analysiert. Dabei werden für jede der beiden abhängigen Variablen zwei Modelle geschätzt. In Modell (1) wird jeweils die $\mathrm{CG}_{t^{-}}$ Variable zur Erfassung des Ideologieeinflusses verwendet und in Modell (2) wird die Modellvariante mit nur kurzzeitiger Wirkung auf das Budgetwachstum, abgebildet durch RCG4 $4_{\mathrm{t}}$, erfasst. Die konkreten Schätzergebnisse sind in Tabelle 5.20 zusammengefasst. ${ }^{339}$

Insgesamt ist die Erklärung der Veränderung der Haushaltspositionen für ein solch komplexes Phänomen wie den gesamtstaatlichen Haushalt mit einem adjustierten $R^{2}$ von 0,36 bis 0,40 relativ gut. ${ }^{340}$ Auch weisen die meisten Koeffi-

${ }^{339}$ Es wurde auch mit unterschiedlichen Lags in mehreren exogenen Variablen $\left(\mathrm{CG}_{\mathrm{t}}, \mathrm{RCG} 4_{\mathrm{t}}\right.$, WIWA $_{t}$ ) experimentiert sowie mit anderen transitorischen RCG-Effekten (RCG2 $2_{t}$ und

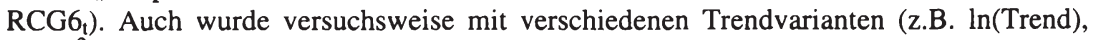
Trend $^{2}$ ) gearbeitet. Bei keiner dieser Modellveränderungen konnten jedoch höhere $t$-Werte und/oder $\mathrm{R}^{2}$-Werte erreicht werden als bei den dargestellten Modellen in Tabelle 5.20. Daher werden diese Regressionen nicht dargestellt.

${ }^{340}$ Da hier der Einfluss der Ideologievariable im Zentrum der Untersuchung steht und nicht die möglichst vollständige Erklärung der Budgetveränderung ist es zu vertreten, dass wahrscheinlich bedeutsame Variablen wie z.B. die relative Preisentwicklung von Trans- 
zienten auf signifikantem Niveau das prognostizierte Vorzeichen auf. Dabei hat erwartungsgemäß die $D_{-} U_{t}-$ Variable in allen Modellen den stärksten Einfluss. Interessant ist auch der hohe Koeffizientenwert der MAAST ${ }_{t}$-Dummyvariable. Da dieser Effekt ausschließlich in den D_GESAUS-Modellen, nicht aber in den D_FLEX-Modellen ein signifikantes Niveau erreicht, ist zu schließen, dass die Vorbereitungen zur europäischen Währungsunion insbesondere zu Reduktionen in den Zinsausgaben führten. Angesichts der Konvergenzkriterien, dort v.a. der Konvergenz der langfristigen Nominalzinsen auf niedrigem Niveau, ist dies nicht überraschend (Art. 109j (1) des EG-Vertrages i.V.m. Art. 4 des Protokolls über die Konvergenzkriterien nach Art. 109j des Vertrags zur Gründung der Europäischen Gemeinschaft). Ferner war in diesem Zeitraum das Weltzinsniveau relativ niedrig. Zwar könnte argumentiert werden, dass die EU im Sinne eines großen Landes dieses Weltzinsniveau nicht nur als exogenen Parameter zu akzeptieren hat, vernachlässigt werden darf die Wirkung des Rests der Welt auf das europäische Zinsniveau jedoch keineswegs. Außerdem hat durch die Abkühlung des Ost-West-Konflikts zu Beginn der 90er Jahre die militärische Bedrohung stark abgenommen. Dieser Effekt ist ebenfalls in der MAAST-Dummy enthalten, hat aber natürlich nichts mit der europäischen Integration zu tun.

Darüber hinaus ist die $\mathrm{UEB}_{\mathrm{t}}-\mathrm{V}$ ariable hoch signifikant. Die Haushaltsprozeduren sind offensichtlich nicht so flexibel, dass nicht erwartbares Wachstum schnell in höhere Ausgaben übersetzt werden kann. Ferner ist ein ökonomisch schwacher, jedoch statistisch signifikanter Trend zu niedrigeren Veränderungen in den Ausgabenaggregaten festzustellen. Der Einfluss des Wirtschaftswachstums ist in der Tat ungewiss, eine deutliche Bestätigung der Wagner-Hypothese ist aber auf jeden Fall nicht zu finden.

Von besonderem Interesse sind hier die Ideologievariablen. In allen vier Modellen haben die Koeffizienten das erwartete Vorzeichen. Linke Regierungsparteien führen tendenziell zu einem stärkeren Anstieg des Ausgabenanteils als rechte Parteien. Es ist jedoch bemerkenswert, dass nur die RCG4,-Variablen in den Modellen (2), nicht jedoch die allgemeine $\mathrm{CG}_{t}$-Variable in den Modellen (1) statistisch signifikant sind. Das bedeutet, dass linke Parteien zwar insgesamt die Staatsausgaben stärker ausweiten, dies jedoch v.a. in den ersten vier Jahren einer Regierungszeit tun. Das kann bedeuten, dass die Parteien tatsächlich ein konkretes Haushaltsziel erreichen möchten und nach dem Erreichen des Zieles wenig Veranlassung sehen, davon weiter abzuweichen.

Die Tatsache, dass die Partisaneffekte in den Modellen zur Erklärung von D_FLEX statistisch besser abgesicherte Werte erbringen, kann als Ausdruck der offensichtlich korrekten Annahme gewertet werden, dass die Militär- und die

ferausgaben und die Veränderung der Altersstruktur der Bevölkerung unberücksichtigt blieben. Zwar könnte dadurch wahrscheinlich das Bestimmtheitsmaß steigen, jedoch ist nicht mit einer absenkenden Wirkung auf die Ideplogiesvariableyzg rechner.5139-8 
Zinsausgaben weitgehend exogenen Charakter haben und folglich nicht systematisch durch ideologische Motive determiniert werden. In folgenden Analysen wird daher auf eine weitere Betrachtung von D_GESAUS verzichtet. ${ }^{341}$

Tabelle 5.20 : Budgetäre Partisaneffekte im Gesamtpanel 1960-1996

\begin{tabular}{|c|c|c|c|c|}
\hline \multirow[b]{2}{*}{$\begin{array}{l}\text { unabhängige } \\
\text { Variable }\end{array}$} & \multicolumn{2}{|c|}{$\begin{array}{c}\text { abhängige Variable: } \\
\text { D_GESAUS }\end{array}$} & \multicolumn{2}{|c|}{$\begin{array}{c}\text { abhängige Variable: } \\
\text { D_FLEX }\end{array}$} \\
\hline & (1) & (2) & (1) & (2) \\
\hline TREND & $\begin{array}{c}-0,012+ \\
(-1,754)\end{array}$ & $\begin{array}{c}-0,023 * \\
(-2,486)\end{array}$ & $\begin{array}{c}-0,014 * \\
(-2,183)\end{array}$ & $\begin{array}{l}-0,013 \\
(-1,532)\end{array}$ \\
\hline $\mathrm{UEB}_{\mathrm{t}}$ & $\begin{array}{l}-0,171 * * \\
(-9,863)\end{array}$ & $\begin{array}{l}-0,138^{* *} \\
(-4,852)\end{array}$ & $\begin{array}{c}-0,164 * * \\
(-10,228)\end{array}$ & $\begin{array}{l}-0,174 * * \\
(-6,580)\end{array}$ \\
\hline$D_{-} U_{t}$ & $\begin{array}{l}0,590 * * \\
(9,202)\end{array}$ & $\begin{array}{l}0,532 * * \\
(7,059)\end{array}$ & $\begin{array}{l}0,458^{* *} \\
(7,722)\end{array}$ & $\begin{array}{l}0,475^{* *} \\
(6,806)\end{array}$ \\
\hline MAAST $_{1}$ & $\begin{array}{l}-0,546^{*} \\
(-2,396)\end{array}$ & $\begin{array}{c}-0,475^{*} \\
(-2,051)\end{array}$ & $\begin{array}{c}-0,346 \\
(-1,639)\end{array}$ & $\begin{array}{c}-0,337 \\
(-1,570)\end{array}$ \\
\hline $\mathrm{CG}_{\mathrm{t}}$ & $\begin{array}{c}-0,056 \\
(-1,313)\end{array}$ & - & $\begin{array}{c}-0,058 \\
(-1,470)\end{array}$ & - \\
\hline $\mathrm{RCG}_{\mathrm{t}}$ & - & $\begin{array}{c}-0,060+ \\
(-1,835)\end{array}$ & - & $\begin{array}{c}-0,071^{*} \\
(-2,371)\end{array}$ \\
\hline WIWA $_{t}$ & - & $\begin{array}{c}-0,059 \\
(-1,582)\end{array}$ & - & $\begin{array}{c}0,012 \\
(0,338)\end{array}$ \\
\hline $\mathbf{R}_{\text {adj. }}^{2}$ & 0,40 & 0,40 & 0,36 & 0,37 \\
\hline
\end{tabular}

Es soll nun untersucht werden, welche Ausgabengruppen besonders gut durch die Ideologievariablen erklärt werden können. Gemäß der Annahme, linke Regierungsparteien würden vornehmlich regressiv verteilungswirksame Ausgaben stärken, ist zu erwarten, dass v.a. die Transferzahlungen und Subventionen überproportional in Zeiten linker Regierungen ansteigen. Dafür werden ähnliche Regressionsgleichungen wie zuvor für die drei endogenen Variablen D_TRANS, D_SUBVENT und D_ZIV für das gesamte Ländersample und den Zeitraum von 1960 bis 1996 gebildet. Die Ergebnisse sind in Tabelle $5.21 \mathrm{zu}$ sammengefasst und zum Teil überraschend.

Erwartungsgemäß reagieren die Transferausgaben am stärksten auf Änderungen der Arbeitslosenquote und auf unerwartete konjunkturelle Entwicklungen. Interessant ist, dass besonders in den Euro-Staaten seit 1992 die Transferzahlungen

${ }^{341}$ Auch in den geschätzten Modellen mit kleineren Länder- und Zeitsamples lieferte die Variable D_FLEX i.d.R. bessere Werte als die D_GESALS-Vlatiable8-3-631-75139-8 
stärker zurückgehen als in den übrigen Ländern; die MAAST ${ }_{1}$-Variable ist signifikant kleiner null. Offenbar wirkten sich hier die fiskalischen Konvergenzkriterien ebenfalls aus. ${ }^{342}$ Unerwartet ist indes, dass keine der beiden Ideologievariablen einen signifikanten Einfluss auf die Änderungen des Anteils der Transferausgaben hat. Der Koeffizient der $\mathrm{CG}_{\mathrm{t}}$-Variable hat sogar das nichttheoriekonforme, positive Vorzeichen. Demnach würden über die gesamte Amtszeit von Regierungen gerechnet rechte Parteien sogar eher Transferausgabenzuwächse realisieren als linke Regierungen, zumindest in Relation zur Entwicklung des BIP. Da Transferleistungen für den Wähler direkt spürbar sind, ist es plausibel, dass rechte Parteien in diesem Bereich nur geringen Handlungsraum haben, falls sie Wahlen gewinnen möchten.

\section{Tabelle 5.21 : Partisaneffekte einzelner Budgetteile im Gesamtpanel 1960-1996}

\begin{tabular}{|c|c|c|c|c|c|c|}
\hline \multirow[b]{2}{*}{$\begin{array}{l}\text { unabhängige } \\
\text { Variable }\end{array}$} & \multicolumn{2}{|c|}{$\begin{array}{c}\text { abhängige Variable: } \\
\text { D_TRANS }\end{array}$} & \multicolumn{2}{|c|}{$\begin{array}{c}\text { abhängige Variable: } \\
\text { D_SUBVENT }\end{array}$} & \multicolumn{2}{|c|}{$\begin{array}{c}\text { abhängige Variable: } \\
\text { D_ZIV }\end{array}$} \\
\hline & (1) & (2) & (1) & (2) & (1) & (2) \\
\hline TREND & $\begin{array}{c}0,002 \\
(0,504)\end{array}$ & $\begin{array}{c}0,003 \\
(0,555)\end{array}$ & $\begin{array}{l}-0,004 * * \\
(-2,643)\end{array}$ & $\begin{array}{l}-0,005^{* *} \\
(-2,671)\end{array}$ & $\begin{array}{l}-0,012^{* *} \\
(-4,461)\end{array}$ & $\begin{array}{l}-0,013 * * \\
(-4,528)\end{array}$ \\
\hline $\mathrm{UEB}_{\mathrm{t}}$ & $\begin{array}{l}-0,047 * * \\
(-3,137)\end{array}$ & $\begin{array}{l}-0,049 * * \\
(-3,153)\end{array}$ & $\begin{array}{l}-0,015^{* *} \\
(-3,211)\end{array}$ & $\begin{array}{l}-0,015^{* *} \\
(-3,205)\end{array}$ & $\begin{array}{l}-0,071 * * \\
(-10,286)\end{array}$ & $\begin{array}{l}-0,072 * * \\
(-10,188)\end{array}$ \\
\hline D_Ut & $\begin{array}{c}0,415^{* *} \\
(10,236)\end{array}$ & $\begin{array}{c}0,418^{* *} \\
(10,183)\end{array}$ & $\begin{array}{c}0,001 \\
(0,073)\end{array}$ & $\begin{array}{c}0,000 \\
(0,019)\end{array}$ & $\begin{array}{l}0,105^{* *} \\
(4,109)\end{array}$ & $\begin{array}{l}0,109 * * \\
(4,172)\end{array}$ \\
\hline MAAST $_{1}$ & $\begin{array}{l}-0,264^{*} \\
(-2,114)\end{array}$ & $\begin{array}{r}-0,263^{*} \\
(-2,085)\end{array}$ & - & - & $\begin{array}{c}0,038 \\
(0,422)\end{array}$ & $\begin{array}{c}0,050 \\
(0,540)\end{array}$ \\
\hline $\mathrm{CG}_{1}$ & $\begin{array}{c}0,012 \\
(0,512)\end{array}$ & - & $\begin{array}{c}0,001 \\
(0,108)\end{array}$ & - & $\begin{array}{l}-0,040^{*} \\
(-2,387)\end{array}$ & - \\
\hline RCG4, & - & $\begin{array}{c}-0,009 \\
(-0,530)\end{array}$ & - & $\begin{array}{c}-0,004 \\
(-0,444)\end{array}$ & - & $\begin{array}{c}-0,032^{*} \\
(-2,433)\end{array}$ \\
\hline WIWA & $\begin{array}{c}-0,011 \\
(-0,579)\end{array}$ & $\begin{array}{c}-0,011 \\
(-0,532)\end{array}$ & - & - & - & - \\
\hline $\mathbf{R}_{\text {adj. }}^{2}$ & 0,36 & 0,36 & 0,02 & 0,02 & 0,31 & 0,31 \\
\hline
\end{tabular}

Anmerkungen: $\quad t$-Werte in Klammern, $+(\alpha<0,1),{ }^{*}(\alpha<0,05),{ }^{* *}(\alpha<0,01)$.

Ein ebenfalls nicht uninteressantes Bild zeichnen die Regressionen zur Erklärung der Änderungen des Anteils der Subventionsausgaben. Auch hier ist kein Zusammenhang zwischen ideologischer Ausrichtung der Regierung und der Hö-

${ }^{342}$ Vgl. Art. 109j i.V.m. Art. c II EG-Vertrag und i.V.m. Art. 1 Protokoll über das Verfahren bei einem übermäßigen Defizit. 
he der Änderung des Subventionsausgabenanteils zu erkennen. Darüber hinaus gibt es offensichtlich klare Planungen für die Entwicklung der Subventionszahlungen, denn im Falle von Überraschungswachstum sinken die Änderungen des Subventionsausgabenanteils. Allerdings scheint ein Großteil der Subventionen nicht auf Änderungen der Arbeitsmarktdaten zu reagieren. Schließlich ist der Wert des adjustierten $\mathrm{R}^{2}$ verschwindend gering. Es kann also geschlussfolgert werden, dass die Subventionsleistungen anderen Gesetzmäßigkeiten unterworfen sind als die übrigen Ausgabengruppen. Im folgenden wird daher auch auf eine weitere Berücksichtigung der Subventionen in den Analysen verzichtet. ${ }^{343}$

Im Falle der zivilen Konsumausgaben verhalten sich die Koeffizienten im wesentlichen erwartungsgemäß. Es gibt einen deutlich negativen Trend im Anstieg der zivilen Konsumausgabenanteile, und diese reagieren statistisch signifikant auf Konjunkturschwankungen. Am interessantesten für diese Arbeit jedoch ist, dass sowohl die $\mathrm{CG}_{\mathrm{t}}$-Variable als auch die $\mathrm{RCG} 4 \mathrm{t}$-Variable auf dem 5\%Signifikanzniveau anzeigt, dass ein Wechsel von einer rechten zu einer linken Regierung zu einem Anstieg der zivilen Konsumausgaben in Relation zum BIP führt. Der ideologische Einfluss auf die Änderung der Staatsquote wird also v.a. durch das unterschiedliches Ausgabenverhalten in der Gruppe der zivilen Konsumausgaben bestimmt.

Es soll im folgenden geprüft werden, inwiefern dieser Zusammenhang in den einzelnen Phasen der Globalisierung bzw. in den unterschiedlich offenen Staaten unterschiedlich ausgeprägt ist, um daraus Rückschlüsse zu ziehen, ob die internationale Öffnung eines Landes tatsächlich den Prägeraum der nationalen Regierungen einengt.

\subsection{Cluster gemäß den ökonomischen Indikatoren}

In der ökonomischen Clusterung soll zunächst geprüft werden, ob es einen systematischen Unterschied zwischen den besonders offenen und den besonders geschlossenen Ländern gibt. Tabelle 5.22 gibt einen Überblick über je drei Regressionen für jeden der drei ökonomischen Ländercluster. Es wird versucht, die drei endogenen Variablen D_FLEX, D_TRANS und D_ZIV durch die oben eingeführten Variablen zu erklären.

Die konjunkturreagiblen Variablen $\left(\mathrm{UEB}_{\mathrm{t}}\right.$ und $\left.\mathrm{D}_{-} \mathrm{U}_{\mathrm{t}}\right)$ verhalten sich in allen drei Clustern gemäß den Erwartungen und haben hoch signifikante Koeffizienten. Allerdings sind im ersten Cluster die Koeffizientenwerte deutlich höher. Dies bedeutet, dass ein plötzlicher Aufschwung in den offenen Ländern nicht so schnell in Ausgabenänderungen übersetzt werden kann und dass ein Anstieg der

${ }^{343}$ Auch in Detailregressionen wurde für die Subventionsausgaben kein anderes Ergebnis gefunden. 
Arbeitslosigkeit in höherem Maße zu Ausgabensteigerungen führt. Ein zweiter wichtiger Unterschied ist offensichtlich. In dem ersten Cluster ist quasi kein Einfluss der Ideologievariable feststellbar. Zwar haben in allen drei Regressionen die Koeffizienten das korrekte Vorzeichen, jedoch kein Koeffizient ist signifikant von null verschieden. ${ }^{344}$ Im zweiten und dritten Cluster sind die Koeffizienten sowohl für die flexiblen Gesamtausgaben als auch für die zivilen Konsumausgaben wenigstens auf dem 10\%-Niveau signifikant, wobei im dritten Cluster die statistische Sicherheit der Schätzung etwas höher ausfällt. Dies könnte insgesamt so interpretiert werden, dass in jenen Ländern, die intensiv Außenhandel betreiben und in hohem Maße internationalen Kapitalverkehr zulassen, tatsächlich geringere Unterschiede in den Veränderungen der Ausgabenanteile bestehen. ${ }^{345}$

Ob tatsächlich die Offenheit der Länder die Ursache dafür ist, dass im ersten Ländercluster die Ideologievariablen insignifikant sind, in den anderen Clustern jedoch nicht, lässt sich anhand der Analyse der einzelnen Zeitphasen überprüfen.

In Tabelle 5.23 werden drei Zeitphasen unterschieden. Der dritte und vierte Zeitcluster wurden hierbei zusammengefasst, da für jeden einzelnen der beiden Zeitcluster ansonsten nur eine sehr geringe Fallzahl in die Regression Eingang gefunden hätte. Auch hier haben die konjunkturreagiblen Variablen (UEB ${ }_{1}$ und $D_{-} U_{1}$ ) fast alle ein signifikantes und annahmegemäßes Vorzeichen. Allerdings ist keine eindeutige Tendenz festzustellen, dass der Erklärungsgehalt dieser Variablen mit zunehmender Offenheit steigt.

Hinsichtlich der Ideologievariable wird das Ergebnis noch schwieriger zu interpretieren, denn nur in der zweiten Zeitphase gibt es deutlich signifikante, theoriekonforme Koeffizientenschätzungen. Von 1960 bis 1973 ist sogar eher auf eine umgekehrte Relation zu schließen. In dieser Zeit wurden besonders nach einem Regierungswechsel von links nach rechts die Ausgabenanteile erhöht. In der dritten Phase von 1986 bis 1996 haben die drei Koeffizienten zwar ein negatives Vorzeichen, sind jedoch nicht signifikant von null verschieden.

Insgesamt lässt sich daraus schlussfolgern, dass zwar die besonders offenen Länder die geringsten Partisaneffekte aufweisen. Es bleibt jedoch unklar, ob dies tatsächlich auf ihre Offenheit zurückzuführen ist, da die Analyse der Zeitcluster nicht zeigen konnte, dass es in den besonders geschlossenen Jahren vor 1973 auch die stärksten Partisaneffekte gab.

${ }^{344}$ Für den ersten Ländercluster wurden für die RCG2,-Variable etwas „,bessere“ Koeffizienten geschätzt als für die RCG4,-Variable. Daher wurde für den ersten Cluster diese Ideologievariable aufgeführt.

${ }^{345} \mathrm{Da}$ bei den Budgetdaten nur Jahresdaten verwendet wurden, wird auf eine detaillierte Analyse der einzelnen Ländercluster für die einzelnen Zeitphasen verzichtet, da die Fallzahl dabei mitunter weit unter 30 sinkt. Die Validität đebErgebrisses wäre-kaum mosh-gesichert. 
Tabelle 5.22 : Partisaneffekte im Budget in der Clusterung gemäß ökonomischen Globalisierungsindikatoren 1960-1996

\begin{tabular}{|c|c|c|c|c|c|c|c|c|c|}
\hline & \multicolumn{3}{|c|}{$\begin{array}{l}\text { (BEL, NL, S, UK) }\end{array}$} & \multicolumn{3}{|c|}{ (CAN, DK, SF, IRL, NOR, CH) } & \multicolumn{3}{|c|}{ (AUS, AU, F, D, ITA, J, E, USA) } \\
\hline & D_FLEX & D_TRANS & D_ZIV & D_FLEX & D_TRANS & D_ZIV & D_FLEX & D_TRANS & D_ZIV \\
\hline TREND & - & - & - & - & - & - & $\begin{array}{l}-0,018 * * \\
(-2,733)\end{array}$ & $\begin{array}{c}-0,002 \\
(-0,492)\end{array}$ & $\begin{array}{l}-0,010 * * \\
(-3,193)\end{array}$ \\
\hline $\mathrm{UEB}_{\mathrm{t}}$ & $\begin{array}{l}-0,374 * * \\
(-5,278)\end{array}$ & $\begin{array}{l}-0,121^{* *} \\
(-3,714)\end{array}$ & $\begin{array}{l}-0,169 * * \\
(-5,795)\end{array}$ & $\begin{array}{c}-0,185 * * \\
(-6,049)\end{array}$ & $\begin{array}{c}-0,041+ \\
(-1,957)\end{array}$ & $\begin{array}{l}-0,103^{* *} \\
(-8,910)\end{array}$ & $\begin{array}{l}-0,139 * * \\
(-7,221)\end{array}$ & $\begin{array}{l}-0,063^{* *} \\
(-6,185)\end{array}$ & $\begin{array}{l}-0,039 * * \\
(-4,070)\end{array}$ \\
\hline D_Ut & $\begin{array}{c}0,634 * * \\
(3,311)\end{array}$ & $\begin{array}{l}0,445^{* *} \\
(5,065)\end{array}$ & $\begin{array}{l}0,270^{* *} \\
(3,424)\end{array}$ & $\begin{array}{c}0,638 * * \\
(6,040)\end{array}$ & $\begin{array}{l}0,612^{* *} \\
(8,360)\end{array}$ & $\begin{array}{c}0,100 * \\
(2,478)\end{array}$ & $\begin{array}{l}0,340 * * \\
(4,280)\end{array}$ & $\begin{array}{l}0,255^{* *} \\
(6,132)\end{array}$ & $\begin{array}{c}0,099 * \\
(2,484)\end{array}$ \\
\hline$R C G 2 t$ & $\begin{array}{c}-0,098 \\
(-1,314)\end{array}$ & $\begin{array}{c}-0,006 \\
(-0,175)\end{array}$ & $\begin{array}{c}-0,022 \\
(-0,710)\end{array}$ & - & - & - & - & - & - \\
\hline $\mathrm{RCG}_{\mathrm{t}}$ & - & - & - & $\begin{array}{c}-0,143+ \\
(-1,705)\end{array}$ & $\begin{array}{c}0,011 \\
(0,190)\end{array}$ & $\begin{array}{l}-0,087 * * \\
(-2,715)\end{array}$ & $\begin{array}{c}-0,088^{*} \\
(-2,365)\end{array}$ & $\begin{array}{c}-0,012 \\
(-0,602)\end{array}$ & $\begin{array}{l}-0,055^{* *} \\
(-2,968)\end{array}$ \\
\hline MAAST $_{t}$ & $\begin{array}{c}-1,188 * * \\
(-2,807)\end{array}$ & $\begin{array}{l}-0,685^{* *} \\
(-3,535)\end{array}$ & $\begin{array}{c}0,006 \\
(0,036)\end{array}$ & $\begin{array}{c}0,771 \\
(1,530)\end{array}$ & $\begin{array}{c}0,383 \\
(1,098)\end{array}$ & $\begin{array}{c}0,123 \\
(0,644)\end{array}$ & - & - & - \\
\hline WIWA $_{t}$ & $\begin{array}{r}0,235^{*} \\
(2,386) \\
\end{array}$ & $\begin{array}{c}0,064 \\
(1,427) \\
\end{array}$ & $\begin{array}{c}0,107 * * \\
(2,645) \\
\end{array}$ & - & - & - & - & - & - \\
\hline $\mathrm{R}_{\mathrm{adj} .}^{2}$ & 0,39 & 0,46 & 0,40 & 0,45 & 0,41 & 0,46 & 0,33 & 0,33 & 0,19 \\
\hline
\end{tabular}


Tabelle 5.23 : Partisaneffekte im Budget in der Clusterung gemäß ökonomischen Globalisierungsindikatoren (alle Länder)

\begin{tabular}{|c|c|c|c|c|c|c|c|c|c|}
\hline & \multicolumn{3}{|c|}{ 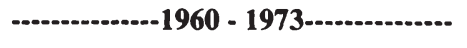 } & \multicolumn{3}{|c|}{ - 1985} & \multicolumn{3}{|c|}{ - } \\
\hline & D_FLEX & D_TRANS & D_ZIV & D_FLEX & D_TRANS & D_ZIV & D_FLEX & D_TRANS & D_ZIV \\
\hline TREND & $\begin{array}{c}-0,032 \\
(-1,350)\end{array}$ & $\begin{array}{c}-0,006 \\
(-0,450)\end{array}$ & $\begin{array}{c}-0,005 \\
(-0,406)\end{array}$ & $\begin{array}{l}-0,130^{* *} \\
(-4,891)\end{array}$ & $\begin{array}{c}-0,021 \\
(-1,459)\end{array}$ & $\begin{array}{l}-0,040 * * \\
(-2,907)\end{array}$ & $\begin{array}{c}0,033 \\
(0,859)\end{array}$ & $\begin{array}{c}0,010 \\
(0,399)\end{array}$ & $\begin{array}{c}0,006 \\
(0,423)\end{array}$ \\
\hline $\mathrm{UEB}_{\mathrm{t}}$ & $\begin{array}{l}-0,128 * * \\
(-5,338)\end{array}$ & $\begin{array}{c}-0,053^{* *} \\
(-4,082)\end{array}$ & $\begin{array}{l}-0,043^{* *} \\
(-3,442)\end{array}$ & $\begin{array}{l}-0,141^{* *} \\
(-5,738)\end{array}$ & $\begin{array}{l}-0,040 * * \\
(-2,969)\end{array}$ & $\begin{array}{c}-0,062^{* *} \\
(-4,918)\end{array}$ & $\begin{array}{c}-0,195^{* *} \\
(-5,983)\end{array}$ & $\begin{array}{l}-0,070^{* *} \\
(-3,440)\end{array}$ & $\begin{array}{l}-0,088^{* *} \\
(-7,143)\end{array}$ \\
\hline D_U, & $\begin{array}{c}0,537 * * \\
(3,671)\end{array}$ & $\begin{array}{c}0,254^{* *} \\
(3,198)\end{array}$ & $\begin{array}{c}0,377 * * \\
(4,939)\end{array}$ & $\begin{array}{c}0,275 * * \\
(2,652)\end{array}$ & $\begin{array}{c}0,314 * * \\
(5,513)\end{array}$ & $\begin{array}{c}0,088 \\
(1,648)\end{array}$ & $\begin{array}{c}0,624 * * \\
(6,319)\end{array}$ & $\begin{array}{l}0,570 * * \\
(9,182)\end{array}$ & $\begin{array}{l}0,104 * * \\
(2,779)\end{array}$ \\
\hline RCG2, & - & - & - & $\begin{array}{c}-0,129 * \\
(-2,116)\end{array}$ & $\begin{array}{c}0,036 \\
(1,081)\end{array}$ & $\begin{array}{c}-0,054+ \\
(-1,721)\end{array}$ & 一 & - & - \\
\hline $\mathrm{RCG}_{4}$ & $\begin{array}{c}0,001 \\
(0,031)\end{array}$ & $\begin{array}{c}-0,008 \\
(-0,373)\end{array}$ & $\begin{array}{c}0,016 \\
(0,790)\end{array}$ & - & - & - & $\begin{array}{c}-0,077 \\
(-1,104)\end{array}$ & $\begin{array}{c}-0,049 \\
(-1,123)\end{array}$ & $\begin{array}{c}-0,013 \\
(-0,504)\end{array}$ \\
\hline MAAST $_{1}$ & - & - & - & - & - & - & $\begin{array}{c}-0,905^{* *} \\
(-2,747)\end{array}$ & $\begin{array}{l}-0,564^{* *} \\
(-2,722)\end{array}$ & $\begin{array}{c}-0,047 \\
(-0,379)\end{array}$ \\
\hline$R_{a d j}^{2}$ & 0,43 & 0,34 & 0,38 & 0,42 & 0,30 & 0,27 & 0,38 & 0,43 & 0,29 \\
\hline
\end{tabular}

Anmerkungen: $\quad \mathrm{t}$-Werte in Klammern, $+(\alpha<0,1),{ }^{*}(\alpha<0,05),{ }^{* *}(\alpha<0,01)$. 
Es sind demnach in dieser Clusterung nur äußerst schwache Hinweise auf die Richtigkeit der Globalisierungsthese $\mathrm{zu}$ finden. Die Partisantheorie wird indes v.a. in den siebziger und achtziger Jahren recht deutlich bestätigt. Es soll im folgenden untersucht werden, ob bei der politischen Clusterung gegebenenfalls eindeutigere Ergebnisse erzielt werden können.

\subsection{Cluster gemäß den politischen Indikatoren}

Analog zu der Vorgehensweise bei der ökonomischen Clusterung wird zunächst die Frage beantwortet, ob in stärker liberalisierten Volkswirtschaften Partisaneffekte im Budget weniger deutlich erkennbar sind als in jenen Volkswirtschaften, die ihre Güter- und Kapitalmärkte weniger liberalisiert haben. In Tabelle 5.24 sind die Ergebnisse für die vier unterschiedlichen Ländercluster und den gesamten Untersuchungszeitraum zusammengestellt.

Auch in diesen Regressionen haben fast alle Koeffizienten der konjunkturreagiblen Variablen das signifikant richtige Vorzeichen. Allerdings ist hier, anders als in der ökonomischen Clusterung, kein eindeutiger Zusammenhang zwischen Öffnungsgrad und Höhe der Koeffizienten erkennbar. Dies gilt sowohl für die Variable $\mathrm{UEB}_{\mathrm{t}}$ als auch für $\mathrm{D}_{-} \mathrm{U}_{\mathrm{t}}$.

Von besonderem Interesse sind die Koeffizienten der Ideologievariablen RCG2 bzw. RCG4. Hier ist eine merkwürdig anmutende Asymmetrie vorhanden. Die Schätzergebnisse, die am besten mit der Partisanthese vereinbar sind, werden im ersten und vierten Cluster ausgewiesen, also in jenen Clustern, die die besonders liberalisierten Länder zum einen und die besonders stark abgeschotteten Länder zum anderen zusammenfassen. Die Koeffizienten sind statistisch hoch signifikant. Dies bedeutet nichts anderes, als dass der gewählte Indikator für die Liberalisierung der Märkte anscheinend keine Erklärung für die Gültigkeit der Partisantheorie liefern kann. Interessant ist darüber hinaus, dass in den beiden anderen Clustern kein einziger Koeffizient der Ideologievariablen signifikant von null abweicht.

Die Analyseergebnisse der Zeitcluster (Tabelle 5.25) ähneln natürlich in diesem Fall sehr stark den Resultaten der ökonomischen Clusterung aus dem vorherigen Abschnitt, da die Cluster eins und zwei sowie die Cluster vier und fünf zu je einer Zeitphase zusammengefasst werden mussten. Dies war nötig, da ansonsten die Fallzahl in den einzelnen Clustern nicht hoch genug gewesen wäre, um gesicherte Schätzungen zu erhalten. Deutlich wird, dass auch hier die stärksten Partisaneffekte in den siebziger und achtziger Jahren erkennbar sind. 
Tabelle 5.24 : Partisaneffekte im Budget in der Clusterung gemäß politischen Globalisierungsindikatoren 1960-1996

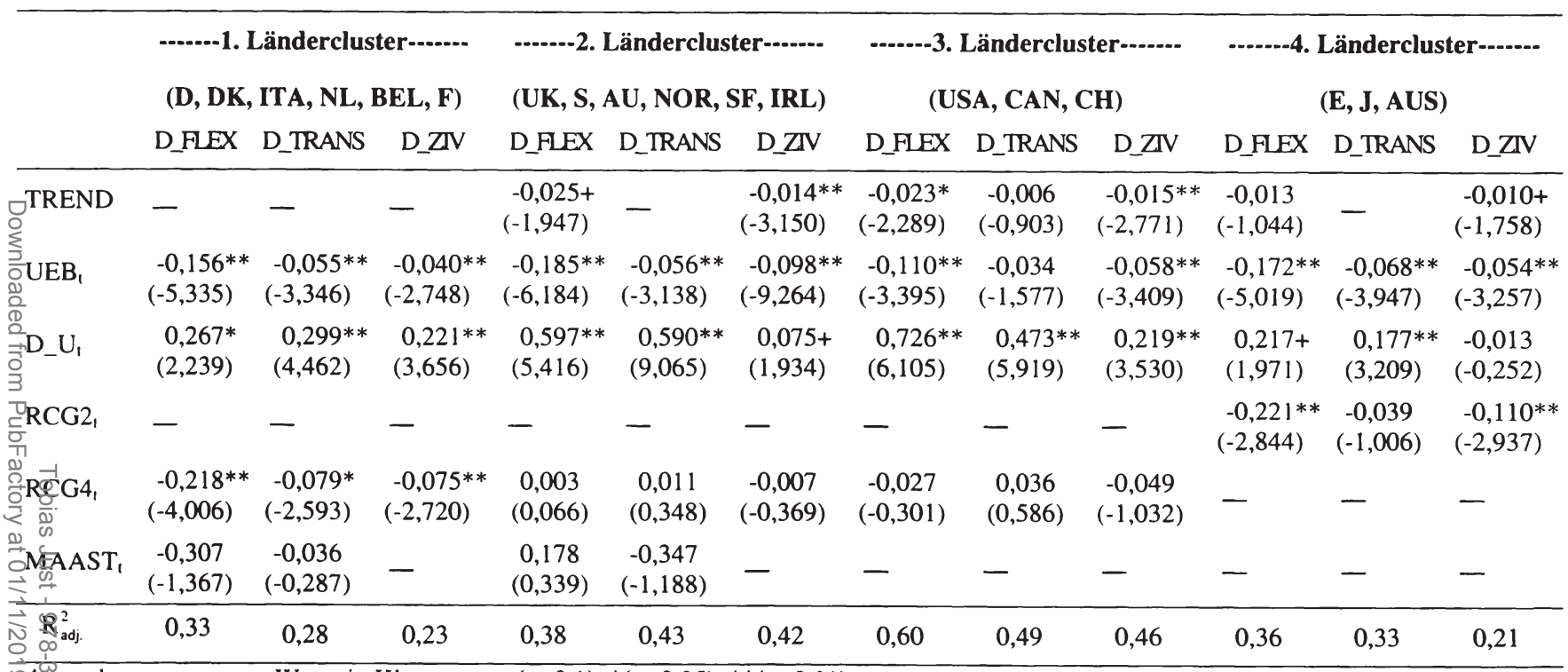

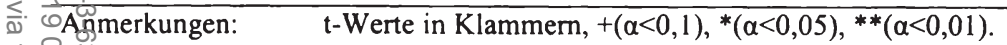


Tabelle 5.25 : Partisaneffekte im Budget in der Clusterung gemäß politischen Globalisierungsindikatoren (alle Länder)

\begin{tabular}{|c|c|c|c|c|c|c|c|c|c|}
\hline & \multicolumn{3}{|c|}{ - } & \multicolumn{3}{|c|}{ 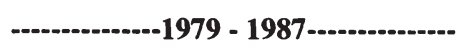 } & \multicolumn{3}{|c|}{ 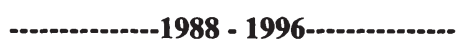 } \\
\hline & D_FLEX & D_TRANS & D_ZIV & D_FLEX & D_TRANS & D_ZIV & D_FLEX & D_TRANS & D_ZIV \\
\hline TREND & $\begin{array}{c}0,023 \\
(1,403)\end{array}$ & $\begin{array}{c}0,015 \\
(1,633)\end{array}$ & $\begin{array}{c}-0,004 \\
(-0,523)\end{array}$ & - & - & - & - & - & - \\
\hline $\mathrm{UEB}_{\mathrm{i}}$ & $\begin{array}{l}-0,171 * * \\
(-8,431)\end{array}$ & $\begin{array}{l}-0,046^{* *} \\
(-4,078)\end{array}$ & $\begin{array}{l}-0,073^{* *} \\
(-8,281)\end{array}$ & $\begin{array}{l}-0,152 * * \\
(-5,562)\end{array}$ & $\begin{array}{l}-0,053^{* *} \\
(-3,932)\end{array}$ & $\begin{array}{l}-0,054 * * \\
(-3,319)\end{array}$ & $\begin{array}{l}-0,174 * * \\
(-4,578)\end{array}$ & $\begin{array}{c}-0,058^{*} \\
(-2,386)\end{array}$ & $\begin{array}{l}-0,089 * * \\
(-6,384)\end{array}$ \\
\hline D_U & $\begin{array}{l}0,364 * * \\
(3,054)\end{array}$ & $\begin{array}{l}0,302^{* *} \\
(4,555)\end{array}$ & $\begin{array}{l}0,159 * * \\
(3,073)\end{array}$ & $\begin{array}{l}0,316^{* *} \\
(3,405)\end{array}$ & $\begin{array}{c}0,325^{* *} \\
(7,087)\end{array}$ & $\begin{array}{c}0,092+ \\
(1,686)\end{array}$ & $\begin{array}{l}0,647 * * \\
(5,762)\end{array}$ & $\begin{array}{c}0,603 * * \\
(8,361)\end{array}$ & $\begin{array}{c}0,093 * * \\
(2,240)\end{array}$ \\
\hline RCG2, & $\begin{array}{c}-0,100+ \\
(-1,823)\end{array}$ & $\begin{array}{c}0,024 \\
(0,778)\end{array}$ & $\begin{array}{c}-0,041+ \\
(-1,711)\end{array}$ & $\begin{array}{l}-0,175 * * \\
(-2,845)\end{array}$ & $\begin{array}{c}-0,026 \\
(-0,863)\end{array}$ & $\begin{array}{c}-0,088^{*} \\
(-2,419)\end{array}$ & - & - & - \\
\hline $\mathrm{RCG}_{\mathrm{t}}$ & - & - & - & - & - & - & $\begin{array}{c}-0,091 \\
(-1,068)\end{array}$ & $\begin{array}{c}-0,056 \\
(-1,029)\end{array}$ & $\begin{array}{c}-0,000 \\
(-0,016)\end{array}$ \\
\hline MAAST $_{t}$ & - & - & - & - & - & - & $\begin{array}{c}-0,835 * * \\
(-2,798)\end{array}$ & $\begin{array}{l}-0,612^{* *} \\
(-3,191)\end{array}$ & $\begin{array}{c}0,020 \\
(0,178)\end{array}$ \\
\hline $\mathbf{R}_{\text {adj. }}^{2}$ & 0,41 & 0,28 & 0,41 & 0,33 & 0,39 & 0,13 & 0,37 & 0,43 & 0,28 \\
\hline
\end{tabular}


Durch die etwas größere Periodenlänge des ersten Clusters werden für den Zeitraum von 1960 bis 1978 auch die Koeffizienten der Variablen D_FLEX und D_ZIV schwach signifikant. Auch hier gibt es keine Hinweise, dass es die Öffnung der Märkte war, die zur Unterschiedslosigkeit der Budgetpolitik in den 90er Jahren nötigte, denn vergleichbare Koeffizienten wurden auch für die 60er Jahre geschätzt.

Ebenso überraschend ist es, dass bei allen Regressionen der politischen Clusterung die Transferleistungen am wenigstens durch die Ideologievariable determiniert werden. Dies kann natürlich daran liegen, dass die Transfers tatsächlich als automatische Stabilisatoren vornehmlich dem Konjunkturverlauf folgen. Es ist aber durchaus auch möglich, dass dies Ausdruck eines systematischen wahltaktischen Vorteils der linken Parteien ist. Sind direkte Transfers für die Wähler am sichtbarsten, so ist anzunehmen, dass rechte Parteien nur sehr zögerlich an dieser Ausgabengruppe ansetzen können, möchten sie zwar eine Kürzung der Gesamtausgaben vornehmen, jedoch dadurch nicht zu große Wählerzahlen verlieren. Die zum Teil erzielten positiven Vorzeichen in den D_TRANSRegressionen könnten sogar dadurch begründet werden, dass rechte Parteien direkte Transfers ausdehnen, um mögliche Wählerverluste zu kompensieren, die sie durch andere Einsparungen erleiden. Freilich sollte diese Interpretation nicht überbewertet werden, da die wenigsten der Koeffizienten mit positivem Vorzeichen auch statistisch signifikant von null abweichen.

\subsubsection{Progressivität als ideologisches Unterscheidungsmoment}

„Keine Experimente!“ (Konrad Adenauer, 1957)

Nach der Definition von Lipset et al. (1954) bedeutet das Attribut links nicht nur eine stärkere Präferenz für Umverteilung, sondern auch eine stärkere Offenheit gegenüber dem gesellschaftlichen Wandel. ${ }^{347}$ Die konkrete Änderungsrichtung des gesellschaftlichen Wandels richtet sich dabei maßgeblich nach der Beschaffenheit des Status quo. Wurde in der Vergangenheit ein hohes Maß an Umverteilung realisiert, so kann eine wandlungsorientierte Linke unter Umständen eher dazu bereit sein, dieses System zu verändern (oder wie man jetzt sagen würde: zu modernisieren) als eine risikoaverse konservative Partei, die womöglich das bestehende Umverteilungssystem mittlerweile als schützenswerten Status quo betrachtet.

\footnotetext{
346 Von einem Wahlplakat für die Bundestagswahl 1957 (vgl. http://www.fortunecity.de/ lindenpark/caesarenstrasse/69/plakate/pp45-69/ppw 1957.html).

${ }^{347} \mathrm{Vgl}$. auch Laponce (1981) und Fuchs und Klingemann (1990). 978-3-631-75139-8
} 
Ergänzend zu den obigen Ergebnissen wird nun die Fragestellung untersucht, ob linke Regierungen generell stärkere Veränderungen in ihrer Haushaltspolitik vornehmen als rechte Parteien und ob diese Unterschiedlichkeit gegebenenfalls in offeneren Staaten weniger deutlich ausgeprägt ist als in den geschlosseneren Ländern.

\subsubsection{Das Modell}

Es werden zwei ähnliche endogene Variable geschätzt, die den Umfang der Änderung der Ausgabenpositionen im Staatshaushalt in jeder Regierungsperiode erfassen sollen. In dem verwendeten Datensatz werden für jedes Land $\mathrm{i}$ insgesamt sieben ( $k$ ) Ausgabenkategorien $\left(A_{i, k, t}\right)$ unterschieden, die in jeder Periode $t$ jeweils einen Anteil $\mathrm{a}_{\mathrm{i}, \mathrm{k}, \mathrm{l}}$ am Gesamtbudget einnehmen, sodass sich der Indikator für die Variabilität der Ausgabenstruktur (AUSG_VAR $\mathrm{R}_{\mathrm{i}, \mathrm{t}}$ ) als die Summe aller absoluten Veränderungen in den Ausgabenanteilen am Gesamthaushalt bestimmt: ${ }^{348}$

$$
\text { AUSG_VAR }{ }_{i, t}=\sum_{k=1}^{7}\left|a_{i, k, t}-a_{i, k, t-1}\right|
$$

Es ist offensichtlich, dass bei dieser Konstruktion der endogenen Variable eine $1 \%$-ige Reduktion eines Ausgabenanteils dieselbe Wirkung für AUSG_VAR ${ }_{i, t}$ bedeutet wie eine äquivalente Erhöhung. Die Variable misst also ausschließlich den Umfang der Änderung in der Budgetstruktur, ohne auf die Änderungsrichtung abzuheben.

Darüber hinaus wird parallel der Logarithmus von AUSG_VAR $R_{i, t}$ als zu erklärende Variable geschätzt. Durch die Konstruktion von AUSG_VAR $R_{i, t}$ als Differenz von Anteilswerten werden die Koeffizienten zum Teil sehr klein, die Logarithmierung führt zu höheren Werten der Koeffizienten. Ausschlaggebend ist aber v.a. ein inhaltlicher Grund für die Logarithmierung der endogenen Variable. Schmidt (1996) kommt in seiner Untersuchung zu dem Ergebnis, dass Parteien der politischen Mitte und des linken Spektrums ähnliche Ausgabenpolitik betreiben und lediglich die rechten Parteien signifikant unterschiedliche Ausga-

348 Zimmermann (1985, S. 241ff) und Zimmermann und Müller (1985, S. 140ff) verwenden einen etwas anderen Indikator für die Variationsmasse. Sie bilden auf der Basis des Vorjahresanteils einer Ausgabengruppe einen hypothetischen Wert dieser Ausgabengruppe für die Ist-Periode, um den empirischen Wert um den hypothetischen Wert zu verringern. Die Summe aller positiven (negativen) Abweichungen des tatsächlichen Ist-Wertes von dem Prognosewert über alle Ausgabengruppen ergibt bei ihnen die Variationsmasse des Budgets. 
benänderungen realisieren. $\mathrm{Da}$ die Logarithmusfunktion konkav verläuft, werden die Änderungen von LOG(AUSG_VAR $R_{i, t}$ ) zunehmend kleiner. Da außerdem AUSG_VAR $R_{i, l}$ stets kleiner als eins ist, wird der Logarithmus stets kleiner als null. Sind rechte Parteien also tatsächlich weniger stark dem Wandel zugeneigt, dann ist ihnen ein geringerer Wert für AUSG_VAR ${ }_{i, t}$ zugeordnet. Dies bedeutet, dass die Logarithmierung der Variable genau das Ergebnis von Schmidt abbilden kann; ein Wechsel von einer rechten zu einer Mitte-Regierung führt zu einer stärkeren Erhöhung von LOG(AUSG_VAR ${ }_{i, t}$ ) als ein äquivalent großer Wechsel von einer Mitte- zu einer linken Regierung.

Als erklärende Variablen werden zusätzlich zu den oben bereits beschriebenen Variablen noch zwei weitere Variablen als Regressoren verwendet. ${ }^{349}$ Zum einen wird die jährliche Änderung des Konsumentenpreisindex (INFL $i, t)$ berücksichtigt. Dies geschieht deshalb, weil bei höheren Inflationsraten auch damit zu rechnen ist, dass die Varianz der Preisänderungsraten der einzelnen Güter steigt. Es ist dann davon auszugehen, dass bei steigenden Inflationsraten Ausgabenkategorieanteile verschoben werden, weil die unterschiedlichen Ausgaben verschieden stark inflationieren. Das erwartete Vorzeichen von $\mathrm{INFL}_{\mathrm{i}, \mathrm{t}}$ ist daher positiv.

Des weiteren wird ein Interaktionsterm betrachtet. Höhere Wachstumsraten können nämlich insbesondere dann rasch in andere Ausgabenpolitik übersetzt werden, wenn die Institutionen flexibel genug dafür sind. Schmidt (1996) stellt einen Indikator für die institutionellen Beschränkungen vor $\left(\mathrm{INST}_{\mathrm{i}}\right)$. Dieser setzt sich zusammen aus sechs Dummyvariablen und nimmt besonders hohe Werte für jene Länder an, die sehr heterogene Entscheidungsstrukturen aufweisen (z.B. hohes $\mathrm{Ma} \beta$ an Föderalismus, Harmonisierungsbedarf aufgrund der EUTeilnahme, 2-Kammersystem). Es sind nun zwei entgegengesetzte Hypothesen formulierbar: Entweder führen diese institutionellen Beschränkungen dazu, dass langsamer auf konjunkturelle Datenänderungen reagiert werden kann; dann müssten stärker beschränkte Systeme im Falle von Wirtschaftswachstum langsamer reagieren können und folglich hätte der Koeffizient des Interaktionsterms WIWA $_{\mathrm{i}, \mathrm{r}} \cdot \mathrm{INST}_{\mathrm{i}}$ ein negatives Vorzeichen. Es ist aber auch vorstellbar, dass die komplizierteren Entscheidungsstrukturen einen größeren Spielraum für logrolling Aktivitäten lassen; dies würde bedeuten, dass gerade in den vermeintlich beschränkten Systemen besonders stark auf Wachstumsänderungen reagiert würde. In diesem Fall hätte der Koeffizient ein positives Vorzeichen. Die Schätzmethode ist wie zuvor eine Panelregression mit fixed effects. Die Schätzgleichungen haben letztlich folgende Form: ${ }^{350}$

349 Jene exogenen Variablen, die nicht mehr Eingang finden, wurden zwar ebenfalls getestet, ergaben jedoch keine signifikanten Ergebnisse. Daher wird auf ihre Darstellung verzichtet.

350 Natürlich wird auch AUSG_VAR ${ }_{i, t}$ auf RCG4 $4_{i, t}$ und LOG(AUSG_VAR $R_{i, t}$ ) auch auf CG $_{i, t}$ regressiert. 


$$
\begin{aligned}
\text { AUSG_VAR }_{\mathrm{i}, \mathrm{t}} & =\alpha_{\mathrm{i}}+\beta_{1} \cdot \mathrm{TREND}_{2} \beta_{2} \cdot \mathrm{D}_{-} \mathrm{U}_{\mathrm{i}, \mathrm{t}}+\beta_{3} \cdot \mathrm{MAAST}_{\mathrm{i}, \mathrm{t}} \\
& +\beta_{4} \cdot\left(\mathrm{WIWA}_{\mathrm{i}, \mathrm{t}} \cdot \mathrm{INST}_{\mathrm{i}}\right)+\beta_{5} \cdot \mathrm{INFL}_{\mathrm{i}, \mathrm{t}}+\gamma_{1} \cdot \mathrm{CG}_{\mathrm{i}, \mathrm{t}}+\varepsilon_{\mathrm{i}, \mathrm{t}} \\
& \text { bzw. }
\end{aligned}
$$

LOG $(\text { AUSG_VAR })_{i, t}=\alpha_{i}+\beta_{1} \cdot$ TREND $+\beta_{2} \cdot D_{-} U_{i, t}+\beta_{3} \cdot$ MAAST $_{i, t}$

\begin{tabular}{|c|c|c|}
\hline Name & Definition & $\begin{array}{l}\text { Vorzeichen- } \\
\text { erwartung }\end{array}$ \\
\hline TREND & Trendvariable & $\beta_{1}<0$ \\
\hline D_U $U_{i, \imath}$ & Erste Veränderung der Arbeitslosenquote & $\beta_{2}>0$ \\
\hline MAAST $_{i, t}$ & Maastricht-Dummyvariable & $\beta_{3}<0$ \\
\hline WIWA $_{\mathrm{i}, \iota} \cdot \mathrm{INST}$ & $\begin{array}{l}\text { Bewertung der realen Wachstumsrate des } \\
\text { BIP mit dem Indikator für institutionelle } \\
\text { Beschränkungen }\end{array}$ & $\beta_{4} ?$ \\
\hline $\mathrm{INFL}_{\mathrm{i}, \mathrm{t}}$ & Änderung des Konsumentenpreisindex & $\beta_{5}>0$ \\
\hline $\mathrm{CG}_{\mathrm{i}, \mathrm{l}}$ & Center of gravity & $\gamma_{1}<0$ \\
\hline $\mathrm{RCG}_{\mathrm{i}, \mathrm{t}}$ & $\mathrm{RCG}_{\mathrm{i}, \mathrm{t}}=\mathrm{CG}_{\mathrm{i}, \mathrm{t}}-\mathrm{CG}_{\mathrm{i}, \mathrm{t}-\mathrm{t}}$ & $v<<0$ \\
\hline $\mathrm{RCG} 2_{\mathrm{i}, \mathrm{t}}$ & $\mathrm{RCG} 2_{\mathrm{i}, \mathrm{t}}=\mathrm{CG}_{\mathrm{i}, \mathrm{t}}-\mathrm{CG}_{\mathrm{i}, \mathrm{t}-2}$ & $\gamma_{2}-0$ \\
\hline
\end{tabular}

$$
+\beta_{4} \cdot\left(\text { WIWA }_{\mathrm{i}, \mathrm{t}} \cdot \mathrm{INST}_{\mathrm{i}}\right)+\beta_{5} \cdot \mathrm{INFL}_{\mathrm{i}, \mathrm{t}}+\gamma_{2} \cdot \mathrm{RCG}_{\mathrm{i}, \mathrm{t}}+\varepsilon_{\mathrm{i}, \mathrm{t}}
$$

wobei für die exogenen Variablen folgendes gilt:

\subsubsection{Schätzergebnisse}

Zunächst werden Regressionen für das Gesamtpanel, also für alle 18 Staaten und den Zeitraum von 1960 bis 1996 geschätzt. Dabei werden je drei Modelle mit den unabhängigen Variablen AUSG_VAR und LOG(AUSG_VAR) regressiert. Insgesamt sind die Schätzergebnisse für die logarithmierten Werte von AUSG_VAR etwas besser. Erstens sind die Koeffizienten höher und zweitens ist das adjustierte $\mathrm{R}^{2}$ in allen Modellvarianten etwas höher. Zwar sind auch in den Modellen (4) bis (6) die Werte des Bestimmtheitsmaßes mit etwa 0,20 nicht sehr hoch, eine Prüfung der Gesamtgüte der Modelle mit Hilfe eines F-Tests zeigt jedoch, dass für alle Modelle auf dem 1\%-Signifikanzniveau die Hypothese, die Variablen besäßen insgesamt keine Erklärungskraft, verworfen werden kann. Außerdem ist es nicht das Ziel, die Ausgabenvariabilität möglichst exakt zu erklären, sondern es sollen v.a. die zentralen Ideologievariablen auf ihren Er- 
klärungsgehalt geprüft werden und dafür ist in erster Linie deren t-Wert von Interesse. In Tabelle 5.26 werden die wesentlichen Ergebnisse zusammengefasst.

Einige bemerkenswerte Ergebnisse sind bereits aus diesen Gesamtpanelregressionen ersichtlich. In fast allen Regressionen wird ein signifikanter Trend zu einer Abnahme der Ausgabenvariabilität deutlich. Dies ist letztlich Ausdruck des bereits zuvor gezeigten Trends (vgl. Tabelle 5.20) zu geringeren Ausgabenzuwächsen. Ähnlich ist auch der signifikant positive Einfluss der D_ $\mathrm{U}_{t}$-Variable bereits in den zuvor durchgeführten Regressionen vorweggenommen, denn dort wurde ja gezeigt, dass die Veränderung der Arbeitslosenquote mit den Veränderungen fast aller Ausgabengruppen positiv korreliert. Dann muss natürlich auch der hier verwendete Indikator für Ausgabenvariabilität positiv mit der Veränderung der Arbeitslosenquote korrelieren.

Tabelle 5.26 : Budgetäre Variabilität im Gesamtpanel 1960-1996

\begin{tabular}{|c|c|c|c|c|c|c|}
\hline \multirow[b]{2}{*}{$\begin{array}{l}\text { unabhängige } \\
\text { Variable }\end{array}$} & \multicolumn{3}{|c|}{$\begin{array}{c}\text { abhängige Variable: } \\
\text { AUSG_VAR }\end{array}$} & \multicolumn{3}{|c|}{$\begin{array}{l}\text { abhängige Variable: } \\
\text { LOG(AUSG_VAR), }\end{array}$} \\
\hline & (1) & (2) & (3) & (4) & (5) & (6) \\
\hline TREND & $\begin{array}{l}-0,000 * * \\
(-2,638)\end{array}$ & $\begin{array}{l}-0,000+ \\
(-1,705)\end{array}$ & $\begin{array}{c}-0,000 \\
(-0,791)\end{array}$ & $\begin{array}{l}-0,009 * * \\
(-3,465)\end{array}$ & $\begin{array}{l}-0,010^{* *} \\
(-3,291)\end{array}$ & $\begin{array}{l}-0,007 * * \\
(-2,666)\end{array}$ \\
\hline D_Ut & $\begin{array}{l}0,005^{* *} \\
(3,885)\end{array}$ & $\begin{array}{l}0,007 * * \\
(5,500)\end{array}$ & $\begin{array}{l}0,007 * * \\
(6,000)\end{array}$ & $\begin{array}{l}0,132 * * \\
(4,793)\end{array}$ & $\begin{array}{l}0,118 * * \\
(3,954)\end{array}$ & $\begin{array}{l}0,143 * * \\
(5,233)\end{array}$ \\
\hline WIWA, & $\begin{array}{c}-0,000 \\
(-0,206)\end{array}$ & - & - & - & $\begin{array}{c}-0,001 \\
(-0,104)\end{array}$ & - \\
\hline WIWA $_{1} \cdot$ INST & - & $\begin{array}{l}0,000 * * \\
(2,845)\end{array}$ & $\begin{array}{l}0,000^{* *} \\
(3,048)\end{array}$ & $\begin{array}{l}0,006^{*} \\
(2,114)\end{array}$ & - & $\begin{array}{c}0,007 * \\
(2,197)\end{array}$ \\
\hline INFL $_{4}$ & $\begin{array}{c}0,000 \\
(1,500)\end{array}$ & $\begin{array}{l}0,001 * \\
(2,197)\end{array}$ & $\begin{array}{l}0,001^{* *} \\
(2,744)\end{array}$ & $\begin{array}{l}0,015^{*} \\
(2,355)\end{array}$ & $\begin{array}{c}0,014^{*} \\
(2,124)\end{array}$ & $\begin{array}{l}0,018^{* *} \\
(2,698)\end{array}$ \\
\hline $\mathrm{CG}_{\mathrm{t}}$ & $\begin{array}{c}0,000 \\
(0,504)\end{array}$ & $\begin{array}{c}0,000 \\
(0,471)\end{array}$ & - & $\begin{array}{c}0,011 \\
(0,642)\end{array}$ & - & - \\
\hline $\mathrm{RCG}_{4}$ & - & - & $\begin{array}{l}-0,002 * * \\
(-2,990)\end{array}$ & - & $\begin{array}{l}-0,055^{* *} \\
(-3,101)\end{array}$ & $\begin{array}{l}-0,053 * * \\
(-2,979)\end{array}$ \\
\hline $\mathbf{R}_{\mathrm{adj} .}^{2}$ & 0,10 & 0,11 & 0,13 & 0,19 & 0,19 & 0,20 \\
\hline
\end{tabular}

Anmerkungen:

t-Werte in Klammern, $+(\alpha<0,1),{ }^{*}(\alpha<0,05),{ }^{* *}(\alpha<0,01)$.

Interessant ist, dass zwar das Wirtschaftswachstum keinen signifikanten Erklärungsgehalt in bezug auf die Ausgabenvariabilität besitzt, wohl aber die mit dem Schmidt'schen Institutionenindikator bewertete Wachstumsrate. Das positive Vorzeichen vor diesem Interaktionsterm könnte dann gemäß der obigen Argumentation dahingehend interpretiert werden, dass gerade in jenen Staaten, die 
komplizierte Entscheidungsinstitutionen haben, Wirtschaftswachstum zu intensiven log-rolling Aktivitäten und folglich zu stärkeren Ausgabenänderungen führt. Der Einfluss der Inflationsrate ist erwartungsgemäß positiv und in den meisten Modellen mindestens auf dem 5\%-Niveau signifikant.

Besonders relevant ist schließlich der Einfluss der Ideologievariablen $\mathrm{CG}_{\mathrm{t}}$ und RCG4. In keiner Modellvariante ist die $\mathrm{CG}_{\mathrm{t}}$-Variable signifikant. Die Koeffizienten haben nicht einmal das erwartete Vorzeichen; angesichts der sehr kleinen Koeffizientenwerte und der verhältnismäßig hohen Streuung lässt sich allerdings die Hypothese der Zufälligkeit dieses Ergebnisses nicht widerlegen. Das bedeutet, über längere Zeiträume unterscheiden sich rechte und linke Parteien nicht in ihrer Veränderungsdynamik. Allerdings zeigen die hoch signifikanten Schätzergebnisse für die RCG4 ${ }^{-}$-Variable, dass zumindest in den ersten vier Jahren nach einem Regierungswechsel linke Regierungen deutlich wandlungsfähiger in bezug auf ihre Haushaltsstrukturen sind. Diese Wandlungsbereitschaft geht danach jedoch zurück, sodass bei der generellen Prüfung des Einflusses der Ideologie kein Unterschied zwischen den Parteien mehr festzustellen ist.

Die These von Schmidt, dass v.a. rechte Parteien sich von linken und MitteParteien unterscheiden, kann hier nicht zufriedenstellend bestätigt werden, da der Einfluss der Ideologievariablen in bezug auf die beiden endogenen Variablen AUSG_VAR und LOG(AUSG_VAR) kaum verschieden ist. Dies könnte natürlich auch daran liegen, dass die Logarithmustransformation nur unzureichend zwischen rechts und nicht-rechts differenziert. Offensichtlich ist allerdings, dass der lineare Zusammenhang zwischen Ideologie und Ausgabenvariabilität zumindest nicht schlechter ist als der nicht-lineare.

Schließlich stellt sich die Frage, ob dieses Ergebnis eines negativen Einflusses von RCG4 a auf AUSG_VAR gegebenenfalls nur das Resultat der höheren Ausgabenneigung linker Parteien in den siebziger und achtziger Jahren ist und nicht deren generelle Offenheit gegenüber Veränderung abbildet. Da der Einfluss von RCG4 ${ }_{\mathrm{t}}$ deutlich negativ ist, muss dies so interpretiert werden, dass den Ausgabensteigerungen der linken Parteien zumindest keine äquivalenten Ausgabenkürzungen der rechten Parteien gegenüber standen, bzw. Strukturveränderungen, die von linken Parteien durchgeführt wurden, wurden nicht in äquivalenter Höhe von rechten Parteien wieder zurückgenommen, da ansonsten kein negatives Vorzeichen vorläge. Grundsätzlich kann diese Beobachtung damit begründet werden, dass linke Parteien einen strategischen Vorteil haben, da es durchaus plausibel ist anzunehmen, dass man dem Wähler Ausgabenerhöhungen leichter erklären kann als deren Kürzungen. Es ist aber auch nicht auszuschließen, dass konservative Parteien bereit sind, einen neu definierten Status quo nach der Veränderung dem Status quo ante vorziehen. Dann wären konservative Parteien in der Tat stärker wandlungsabgeneigt. 


\subsection{Cluster gemäß den ökonomischen Indikatoren}

Im weiteren werden nur noch die Ergebnisse der LOG(AUSG_VAR)-Variable dargestellt, da die Bestimmtheitsmaße dieser Regressionen i.d.R. höher ausgefallen sind und die Koeffizientenwerte besser darstellbar sind.

In Tabelle 5.27 sind die Regressionsergebnisse für die drei verschiedenen Ländercluster und den gesamten Untersuchungszeitraum dargestellt. Es werden für jeden Cluster zwei Modellvarianten geschätzt. Zum einen wird ein Modell mit der $\mathrm{CG}_{\mathbf{t}}$-Variable und zum anderen ein Modell mit der $\mathrm{RCG} 4_{\mathrm{t}}$-Variable geschätzt. Einige der Aussagen von zuvor lassen sich auch hier treffen, andere sind einzuschränken.

\section{Tabelle 5.27 : Ausgabenvariabilität in den ökonomischen Clustern 1960-1996}

\begin{tabular}{|c|c|c|c|c|c|c|}
\hline \multirow[b]{4}{*}{$\begin{array}{l}\text { unabhängige } \\
\text { Variable }\end{array}$} & \multicolumn{4}{|c|}{ abhängige Variable: LOG(AUS_VAR) } & & \\
\hline & \multirow{2}{*}{\multicolumn{2}{|c|}{$\begin{array}{c}\text { 1. Cluster } \\
(B E L, N L, N Z L, S, U K)\end{array}$}} & \multirow{2}{*}{\multicolumn{2}{|c|}{$\begin{array}{c}\text { 2. Cluster } \\
\text { (CAN, DK, SF, IRL, } \\
\text { NOR, CH) }\end{array}$}} & \multirow{2}{*}{\multicolumn{2}{|c|}{$\begin{array}{c}\text { 3. Cluster } \\
\text { (AUS, AU, F, D, ITA, J, } \\
\text { E, USA) }\end{array}$}} \\
\hline & & & & & & \\
\hline & (1) & (2) & (1) & (2) & (1) & (2) \\
\hline TREND & - & 一 & - & - & $\begin{array}{l}-0,016 * * \\
(-4,940)\end{array}$ & $\begin{array}{l}-0,016 * * \\
(-4,435)\end{array}$ \\
\hline D_U, & $\begin{array}{c}0,115+ \\
(1,933)\end{array}$ & $\begin{array}{c}0,148^{*} \\
(2,524)\end{array}$ & $\begin{array}{c}0,159 * * \\
(3,672)\end{array}$ & $\begin{array}{l}0,164^{* *} \\
(3,867)\end{array}$ & $\begin{array}{c}0,052 \\
(1,289)\end{array}$ & $\begin{array}{c}0,056 \\
(1,346)\end{array}$ \\
\hline WIWA $\cdot$ - INST & $\begin{array}{c}-0,009 \\
(-0,573)\end{array}$ & $\begin{array}{c}-0,010 \\
(-0,694)\end{array}$ & $\begin{array}{c}0,005 \\
(0,797)\end{array}$ & $\begin{array}{c}0,005 \\
(0,826)\end{array}$ & - & - \\
\hline MAAST & $\begin{array}{l}-0,269 \\
(-1,660)\end{array}$ & $\begin{array}{c}-0,297+ \\
(-1,943)\end{array}$ & $\begin{array}{c}-0,164 \\
(-0,834)\end{array}$ & $\begin{array}{l}-0,260 \\
(-1,309)\end{array}$ & - & - \\
\hline $\mathrm{INFL}_{1}$ & - & - & - & - & $\begin{array}{l}0,025 * * \\
(2,873)\end{array}$ & $\begin{array}{l}0,022^{*} \\
(2,395)\end{array}$ \\
\hline $\mathrm{CG}_{\mathrm{t}}$ & $\begin{array}{l}-0,015 \\
(-0,525)\end{array}$ & 一 & $\begin{array}{c}0,017 \\
(0,370)\end{array}$ & - & $\begin{array}{c}-0,011 \\
(-0,448)\end{array}$ & - \\
\hline RCG2, & - & $\begin{array}{l}-0,072 * * \\
(-2,685)\end{array}$ & - & $\begin{array}{l}-0,070+ \\
(-1,670)\end{array}$ & - & - \\
\hline RCG4, & - & - & - & - & - & $\begin{array}{c}-0,036+ \\
(-1,721)\end{array}$ \\
\hline $\mathbf{R}_{\text {adj. }}^{2}$ & 0,13 & 0,19 & 0,07 & 0,09 & 0,27 & 0,28 \\
\hline
\end{tabular}

Anmerkungen:

t-Werte in Klammern, $+(\alpha<0,1),{ }^{*}(\alpha<0,05),{ }^{* *}(\alpha<0,01)$. 
So ist der Trend eines deutlichen Rückgangs der Ausgabenvariabilität nur im dritten Ländercluster zu erkennen. In den anderen beiden Clustern gab es keine signifikanten Schätzergebnisse, daher wurde auf die Darstellung der Koeffizienten verzichtet. Dieses Ergebnis ist im Einklang mit den Resultaten aus Tabelle 5.22. Auch dort wurde lediglich für den dritten Cluster ein klarer Trend zu geringeren Ausgabenzuwächsen gefunden. Beide Ergebnisse sind sicherlich erstaunlich, da im dritten Cluster gerade die besonders geschlossenen Länder enthalten sind. Der Einfluss des Arbeitsmarktes ist wie erwartet deutlich positiv in allen Regressionen, die Inflationsrate jedoch lediglich in dem dritten Cluster.

Interessanterweise ist in der ökonomischen Clusterung der Einfluss des Interaktionsterms aus Wachstumsrate und dem Schmidt-Indikator nicht mehr signifikant. Offensichtlich ist hier das Schätzergebnis nicht sehr robust hinsichtlich des Ländersamples. Daher sollte die obige Aussage von stärkerem log-rolling in Staaten mit starken institutionellen Restriktionen nicht zu stark verallgemeinert werden. ${ }^{351}$

Die $\mathrm{CG}_{\mathrm{t}}$-Variable besitzt auch hier in keiner der drei Clusterungen signifikanten Erklärungsgehalt. Allerdings deuten die einheitlich signifikanten Koeffizienten für die $\mathrm{RCG}_{\mathrm{t}}$-Variable darauf hin, dass tatsächlich linke Parteien vor allem in den ersten Jahren nach der Regierungsübernahme besondere Dynamik entwickeln. Bemerkenswert ist, dass dieser Zusammenhang besonders deutlich sowohl hinsichtlich des Signifikanzniveaus als auch der Koeffizientenhöhe gerade in den besonders offenen Ländern zu sein scheint. Sieht man also in der unterschiedlichen Ausgabendynamik ein konstituierendes Merkmal zur Unterscheidung zwischen linker und rechter Politik, so wird dies in den offenen Ländern eher akzentuiert denn restringiert.

Dieses Ergebnis wird durch die Regressionen in Tabelle 5.28 gestützt. Dort werden ähnliche Schätzmodelle für die drei ökonomisch unterschiedlichen Zeitphasen präsentiert. ${ }^{352}$ Es soll auf drei Sachverhalte besonders geachtet werden. Erstens nimmt der Interaktionsterm nur in der Zeit nach 1986 ein deutlich positives Vorzeichen an, folglich hätte die log-rolling-Hypothese nur für diesen Zeitraum Bestätigung. Zweitens haben auch hier die RCG-Koeffizienten eindeutig die höhere Erklärungskraft im Vergleich zu den $\mathrm{CG}_{\mathrm{t}}$-Variablen, und die beste Bestätigung findet sich gerade für die Zeitphase, die mit der stärksten internatio-

351 Erklärbar wird dieses Abweichen von dem vorherigen Ergebnis aus dem Gesamtpanel, wenn man sich vergegenwärtigt, dass der Indikator von Schmidt die Werte 0 bis 5 annimmt (eigentlich bis 6, dies kommt aber empirisch nicht vor). Da in kleineren Stichproben Ausreißer zu stärkeren Verzerrungen führen, bedeutet die Multiplikation mit der INST-Variable, dass Zufallsschwankungen bei kleinen Stichproben sehr stark ins Gewicht fallen können.

352 Der dritte und vierte Cluster wurden zusammengefasst, um die Anzahl der Beobachtungen und somit die statistische Validität zu erhöhen. Tobias Just - 978-3-631-75139-8 
nalen Integration verbunden wird. Drittens wird hier an einigen Stellen deutlich, dass linke Parteien nicht nur bemüht sind, durch Ausgabensteigerungen ein anderes Verteilungsziel zu erreichen, sondern scheinbar auch grundsätzlich rascher bereit bzw. in der Lage sind, einen Strukturwandel durchzuführen.

Tabelle 5.28 : Ausgabenvariabilität in den ökonomischen Clustern (alle Länder)

\begin{tabular}{|c|c|c|c|c|c|c|}
\hline \multicolumn{7}{|c|}{ abhängige Variable: LOG(AUS_VAR) } \\
\hline \multirow[b]{3}{*}{$\begin{array}{l}\text { unabhängige } \\
\text { Variable }\end{array}$} & \multirow{2}{*}{\multicolumn{2}{|c|}{$\begin{array}{c}1961-1973 \\
(1 . \text { Zeitcluster })\end{array}$}} & \multirow{2}{*}{\multicolumn{2}{|c|}{$\begin{array}{c}1974-1985 \\
(2 . \text { Zeitcluster })\end{array}$}} & \multirow{2}{*}{\multicolumn{2}{|c|}{$\begin{array}{c}1986-1996 \\
(3 .+4 . \text { Zeitcluster })\end{array}$}} \\
\hline & & & & & & \\
\hline & (1) & $(2)$ & (1) & (2) & (1) & $(2)$ \\
\hline TREND & - & - & - & - & $\begin{array}{c}0,034^{*} \\
(2,542)\end{array}$ & $\begin{array}{c}0,029 * \\
(2,268)\end{array}$ \\
\hline D_Ut & $\begin{array}{c}0,017 \\
(0,211)\end{array}$ & $\begin{array}{c}-0,004 \\
(-0,043)\end{array}$ & $\begin{array}{c}0,125^{*} \\
(2,284)\end{array}$ & $\begin{array}{c}0,129 * \\
(2,331)\end{array}$ & $\begin{array}{l}0,155^{* *} \\
(4,120)\end{array}$ & $\begin{array}{c}0,185^{* *} \\
(4,983)\end{array}$ \\
\hline WIWA $_{t} \cdot$ INST & $\begin{array}{c}-0,005 \\
(-0,976)\end{array}$ & $\begin{array}{c}-0,004 \\
(-0,838)\end{array}$ & $\begin{array}{c}0,007 \\
(1,070)\end{array}$ & $\begin{array}{c}0,007 \\
(1,069)\end{array}$ & $\begin{array}{l}0,018^{* *} \\
(3,145)\end{array}$ & $\begin{array}{l}0,018^{* *} \\
(3,374)\end{array}$ \\
\hline $\mathrm{INFL}_{4}$ & $\begin{array}{c}0,007 \\
(0,374)\end{array}$ & $\begin{array}{c}0,023 \\
(1,278)\end{array}$ & $\begin{array}{c}0,031 * \\
(2,271)\end{array}$ & $\begin{array}{c}0,030^{*} \\
(2,154)\end{array}$ & $\begin{array}{l}0,067 * * \\
(2,734)\end{array}$ & $\begin{array}{l}0,062 * * \\
(2,645)\end{array}$ \\
\hline $\mathrm{CG}_{\mathbf{1}}$ & $\begin{array}{c}0,020 \\
(0,605)\end{array}$ & - & $\begin{array}{c}-0,041 \\
(-1,354)\end{array}$ & - & $\begin{array}{c}0,038 \\
(0,933)\end{array}$ & - \\
\hline RCG2, & - & - & - & - & - & $\begin{array}{l}-0,094 * * \\
(-2,967)\end{array}$ \\
\hline RCG4, & - & $\begin{array}{c}-0,035 \\
(-1,602)\end{array}$ & - & $\begin{array}{c}-0,032 \\
(-1,437)\end{array}$ & - & - \\
\hline $\mathrm{R}_{\mathrm{adj} .}^{2}$ & 0,12 & 0,23 & 0,15 & 0,15 & 0,30 & 0,33 \\
\hline
\end{tabular}

Anmerkungen:

$\mathrm{t}$-Werte in Klammern, $+(\alpha<0,1),{ }^{*}(\alpha<0,05),{ }^{* *}(\alpha<0,01)$.

Vergleicht man z.B. die Regressionsergebnisse des ersten Länderclusters in Tabelle 5.27 mit den Regressionsergebnissen für denselben Ländercluster in Tabelle 5.22, so erkennt man, dass für diese Länder zwar ein signifikanter $\mathrm{Zu}$ sammenhang zwischen Ideologie und Ausgabenvariabilität besteht, jedoch nicht zwischen Ideologie und Höhe der Ausgabensteigerung. Das bedeutet, dass sich in diesen Ländern linke Parteien nicht ausschließlich durch Ausgabenerhöhungen, sondern v.a. durch Änderungen der Ausgabenstrukturen positioniert haben. Diese Strukturveränderungen waren nicht systematisch immer zu den idealtypisch linken Ausgabenpositionen, denn sonst wären diese Ausgabengruppen signifikant schneller gewachsen. Dem war aber gerade nicht so. 
Ähnliches gilt für die Zeitphase von 1986 bis 1996. Auch hier ist zwar ein signifikanter Zusammenhang zwischen der Ideologievariable und der Bereitschaft, Ausgabenanteile zu verändern festzustellen. Es lässt sich jedoch kein systematischer Zusammenhang zwischen der Ideologievariable und dem Anstieg einer Ausgabengruppe ablesen (Tabelle 5.23).

Es scheint also durchaus gerechtfertigt zu sein, Linkssein mit einer tendenziell höheren Neigung für budgetäre Experimente zu verbinden. Es wird im folgenden untersucht, ob das zentrale Ergebnis des Einflusses der Ideologievariablen auf den Indikator für Ausgabenvariabilität robust hinsichtlich der Clusterung ist.

\subsection{Cluster gemäß den politischen Indikatoren}

Im wesentlichen werden die zuvor gefundenen Ergebnisse auch in dieser Clusterung bestätigt.

Sowohl in der Länder- als auch in der zeitlichen Clusterung ist ein negativer Trend festzustellen. Außerdem bestätigt sich der starke Einfluss des Arbeitsmarktes und vereinzelt der Einfluss der Preisentwicklung. Hinsichtlich des Interaktionsterms zeigt sich auch hier, dass die Wirkungsweise nicht sehr robust hinsichtlich der Ländersample zu sein scheint. Man sollte diese Ergebnisse folglich nicht überinterpretieren.

Es ist aber v.a. darauf hinzuweisen, dass auch in dieser Clusterung die $\mathrm{RCG}^{-}$ Variable eindeutig bessere Ergebnisse liefert als die allgemeinere $\mathbf{C G}_{t}-$ Variable. Auch wird deutlich, dass der Zusammenhang zwischen einem Regierungswechsel nach links und der daran anschließenden Ausgabendynamik in offenen Volkswirtschaften nicht deutlich schwächer ausfällt als in besonders geschlossenen Ländern. Zwar ist das Vorzeichen für den ersten Cluster in Tabelle 5.29 nicht signifikant, doch dafür ist der Zusammenhang für den dritten Cluster sogar umgekehrt ${ }^{353}$ und für den zweiten Cluster wurde ein vergleichbarer Koeffizientenwert geschätzt wie für den besonders geschlossenen vierten Cluster. Außerdem ist auch in der letzten Zeitphase das Vorzeichen eindeutig negativ (vgl. Tabelle 5.30). ${ }^{354}$

${ }^{353}$ Dies lässt sich dadurch begründen, dass im dritten Cluster lediglich drei Länder enthalten sind, von denen die USA z.B. unter dem Republikaner Ronald Reagan einen deutlichen Strukturbruch und unter dem Demokraten Clinton eher eine Fortsetzung der konservativen Politik erlebte. Eine Regression ohne die USA im dritten Cluster führt tatsächlich auch für den dritten Cluster zu dem theoriekonformen Vorzeichen.

${ }^{354}$ Auch hier wurden Zeitphasen zusammengefasst, um die Anzahl der Beobachtungen in den Regressionen zu erhöhen. 
Tabelle 5.29 : Ausgabenvariabilität in der Clusterung gemäß politischen Globalisierungsindikatoren 1960-1997

abhängige Variable: LOG(AUS_VAR)

$\begin{array}{llll}\text { 1. Cluster } & \text { 2. Cluster } & \text { 3. Cluster } & \text { 4. Cluster }\end{array}$

unabh.

Variable (D, DK, ITA, NL, BEL, F) (UK, S, AU, NOR, SF, IRL) (USA, CAN, CH) (E, J, AUS, NZL)

(1)

$-0,015 * * \quad-0,013 *$

$(-2,806)$

$0,113+$

$(1,744)$

$0,010^{*}$

$(2,100)$

$0,033 * *$

$(2,700)$

$-0,012$

$(-0,346)$

(2)

(1)

(2)

(1)

(2)

(1)

(2)

$-0,006 \quad-0,009$

$-0,008+\quad-0,006$

$-0,009$

$-0,009$
$(-1,449)$

$0,206 *$

$(2,558)$

$(3,456)$

$0,154 * *$

$(1,793)$

$-0,008$

$(4,070)$

0,010

$(1,908)$

$(-0,864)$

$-0,008$

$(-0,908)$

$(1,534)$

$0,033 * *$

$(2,663)$

-

- $\quad 0,022$

0,022
$(0,835)$

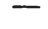

-

-

$-0,070 * *$

$(-2,883)$

$-0,044$

$(-1,593)$

0,24

0,25

0,13

0,121

$(1,599)$

-

$0,215^{* *}$

$(2,823)$

$0,014 *$

$(2,577)$

0,003

$(0,142)$

$-$

$0,146+$

$(1,923)$

$\begin{array}{ll}-0,025^{* *} & -0,021^{* *} \\ (-2,870) & (-2,879)\end{array}$

0,043

0,009

$(0,673)$

$(0,136)$

$-0,014$

$(-1,537)$

$-0,016+$

$-1,801)$

(1)

0,23

0,21

t-Werte in Klammern, $+(\alpha<0,1),{ }^{*}(\alpha<0,05),{ }^{* *}(\alpha<0,01)$. 
Tabelle 5.30 : Ausgabenvariabilität in den politischen Clustern (alle Länder)

\begin{tabular}{|c|c|c|c|c|c|c|}
\hline \multicolumn{7}{|c|}{ abhängige Variable: LOG(AUS_VAR) } \\
\hline \multirow[b]{3}{*}{$\begin{array}{l}\text { unabhängige } \\
\text { Variable }\end{array}$} & \multirow{2}{*}{\multicolumn{2}{|c|}{$\begin{array}{c}1960-1978 \\
(1 .+2 . \text { Cluster })\end{array}$}} & \multirow{2}{*}{\multicolumn{2}{|c|}{$\begin{array}{l}1979-1987 \\
\text { (3. Cluster) }\end{array}$}} & \multirow{2}{*}{\multicolumn{2}{|c|}{$\begin{array}{c}1988-1996 \\
(4 .+5 . \text { Cluster })\end{array}$}} \\
\hline & & & & & & \\
\hline & (1) & (2) & (1) & (2) & (1) & (2) \\
\hline TREND & $\begin{array}{l}-0,039 * * \\
(-4,370)\end{array}$ & $\begin{array}{c}-0,039 * * \\
(-3,633)\end{array}$ & - & 一 & - & - \\
\hline D_Ut & $\begin{array}{c}0,043 \\
(0,848)\end{array}$ & $\begin{array}{c}0,026 \\
(0,498)\end{array}$ & $\begin{array}{l}0,182 * * \\
(3,027)\end{array}$ & $\begin{array}{l}0,196^{* *} \\
(3,186)\end{array}$ & $\begin{array}{c}0,167 * * \\
(4,167)\end{array}$ & $\begin{array}{c}0,192^{* *} \\
(4,850)\end{array}$ \\
\hline WIWA $_{t} \cdot$ INST & - & - & $\begin{array}{c}0,014+ \\
(1,695)\end{array}$ & $\begin{array}{c}0,015+ \\
(1,898)\end{array}$ & $\begin{array}{l}0,017 * * \\
(2,923)\end{array}$ & $\begin{array}{l}0,018^{* *} \\
(3,172)\end{array}$ \\
\hline $\mathrm{INFL}_{4}$ & $\begin{array}{c}0,052 * * \\
(4,721)\end{array}$ & $\begin{array}{c}0,053 * * \\
(4,777)\end{array}$ & $\begin{array}{c}0,013 \\
(0,829)\end{array}$ & $\begin{array}{c}0,018 \\
(1,113)\end{array}$ & $\begin{array}{c}0,021 \\
(0,857)\end{array}$ & $\begin{array}{c}0,026 \\
(1,062)\end{array}$ \\
\hline $\mathrm{CG}_{\mathrm{t}}$ & $\begin{array}{c}-0,014 \\
(-0,485)\end{array}$ & - & $\begin{array}{c}-0,097 * \\
(-2,510)\end{array}$ & - & $\begin{array}{c}0,022 \\
(0,514)\end{array}$ & - \\
\hline RCG2, & - & - & - & - & - & $\begin{array}{c}-0,078^{*} \\
(-2,203)\end{array}$ \\
\hline RCG4, & - & $\begin{array}{c}-0,038+ \\
(-1,905)\end{array}$ & - & $\begin{array}{c}-0,064^{*} \\
(-2,418)\end{array}$ & - & - \\
\hline $\mathbf{R}_{\text {adj. }}^{2}$ & 0,15 & 0,20 & 0,25 & 0,24 & 0,30 & 0,32 \\
\hline
\end{tabular}

Anmerkungen:

t-Werte in Klammern, $+(\alpha<0,1),{ }^{*}(\alpha<0,05),{ }^{* *}(\alpha<0,01)$.

Dieses negative Vorzeichen für den Zeitraum von 1988 bis 1996 ist nicht zuletzt deshalb von Interesse, weil es nicht allein durch stärkeres Ausgabenwachstum der linken Parteien in dieser Periode erklärt werden kann (vgl. Tabelle 5.25). Folglich nahmen linke Parteien stärkere Veränderungen in der Haushaltsstruktur vor, dies jedoch nicht immer zugunsten zunehmender Ausgabenpositionen.

Grundsätzlich lässt sich die Vermutung, dass linke Parteien progressiver wären und demnach rascher Kurswenden durchführten, eher bestätigen als ablehnen. Allerdings muss man sich vergegenwärtigen, dass die Ursache, warum dies in den Regressionen gemessen werden konnte, damit noch nicht geklärt ist. Es sind drei plausible Begründungen anführbar:

1. Linke Parteien definieren sich tatsächlich zumindest teilweise über die größere Affinität zu neuen Themen und Projekten. Dies kann dann mitunter auch dazu führen, dass dabei traditionell linke Themen geopfert werden wie z.B. in Neuseeland seit 1984 (Evans et al, 1996). Konservative Parteien brauchen

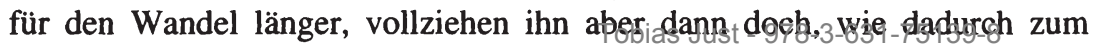


Ausdruck kommt, dass für die allgemeine $\mathrm{CG}_{\mathrm{t}}$-Variable kaum signifikante Werte geschätzt wurden. Konservative Parteien sind lediglich in den ersten Jahren zögerlicher.

2. Die in den Schätzungen gefundene höhere Variabilität der Ausgabenanteile während linker Regierungen kann aber auch daran liegen, dass linke Parteien mitunter einen strategischen Vorteil besitzen. Zum einen lassen sich einige Wählergruppen besser durch Ausgabenerhöhungen ködern als durch deren Senkungen. Zum anderen haben linke Parteien einen deutlichen Glaubwürdigkeitsvorteil in der Situation, wenn Ausgabenkürzungen die einzige Handlungsmöglichkeit der Politik bleiben. Nach dem Diktum von Cukierman und Tommasi (1998): „When Does it Take a Nixon to Go to China“ ist zu erwarten, dass die Handlungsnotwendigkeit zu Ausgabenkürzungen besonders dann glaubwürdig erscheint, wenn sie von jenen propagiert wird, die eher gegen Kürzungen sind. Das heißt, linke Parteien können solche unwillkommenen Projekte schneller durchsetzen, weil man ihnen glaubt, dass sie keine andere Wahl haben.

3. Es ließe sich auch argumentieren, dass gerade in Zeiten reduzierter Handlungsspielräume der letzten Jahre die Ausgabenvariabilität der Linken deshalb deutlicher ausfiel als ihre Ausgabensteigerungen, weil die Globalisierung Ausgabenwachstum nur noch begrenzt zulässt und dass daher ,linke“ Projekte zu Lasten ,rechter“ Ausgaben gestärkt werden. Immerhin wurde gezeigt, dass die Differenzen zwischen den Ausgabenänderungen linker und rechter Regierungen in den letzten Jahren nicht mehr so stark ausfallen wie in den 70er und 80er Jahren. Die höhere Variabilität wäre dann Substitut für nicht mehr mögliche Steigerungsraten. Allerdings benötigt man für diese Argumentationslinie dann wieder Erklärung 1. oder 2., um zu begründen, warum bei einem Regierungswechsel nach rechts nicht wieder der alte Zustand angestrebt wird.

\subsubsection{Zusammenfassung}

In diesem Abschnitt wurde untersucht, inwiefern linke und rechte Regierungen unterschiedliche Budgetpolitik betrieben haben bzw. noch immer betreiben. Es wurden eine Reihe teilweise erwarteter und zum Teil überraschender Ergebnisse erzielt. Es soll an dieser Stelle ausschließlich auf die besonders interessierenden Ideologievariablen fokussiert werden.

Hinsichtlich der Hypothese, linke Parteien würden vornehmlich jene Ausgabenpositionen stärken, die verteilungsmäßig besonders regressiv wirken, wurde gezeigt, dass die Gesamtausgaben, besonders aber die um die Militär- und Zinsausgaben verringerten Gesamtausgaben, insbesondere unter linken Regierungen schneller anstiegen als das Bruttoinlandsprodukitas Auch die_zixilen Konsumaus- 
gaben wiesen deutlich diese Struktur auf. Wider die Erwartungen konnte jedoch weder für die Transferausgaben noch für die Subventionen dasselbe Resultat gezeigt werden, dass deren Anstieg v.a. unter linken Regierungen erfolgte.

Diese Differenz des Ausgabenwachstums ist aber v.a. in den ersten vier Jahren nach dem Regierungswechsel zu finden (teilweise sogar in noch kürzeren Zeitspannen). Linke Parteien schaffen es besser, ihre gewünschten Budgetstrukturen bereits in den ersten Jahren zu erreichen, rechte Regierungen gelingt dies offensichtlich nicht in äquivalentem $\mathrm{Maß}$.

Bezüglich der Globalisierungsthese wurde deutlich, dass die über das gesamte Länderpanel geschätzte Differenz der Parteien im letzten Jahrzehnt deutlich abgenommen hat. In der Zeitphase nach 1986 konnte die Hypothese einer Unterschiedslosigkeit des Budgetwachstums nicht widerlegt werden. Ob dies nun jedoch Ausdruck von Handlungsbegrenzungen oder von Lernprozessen ist, kann nicht eindeutig beantwortet werden, denn in der politischen Clusterung sind es insbesondere die besonders geöffneten Länder, in denen Partisaneffekte im Haushalt isoliert werden können. Allein die marktliche Öffnung eines Landes scheint demnach nicht alle haushaltspolitischen Spielräume einzuebnen. Im Falle des ersten politischen Clusters könnte eingeräumt werden, dass die dort zusammengefassten Länder sämtlich Mitglieder der Europäischen Union sind. Durch dieses supranationale Organ wird gegebenenfalls die Aufgabe von Souveränität in Folge der Öffnung der Märkte durch die politische und auch wirtschaftliche Macht dieses weitgehend autarken Akteurs ausgeglichen. Mit anderen Worten: die Institution der EU gibt den Nationalstaaten budgetären Handlungsspielraum zurück, der durch die globalen Märkte verloren zu gehen droht. Diese Interpretation wird dadurch gestützt, dass in der ökonomischen Clusterung die besonders offenen Länder auch in der Tat kaum Partisaneffekte in den Haushalten aufweisen.

Ein zweites Argument folgt aus der Analyse der Haushaltsentwicklungen der 18 OECD-Staaten auf ihre generelle Flexibilität. Es konnte gezeigt werden, dass die Hypothese, linke Regierungen wären eher zu Strukturveränderungen bereit, tatsächlich Berechtigung hat, jedoch lediglich in der kurzen Frist. In den ersten vier Jahren agieren linke Parteien tendenziell „experimentierfreudiger" als rechte Regierungen. Diese Verschiedenheit verschwindet jedoch, wenn man nicht nur die ersten vier Jahre nach einem Regierungswechsel betrachtet.

Die Tatsache, dass diese unterschiedliche Änderungsdynamik gerade in den letzten Jahren besonders deutlich wurde, mag man so interpretieren, dass linke Parteien auch heute noch deutliches Profilierungspotential besitzen. Eine andere mögliche Erklärung könnte darin bestehen, dass durch diese deutlich unterschiedliche Änderungsintensität der Parteien die Handlungsrestriktionen der Globalisierung besonders deutlich werden. Geht man davon aus, dass linke Parteien einen grundsätzlichen, strategischen Glaubwürdigkeitsvorteil hinsichtlich

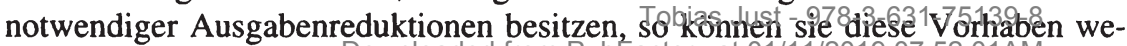


sentlich schneller durchführen als rechte Parteien. Dies bedeutet jedoch nicht, dass in den 90er Jahren alle linken Parteien nur noch idealtypisch rechte Politik betrieben haben, denn die Daten für die Zeit von 1986 bis 1996 zeigen keineswegs einen positiven Zusammenhang zwischen der Ideologievariable und der Ausdehnung der Staatsausgabenanteile. Dieser wäre aber die logische Konsequenz, falls die linken Regierungen wirklich erfolgreichere rechte Politik betrieben hätten.

Insgesamt muss sicherlich eingeräumt werden, dass sich in den Jahren nach 1996 der Globalisierungsprozess im Vergleich zu allen Vorjahren eher weiter beschleunigt hat. Es ist durchaus plausibel, dass sich die hier bereits angedeuteten Ergebnisse noch stärker abzeichnen. Der Staatshaushalt würde zunehmend an Bedeutung für die parteiliche Profilierung verlieren, und es würde allenfalls die Umsetzungsgeschwindigkeit von Projekten variiert. Sollte jedoch allein der Glaubwürdigkeitsvorsprung der Linken dafür ursächlich sein, dass Linke rascher Sparprogramme durchsetzen können, so wird auch diese Unterschiedlichkeit zwischen den Parteien um so stärker verschwinden, je mehr die Wahlbürger diesen überparteilichen Konsens in der Budgetpolitik verstehen, denn dann verlieren die Linken ihren relativen Glaubwürdigkeitsvorsprung. 
Tobias Just - 978-3-631-75139-8

Downloaded from PubFactory at 01/11/2019 07:52:01AM

via free access 


\section{Kapitel 6}

\section{Schlussbemerkungen}

Die Kernfrage der vorliegenden Arbeit war, ob und inwiefern ideologisch motivierte Wirtschaftspolitik durch die internationale Integration der Faktor- und Gütermärkte unmöglich geworden ist. Dafür musste in einem ersten Schritt geklärt werden, wie der Begriff Ideologie zu definieren ist und ob diese Ideologievorstellung kompatibel mit dem ökonomischen „model of man“ eines rational eigennutzorientierten Individuums ist. Zweitens musste für eine empirische Analyse der sehr weite Ideologiebegriff operationalisiert werden. Es galt also die Frage zu beantworten, wie Ideologie im wirtschaftspolitischen Bereich am exaktesten erfasst werden kann. Da sowohl in der wissenschaftlichen Literatur als auch in der politischen Diskussion der Begriff der Globalisierung sehr unterschiedlich definiert wird, musste zum dritten eine Definition gegeben werden, die geeignet für die empirische Hypothesenprüfung ist. Schließlich wurde in einem vierten und letzten Schritt untersucht, ob die gewählten Ideologieindikatoren empirische Erklärungskraft für die Erklärung der Arbeitslosenquote, der Inflationsrate und der Budgetstruktur in einem Land besitzen. Anschließend wurde geprüft, inwiefern dieser Zusammenhang von der Offenheit eines Landes abhängt. In diesem letzten Kapitel sollen nun nicht nur die in dieser Arbeit gegebenen Antworten auf die obigen Fragen zusammengefasst und bewertet werden, sondern auch die daraus zum Teil erwachsenden Problemstellungen skizziert werden.

Die erste Frage nach der Möglichkeit der Integration des soziologischen Ideologiebegriffs in die ökonomische Analyse wurde eindeutig bejaht. Fasst man Ideologie als die Orientierung der (politischen) Ziele an einem höheren Wertemaßstab hinsichtlich des gesellschaftlich Wünschenswerten auf, so wird deutlich, dass eine einfache Integration einer Ideologievariable in die Nutzenfunktion, wie es üblicherweise in politökonomischen Texten geschieht, mikroökonomisch erst zu rechtfertigen ist, da dies zunächst im Widerspruch sowohl zu der Rationalitätsannahme als auch zum Postulat des methodologischen Individualismus zu sein scheint. ${ }^{355}$

${ }^{355}$ Definiert man das Verfolgen einer bestimmten Ideologie als nutzenstiftend, ergibt sich allerdings ex definitione kein Widerspruch zur Annahme der Eigennutzorientierung. Es wurde allerdings argumentiert, dass Werte auch als Restriktionen aufgefasst werden können; aber auch dann sind sie nicht im Widerspruch zur Ejigennugzannahme $75139-8$ 
Drei Ansätze wurden für diese Rechtfertigung dargestellt und diskutiert. Der erste Ansatz von Taylor und Kirsch basierte auf der Grundannahme, dass der Mensch nicht nur selbstorientiert im Sinne reiner materieller Bedürfnisbefriedigung ist, sondern darüber hinaus auch Interesse daran hat, das durch seine Handlungen entworfene Selbstbildnis zu akzeptieren. Dies, so die Annahme, erreicht er nur, wenn er sich an den gesellschaftlichen Wertekanon hält oder zumindest nicht zu weit davon abweicht, ${ }^{356}$ und hat zur Folge, dass sich Individuen eigennützig an die Verfolgung von Gruppeninteressen halten, die durch den Wertekanon bestimmt sind.

Der zweite Ansatz, der auf Harsanyi, Sen und Margolis zurückgeht, beruhte auf der grundsätzlichen Annahme, dass der Mensch eine Art Fair Share Regel als genetische Mitgift verfolgt, die besagt, dass der Mensch sowohl Selbst- als auch Gruppeninteressen berücksichtigt und stets gegeneinander abwägt. Das bedeutet, jeder Mensch verfolgt in unterschiedlichem Ausmaß völlig uneigennützig auch Gruppeninteressen. Damit wird das ökonomische Konzept der Eigennutzorientierung sozusagen zu großen Teilen über Bord geworfen, da die Annahme des Eigennutzes nicht den Anspruch der Allgemeingültigkeit erheben kann.

Beiden Ansätzen ist inhärent, dass der oben angesprochene vermeintliche Widerspruch durch Aufnahme eines neuen Elements in der Nutzenfunktion wegdefiniert wird. Danach verhält sich der Mensch gruppenorientiert bzw. wertefundiert, weil es ihm Nutzen stiftet bzw. Nutzenverluste verhindert. Dies muss nicht notwendigerweise abwegig sein, grenzt aber sehr stark an Tautologien und eignet sich daher kaum für empirische Untersuchungen oder Verhaltensprognosen; v.a. erfordern beide Theorien eine sehr weitgehende Neuschreibung der ökonomischen Theorie.

Der dritte Ansatz, der eine Übertragung des Becker'schen Konzepts der Haushaltsproduktionsfunktion darstellt, bietet bessere Prognosemöglichkeiten. Er fußt auf der Annahme, dass menschliche Bedürfnisbefriedigung in hohem Maße von den vergangenen Erfahrungen einer Person abhängt. Diese eigenen Erfahrungen gehen als Privatkapital in die Haushaltsproduktionsfunktion ein und bestimmen den Umfang der Nutzenstiftung von Gütern und Aktivitäten. Außerdem gehen verschiedene soziale Interaktionskomponenten als soziales Kapital in die individuelle Haushaltsproduktionsfunktion ein. Innerhalb seines Lebens ist jeder Mensch gezwungen, zentralen Personen, den Sozialisanden, zu folgen, da prohibitiv hohe Informationskosten einen Informationsstand, der ein individuell rationales Abwägen ermöglichen würde, verhindern. Die Sozialisanden haben wiederum ein eigenes Interesse, das Individuum in bestimmter, gezielter Weise

${ }^{356}$ Es ist allerdings auch möglich, dass für einzelne nicht der Wertekanon der Gesamtgesellschaft, sondern lediglich von Subgruppen gilt. Diese Individuen definieren sich dann gegebenenfalls durch eine möglichst weite Abweichung von der gesellschaftlichen Norm, jedoch durch die sehr enge Anbindung an die Nornem der geseldschaftichent/atergruppe. 
zu prägen. Durch die von anderen motivierten Erfahrungen sammelt das sozialisierte Individuum Sozialkapital an, das lange seine Entscheidungen beeinflusst. Werte und Ideologien sind demzufolge Ausdruck dieser fortdauernden Kapitalbestände und hätten danach handlungsleitende Bedeutung.

Da dieser Ansatz darauf aufbaut, dass Dritte ein Interesse haben, in das soziale Kapital einer Person zu investieren, müsste sich Wertewandel darauf zurückführen lassen, dass sich die Parameter im Investitionskalkül der Sozialisanden verändert haben. Insbesondere, weil diese Parameter zumindest zum Teil operationalisierbar scheinen, könnte dieser Humankapitalansatz eine wertvolle Befruchtung der soziologischen Wertewandeldiskussion bieten und kann erklären, wie es bei rein rational unterinformierten und eigennutzmaximierenden Individuen trotzdem zur Herausbildung von Werten und Ideologien kommen kann.

Es ist also durchaus gerechtfertigt, in ökonomischen Modellen von einer ideologischen Komponente auszugehen. In Kapitel 3 wurde dieser ideologische Aspekt in der Politik anhand des traditionellen Links-Rechts-Schemas operationalisiert. Dabei wurde ein etwas komplizierterer, stetiger Indikator gewählt, als üblicherweise in politökonomischen Modellen verwendet wird, um die Realität von sich ändernden Kabinetten und Koalitionsregierungen exakter abbilden zu können. ${ }^{357}$ Außerdem wurde ausführlich gezeigt, dass sich ideologische Unterschiede in der Wirtschaftspolitik relativ gut auf ein einziges Unterscheidungsmerkmal zurückführen lassen, nämlich auf das Kriterium des Gleichheitskonzepts. Die Diskussion wurde für unterschiedliche Konzeptionen der Arbeitsmarkt- und der Preispolitik sowie für verschiedene Präferenzen hinsichtlich der Struktur der staatlichen Ausgabenpositionen geführt. Diese Argumentation war zentral für die Formulierung der zu prüfenden Hypothesen.

Das vierte Kapitel thematisierte den Globalisierungsprozess. Es wurde argumentiert, dass insbesondere zwei Kräfte dazu führten, dass die Märkte als stärker integriert bezeichnet werden können als zu irgendeinem anderen Zeitpunkt der Weltgeschichte. Zum einen wurden zahlreiche technische sowie institutionelle Innovationen getätigt, die zu einem starken Absinken der Transport- v.a. aber der Kommunikations- und Koordinationskosten geführt haben. Dies bedeutet, dass eine Desintegration der Produktionsprozesse aus transaktionskostentheoretischen Argumenten zunehmend rational wurde. Um gleichzeitig Vorteile der internationalen Arbeitsteilung zu nutzen, wurde diese Desintegration des Produktionsprozesses auch in steigendem Maße international organisiert. Allerdings erforderte dies die rechtliche Möglichkeit der Zergliederung des Produktionsprozesses. Es mussten also zunächst die politischen Wege bereitet werden. Um zu zeigen, warum es für wiederwahlorientierte Politiker rational gewe-

${ }^{357}$ Die meisten ökonomischen Modelle unterscheiden Regierungen schematisch in links oder rechts gemäß einer binären Variable, selten mit einer diskreten Variable mit sehr wenigen Ausprägungsmöglichkeiten. 
sen ist, selbst dann als Globalisierungsbefürworter zu agieren, wenn durch die internationale Integration ihre eigenen Handlungsspielräume eingeengt werden, wurde ein einfaches polit-ökonomisches Modell präsentiert, in dem die angebotenen Protektionsniveaus endogene Parameter des politischen Marktes waren. Ziel war es, zu zeigen, warum rational wiederwahlorientierte Politiker für die Öffnung der Märkte eintreten werden, selbst wenn dadurch gegebenenfalls Handlungsfreiräume verringert werden.

Kann der Globalisierungsprozess also insgesamt recht gut begründet werden, so wurde in Kapitel 4 auch deutlich, dass es nicht so einfach ist, die Globalisierung in einem Indikator zu erfassen, denn die Liberalisierung der Märkte erklärt noch nicht, dass die internationale Konkurrenz für ein konkretes Land auch besondere Bedeutung hat. Es wurde gezeigt, dass es durchaus Länder gibt, die zwar ihre Märkte deutlich liberalisiert haben, für die der Offenheitsgrad (der Anteil des Außenhandels am Bruttoinlandsprodukt) verhältnismäßig klein ausfällt. Gleichzeitig gibt es Länder, für die der Offenheitsgrad deutlich höher ausfällt, die jedoch deutlich geringere Liberalisierungsbemühungen vorgenommen haben. Möchte man also die Integration eines Landes kennzeichnen, muss sowohl der ökonomische als auch der politische Aspekt in Betracht gezogen werden. Die durchgeführten Clusteranalysen ergaben deutlich unterschiedliche Länder- und Zeitcluster, je nachdem ob nach ökonomischen Variablen oder nach politischen Variablen geclustert wurde.

Dieser Befund, dass zwischen politischen und ökonomischen Clustern unterschieden werden kann, wurde dann in Kapitel 5, der empirischen Hypothesenprüfung, verwandt. In den drei wirtschaftspolitischen Feldern (Arbeitsmarkt, Inflation und staatliche Budgetstruktur) wurden mit Hilfe der Panelregressionsanalyse z.T. bemerkenswerte Ergebnisse erzielt. Wichtigste Resultate waren:

1. Es gab keine Hinweise dafür, dass linke und rechte Regierungen tatsächlich dauerhaft unterschiedliche Arbeitsmarktperformance zeigen. Lediglich in einigen Regressionen für einzelne Ländergruppen gab es Hinweise, dass die Partisantheorie ein angemessenes Bild liefert. Diese Ländergruppen schienen jedoch nicht durch den ökonomischen Offenheitsgrad oder die politischen Liberalisierungsbemühungen gekennzeichnet zu sein. Einflüsse der Globalisierung konnten also nicht isoliert werden.

2. Die rationale Partisantheorie mit nur temporären Partisaneffekten auf dem Arbeitsmarkt konnte anhand der untersuchten Daten indes nicht widerlegt werden. Es scheint in den ersten sechs bis acht Quartalen nach einem Regierungswechsel nach links tendenziell eher eine kurzfristige Entspannung auf dem Arbeitsmarkt zu geben als bei einem Regierungswechsel nach rechts. Dieser Befund war für einige Länder deutlich stärker ausgeprägt als für andere. Es scheint jedoch auch hier so zu sein, dass die wirtschaftliche Integration eines Landes in die Weltwirtschaft ein unzureichender Indikator für die Gültigkeit der rationalen Partisantheorie ist. Diejes Alissagen ${ }^{3}$ gelteh sowolhl für 
die ökonomischen als auch für die politischen Cluster, sodass die Vermutung erhärtet wird, dass in den Ländern, in denen tatsächlich eine Annäherung der Arbeitsmarktperformance stattgefunden hat, eher Lernprozesse stattgefunden haben, als dass Restriktionen durch die internationalen Märkte als Ursache gelten können. Schäfer (2000, S. 121) benutzt den Begriff des „pathologischen Lernens", für den Tatbestand, dass einige Politiker aus gemachten Fehlern schmerzhaft gelernt haben. Diese Vermutung lässt sich aber mit den hier verwendeten Daten nicht exakter formulieren.

3. Die Prüfung der Unterschiedlichkeit der Inflationsraten verschiedener Regierungen ergab ein unerwartetes Resultat. In der Regel ergaben die Schätzergebnisse keine oder nicht sehr deutliche Partisaneffekte. Hinsichtlich der Globalisierungshypothese ist sogar eher ein entgegengesetzter Zusammenhang gefunden worden. Es waren eher die besonders offenen Länder oder die Jahre besonders intensiver Öffnung, für die Partisaneffekte gefunden wurden. Diese Beobachtung ist allerdings ebenfalls statistisch äußerst wenig abgesichert und sollte nicht überinterpretiert werden.

4. Ein wichtiges Instrument zur parteipolitischen Profilierung scheint tatsächlich das staatliche Budget zu sein. Es wurden v.a. für die ersten vier Jahre nach einem Regierungswechsel deutliche Partisaneffekte dergestalt gefunden, dass linke Parteien tendenziell den Anteil der zivilen Konsumausgaben und der Gesamtausgaben zum Bruttoinlandsprodukt stärker ansteigen ließen als rechte Parteien. Für die Transferausgaben und Subventionen konnte dieser Zusammenhang indes nicht gefunden werden. Die Vermutung liegt nahe, dass Arbeitsmarktziele zumindest zum Teil auch über staatliche Auffangmaßnahmen realisiert wurden. Der Staat hätte dann v.a. unter linken Regierungen deutlicher die Funktion eines employers of last resort. Es gibt allerdings ebenfalls deutliche Hinweise, dass dieser Zusammenhang seit 1986 an Klarheit verloren hat. $\mathrm{Ob}$ dies jedoch auf die oben skizzierten (pathologischen) Lernprozesse seitens der Politik oder auf restringierende Wirkungen der Weltmärkte zurückzuführen ist, bleibt unklar. Es ist durchaus denkbar, dass beides parallel wirkt: aufgrund der Globalisierung werden die Politiker zu schnellerem und schmerzhafterem Lernen gezwungen. Allerdings scheint es so zu sein, dass die Kern-EU-Länder, die untereinander vollständig offen sind, weite Teile ihrer budgetären Profilierungsmöglichkeiten erhalten haben. Inwiefern tatsächlich die supranationale Organisation Europäische Union den Ländern diese Handlungsmöglichkeiten lässt, oder ob diese Beobachtung eher darauf zurückzuführen ist, dass die EU ein Zusammenschluss ähnlich funktionierender Einzelstaaten ist, kann an dieser Stelle nicht geprüft werden.

5. Schließlich wurde auch gezeigt, dass linke Regierungen zumindest in den ersten Jahren nach der Regierungsübernahme deutlich stärkere budgetäre Veränderungsimpulse setzen. Dabei war bemerkenswert, dass dies nicht nur für die Zeit der stärkeren Ausgabenanteilssteigernungę - y951 1986 galt, son- 
dern auch in der Zeit, in der linke Regierungen nicht durch ihre Ausgabensteigerungen unterscheidbar waren. Die Tatsache, dass sie in dieser Zeit die Budgets deutlich stärker umstrukturierten, könnte Ausdruck einer höheren Reformbereitschaft linker Regierungen sein. Es könnte jedoch auch so interpretiert werden, dass linke Regierungen bislang einen Signalingvorteil gegenüber rechten Parteien hatten, wenn unwillkommene Reformen erforderlich wurden, da ihr Engagement glaubwürdiger und dadurch leichter dem Wähler vermittelbar war.

Insgesamt sollten bei all den gefundenen Ergebnissen zwei Aspekte bedacht werden: Erstens wurde ausdrücklich der Bereich der Ordnungspolitik von der Analyse ausgeschlossen. Selbst wenn man davon ausgeht, dass durch die Globalisierung ein Anpassungsdruck der nationalen Institutionen entstanden ist, so ist das Verständnis über das korrekte Design der bestmöglichen Institutionen keineswegs gesichert. Dies impliziert, dass weitreichende politische Handlungsmöglichkeiten nicht nur bestehen bleiben, sondern durch die Globalisierung gerade gefördert werden. Es ist durchaus plausibel, dass v.a. im ordnungspolitischen Bereich, die Anpassungsstrategie der innovativen Pionierstrategie im Sinne eines Schumpeter'schen entrepreneurs unterlegen ist. Dann käme es aber gerade nicht $\mathrm{zu}$ dem race to the bottom in der Ordnungspolitik, sondern nur $\mathrm{zu}$ einem ergebnisoffenen race.

Zweitens muss berücksichtigt werden, dass in dieser Studie lediglich Daten bis 1997 benutzt wurden. Da seitdem die internationalen Märkte, insbesondere die Kapitalmärkte, noch deutlich an Umfang gewonnen haben, sollten diese Ergebnisse, die zum Teil nur ausgesprochen schwachen Befund für die Globalisierungsthese liefern konnten, vorsichtig interpretiert werden und gegebenenfalls später mit einem größeren Datensatz nachgeschätzt werden. Denn falls Donges (1998, S. 3) Recht behält, dass die Globalisierung unumkehrbar ist, so wird der Umfang der weltweiten Marktintegration in der Zukunft eher zunehmen als geringer werden. Andererseits können Skeptiker der Unumkehrbarkeitsthese einräumen, dass die Zukunft ex definitione ungewiss ist. Letztlich ist die Geschichte voller Belege dafür, dass Entwicklungen selten linear verlaufen. 


\section{Anhang A \\ Abstimmungsentscheidung und expressive Nutzenelemente}

Tabelle A.1: Wahlentscheidung und Entscheidungskommunikation

301

Die Basis für das folgende Schema ist die Situation in 2.3.2.2: Es wird ein Wähler betrachtet, dessen monetäres Parteidifferential zugunsten der C-Partei ausfällt. Es soll gezeigt werden, dass durch die Ergänzung dieser instrumentellen Nutzen durch interne und externe expressive Nutzen gravierende Unterschiede sowohl im Wahl- als auch im Kommunikationsverhalten auftreten können. AuBerdem wird hier gezeigt, dass die Berücksichtigung zusätzlicher interner und externer Kosten der Unaufrichtigkeit in der Kommunikation der Wahlentscheidung (Kosten des Lügens), Ergebnisänderungen bewirken. Eine explizite Berücksichtigung der instrumentellen Nutzen ist dann nicht erforderlich, wenn von einem hinreichend großen Kollektiv ausgegangen wird, denn dann sind die internen expressiven Nutzenelemente wahrscheinlich immer größer als das mit der Eintrittswahrscheinlichkeit der entscheidende Wähler zu sein bewertete Parteidifferential - die Wahlentscheidung beeinflusst es dann also nicht. Es werden im folgenden die folgenden Abkürzungen verwandt:

$I_{C}$ : Interne expressive Nutzen aus der Wahl für $\mathrm{C}$

$I_{S}$ : Interne expressive Nutzen aus der Wahl für $\mathrm{S}$

$\mathrm{E}_{\mathrm{C}}$ : Externe expressive Nutzen aus der Wahl für $\mathrm{C}$

$\mathrm{E}_{S}$ : Externe expressive Nutzen aus der Wahl für $\mathrm{S}$

L: Kosten, die durch unwahre Aussagen entstehen - entweder intern durch Gewissensbisse oder extern durch mit der Entdeckungswahrscheinlichkeit bewertete Sanktionen.

Die folgende Abbildung kennzeichnet die Entscheidungssituation des Wählers: 


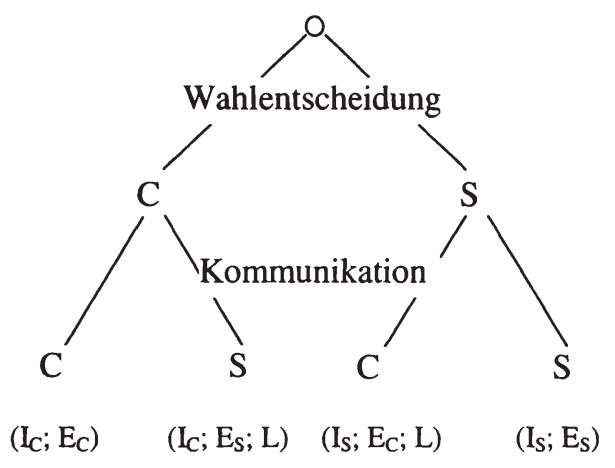

Ein Wähler befindet sich also in einem zweistufigen Optimierungsmodell. Er muss sich zunächst entscheiden, welche Partei er wählt und anschließend die Entscheidung treffen, ob er seine Entscheidung auch wahrheitsgemäß kommuniziert. Es wird angenommen, dass alle diese Nutzenelemente positiv sind und die Nettonutzen in jedem Fall die Wahlkosten übersteigen. In Tabelle A.1 sind die Entscheidungskriterien und die Entscheidungen zusammengestellt. Diese Übersicht macht deutlich, dass es nicht nur bei der Entscheidungsäußerung, sondern auch bei der Wahlentscheidung selbst auf die internen und externen Sanktionsmechanismen ankommt.

Außerdem lässt sich so auch die Existenz von Nichtwählern erklären. Natürlich werden jene Personen mit relativ geringen expressiven Nutzenelementen nicht zur Wahl gehen (mit $\left|\left(I_{C}-I_{S}\right)\right|+\left|\left(E_{C}-E_{S}\right)\right|<C$, wobei $C$ für die Kosten des Wahlaktes steht). Dies ist aber nicht Hauptaugenmerk dieser Darstellung und soll daher auch nicht ausführlicher betrachtet werden. Eine darauf aufbauende Wählertypologie scheint aber möglich.

In der folgenden Übersicht gilt es zu beachten, dass es zwar gemäß dem obigen Entscheidungsbaum im Falle von $\mathrm{L}>0$ immer vier Handlungsalternativen gibt. Im Fall II ist jedoch die Auszahlung $\mathrm{I}_{S}+\mathrm{E}_{\mathrm{C}}-\mathrm{L}$ wegen $\mathrm{I}_{\mathrm{C}}>\mathrm{I}_{\mathrm{S}}$ und $\mathrm{E}_{\mathrm{C}}<\mathrm{E}_{\mathrm{S}}$ immer eine unterlegene Wahl. Analog kann im Fall III die Auszahlung $\mathrm{I}_{S}+\mathrm{E}_{\mathrm{C}}-\mathrm{L}$ wegen $\mathrm{I}_{\mathrm{C}}<\mathrm{I}_{\mathrm{S}}$ und $\mathrm{E}_{\mathrm{C}}>\mathrm{E}_{\mathrm{S}}$ niemals dominante Strategie sein. Daher wird auf die Darstellung dieser beiden Scheinalternativen verzichtet. 
Tabelle A.1 : Wahlentscheidung und Entscheidungskommunikation

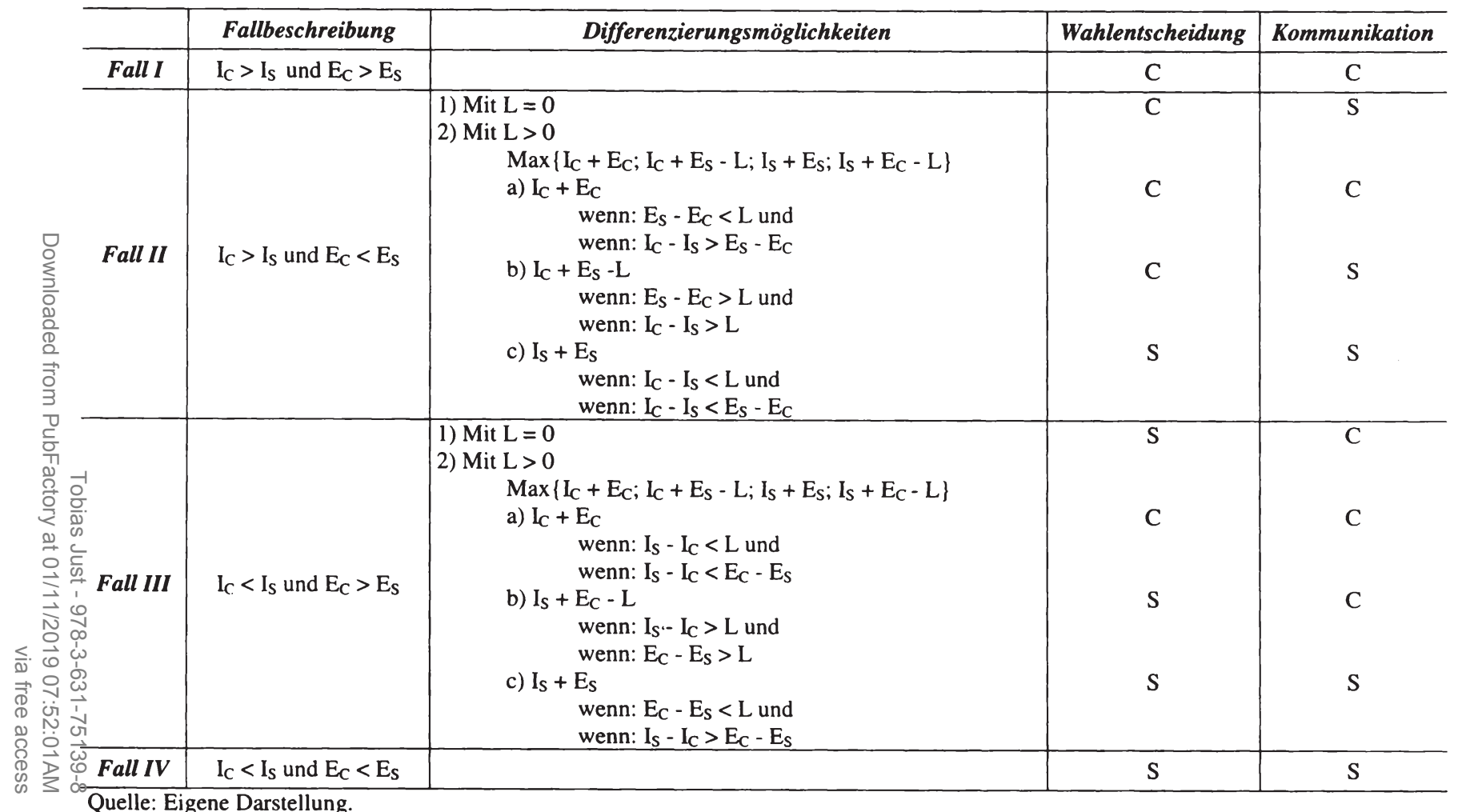


Tobias Just - 978-3-631-75139-8

Downloaded from PubFactory at 01/11/2019 07:52:01AM

via free access 


\section{Anhang B \\ Die Ideologievariablen für 19 OECD-Staaten}

Tabelle B.1 : Parteiverortung im Links-Rechts-Kontinuum....................... 304

Tabelle B.2 : Center of Gravity: Quartalsdaten Australien ........................ 308

Tabelle B.3 : Center of Gravity: Quartalsdaten Österreich........................ 309

Tabelle B.4 : Center of Gravity: Quartalsdaten Belgien ............................. 310

Tabelle B.5 : Center of Gravity: Quartalsdaten Kanada ............................. 311

Tabelle B.6 : Center of Gravity: Quartalsdaten Dänemark ......................... 312

Tabelle B.7 : Center of Gravity: Quartalsdaten Finnland .......................... 313

Tabelle B.8: Center of Gravity: Quartalsdaten Frankreich ...................... 314

Tabelle B.9 : Center of Gravity: Quartalsdaten Deutschland .................... 315

Tabelle B.10 : Center of Gravity: Quartalsdaten Irland .............................. 316

Tabelle B.11 : Center of Gravity: Quartalsdaten Italien .............................. 317

Tabelle B.12 : Center of Gravity: Quartalsdaten Japan ............................... 318

Tabelle B.13 : Center of Gravity: Quartalsdaten Niederlande..................... 319

Tabelle B.14 : Center of Gravity: Quartalsdaten Neuseeland....................... 320

Tabelle B.15 : Center of Gravity: Quartalsdaten Norwegen........................ 321

Tabelle B.16 : Center of Gravity: Quartalsdaten Schweden ........................ 322

Tabelle B.17 : Center of Gravity: Quartalsdaten Schweiz ............................ 323

Tabelle B.18 : Center of Gravity: Quartalsdaten Spanien............................ 324

Tabelle B.19 : Center of Gravity: Quartalsdaten Großbritannien ................. 325

Tabelle B.20 : Center of Gravity: Quartalsdaten USA................................. 326 


\section{Positionierung der Parteien aus 19 OECD-Staaten im Links- Rechts-Kontinuum}

Die Verortung folgt weitgehend der Darstellung bei Castles und Mair (1984). Damit Parteien, die (noch) nicht in der Studie von Castles und Mair berücksichtigt wurden, Eingang in diese Arbeit finden können, wurden diese durch eine eigene Einschätzung gemäß der verbalen Darstellung in Gorvin (1989), Mackie und Rose (1974) sowie Mackie und Rose (1997) vorgenommen. Für die Länder Japan und Schweiz wurde darüber hinaus die Einschätzung von Cusack (1995) herangezogen.

Tabelle B.1 : Parteiverortung im Links-Rechts-Kontinuum

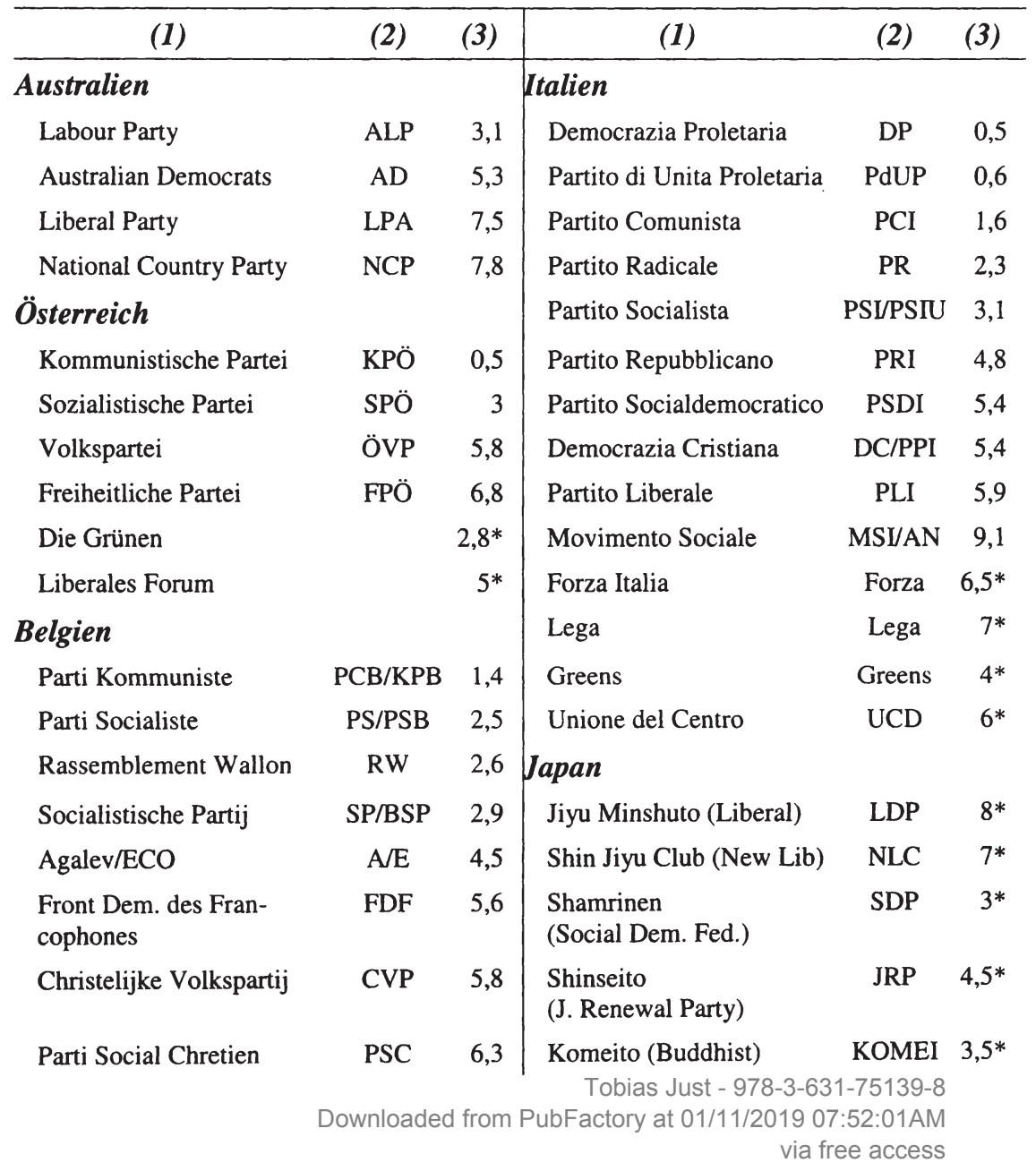


Volkunie

Partie Reformateur

Liberal

P. voor Frijheid en

Vooruitg.

Respect voor Arbeid en Dem.

Vlaams Blok

\section{Kanada}

New Democratic Party

Liberal Party

Progressive Cons. Party

Social Credit Party

Reform Party

Bloc Quebecois

\section{Dänemark}

Venstre Socialisterne

Kommunistiske Parti

Socialistisk Folkeparti

Socialdemokratiet

Radikale Venstre

Centrumsdemokraterne

Kristeligt Folkeparti

Venstre

Konservative Folkeparti

Fremskridtspartiet

Danmarks Retsforbund

Faelles Kurs

\section{Finnland}

Suomen Kansan Dem.

Puolue

\begin{tabular}{|c|c|c|c|c|}
\hline VU & 6,8 & $\begin{array}{l}\text { Nihon Shinto } \\
\text { (J. New Party) }\end{array}$ & JNP & $4,5^{*}$ \\
\hline PRL & 7,6 & $\begin{array}{l}\text { Minshato (Dem. Socialist } \\
\text { Party) }\end{array}$ & DSP & $3 *$ \\
\hline PVV & 7,8 & New Harbinger Party & Sakigake & $5^{*}$ \\
\hline $\begin{array}{l}\text { AD/ } \\
\text { DRT }\end{array}$ & 9,2 & Kommunistische Partei & JCP & $1,5^{*}$ \\
\hline \multirow[t]{2}{*}{ VB } & 9,8 & Dem. Party of Japan & DPJ & $3,5^{*}$ \\
\hline & & Niederlande & & \\
\hline NDP & 3,2 & Pacifistisch Soc. Partij & PSP & 0,6 \\
\hline $\mathrm{L}$ & 5,3 & Communistische Partij & $\mathrm{CPN}$ & 0,8 \\
\hline PC & 6,5 & Politieke Partij Radikalen & PPR & 1,6 \\
\hline PCS & 7,8 & Partij van de Arbeid & PvdA & 2,6 \\
\hline RP & $6,5^{*}$ & Democraten '66 & D66/DS 70 & $0 \quad 4,4$ \\
\hline \multirow[t]{2}{*}{ BQ } & $7 *$ & $\begin{array}{l}\text { Christen Democratisch } \\
\text { Appel }\end{array}$ & $\begin{array}{l}\text { CDA/KVP } \\
\text { CHU/ARP }\end{array}$ & 5,7 \\
\hline & & $\begin{array}{l}\text { Volkspartij voor Vrijheid } \\
\text { en Dem. }\end{array}$ & VVD & 7,4 \\
\hline vs & 0,8 & $\begin{array}{l}\text { Gereformeerd Politiek } \\
\text { Verbond }\end{array}$ & GPV & 9 \\
\hline DKP & 1 & $\begin{array}{l}\text { Reform. Politieke Fede- } \\
\text { ratie }\end{array}$ & RPF & 9,2 \\
\hline SF & 1,9 & $\begin{array}{l}\text { Staatkundig Gerefor- } \\
\text { meerde Partij }\end{array}$ & SGP & 9,2 \\
\hline SD & 3,8 & Boerenpartij & $\mathrm{BP} / \mathrm{RVP}$ & 9* \\
\hline RV & 4,8 & Neuseeland & & \\
\hline $\mathrm{CD}$ & 5,7 & Labour Party & LAB & 3,8 \\
\hline $\mathrm{KRF}$ & 6,2 & $\begin{array}{l}\text { Social Credit Political } \\
\text { League }\end{array}$ & SC & 5,6 \\
\hline $\mathrm{v}$ & 6,7 & National Party & NP & 6 \\
\hline $\mathrm{KF}$ & 7,3 & New Zealand Party & NZP & $6,5^{*}$ \\
\hline FRP & 8,7 & Norwegen & & \\
\hline ST & $5^{*}$ & Sosialistisk Venstreparti & SV/COM & 1,2 \\
\hline \multirow[t]{2}{*}{ FK } & $5^{*}$ & Det Norske Arbeiderparti & DNA & 3 \\
\hline & & Venstre & $\mathrm{V}$ & 4 \\
\hline \multirow[t]{2}{*}{ SKDL } & 1,8 & Senterpartiet & SP & 5,8 \\
\hline & & Tobias Jus & 139-8 & \\
\hline
\end{tabular}




\begin{tabular}{|c|c|c|c|c|c|}
\hline $\begin{array}{l}\text { Suomen Sosialidem. } \\
\text { Puolue }\end{array}$ & SDP & 3 & Kristelig Folkeparti & $\mathrm{KrF}$ & 6,1 \\
\hline Keskustapuolue & KESK & 5,2 & Hoyre & $\mathrm{H}$ & 7,7 \\
\hline $\begin{array}{l}\text { Liberaalinen } \\
\text { Kansanpuolue }\end{array}$ & LKP & 5,6 & Fremskrittspartiet & $\mathrm{Fp}$ & 9,4 \\
\hline $\begin{array}{l}\text { Suomen Maaseuden Pu- } \\
\text { olue }\end{array}$ & SMP & 5,8 & Det Liberale Folkepartiet & DFP & $4^{*}$ \\
\hline $\begin{array}{l}\text { Ruotsalainen Kansanpu- } \\
\text { olue }\end{array}$ & RKP & 6,1 & Schweden & & \\
\hline Suomen Kristillinen Liitto & SKL & 6,8 & $\begin{array}{l}\text { Vansterpartiet Kommu- } \\
\text { nisterna }\end{array}$ & VPK & 1,2 \\
\hline Kansallinen Kokoomus & KOK & 7,2 & Socialdem. Arbeitarpartiet & SD & 2,9 \\
\hline $\begin{array}{l}\text { Työväen ja Pienviljelijäin } \\
\text { Sosialidemo. Liitto }\end{array}$ & TPSL & $3 *$ & Folkpartiet & FP & 5,5 \\
\hline Vihreä Liitto & VIHR & $4^{*}$ & Centerpartiet & $\mathrm{CP}$ & 5,9 \\
\hline Vassemmisto Liitto & VAS & $3^{*}$ & Moderata Samlingspartiet & $\mathbf{M}$ & 7,7 \\
\hline $\begin{array}{l}\text { Demokraatinen Vaih- } \\
\text { toehto }\end{array}$ & DEVA & $1^{*}$ & $\begin{array}{l}\text { Kristendem. Sam- } \\
\text { hällspartiet }\end{array}$ & KDS & $5^{*}$ \\
\hline Frankreich & & & Schweiz & & \\
\hline Parti Communiste & PCF & 1,4 & $\begin{array}{l}\text { Schweizerische } \\
\text { Volkspartei }\end{array}$ & SVP & $5^{*}$ \\
\hline Parti Socialiste & $\begin{array}{l}\text { PS/RSP/ } \\
\text { PSU }\end{array}$ & 2,6 & Freisinning-dem. Partei & FDP & $4^{*}$ \\
\hline Generation Ecologiste & $\mathrm{E}$ & 3,5 & $\begin{array}{l}\text { Christlich Dem. Volks- } \\
\text { partei }\end{array}$ & CVP & $6^{*}$ \\
\hline Radicaux de Gauche & MRG/PRG & 3,8 & $\begin{array}{l}\text { Sozialdemo. Partei der } \\
\text { Schweiz }\end{array}$ & SP & $3^{*}$ \\
\hline $\begin{array}{l}\text { Union pour la Demo. } \\
\text { Francaise }\end{array}$ & UDF & 6,6 & $\begin{array}{l}\text { Landesring der Unabhän- } \\
\text { gigen }\end{array}$ & LdU & $6,6^{*}$ \\
\hline $\begin{array}{l}\text { Rassemblement pour la } \\
\text { Republique. }\end{array}$ & $\begin{array}{l}\text { RPR/UNR/ } \\
\text { UDT/UDR }\end{array}$ & 8,2 & $\begin{array}{l}\text { Liberale Partei der } \\
\text { Schweiz }\end{array}$ & LPS & $4^{*}$ \\
\hline $\begin{array}{l}\text { Centre Nat. des Indep. et } \\
\text { Paysans }\end{array}$ & CNIP/IND & 8,6 & Sozialisten & PdA/PSA & $2 *$ \\
\hline Front National & FN & 9,8 & Grünes Bündnis & Grüne & $3 *$ \\
\hline $\begin{array}{l}\text { Parti des Forces } \\
\text { Nouvelles }\end{array}$ & PFN & 9,8 & Republikaner & $\underset{\text { ar }}{\mathrm{Rep} / \mathrm{NA} / \mathrm{C}}$ & $8^{*}$ \\
\hline $\begin{array}{l}\text { Union Nouvelle pour la } \\
\text { Republique. }\end{array}$ & MRP & $6,2^{*}$ & Spanien & & \\
\hline \multirow[t]{2}{*}{ Centre Democratique } & \multirow[t]{2}{*}{$\begin{array}{l}\text { CD/PDM/G } \\
\text { D/VREP }\end{array}$} & \multirow{2}{*}{7,2} & Herri Batasuna & HB & 0,5 \\
\hline & & & \multicolumn{2}{|c|}{ Tobias Just - 978-3-631-75139-8 } & \\
\hline
\end{tabular}




\section{Deutschland}

Partei des dem. Soz.

Die Grünen

Sozialdemokratische

Partei Deutschlands

Freie Demokratische.

Partei

Christlich Dem. Union

Christlich Soziale Union

\section{Irland}

Worker Party

Labour Party

Fianna Fail

Fine Gael

Progressive Democrats

Clann na Talmhan

Clann na Poblachta

Grüne
PDS $1,8^{*}$

G $\quad 2,8$

SPD

3,3

FDP

5,1

CDU

CSU $\quad 7,9$

6,7

nya

Partido Socialista de

Andalucia

Convergencia y Union

Partido Nacionalista

Vasco

WP 1,8 Union des Centro Dem. UCD 7,1

LAB $\quad 3,6$

$\mathrm{FF}$

FG

6,3

P. Aragones Regionalista

Alianza Popular

PAR 8,2

AP $\quad 8,4$

Union Nacional/Fuerza Nueva

PD 5 Grossbritannien

CT $\quad 7 *$

$\mathrm{CP} \quad 8^{*}$

G
Labour Party

Conservative Party

3*

Plaid Cymru
Scottish National Party

Social Democratic Party

Liberal Party

Ulster Unionists

USA

\begin{tabular}{l|lll} 
& USA & $\mathrm{D}$ & 4,8 \\
& Democrats & $\mathrm{R}$ & 6,8 \\
\hline & Republicans & & \\
\hline
\end{tabular}

(1) Parteiname.

(2) Parteikürzel, wie sie bei Castles/Mair (1984) verwandt werden.

(3) Expertenurteil hinsichtlich der Einstufung auf einem Links-Rechts-Kontinuum von 0-10. Der Wert 0 entspricht einer äußerst linken Position, der Wert 10 einer extrem rechten.

(*) Diese Parteien wurden nicht von durch die Experten in der Castles/Mair-Studie eingestuft. Ihre Bewertung erfolgte gemäß der Angaben bei Gorvin (1989), und Mackie und Rose (1997) und Mackie und Rose (1974). 
Tabelle B.2 : Center of Gravity: Quartalsdaten Australien

\begin{tabular}{|c|c|c|c|c|c|c|c|c|c|c|c|c|c|c|c|c|c|}
\hline & & $C G$ & & & $C G$ & & & $C G$ & & & $C G$ & & & $C G$ & & & $C G$ \\
\hline 1960 & 1 & 7,53 & 1967 & 1 & 7,58 & 1974 & 1 & 3,10 & 1981 & 1 & 7,56 & 1988 & 1 & 3,10 & 1995 & 1 & 3,10 \\
\hline & 2 & 7,53 & & 2 & 7,58 & & 2 & 3,10 & & 2 & 7,56 & & 2 & 3,10 & & 2 & 3,10 \\
\hline & 3 & 7,53 & & 3 & 7,58 & & 3 & 3,10 & & 3 & 7,56 & & 3 & 3,10 & & 3 & 3,10 \\
\hline 1961 & 1 & 7,53 & 1968 & 1 & 7,58 & 1975 & 1 & 3,10 & 1982 & 1 & 7,56 & 1989 & 1 & 3,10 & 1996 & 1 & 3,10 \\
\hline & 2 & 7,53 & & 2 & 7,58 & & 2 & 3,10 & & 2 & 7,56 & & 2 & 3,10 & & 2 & 7,56 \\
\hline 망 & 3 & 7,53 & & 3 & 7,58 & & 3 & 3,10 & & 3 & 7,56 & & 3 & 3,10 & & 3 & 7,56 \\
\hline$\frac{2}{10}$ & 2 & 7,55 & & 2 & 7,58 & & 2 & 7,58 & & 2 & 3,10 & & 2 & 3,10 & & 2 & 7,56 \\
\hline$\stackrel{1}{2}$ & 3 & 7,55 & & 3 & 7,58 & & 3 & 7,58 & & 3 & 3,10 & & 3 & 3,10 & & 3 & 7,56 \\
\hline $\overrightarrow{\mathrm{O}}$ & 4 & 7,55 & & 4 & 7,58 & & 4 & 7,58 & & 4 & 3,10 & & 4 & 3,10 & & 4 & 7,56 \\
\hline 1963 & 1 & 7,55 & 1970 & 1 & 7,58 & 1977 & 1 & 7,58 & 1984 & 1 & 3,10 & 1991 & 1 & 3,10 & & & \\
\hline ᄃ & 2 & 7,55 & & 2 & 7,58 & & 2 & 7,58 & & 2 & 3,10 & & 2 & 3,10 & & & \\
\hline ग & 3 & 7,55 & & 3 & 7,58 & & 3 & 7,58 & & 3 & 3,10 & & 3 & 3,10 & & & \\
\hline 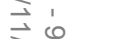 & 4 & 7,58 & & 4 & 7,57 & & 4 & 7,56 & & 4 & 3,10 & & 4 & 3,10 & & & \\
\hline సু তু 1965 & 1 & 7,58 & 1972 & 1 & 7,57 & 1979 & 1 & 7,56 & 1986 & 1 & 3,10 & 1993 & 1 & 3,10 & & & \\
\hline $\overrightarrow{0} \omega$ & 2 & 7,58 & & 2 & 7,57 & & 2 & 7,56 & & 2 & 3,10 & & 2 & 3,10 & & & \\
\hline 이 ట్ & 3 & 7,58 & & 3 & 7,57 & & 3 & 7,56 & & 3 & 3,10 & & 3 & 3,10 & & & \\
\hline$\ddot{v} \vec{i}$ & 4 & 7,58 & & 4 & 7,57 & & 4 & 7,56 & & 4 & 3,10 & & 4 & 3,10 & & & \\
\hline 1966 & 1 & 7,58 & 1973 & 1 & 3,10 & 1980 & 1 & 7,56 & 1987 & 1 & 3,10 & 1994 & 1 & 3,10 & & & \\
\hline$\vec{\nabla} \omega$ & 2 & 7,58 & & 2 & 3,10 & & 2 & 7,56 & & 2 & 3,10 & & 2 & 3,10 & & & \\
\hline $3 \infty$ & 3 & 7,58 & & 3 & 3,10 & & 3 & 7,56 & & 3 & 3,10 & & 3 & 3,10 & & & \\
\hline & 4 & 7,58 & & 4 & 3,10 & & 4 & 7,56 & & 4 & 3,10 & & 4 & 3,10 & & & \\
\hline
\end{tabular}


Tabelle B.3 : Center of Gravity: Quartalsdaten Österreich

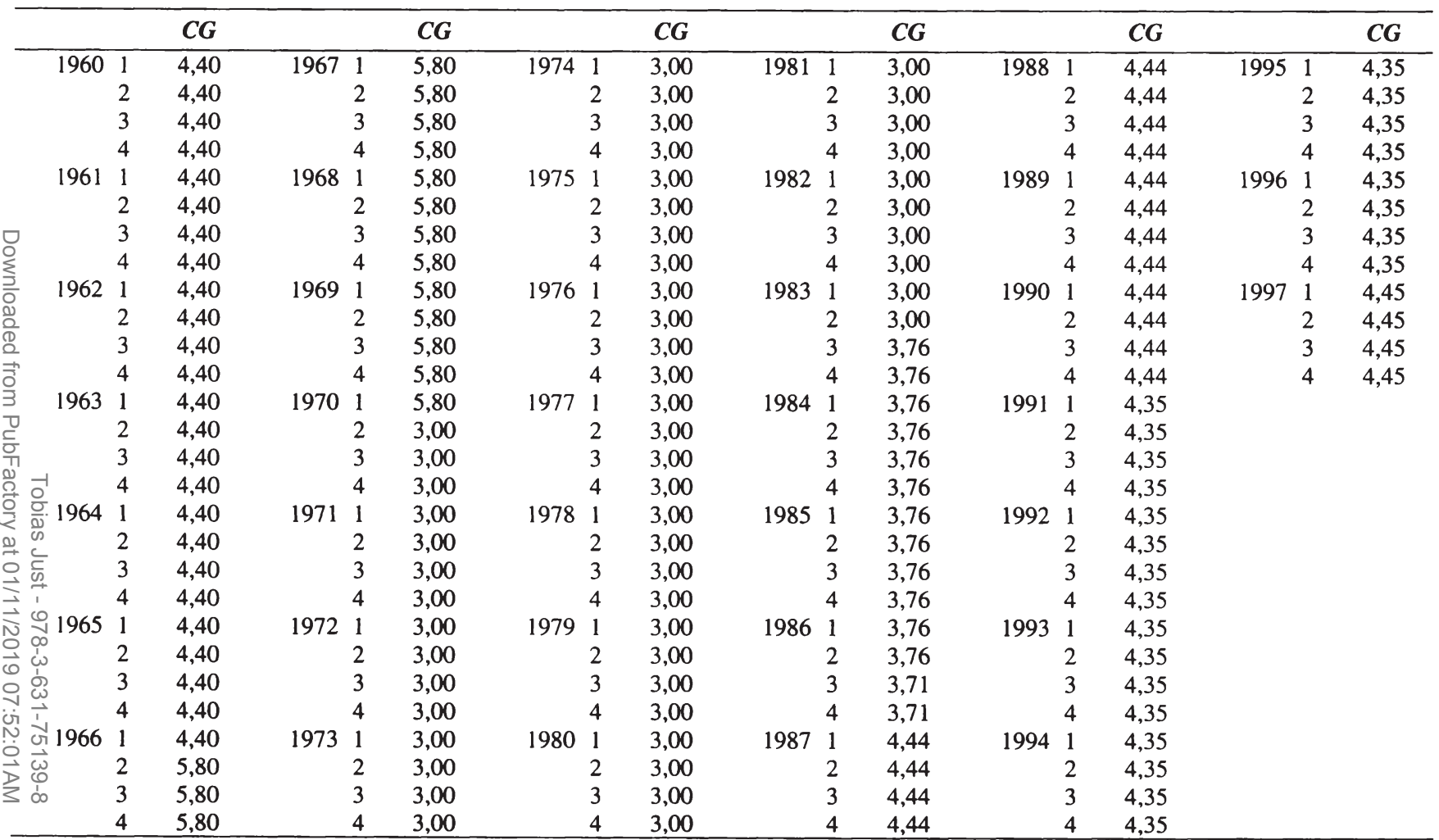


Tabelle B.4 : Center of Gravity: Quartalsdaten Belgien

\begin{tabular}{|c|c|c|c|c|c|c|c|c|c|c|c|c|c|c|c|c|c|}
\hline & & $C G$ & & & $C G$ & & & $C G$ & & & $C G$ & & & $C G$ & & & $C G$ \\
\hline 1960 & 1 & 6,47 & 1967 & 1 & 6,54 & 1974 & 1 & 5,07 & 1981 & 1 & 4,46 & 1988 & 1 & 6,43 & 1995 & 1 & 4,19 \\
\hline & 2 & 6,47 & & 2 & 6,54 & & 2 & 6,36 & & 2 & 4,50 & & 2 & 6,43 & & 2 & 4,19 \\
\hline & 3 & 6,50 & & 3 & 6,54 & & 3 & 6,36 & & 3 & 4,50 & & 3 & 4,47 & & 3 & 4,19 \\
\hline & 4 & 6,50 & & 4 & 6,54 & & 4 & 6,36 & & 4 & 4,50 & & 4 & 4,47 & & 4 & 4,19 \\
\hline 1961 & 1 & 6,50 & 1968 & 1 & 6,54 & 1975 & 1 & 6,36 & 1982 & 1 & 6,94 & 1989 & 1 & 4,47 & 1996 & 1 & 4,19 \\
\hline & 2 & 4,67 & & 2 & 6,54 & & 2 & 6,36 & & 2 & 6,94 & & 2 & 4,47 & & 2 & 4,19 \\
\hline 8 & 3 & 4,67 & & 3 & 4,62 & & 3 & 6,36 & & 3 & 6,94 & & 3 & 4,47 & & 3 & 4,19 \\
\hline$\sum_{\Sigma}^{0}$ & 4 & 4,67 & & 4 & 4,62 & & 4 & 6,36 & & 4 & 6,94 & & 4 & 4,47 & & 4 & 4,19 \\
\hline 1962 & 1 & 4,67 & 1969 & 1 & 4,62 & 1976 & 1 & 6,36 & 1983 & 1 & 6,94 & 1990 & 1 & 4,47 & 1997 & 1 & 4,19 \\
\hline ڤ & 2 & 4,67 & & 2 & 4,62 & & 2 & 6,36 & & 2 & 6,94 & & 2 & 4,47 & & 2 & 4,19 \\
\hline बे & 3 & 4,67 & & 3 & 4,62 & & 3 & 6,36 & & 3 & 6,94 & & 3 & 4,47 & & 3 & 4,19 \\
\hline $\overrightarrow{0}$ & 4 & 4,67 & & 4 & 4,62 & & 4 & 6,36 & & 4 & 6,94 & & 4 & 4,47 & & 4 & 4,19 \\
\hline 1963 & 1 & 4,67 & 1970 & 1 & 4,62 & 1977 & 1 & 6,31 & 1984 & 1 & 6,94 & 1991 & 1 & 4,47 & & & \\
\hline$\underset{c}{0}$ & 2 & 4,67 & & 2 & 4,62 & & 2 & 6,31 & & 2 & 6,94 & & 2 & 4,47 & & & \\
\hline 휘 & 3 & 4,67 & & 3 & 4,62 & & 3 & 4,82 & & 3 & 6,94 & & 3 & 4,47 & & & \\
\hline$\stackrel{2}{\stackrel{2}{2}}$ & 4 & 4,67 & & 4 & 4,62 & & 4 & 4,82 & & 4 & 6,94 & & 4 & 4,24 & & & \\
\hline$\frac{\sigma^{\prime}}{\frac{0}{2}} \cdot 1964$ & 1 & 4,67 & 1971 & 1 & 4,62 & 1978 & 1 & 4,82 & 1985 & 1 & 6,94 & 1992 & 1 & 4,24 & & & \\
\hline 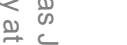 & 2 & 4,67 & & 2 & 4,62 & & 2 & 4,82 & & 2 & 6,94 & & 2 & 4,19 & & & \\
\hline 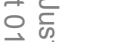 & 3 & 4,67 & & 3 & 4,62 & & 3 & 4,82 & & 3 & 6,94 & & 3 & 4,19 & & & \\
\hline 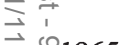 & 4 & 4,67 & & 4 & 4,62 & & 4 & 4,96 & & 4 & 6,94 & & 4 & 4,19 & & & \\
\hline $\overrightarrow{\mathrm{N}} \stackrel{\sim}{N}_{1} 1965$ & 1 & 4,67 & 1972 & 1 & 4,53 & 1979 & 1 & 4,96 & 1986 & 1 & 6,47 & 1993 & 1 & 4,19 & & & \\
\hline $\begin{array}{c}0 \\
\stackrel{1}{c}\end{array}$ & 2 & 4,67 & & 2 & 4,53 & & 2 & 4,58 & & 2 & 6,47 & & 2 & 4,19 & & & \\
\hline $\begin{array}{ll}0 & 1 \\
0 & 5\end{array}$ & 3 & 4,50 & & 3 & 4,53 & & 3 & 4,58 & & 3 & 6,47 & & 3 & 4,19 & & & \\
\hline$\underset{\omega}{\sim} \frac{\omega}{1}$ & 4 & 4,50 & & 4 & 4,53 & & 4 & 4,58 & & 4 & 6,47 & & 4 & 4,19 & & & \\
\hline ज̃'1966 & 1 & 4,50 & 1973 & 1 & 5,07 & 1980 & 1 & 4,15 & 1987 & 1 & 6,47 & 1994 & 1 & 4,19 & & & \\
\hline$\stackrel{\ominus}{\stackrel{\omega}{\triangleright}}$ & 2 & 6,54 & & 2 & 5,07 & & 2 & 4,15 & & 2 & 6,47 & & 2 & 4,19 & & & \\
\hline 3 & 3 & 6,54 & & 3 & 5,07 & & 3 & 5,09 & & 3 & 6,47 & & 3 & 4,19 & & & \\
\hline & 4 & 6,54 & & 4 & 5,07 & & 4 & 4,46 & & 4 & 6,43 & & 4 & 4,19 & & & \\
\hline
\end{tabular}


Tabelle B.5 : Center of Gravity: Quartalsdaten Kanada

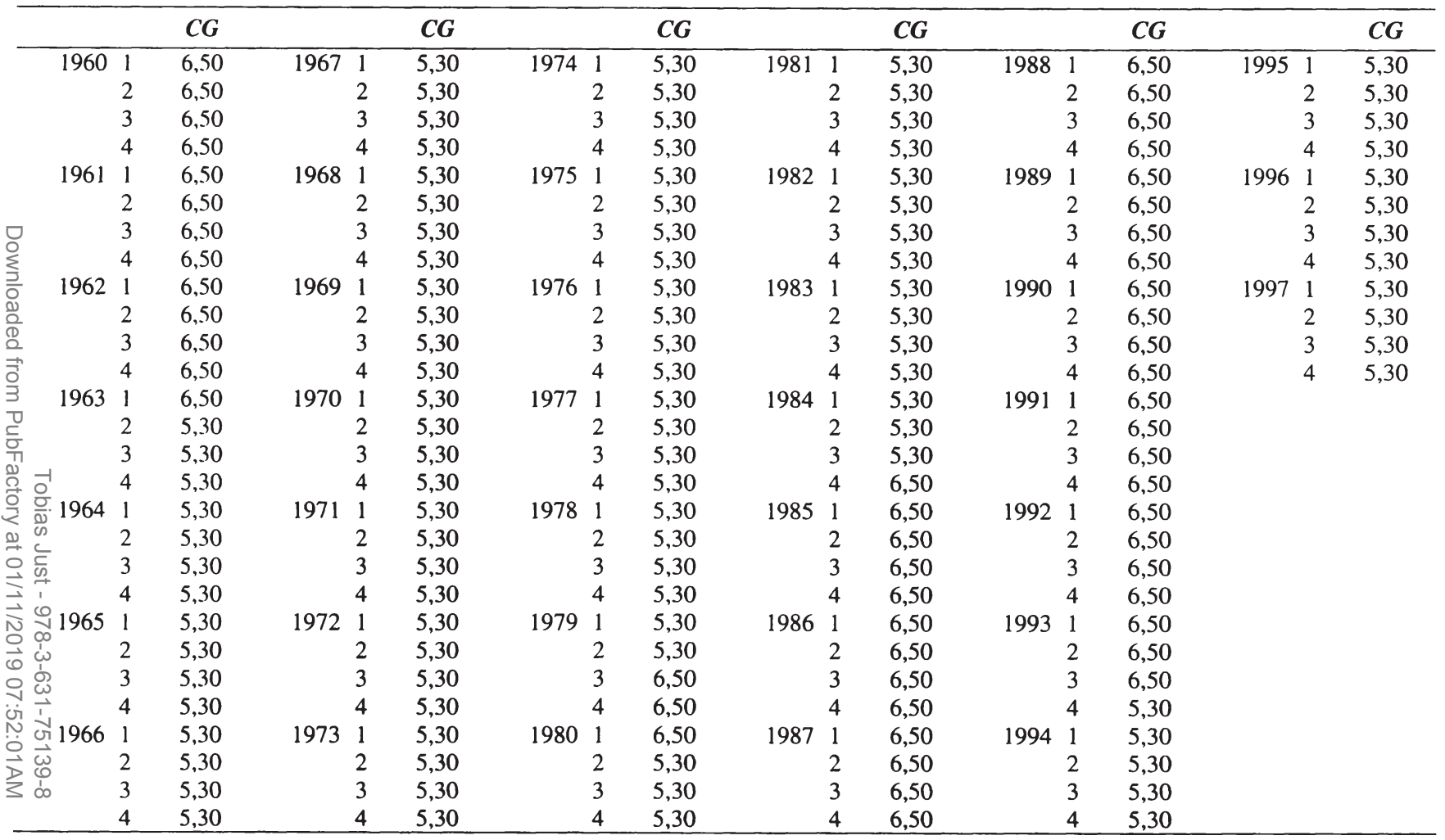


Tabelle B.6 : Center of Gravity: Quartalsdaten Dänemark

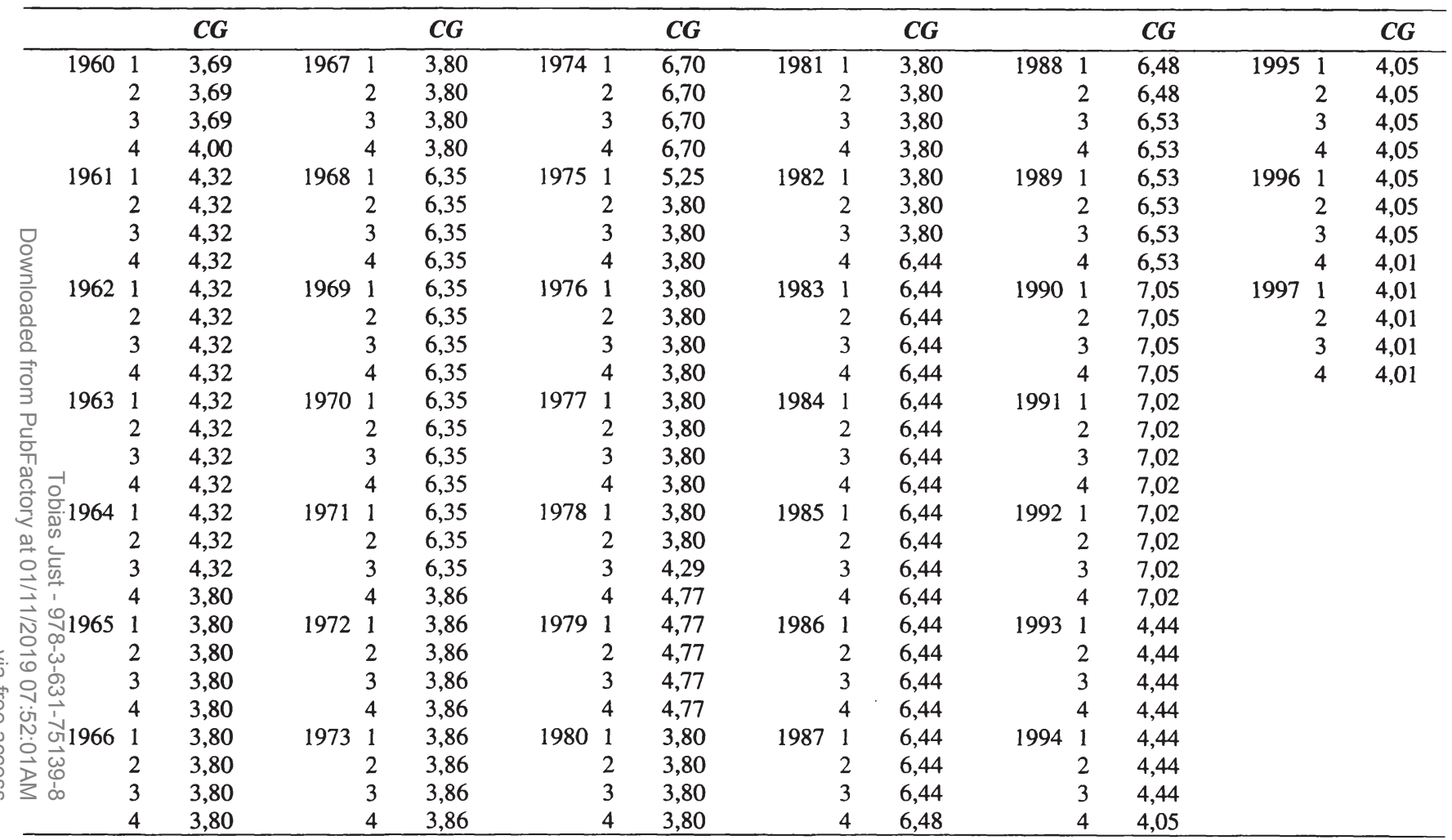


Tabelle B.7 : Center of Gravity: Quartalsdaten Finnland

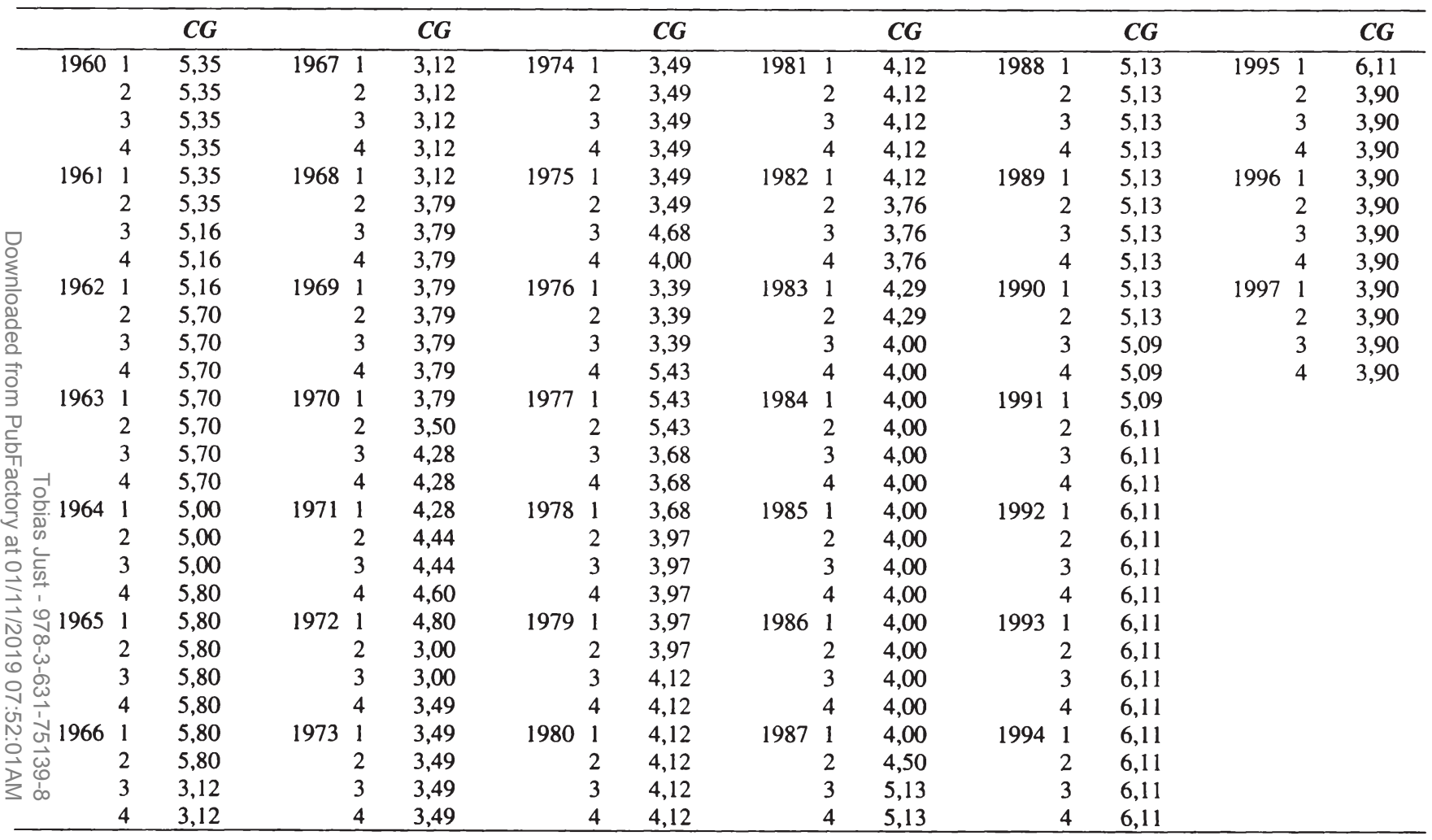


Tabelle B.8 : Center of Gravity: Quartalsdaten Frankreich

\begin{tabular}{|c|c|c|c|c|c|c|c|c|c|c|c|c|c|c|c|c|c|}
\hline \multirow{2}{*}{1960} & & \multirow{2}{*}{$\begin{array}{c}C G \\
6,50\end{array}$} & & & \multirow{2}{*}{$\frac{\boldsymbol{C} \boldsymbol{G}}{7,16}$} & \multicolumn{4}{|c|}{$C G$} & \multicolumn{2}{|r|}{$C G$} & \multicolumn{4}{|c|}{$C G$} & & \multirow{2}{*}{$\begin{array}{c}\boldsymbol{C} \boldsymbol{G} \\
7,39\end{array}$} \\
\hline & 1 & & 1967 & 1 & & 1974 & 1 & 7,75 & 1981 & 1 & 6,15 & 1988 & 1 & 7,30 & 1995 & 1 & \\
\hline & 2 & 6,50 & & 2 & 7,28 & & 2 & 6,80 & & 2 & 5,00 & & 2 & 3,24 & & 2 & 7,39 \\
\hline & 3 & 6,50 & & 3 & 7,28 & & 3 & 6,80 & & 3 & 2,53 & & 3 & 2,79 & & 3 & 7,66 \\
\hline & 4 & 6,50 & & 4 & 7,28 & & 4 & 6,80 & & 4 & 2,53 & & 4 & 2,79 & & 4 & 7,66 \\
\hline 1961 & 1 & 6,50 & 1968 & 1 & 7,28 & 1975 & 1 & 6,80 & 1982 & 1 & 2,53 & 1989 & 1 & 2,79 & 1996 & 1 & 7,63 \\
\hline & 2 & 6,50 & & 2 & 7,28 & & 2 & 6,80 & & 2 & 2,53 & & 2 & 2,79 & & 2 & 7,63 \\
\hline ס & 3 & 6,50 & & 3 & 6,67 & & 3 & 6,80 & & 3 & 2,53 & & 3 & 2,79 & & 3 & 7,63 \\
\hline § & 4 & 6,50 & & 4 & 6,67 & & 4 & 6,80 & & 4 & 2,53 & & 4 & 2,79 & & 4 & 7,63 \\
\hline 1962 & 1 & 6,50 & 1969 & 1 & 6,67 & 1976 & 1 & 6,80 & 1983 & 1 & 2,53 & 1990 & 1 & 2,79 & 1997 & 1 & 7,63 \\
\hline ڤ & 2 & 7,25 & & 2 & 6,67 & & 2 & 6,80 & & 2 & 2,53 & & 2 & 2,79 & & 2 & 7,63 \\
\hline व & 3 & 7,25 & & 3 & 7,12 & & 3 & 6,22 & & 3 & 2,53 & & 3 & 2,79 & & 3 & 2,41 \\
\hline $\overrightarrow{\text { Oे }}$ & 4 & 7,25 & & 4 & 7,12 & & 4 & 6,22 & & 4 & 2,53 & & 4 & 2,79 & & 4 & 2,41 \\
\hline 1963 & 1 & 7,32 & 1970 & 1 & 7,12 & 1977 & 1 & 6,22 & 1984 & 1 & 2,53 & 1991 & 1 & 2,79 & & & \\
\hline$\underset{C}{\complement}$ & 2 & 7,32 & & 2 & 7,12 & & 2 & 7,12 & & 2 & 2,53 & & 2 & 2,80 & & & \\
\hline 후 & 3 & 7,32 & & 3 & 7,12 & & 3 & 7,12 & & 3 & 2,65 & & 3 & 2,95 & & & \\
\hline กี & 4 & 7,32 & & 4 & 7,12 & & 4 & 7,12 & & 4 & 2,65 & & 4 & 2,95 & & & \\
\hline$\frac{\overrightarrow{0}}{2} \frac{\sigma}{\partial} \cdot 1964$ & 1 & 7,32 & 1971 & 1 & 7,12 & 1978 & 1 & 7,12 & 1985 & 1 & 2,65 & 1992 & 1 & 2,95 & & & \\
\hline $\begin{array}{ll}\infty \\
\approx\end{array}$ & 2 & 7,32 & & 2 & 7,12 & & 2 & 6,15 & & 2 & 2,65 & & 2 & 3,09 & & & \\
\hline $\begin{array}{l}\vec{c} \\
\circ \\
\end{array}$ & 3 & 7,32 & & 3 & 7,12 & & 3 & 6,15 & & 3 & 2,65 & & 3 & 3,09 & & & \\
\hline $\overrightarrow{1}$ & 4 & 7,32 & & 4 & 7,12 & & 4 & 6,15 & & 4 & 2,65 & & 4 & 3,09 & & & \\
\hline $\overrightarrow{\mathrm{N}} \underset{\sim}{\vec{V}} 1965$ & 1 & 7,32 & 1972 & 1 & 7,12 & 1979 & 1 & 6,15 & 1986 & 1 & 2,65 & 1993 & 1 & 3,09 & & & \\
\hline $\begin{array}{l}\infty \\
\omega \\
\omega\end{array}$ & 2 & 7,32 & & 2 & 7,12 & & 2 & 6,15 & & 2 & 7,30 & & 2 & 7,39 & & & \\
\hline $\begin{array}{ll}6 & 1 \\
0 & 0\end{array}$ & 3 & 7,32 & & 3 & 6,99 & & 3 & 6,15 & & 3 & 7,30 & & 3 & 7,39 & & & \\
\hline$\underset{\omega}{\sim} \stackrel{\omega}{1}$ & 4 & 7,32 & & 4 & 6,99 & & 4 & 6,15 & & 4 & 7,30 & & 4 & 7,39 & & & \\
\hline ํㅓㅇ जั1966 & 1 & 7,16 & 1973 & 1 & 6,99 & 1980 & 1 & 6,15 & 1987 & 1 & 7,30 & 1994 & 1 & 7,39 & & & \\
\hline$\stackrel{\circ}{\vec{\omega}}$ & 2 & 7,16 & & 2 & 7,75 & & 2 & 6,15 & & 2 & 7,30 & & 2 & 7,39 & & & \\
\hline $\begin{array}{l}1 \\
3 \\
1\end{array}$ & 3 & 7,16 & & 3 & 7,75 & & 3 & 6,15 & & 3 & 7,30 & & 3 & 7,39 & & & \\
\hline & 4 & 7,16 & & 4 & 7,75 & & 4 & 6,15 & & 4 & 7,30 & & 4 & 7,39 & & & \\
\hline
\end{tabular}


Tabelle B.9 : Center of Gravity: Quartalsdaten Deutschland

\begin{tabular}{|c|c|c|c|c|c|c|c|c|c|c|c|c|c|c|c|c|c|}
\hline & & $C G$ & & & $C G$ & & & $C G$ & & & $C G$ & & & $\boldsymbol{C G}$ & & & $C G$ \\
\hline 1960 & 1 & 6,79 & 1967 & 1 & 5,29 & 1974 & 1 & 3,75 & 1981 & 1 & 3,75 & 1988 & 1 & 6,47 & 1995 & 1 & 6,63 \\
\hline & 2 & 6,79 & & 2 & 5,29 & & 2 & 3,75 & & 2 & 3,75 & & 2 & 6,47 & & 2 & 6,63 \\
\hline & 3 & 6,97 & & 3 & 5,29 & & 3 & 3,75 & & 3 & 3,75 & & 3 & 6,47 & & 3 & 6,63 \\
\hline 1961 & 1 & 6,97 & 1968 & 1 & 5,29 & 1975 & 1 & 3,75 & 1982 & 1 & 6,37 & 1989 & 1 & 6,47 & 1996 & 1 & 6,63 \\
\hline & 2 & 6,97 & & 2 & 5,29 & & 2 & 3,75 & & 2 & 6,61 & & 2 & 6,47 & & 2 & 6,63 \\
\hline ס & 3 & 6,97 & & 3 & 5,29 & & 3 & 3,75 & & 3 & 6,61 & & 3 & 6,47 & & 3 & 6,63 \\
\hline ڤ & 2 & 6,55 & & 2 & 5,29 & & 2 & 3,75 & & 2 & 6,61 & & 2 & 6,47 & & 2 & 6,63 \\
\hline$\stackrel{1}{\varrho}$ & 3 & 6,55 & & 3 & 5,29 & & 3 & 3,75 & & 3 & 6,61 & & 3 & 6,47 & & 3 & 6,63 \\
\hline 疋 & 4 & 6,55 & & 4 & 3,74 & & 4 & 3,75 & & 4 & 6,61 & & 4 & 6,47 & & 4 & 6,63 \\
\hline 1963 & 1 & 6,55 & 1970 & 1 & 3,74 & 1977 & 1 & 3,75 & 1984 & 1 & 6,61 & 1991 & 1 & 6,47 & & & \\
\hline$\underset{ᄃ}{\nearrow}$ & 2 & 6,55 & & 2 & 3,74 & & 2 & 3,75 & & 2 & 6,61 & & 2 & 6,47 & & & \\
\hline 宁 & 3 & 6,55 & & 3 & 3,74 & & 3 & 3,75 & & 3 & 6,61 & & 3 & 6,47 & & & \\
\hline$\stackrel{+}{\perp}$ & 4 & 6,36 & & 4 & 3,74 & & 4 & 3,75 & & 4 & 6,61 & & 4 & 6,47 & & & \\
\hline $\overrightarrow{\vec{N}} \underset{\infty}{\bullet} 1965$ & 1 & 6,36 & 1972 & 1 & 3,74 & 1979 & 1 & 3,75 & 1986 & 1 & 6,61 & 1993 & 1 & 6,47 & & & \\
\hline $\begin{array}{l}0 \\
0 \\
0 \\
\omega\end{array}$ & 2 & 6,36 & & 2 & 3,74 & & 2 & 3,75 & & 2 & 6,61 & & 2 & 6,47 & & & \\
\hline $\begin{array}{l}0 \\
0 \\
0\end{array}$ & 3 & 6,36 & & 3 & 3,64 & & 3 & 3,75 & & 3 & 6,61 & & 3 & 6,47 & & & \\
\hline نे & 4 & 6,62 & & 4 & 3,64 & & 4 & 3,75 & & 4 & 6,61 & & 4 & 6,47 & & & \\
\hline 1966 & 1 & 6,62 & 1973 & 1 & 3,75 & 1980 & 1 & 3,75 & 1987 & 1 & 6,61 & 1994 & 1 & 6,47 & & & \\
\hline$\vec{D} \vec{\omega}$ & 2 & 6,62 & & 2 & 3,75 & & 2 & 3,75 & & 2 & 6,47 & & 2 & 6,47 & & & \\
\hline $\begin{array}{l}1 \\
3 \\
3\end{array}$ & 3 & 6,62 & & 3 & 3,75 & & 3 & 3,75 & & 3 & 6,47 & & 3 & 6,47 & & & \\
\hline & 4 & 6,62 & & 4 & 3,75 & & 4 & 3,75 & & 4 & 6,47 & & 4 & 6,47 & & & \\
\hline
\end{tabular}


Tabelle B.10 : Center of Gravity: Quartalsdaten Irland

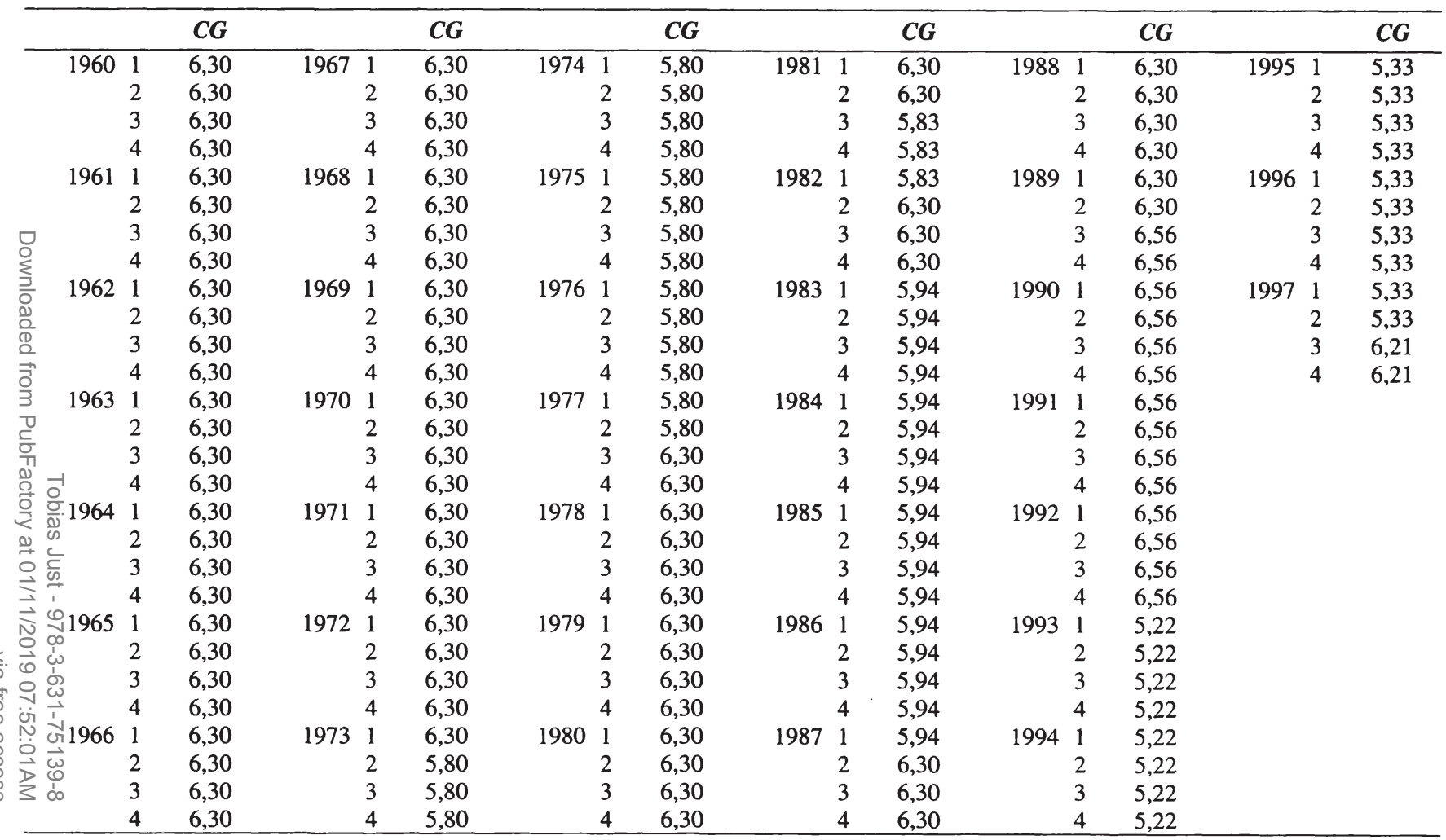


Tabelle B.11 : Center of Gravity: Quartalsdaten Italien

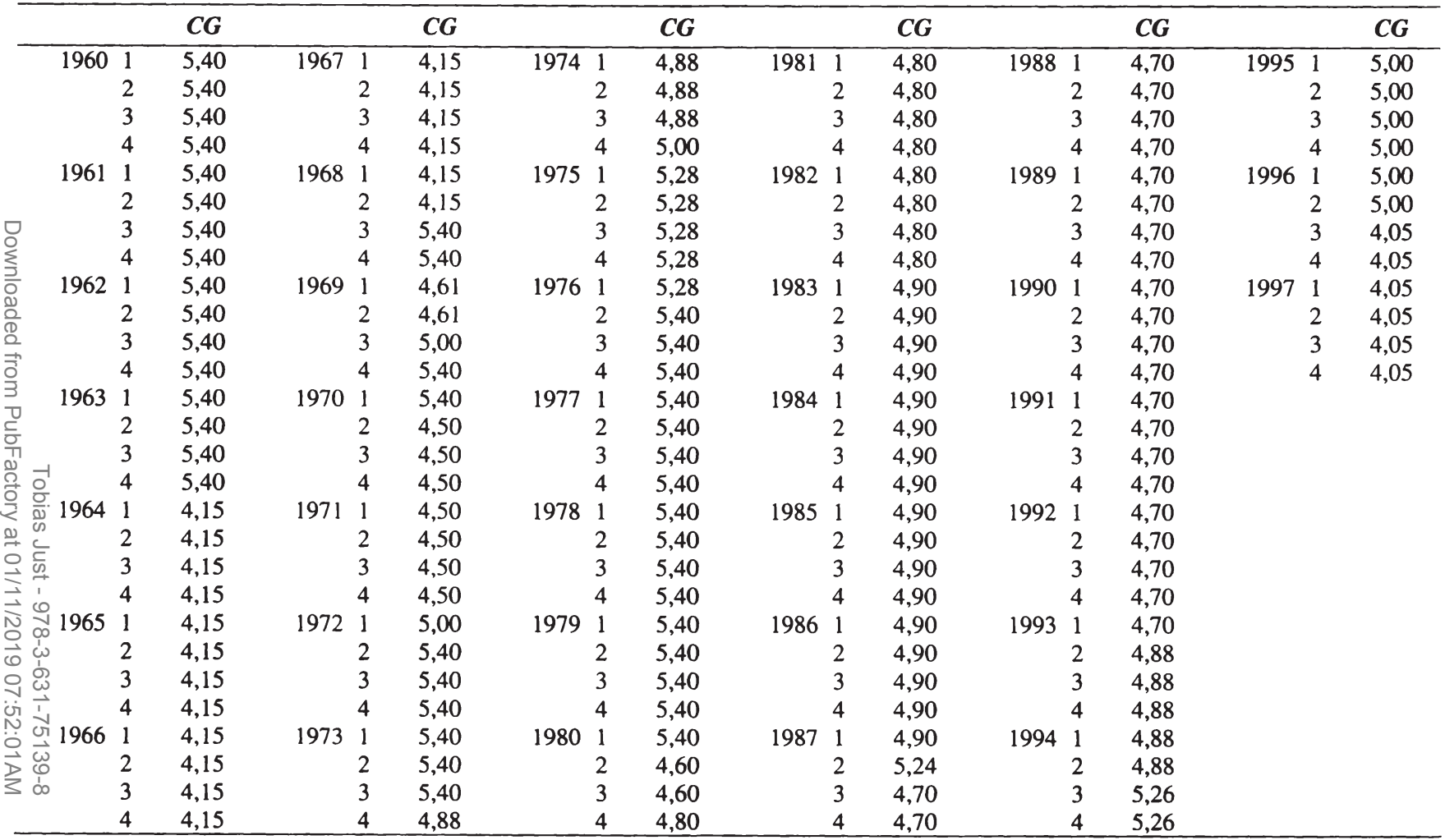


Tabelle B.12 : Center of Gravity: Quartalsdaten Japan

\begin{tabular}{|c|c|c|c|c|c|c|c|c|c|c|c|c|c|c|c|c|c|}
\hline & & $C G$ & & & $C G$ & & & $C G$ & & & $C G$ & & & $C G$ & & & $C G$ \\
\hline 1960 & 1 & 8,00 & 1967 & 1 & 8,00 & 1974 & 1 & 8,00 & 1981 & 1 & 8,00 & 1988 & 1 & 8,00 & 1995 & 1 & 6,29 \\
\hline & 2 & 8,00 & & 2 & 8,00 & & 2 & 8,00 & & 2 & 8,00 & & 2 & 8,00 & & 2 & 6,29 \\
\hline & 3 & 8,00 & & 3 & 8,00 & & 3 & 8,00 & & 3 & 8,00 & & 3 & 8,00 & & 3 & 6,29 \\
\hline & 4 & 8,00 & & 4 & 8,00 & & 4 & 8,00 & & 4 & 8,00 & & 4 & 8,00 & & 4 & 6,29 \\
\hline 1961 & 1 & 8,00 & 1968 & 1 & 8,00 & 1975 & 1 & 8,00 & 1982 & 1 & 8,00 & 1989 & 1 & 8,00 & 1996 & 1 & 4,58 \\
\hline & 2 & 8,00 & & 2 & 8,00 & & 2 & 8,00 & & 2 & 8,00 & & 2 & 8,00 & & 2 & 5,58 \\
\hline 망 & 3 & 8,00 & & 3 & 8,00 & & 3 & 8,00 & & 3 & 8,00 & & 3 & 8,00 & & 3 & 5,58 \\
\hline \ั & 4 & 8,00 & & 4 & 8,00 & & 4 & 8,00 & & 4 & 8,00 & & 4 & 8,00 & & 4 & 6,50 \\
\hline 1962 & 1 & 8,00 & 1969 & 1 & 8,00 & 1976 & 1 & 8,00 & 1983 & 1 & 8,00 & 1990 & 1 & 8,00 & 1997 & 1 & 8,00 \\
\hline ڤ్రి & 2 & 8,00 & & 2 & 8,00 & & 2 & 8,00 & & 2 & 8,00 & & 2 & 8,00 & & 2 & 8,00 \\
\hline$\frac{2}{8}$ & 3 & 8,00 & & 3 & 8,00 & & 3 & 8,00 & & 3 & 8,00 & & 3 & 8,00 & & 3 & 8,00 \\
\hline $\overrightarrow{0}$ & 4 & 8,00 & & 4 & 8,00 & & 4 & 8,00 & & 4 & 8,00 & & 4 & 8,00 & & 4 & 8,00 \\
\hline 1963 & 1 & 8,00 & 1970 & 1 & 8,00 & 1977 & 1 & 8,00 & 1984 & 1 & 8,00 & 1991 & 1 & 8,00 & & & \\
\hline$\underset{c}{0}$ & 2 & 8,00 & & 2 & 8,00 & & 2 & 8,00 & & 2 & 8,00 & & 2 & 8,00 & & & \\
\hline 후 & 3 & 8,00 & & 3 & 8,00 & & 3 & 8,00 & & 3 & 8,00 & & 3 & 8,00 & & & \\
\hline$\stackrel{-1}{\circ}$ & 4 & 8,00 & & 4 & 8,00 & & 4 & 8,00 & & 4 & 8,00 & & 4 & 8,00 & & & \\
\hline$\frac{0}{\frac{0}{2}} \frac{0}{2} \cdot 1964$ & 1 & 8,00 & 1971 & 1 & 8,00 & 1978 & 1 & 8,00 & 1985 & 1 & 8,00 & 1992 & 1 & 8,00 & & & \\
\hline की & 2 & 8,00 & & 2 & 8,00 & & 2 & 8,00 & & 2 & 8,00 & & 2 & 8,00 & & & \\
\hline$\stackrel{\overrightarrow{0}}{\circ} \frac{c}{\infty}$ & 3 & 8,00 & & 3 & 8,00 & & 3 & 8,00 & & 3 & 8,00 & & 3 & 8,00 & & & \\
\hline 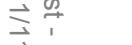 & 4 & 8,00 & & 4 & 8,00 & & 4 & 8,00 & & 4 & 8,00 & & 4 & 8,00 & & & \\
\hline$\stackrel{\vec{N}}{*} 1965$ & 1 & 8,00 & 1972 & 1 & 8,00 & 1979 & 1 & 8,00 & 1986 & 1 & 8,00 & 1993 & 1 & 8,00 & & & \\
\hline $\begin{array}{ll} & 0 \\
0 & 1 \\
1 & 1\end{array}$ & 2 & 8,00 & & 2 & 8,00 & & 2 & 8,00 & & 2 & 8,00 & & 2 & 8,00 & & & \\
\hline$\because$ o & 3 & 8,00 & & 3 & 8,00 & & 3 & 8,00 & & 3 & 8,00 & & 3 & 6,00 & & & \\
\hline$\nu \omega$ & 4 & 8,00 & & 4 & 8,00 & & 4 & 8,00 & & 4 & 8,00 & & 4 & 3,88 & & & \\
\hline Nㅓำ जั1966 & 1 & 8,00 & 1973 & 1 & 8,00 & 1980 & 1 & 8,00 & 1987 & 1 & 8,00 & 1994 & 1 & 3,88 & & & \\
\hline$\stackrel{\vec{\omega}}{\vec{\omega}}$ & 2 & 8,00 & & 2 & 8,00 & & 2 & 8,00 & & 2 & 8,00 & & 2 & 4,71 & & & \\
\hline $\begin{array}{l}1 \\
3 \\
3\end{array}$ & 3 & 8,00 & & 3 & 8,00 & & 3 & 8,00 & & 3 & 8,00 & & 3 & 6,29 & & & \\
\hline & 4 & 8,00 & & 4 & 8,00 & & 4 & 8,00 & & 4 & 8,00 & & 4 & 6,29 & & & \\
\hline
\end{tabular}


Tabelle B.13 : Center of Gravity: Quartalsdaten Niederlande

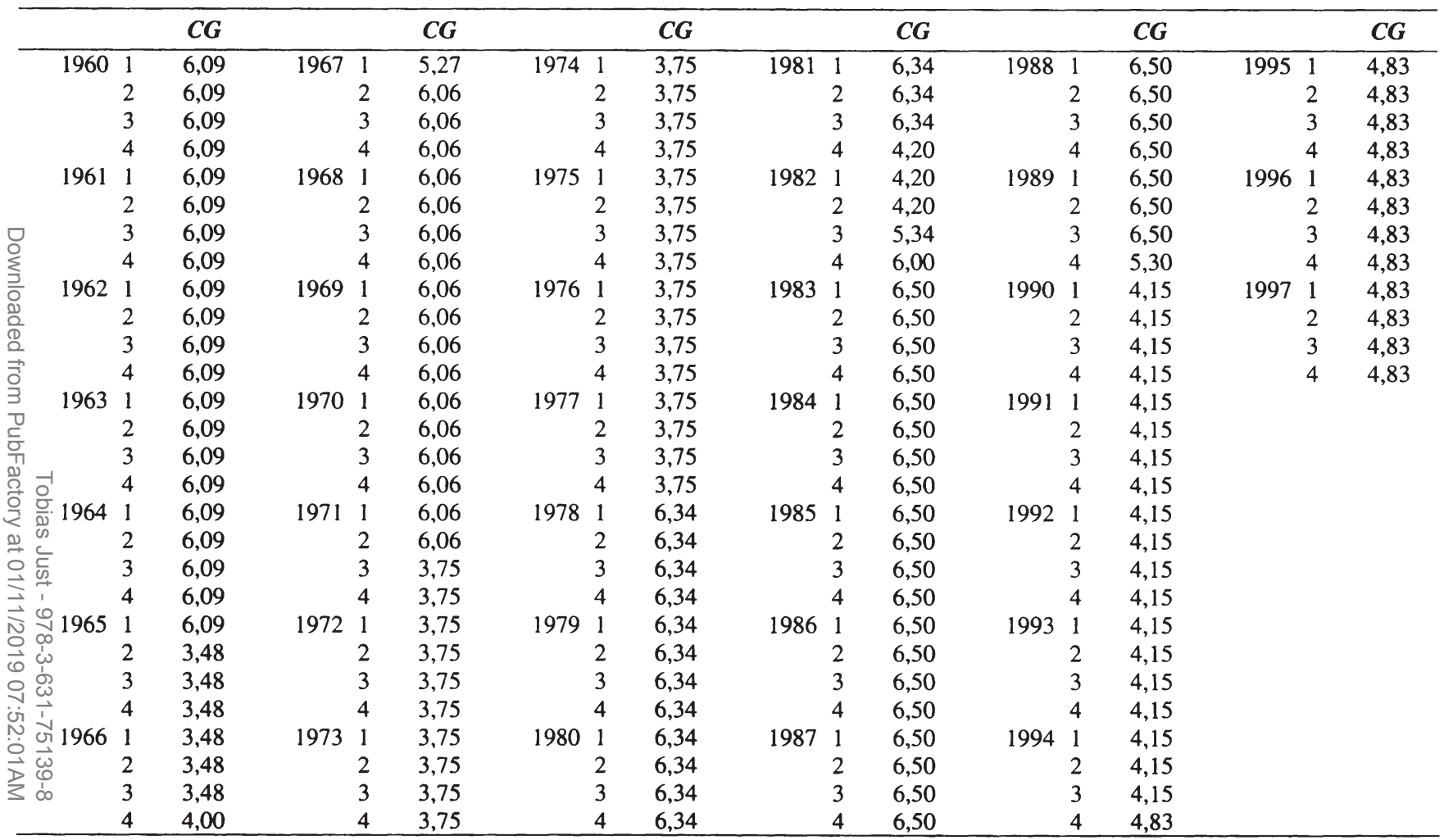


Tabelle B.14 : Center of Gravity: Quartalsdaten Neuseeland

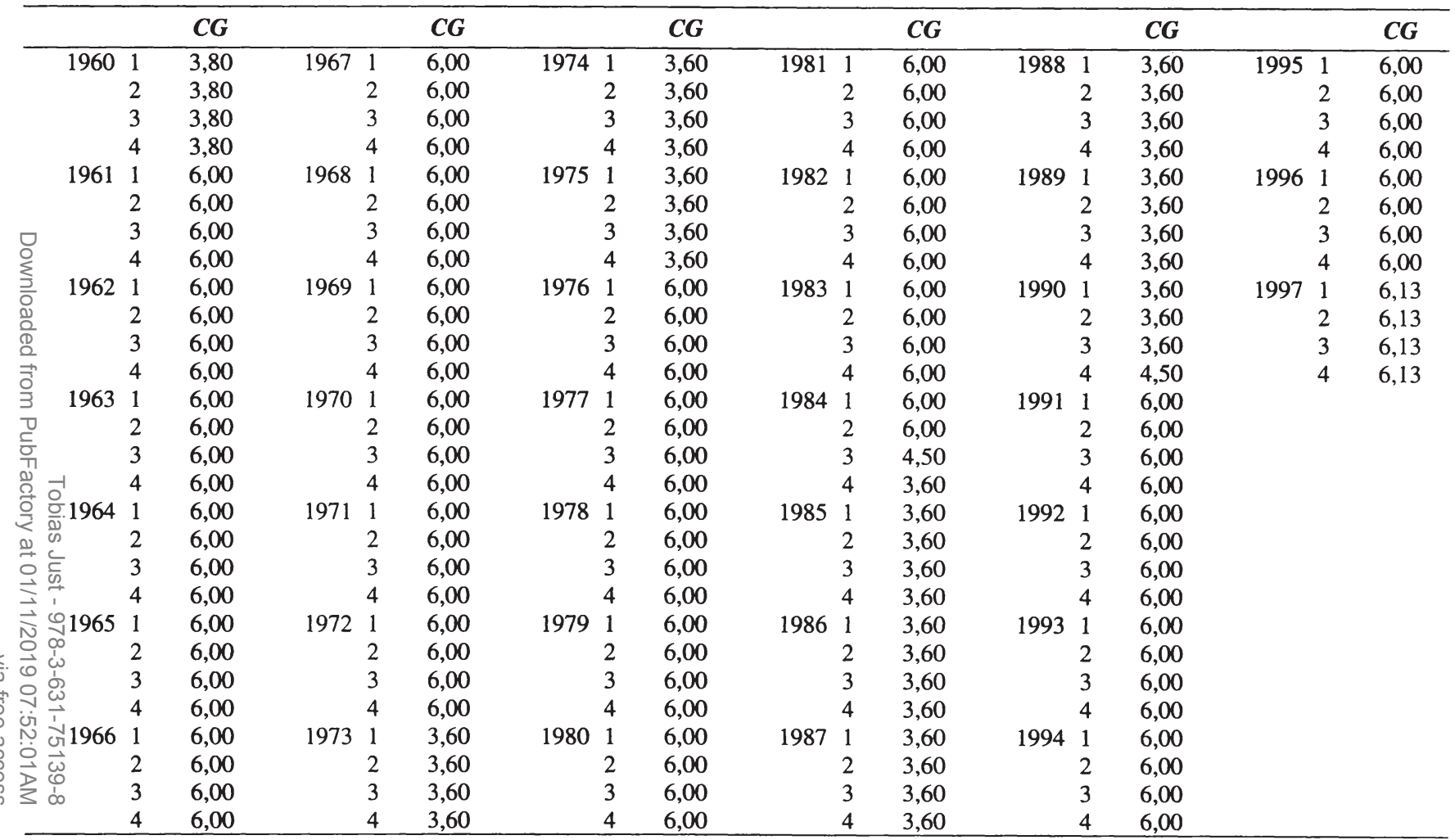


Tabelle B.15 : Center of Gravity: Quartalsdaten Norwegen

\begin{tabular}{|c|c|c|c|c|c|c|c|c|c|c|c|c|c|c|c|c|c|c|}
\hline & & & $C G$ & & & $C G$ & & & $C G$ & & & $C G$ & & & $C G$ & & & $C G$ \\
\hline & 1960 & 1 & 3,00 & 1967 & 1 & 6,26 & 1974 & 1 & 3,00 & 1981 & 1 & 3,00 & 1988 & 1 & 3,00 & 1995 & 1 & 3,00 \\
\hline & & 2 & 3,00 & & 2 & 6,26 & & 2 & 3,00 & & 2 & 3,00 & & 2 & 3,00 & & 2 & 3,00 \\
\hline & & 3 & 3,00 & & 3 & 6,26 & & 3 & 3,00 & & 3 & 3,00 & & 3 & 3,00 & & 3 & 3,00 \\
\hline & & 4 & 3,00 & & 4 & 6,26 & & 4 & 3,00 & & 4 & 7,70 & & 4 & 3,00 & & 4 & 3,00 \\
\hline & 1961 & 1 & 3,00 & 1968 & 1 & 6,26 & 1975 & 1 & 3,00 & 1982 & 1 & 7,70 & 1989 & 1 & 3,00 & 1996 & 1 & 3,00 \\
\hline & & 2 & 3,00 & & 2 & 6,26 & & 2 & 3,00 & & 2 & 7,70 & & 2 & 3,00 & & 2 & 3,00 \\
\hline$\nabla$ & & 3 & 3,00 & & 3 & 6,26 & & 3 & 3,00 & & 3 & 7,70 & & 3 & 3,00 & & 3 & 3,00 \\
\hline$\sum$ & & 4 & 3,00 & & 4 & 6,26 & & 4 & 3,00 & & 4 & 7,70 & & 4 & 6,71 & & 4 & 3,00 \\
\hline$\overline{\bar{O}}$ & 1962 & 1 & 3,00 & 1969 & 1 & 6,26 & 1976 & 1 & 3,00 & 1983 & 1 & 7,70 & 1990 & 1 & 6,71 & 1997 & 1 & 3,00 \\
\hline$\stackrel{0}{\circ}$ & & 2 & 3,00 & & 2 & 6,26 & & 2 & 3,00 & & 2 & 7,70 & & 2 & 6,71 & & 2 & 3,00 \\
\hline$\stackrel{2}{2}$ & & 3 & 3,00 & & 3 & 6,26 & & 3 & 3,00 & & 3 & 7,12 & & 3 & 6,71 & & 3 & 3,00 \\
\hline$\underline{\overrightarrow{0}}$ & & 4 & 3,00 & & 4 & 6,26 & & 4 & 3,00 & & 4 & 7,12 & & 4 & 6,71 & & 4 & 5,16 \\
\hline 3 & 1963 & 1 & 3,00 & 1970 & 1 & 6,26 & 1977 & 1 & 3,00 & 1984 & 1 & 7,12 & 1991 & 1 & 3,00 & & & \\
\hline$\frac{0}{\delta}$ & & 2 & 3,00 & & 2 & 6,26 & & 2 & 3,00 & & 2 & 7,12 & & 2 & 3,00 & & & \\
\hline ग1 & & 3 & 3,00 & & 3 & 6,26 & & 3 & 3,00 & & 3 & 7,12 & & 3 & 3,00 & & & \\
\hline$\frac{0}{0}$ & & 4 & 3,00 & & 4 & 6,26 & & 4 & 3,00 & & 4 & 7,12 & & 4 & 3,00 & & & \\
\hline$\frac{2}{0}$ & 1964 & 1 & 3,00 & 1971 & 1 & 6,26 & 1978 & 1 & 3,00 & 1985 & 1 & 7,12 & 1992 & 1 & 3,00 & & & \\
\hline$\stackrel{n}{\Rightarrow}$ & & 2 & 3,00 & & 2 & 3,00 & & 2 & 3,00 & & 2 & 7,12 & & 2 & 3,00 & & & \\
\hline$\stackrel{\circ}{\rightleftharpoons} \stackrel{5}{=}$ & & 3 & 3,00 & & 3 & 3,00 & & 3 & 3,00 & & 3 & 7,12 & & 3 & 3,00 & & & \\
\hline$\vec{P}$ & & 4 & 3,00 & & 4 & 3,00 & & 4 & 3,00 & & 4 & 7,12 & & 4 & 3,00 & & & \\
\hline जे & 1965 & 1 & 3,00 & 1972 & 1 & 3,00 & 1979 & 1 & 3,00 & 1986 & 1 & 6,92 & 1993 & 1 & 3,00 & & & \\
\hline $\overrightarrow{0}$ & & 2 & 3,00 & & 2 & 3,00 & & 2 & 3,00 & & 2 & 6,92 & & 2 & 3,00 & & & \\
\hline ○ c & & 3 & 3,00 & & 3 & 3,00 & & 3 & 3,00 & & 3 & 3,00 & & 3 & 3,00 & & & \\
\hline ن I & & 4 & 6,26 & & 4 & 5,28 & & 4 & 3,00 & & 4 & 3,00 & & 4 & 3,00 & & & \\
\hline$\because 0$ & 1966 & 1 & 6,26 & 1973 & 1 & 5,28 & 1980 & 1 & 3,00 & 1987 & 1 & 3,00 & 1994 & 1 & 3,00 & & & \\
\hline$\vec{D}$ & & 2 & 6,26 & & 2 & 5,28 & & 2 & 3,00 & & 2 & 3,00 & & 2 & 3,00 & & & \\
\hline $3 a^{\prime}$ & & 3 & 6,26 & & 3 & 5,28 & & 3 & 3,00 & & 3 & 3,00 & & 3 & 3,00 & & & \\
\hline & & 4 & 6,26 & & 4 & 3,00 & & 4 & 3,00 & & 4 & 3,00 & & 4 & 3,00 & & & \\
\hline
\end{tabular}


Tabelle B.16 : Center of Gravity: Quartalsdaten Schweden

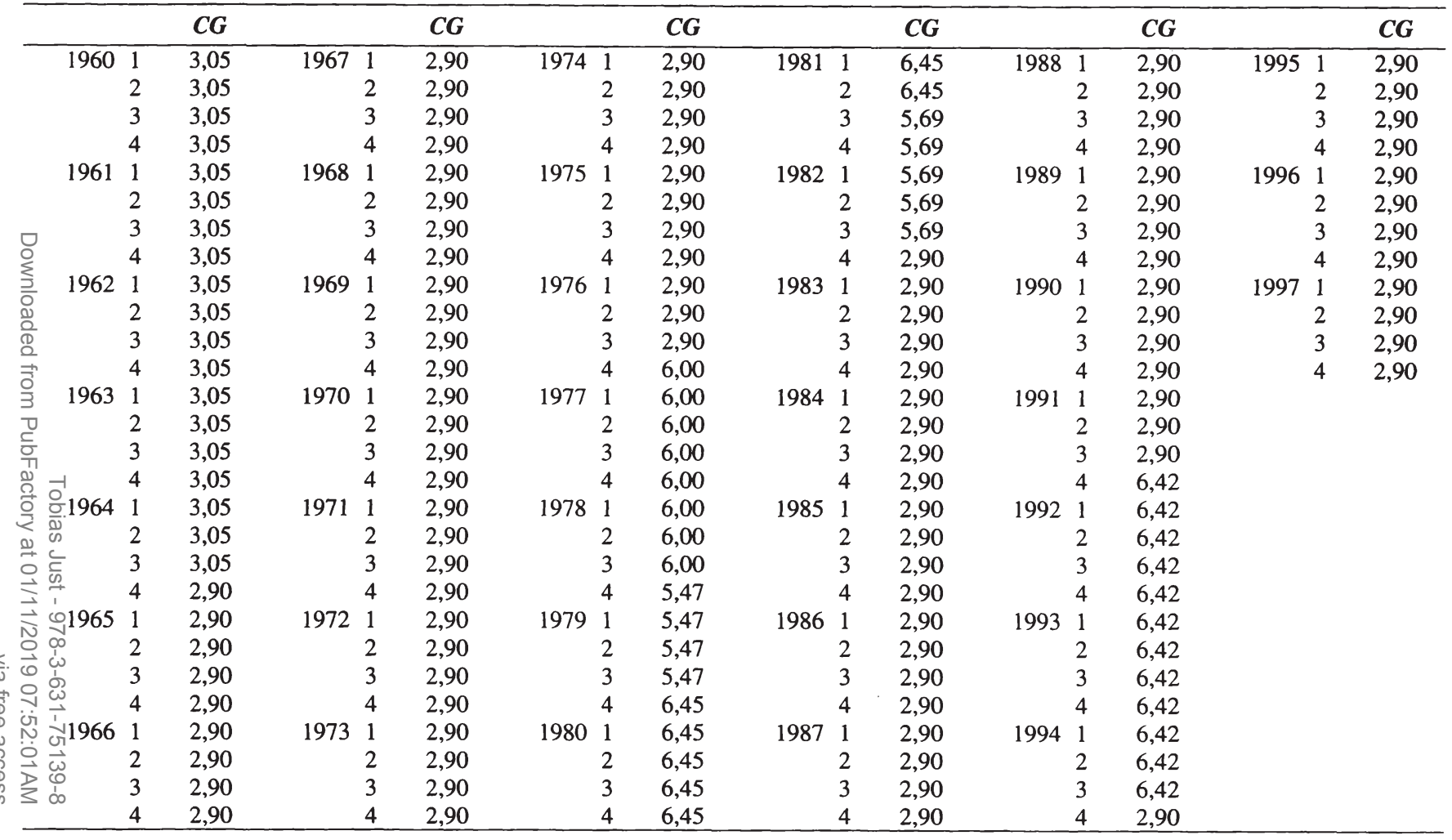


Tabelle B.17 : Center of Gravity: Quartalsdaten Schweiz

\begin{tabular}{|c|c|c|c|c|c|c|c|c|c|c|c|c|c|c|c|c|c|c|}
\hline & & & $C G$ & & & $C G$ & & & $C G$ & & & $C G$ & & & $C G$ & & & $C G$ \\
\hline & 1960 & 1 & 4,43 & 1967 & 1 & 4,43 & 1974 & 1 & 4,43 & 1981 & 1 & 4,43 & 1988 & 1 & 4,43 & 1995 & 1 & 4,43 \\
\hline & & 2 & 4,43 & & 2 & 4,43 & & 2 & 4,43 & & 2 & 4,43 & & 2 & 4,43 & & 2 & 4,43 \\
\hline & & 3 & 4,43 & & 3 & 4,43 & & 3 & 4,43 & & 3 & 4,43 & & 3 & 4,43 & & 3 & 4,43 \\
\hline & & 4 & 4,43 & & 4 & 4,43 & & 4 & 4,43 & & 4 & 4,43 & & 4 & 4,43 & & 4 & 4,43 \\
\hline & 1961 & 1 & 4,43 & 1968 & 1 & 4,43 & 1975 & 1 & 4,43 & 1982 & 1 & 4,43 & 1989 & 1 & 4,43 & 1996 & 1 & 4,43 \\
\hline & & 2 & 4,43 & & 2 & 4,43 & & 2 & 4,43 & & 2 & 4,43 & & 2 & 4,43 & & 2 & 4,43 \\
\hline ஜ & & 3 & 4,43 & & 3 & 4,43 & & 3 & 4,43 & & 3 & 4,43 & & 3 & 4,43 & & 3 & 4,43 \\
\hline$\stackrel{0}{\vdots}$ & & 4 & 4,43 & & 4 & 4,43 & & 4 & 4,43 & & 4 & 4,43 & & 4 & 4,43 & & 4 & 4,43 \\
\hline$\frac{\partial}{\bar{O}}$ & 1962 & 1 & 4,43 & 1969 & 1 & 4,43 & 1976 & 1 & 4,43 & 1983 & 1 & 4,43 & 1990 & 1 & 4,43 & 1997 & 1 & 4,43 \\
\hline$\stackrel{0}{\circ}$ & & 2 & 4,43 & & 2 & 4,43 & & 2 & 4,43 & & 2 & 4,43 & & 2 & 4,43 & & 2 & 4,43 \\
\hline$\stackrel{1}{2}$ & & 3 & 4,43 & & 3 & 4,43 & & 3 & 4,43 & & 3 & 4,43 & & 3 & 4,43 & & 3 & 4,43 \\
\hline $\overrightarrow{\overrightarrow{0}}$ & & 4 & 4,43 & & 4 & 4,43 & & 4 & 4,43 & & 4 & 4,43 & & 4 & 4,43 & & 4 & 4,43 \\
\hline 3 & 1963 & 1 & 4,43 & 1970 & 1 & 4,43 & 1977 & 1 & 4,43 & 1984 & 1 & 4,43 & 1991 & 1 & 4,43 & & & \\
\hline ᄃ & & 2 & 4,43 & & 2 & 4,43 & & 2 & 4,43 & & 2 & 4,43 & & 2 & 4,43 & & & \\
\hline 员 & & 3 & 4,43 & & 3 & 4,43 & & 3 & 4,43 & & 3 & 4,43 & & 3 & 4,43 & & & \\
\hline م & & 4 & 4,43 & & 4 & 4,43 & & 4 & 4,43 & & 4 & 4,43 & & 4 & 4,43 & & & \\
\hline$\frac{0}{2}$ & . 1964 & 1 & 4,43 & 1971 & 1 & 4,43 & 1978 & 1 & 4,43 & 1985 & 1 & 4,43 & 1992 & 1 & 4,43 & & & \\
\hline$\stackrel{\infty}{\rightleftharpoons}$ & & 2 & 4,43 & & 2 & 4,43 & & 2 & 4,43 & & 2 & 4,43 & & 2 & 4,43 & & & \\
\hline$\stackrel{\circ}{\rightleftharpoons}$ & & 3 & 4,43 & & 3 & 4,43 & & 3 & 4,43 & & 3 & 4,43 & & 3 & 4,43 & & & \\
\hline$\vec{\exists}$ & & 4 & 4,43 & & 4 & 4,43 & & 4 & 4,43 & & 4 & 4,43 & & 4 & 4,43 & & & \\
\hline $\overrightarrow{\mathrm{N}}$ & D. 1965 & 1 & 4,43 & 1972 & 1 & 4,43 & 1979 & 1 & 4,43 & 1986 & 1 & 4,43 & 1993 & 1 & 4,43 & & & \\
\hline $\overrightarrow{0}$ & & 2 & 4,43 & & 2 & 4,43 & & 2 & 4,43 & & 2 & 4,43 & & 2 & 4,43 & & & \\
\hline$\circ$ & & 3 & 4,43 & & 3 & 4,43 & & 3 & 4,43 & & 3 & 4,43 & & 3 & 4,43 & & & \\
\hline जे. & & 4 & 4,43 & & 4 & 4,43 & & 4 & 4,43 & & 4 & 4,43 & & 4 & 4,43 & & & \\
\hline Nִ & 1966 & 1 & 4,43 & 1973 & 1 & 4,43 & 1980 & 1 & 4,43 & 1987 & 1 & 4,43 & 1994 & 1 & 4,43 & & & \\
\hline$\vec{D}$ & ڤ્. & 2 & 4,43 & & 2 & 4,43 & & 2 & 4,43 & & 2 & 4,43 & & 2 & 4,43 & & & \\
\hline 3 & & 3 & 4,43 & & 3 & 4,43 & & 3 & 4,43 & & 3 & 4,43 & & 3 & 4,43 & & & \\
\hline & & 4 & 4,43 & & 4 & 4,43 & & 4 & 4,43 & & 4 & 4,43 & & 4 & 4,43 & & & \\
\hline
\end{tabular}


Tabelle B.18 : Center of Gravity: Quartalsdaten Spanien

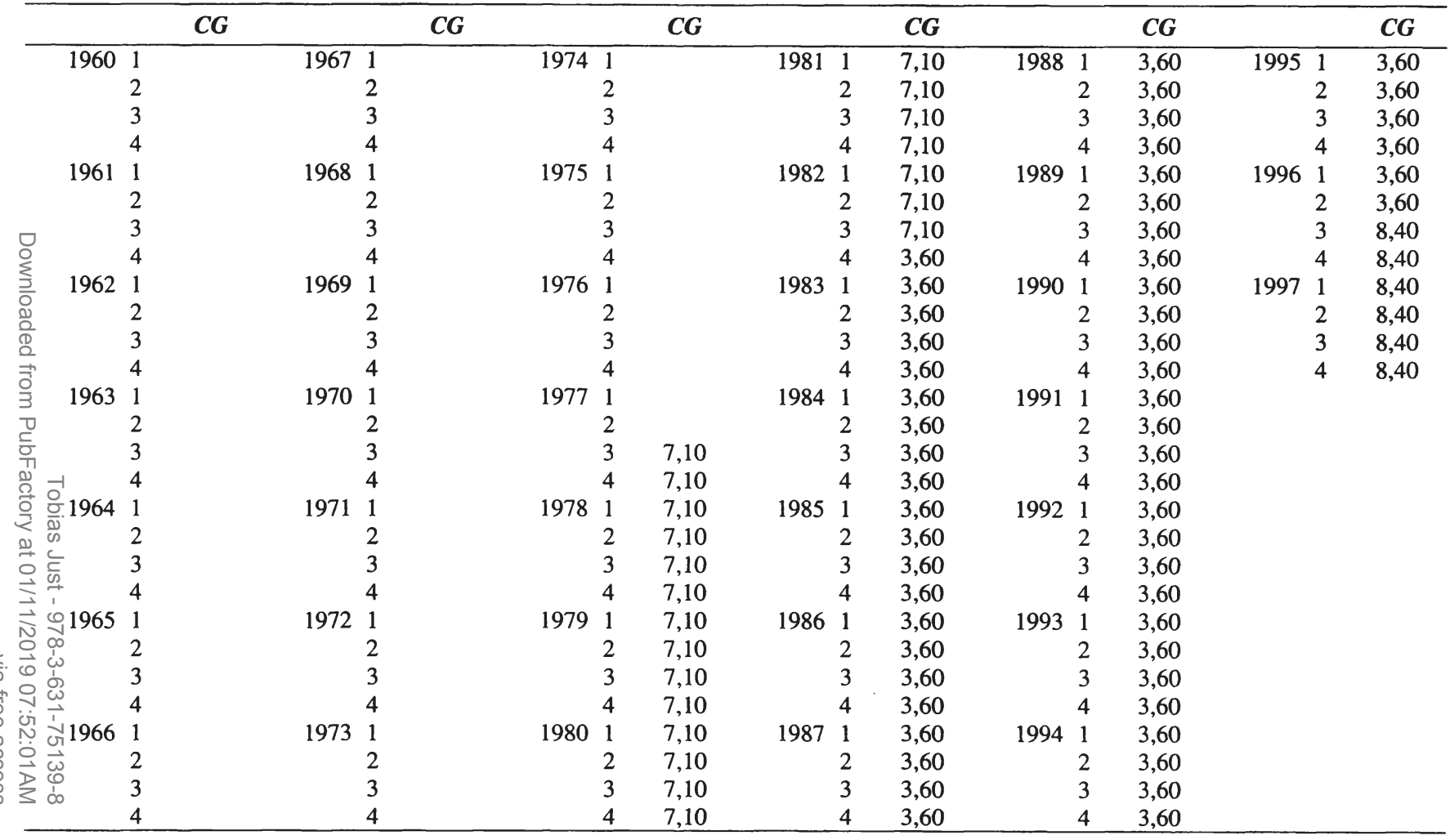


Tabelle B.19 : Center of Gravity: Quartalsdaten Großbritannien

\begin{tabular}{|c|c|c|c|c|c|c|c|c|c|c|c|c|c|c|c|c|c|c|}
\hline & & & $C G$ & & & $C G$ & & & $C G$ & & & $C G$ & & & $C G$ & & & $C G$ \\
\hline & 1960 & 1 & 7,80 & 1967 & 1 & 2,30 & 1974 & 1 & 7,80 & 1981 & 1 & 7,80 & 1988 & 1 & 7,80 & 1995 & 1 & 7,80 \\
\hline & & 2 & 7,80 & & 2 & 2,30 & & 2 & 2,30 & & 2 & 7,80 & & 2 & 7,80 & & 2 & 7,80 \\
\hline & & 3 & 7,80 & & 3 & 2,30 & & 3 & 2,30 & & 3 & 7,80 & & 3 & 7,80 & & 3 & 7,80 \\
\hline & & 4 & 7,80 & & 4 & 2,30 & & 4 & 2,30 & & 4 & 7,80 & & 4 & 7,80 & & 4 & 7,80 \\
\hline & 1961 & 1 & 7,80 & 1968 & 1 & 2,30 & 1975 & 1 & 2,30 & 1982 & 1 & 7,80 & 1989 & 1 & 7,80 & 1996 & 1 & 7,80 \\
\hline & & 2 & 7,80 & & 2 & 2,30 & & 2 & 2,30 & & 2 & 7,80 & & 2 & 7,80 & & 2 & 7,80 \\
\hline 무 & & 3 & 7,80 & & 3 & 2,30 & & 3 & 2,30 & & 3 & 7,80 & & 3 & 7,80 & & 3 & 7,80 \\
\hline$\sum$ & & 4 & 7,80 & & 4 & 2,30 & & 4 & 2,30 & & 4 & 7,80 & & 4 & 7,80 & & 4 & 7,80 \\
\hline $\bar{\partial}$ & 1962 & 1 & 7,80 & 1969 & 1 & 2,30 & 1976 & 1 & 2,30 & 1983 & 1 & 7,80 & 1990 & 1 & 7,80 & 1997 & 1 & 7,80 \\
\hline ำ & & 2 & 7,80 & & 2 & 2,30 & & 2 & 2,30 & & 2 & 7,80 & & 2 & 7,80 & & 2 & 7,80 \\
\hline$\stackrel{10}{\varrho}$ & & 3 & 7,80 & & 3 & 2,30 & & 3 & 2,30 & & 3 & 7,80 & & 3 & 7,80 & & 3 & 2,30 \\
\hline 옴 & & 4 & 7,80 & & 4 & 2,30 & & 4 & 2,30 & & 4 & 7,80 & & 4 & 7,80 & & 4 & 2,30 \\
\hline 3 & 1963 & 1 & 7,80 & 1970 & 1 & 2,30 & 1977 & 1 & 2,30 & 1984 & 1 & 7,80 & 1991 & 1 & 7,80 & & & \\
\hline ¿ & & 2 & 7,80 & & 2 & 2,30 & & 2 & 2,30 & & 2 & 7,80 & & 2 & 7,80 & & & \\
\hline 吊 & & 3 & 7,80 & & 3 & 7,80 & & 3 & 2,30 & & 3 & 7,80 & & 3 & 7,80 & & & \\
\hline 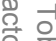 & & 4 & 7,80 & & 4 & 7,80 & & 4 & 2,30 & & 4 & 7,80 & & 4 & 7,80 & & & \\
\hline$\frac{0}{2}$ & 1964 & 1 & 7,80 & 1971 & 1 & 7,80 & 1978 & 1 & 2,30 & 1985 & 1 & 7,80 & 1992 & 1 & 7,80 & & & \\
\hline$\stackrel{\oplus}{\rightleftharpoons} \stackrel{\infty}{\leftrightarrows}$ & & 2 & 7,80 & & 2 & 7,80 & & 2 & 2,30 & & 2 & 7,80 & & 2 & 7,80 & & & \\
\hline$\stackrel{\circ}{\stackrel{c}{中}}$ & & 3 & 7,80 & & 3 & 7,80 & & 3 & 2,30 & & 3 & 7,80 & & 3 & 7,80 & & & \\
\hline $\begin{array}{ll} & \\
& 1 \\
\end{array}$ & & 4 & 2,30 & & 4 & 7,80 & & 4 & 2,30 & & 4 & 7,80 & & 4 & 7,80 & & & \\
\hline Nু & 1965 & 1 & 2,30 & 1972 & 1 & 7,80 & 1979 & 1 & 2,30 & 1986 & 1 & 7,80 & 1993 & 1 & 7,80 & & & \\
\hline$\vec{\omega} \omega_{1}^{1}$ & & 2 & 2,30 & & 2 & 7,80 & & 2 & 5,00 & & 2 & 7,80 & & 2 & 7,80 & & & \\
\hline 이 డి & & 3 & 2,30 & & 3 & 7,80 & & 3 & 7,80 & & 3 & 7,80 & & 3 & 7,80 & & & \\
\hline if & & 4 & 2,30 & & 4 & 7,80 & & 4 & 7,80 & & 4 & 7,80 & & 4 & 7,80 & & & \\
\hline ஸִ G & 1966 & 1 & 2,30 & 1973 & 1 & 7,80 & 1980 & 1 & 7,80 & 1987 & 1 & 7,80 & 1994 & 1 & 7,80 & & & \\
\hline $\overrightarrow{\vec{D}} \omega$ & & 2 & 2,30 & & 2 & 7,80 & & 2 & 7,80 & & 2 & 7,80 & & 2 & 7,80 & & & \\
\hline $3 \infty$ & & 3 & 2,30 & & 3 & 7,80 & & 3 & 7,80 & & 3 & 7,80 & & 3 & 7,80 & & & \\
\hline & & 4 & 2,30 & & 4 & 7,80 & & 4 & 7,80 & & 4 & 7,80 & & 4 & 7,80 & & & \\
\hline
\end{tabular}


Tabelle B.20 : Center of Gravity: Quartalsdaten USA

\begin{tabular}{|c|c|c|c|c|c|c|c|c|c|c|c|c|c|c|c|c|c|c|}
\hline & & & $C G$ & & & $C G$ & & & $C G$ & & & $C G$ & & & $C G$ & & & $C G$ \\
\hline & 1960 & 1 & 6,80 & 1967 & 1 & 4,80 & 1974 & 1 & 6,80 & 1981 & 1 & 6,80 & 1988 & 1 & 6,80 & 1995 & 1 & 4,80 \\
\hline & & 2 & 6,80 & & 2 & 4,80 & & 2 & 6,80 & & 2 & 6,80 & & 2 & 6,80 & & 2 & 4,80 \\
\hline & & 3 & 6,80 & & 3 & 4,80 & & 3 & 6,80 & & 3 & 6,80 & & 3 & 6,80 & & 3 & 4,80 \\
\hline & & 4 & 6,80 & & 4 & 4,80 & & 4 & 6,80 & & 4 & 6,80 & & 4 & 6,80 & & 4 & 4,80 \\
\hline & 1961 & 1 & 4,80 & 1968 & 1 & 4,80 & 1975 & 1 & 6,80 & 1982 & 1 & 6,80 & 1989 & 1 & 6,80 & 1996 & 1 & 4,80 \\
\hline & & 2 & 4,80 & & 2 & 4,80 & & 2 & 6,80 & & 2 & 6,80 & & 2 & 6,80 & & 2 & 4,80 \\
\hline & & 3 & 4,80 & & 3 & 4,80 & & 3 & 6,80 & & 3 & 6,80 & & 3 & 6,80 & & 3 & 4,80 \\
\hline \' & & 4 & 4,80 & & 4 & 6,80 & & 4 & 6,80 & & 4 & 6,80 & & 4 & 6,80 & & 4 & 4,80 \\
\hline$\leqq$ & 1962 & 1 & 4,80 & 1969 & 1 & 6,80 & 1976 & 1 & 6,80 & 1983 & 1 & 6,80 & 1990 & 1 & 6,80 & 1997 & 1 & 4,80 \\
\hline$\overline{\mathrm{D}}$ & & 2 & 4,80 & & 2 & 6,80 & & 2 & 6,80 & & 2 & 6,80 & & 2 & 6,80 & & 2 & 4,80 \\
\hline ㅇ & & 3 & 4,80 & & 3 & 6,80 & & 3 & 6,80 & & 3 & 6,80 & & 3 & 6,80 & & 3 & 4,80 \\
\hline $\begin{array}{l}2 \\
\Rightarrow\end{array}$ & & 4 & 4,80 & & 4 & 6,80 & & 4 & 6,80 & & 4 & 6,80 & & 4 & 6,80 & & 4 & 4,80 \\
\hline$\frac{0}{3}$ & 1963 & 1 & 4,80 & 1970 & 1 & 6,80 & 1977 & 1 & 4,80 & 1984 & 1 & 6,80 & 1991 & 1 & 6,80 & & & \\
\hline ர & & 2 & 4,80 & & 2 & 6,80 & & 2 & 4,80 & & 2 & 6,80 & & 2 & 6,80 & & & \\
\hline ᄒิ & & 3 & 4,80 & & 3 & 6,80 & & 3 & 4,80 & & 3 & 6,80 & & 3 & 6,80 & & & \\
\hline อి. & $\Rightarrow$ & 4 & 4,80 & & 4 & 6,80 & & 4 & 4,80 & & 4 & 6,80 & & 4 & 6,80 & & & \\
\hline 울 & 음 1964 & 1 & 4,80 & 1971 & 1 & 6,80 & 1978 & 1 & 4,80 & 1985 & 1 & 6,80 & 1992 & 1 & 6,80 & & & \\
\hline Z & $\frac{\overline{0}}{\infty}$ & 2 & 4,80 & & 2 & 6,80 & & 2 & 4,80 & & 2 & 6,80 & & 2 & 6,80 & & & \\
\hline$\stackrel{9}{\circ}$ & ¿ & 3 & 4,80 & & 3 & 6,80 & & 3 & 4,80 & & 3 & 6,80 & & 3 & 6,80 & & & \\
\hline 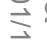 & & 4 & 4,80 & & 4 & 6,80 & & 4 & 4,80 & & 4 & 6,80 & & 4 & 6,80 & & & \\
\hline $\overrightarrow{\vec{N}}$ & 1965 & 1 & 4,80 & 1972 & 1 & 6,80 & 1979 & 1 & 4,80 & 1986 & 1 & 6,80 & 1993 & 1 & 4,80 & & & \\
\hline 응 & $\infty$ & 2 & 4,80 & & 2 & 6,80 & & 2 & 4,80 & & 2 & 6,80 & & 2 & 4,80 & & & \\
\hline $\overrightarrow{0}$ & $\begin{array}{l}\omega \\
\dot{b}\end{array}$ & 3 & 4,80 & & 3 & 6,80 & & 3 & 4,80 & & 3 & 6,80 & & 3 & 4,80 & & & \\
\hline$\stackrel{ }{0}$ & $\stackrel{\omega}{\omega}$ & 4 & 4,80 & & 4 & 6,80 & & 4 & 4,80 & & 4 & 6,80 & & 4 & 4,80 & & & \\
\hline N & 난 1966 & 1 & 4,80 & 1973 & 1 & 6,80 & 1980 & 1 & 4,80 & 1987 & 1 & 6,80 & 1994 & 1 & 4,80 & & & \\
\hline$\stackrel{0}{\longrightarrow}$ & $\vec{\omega}$ & 2 & 4,80 & & 2 & 6,80 & & 2 & 4,80 & & 2 & 6,80 & & 2 & 4,80 & & & \\
\hline$\frac{D}{2}$ & 0 & 3 & 4,80 & & 3 & 6,80 & & 3 & 4,80 & & 3 & 6,80 & & 3 & 4,80 & & & \\
\hline & & 4 & 4,80 & & 4 & & & 4 & 4,80 & & 4 & 6,80 & & 4 & 4,80 & & & \\
\hline
\end{tabular}




\section{Anhang C \\ Detaillierte Regressionsergebnisse}

Tabelle C.1 : Traditionelle Partisantheorie auf dem Arbeitsmarkt bei

ökonomischen Clustern (1. Ländercluster)

Tabelle C.2 : Traditionelle Partisantheorie auf dem Arbeitsmarkt bei ökonomischen Clustern (2. Ländercluster)

Tabelle C.3 : Traditionelle Partisantheorie auf dem Arbeitsmarkt bei ökonomischen Clustern (3. Ländercluster) ...

Tabelle C.4 : Traditionelle Partisantheorie auf dem Arbeitsmarkt bei politischen Clustern (1. Ländercluster)

Tabelle C.5 : Traditionelle Partisantheorie auf dem Arbeitsmarkt bei politischen Clustern (2. Ländercluster)

Tabelle C.6 : Traditionelle Partisantheorie auf dem Arbeitsmarkt bei politischen Clustern (3. Ländercluster)

Tabelle C.7 : Traditionelle Partisantheorie auf dem Arbeitsmarkt bei politischen Clustern (4. Ländercluster)

Tabelle C.8 : Rationale Partisaneffekte auf dem Arbeitsmarkt bei ökonomischen Clustern (1. Ländercluster)

Tabelle C.9 : Rationale Partisaneffekte auf dem Arbeitsmarkt bei ökonomischen Clustern (2. Ländercluster)

Tabelle C.10 : Rationale Partisaneffekte auf dem Arbeitsmarkt bei ökonomischen Clustern (3. Ländercluster)

Tabelle C.11 : Rationale Partisaneffekte auf dem Arbeitsmarkt bei politischen Clustern (1. Ländercluster)

Tabelle C.12 : Rationale Partisaneffekte auf dem Arbeitsmarkt bei politischen Clustern (2. Ländercluster)

Tabelle C.13 : Rationale Partisaneffekte auf dem Arbeitsmarkt bei politischen Clustern (3. Ländercluster)

Tabelle C.14 : Rationale Partisaneffekte auf dem Arbeitsmarkt bei politischen Clustern (4. Ländercluster)

Tabelle C.15 : Partisaneffekte hinsichtlich der Inflationsraten bei ökonomischen Clustern (1. Ländercluster)

Tabelle C.16 : Partisaneffekte hinsichtlich der Inflationsraten bei ökonomischen Clustern (2. Ländercluster)

Tabelle C.17 : Partisaneffekte hinsichtlich der Inflationsraten bei ökonomischen Clustern (3. Ländiercluster97.8-3-6.31-7.5.13.9-8... 
Tabelle C.18 : Partisaneffekte hinsichtlich der Inflationsraten bei politischen Clustern (1. Ländercluster) ............................... 346

Tabelle C.19 : Partisaneffekte hinsichtlich der Inflationsraten bei politischen Clustern (2. Ländercluster) ................................ 347

Tabelle C.20 : Partisaneffekte hinsichtlich der Inflationsraten bei politischen Clustern (3. Ländercluster) .............................. 348

Tabelle C.21 : Partisaneffekte hinsichtlich der Inflationsraten bei politischen Clustern (4. Ländercluster) 
Tabelle C.1 : Traditionelle Partisantheorie auf dem Arbeitsmarkt bei ökonomischen Clustern (1. Ländercluster)

abhängige Variable: $U_{1}^{\text {Diff }}$ (Belgien, Niederlande, Neuseeland, Schweden, Großbritannien)

unabh. $1960-1973$ (1. Zeitcluster) $1974-1985$ (2. Zeitcluster) $\quad 1986-1992$ (3. Zeitcluster) $1993-1997$ (4. Zeitcluster)

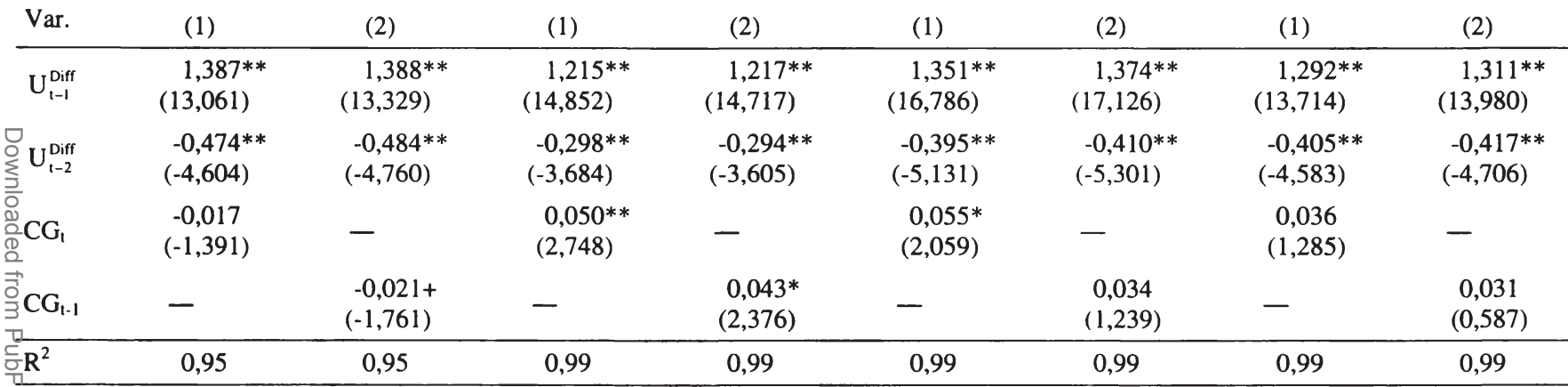

Afimerkungen: $\quad \mathrm{t}$-Werte in Klammern, $+(\alpha<0,1),{ }^{*}(\alpha<0,05),{ }^{* *}(\alpha<0,01)$. 
Tabelle C.2 : Traditionelle Partisantheorie auf dem Arbeitsmarkt bei ökonomischen Clustern (2. Ländercluster)

abhängige Variable: U $U_{t}^{\text {Diff }}$ (Kanada, Dänemark, Finnland, Irland, Norwegen, Schweiz)

\begin{tabular}{|c|c|c|c|c|c|c|c|c|}
\hline \multirow{2}{*}{$\begin{array}{l}\text { unabh. } \\
\text { Var. }\end{array}$} & \multicolumn{2}{|c|}{1960 - 1973 (1. Zeitcluster) } & \multicolumn{2}{|c|}{1974 - 1985 (2. Zeitcluster) } & \multicolumn{2}{|c|}{1986 - 1992 (3. Zeitcluster) } & \multicolumn{2}{|c|}{1993 - 1997 (4. Zeitcluster) } \\
\hline & $(1)$ & $(2)$ & $(1)$ & $(2)$ & (1) & (2) & (1) & (2) \\
\hline$U_{t-1}^{\text {Diff }}$ & $\begin{array}{c}1,233 * * \\
(14,654)\end{array}$ & $\begin{array}{c}1,226^{* *} \\
(14,492)\end{array}$ & $\begin{array}{c}1,390 * * \\
(22,599)\end{array}$ & $\begin{array}{c}1,394 * * \\
(22,672)\end{array}$ & $\begin{array}{c}1,645^{* *} \\
(26,266)\end{array}$ & $\begin{array}{c}1,642 * * \\
(26,191)\end{array}$ & $\begin{array}{c}1,444 * * \\
(17,707)\end{array}$ & $\begin{array}{c}1,345^{* *} \\
(16,322)\end{array}$ \\
\hline$U_{t-2}^{\text {Diff }}$ & $\begin{array}{l}-0,309 * * \\
(-3,698)\end{array}$ & $\begin{array}{l}-0,302 * * \\
(-3,626)\end{array}$ & $\begin{array}{l}-0,480 * * \\
(-8,044)\end{array}$ & $\begin{array}{c}-0,485^{* *} \\
(-8,061)\end{array}$ & $\begin{array}{c}-0,655^{* *} \\
(-9,788)\end{array}$ & $\begin{array}{c}-0,650 * * \\
(-9,699)\end{array}$ & $\begin{array}{l}-0,495^{* *} \\
(-6,149)\end{array}$ & $\begin{array}{l}-0,428 * * \\
(-5,457)\end{array}$ \\
\hline 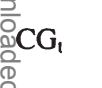 & $\begin{array}{c}-0,043 \\
(-1,351)\end{array}$ & - & $\begin{array}{c}-0,007 \\
(-0,342)\end{array}$ & - & $\begin{array}{c}0,005 \\
(0,197)\end{array}$ & - & $\begin{array}{c}0,069 \\
(1,179)\end{array}$ & - \\
\hline $\mathrm{R}^{2}$ & 0,98 & 0,98 & 0,99 & 0,99 & 0,99 & 0,99 & 0,99 & 0,99 \\
\hline
\end{tabular}




\section{Tabelle C.3 : Traditionelle Partisantheorie auf dem Arbeitsmarkt bei ökonomischen Clustern (3. Ländercluster)}

abhängige Variable: $U_{1}^{\text {Dit }}$ (Australien, Österreich, Frankreich, Deutschland, Italien, Japan, Spanien, USA)

unabh. $1960-1973$ (1. Zeitcluster) $1974-1985$ (2. Zeitcluster) $1986-1992$ (3. Zeitcluster) $1993-1997$ (4. Zeitcluster)

\begin{tabular}{|c|c|c|c|c|c|c|c|c|}
\hline Var. & (1) & (2) & (1) & (2) & (1) & (2) & (1) & (2) \\
\hline$U_{t-1}^{\text {Diff }}$ & $\begin{array}{c}1,287 * * \\
(23,830)\end{array}$ & $\begin{array}{c}1,290 * * \\
(23,874)\end{array}$ & $\begin{array}{c}1,369 * * \\
(29,257)\end{array}$ & $\begin{array}{c}1,418 * * \\
(29,938)\end{array}$ & $\begin{array}{c}1,107 * * \\
(13,529)\end{array}$ & $\begin{array}{c}1,107 * * \\
(13,532)\end{array}$ & $\begin{array}{c}1,212 * * \\
(19,004)\end{array}$ & $\begin{array}{c}1,221^{* *} \\
(19,286)\end{array}$ \\
\hline$U_{t-2}^{\text {Diff }}$ & $\begin{array}{c}-0,365 * * \\
(-6,763)\end{array}$ & $\begin{array}{c}-0,367 * * \\
(-6,800)\end{array}$ & $\begin{array}{l}-0,394 * * \\
(-8,362)\end{array}$ & $\begin{array}{l}-0,442 * * \\
(-9,270)\end{array}$ & $\begin{array}{c}0,308^{*} \\
(2,429)\end{array}$ & $\begin{array}{c}0,308^{*} \\
(2,431)\end{array}$ & $\begin{array}{c}-0,203 * \\
(-2,086)\end{array}$ & $\begin{array}{c}-0,206^{*} \\
(-2,108)\end{array}$ \\
\hline$U_{t-3}^{\text {Diff }}$ & - & - & - & - & $\begin{array}{c}-0,300 * \\
(-2,324)\end{array}$ & $\begin{array}{c}-0,300 * \\
(-2,324)\end{array}$ & $\begin{array}{c}-0,131^{*} \\
(-2,253)\end{array}$ & $\begin{array}{c}-0,130^{*} \\
(-2,245)\end{array}$ \\
\hline $\begin{array}{c}\mathrm{C}_{0} \\
\mathrm{CG}_{\mathrm{t}}\end{array}$ & $\begin{array}{c}0,013 \\
(0,898)\end{array}$ & - & $\begin{array}{c}-0,003 \\
(-0,289)\end{array}$ & - & $\begin{array}{c}0,006 \\
(0,272)\end{array}$ & - & $\begin{array}{c}-0,025+ \\
(-1,902)\end{array}$ & - \\
\hline$\frac{C_{0}}{c^{t}-2}$ & - & $\begin{array}{c}0,006 \\
(0,382) \\
\end{array}$ & - & $\begin{array}{c}0,001 \\
(0,071) \\
\end{array}$ & - & $\begin{array}{c}0,002 \\
(0,114)\end{array}$ & - & $\begin{array}{l}-0,024+ \\
(1,722)\end{array}$ \\
\hline$R_{0}^{a}$ & 0,98 & 0,98 & 0,99 & 0,99 & 0,99 & 0,99 & 0,99 & 0,99 \\
\hline
\end{tabular}

Anmerkungen: $\quad \mathrm{t}$-Werte in Klammern, $+(\alpha<0,1),{ }^{*}(\alpha<0,05),{ }^{* *}(\alpha<0,01)$. 
Tabelle C.4 : Traditionelle Partisantheorie auf dem Arbeitsmarkt bei politischen Clustern (1. Ländercluster)

abhängige Variable: $U_{1}^{\text {Diff }}$ (Deutschland, Dänemark, Italien, Niederlande, Belgien, Frankreich)

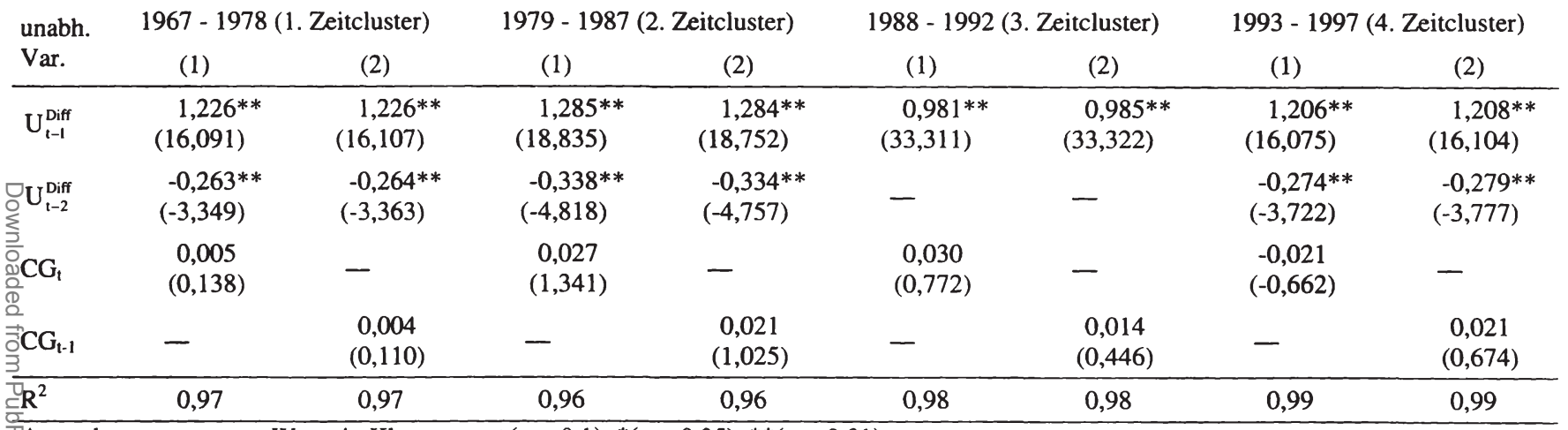


Tabelle C.5 : Traditionelle Partisantheorie auf dem Arbeitsmarkt bei politischen Clustern (2. Ländercluster)

abhängige Variable: Ut $U_{t}^{\text {Diff }}$ (Großbritannien, Schweden, Österreich, Norwegen, Finnland, Irland)

unabh. $1967-1978$ (1. Zeitcluster) $1979-1987$ (2. Zeitcluster) 1988 - 1992 (3. Zeitcluster) $1993-1997$ (4. Zeitcluster)

\begin{tabular}{|c|c|c|c|c|c|c|c|c|}
\hline Var. & (1) & (2) & (1) & (2) & (1) & (2) & (1) & (2) \\
\hline$U_{t-1}^{\text {Diff }}$ & $\begin{array}{c}1,474 * * \\
(24,418)\end{array}$ & $\begin{array}{c}1,472 * * \\
(24,267)\end{array}$ & $\begin{array}{c}1,282^{* *} \\
(19,257)\end{array}$ & $\begin{array}{c}1,274 * * \\
(19,204)\end{array}$ & $\begin{array}{c}1,743 * * \\
(25,330)\end{array}$ & $\begin{array}{c}1,749 * * \\
(25,722)\end{array}$ & $\begin{array}{c}1,446^{* *} \\
(18,227)\end{array}$ & $\begin{array}{c}1,421 * * \\
(18,128)\end{array}$ \\
\hline$U_{t-2}^{\text {Diff }}$ & $\begin{array}{c}-0,530 * * \\
(-8,650)\end{array}$ & $\begin{array}{l}-0,528 * * \\
(-8,583)\end{array}$ & $\begin{array}{c}-0,365 * * \\
(-5,813)\end{array}$ & $\begin{array}{c}-0,359 \\
(-5,761)\end{array}$ & $\begin{array}{c}-0,762 * * \\
(-10,414)\end{array}$ & $\begin{array}{c}-0,759 * * \\
(-10,329)\end{array}$ & $\begin{array}{c}-0,516^{* *} \\
(-6,583)\end{array}$ & $\begin{array}{l}-0,491 * * \\
(-6,307)\end{array}$ \\
\hline $\mathrm{CG}_{\mathrm{t}}$ & $\begin{array}{c}0,016 \\
(1,220)\end{array}$ & - & $\begin{array}{c}0,007 \\
(0,509)\end{array}$ & - & $\begin{array}{c}0,014 \\
(0,487)\end{array}$ & - & $\begin{array}{c}0,033 \\
(1,110)\end{array}$ & - \\
\hline $\mathbf{R}^{2}$ & 0,96 & 0,96 & 0,99 & 0,99 & 0,99 & 0,99 & 0,99 & 0,99 \\
\hline
\end{tabular}

Aylmerkungen: $\quad \mathrm{t}$-Werte in Klammern, $+(\alpha<0,1),{ }^{*}(\alpha<0,05),{ }^{* *}(\alpha<0,01)$. 
Tabelle C.6 : Traditionelle Partisantheorie auf dem Arbeitsmarkt bei politischen Clustern (3. Ländercluster)

\begin{tabular}{|c|c|c|c|c|c|c|c|c|}
\hline \multirow{3}{*}{$\begin{array}{l}\text { unabh. } \\
\text { Var. }\end{array}$} & \multicolumn{6}{|c|}{ abhängige Variable: $U_{t}^{\text {Diff }}$ (USA, Kanada, Schweiz) } & \multirow{2}{*}{\multicolumn{2}{|c|}{1988 - 1997 (4. Zeitcluster) }} \\
\hline & \multicolumn{2}{|c|}{1960 - 1966 (1. Zeitcluster) } & \multicolumn{2}{|c|}{$1967-1978$ (2. Zeitcluster) } & \multicolumn{2}{|c|}{$1979-1987$ (3. Zeitcluster) } & & \\
\hline & $(1)$ & $(2)$ & (1) & (2) & (1) & (2) & (1) & (2) \\
\hline$U_{1-1}^{\text {Diff }}$ & $\begin{array}{l}0,818 * * \\
(6,300)\end{array}$ & $\begin{array}{l}0,806 * * \\
(6,283)\end{array}$ & $\begin{array}{l}1,253^{* *} \\
(12,828)\end{array}$ & $\begin{array}{c}1,252 * * \\
(12,734)\end{array}$ & $\begin{array}{c}1,441^{* *} \\
(15,210)\end{array}$ & $\begin{array}{l}1,441 * * \\
(15,583)\end{array}$ & $\begin{array}{l}1,423 * * \\
(17,615)\end{array}$ & $\begin{array}{l}1,435^{* *} \\
(17,807)\end{array}$ \\
\hline 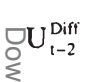 & $\begin{array}{c}0,038 \\
(0,294)\end{array}$ & $\begin{array}{c}0,049 \\
(0,387)\end{array}$ & $\begin{array}{l}-0,341 * * \\
(-3,523)\end{array}$ & $\begin{array}{l}-0,344^{* *} \\
(-3,562)\end{array}$ & $\begin{array}{l}-0,539 * * \\
(-5,729)\end{array}$ & $\begin{array}{l}-0,545^{* *} \\
(-5,955)\end{array}$ & $\begin{array}{l}-0,467 * * \\
(-5,757)\end{array}$ & $\begin{array}{l}-0,474 * * \\
(-5,890)\end{array}$ \\
\hline $\begin{array}{l}\frac{\partial}{2} \\
\frac{2}{D} \\
\frac{2}{D}\end{array}$ & $\begin{array}{c}0,088+ \\
(1,751)\end{array}$ & - & $\begin{array}{l}0,072 * \\
(2,325)\end{array}$ & - & $\begin{array}{c}-0,054 \\
(-1,310)\end{array}$ & - & $\begin{array}{c}0,064^{*} \\
(2,467)\end{array}$ & - \\
\hline$\frac{2}{\Rightarrow} \mathrm{CG}_{1-1}$ & - & $\begin{array}{c}0,094^{*} \\
(2,134)\end{array}$ & - & $\begin{array}{c}0,071^{*} \\
(2,242)\end{array}$ & - & $\begin{array}{l}-0,067+ \\
(-1,691)\end{array}$ & - & $\begin{array}{c}0,064 * \\
(2,450)\end{array}$ \\
\hline $\mathrm{R}^{2}$ & 0,87 & 0,87 & 0,93 & 0,93 & 0,99 & 0,99 & 0,99 & 0,99 \\
\hline
\end{tabular}

TAnmerkungen: $\quad$-Werte in Klammern, $+(\alpha<0,1),{ }^{*}(\alpha<0,05),{ }^{* *}(\alpha<0,01)$.

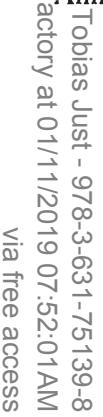


Tabelle C.7 : Traditionelle Partisantheorie auf dem Arbeitsmarkt bei politischen Clustern (4. Ländercluster)

abhängige Variable: $U_{t}^{\text {Diff }}$ (Spanien, Japan, Australien, Neuseeland)

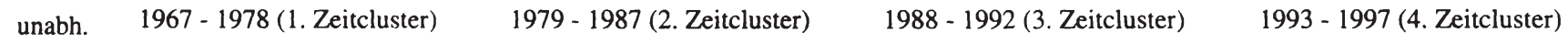

\begin{tabular}{|c|c|c|c|c|c|c|c|c|}
\hline Var. & (1) & (2) & (1) & (2) & (1) & (2) & (1) & (2) \\
\hline$U_{t-1}^{\text {Diff }}$ & $\begin{array}{c}1,263 * * \\
(13,165)\end{array}$ & $\begin{array}{c}1,264 * * \\
(13,198)\end{array}$ & $\begin{array}{c}1,339 * * \\
(16,329)\end{array}$ & $\begin{array}{c}1,346 * * \\
(16,580)\end{array}$ & $\begin{array}{c}1,351 * * \\
(11,381)\end{array}$ & $\begin{array}{c}1,349 * * \\
(11,363)\end{array}$ & $\begin{array}{c}1,421 * * \\
(15,498)\end{array}$ & $\begin{array}{c}1,416^{* *} \\
(15,811)\end{array}$ \\
\hline$U_{t-2}^{\text {Diff }}$ & $\begin{array}{l}-0,293^{* *} \\
(-2,895)\end{array}$ & $\begin{array}{l}-0,294 * * \\
(-2,907)\end{array}$ & $\begin{array}{l}-0,394 * * \\
(-5,050)\end{array}$ & $\begin{array}{l}-0,401 * * \\
(-5,165)\end{array}$ & $\begin{array}{l}-0,437 * * \\
(-3,835)\end{array}$ & $\begin{array}{l}-0,427 * * \\
(-3,711)\end{array}$ & $\begin{array}{l}-0,527 * * \\
(-6,602)\end{array}$ & $\begin{array}{c}-0,526^{* *} \\
(-6,709)\end{array}$ \\
\hline $\mathrm{CG}_{\mathrm{t}}$ & $\begin{array}{c}0,005 \\
(0,263)\end{array}$ & - & $\begin{array}{c}-0,037+ \\
(-1,761)\end{array}$ & - & $\begin{array}{c}0,011 \\
(0,132)\end{array}$ & - & $\begin{array}{c}-0,019 \\
(-1,056)\end{array}$ & - \\
\hline $\mathrm{R}^{2}$ & 0,97 & 0,97 & 0,99 & 0,99 & 0,99 & 0,99 & 0,99 & 0,99 \\
\hline
\end{tabular}

Aglmerkungen: $\quad \mathrm{t}$-Werte in Klammern, $+(\alpha<0,1),{ }^{*}(\alpha<0,05),{ }^{* *}(\alpha<0,01)$. 
Tabelle C.8 : Rationale Partisaneffekte auf dem Arbeitsmarkt bei ökonomischen Clustern (1. Ländercluster)

abhängige Variable: $U_{t}^{\text {Diff }}$ (Belgien, Niederlande, Neuseeland, Schweden, Großbritannien)

\begin{tabular}{|c|c|c|c|c|c|c|c|c|}
\hline \multirow{2}{*}{$\begin{array}{l}\text { unabh. } \\
\text { Var. }\end{array}$} & \multicolumn{2}{|c|}{$1960-1973$ (1. Zeitcluster) } & \multicolumn{2}{|c|}{1974 - 1985 (2. Zeitcluster) } & \multicolumn{2}{|c|}{1986 - 1992 (3. Zeitcluster) } & \multicolumn{2}{|c|}{1993 - 1997 (4. Zeitcluster) } \\
\hline & $(1)$ & $(2)$ & (1) & $(2)$ & (1) & (2) & (1) & (2) \\
\hline$U_{t-1}^{\text {Diff }}$ & $\begin{array}{c}1,253^{* *} \\
(10,811)\end{array}$ & $\begin{array}{c}1,240 * * \\
(9,879)\end{array}$ & $\begin{array}{c}1,229 * * \\
(14,727)\end{array}$ & $\begin{array}{c}1,207 * * \\
(13,889)\end{array}$ & $\begin{array}{c}1,349 * * \\
(16,862)\end{array}$ & $\begin{array}{c}1,369 * * \\
(13,541)\end{array}$ & $\begin{array}{c}1,278^{* *} \\
(13,991)\end{array}$ & $\begin{array}{c}1,265^{* *} \\
(13,480)\end{array}$ \\
\hline$U_{t-2}^{\text {Diff }}$ & $\begin{array}{l}-0,377^{* *} \\
(-3,520)\end{array}$ & $\begin{array}{l}-0,368 * * \\
(-3,305)\end{array}$ & $\begin{array}{l}-0,278 * * \\
(-3,300)\end{array}$ & $\begin{array}{c}-0,256 * * \\
(-2,940)\end{array}$ & $\begin{array}{l}-0,385^{* *} \\
(-4,958)\end{array}$ & $\begin{array}{l}-0,408 * * \\
(-5,350)\end{array}$ & $\begin{array}{l}-0,398 * * \\
(-4,640)\end{array}$ & $\begin{array}{l}-0,375^{* *} \\
(-4,238)\end{array}$ \\
\hline 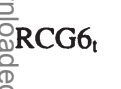 & $\begin{array}{c}0,003 \\
(0,352)\end{array}$ & - & $\begin{array}{c}0,025 \\
(1,645)\end{array}$ & - & $\begin{array}{c}0,058 * \\
(2,236)\end{array}$ & 一 & $\begin{array}{c}0,063 * \\
(2,436)\end{array}$ & 一 \\
\hline $\begin{array}{l}\mathrm{e} \\
\mathrm{c} \\
\mathrm{C}\end{array}$ & - & $\begin{array}{c}0,003 \\
(0,284) \\
\end{array}$ & - & $\begin{array}{c}0,027+ \\
(1,756)\end{array}$ & - & $\begin{array}{c}0,058 * \\
(2,182)\end{array}$ & - & $\begin{array}{c}0,058 * \\
(2,146)\end{array}$ \\
\hline $\mathrm{R}^{2}$ & 0,95 & 0,93 & 0,99 & 0,99 & 0,99 & 0,99 & 0,99 & 0,99 \\
\hline
\end{tabular}

TAnmerkungen: $\quad \mathrm{t}$-Werte in Klammern, $+(\alpha<0,1),{ }^{*}(\alpha<0,05),{ }^{* *}(\alpha<0,01)$.

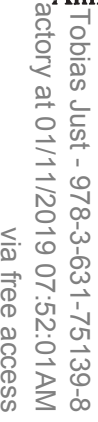


Tabelle C.9 : Rationale Partisaneffekte auf dem Arbeitsmarkt bei ökonomischen Clustern (2. Ländercluster)

abhängige Variable: $U_{t}^{\text {Diff }}$ (Kanada, Dänemark, Finnland, Irland, Norwegen, Schweiz)

unabh. $\quad 1960-1973$ (1. Zeitcluster) $1974-1985$ (2. Zeitcluster) $1986-1992$ (3. Zeitcluster) $1993-1997$ (4. Zeitcluster)

\begin{tabular}{|c|c|c|c|c|c|c|c|c|}
\hline Var. & (1) & (2) & (1) & (2) & (1) & (2) & (1) & (2) \\
\hline$U_{t-1}^{\text {Diff }}$ & $\begin{array}{c}1,150^{* *} \\
(12,219)\end{array}$ & $\begin{array}{c}1,118^{* *} \\
(11,761)\end{array}$ & $\begin{array}{c}1,391 * * \\
(22,139)\end{array}$ & $\begin{array}{c}1,388^{* *} \\
(22,038)\end{array}$ & $\begin{array}{c}1,645^{* *} \\
(26,262)\end{array}$ & $\begin{array}{c}1,644 * * \\
(26,249)\end{array}$ & $\begin{array}{c}1,451 * * \\
(19,318)\end{array}$ & $\begin{array}{c}1,477 * * \\
(19,044)\end{array}$ \\
\hline$U_{t-2}^{\text {Diff }}$ & $\begin{array}{c}-0,227^{*} \\
(-2,504)\end{array}$ & $\begin{array}{c}-0,206 * \\
(-2,249)\end{array}$ & $\begin{array}{c}-0,477 * * \\
(-7,761)\end{array}$ & $\begin{array}{l}-0,473 * * \\
(-7,640)\end{array}$ & $\begin{array}{l}-0,654 * * \\
(-9,770)\end{array}$ & $\begin{array}{c}-0,653 * * \\
(-9,710)\end{array}$ & $\begin{array}{c}-0,531 * * \\
(-7,083)\end{array}$ & $\begin{array}{l}-0,524 * * \\
(-6,777)\end{array}$ \\
\hline RCG6 & $\begin{array}{c}-0,046 \\
(-1,564)\end{array}$ & - & $\begin{array}{c}0,008 \\
(0,410)\end{array}$ & - & $\begin{array}{c}-0,001 \\
(-0,050)\end{array}$ & - & $\begin{array}{l}-0,115^{* *} \\
(-2,651)\end{array}$ & - \\
\hline$R^{2}$ & 0,98 & 0,98 & 0,99 & 0,99 & 0,99 & 0,99 & 0,99 & 0,99 \\
\hline
\end{tabular}

Aylmerkungen: $\quad \mathrm{t}$-Werte in Klammern, $+(\alpha<0,1),{ }^{*}(\alpha<0,05),{ }^{* *}(\alpha<0,01)$. 
Tabelle C.10 : Rationale Partisaneffekte auf dem Arbeitsmarkt bei ökonomischen Clustern (3. Ländercluster)

abhängige Variable: $U_{t}^{\text {Diff }}$ (Australien, Österreich, Frankreich, Deutschland, Italien, Japan, Spanien, USA)

unabh. $1960-1973$ (1. Zeitcluster) $1974-1985$ (2. Zeitcluster) $1986-1992$ (3. Zeitcluster) $1993-1997$ (4. Zeitcluster)

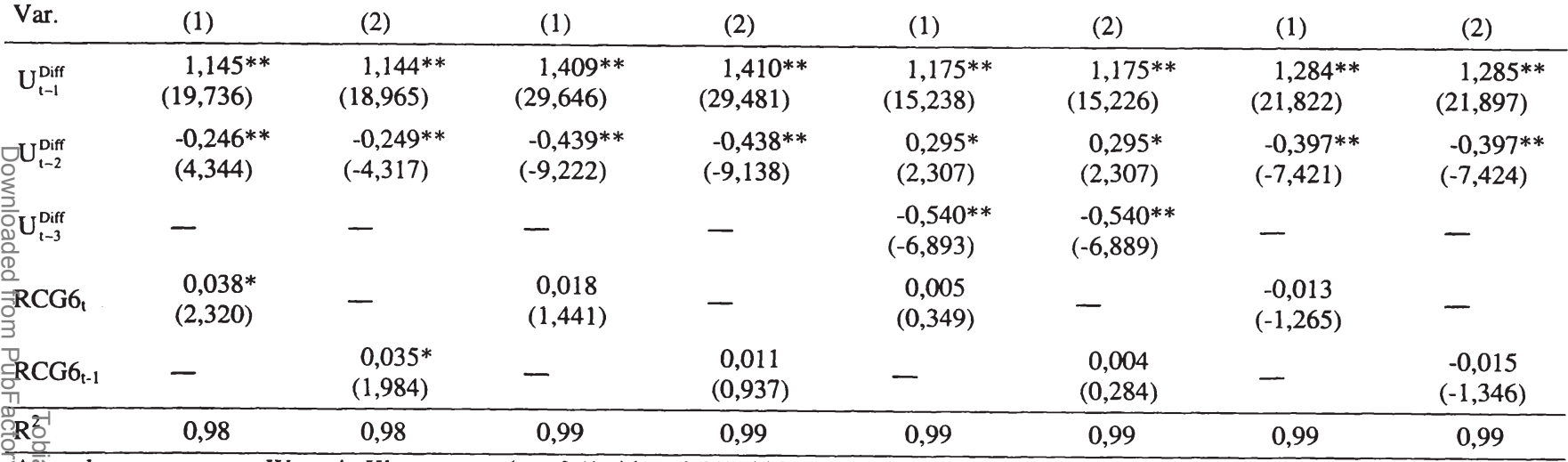

A

$\vec{\circ} \subsetneq$ 
Tabelle C.11 : Rationale Partisaneffekte auf dem Arbeitsmarkt bei politischen Clustern (1. Ländercluster)

abhängige Variable: $U_{t}^{\text {Diff }}$ (Deutschland, Dänemark, Italien, Niederlande, Belgien, Frankreich)

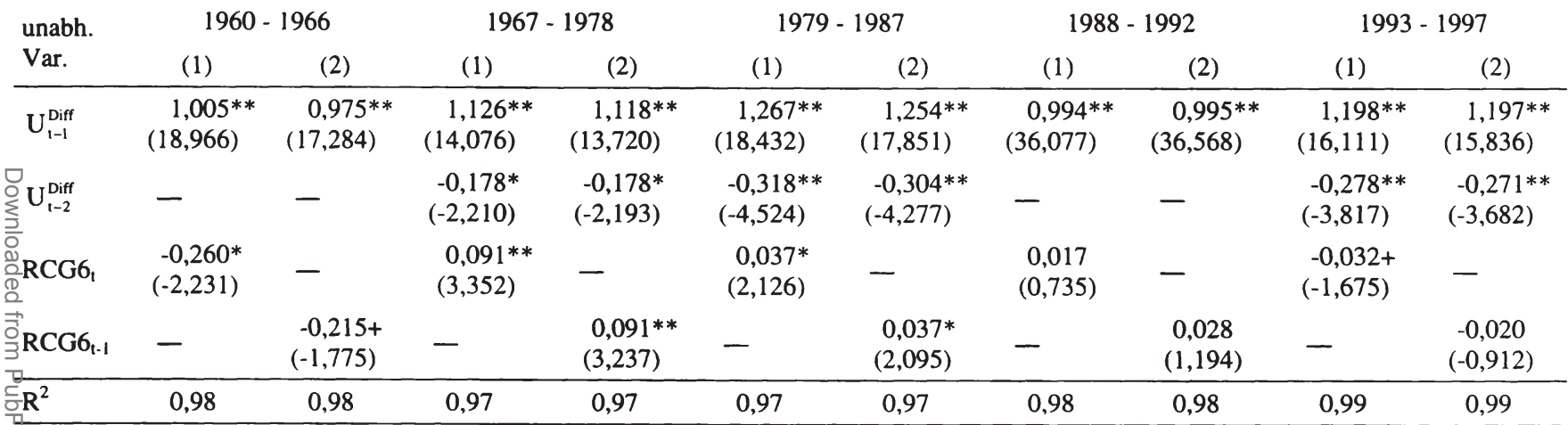

Afimerkungen: $\quad \mathrm{t}$-Werte in Klammern, $+(\alpha<0,1),{ }^{*}(\alpha<0,05),{ }^{* *}(\alpha<0,01)$.

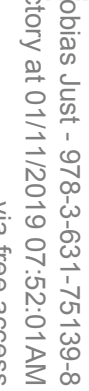


Tabelle C.12 : Rationale Partisaneffekte auf dem Arbeitsmarkt bei politischen Clustern (2. Ländercluster)

abhängige Variable: $U_{t}^{\text {Diff }}$ (Großbritannien, Schweden, Österreich, Norwegen, Finnland, Irland)

\begin{tabular}{|c|c|c|c|c|c|c|c|c|c|c|}
\hline \multirow{2}{*}{$\begin{array}{l}\text { unabh. } \\
\text { Var. }\end{array}$} & \multicolumn{2}{|c|}{$1960-1966$} & \multicolumn{2}{|c|}{$1967-1978$} & \multicolumn{2}{|c|}{$1979-1987$} & \multicolumn{2}{|c|}{$1988-1992$} & \multicolumn{2}{|c|}{$1993-1997$} \\
\hline & (1) & (2) & (1) & (2) & (1) & (2) & (1) & (2) & (1) & (2) \\
\hline$U_{t-1}^{\text {Diff }}$ & $\begin{array}{c}0,842 * * \\
(23,339)\end{array}$ & $\begin{array}{c}0,777 * * \\
(18,970)\end{array}$ & $\begin{array}{c}1,487 * * \\
(24,321)\end{array}$ & $\begin{array}{c}1,482 * * \\
(24,000)\end{array}$ & $\begin{array}{c}1,254 * * \\
(18,214)\end{array}$ & $\begin{array}{c}1,271 * * \\
(18,474)\end{array}$ & $\begin{array}{c}1,745 * * \\
(25,443)\end{array}$ & $\begin{array}{c}1,748 * * \\
(25,612)\end{array}$ & $\begin{array}{c}1,466 * * \\
(19,137)\end{array}$ & $\begin{array}{c}1,450 * * \\
(18,867)\end{array}$ \\
\hline$U_{t-2}^{\text {Diff }}$ & - & - & $\begin{array}{l}-0,542 * * \\
(-8,736)\end{array}$ & $\begin{array}{c}-0,539 * * \\
(-8,614)\end{array}$ & $\begin{array}{c}-0,338 * * \\
(-5,191)\end{array}$ & $\begin{array}{l}-0,353 * * \\
(-5,408)\end{array}$ & $\begin{array}{c}-0,761 * * \\
(-10,381)\end{array}$ & $\begin{array}{c}-0,763 * * \\
(-10,429)\end{array}$ & $\begin{array}{l}-0,530 * * \\
(-6,886)\end{array}$ & $\begin{array}{l}-0,511 * * \\
(-6,574)\end{array}$ \\
\hline$\frac{O}{\frac{O}{D}}$ RCG6 $_{1}$ & $\begin{array}{c}0,012 \\
(0,661)\end{array}$ & - & $\begin{array}{c}0,013 \\
(1,074)\end{array}$ & 一 & $\begin{array}{c}-0,020 \\
(-1,650)\end{array}$ & - & $\begin{array}{c}0,008 \\
(0,393)\end{array}$ & - & $\begin{array}{c}0,019 \\
(0,703)\end{array}$ & - \\
\hline $\mathrm{C}^{2}$ & 0,94 & 0,93 & 0,96 & 0,96 & 0,99 & 0,99 & 0,99 & 0,99 & 0,99 & 0,99 \\
\hline
\end{tabular}

TAnmerkungen: $\quad \mathrm{t}$-Werte in Klammern, $+(\alpha<0,1),{ }^{*}(\alpha<0,05),{ }^{* *}(\alpha<0,01)$.

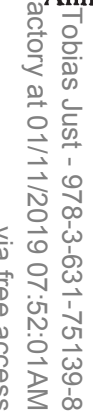


Tabelle C.13 : Rationale Partisaneffekte auf dem Arbeitsmarkt bei politischen Clustern (3. Ländercluster)

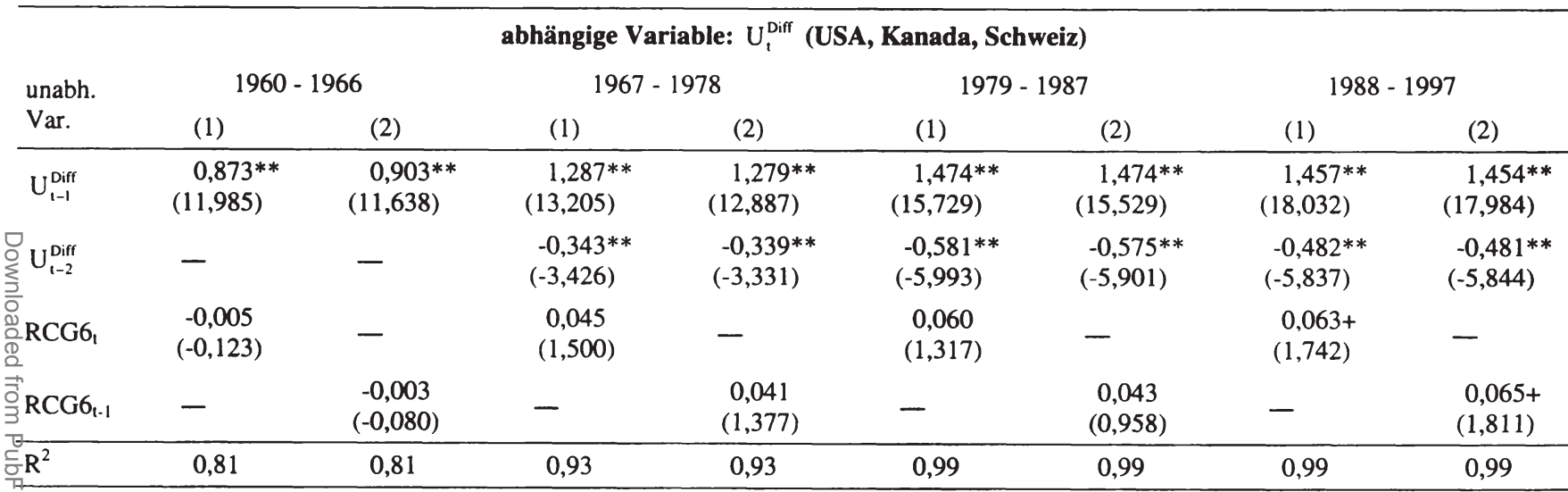

Arimerkungen: t-Werte in Klammern, $+(\alpha<0,1),{ }^{*}(\alpha<0,05),{ }^{* *}(\alpha<0,01)$. 
Tabelle C.14 : Rationale Partisaneffekte auf dem Arbeitsmarkt bei politischen Clustern (4. Ländercluster)

abhängige Variable: $U_{t}^{\text {Diff }}$ (Spanien, Australien Japan, Neuseeland)

\begin{tabular}{|c|c|c|c|c|c|c|c|c|}
\hline \multirow{2}{*}{$\begin{array}{l}\text { unabh. } \\
\text { Var. }\end{array}$} & \multicolumn{2}{|c|}{$1960-1978$} & \multicolumn{2}{|c|}{$1979-1987$} & \multicolumn{2}{|c|}{$1988-1992$} & \multicolumn{2}{|c|}{ 1993- 1997} \\
\hline & $(1)$ & $(2)$ & $(1)$ & $(2)$ & (1) & (2) & (1) & (2) \\
\hline$U_{t-1}^{\text {Diff }}$ & $\begin{array}{c}1,262^{* *} \\
(14,400)\end{array}$ & $\begin{array}{c}1,292 * * \\
(14,560)\end{array}$ & $\begin{array}{c}1,358 * * \\
(16,708)\end{array}$ & $\begin{array}{c}1,353 * * \\
(16,372)\end{array}$ & $\begin{array}{c}1,348 * * \\
(11,113)\end{array}$ & $\begin{array}{c}1,353 * * \\
(11,399)\end{array}$ & $\begin{array}{c}1,438 * * \\
(15,873)\end{array}$ & $\begin{array}{c}1,430 * * \\
(16,080)\end{array}$ \\
\hline$\sum_{\underline{\underline{t}}}^{O} \mathrm{U}_{\mathrm{t}-2}^{\text {Diff }}$ & $\begin{array}{l}-0,323 * * \\
(-3,671)\end{array}$ & $\begin{array}{c}-0,349 * * \\
(-3,900)\end{array}$ & $\begin{array}{c}-0,397 * * \\
(-5,046)\end{array}$ & $\begin{array}{c}-0,393 * * \\
(-4,921)\end{array}$ & $\begin{array}{c}-0,434 * * \\
(-3,846)\end{array}$ & $\begin{array}{l}-0,430 * * \\
(-3,799)\end{array}$ & $\begin{array}{c}-0,537 * * \\
(-6,720)\end{array}$ & $\begin{array}{l}-0,533^{* *} \\
(-6,777)\end{array}$ \\
\hline $\begin{array}{l}\bar{d} \\
\frac{0}{d} \\
\text { RCG6, }\end{array}$ & $\begin{array}{c}0,019 \\
(1,247)\end{array}$ & - & $\begin{array}{c}-0,017 \\
(-0,774)\end{array}$ & 一 & $\begin{array}{c}0,010 \\
(0,122)\end{array}$ & - & $\begin{array}{c}-0,009 \\
(-0,539)\end{array}$ & - \\
\hline $\begin{array}{l}\frac{e}{2} \\
R C G 6 \\
t-1\end{array}$ & - & $\begin{array}{c}0,019 \\
(1,221) \\
\end{array}$ & - & $\begin{array}{c}-0,272 \\
(-1,236) \\
\end{array}$ & - & $\begin{array}{c}-0,034 \\
(-0,389)\end{array}$ & - & $\begin{array}{c}-0,019 \\
(-1,166)\end{array}$ \\
\hline $\mathrm{R}^{2}$ & 0,97 & 0,97 & 0,99 & 0,99 & 0,99 & 0,99 & 0,99 & 0,99 \\
\hline
\end{tabular}

TAnmerkungen: $\quad \mathrm{t}$-Werte in Klammern, $+(\alpha<0,1),{ }^{*}(\alpha<0,05),{ }^{* *}(\alpha<0,01)$.

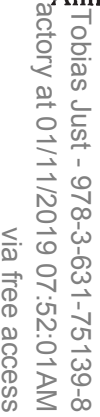


Tabelle C.15 : Partisaneffekte hinsichtlich der Inflationsraten bei ökonomischen Clustern (1. Ländercluster)

abhängige Variable: $\pi$ (Belgien, Niederlande, Neuseeland, Schweden, Großbritannien)

\begin{tabular}{|c|c|c|c|c|c|c|c|c|}
\hline \multirow{2}{*}{$\begin{array}{l}\text { unabh. } \\
\text { Var. }\end{array}$} & \multicolumn{2}{|c|}{$1960-1973$ (1. Zeitcluster) } & \multicolumn{2}{|c|}{1974 - 1985 (2. Zeitcluster) } & \multicolumn{2}{|c|}{1986 - 1992 (3. Zeitcluster) } & \multicolumn{2}{|c|}{1993 - 1997 (4. Zeitcluster) } \\
\hline & (1) & (2) & (1) & (2) & (1) & (2) & (1) & (2) \\
\hline$\pi_{t-1}$ & $\begin{array}{c}0,946^{* *} \\
(15,839)\end{array}$ & $\begin{array}{c}0,946 * * \\
(15,842)\end{array}$ & $\begin{array}{c}1,271^{* *} \\
(21,897)\end{array}$ & $\begin{array}{c}1,269 * * \\
(22,043)\end{array}$ & $\begin{array}{c}1,142 * * \\
(13,526)\end{array}$ & $\begin{array}{c}1,171 * * \\
(13,691)\end{array}$ & $\begin{array}{c}1,025 * * \\
(10,270)\end{array}$ & $\begin{array}{l}0,977 * * \\
(9,929)\end{array}$ \\
\hline$\pi_{1-2}$ & $\begin{array}{l}-0,191 * * \\
(-3,289)\end{array}$ & $\begin{array}{l}-0,192 * * \\
(-3,295)\end{array}$ & $\begin{array}{l}-0,452 * * \\
(-8,281)\end{array}$ & $\begin{array}{c}-0,455 \\
(-8,392)\end{array}$ & $\begin{array}{l}-0,308^{* *} \\
(-3,806)\end{array}$ & $\begin{array}{l}-0,324 * * \\
(-3,934)\end{array}$ & $\begin{array}{l}-0,301 * * \\
(-2,995)\end{array}$ & $\begin{array}{l}-0,322 * * \\
(-3,331)\end{array}$ \\
\hline$\pi_{1}^{\mathrm{w}}$ & $\begin{array}{l}0,281 * * \\
(6,098)\end{array}$ & $\begin{array}{l}0,283 * * \\
(6,131)\end{array}$ & $\begin{array}{l}0,170 * * \\
(5,345)\end{array}$ & $\begin{array}{l}0,169 * * \\
(5,334)\end{array}$ & $\begin{array}{c}0,021 \\
(0,187)\end{array}$ & $\begin{array}{c}0,039 \\
(0,345)\end{array}$ & $\begin{array}{c}0,341 \\
(1,523)\end{array}$ & $\begin{array}{c}0,276 \\
(1,290)\end{array}$ \\
\hline $\mathrm{CG}_{1.2}$ & - & $\begin{array}{c}0,032 \\
(0,774) \\
\end{array}$ & - & $\begin{array}{c}-0,108^{*} \\
(-2,200)\end{array}$ & - & $\begin{array}{c}-0,196 \\
(-1,544)\end{array}$ & - & $\begin{array}{c}0,203^{*} \\
(2,569)\end{array}$ \\
\hline $\mathrm{R}_{\frac{2}{2}}^{\frac{2}{2}}$ & 0,85 & 0,85 & 0,94 & 0,94 & 0,91 & 0,90 & 0,70 & 0,72 \\
\hline
\end{tabular}

Anmerkungen: $\quad \mathrm{t}$-Werte in Klammern, $+(\alpha<0,1),{ }^{*}(\alpha<0,05),{ }^{* *}(\alpha<0,01)$. 
Tabelle C.16 : Partisaneffekte hinsichtlich der Inflationsraten bei ökonomischen Clustern (2. Ländercluster)

abhängige Variable: $\pi_{t}$ (Kanada, Dänemark, Finnland, Irland, Norwegen, Schweiz)

\begin{tabular}{|c|c|c|c|c|c|c|c|c|}
\hline \multirow{2}{*}{$\begin{array}{l}\text { unabh. } \\
\text { Var. }\end{array}$} & \multicolumn{2}{|c|}{1960 - 1973 (1. Zeitcluster) } & \multicolumn{2}{|c|}{1974 - 1985 (2. Zeitcluster) } & \multicolumn{2}{|c|}{1986 - 1992 (3. Zeitcluster) } & \multicolumn{2}{|c|}{1993 - 1997 (4. Zeitcluster) } \\
\hline & (1) & (2) & (1) & (2) & (1) & (2) & (1) & (2) \\
\hline$\pi_{t-1}$ & $\begin{array}{c}0,996 * * \\
(18,217)\end{array}$ & $\begin{array}{c}0,991 * * \\
(18,064)\end{array}$ & $\begin{array}{c}0,928 * * \\
(15,758)\end{array}$ & $\begin{array}{c}0,932 * * \\
(15,184)\end{array}$ & $\begin{array}{c}1,244 * * \\
(16,506)\end{array}$ & $\begin{array}{c}1,234 * * \\
(16,387)\end{array}$ & $\begin{array}{c}1,054 * * \\
(12,183)\end{array}$ & $\begin{array}{c}1,036 * * \\
(12,126)\end{array}$ \\
\hline$\pi_{t-2}$ & $\begin{array}{l}-0,240 * * \\
(-4,441)\end{array}$ & $\begin{array}{c}-0,238 * * \\
(-4,400)\end{array}$ & $\begin{array}{l}-0,148 * * \\
(-2,710)\end{array}$ & $\begin{array}{l}-0,149 * * \\
(-2,738)\end{array}$ & $\begin{array}{l}-0,347 * * \\
(-4,495)\end{array}$ & $\begin{array}{l}-0,329 * * \\
(-4,217)\end{array}$ & $\begin{array}{l}-0,410 * * \\
(-4,876)\end{array}$ & $\begin{array}{l}-0,380 * * \\
(-4,575)\end{array}$ \\
\hline$\pi_{1}^{\omega}$ & $\begin{array}{l}0,279 * * \\
(5,886)\end{array}$ & $\begin{array}{l}0,286 * * \\
(6,013)\end{array}$ & $\begin{array}{l}0,274 * * \\
(7,697)\end{array}$ & $\begin{array}{c}0,266^{* *} \\
(7,600)\end{array}$ & $\begin{array}{c}-0,016 \\
(-0,310)\end{array}$ & $\begin{array}{c}-0,004 \\
(-0,079)\end{array}$ & $\begin{array}{c}0,188 \\
(1,169)\end{array}$ & $\begin{array}{c}0,419 * \\
(2,364)\end{array}$ \\
\hline${ }^{5} \mathrm{CG}_{\mathrm{t}-2}$ & - & $\begin{array}{c}-0,150 * \\
(-2,049)\end{array}$ & - & $\begin{array}{c}0,061 \\
(0,767) \\
\end{array}$ & - & $\begin{array}{c}0,077 \\
(1,298)\end{array}$ & - & $\begin{array}{c}-0,159^{*} \\
(-2,196)\end{array}$ \\
\hline${ }_{0}^{\bar{\theta}}$ & 0,82 & 0,82 & 0,93 & 0,93 & 0,88 & 0,88 & 0,72 & 0,73 \\
\hline
\end{tabular}

Añmerkungen: $\quad t$-Werte in Klammern, $+(\alpha<0,1),{ }^{*}(\alpha<0,05),{ }^{* *}(\alpha<0,01)$.

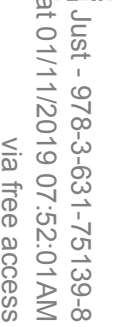


Tabelle C.17 : Partisaneffekte hinsichtlich der Inflationsraten bei ökonomischen Clustern (3. Ländercluster)

abhängige Variable: $\pi_{1}$ (Australien, Österreich, Frankreich, Deutschland, Italien, Japan, Spanien, USA)

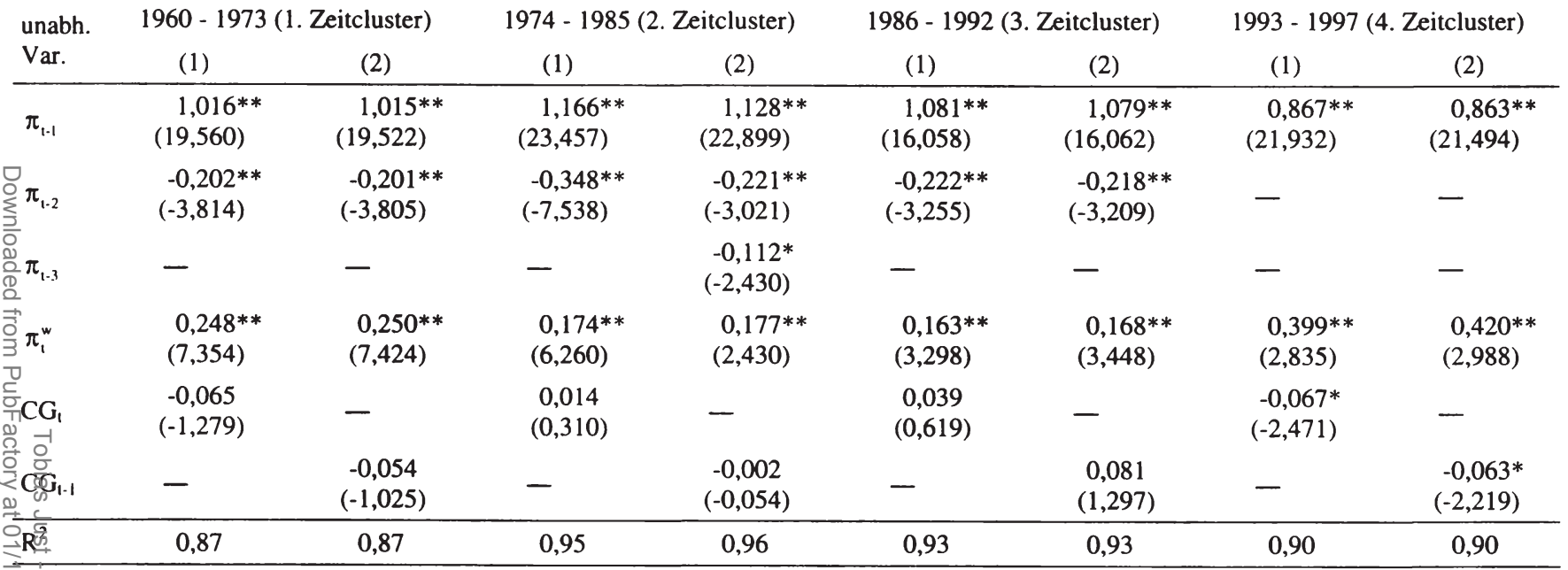


Tabelle C.18 : Partisaneffekte hinsichtlich der Inflationsraten bei politischen Clustern (1. Ländercluster)

abhängige Variable: $\pi_{t}$ (Deutschland, Dänemark, Italien, Niederlande, Belgien, Frankreich)

\begin{tabular}{|c|c|c|c|c|c|c|c|c|c|c|}
\hline \multirow{2}{*}{$\begin{array}{l}\text { unabh. } \\
\text { Var. }\end{array}$} & \multicolumn{2}{|c|}{$1960-1966$} & \multicolumn{2}{|c|}{$1967-1978$} & \multicolumn{2}{|c|}{$1979-1987$} & \multicolumn{2}{|c|}{$1988-1992$} & \multicolumn{2}{|c|}{$1993-1997$} \\
\hline & (1) & (2) & (1) & (2) & (1) & (2) & (1) & (2) & (1) & (2) \\
\hline$\pi_{t-1}$ & $\begin{array}{c}0,771 * * \\
(15,021)\end{array}$ & $\begin{array}{c}0,767 * * \\
(15,027)\end{array}$ & $\begin{array}{c}1,150 * * \\
(19,834)\end{array}$ & $\begin{array}{c}1,151 * * \\
(19,853)\end{array}$ & $\begin{array}{c}1,056 * * \\
(15,395)\end{array}$ & $\begin{array}{c}1,048 * * \\
(15,343)\end{array}$ & $\begin{array}{c}1,064 * * \\
(11,745)\end{array}$ & $\begin{array}{c}1,070 * * \\
(11,507)\end{array}$ & $\begin{array}{c}0,872 * * \\
(18,488)\end{array}$ & $\begin{array}{c}0,871 * * \\
(18,691)\end{array}$ \\
\hline & - & - & $\begin{array}{c}-0,332 * * \\
(-6,125)\end{array}$ & $\begin{array}{l}-0,331 * * \\
(-6,118)\end{array}$ & $\begin{array}{l}-0,208 * * \\
(-3,330)\end{array}$ & $\begin{array}{l}-0,200 * * \\
(-3,210)\end{array}$ & $\begin{array}{l}-0,348^{* *} \\
(-3,924)\end{array}$ & $\begin{array}{l}-0,340 * * \\
(-3,761)\end{array}$ & - & - \\
\hline$\pi^{\mathrm{w}}$ & $\begin{array}{c}0,344+ \\
(1,969)\end{array}$ & $\begin{array}{c}0,309+ \\
(1,741)\end{array}$ & $\begin{array}{l}0,177^{* *} \\
(4,892)\end{array}$ & $\begin{array}{l}0,174^{* *} \\
(4,807)\end{array}$ & $\begin{array}{l}0,196 * * \\
(7,429)\end{array}$ & $\begin{array}{l}0,199 * * \\
(7,676)\end{array}$ & $\begin{array}{c}0,097 \\
(1,548)\end{array}$ & $\begin{array}{c}0,100 \\
(1,557)\end{array}$ & $\begin{array}{l}0,362 * * \\
(2,889)\end{array}$ & $\begin{array}{c}0,366 * * \\
(2,917)\end{array}$ \\
\hline${ }_{5} C_{t}$ & $\begin{array}{c}0,024 \\
(0,177)\end{array}$ & - & $\begin{array}{c}0,034 \\
(0,401)\end{array}$ & - & $\begin{array}{c}0,081+ \\
(1,769)\end{array}$ & - & $\begin{array}{l}-0,211 * * \\
(-2,710)\end{array}$ & - & $\begin{array}{c}-0,000 \\
(-0,009)\end{array}$ & - \\
\hline $\mathrm{CG}_{\mathrm{t}-1}$ & - & $\begin{array}{c}-0,070 \\
(-0,500)\end{array}$ & - & $\begin{array}{c}-0,009 \\
(-0,112)\end{array}$ & - & $\begin{array}{c}0,108 * \\
(2,405)\end{array}$ & - & $\begin{array}{c}-0,123+ \\
(-1,795)\end{array}$ & - & $\begin{array}{c}0,012 \\
(0,280)\end{array}$ \\
\hline${ }_{0}^{2}$ & 0,74 & 0,74 & 0,92 & 0,92 & 0,98 & 0,98 & 0,90 & 0,90 & 0,90 & 0,90 \\
\hline
\end{tabular}


Tabelle C.19 : Partisaneffekte hinsichtlich der Inflationsraten bei politischen Clustern (2. Ländercluster)

abhängige Variable: $\pi$, (Großbritannien, Schweden, Österreich, Norwegen, Finnland, Irland)

\begin{tabular}{|c|c|c|c|c|c|c|c|c|c|c|}
\hline \multirow{2}{*}{$\begin{array}{l}\text { unabh. } \\
\text { Var. }\end{array}$} & \multicolumn{2}{|c|}{$1960-1966$} & \multicolumn{2}{|c|}{$1967-1978$} & \multicolumn{2}{|c|}{$1979-1987$} & \multicolumn{2}{|c|}{$1988-1992$} & \multicolumn{2}{|c|}{$1993-1997$} \\
\hline & (1) & (2) & (1) & (2) & (1) & (2) & (1) & (2) & (1) & (2) \\
\hline$\pi_{t-1}$ & $\begin{array}{c}1,005^{* *} \\
(12,758)\end{array}$ & $\begin{array}{c}1,009 * * \\
(12,868)\end{array}$ & $\begin{array}{c}1,098^{* *} \\
(19,368)\end{array}$ & $\begin{array}{c}1,097 * * \\
(19,408)\end{array}$ & $\begin{array}{c}0,808 * * \\
(26,172)\end{array}$ & $\begin{array}{c}0,808^{* *} \\
(26,136)\end{array}$ & $\begin{array}{c}1,172 * * \\
(12,582)\end{array}$ & $\begin{array}{c}1,159 * * \\
(13,080)\end{array}$ & $\begin{array}{c}0,925 * * \\
(10,028)\end{array}$ & $\begin{array}{c}0,916 * * \\
(9,981)\end{array}$ \\
\hline$\pi_{t-2}$ & $\begin{array}{l}-0,336^{* *} \\
(-4,225)\end{array}$ & $\begin{array}{c}-0,339 * * \\
(-4,274)\end{array}$ & $\begin{array}{l}-0,290 * * \\
(-5,540)\end{array}$ & $\begin{array}{l}-0,289 * * \\
(-5,531)\end{array}$ & - & - & $\begin{array}{l}-0,382^{* *} \\
(-4,353)\end{array}$ & $\begin{array}{l}-0,396^{* *} \\
(-4,746)\end{array}$ & $\begin{array}{l}-0,257^{* *} \\
(-2,754)\end{array}$ & $\begin{array}{l}-0,265^{* *} \\
(-2,869)\end{array}$ \\
\hline$\pi_{1}^{w}$ & $\begin{array}{c}0,327+ \\
(1,788)\end{array}$ & $\begin{array}{c}0,332+ \\
(1,821)\end{array}$ & $\begin{array}{c}0,249 * * \\
(6,720)\end{array}$ & $\begin{array}{c}0,250 * * \\
(6,799)\end{array}$ & $\begin{array}{c}0,259 * * \\
(6,803)\end{array}$ & $\begin{array}{c}0,248 * * \\
(6,754)\end{array}$ & $\begin{array}{l}0,277 * * \\
(2,750)\end{array}$ & $\begin{array}{l}0,316 * * \\
(3,174)\end{array}$ & $\begin{array}{c}0,307 \\
(1,385)\end{array}$ & $\begin{array}{c}0,261 \\
(1,170)\end{array}$ \\
\hline $\mathrm{CG}_{1}$ & $\begin{array}{c}-0,043 \\
(-0,572)\end{array}$ & - & $\begin{array}{c}0,044 \\
(0,802)\end{array}$ & - & $\begin{array}{c}-0,083 \\
(-1,150)\end{array}$ & - & $\begin{array}{c}-0,189 * \\
(-2,433)\end{array}$ & - & $\begin{array}{c}0,050 \\
(0,953)\end{array}$ & - \\
\hline $\mathrm{CG}_{t-1}$ & - & $\begin{array}{c}-0,026 \\
(-0,323)\end{array}$ & - & $\begin{array}{c}0,065 \\
(1,195)\end{array}$ & - & $\begin{array}{c}-0,070 \\
(-1,003)\end{array}$ & - & $\begin{array}{l}-0,273^{* *} \\
(-3,478)\end{array}$ & - & $\begin{array}{c}0,097 \\
(1,611)\end{array}$ \\
\hline $\mathrm{R}_{0}^{0}$ & 0,68 & 0,68 & 0,93 & 0,93 & 0,92 & 0,92 & 0,91 & 0,92 & 0,71 & 0,71 \\
\hline
\end{tabular}

Anmerkungen: $\quad \mathrm{t}$-Werte in Klammern, $+(\alpha<0,1),{ }^{*}(\alpha<0,05),{ }^{* *}(\alpha<0,01)$. 
Tabelle C.20 : Partisaneffekte hinsichtlich der Inflationsraten bei politischen Clustern (3. Ländercluster)

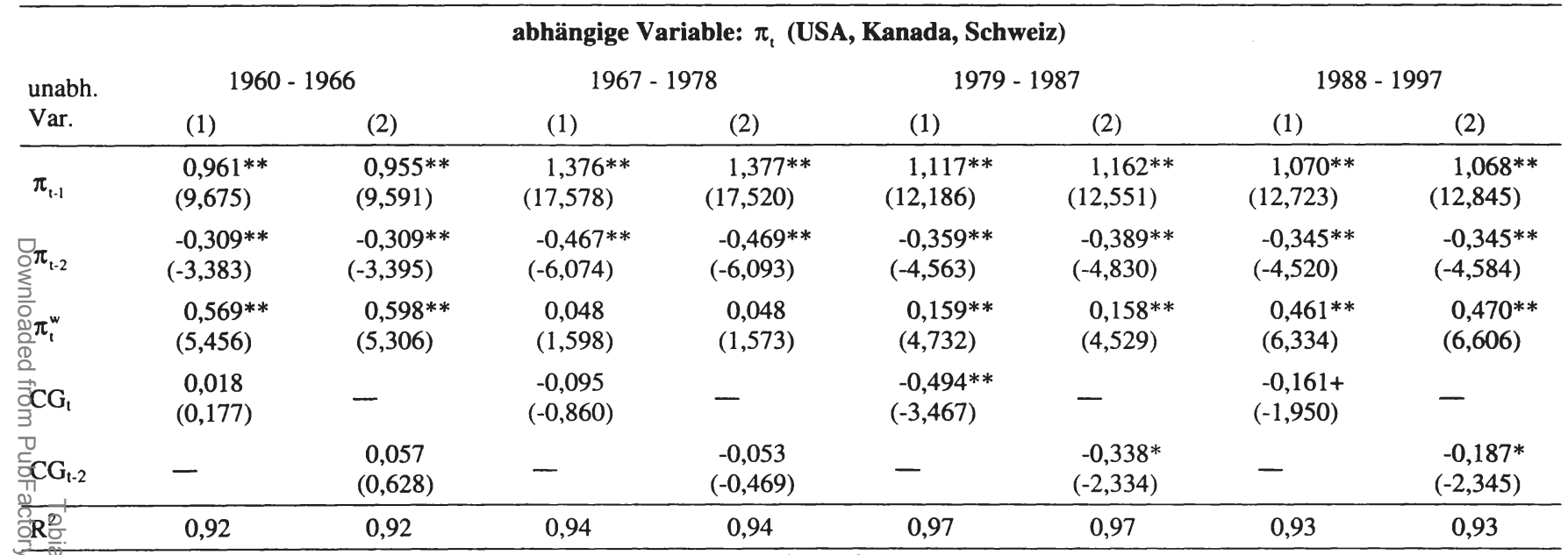

An̂merkungen: $\quad$ t-Werte in Klammern, $+(\alpha<0,1),{ }^{*}(\alpha<0,05),{ }^{* *}(\alpha<0,01)$.

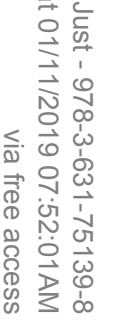


Tabelle C.21 : Partisaneffekte hinsichtlich der Inflationsraten bei politischen Clustern (4. Ländercluster)

abhängige Variable: $\pi_{\mathrm{t}}$ (Spanien, Japan, Australien, Neuseeland)

\begin{tabular}{|c|c|c|c|c|c|c|c|c|c|c|}
\hline \multirow{2}{*}{$\begin{array}{l}\text { unabh. } \\
\text { Var. }\end{array}$} & \multicolumn{2}{|c|}{$1960-1966$} & \multicolumn{2}{|c|}{$1967-1978$} & \multicolumn{2}{|c|}{$1979-1987$} & \multicolumn{2}{|c|}{$1988-1992$} & \multicolumn{2}{|c|}{$1993-1997$} \\
\hline & $(1)$ & $(2)$ & $(1)$ & (2) & (1) & (2) & (1) & (2) & (1) & (2) \\
\hline$\pi_{t-1}$ & $\begin{array}{l}0,998 * * \\
(8,749)\end{array}$ & $\begin{array}{l}0,996 * * \\
(8,802)\end{array}$ & $\begin{array}{c}1,063^{* *} \\
(12,993)\end{array}$ & $\begin{array}{c}1,097 * * \\
(14,434)\end{array}$ & $\begin{array}{c}1,317 * * \\
(16,371)\end{array}$ & $\begin{array}{c}1,317 * * \\
(16,440)\end{array}$ & $\begin{array}{c}0,835^{* *} \\
(14,176)\end{array}$ & $\begin{array}{c}0,838 * * \\
(14,290)\end{array}$ & $\begin{array}{c}1,143 * * \\
(10,230)\end{array}$ & $\begin{array}{c}1,142 * * \\
(10,351)\end{array}$ \\
\hline$\pi_{1.2}$ & $\begin{array}{l}-0,290 * \\
(2,554)\end{array}$ & $\begin{array}{c}-0,293^{*} \\
(-2,581)\end{array}$ & $\begin{array}{l}-0,305^{* *} \\
(-4,011)\end{array}$ & $\begin{array}{l}-0,309^{* *} \\
(-4,381)\end{array}$ & $\begin{array}{c}-0,549 * * \\
(-6,907)\end{array}$ & $\begin{array}{c}-0,549 * * \\
(-6,926)\end{array}$ & - & - & $\begin{array}{c}-0,337 * * \\
(-2,994)\end{array}$ & $\begin{array}{l}-0,345^{* *} \\
(-3,100)\end{array}$ \\
\hline$\pi_{1}^{\omega}$ & $\begin{array}{c}0,111 \\
(0,553)\end{array}$ & $\begin{array}{c}0,091 \\
(0,446)\end{array}$ & $\begin{array}{c}0,311^{* *} \\
(3,419)\end{array}$ & $\begin{array}{c}0,245^{* *} \\
(2,939)\end{array}$ & $\begin{array}{c}0,140^{* *} \\
(2,751)\end{array}$ & $\begin{array}{l}0,137 * * \\
(2,784)\end{array}$ & $\begin{array}{c}0,318^{*} \\
(2,488)\end{array}$ & $\begin{array}{c}0,303^{*} \\
(2,379)\end{array}$ & $\begin{array}{c}0,152 \\
(0,645)\end{array}$ & $\begin{array}{c}0,190 \\
(0,828)\end{array}$ \\
\hline $\mathrm{CG}_{1}$ & $\begin{array}{c}0,000 \\
(0,001)\end{array}$ & - & $\begin{array}{c}-0,120 \\
(-0,143)\end{array}$ & - & $\begin{array}{c}0,011 \\
(0,112)\end{array}$ & - & $\begin{array}{c}-0,252 \\
(-1,362)\end{array}$ & - & $\begin{array}{c}-0,078+ \\
(-1,933)\end{array}$ & - \\
\hline${ }_{1} \mathrm{CG}_{\mathrm{t}-2}$ & - & $\begin{array}{c}0,113 \\
(0,414)\end{array}$ & - & $\begin{array}{c}-0,093 \\
(-0,718)\end{array}$ & - & $\begin{array}{c}0,018 \\
(0,188)\end{array}$ & - & $\begin{array}{c}-0,249 \\
(-1,306)\end{array}$ & - & $\begin{array}{c}-0,088^{*} \\
(-2,132)\end{array}$ \\
\hline$R_{0}^{2}$ & 0,84 & 0,84 & 0,92 & 0,93 & 0,94 & 0,94 & 0,90 & 0,90 & 0,90 & 0,90 \\
\hline
\end{tabular}

Ahmerkungen: $\quad$ t-Werte in Klammern, $+(\alpha<0,1),{ }^{*}(\alpha<0,05),{ }^{* *}(\alpha<0,01)$. 
Tobias Just - 978-3-631-75139-8

Downloaded from PubFactory at 01/11/2019 07:52:01AM

via free access 


\section{Literaturverzeichnis}

Abramson, P.R., R. Inglehart (1995), Value Change in Global Perspective, Ann Arbor.

Akerlof, G.A., R.E. Kranton (2000), Economics and Identity, in: Quarterly Journal of Economics 115, S. 715-753.

Åkerman, J. (1947), Political Economic Cycles, in: Kyklos 1, S. 101-117.

Alesina, A. (1987), Macroeconomic Policy in a Two-Party System as a Repeated Game, in: Quarterly Journal of Economics 102, S. 651-678.

Alesina, A. (1991), Political Models of Macroeconomic Policy and Fiscal Reforms. Policy Research Working Paper No. 570, World Bank, New York.

Alesina, A., G.D. Cohen, N. Roubini (1992), Macroeconomic Policy and Elections in OECD Democracies, in: Economics and Politics 4, S. 1-30.

Alesina, A., G.D. Cohen, N. Roubini (1993), Electoral Business Cycles in Industrial Democracies, in: European Journal of Political Economy 9, S. 1-23.

Alesina, A., G.D. Cohen, N. Roubini (1997), Political Cycles and the Macroeconomy, Cambridge, London.

Alesina, A., H. Rosenthal (1995), Partisan Politics, Divided Government, and the Economy, Cambridge.

Alesina, A., N. Roubini (1990), Political Cycles in OECD Economies, NBER Working Paper No. 3478, Cambridge.

Alesina, A., J. Sachs (1988), Political Parties and the Business Cycle in the United States, in: Journal of Money, Credit and Banking 20, S. 63-84.

Alexander, V., H.E. Loef (1975), Die Messung der sozialen Kosten der Inflation. Eine theoretische und empirische Problemanalyse, in: P. Harbusch, D. Wiek [Hrsg.], Marktwirtschaft. Eine Einfuihrung in das Konzept der freiheitlichen Wirtschaftsordnung, Stuttgart.

Alogoskoufis, G.S., B. Lockwood, A. Philippopoulos (1992), Wage Inflation, Electoral Uncertainty and the Exchange Rate Regime Theory and UK Evidence, in: The Economic Journal 102, S. 1370-1394.

Amelung, T. (1989), Die politische Ökonomie der Importsubstitution und der Handelsliberalisierung. Das Beispiel Türkei, Tübingen.

Andreoni, J. (1988), Why Free Ride? Strategies and Learning in Public Goods Experiments, in: Journal of Public Economics 37, S. 291-304.

Andreoni, J. (1995), Cooperation in Public-Goods Experiments: Kindness or

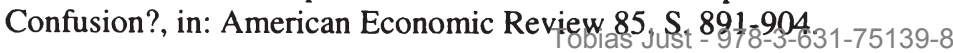


Aron, $R$. (1968), The End of the Ideological Age?, in: C.I. Waxman [Hrsg.], The End of Ideolgy Debate, New York, S. 27-47.

Arrow, K.J. (1963), Social Choice and Individual Values, 2. Aufl., New York.

Arrow, K.J. (1990), Interview, in: R. Swedberg, Economics and Sociology, Princeton, S. 133-151.

Asch, S.E. (1951), Effects of Group Pressure upon the Modification and Distortion of Judgments, in: H. Guetzkow [Hrsg.], Groups, Leadership and Men, New York, S. 177-190.

Backes, U., E. Jesse (1997), Die Rechts-Links-Unterscheidung. Betrachtungen zu ihrer Geschichte, Logik, Leistungsfähigkeit und Problematik, in: U. Backes, E. Jesse [Hrsg.], Jahrbuch Extremismus und Demokratie 9, S. 13-38.

Backhaus, K., B. Erichson, W. Plinke, R. Weiber (1996), Multivariate Analysemethoden. Eine anwendungsorientierte Einführung, 8. Aufl., Berlin et al.

Bairoch, P. (1996), Globalization Myths and Reality: One Century of External Trade and Foreign Investment, in: R. Boyer, D. Drache [Hrsg.], States Against Markets. The Limits of Globalization, London, New York, S. 173-192.

Bakshi, H., A.G. Haldane, N. Hatch (1997), Some Costs and Benefits of Price Stability in the United Kingdom, Bank of England, London.

Baldwin, R.E. (1982), The Political Economy of Protectionism, in: J. Bhagwati [Hrsg.], Import Competition and Response, Chicago, S. 263-286.

Baldwin, R.E., P. Martin (1999), Two Waves of Globalization: Superficial Similarities, Fundamental Differences, in: H. Siebert [Hrsg.], Globalization and Labor, Tübingen, S. 3-58.

Banerjee, A.V. (1992), A Simple Model of Herd Behavior, in: Quarterly Journal of Economics 107, S. 797-817.

Bar-Gill, O., C. Fershtman (2000), The Limit of Public Policy: Endogenous Preferences. CentER Working Paper No. 71, Tilburg.

Baron, J. (1997), The Illusion of Morality as Self-Interest: A Reason to Co-operate in Social Dilemmas, in: Psychological Science 8, S. 330-335.

Barro, R.J. (1973), The Control of Politicians: An Economic Model, in: Public Choice 14, S. 19-42.

Barro, R.J. (1995), Inflation and Economic Growth, in: Bank of England Quarterly Bulletin 35, S. 166-175.

Beck, N. (1982), Parties, Administrations, and American Macroeconomic Outcomes, in: The American Political Science Review 76, S. 83-94.

Beck, U. (1997), Was ist Globalisierung? Irrtümer des Globalismus, Antworten auf Globalisierung, Frankfurt a.M. 
Becker, G.S. (1967), Human Capital and the Personal Income Distribution. An Analytical Approach, Ann Arbor.

Becker, G.S. (1974), A Theory of Social Interactions, in: Journal of Political Economy 82, S. 1063-1091.

Becker, G.S. (1976), The Economic Approach to Human Behaviour, Chicago.

Becker, G.S. (1981), A Treatise on the Family, Cambridge.

Becker, G.S. (1992), Habits, Addictions and Traditions, in: Kyklos 45, S. 327-345.

Becker, G.S. (1993), Human Capital, A Theoretical and Empirical Analysis with Special Reference to Education, 3. ed., Chicago, London.

Becker, G.S. (1996), Accounting for Tastes, Cambridge, London.

Beckmann, K. (1998), Analytische Grundlagen einer Finanzverfassung, Frankfurt a.M.

Belke, A. (1996), Politische Konjunkturzyklen in Theorie und Praxis, Tübingen.

Belke, A. (2000), Partisan Political Business Cycles in the German Labour Market? Empirical Tests in the Light of the Lucas-Critique, in: Public Choice 104, S. 225-283.

Bell, D. (1960), The End of Ideology: On the Exhaustion of Political Ideas in the Fifties, Glencoe Illinois.

Bell, D. (1968), The End of Ideology in the West, in: C.I. Waxman [Hrsg.], The End of Ideology Debate, New York, S. 87-106.

Berlemann, M. (1999), Politökonomische Theorien der Inflation und der Konjunktur. Eine theoretische und empirische Analyse unter besonderer Berücksichtigung des Zeitinkonsistenzproblems, Marburg.

Bernholz, P., F. Breyer (1994), Grundlagen der politischen Ökonomie, Band 2, 3. Aufl., Tübingen.

Berthold, N. (1997), Der Sozialstaat im Zeitalter der Globalisierung, Tübingen.

Berthold, N., R. Fehn (1994), Verursachen Wahlen einen Politischen Konjunkturzyklus?, in: WiSt 23, S. 166-175.

Beyme, K. von (1985), Political Parties in Western Democracies, Aldershot.

Bhagwati, J. (1988), Protectionism, Cambridge, London.

Bhagwati, J. (1999), Globalization: Who Gains, who Loses?, in: H. Siebert [Hrsg.], Globalization and Labor, Kiel, S. 225-236.

Blankart, C.B. (1998), Öffentliche Finanzen in der Demokratie, 3. Aufl., München.

Blinder, A.S. (1987), Hard Heads, Soft Hearts. Tough-Minded Economists for a Just Society, Reading. 
Blinder, A.S., H.Y. Esaki (1978), Macroeconomic Activity and Income Distribution in the Postwar United States, in: The Review of Economics and Statistics 60, S. 604-609.

Blomberg, S.B, (1997), Economics and Politics: Introduction to the Literature and Basic Modeling of Political Macroeconomics, Wellesley College Working Paper No. 97-06.

Blümel, W., R. Pethig, O. von dem Hagen (1986), The Theory of Public Goods: A Survey of Recent Issues, in: Zeitschrift für die gesamte Staatswissen-schaft 142, S. 241-309.

Bobbio, N. (1994), Rechts und Links. Gründe und Bedeutungen einer politischen Unterscheidung, Berlin.

Bordo, M.D., B. Eichengreen, D.A. Irwin (1999), Is Globalization Today Really Different than Globalization a 100 Years Ago? NBER Working Paper 7195, Cambridge.

Boulding, K.E. (1969), Economics as a Moral Science, in: American Economic Review 59, S. 1-12.

Bowles, S. (1998), Endogenous Preferences: The Cultural Consequences of Markets and other Economic Institutions, in: Journal of Economic Litera-ture 36, S. 75-111.

Brandts, J., A. Schram (1995), The Bid Function Approach to VCM Experiments, Working Paper, University of Amsterdam.

Brennan, G., J.M. Buchanan (1993), Die Begründung von Regeln, Konstitutionelle Politische Ökonomie, Tübingen.

Brennan, G., L. Lomasky (1993), Democracy and Decisions, Cambridge.

Brettschneider, F., K. Ahlstich; B. Klett; A. Vetter (1994), Materialien zu Gesellschaft, Wirtschaft und Politik in den Mitgliedstaaten der Europäischen Gemeinschaft, in: O.W. Gabriel, F. Brettschneider [Hrsg.], Die EU-Staaten im Vergleich - Strukturen, Prozesse, Politikinhalte, 2. Aufl., Opladen, S. 445624.

Brümmerhoff, D. (1992), Finanzwissenschaft, 6. Aufl., München.

Brunner, E.J. (1998), Free Riders or Easy Riders? An Examination of the Voluntary Provision of Public Radio, in: Public Choice 97, S. 587-604.

Brunner, K., H.K. Meckling (1977), The Perception of Man and the Conception of Government, in: Journal of Money, Credit, and Banking 9, S. 70-85.

Budge, I., R.I. Hofferbert (1990), Mandates and Policy Outputs: U.S. Party Platforms and Federal Expenditures, in: American Political Science Review 84, S. 111-131.

Bundeswahlleiter, (1999a), Wahlergebnisse in Hamburg, http://www.statistikbund.de/wahlen/ergebalt/d/t/twint02.htm, Aaptib 1999. 978-3-631-75139-8 
Bundeswahlleiter, (1999b), Wahlergebnisse in Deutschland, http://www.statistikbund.de/wahlen/ergeb98/d/t/bun999_02.htm, April 1999.

Bürklin, W., M. Klein, A. Ruß (1994), Dimension des Wertewandels. Eine empirische Längsschnittanalyse zwischen Dimensionalität und der Wandlungsdynamik gesellschaftlicher Wertorientierungen, in: Politische Vierteljahresschrift 35, S. 579-606.

Cairncross, F. (1997), The Death of Distance. How the Communications Revolution Will Change Our Lives, London.

Calvert, R.L. (1985), Robustness of the Multidimensional Voting Model: Candidate Motivations, Uncertainty, and Convergence, in: American Journal of Political Science 29, S. 69-95.

Cameron, D. (1978), The Expansion of the Public Economy: A Comparative Analysis, in: American Political Science Review 72, S. 1243-1261.

Carlsen, F. (1997), Counterfiscal Policies and Partisan Politics: Evidence from Industrialized Countries, in: Applied Economics 29, S. 145-151.

Cassel, D. (1988), Wirtschaftspolitik als Ordnungspolitik, in: D. Cassel et al. [Hrsg.], Ordnungspolitik, München.

Cassel, D. (1992), Inflation, in: D. Bender et al. [Hrsg.], Vahlens Kompendium der Wirtschaftstheorie und Wirtschaftspolitik, Band 1, 5. Aufl., München, S. 265322.

Castles, F.G. [Hrsg.], (1982), The Impact of Parties, London.

Castles, F.G. (1989), Explaining Public Education Expenditure in OECD Nations, in: European Journal of Political Research 17, S. 431-448.

Castles, F.G., P. Mair (1984), Left-Right Political Scales: Some 'Expert' Judgements, in: European Journal of Political Research 12, S. 73-88.

Cayrol, $R$. (1992), La droite, la gauche et les références idéologiques, in: L'état de l'opinion, Paris, SOFRES, S. 58-63.

Chappell, H.W., W.R. Keech (1986), Party Differences in Macroeconomic Policies and Outcomes, in: American Economic Review, Papers and Procee-dings 76, S. 71-74.

Chappell, H.W., W.R. Keech (1988), The Unemployment Rate Consequences of Partisan Monetary Policies, in: Southern Economic Journal 55, S. 107-122.

Chirinko, R.S. (1990), Altruism, Egoism and the Role of Social Capital in the Private Provision of Public Goods, in: Economics and Politics 2, S. 75-90.

Coase, $R$. (1960), The Problem of Social Cost, in: Journal of Law and Economics 3, S. 1-44.

Coleman, J.S. (1988), Social Capital in the Creation of Human Capital, in: American Journal of Sociology 94 Supplement, S. S95-S120. 
Coleman, J.S. (1991), Grundlagen der Sozialtheorie, Band 1, München.

Crone, L., J. Tschirhart (1998), Separating Economic From Political Influences on Government Decisions, in: Journal of Economic Behavior and Organi-zation 35, S. 405-425.

Crosby, M., D. Brown, L. Malady (1995), Political and Partisan Cycles in the Australian Economy, Working Paper 2/95, Centre for Applied Economic Research, Sydney.

Cukierman, A., A.H. Meltzer (1986), A Theory of Ambiguity, Credibility and Inflation under Discretion and Asymmetric Information, in: Econometrica 54, S. 1099-1128.

Cukierman, A., M. Tommasi (1998), When Does it Take a Nixon to Go to China?, in: American Economic Review 88, S. 180-197.

Cusack, T.R. (1991), The Changing Contours of Government, WZB Discussion Paper P91-304, Berlin.

Cusack, T.R. (1992), The Dynamics of Public Spending, WZB Discussion Paper P92-306, Berlin.

Cusack, T.R. (1995), Politics and Macroeconomic Performance in the OECD Countries, WZB Discussion Paper FS I 95-315, Berlin.

Cusack, T.R. (1997a), Partisan Politics and Public Finance: Changes in Public Spending in the Industrialized Democracies 1955 - 1989, in: Public Choice 91, S. 375-395.

Cusack, T.R. (1997b), Partisan Politics and Fiscal Policy, WZB Discussion Paper FS I 97-306, Berlin.

Daalder, H. (1966), Parties, Elites and Political Developments, in: J. LaPalombara, M. Weiner [Hrsg.], Political Parties and Political Development, Princeton, S. 43-77.

Dahrendorf, $R$. (1992), Der moderne soziale Konflikt. Essay zur Politik der Freiheit, Stuttgart.

Dahrendorf, $R$. (1998), Die neue Parteienlandschaft, in: Die ZEIT, 25.06.1998, S. 7-8.

Dawes, R.M. (1980), Social Dilemmas, in: Annual Review of Psychology 31, S. 169-193.

Decker, F. (1998), Jenseits von rechts und links? Zum Bedeutungswandel von politischen Richtungsbegriffen in: U. Backes, E. Jesse [Hrsg.], Jahrbuch Extremismus und Demokratie 10, S. 33-49.

Decker, F. (2000), Parteien unter Druck. Der neue Rechtspopulismus in den westlichen Demokratien, Opladen. 
Demsetz, H. (1967), Toward a Theory of Property Rights, in: American Economic Review Papers and Proceedings 57, S. 347-359.

Denzau, A.T., D.C. North (1994), Shared Mental Models: Ideologies and Institutions, in: Kyklos 47, S. 3-31.

Deutsche Bundesbank, (2000), Monatsbericht März 2000, Frankfurt a.M.

Deutsches Institut für Wirtschaftsforschung, (diverse Jahrgänge), DIW-Wochenbericht, Berlin.

Devarajan, S., S.I. Hossain (1995), The Combined Incidence of Taxes and Public Expenditures in the Phillippines, Policy Research Working Paper 1543, World Bank, Washington D.C.

Dinkel, $R$. (1977), Der Zusammenhang zwischen der ökonomischen und politischen Entwicklung in einer Demokratie. Eine Untersuchung mit Hilfe der ökonomischen Theorie der Politik, Berlin.

Dodd, L.C. (1976), Coalitions in Parliamentary Government, Princeton.

Donges, J.B. (1998), Was heißt Globalisierung?, in: J.B. Donges, A. Freytag [Hrsg.], Die Rolle des Staates in einer globalisierten Wirtschaft, Stuttgart et al., S. 1-7.

Dörrenbacher, C. (1999), Vom Hoflieferanten zum Global Player. Unternehmensstrategien und nationale Politik in der Welttelekommunikationsindustrie, Berlin.

Dowell, R.S., R.S. Goldfarb, W.B. Griffith (1998), Economic Man as a Moral Individual, in: Economic Inquiry 36, S. 645-653.

Downs, A. (1957), An Economic Theory of Democracy, New York.

Downs, A. (1965), A Theory of Bureaucracy, in: American Economic Review 55, S. 439-446.

Drèze, J.H., H. Sneessens (1997), Technological Development, Competition from Low-Wage Economies and Low-Skilled Unemployment, in: D.J. Snower, G. de la Dehesa [Hrsg.], Unemployment Policy. Government Options for the Labour Market, Cambridge, S. 250-277.

Duesenberry, J. (1960), Comment on Economic Analysis of Fertility in Demographic Change in Developed Countries, in: A Conference of the Universities - NBER, Princeton.

Eekhoff, J. (1998), Bedroht die Globalisierung eine nationale Sozialpolitik?, in: J.B. Donges, A. Freytag [Hrsg.], Die Rolle des Staates in einer globalisierten Wirtschaft, Stuttgart, S. 199-215.

Endres, A., I. Querner (2000), Die Ökonomie natürlicher Ressourcen, 2. Aufl., Stuttgart et al. 
Engelhardt, G. (1989), Imperialismus der Ökonomie, in: H.B. Schäfer, K. Wehrt [Hrsg.], Die Ökonomisierung der Sozialwissenschaften. Sechs Wortmeldungen, Frankfurt a.M., New York, S. 19-49.

European Journal of Political Research, (1993), Political Data 24, S. 44-91.

Evans, L., et al. (1996), Economic Reform in New Zealand 1984-1995: The Pursuit of Efficiency, in: Journal of Economic Literature 34, S. 1856-1902.

Fair, R. (1978), The Effects of Economic Events on Votes for Presidents, in: Review of Economics and Statistics 60, S. 159-172.

Falter, J.W., M. Klein, S. Schumann (1994), Politische Konflikte, Wählerverhalten und die Struktur des Parteienwettbewerbs, in: O.W. Gabriel, F. Brettschneider [Hrsg.], Die EU-Staaten im Vergleich - Strukturen, Prozesse, Politikinhalte, 2. Aufl., Opladen, S. 194-220.

Fehn, R. (1993), Political Business Cycles and the Reelection Probabilities of Partisan Governments: The Effects of Unemployment Persistence, Kiel Advanced Studies Working Paper No. 251.

Feld, L.P. (1997), Exit, Voice and Income Taxes: The Loyalty of Voters, in: European Journal of Political Economy 13, S. 455-478.

Feld, L.P. (2000a), Fiskalischer Wettbewerb und Einkommensverteilung, in: Perspektiven der Wirtschaftspolitik 1, S. 181-198.

Feld, L.P. (2000b), Steuerwettbewerb und seine Auswirkungen auf die Allokation und Distribution: Eine empirische Analyse für die Schweiz, Tübingen.

Feld, L.P., J.G. Matsusaka (2000), Budget Referendums and Government Spending: Evidence from Swiss Cantons, mimeo.

Felderer, B., S. Homburg (1994), Makroökonomik und neue Makroökonomik, 6. Aufl., Berlin et al.

Feldstein, M. (1996), The Costs and Benefits of Going from Low Inflation to Price Stability, NBER Working Paper, No. 5469, Cambridge.

Ferejohn, J.A., M.P. Fiorina (1974), The Paradox of Not Voting: A Decision Theoretic Analysis, in: American Political Science Review 68, S. 525-536.

Fine, B. (1999), The Developmental State is Dead - Long Live Social Capital?, in: Development and Change 30, S. 1-19.

Fischer, S. (1977), Long-Term Contracts, Rational Expectations, and the Optimal Money Supply Rule, in: Journal of Political Economy 85, S. 191-206.

Frank, R.H. (1987), If Homo Oeconomicus Could Choose his Own Utility Function, Would He Want One with a Conscience?, in: American Economic Review 77, S. 593-604. 
Freeman, R.B., R. Schettkat (1999), Zwischen Fastfood und Excellence! Die Beschäftigungslücke in Deutschland im Vergleich zu den USA, in: Hamburger Jahrbuch für Wirtschafts- und Gesellschaftspolitik 44, Hamburg, S. 95-113.

Frey, B.S. (1976), Theorie und Empirie politischer Konjunkturzyklen, in: Zeitschrift für Nationalökonomie 36, S. 95-120.

Frey, B.S. (1978), Politico-Economic Models and Cycles, in: Journal of Public Economics 9, S. 203-220.

Frey, B.S. (1981), Theorie demokratischer Wirtschaftspolitik, München.

Frey, B.S. (1985), Internationale Politische Ökonomie, München.

Frey, B.S. (2000), Was bewirkt die Volkswirtschaftslehre?, in: Perspektiven der Wirtschaftspolitik 1, S. 5-33.

Frey, B.S., L.J. Lau (1968), Towards a Mathematical Model of Government Behaviour, in: Zeitschrift für Nationalökonomie 28, S. 355-380.

Frey, B.S., F. Schneider (1978a), An Empirical Study of Politico-Economic Interaction in the United States, in: Review of Economics and Statistics 60, S. 174-183.

Frey, B.S., F. Schneider (1978b), A Politico-Economic Model of the United Kingdom, in: The Economic Journal 88, S. 243-253.

Frey, B.S., F. Schneider (1979), An Econometric Model with an Endogenous Government Sector, in: Public Choice 34, S. 29-43.

Freytag, A., C.-P. Meier, P. Weiß (1998), Befund: Globalisierung in verschiedenen Facetten, in: J.B. Donges, A. Freytag [Hrsg.], Die Rolle des Staates in einer globalisierten Wirtschaft, Stuttgart, S. 9-36.

Friedman, M. (1953), Choice, Chance, and the Personal Distribution of Income, in: Journal of Political Economy 61, S. 277-290.

Friedman, M., A.J. Schwartz (1963), A Monetary History of the United States, 1867-1960, Princeton.

Fritsch, M., T. Wein, H.-J. Ewers (1993), Marktversagen und Wirtschaftspolitik. Mikroökonomische Grundlagen staatlichen Handelns, München.

Frohlich, N., J. Oppenheimer (1984), Beyond Economic Man, Altruism, Egalitarianism and Difference Maximizing, in: Journal of Conflict Resolution 28, S. 3-24.

Fuchs, V.R. (1994), Nobel Laureate Gary S. Becker: Ideas about Facts, in: Journal of Economic Perspectives 8, S. 183-192.

Fuchs, D., H.-D. Klingemann (1990), The Left-Right-Schema, in: M.K. Jennings, J.W. van Deth et al. [Hrsg.], Continuities in Political Action, Berlin, New York, S. 203-234.

Fudenberg, D., J. Tirole (1991), Game Theory Camebridge, London 
Fukuyama, F. (1992), Das Ende der Geschichte. Wo stehen wir?, München.

Fukuyama, F. (1995), Trust: The Social Virtues and the Creation of Prosperity, New York.

Furobotn, E.G., R. Richter (1994), The New Institutional Economics: Bounded Rationality and the Analysis of State and the Society, in: Journal of Institutional and Theoretical Economics 150, S. 11-17.

Gärtner, M. (1994), Democracy, Elections, and Macroeconomic Policy: Two Decades of Progress, in: European Journal of Political Economy 10, S. 85109.

Geißler, R. (1996), Politische Sozialisation in der Familie, in: B. Claußen, R. Geißler [Hrsg.], Die Politisierung des Menschen. Instanzen der politischen Sozialisation. Ein Handbuch, Opladen, S. 51-70.

Gesellschaft für deutsche Sprache, (2000), Gesellschaft für deutsche Sprache Online, http:///www.geist.de/gfds/Verlag-D.htm, Februar 2000.

Gibrat, R. (1931), Les inégalités économique, Paris.

Giddens, A. (1997), Jenseits von Links und Rechts - Die Zukunft radikaler Demokratie, Frankfurt a.M.

Gorvin, I. (1989), Elections since 1945: A Worldwide Reference Compendium, Chicago.

Gramlich, H. (1974), The Distributional Effects of Higher Unemployment, in: Brookings Papers on Economic Activity, S. 293-341.

Gramlich, H., D. Laren (1984), How Widespread are Income Losses in a Recession? in: D.L. Bawden [Hrsg.], The Social Contract Revisited, Washington D.C., S. 157-180.

Grill, B. (2000), Paranoia im Paradies, in: DIE ZEIT, Nr. 21 vom 18.05.2000.

Gross, D.A., L. Sigelman (1984), Comparing Party Systems - A Multidimensional Approach, in: Comparative Politics 16, S. 463-479.

Groys, B. (1993), Abschied vom Homogenen. Die Mehrheit der Minderheiten, in: Rotbuch-Verlag [Hrsg.], What's left? Prognosen zur Linken, Berlin, S. 60-65.

Grubel, H.G., P.J. Lloyd (1975), Intra Industry Trade: The Theory and Measurement of International Trade in Differentiated Products, London.

Grunberg, G., E. Schweisguth (1990), Libéralisme culturel et libéralisme économique, in: D. Boy, N. Mayer [Hrsg.], L'électeure francais en question, Paris, S. 46-55.

Grüske, K.-D. (1985), Personale Verteilung und Effizienz der Umverteilung Analyse und Synthese, Göttingen.

Gylfason, T., T.T. Herbertsson (1996), Does Inflation Matter for Growth? CEPR Discussion Paper No. 1503, London. 
Haber, R.A. (1968), The End of Ideology as Ideology, in: C.I. Waxman [Hrsg.], The End of Ideology Debate, New York, S. 182-206.

Habermas, J. (1998), Es gibt doch Alternativen, in: DIE ZEIT, 8.10.1998, S. 12-15.

Hansmeyer, K.H., B. Rürup (1975), Staatswirtschaftliche Planungsinstrumente, Tübingen, Düsseldorf.

Hanusch, H. (1976), Verteilung öffentlicher Leistungen. Eine Studie zur personalen Inzidenz, Göttingen.

Harbaugh, W.T. (1996), If People Vote because They Like to, Then Why Do So Many of Them Lie?, in: Public Choice 89, S. 63-76.

Harberger, A.C. (1962), The Incidence of the Corporation Income Tax, in: Journal of Political Economy 70, S. 215-240.

Hardin, G. (1968), The Tragedy of the Commons - The Population Problem Has No Technical Solution; It Requires a Fundamental Extension in Morality, in: Science 162, S. 1243-1248.

Harmel, R., K. Janda (1966), Comparing Political Parties, Empirical Teaching Units in Political Science, Washington D.C.

Harsanyi, J.C. (1955), Cardinal Welfare, Individualistic Ethics, and Interpersonal Comparison of Utility, in: Journal of Political Economy 63, S. 309-321.

Haslinger, $F$. (1997), Individuum und Verteilung in einer unsicheren Welt - Zur Rolle der Moral in der Ökonomik, in: M. Held [Hrsg.], Normative Grundfragen der Ökonomik: Folgen für die Theoriebildung, Frankfurt a.M., New York, S. 150-167.

Hasse, R.H. (1995), Globalisierung versus Protektionismus, Discussion Paper in Economic Policy Nr. 59 der Universität der Bundeswehr, Hamburg.

Hayek, F.A. von (1968), Der Wettbewerb als Entdeckungsverfahren, Kiel.

Haynes, S.E., J.E. Stone (1994), Political Parties and the Variable Duration of Business Cycles, in: Southern Economic Journal 60, S. 869-885.

Hechter, M. (1994), The Role of Values in Rational Choice Theory, in: Rationality and Society 6, S. 318-333.

Heiner, R.A. (1983), The Origin of Predictable Behaviour, in: American Economic Review 73, S. 560-595.

Held, M. (1991), "Die Ökonomik hat kein Menschenbild" - Institutionen, Normen, Menschenbild, in: B. Biervert, M. Held [Hrsg.], Das Menschenbild der ökonomischen Theorie: zur Natur des Menschen, Frankfurt a.M., New York, S. 1041.

Helliwell, J.F. (1996a), Economic Growth and Social Capital in Asia, NBER Working Paper No. 5470, Cambridge. 
Helliwell, J.F. (1996b), Do Borders Matter for Social Capital? Economic Growth and Civic Culture in U.S. States and Canadian Provinces, NBER Working Paper No. 5863, Cambridge.

Helliwell, J.F., R.D. Putnam (1995), Economic Growth and Social Capital in Italy, in: Eastern Economic Journal 21, S. 295-307.

Hendry, D.F. (1980), Econometrics - Alchemy or Science?, in: Economica 47, S. 387-406.

Hess, R.D., J.V. Torney (1966), The Development of Political Attitudes in Children, Chicago, 1966.

Heubes, J. (1991), Konjunktur und Wachstum, München.

Hey, J.D. (1991), Experiments in Economics, Oxford, Cambridge.

Hibbs, D.A. (1977), Political Parties and Macroeconomic Policy, in: American Political Science Review 71, S. 1467-1487.

Hibbs, D.A. (1979), Communication, in: American Political Science Review 73, S. 185-190.

Hibbs, D.A. (1987), The American Political Economy. Macroeconomics and Electoral Politics, Cambridge, London.

Hibbs, D.A. (1992), Partisan Theory after Fifteen Years, in: European Journal of Political Economy 8, S. 361-373.

Hibbs, D.A. (1994), The Partisan Model of Macroeconomic Cycles: More Theory and Evidence for the United States, in: Economics and Politics 6, S. 1-23.

Hirst, P., G. Thompson (1996), Globalization in Question, Cambridge.

Hochman, A., J. Rodgers (1969), Pareto Optimal Redistribution, in: American Economic Review 59, S. 542-557.

Hofferbert, R.I., H.-D. Klingemann (1990), The Policy-Impact of Party Programmes and Government Declarations in the Federal Republic of Germany, in: European Journal of Political Research 17, S. 277-304.

Hofferbert, R.I., H.-D. Klingemann, A. Volkens (1996), Election Programmes, Government Statements and Political Action - Political Parties and their Programmes, in: J. Thesing, W. Hofmeister [Hrsg.], Political Parties in Democracy, St. Augustin, Konrad-Adenauer-Stiftung, S. 321-331.

Holländer, H. (1990), A Social Exchange Approach to Voluntary Cooperation, in: American Economic Review 80, S. 1157-1167.

Hotelling, H. (1929), Stability in Competition, in: Economic Journal 39, S. 41-57.

Hübner, K. (1998), Der Globalisierungskomplex. Grenzenlose Ökonomie - grenzenlose Politik?, Berlin.

Hurrelmann, K. (1998), Einführung in die Sozialisationstheorie, 6. Aufl., Weinheim, Basel. 
IMF, (1993), World Economic Outlook, May 1993, Washington D.C.

$I M F$, (diverse Jahrgänge), Balance of Payments Yearbook, Washington D.C.

$I M F$, (diverse Jahrgänge), International Financial Statistics, Washington D.C.

Inglehart, $R$. (1971), The Silent Revolution in Europe: Intergenerational Change in Post Industrial Societies, in: American Political Science Review 65, S. 9911017.

Inglehart, $R$. (1977), The Silent Revolution: Changing Values and Political Styles Among Western Publics, Princeton.

Inglehart, $R$. (1990a), Culture Shift in Advanced Industrial Society, New Jersey.

Inglehart, $R$. (1990b), From Class Based to Value Based Politics, in: P. Mair [Hrsg.], The West European Party System, Oxford, S. 266-282.

Inglehart, R. (1990c), Political Value Orientations, in: M.K. Jennings, J.W. van Deth et al. [Hrsg.], Continuities in Political Action, Berlin, S. 67-102.

Inglehart, R., H.-D. Klingemann (1979), Ideological Conceptualization and Value Priorities, in: S.H. Barnes, M. Kaase et al. [Hrsg.], Political Action. Mass Participation in Five Western Democracies, London, S. 203-213.

Inglehart, R., H.-D. Klingemann (1996), Dimensionen des Wertewandels. Theoretische und methodische Relexionen anläßlich einer neuerlichen Kritik, in: Politische Vierteljahresschrift 37, S. 319-340.

International Parliamentary Union (1999), International Parliamentary Union Database, http://www.ipu.org/parline-e/parlinesearch.asp, 21.07.1999.

Ireland, T.R., D.B. Johnson (1970), The Economics of Charity, Blacksburg.

Isaac, R.M., J.M. Walker, S.H. Thomas (1984), Divergent Evidence on Free Riding: An Experimental Examination of Possible Explanations, in: Public Choice 43, S. 113-149.

Isaac, R.M., J.M. Walker, A.W. Williams (1994), Group Size and the Voluntary Provision of Public Goods, in: Journal of Public Economics 54, S. 1-36.

Jennings, M.K. (1990), The Crystallization of Orientations, in: M.K. Jennings, J.W. van Deth et al. [Hrsg.], Continuities in Political Action, Berlin, S. 313-348.

Jones, P., J. Hudson (2000), Civic Duty and Expressive Voting: Is Virtue its Own Reward?, in: Kyklos 53, S. 3-16.

Judge, G.G., et al. (1988), Introduction to the Theory and Practice of Econometrics, New York u.a.

Judson, R.A., A.L. Owen (1999), Estimating Dynamic Panel Data Models: A Guide for Macroeconomists, in: Economics Letters 65, S. 9-15.

Kahnemann, D., J.L. Knetsch, R.H. Thaler (1986a), Fairness and the Assumptions of Economics, in: Journal of Business 59, S. 285-300. 
Kahnemann, D., J.L. Knetsch, R. Thaler (1986b), Fairness as a Constraint on Profit Seeking: Entitlements in the Market, in: American Economic Review 76, S. 728-741.

Kalecki, M. (1943), Political Aspects of Full Employment, in: Political Quarterly 14, S. 322-331.

Kalt, J.P. (1981), The Economics and Politics of Oil Price Regulation, Cambridge.

Kalt, J.P. (1983), The Costs and Benefits of Federal Regulation of Coal Strip Mining, in: Natural Resources Journal 23, S. 893-915.

Kalt, J.P., M.A. Zupan (1984), Capture and Ideology in the Economic Theory of Politics, in: American Economic Review 74, S. 279-300.

Katterle, S. (1991), Methodologischer Individualismus and Beyond, in: B. Biervert, M. Held [Hrsg.], Das Menschenbild der ökonomischen Theorie: Zur Natur des Menschen, Frankfurt a.M., New York, S. 132-152.

Kau, J.B., P.H. Rubin (1979), Self-interest, Ideology, and Logrolling in Congressional Voting, in: Journal of Law and Economics 22, S. 365-384.

Kim, O., M. Walker (1984), The Free Rider Problem: Experimental Evidence, in: Public Choice 43, S. 3-24.

Kirchgässner, G. (1986), Rationale Erwartungen und ökonomische Theorie der Politik, in: Jahrbuch für Neue Politische Ökonomie 5, S. 103-121.

Kirchgässner, G. (1990), Hebt ein "knapper" Wahlausgang die Wahlbeteiligung?, in: M. Kaase, H.-D. Klingemann [Hrsg.], Wahlen und Wähler, Opladen, S. 445-477.

Kirchgässner, G. (1991), Homo Oeconomicus: Das ökonomische Modell individuellen Verhaltens und seine Anwendung in den Wirtschafts- und Sozialwissenschaften, Tübingen.

Kirchgässner, G. (2000), Wirtschaftliche Auswirkungen der direkten Demokratie, in: Perspektiven der Wirtschaftspolitik 2, S. 161-180.

Kirsch, G. (1991), Umweltbewußtsein und Umweltverhalten. Eine theoretische Skizze eines empirischen Problems, in: Zeitschrift für Umweltpolitik und Umweltrecht 3/91, S. 249-261.

Kirsch, G. (1994), Umweltmoral - Ein Ersatz für staatliche Umweltpolitik?, in: K. Mackscheidt, D. Ewringmann, E. Gawel [Hrsg.], Umweltpolitik mit hoheitlichen Zwangsabgaben?, Karl-Heinrich Hansmeyer zur Vollendung seines 65. Lebensjahres, Berlin, S. 261-271.

Kirsch, G. (1997), Neue Politische Ökonomie, 4. Aufl., Düsseldorf.

Klages, H. (1992), Die gegenwärtige Situation der Wert- und Wertwandelforschung - Probleme und Perspektiven, in: H. Klages, H.J. Hilpert, W. Herbert [Hrsg.], Werte und Wandel, Frankfurt a.M., New York, S. 5-39. 
Klingemann, H.-D. (1979), Measuring Ideological Conceptualizations, in: S.H. Barnes, M. Kaase et al. [Hrsg.], Political Action. Mass Participation in Five Western Democracies, Beverly Hills, S. 215-255.

Kluckhohn, C. (1951), Values and Value Orientation in the Theory of Action: An Exploration in Definition and Classification, in: T. Parsons, E. Shils [Hrsg.], Toward a General Theory of Action, Cambridge, S. 388-433.

Kmieciak, P. (1976), Wertstrukturen und Wertwandel in der Bundesrepublik Deutschland, Göttingen.

Knack, S., P. Keefer (1997), Does Social Capital have an Economic Payoff? A Cross-Country Investigation, in: The Quarterly Journal of Economics 112, S. 1251-1288.

Kohler-Koch, B., W. Woyke (1996), Die Europäische Union, in: D. Nohlen [Hrsg.], Lexikon der Politik, Band 5, München.

Krueger, A.B. (2000), From Bismarck to Maastricht: The March to European Union and the Labour Compact, in: Labour Economics 7, S. 117-134.

Krugman, P.R., M. Obstfeld (2000), International Economics. Theory and Policy, 5. ed., Reading.

Kubon-Gilke, G. (1995), Nützlichkeit versus Moral, in: Ökonomie und Gesellschaft Jahrbuch 11, Markt, Norm und Moral, Frankfurt a.M., New York, S. 210-239.

Külp, B. (1994), Verteilung - Theorie und Praxis, 3. Aufl., Stuttgart, Jena.

Kuran, T. (1990), Private and Public Preferences, in: Economics and Philosophy 6, S. 1-26.

Kydland F.E., E.C. Prescott (1982), Time to Build and Aggregate Fluctuations, in: Econometrica 50, S. 1345-1370.

Lächler, U. (1984), The Political Business Cycle under Rational Behaviour, in: Public Choice 44, S. 411-430.

Lancaster, K. (1971), Consumer Demand. A New Approach, New York, London.

Landmann, O. (1999), Die Globalisierung: Wachstumsmotor oder Jobkiller?, in Hamburger Jahrbuch für Wirtschafts- und Gesellschaftspolitik 44, Hamburg, S. 133-152.

LaPalombara, J. (1968), Decline of Ideology: Dissent and Interpretation, in: C.I. Waxman [Hrsg.], The End of Ideology Debate, New York, S. 315-342.

Laponce, J.A. (1981), Left and Right, Toronto.

Lautmann, R. (1969), Wert und Norm. Begriffsanalyse für die Soziologie, Opladen.

Laver, M.J., B.W. Hunt (1992), Policy and Party Competition, London.

Laver, M.J., M. Taylor (1973), Government Coalitions in Western Europe, in: European Journal of Political Research 1,TSbi237-2248.978-3-631-75139-8 
Ledyard, J.O. (1995), Public Goods: A Survey of Experimental Research, in: J.H. Kagel, A.E. Roth [Hrsg.], The Handbook of Experimental Economics, New Jersey, S. 111-194.

Lehner, F. (1979), Die "stille Revolution": Zur Theorie und Realität des Wertwandels, in: H. Klages, P. Kmieciak [Hrsg.], Wertwandel und gesellschaftlicher Wandel, Frankfurt a.M., New York, S. 317-327.

Li, H., L. Squire, H. Zou (1998), Explaining International and Intertemporal Variations in Income Inequality, in: The Economic Journal 108, S. 26-43.

Lijphart, A. (1984), Democracies, New Haven and London.

Lijphart, A. (1990), Dimensions of Ideology in European Party Systems, in: P. Mair [Hrsg.], The West European Party Systems, Oxford, S. 253-265.

Lipset, S.M., P.F. Lazarsfeld, A.H. Barton, J. Linz (1954), The Psychology of Voting: An Analysis of Voting Behavior, in: G. Lindzey [Hrsg.], Handbook of Social Psychology, Vol. 2, Reading.

Lipset, S.M., S. Rokkan (1967), Cleavage Structures, Party Systems and Voter Alignments, in: S.M. Lipset, S. Rokkan [Hrsg.], Party Systems and Voter Alignments, New York, S. 1-64.

Litz, H.P. (1999), Nationale und supranationale Konzepte der Messung der Arbeitslosigkeit und Probleme des internationalen Vergleichs, in: W. von Bülow et al. [Hrsg.], Globalisierung und Wirtschaftspolitik, Marburg, S. 159-182.

López, E.J., C.D. Ramirez (2000), Is Political Ideology Endogenous? Evidence from the Business Cycle, University of North Texas Working Paper No. 0005 .

Lott, J.R. (1990), An Explanation for Public Provision of Schooling: The Importance of Indoctrination, in: Journal of Law and Economics 33, S. 199-231.

Loury, G. (1977), A Dynamic Theory of Racial Income Differences, in: P.A. Wallace, A. Le Mund [Hrsg.], Women, Minorities and Employment Discrimination, Lexington.

Loury, G. (1987), Why Should We Care About Group Inequality, in: Social Philosophy and Policy 5, S. 249-271.

Lucas, R. E. (1973), Some International Evidence on Output-Inflation Tradeoffs, in: American Economic Review 63, S. 326-334.

Maag, G. (1991), Gesellschaftliche Werte - Strukturen, Stabilität, Funktion, Opladen.

Mackie, T.T., R. Rose (1974), The International Almanac of Electoral History, New York et al.

Mackie, T.T., R. Rose (1997), A Decade of Election Results: Updating the International Almanac, Glasgow. 
MacRae, C.D. (1977), A Political Model of the Business Cycle, in: Journal of Political Economy 85, S. 239-263.

Maennig, W., B. Wilfling (1998), Außenwirtschaft, Theorie und Praxis, München.

Magee, S.P. (1980), Three Simple Tests of the Stolper-Samuelson-Theorem, in: P. Oppenheimer [Hrsg.], Issues in International Economics, Stocksfield, S. 138153.

Magee, S.P., W.A. Brock (1983), A Model of Politics, Tariffs and Rent-Seeking, in: B. Weisbrod, H. Hughes [Hrsg.]. Human Resources, Employment and Development, Vol. 3, London, S. 497-523.

Mankiw, G.N. (1999), Macroeconomics, 4. ed., New York.

Mannheim, K. (1955), Ideology and Utopia, New York.

Margolis, H. (1981), A New Model of Rational Choice, in: Ethics 91, S. 265-279.

Margolis, H. (1982), Selfishness, Altruism and Rationality: A Theory of Social Change, Cambridge.

Marshall, A. (1962), Principles of Economics, 8. ed., London.

Marshall, T.H. (1950), Citizenship and Social Class, Cambridge.

Martin, H.-P., J. Schumann (1997), Die Globalisierungsfalle. Der Angriff auf Demokratie und Wohlstand, Reinbek.

Marwell, G., R.E. Ames (1981), Economists Free Ride. Does Anyone Else?, in: Journal of Public Economics 15, S. 295-310.

Maslow, A.H. (1981), Motivation und Persönlichkeit, Reinbek.

Mayer, N., P. Perrineau (1992), Les comportements politiques, Paris.

McCallum, B.T. (1978), The Political Business Cycle: An Empirical Test, in: Southern Economic Journal 44, S. 504-515.

Meckling, W.H. (1976), Values and the Choice of the Model of the Individual in the Social Sciences, in: Schweizerische Zeitschrift für Volkswirtschaft und Statistik 112, S. 545-560.

Michael, R.T., G.S. Becker (1973), On the New Theory of Consumer Behaviour, in: Swedish Journal of Economics 75, S. 378-395.

Minford, P., D. Peel (1982), The Political Theory of the Business Cycle, in: European Economic Review 17, S. 253-270.

Moore, B.J. (1989), The Effects of Monetary Policy on Income Distribution, in: P. Davidson, J. Kregel [Hrsg.], Macroeconomic Problems and Policies of Income Distribution. Functional, Personal, International, Aldershot, S. 18-41.

Mueller, D.C. (1986), Rational Egoism versus Adaptive Egoism as Fundamental Postulate for a Descriptive Theory of Human Behavior, in: Public Choice 51, S. 3-23. 
Mueller, D.C. (1989), Public Choice II, Cambridge.

Murck, M. (1982), Voraussetzungen und Methoden planungsrelevanter Bedürfnisforschung, in: H. Stachowiak et al. [Hrsg.], Bedürfnisse, Werte und Normen im Wandel, Band II, Methoden und Analysen, München et al., S. 131-162.

Musgrave, R.A. (1959), The Theory of Public Finance, New York.

Muth, J.F. (1961), Rational Expectation and the Theory of Price Movements, in: Econometrica 29, S. 315-335.

Nannestad, P., M. Paldam (1994), The VP-function: A Survey of the Literature on Vote and Popularity Functions after 25 Years, in: Public Choice 79, S. 213244.

Naßmacher, H. (1994), Parteiorganisation, Parteiprogramme und Strukturen innerparteilicher Willensbildung, in: O.W. Gabriel, F. Brettschneider [Hrsg.], Die EU-Staaten im Vergleich - Strukturen, Prozesse, Politikinhalte, 2. Aufl., Opladen, S. 221-258.

Neumann, M.J.M. (1989), Discussion, in: Economic Policy 8, S. 90-92.

Nickell, S. (1981), Biases in Dynamic Models with Fixed Effects, in: Econometrica 49, S. 1417-1426.

Niskanen, W.A. (1971), Bureaucracy and Representative Government, Chicago.

Niskanen, W.A. (1975), Bureaucrats and Politicians, in: Journal of Law and Economics 18, S. 617-643.

Noelle-Neumann, E. (1980), Die Schweigespirale. Öffentliche Meinung - unsere soziale Haut, München, Zürich.

Noll, R.G. (1989), Economic Perspectives on the Politics of Regulation, in: R. Schmalensee, R.D. Willig [Hrsg.], Handbook of Industrial Organization, Vol. II, New York, S. 1253-1287.

Nordhaus, W.D. (1975), The Political Business Cycle, in: Review of Economic Studies 42, S. 169-190.

North, D.C. (1988), Theorie des institutionellen Wandels, Tübingen.

North, D.C. (1992), Institutionen, institutioneller Wandel und Wirtschaftsleistung, Tübingen.

Obstfeld, M. (1998), The Global Capital Market: Benefactor or Menace, in: Journal of Economic Perspectives 12, S. 9-30.

Ockenfels, A., Weimann, J. (1999), Types and Patterns: An Experimental EastWest-German Comparison of Cooperation and Solidarity, in: Journal of Public Economics 71, S. 275-287.

OECD (1996), Globalization of Industry. Overview and Sector Reports, Paris. OECD (1999), National Accounts, Vol. 1, Paris. 
OECD (diverse Jahrgänge), Main Economic Indicators, Paris.

Okun, A. (1962), Potential GNP: Its Measurement and Significance, American Statistical Association, Proceedings of the Business and Economics Statistics Section.

Olson, M. (1965), The Logic of Collective Action, Cambridge.

O'Rourke, K.H., J.G. Williamson (1999), Globalization and History. The Evolution of a 19th Century Atlantic Economy, Cambridge.

Paldam, M. (1979), Is there an Electional Cycle? A Comparative Study of National Accounts, in: Scandinavian Journal of Economics 81, S. 201-220.

Paldam, M. (1989a), Politics Matters After All (2) Testing Hibbs' Theory of Partisan Cycles on Data for 17 Countries, Memo 1989-8, Aarhus (DK).

Paldam, M. (1989b), Politics matters after all (1) Testing Alesina's Theory of RE Partisan Cycles on Data for 17 Countries, Memo, 1989-6, Aarhus (DK).

Paldam, M. (1997), Political Business Cycles, in: D.C. Mueller [Hrsg.], Perspectives on Public Choice: A Handbook, Cambridge.

Pappi, F.U. (1987), Klassenstruktur und Wahlverhalten im sozialen Wandel, in: M. Kaase, H.-D. Klingemann [Hrsg.], Wahlen und Wähler. Analysen aus Anlass der Bundestagswahl 1987, S. 15-30.

Pawelka, P. (1977), Politische Sozialisation, Wiesbaden.

Payne, J.L. (1979), Inflation, Unemployment, and Left-Wing Political Parties: A Reanalysis, in: American Political Science Review 73, S. 181-185.

Peltzman, S. (1976), Towards a More General Theory of Regulation?, in: Journal of Law and Economics 19, S. 211-240.

Peltzman, S. (1984), Constituent Interest and Congressional Voting, in: Journal of Law and Economics 27, S. 181-210.

Persson, T., G. Tabellini (1990), Macroeconomic Policy, Credibility and Politics, Chur.

Pommerehne, W.W., H. Weck-Hannemann (1996), Tax Rates, Tax Administration and Income Tax Evasion in Switzerland, in: Public Choice 88, S. 161-170.

Poole, K.T., R.S. Daniels (1985), Ideology, Party, and Voting in the U.S. Congress, in: American Political Science Review 79, S. 373-399.

Poole, K.T., H. Rosenthal (1991), Patterns of Congressional Voting, in: American Journal of Political Science 35, S. 228-278.

Posner, R.A. (1973), Economic Analysis of Law, Boston.

Prätorius, R. (1987), Ideologie, in: A. Görlitz, R. Prätorius [Hrsg.], Handbuch Politikwissenschaft, Grundlagen - Forschungsstand - Perspektiven, Reinbek, S. 139-144. 
Pressac, P. de (1931), Réponse à l'enquête. Qu'appelez-vous droite et gauche? Sous la direction de Emmanuel Beau de Loménie, Paris.

Putnam, R.D. (1993), Making Democracy Work, Princeton.

Putnam, R.D. (1995), Bowling Alone. America's Declining Social Capital, in: Journal of Democracy 6, S. 65-78.

Quinn, D.P., C. Inclan (1997), The Origins of Financial Openness: A Study of Current and Capital Account Liberalization, in: American Journal of Political Science 41, S. 771-813.

Ramanathan, $R$. (1992), Introductory Econometrics, 2. ed., Fort Worth et al.

Rawls, J.A. (1971), A Theory of Justice, Cambridge.

Reichart-Dreyer, I. (1977), Werte-Systeme-Programme: Modell, Praxis und Alternativen politischer Willensbildung und Programmatik entwickelt am Beispiel der CDU, Bonn.

Rejai, M. (1991), Political Ideologies - A Comparative Approach, London.

Riker, W.H., P.C. Ordeshook (1968), A Theory of the Calculus of Voting, in: American Political Science Review 62, S. 25-42.

Ritter, H. (1993), Einkehr zur Weltfremdheit. Hat die Linke noch eine Zukunft?, in: Rotbuch-Verlag [Hrsg.], What's left? Prognosen zur Linken, Berlin, S. 41-47.

Rodrik, D. (1997), Has Globalization Gone Too Far?, Institute for International Economics, Washington D.C.

Rogoff, K. (1990), Equilibrium Political Budget Cycles, in: American Economic Review 80, S. 21-36.

Rogoff, K., A. Sibert (1988), Elections and Macroeconomic Policy Cycles, in: Review of Economic Studies 55, S. 1-16.

Rose, R. (1984), Do Parties Make a Difference, London.

Roubini, N., J.D. Sachs (1989), Political and Economic Determinants of Budget Deficits in the Industrial Democracies, in: European Economic Review 33, S. 903-938.

Sachverständigenrat zur Begutachtung der gesamtwirtschaftlichen Entwicklung (1998), Vor weitreichenden Entscheidungen, Jahresgutachten 1998/1999, Stuttgart.

Samuelson, P.A. (1938), A Note on the Pure Theory of Consumer's Behaviour, in: Economica 5, S. 61-71.

Sargent, T.J., N. Wallace (1975), "Rational" Expectations, the Optimal Money Instrument, and the Optimal Money Supply Rule, in: Journal of Political Economy 83, S. 241-254.

Sartori, G. (1976), Parties and Party Systems: A Framework for Analysis, Vol. I, Cambridge. 
Schäfer, W. (1999), Globalisierung: Entmonopolisierung des Nationalen?, in: H. Berg et al. [Hrsg.], Globalisierung der Wirtschaft: Ursachen - Formen Konsequenzen, Berlin, S. 11-21.

Schäfer, W. (2000), Zukunftsperspektiven des europäischen Modells der Sozialen Marktwirtschaft, in: List Forum für Wirtschafts- und Finanzpolitik 26, S. 121132.

Scharpf, F.W. (1997), Globalisierung als Beschränkung der Handlungsmöglichkeiten nationalstaatlicher Politik, Max Planck Institut für Gesellschaftsforschung, Discussion Paper 97/1, Köln.

Schenk, M. (1994), Strukturelle Entwicklung der Staatseinnahmen - Eine theoretische und empirische Analyse aus politisch-institutioneller Sicht, in: K.-D. Grüske [Hrsg.], Forum Finanzwissenschaft Band 9, Nürnberg.

Scherf, $H$. (1986), Enttäuschte Hoffnungen - vergebene Chancen. Die Wirtschaftspolitik der Sozial-Liberalen Koalition 1969-1982, Göttingen.

Schlittgen, $R$. (1993), Einführung in die Statistik. Analyse und Modellierung von Daten, 4. Aufl., München, Wien.

Schmidt, M.G. (1980), CDU und SPD an der Regierung. Ein Vergleich ihrer Politik in den Ländern. Frankfurt a.M.

Schmidt, M.G. (1995), The-Parties-Do-Matter Hypothesis and the Case of the Federal Republic of Germany, in: German Politics 4, S. 1-21.

Schmidt, M.G. (1996), When Parties Matter: A Review of the Possibilities and Limits of Partisan Influence on Public Policy, in: European Journal of Political Research 30, S. 155-183.

Schmitt, H., J. Hofrichter (1992), One or Two Ideological Dimensions? On the Relationship of New Politics and Left-Right-Orientations in Western Europe, in: H. Klages, H.J. Hippler, W. Herbert [Hrsg.], Werte und Wandel, Frankfurt a.M., New York, S. 187-207.

Schneider, F., W.W. Pommerehne (1981), Free Riding and Collective Action: An Experiment in Public Microeconomics, in: Quarterly Journal of Economics 96, S. 689-704.

Schotter, A. (1983), Selfishness, Altruism and Rationality: A Theory of Social Change by Howard Margolis, Book Review, in: Journal of Economic Literature 21, S. 555-557.

Schuknecht, L. (1996), Political Business Cycles and Fiscal Policies in Developing Countries, in: Kyklos 49, S. 155-170.

Schumann, J. (1992), Grundzüge der mikroökonomischen Theorie, 6. Aufl., Berlin et al.

Schumpeter, J.A. (1908), Das Wesen und der Hauptinhalt der theoretischen Nationalökonomie, Leipzig. 
Schumpeter, J.A. (1950), Kapitalismus, Sozialismus und Demokratie, 2. Aufl., Bern.

Scott, A. (1997), The Limits of Globalization. Cases and Arguments, New York.

Sell, F.L. (1999), Anforderungen an immobile Produktionsfaktoren vor dem Hintergrund der Globalisierung, in: H. Berg [Hrsg.], Globalisierung der Wirtschaft: Ursachen - Formen - Konsequenzen, Berlin, S. 69-101.

Selten, $R$. (1971), Anwendung der Spieltheorie auf die politische Wissenschaft, in: H. Maier, K. Ritter, U. Matz [Hrsg.], Politik und Wissenschaft, München, S. 287-320.

Sen, A.K. (1973), Behaviour and the Concept of Performance, in: Economica 40, S. 241-259.

Sen, A.K. (1977), Rational Fools: A Critique of The Behavioural Foundations of Economic Theory, in: Philosophy and Public Affairs 6, S. 317-344.

Sen, A.K. (1987), On Ethics and Economics, Oxford.

Sheffrin, S.M. (1989), Evaluating Rational Partisan Business Cycle Theory, in: Economics and Politics 1, S. 239-259.

Siebenhüner, B. (1997), Vom homo oeconomicus zum homo oecologicus - Zum Menschenbild der ökologischen Ökonomik, in: F. Biermann, S. Büttner, C. Helm [Hrsg.], Zukunftsfähige Entwicklung, Herausforderungen an Wissenschaft und Politik, Festschrift für Udo E. Simonis, Berlin.

Siebert, H., M.J. Koop (1990), Institutional Competition. A Concept for Europe?, Kiel Working Paper No. 440, Kiel.

Siebert, H., M. Rauscher (1991), Neuere Entwicklungen der Außenhandelstheorie, in: WiSt 20, S. 503-509.

Siebke, J. (1992), Verteilung, in: D. Bender et al. [Hrsg.], Vahlens Kompendium der Wirtschaftstheorie und Wirtschaftspolitik Band 1, München, S. 383-415.

Simon, H.A. (1955), A Behavioural Model of Rational Choice, in: Quarterly Journal of Economics 69, S. 99-118.

Simon, H.A. (1957), Models of Man, New York.

Simon, H.A. (1995), Rationality in Political Behavior, in: Political Psychology 16, S. 99-118.

Sinn, H.-W. (1990), Tax Harmonization and Tax Competition in Europe, in: European Economic Review 34, S. 489-504.

Smith, A. (1976a [1776]), An Inquiry into the Nature and Causes of the Wealth of Nations, wiederabgedruck in: R.H. Campbell, A.S. Skinner [Hrsg.], The Glasgow Edition of the Works and Correspondence of Adam Smith, Vol. II, Oxford. 
Smith, A. (1976b [1759]), The Theory of Moral Sentiments, London, wiederabgedruckt in: D.D. Raphael, A.L. Macfie [Hrsg.], The Glasgow Edition of the Works and Correspondence of Adam Smith, Vol. I, Oxford.

Smith, V., M. Kehoe, M. Cremer (1995), The Private Provision of Public Goods: Altruism and Voluntary Giving, in: Journal of Public Economics 58, S. 107. 126.

Snowdon, B., H.R. Vane (1997), Politics and the Macroeconomy: Endogenous Politicians and Aggregate Instability, in: B. Snowdon, H.R. Vane [Hrsg.], Reflections on the Development of Modern Macroeconomics, Cheltenham.

Söllner, F. (1998), Geht Homo Oeconomicus zur Wahl?, in: WiSt 27, S. 14-19.

Stalder, I. (1995), Staatsverschuldung in der Demokratie. Eine polit-ökonomische Analyse, Frankfurt a.M. et al.

Statistisches Bundesamt, (1999), Statistisches Jahrbuch für die Bundesrepublik Deutschland 1999, Stuttgart, Mainz.

Stigler, G.J. (1961), The Economics of Information, in: Journal of Political Economy 69, S. 213-225

Stigler, G.J. (1971), The Theory of Regulation, in: Bell Journal of Economics and Management Science 2, S. 3-21.

Stigler, G.J. (1976), Do Economists Matter, in: Southern Economic Journal 42, S. 347-354.

Stigler, G.J., G.S. Becker (1977), De Gustibus Non Est Disputandum, in: American Economic Review 67, S. 76-90.

Stiglitz, J. (1998), The Private Use of Public Interests: Incentives and Institutions, in: Journal of Economic Perspectives 12, S. 3-22.

Stokes, D.E. (1963), Spatial Models of Party Competition, in: American Political Science Review 57, S. 368-377.

Stolz, I. (1983), Die Einkommensumverteilung in der Bundesrepublik Deutschland, Frankfurt a.M.

Straubhaar, T. (1998), Empirische Indikatoren für den Systemwettbewerb - Moderne und historische Befunde, in: Jahrbuch für Neue Politische Ökonomie 17, S. 243-272.

Sugden, $R$. (1989), Spontaneous Order, in: Journal of Economic Perspectives 3, S. 85-97.

Swank, O.H. (1998), Towards an Economic Theory of Party Ideology, in: European Journal of Political Economy 14, S. 223-240.

Taylor, C. (1996), Quellen des Selbst. Die Entstehung der neuzeitlichen Identität, Berlin. 
Taylor, M. (1983), Selfishness, Altruism and Rationality: A Theory of Social Change by Howard Margolis, Book Review, in: Ethics 93, S. 150-152.

Teichmann, U. (1976), Grundriss der Konjunkturpolitik, München.

Theurl, T. (1999), Globalisierung als Selektionsprozeß ordnungspolitischer Paradigmen, in: H. Berg et al. [Hrsg.], Globalisierung der Wirtschaft: Ursachen Formen - Konsequenzen, Berlin, S. 23-49.

Thome, H. (1985), Wertewandel in der Politik? Eine Auseinandersetzung mit Inglehart's Thesen zum Postmaterialismus, Berlin.

Thorbecke, W. (1995), The Distributional Effects of Disinflationary Monetary Policy, George Mason University Working Paper No. 144, Fairfax.

Thurow, L.W. (1971), The Income Distribution as a Pure Public Good, in: Quarterly Journal of Economics 85, S. 327-336.

Tiebout, C.M. (1956), A Pure Theory of Local Expenditure, in: Journal of Political Economy 64, S. 416-424.

Tietz, R. (1996), Experimentelle Wirtschaftsforschung - Wege zur Modellierung eingeschränkter Rationalität, in: Ökonomie und Gesellschaft 13, Experiments in Economics, Frankfurt a.M., S. 120-155.

Tietzel, M. (1981), Die Rationalitätsannahme in den Wirtschaftswissenschaften oder: Der homo oeconomicus und seine Verwandten, in: Jahrbuch für Sozialwissenschaft 32, S. 115-138.

Tobin, J. (1972), Inflation and Unemployment, in: American Economic Review 62, S. 1-18.

Torre, A. de la, M.R. Kelly (1992), Regional Trade Arrangements, Washington D.C.

Tullock, G. (1974), The Social Dilemma. Economics of War and Revolution, Blacksburg.

U.S. Department of Commerce, (1998), The Emerging Digital Economy. Report to the President, Washington, D.C.

Valois, G. (1931), Réponse à l'enquête. Qu'appelez-vous droite et gauche? Sous la direction de Emmanuel Beau de Loménie, Paris S. 39-45.

Vosgerau, H.J. (1978), Konjunkturtheorie, in: Handwörterbuch der Wirtschaftswissenschaften, Band 4, Stuttgart, S. 478-507.

Wagner, A. (1893), Grundlegung der politischen Ökonomie, Theil I: Grundlagen der Volkswirtschaft, 2. Halbband, 3. Aufl., Leipzig.

Wagner, H. (1996), Stabilitätspolitik. Theoretische Grundlagen und institutionelle Alternativen, 3. Aufl., München, Wien.

Wagschal, U. (1996), Staatsverschuldung - Ursachen im internationalen Vergleich, Opladen. 
Wartenberg, U. (1979), Verteilungswirkungen staatlicher Aktivitäten. Ein Beitrag zur Untersuchung der personellen Budgetinzidenz, Berlin.

Weimann, J. (1995), Freifahrer im Test: - Ein Überblick über 20 Jahre Freifahrerexperimente, in: Ökonomie und Gesellschaft 12, Soziale Kooperation, Frankfurt a.M., New York, S. 168-241.

Weise, P. (1995), Moral: Die Handlungsbeschränkung für den Deppen?, in: E.K. Seifert, B.P. Priddat [Hrsg.], Neuorientierungen in der ökonomischen Theorie. Zur moralischen, institutionellen und evolutorischen Dimension des Wirtschaftens, Marburg, S. 71-105.

Weizsäcker, C.C. von (1971), Notes on Endogenous Change of Tastes, in: Journal of Economic Theory 3, S. 345-372.

Weizsäcker, C.C von (1999), Logik der Globalisierung, Göttingen.

Weltbank, (1995), Weltentwicklungsbericht, Washington, D.C.

Wheeler, M., S. Pozo (1997), Is the World Economy More Integrated Today Than a Century Ago, in: American Economic Journal 25, S. 139-154.

Wiehn, E.R. (1972), Individuelle Bedürfnisse und soziale Werte. Zur Konstitution sozioindividuellen Verhaltens, Tübingen.

Wilensky, H.L. (1975), The Welfare State and Equality. Structural and Ideological Roots of Public Expenditure, Berkeley.

Willgerodt, $H$. (1998), Neue Kontrollen für den internationalen Kapitalverkehr?, in: J.B. Donges, A. Freytag [Hrsg.], Die Rolle des Staates in einer globalisierten Wirtschaft, Stuttgart, S. 119-171.

Williamson, O. (1990), Die ökonomischen Institutionen des Kapitalismus, Tübingen.

Wilson, R.K., J. Sell (1990), "Liar, Liar, Pants on Fire": Cheap Talk and Signaling in Repeated Public Good Settings, Texas A\&M University, Discussion Paper.

Winden, F. van, R. Bosman (1996), Experimental Research in Public Eco-nomics, in: Ökonomie und Gesellschaft 13, Experiments in Economics, Frankfurt a.M.

Wittman, D. (1983), Candidate Motivation: A Synthesis of Alternative Theories, in: American Political Science Review 77, S. 142-157.

Wolf, C. (1993), Markets or Government. Choosing between Imperfect Alternatives, 2. ed., Cambridge, London.

Woll, A. (1990), Allgemeine Volkswirtschaftslehre, 10. Aufl., München.

Woll, $H$. (1994), Menschenbilder in der Ökonomie, München.

Woolcock, M. (1998), Social Capital and Economic Development: Toward a Theoretical Synthesis and Policy Framework, in: Theory and Society 27, S. 151-208. 
WTO (2000), GATT-Members, http://www.wto.org/wto/about/gattmem.htm, 29.05.2000.

Zängle, M. (1978), Einführung in die politische Sozialisationsforschung, Paderborn.

Zevin, R.B. (1992), Are World Financial Markets More Open? If so, Why and With What Effects?, in: T. Banuri, J.B. Schor [Hrsg.], Financial Openness and National Autonomy. Opportunities and Constraints, Oxford.

Zimmermann, $K$. (1985), Umweltpolitik und Verteilung. Eine Analyse der Verteilungswirkungen des öffentlichen Gutes Umwelt, Berlin.

Zimmermann, K.W., T. Just, (2000), Interest Groups, Referenda, and the Political Process: On the Efficiency of Direct Democracy, in: Constitutional Political Economy 11, S. 147-163.

Zimmermann, K.W., F.G. Müller (1985), Umweltschutz als neue Aufgabe, Substitutionseffekte in öffentlichen Budgets, Frankfurt a.M., New York. 


\title{
SCHRIFTEN ZUR WIRTSCHAFTSTHEORIE UND WIRTSCHAFTSPOLITIK
}

\author{
Herausgegeben von Rolf Hasse, Jörn Kruse, Wolf Schäfer,
} Thomas Straubhaar, Klaus W. Zimmermann

Band 1 Lars Bünning: Die Konvergenzkriterien des Maastricht-Vertrages unter besonderer Berücksichtigung ihrer Konsistenz. 1997.

Band 2 Andreas Henning: Beveridge-Kurve, Lohnsetzung und Langzeitarbeitslosigkeit. Eine theoretische Untersuchung unter Berücksichtigung des Insider-Outsider-Ansatzes und der Entwertung des Humankapitals. 1997.

Band 3 Iris Henning: Die Reputation einer Zentralbank. Eine theoretische Untersuchung unter besonderer Berücksichtigung der Europäischen Zentralbank. 1997.

Band 4 Rüdiger Hermann: Ein gemeinsamer Markt für Elektrizität in Europa. Optionen einer Wettbewerbsordnung zwischen Anspruch und Wirklichkeit. 1997.

Band 5 Alexander Tiedtke: Japan und der Vorwurf des Trittbrettfahrerverhaltens in der US-amerikanisch-japanischen Allianz. 1997.

Band 6 Wolfgang Grimme: Ordnungspolitisches Konzept der Regionalpolitik. Darstellung der Defizite und des Reformbedarfs der Regionalpolitik am Beispiel Mecklenburg-Vorpommerns. 1997.

Band 7 Christian Ricken: Determinanten der Effektivität der Umweltpolitik. Der nationale Politikstil im Spannungsfeld von Ökonomie, Politik und Kultur. 1997.

Band 8 Christian Schmidt: Real Convergence in the European Union. An Empirical Analysis. 1997.

Band 9 Silvia Marengo: Exchange Rate Policy for MERCOSUR: Lessons from the European Union. 1998.

Band 10 Jens Kleinemeyer: Standardisierung zwischen Kooperation und Wettbewerb. Eine spieltheoretische Betrachtung. 1998.

Band 11 Stefan M. Golder: Migration und Arbeitsmarkt. Eine empirische Analyse der Performance von Ausländem in der Schweiz. 1999.

Band 12 Stefan Kramer: Die Wirkung einer Internationalisierung des Yen auf die japanischen Finanzmärkte, die japanische Geldpolitik und die Usancen der Fakturierung. 1999.

Band 13 Antje Marielle Gerhold: Wirtschaftliche Integration und Kooperation im asiatisch-pazifischen Raum. Die APEC. 1999.

Band 14 Tamim Achim Dawar. Deutsche Direktinvestitionen in Australien. Eine Evaluation der theoretischen Erklärungsansätze und der Standortattraktivität des Produktions- und Investitionsstandortes Australien. 1999.

Band 15 Hans-Markus Johannsen: Die ordnungspolitische Haltung Frankreichs im Prozeß der europäischen Einigung. 1999.

Band 16 Annette Schönherr. Vereinigungsbedingte Dimensionen regionaler Arbeitsmobilität. Wirkungen unter analytisch einfachen Bedingungen und potentielle individuelle Migrationsgewinne in Deutschland nach der Vereinigung. 1999.

Band 17 Henrik Müller. Wechselkurspolitik des Eurolandes. Konfliktstoff für die neue währungspolitische Ära. 1999.

Band 18 Lars H. Wengorz: Die Bedeutung von Untemehmertum und Eigentum für die Existenz von Unternehmen. Eine methodenkritische Analyse der Transformation des Wirtschaftssystems in Russland. 2000.

Band 19 Eckart Bauer: Konzeptionelle Grundfragen eines Kinderleistungsausgleichs im Rahmen einer umlagefinanzierten zwangsweisen Rentenversicherung. 2000. 
Band 20 Hubertus Hille: Enlarging the European Union. A Computable General Equilibrium Assessment of Different Integration Scenarios of Central and Eastern Europe. 2001.

Band 21 Tobias Just: Globalisierung und Ideologie. Eine Analyse der Existenz und Persistenz von Partisaneffekten bei zunehmender Internationalisierung der Märkte. 2001. 


\section{Ali Sait Yüksel \\ Welthandelsorganisation WTO (GATT)}

\section{Aufgaben / Aktivitäten / EU-Beziehungen Ein Hand- und Lehrbuch}

Frankfurt/M., Berlin, Bern, Bruxelles, New York, Oxford, Wien, 2001. IV, 300 S., zahlr. Tab. u. Graf.

ISBN 3-631-37865-3 · br. DM 49.-*

Die WTO ist die einzige internationale Wirtschaftsinstitution, deren Entscheidungen mit Sanktionen völkerrechtlich bindende Wirkung haben und selbst von den großen Wirtschaftsmächten der Welt akzeptiert werden. Deshalb zählt sie zu den mächtigsten Akteuren auf der internationalen Bühne. Der krasse Wandel durch die Neuordnung des GATT-Systems in der Weltwirtschaft ist ebenso unüberschaubar wie die Tatsache, dass die Welt von einem freien Warenaustausch noch weit entfernt ist. Industriestaaten verteuern nicht nur agrarische Importgüter, auch für Industriegüterimporte werden zusätzliche Handelshemmnisse ausgedacht. Der Multilateralismus erweist sich als positiv bezüglich des Interessenschutzes wirtschaftlich schwächerer Länder, trotz der derzeitigen strukturellen Krise. Der WTO wird deshalb in der zukünftigen Architektur der Weltwirtschaft eine tragende Funktion zugewiesen. Alle Probleme der "wilden Globalisierung" unmittelbar der WTO anzulasten, ändert nichts an der Tatsache, dass sie heute wichtigster Bestandteil der Weltwirtschaftsordnung geworden ist. Die am Export orientierten Unternehmen müssen das internationale Marketingumfeld, insbesondere das internationale Handelssystem verstehen können. Der Freihandel bringt denjenigen Wirtschaften, die sich auf dem gesunden Wege eines Strukturwandels befinden, wirtschaftliche Vorteile. Sein Beitrag zum dauerhaften Weltfrieden ist jedoch noch wichtiger.

Aus dem Inhalt. WTO - Weltwirtschaft - Europäische Union · Freihandel - Protektionismus · Handel und Umwelt · Handel und Wettbewerb - Internationale Wirtschaftsstandards · Internationale Korruption und Geldwäsche · Entwicklungsländer $\cdot$ Handelshemmnisse

FrankfurtM - Berlin - Bern - Bruxelles - New York - Oxford - Wien

Auslieferung: Verlag Peter Lang AG

Jupiterstr. 15, CH-3000 Bern 15

Telefax (004131) 9402131

*inklusive Mehrwertsteuer

Preisänderungen vorbehalten

Homepage http://umw.peterlang.de 
Tobias Just - 978-3-631-75139-8

Downloaded from PubFactory at 01/11/2019 07:52:01AM

via free access 